\title{
Water-Quality, Streambed-Sediment, and Biological Data from the Clark Fork-Pend Oreille and Spokane River Basins, Montana, Idaho, and Washington, 1998-2001
}

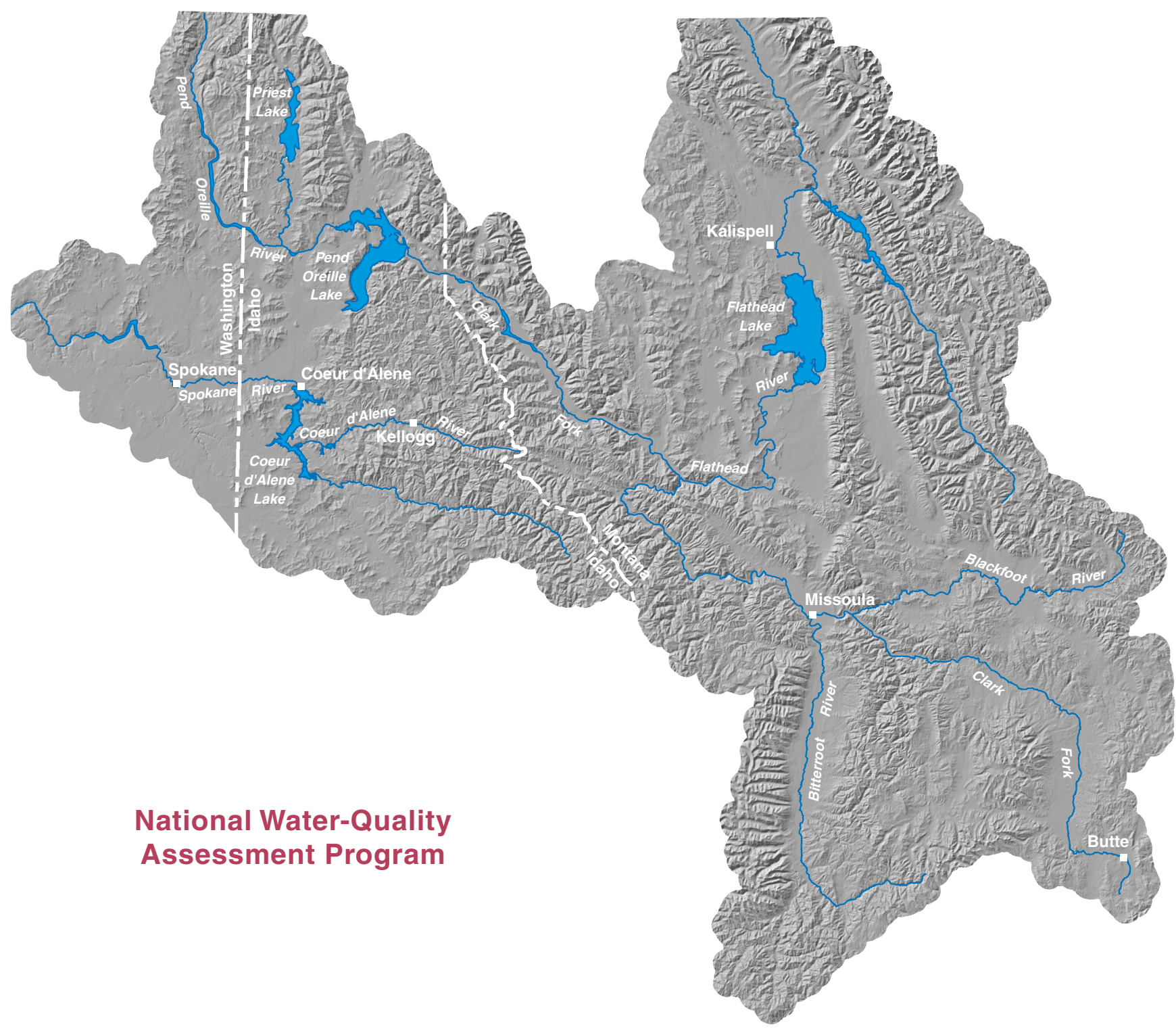


U.S. Department of the Interior

U.S. Geological Survey

Water-Quality, Streambed-Sediment, and Biological

Data from the Clark Fork-Pend Oreille and Spokane

River Basins, Montana, Idaho, and Washington, 1998-2001

\section{By Craig L. Bowers, Rodney R. Caldwell, and DeAnn M. Dutton}

Open-File Report 03-292 


\section{U.S. Department of the Interior}

GALE A. NORTON, Secretary

U.S. Geological Survey

Charles G. Groat, Director

Any use of trade, product, or firm name in this publication is for descriptive purposes only and does not imply endorsement by the U.S. Government

For additional information write to:

District Chief

U.S. Geological Survey

3162 Bozeman Avenue

Helena, MT 59601-6456

Copies of this report may be purchased from:

\section{U.S. Geological Survey}

Branch of Information Services

Box 25286

Denver, CO 80225-0286

Information regarding the National Water-Quality Assessment (NAWQA) Program is available on the Internet via the world wide web at http://water.usgs.gov/nawqa 


\section{FOREWORD}

The U.S. Geological Survey (USGS) is committed to serve the Nation with accurate and timely scientific information that helps enhance and protect the overall quality of life, and facilitates effective management of water, biological, energy, and mineral resources (http://www.usgs.gov/ ). Information on the quality of the Nation's water resources is of critical interest to the USGS because it is so integrally linked to the long-term availability of water that is clean and safe for drinking and recreation and that is suitable for industry, irrigation, and habitat for fish and wildlife. Escalating population growth and increasing demands for the multiple water uses make water availability, now measured in terms of quantity and quality, even more critical to the long-term sustainability of our communities and ecosystems.

The USGS implemented the National Water-Quality Assessment (NAWQA) Program to support national, regional, and local information needs and decisions related to water-quality management and policy (http://water.usgs.gov/ nawqa/). Shaped by and coordinated with ongoing efforts of other Federal, State, and local agencies, the NAWQA Program is designed to answer: What is the condition of our Nation's streams and ground water? How are the conditions changing over time? How do natural features and human activities affect the quality of streams and ground water, and where are those effects most pronounced? By combining information on water chemistry, physical characteristics, stream habitat, and aquatic life, the NAWQA Program aims to provide science-based insights for current and emerging water issues and priorities. NAWQA results can contribute to informed decisions that result in practical and effective water-resource management and strategies that protect and restore water quality.

Since 1991, the NAWQA Program has implemented interdisciplinary assessments in more than 50 of the Nation's most important river basins and aquifers, referred to as Study Units (http://water.usgs.gov/nawqa/nawqamap.html). Collectively, these Study Units account for more than 60 percent of the overall water use and population served by public water supply, and are representative of the Nation's major hydrologic landscapes, priority ecological resources, and agricultural, urban, and natural sources of contamination.

Each assessment is guided by a nationally consistent study design and methods of sampling and analysis. The assessments thereby build local knowledge about water-quality issues and trends in a particular stream or aquifer while providing an understanding of how and why water quality varies regionally and nationally. The consistent, multi-scale approach helps to determine if certain types of water-quality issues are isolated or pervasive, and allows direct comparisons of how human activities and natural processes affect water quality and ecological health in the Nation's diverse geographic and environmental settings. Comprehensive assessments on pesticides, nutrients, volatile organic compounds, trace metals, and aquatic ecology are developed at the national scale through comparative analysis of the Study-Unit findings (http://water.usgs.gov/nawqa/natsyn.html). 
The USGS places high value on the communication and dissemination of credible, timely, and relevant science so that the most recent and available knowledge about water resources can be applied in management and policy decisions. We hope this NAWQA publication will provide you the needed insights and information to meet your needs, and thereby foster increased awareness and involvement in the protection and restoration of our Nation's waters.

The NAWQA Program recognizes that a national assessment by a single program cannot address all water-resource issues of interest. External coordination at all levels is critical for a fully integrated understanding of watersheds and for cost-effective management, regulation, and conservation of our Nation's water resources. The Program, therefore, depends extensively on the advice, cooperation, and information from other Federal, State, interstate, Tribal, and local agencies, non-government organizations, industry, academia, and other stakeholder groups. The assistance and suggestions of all are greatly appreciated.

Robert M. Hirsch

Associate Director for Water

iv Water-Quality, Streambed-Sediment,and Biological Data from the Clark Fork-Pend Oreille and Spokane River Basins, Montana, Idaho, and Washington, 1998-2001 


\section{CONTENTS}

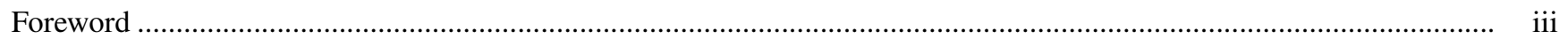

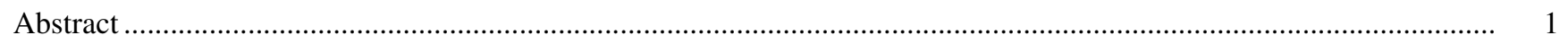

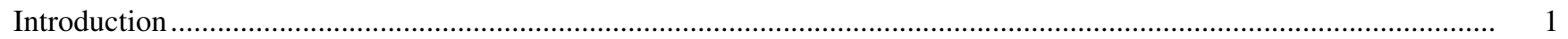

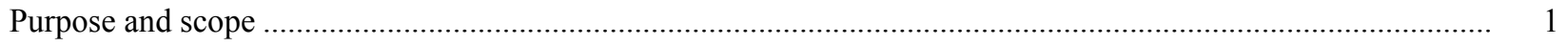

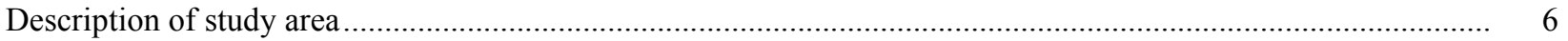

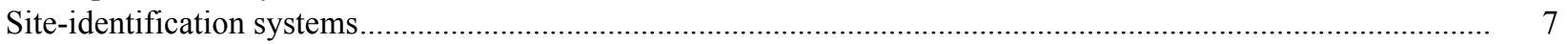

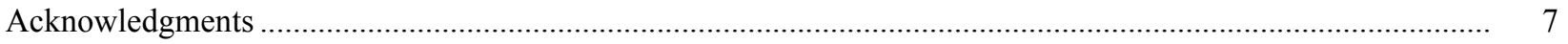

Methods for the collection of water-quality, streambed-sediment, and biological samples ..........................................

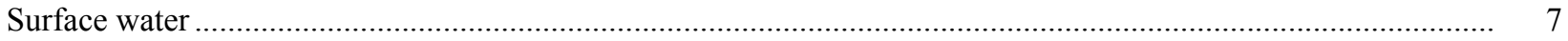

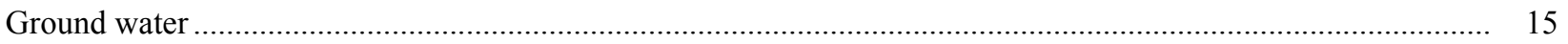

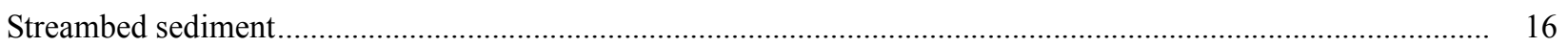

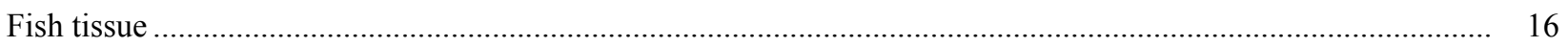

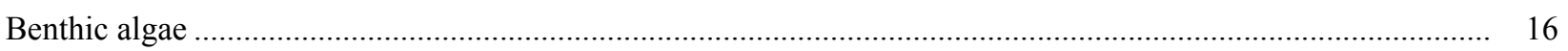

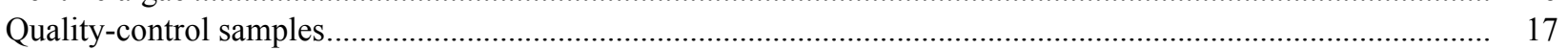

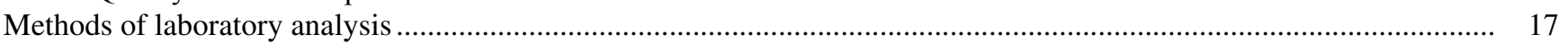

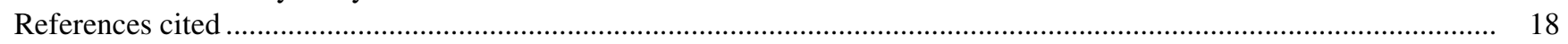

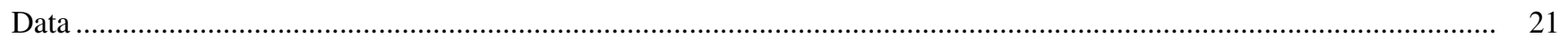

\section{ILLUSTRATIONS}

Figure $\quad$ 1. Map showing surface-water-quality, streambed-sediment, and biological sampling sites in the Northern Rockies Intermontane Basins study unit, Montana, Idaho, and Washington

2. Map showing ground-water study areas within the Northern Rockies Intermontane Basins study unit, Montana, Idaho, and Washington ................................................................................... 10

3. Map showing locations of sampled wells in subunit survey 1, Idaho and Washington ........................... 11

4. Map showing locations of sampled wells in subunit survey 2, Montana .............................................. 12

5. Map showing locations of selected wells in the surface-water/ground-water interaction study area, Idaho and Washington

6. Diagram showing location-numbering system for wells and springs ................................................. 14

\section{TABLES}

Table 1. Types of data collected at surface-water sites for the Northern Rockies Intermontane Basins study unit in Montana, Idaho, and Washington, 1998-2001

2. Physical data for wells sampled in subunit survey 1 in Idaho and Washington, Northern Rockies Intermontane Basins study unit

3. Physical data for wells sampled in subunit survey 2 in Montana, Northern Rockies Intermontane Basins study unit

4. Physical data for monitoring wells sampled for the surface-water/ground-water interaction study of the Spokane River in Idaho and Washington, Northern Rockies Intermontane Basins study unit .....

5. Summary of analytical method references, analyzing laboratories, and field processing of samples for chemical constituents in water, streambed sediment, fish tissue, and benthic algae, Northern Rockies Intermontane Basins study unit.

6. Streamflow, physical, and major-ion concentration data for surface-water samples collected at fixed water-quality sites, Northern Rockies Intermontane Basins study unit, 1998-2001.

7. Nutrient and organic-carbon concentration data for surface-water samples collected at fixed water-quality sites, Northern Rockies Intermontane Basins study unit, 1998-2001

8. Trace-element concentration data for surface-water samples collected at fixed water-quality sites, Northern Rockies Intermontane Basins study unit, 1998-2001 


\section{TABLES--continued}

Table 9. Pesticide concentration data for filtered surface-water samples collected at selected fixed waterquality sites, Northern Rockies Intermontane Basins study unit, 1999-2000 …................................... 68

10. Volatile organic-compound concentration data for surface-water samples collected at fixed waterquality sites along the Spokane River, Idaho and Washington, 1999-2000

11. Streamflow, physical, and major-ion concentration data for surface-water samples collected for the synoptic study of mining-affected areas, Northern Rockies Intermontane Basins study unit, August-September 2000

12. Nutrient data for surface-water samples collected for the synoptic study of mining-affected areas, Northern Rockies Intermontane Basins study unit, August-September 2000

13. Trace-element concentration data for surface-water samples collected for the synoptic study of mining-affected areas, Northern Rockies Intermontane Basins study unit, August-September 2000 .

14. Stable-isotope data for surface-water samples along the Spokane River, Idaho and Washington, 1999-2001

15. Physical and major-ion concentration data for ground-water samples collected from basin-fill aquifers, Northern Rockies Intermontane Basins study unit, 1999 and 2001

16. Nutrient and dissolved organic-carbon concentration data for ground-water samples collected from basin-fill aquifers, Northern Rockies Intermontane Basins study unit, 1999 and 2001

17. Trace-element concentration data for ground-water samples collected from basin-fill aquifers, Northern Rockies Intermontane Basins study unit, 1999 and 2001

18. Pesticide-concentration data for filtered ground-water samples collected from basin-fill aquifers, Northern Rockies Intermontane Basins study unit, 1999 and 2001

19. Pesticide and pesticide-degradate concentration data for filtered ground-water samples collected from basin-fill aquifers in Montana, Northern Rockies Intermontane Basins study unit, 2001 ....

20. Volatile organic-compound concentration data for ground-water samples collected from basin-fill aquifers, Northern Rockies Intermontane Basins study unit, 1999 and 2001

21. Tritium and radon concentration and stable-isotope data for ground-water samples collected from basin-fill aquifers, Northern Rockies Intermontane Basins study unit, 1999 and 2001

22. Radionuclide data from filtered ground-water samples collected from basin-fill aquifers in Idaho and Washington, Northern Rockies Intermontane Basins study unit, 1999

23. Water-level, specific-conductance, and ancillary data for selected ground-water sites inventoried for the surface-water/ground-water interaction study of the Spokane River, Idaho and Washington

24. Physical and major-ion concentration data for ground-water samples collected for the surface-water/ground-water interaction study of the Spokane River and the surrounding basin-fill aquifers, Idaho and Washington, 2000-01

25. Trace-element concentration data for ground-water samples collected for the surface-water/groundwater interaction study of the Spokane River and surrounding basin-fill aquifers, Idaho and Washington, 2000-01

26. Stable-isotope data for ground-water samples collected during the surface-water/ground-water interaction study of the Spokane River and the surrounding basin-fill aquifers, Idaho and Washington, 1999-2001

27. Trace-element and carbon concentration data for streambed-sediment samples, Northern Rockies Intermontane Basins study unit, 1998-99

28. Trace-element and carbon concentration data for streambed-sediment samples collected for a synoptic study of mining-affected areas, Northern Rockies Intermontane Basins study unit, August-September 2000 .

29. Organochlorine-compound and carbon concentration data for streambed-sediment samples, Northern Rockies Intermontane Basins study unit, 1998-99.

30. Trace-element concentration data for fish-tissue samples, Northern Rockies Intermontane Basins study unit, 1998-99. 


\section{TABLES--continued}

Table 31. Organochlorine-compound concentration data for fish-tissue samples, Northern Rockies

Intermontane Basins study unit, 1998-99

32. Benthic algae data for samples, Northern Rockies Intermontane Basins study unit, 1999-2001

\section{CONVERSION FACTORS, DATUM, ABBREVIATED UNITS, AND ACRONYMS}

\begin{tabular}{rll}
\hline Multiply & By & \multicolumn{1}{c}{ To obtain } \\
\hline acre & 0.4047 & square hectometer \\
cubic foot per second $\left(\mathrm{ft}^{3} / \mathrm{s}\right)$ & 0.028317 & cubic meter per second \\
foot & 0.3048 & meter \\
gallon & 3.7850 & liter $(\mathrm{L})$ \\
inch (in.) & 2.54 & centimeter $(\mathrm{cm})$ \\
& micrometer $(\mu \mathrm{m})$ \\
square mile $\left(\mathrm{mi}^{2}\right)$ & 25,400 & square kilometer \\
\hline
\end{tabular}

Temperature can be converted to degrees Celsius $\left({ }^{\circ} \mathrm{C}\right)$ or degrees Fahrenheit $\left({ }^{\circ} \mathrm{F}\right)$ by the following equations:

$$
\begin{aligned}
& { }^{\circ} \mathrm{C}=5 / 9\left({ }^{\circ} \mathrm{F}-32\right) \\
& { }^{\circ} \mathrm{F}=9 / 5\left({ }^{\circ} \mathrm{C}\right)+32
\end{aligned}
$$

Horizontal coordinate information for surface-water sites is referenced to the North American Datum of 1927 (NAD 27). Horizontal coordinate information for ground-water sites is referenced to North American Datum of 1983 (NAD 83).

Abbreviated units used in this report:

$\begin{array}{ll}\mathrm{cm} & \text { centimeter } \\ \mathrm{g} / \mathrm{kg} & \text { grams per kilogram (equivalent to parts per thousand) } \\ \mathrm{g} / \mathrm{m}^{2} & \text { grams per square meter } \\ \mu \mathrm{g} / \mathrm{g} & \text { micrograms per gram (equivalent to parts per million) } \\ \mu \mathrm{g} / \mathrm{kg} & \text { micrograms per kilogram (equivalent to parts per billion) } \\ \mu \mathrm{g} / \mathrm{L} & \text { micrograms per liter (equivalent to parts per billion) } \\ \mu \mathrm{m} & \text { micrometer } \\ \mu \mathrm{S} / \mathrm{cm} & \text { microsiemens per centimeter at } 25 \text { degrees Celsius } \\ \mathrm{mg} / \mathrm{L} & \text { milligrams per liter (equivalent to parts per million) } \\ \mathrm{mg} / \mathrm{m}^{2} & \text { milligrams per square meter } \\ \mathrm{mL} & \text { milliliter } \\ \mathrm{mm} & \text { millimeter } \\ \mathrm{pCi} / \mathrm{L} & \text { picocuries per liter }\end{array}$

Acronyms used in this report:

$\begin{array}{ll}\text { DOC } & \text { dissolved organic carbon } \\ \text { LRL } & \text { laboratory reporting level } \\ \text { LT-MDL } & \text { long-term method-detection level } \\ \text { MDC } & \text { minimum detectable concentration } \\ \text { MRL } & \text { minimum reporting level } \\ \text { NAWQA } & \text { National Water-Quality Assessment } \\ \text { NROK } & \text { Northern Rockies Intermontane Basins } \\ \text { NWQL } & \text { National Water Quality Laboratory of the U.S. Geological Survey, Denver, Colo. }\end{array}$


Acronyms used in this report (continued)

$\begin{array}{ll}\text { o,p'-DDD } & \text { 1,1-dichloro-2-(o-chlorophenyl)-2-(p-chlorophenyl)ethane } \\ \text { o,p'-DDE } & \text { 1,1-dichloro-2-(o-chlorophenyl)-2-(p-chlorophenyl)ethylene } \\ \text { o,p'-DDT } & \text { 1,1,1-trichloro-2-(o-chlorophenyl)-2-(p-chlorophenyl)ethane } \\ \text { p,p'-DDD } & \text { 1,1-dichloro-2,2-bis(p-chlorophenyl)ethane } \\ \text { p,p'-DDE } & \text { 1,1-dichloro-2,2-bis(p-chlorophenyl)ethylene } \\ \text { p',p'-DDT } & \text { 1,1,1-trichloro-2,2-bis(p-chlorophenyl)ethane } \\ \text { PVC } & \text { polyvinyl chloride } \\ \text { RL } & \text { reporting level } \\ \text { SMOW } & \text { Standard Mean Ocean Water } \\ \text { SUS } & \text { subunit survey } \\ \text { SVRP } & \text { Spokane Valley/Rathdrum Prairie } \\ \text { USEPA } & \text { U.S. Environmental Protection Agency } \\ \text { USGS } & \text { U.S. Geological Survey } \\ \text { VOC } & \text { volatile organic compound } \\ \text { VSMOW } & \text { Vienna Standard Mean Ocean Water }\end{array}$

viii Water-Quality, Streambed-Sediment, and Biological Data from the Clark Fork-Pend Oreille and Spokane River Basins, Montana, Idaho, and Washington, 1998-2001 


\title{
Water-Quality, Streambed-Sediment, and Biological Data from the Clark Fork-Pend Oreille and Spokane River Basins, Montana, Idaho, and Washington, 1998-2001
}

\author{
By Craig L. Bowers, Rodney R. Caldwell, and DeAnn M. Dutton
}

\begin{abstract}
Water-quality, streambed-sediment, and biological data were collected in the Clark Fork-Pend Oreille and Spokane River basins as part of the U.S. Geological Survey's National Water-Quality Assessment Program and are presented in this report. These river basins compose the Northern Rockies Intermontane Basins study unit which was selected to include a river system that has a mixture of forested, agricultural, urban, and developing areas. Waterquality samples were collected from 28 surface-water sites and 86 ground-water sites from June 1998 to September 2001. Data collected included measurements of physical properties and chemical analyses of concentrations of major ions, trace elements, nutrients, organic carbon, pesticides, volatile organic compounds, and radiochemical constituents. Streambed-sediment and biological tissue samples were collected from 41 sites and analyzed for trace elements and organochlorine compounds. Benthic algae were collected to determine chlorophyll concentration and areal density.
\end{abstract}

\section{INTRODUCTION}

The U.S. Geological Survey (USGS) National Water-Quality Assessment (NAWQA) Program is designed to produce a comprehensive assessment of the quality of the Nation's stream and aquifer systems (Hirsch and others, 1988; Leahy and others, 1990). Investigations in more than 50 NAWQA study units use an integrated approach to assess the status and trends of water quality in major river basins of the Nation (Gurtz, 1994). These assessments consist of the collection of physical, chemical, and biological data at sites representing a wide range of environmental conditions.

The Northern Rockies Intermontane Basins (NROK) study area was selected to include several large river systems that represent a mixture of forested, agricultural, urban, and developing areas. The study area also contains two major sole-source aquifers - the Spokane Valley/Rathdrum Prairie (SVRP) and Missoula sole-source aquifers.

The effects of land-use and water-use practices on surface- and ground-water resources are a concern to local resource managers, citizen groups, and Federal, State, Tribal, and local governments. Information obtained for the NROK NAWQA program will increase the scientific understanding of surface- and ground-water quality and health of aquatic biological communities of this area.

\section{Purpose and Scope}

This report presents water-quality, streambedsediment, and biological data for samples collected in the Clark Fork-Pend Oreille and Spokane River basins that compose the NROK study unit. These data (tables 1-4 in main body of report; tables 5-32, back of the report) were collected between June 1998 and September 2001 at 28 surface-water-quality sites, 86 groundwater sites, 37 streambed-sediment sites, and 19 fishtissue sample sites. Surface-water-quality sites (table 1) consisted of fixed water-quality and synoptic waterquality sites. Fixed water-quality sites were sampled on a routine basis for about 2 years. All synoptic waterquality sites were sampled once over a short interval of time as part of a targeted characterization in miningaffected areas; some synoptic sites subsequently were sampled more than once. Ground-water-quality sites consisted of 86 wells (tables 2, 3, and 4) that were sampled to assess the quality of water in the Spokane Valley/Rathdrum Prairie (SVRP) and Missoula solesource aquifers and nearby basin-fill aquifers. In addition, physical and chemical data were collected and compiled to supplement ground-water-quality 


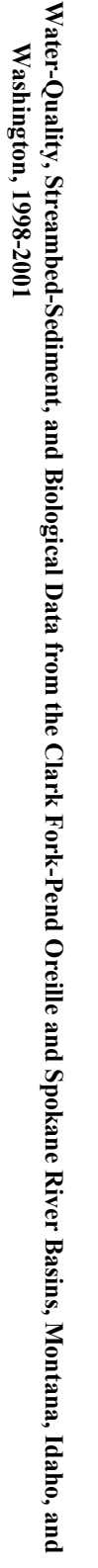

Table 1. Types of data collected at surface-water sites for the Northern Rockies Intermontane Basins study unit in Montana, Idaho, and Washington, 1998-2001 [Site locations shown on figure 1. Site identification number described in text. Latitude and longitude given in degrees, minutes, and seconds referenced to North American Datum of 1927]

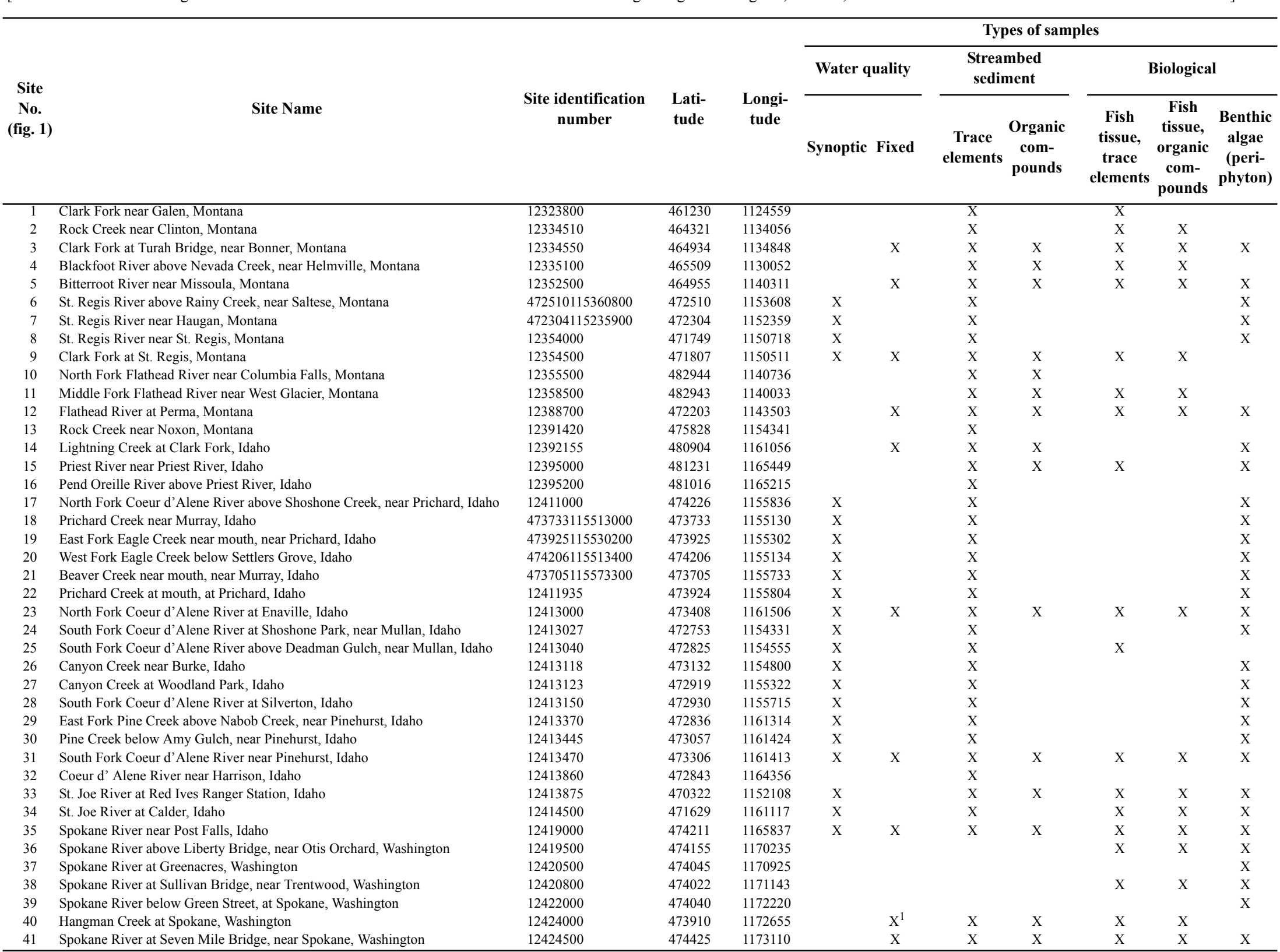

${ }^{1}$ Samples collected only for pesticide analysis at this site. 
Table 2. Physical data for wells sampled in subunit survey 1 in Idaho and Washington, Northern Rockies Intermontane Basins study unit

[Well number (used for cross reference to wells plotted on figure 3), location number, and well identification number described in text. Latitude and longitude reported in degrees, minutes, seconds, and tenths of seconds. Latitude and longitude referenced to North American Datum of 1983]

\begin{tabular}{|c|c|c|c|c|c|c|}
\hline $\begin{array}{c}\text { Well } \\
\text { number } \\
\text { (fig. 3) }\end{array}$ & Location number & $\begin{array}{c}\text { Well } \\
\text { identification } \\
\text { number }\end{array}$ & Latitude & Longitude & $\begin{array}{c}\text { Well depth } \\
\text { (feet below } \\
\text { land surface) }\end{array}$ & $\begin{array}{c}\text { County and } \\
\text { State }\end{array}$ \\
\hline 1 & 56N01W10BBBB01 & 481320116261101 & 481320.7 & 1162611.6 & 139 & Bonner, Idaho \\
\hline 2 & 56N02W35BBBD01 & 480950116324401 & 480949.9 & 1163243.6 & 135 & Bonner, Idaho \\
\hline 3 & 31N45E27DAAD01 & 480928117053201 & 480928.4 & 1170532.2 & 56 & Pend Oreille, Wash \\
\hline 4 & 55N05W08BABA01 & 480813116593701 & 480813.0 & 1165937.9 & 162 & Bonner, Idaho \\
\hline 5 & 55N06W13AAAD01 & 480718117012501 & 480718.0 & 1170125.4 & 130 & Bonner, Idaho \\
\hline 6 & 54N04W08AADA01 & 480249116510001 & 480252.2 & 1165103.8 & 198 & Bonner, Idaho \\
\hline 7 & 54N04W10ADDD01 & 480235116483001 & 480235.9 & 1164830.0 & 80 & Bonner, Idaho \\
\hline 8 & 29N43E02DCBC01 & 480203117200601 & 480204.6 & 1172019.0 & 60 & Spokane, Wash. \\
\hline 9 & 54N02W18CCDD01 & 480128116374601 & 480127.6 & 1163746.1 & 76 & Bonner, Idaho \\
\hline 10 & 29N44E07CBCD01 & 480119117182101 & 480119.0 & 1171821.1 & 123 & Spokane, Wash. \\
\hline 11 & 29N44E21CCCB01 & 475925117153001 & 475920.9 & 1171544.2 & 77 & Spokane, Wash. \\
\hline 12 & 29N41E34ADDB01 & 475825117364401 & 475809.8 & 1173638.2 & 67 & Stevens, Wash. \\
\hline 13 & 53N02W07ABDD01 & 475731116371301 & 475730.6 & 1163713.2 & 431 & Kootenai, Idaho \\
\hline 14 & 28N42E12ABDD01 & 475637117262501 & 475636.8 & 1172625.3 & 90 & Spokane, Wash. \\
\hline 15 & 53N03W24CCCD01 & 475510116391201 & 475509.6 & 1163912.3 & 339 & Kootenai, Idaho \\
\hline 16 & 53N03W34ADAA01 & 475400116404201 & 475400.0 & 1164042.0 & 237 & Kootenai, Idaho \\
\hline 17 & 28N43E28CDAD01 & 475343117225201 & 475329.4 & 1172250.5 & 150 & Spokane, Wash. \\
\hline 18 & 52N04W06AAAA01 & 475322116522201 & 475322.1 & 1165222.1 & 443 & Kootenai, Idaho \\
\hline 19 & 27N42E12ABAA01 & 475130117262201 & 475134.3 & 1172619.0 & 57 & Spokane, Wash. \\
\hline 20 & 52N03W21BACD01 & 475035116424801 & 475034.6 & 1164248.1 & 338 & Kootenai, Idaho \\
\hline 21 & 51N04W06CDDD01 & 474718116530201 & 474718.4 & 1165302.0 & 298 & Kootenai, Idaho \\
\hline 22 & 26N43E06ACBD01 & 474708117250501 & 474655.3 & 1172514.8 & 45 & Spokane, Wash. \\
\hline 23 & 26N42E04CCDB01 & 474629117305101 & 474627.0 & 1173054.7 & 321 & Spokane, Wash. \\
\hline 24 & 51N04W20CBCD01 & 474456116522001 & 474456.1 & 1165220.0 & 290 & Kootenai, Idaho \\
\hline 25 & 26N42E20ABAC01 & 474427117312101 & 474434.5 & 1173132.5 & 60 & Spokane, Wash. \\
\hline 26 & 26N43E28CABA01 & 474317117225301 & 474317.2 & 1172253.3 & 260 & Spokane, Wash. \\
\hline 27 & 50N03W06DACC01 & 474218116445601 & 474218.4 & 1164456.5 & 209 & Kootenai, Idaho \\
\hline 28 & 50N05W12BCAD01 & 474147116544001 & 474147.4 & 1165440.4 & 200 & Kootenai, Idaho \\
\hline 29 & 50N06W12DBCD01 & 474130117015401 & 474129.7 & 1170154.4 & 137 & Kootenai, Idaho \\
\hline 30 & 25N45E08BDAA01 & 474050117084101 & 474054.0 & 1170839.1 & 128 & Spokane, Wash. \\
\hline 31 & 24N43E05CBBD01 & 473612117243601 & 473603.6 & 1172432.0 & 59 & Spokane, Wash. \\
\hline
\end{tabular}


Table 3. Physical data for wells sampled in subunit survey 2 in Montana, Northern Rockies Intermontane Basins study unit

[Well number (used for cross reference to wells plotted on figure 4), location number, and well identification number described in text. Latitude and longitude reported in degrees, minutes, seconds, and tenths of seconds. Latitude and longitude referenced to North American Datum of 1983]

\begin{tabular}{|c|c|c|c|c|c|c|}
\hline $\begin{array}{c}\begin{array}{c}\text { Well } \\
\text { number } \\
\text { (fig. 4) }\end{array} \\
\text { (fig) }\end{array}$ & Location number & $\begin{array}{c}\text { Well } \\
\text { identification } \\
\text { number }\end{array}$ & Latitude & Longitude & $\begin{array}{l}\text { Well depth, } \\
\text { (feet below } \\
\text { land surface) }\end{array}$ & County and State \\
\hline 32 & 16N23W27BBCC01 & 470719114301401 & 470714.1 & 1143013.7 & 40 & Missoula, Mont. \\
\hline 33 & 15N23W01DCAC01 & 470502114265301 & 470502.1 & 1142653.1 & 60 & Missoula, Mont. \\
\hline 34 & 15N21W17DBAD01 & 470328114164301 & 470327.6 & 1141642.6 & 79 & Missoula, Mont. \\
\hline 35 & $15 \mathrm{~N} 21 \mathrm{~W} 34 \mathrm{BACC} 01$ & 470112114144001 & 470111.9 & 1141440.1 & 174 & Missoula, Mont. \\
\hline 36 & 14N20W16AACB01 & 465838114074501 & 465838.3 & 1140744.7 & 70 & Missoula, Mont. \\
\hline 37 & 14N20W19BBDC01 & 465741114110601 & 465741.4 & 1141105.6 & 141.5 & Missoula, Mont. \\
\hline 38 & 13N19W05CBCC01 & 465440114022101 & 465440.2 & 1140221.4 & 132 & Missoula, Mont. \\
\hline 39 & 13N20W14BACA01 & 465323114054301 & 465322.7 & 1140543.2 & 119 & Missoula, Mont. \\
\hline 40 & 13N20W26BCDA01 & 465127114055401 & 465127.1 & 1140553.8 & 51 & Missoula, Mont. \\
\hline 41 & 12N19W06BBCD01 & 464951114023701 & 464951.1 & 1140237.4 & 82 & Missoula, Mont. \\
\hline 42 & 10N20W02AADA01 & 463932114035901 & 463931.6 & 1140359.4 & 50 & Ravalli, Mont. \\
\hline 43 & 10N19W08ADAD01 & 463827114001201 & 463825.7 & 1140014.8 & 340 & Ravalli, Mont. \\
\hline 44 & 09N19W05CCDC01 & 463335114011701 & 463334.9 & 1140116.7 & 43 & Ravalli, Mont. \\
\hline 45 & 09N20W21CBBB01 & 463122114074701 & 463121.9 & 1140747.0 & 60 & Ravalli, Mont. \\
\hline 46 & 09N20W34BDAB01 & 462948114060101 & 462948.3 & 1140601.0 & 50 & Ravalli, Mont. \\
\hline 47 & 08N19W04BDAA01 & 462859113574401 & 462858.6 & 1135847.3 & 56 & Ravalli, Mont. \\
\hline 48 & 08N20W08BAAB01 & 462818114074101 & 462817.7 & 1140741.1 & 50 & Ravalli, Mont. \\
\hline 49 & 08N19W08ADDD01 & 462754113592701 & 462754.3 & 1135927.3 & 234 & Ravalli, Mont. \\
\hline 50 & 08N21W24ACDB01 & 462616114094301 & 462614.3 & 1140945.9 & 49 & Ravalli, Mont. \\
\hline 51 & 08N20W23CDDD01 & 462545114034301 & 462545.0 & 1140346.7 & 231.5 & Ravalli, Mont. \\
\hline 52 & 07N21W11BBCD01 & 462256114114501 & 462256.2 & 1141144.7 & 29 & Ravalli, Mont. \\
\hline 53 & 07N20W12CBDA01 & 462228114030301 & 462230.8 & 1140246.3 & 79 & Ravalli, Mont. \\
\hline 54 & 06N20W03BDCC01 & 461823114050901 & 461822.8 & 1140512.7 & 53.5 & Ravalli, Mont. \\
\hline 55 & 06N21W03CDAA01 & 461807114123001 & 461807.1 & 1141230.1 & 91 & Ravalli, Mont. \\
\hline 56 & 06N20W13BDCD01 & 461638114023401 & 461638.3 & 1140234.0 & 120 & Ravalli, Mont. \\
\hline 57 & 06N20W30CBAB01 & 461451114090801 & 461451.0 & 1140907.9 & 40 & Ravalli, Mont. \\
\hline 58 & 05N21W03ACBA01 & 461320114121501 & 461318.2 & 1141218.2 & 55 & Ravalli, Mont. \\
\hline 59 & 05N21W23BABB01 & 461055114112601 & 461055.1 & 1141125.0 & 54 & Ravalli, Mont. \\
\hline 60 & 03N21W15ABDA01 & 460110114110901 & 460110.2 & 1141109.5 & 85 & Ravalli, Mont. \\
\hline 61 & 02N20W18DBAC01 & 455521114074801 & 455520.8 & 1140747.6 & 50 & Ravalli, Mont. \\
\hline
\end{tabular}


Table 4. Physical data for monitoring wells sampled for the surface-water/ground-water interaction study of the Spokane River in Idaho and Washington, Northern Rockies Intermontane Basins study unit

[Well number (used for cross reference to wells plotted on figure 5), location number, and well identification number described in text. Latitude and longitude reported in degrees, minutes, seconds, and tenths of seconds. Latitude and longitude referenced to North American Datum of 1983]

\begin{tabular}{|c|c|c|c|c|c|c|}
\hline $\begin{array}{c}\text { Well } \\
\text { number } \\
\text { (fig. 5) }\end{array}$ & Location number & $\begin{array}{c}\text { Well } \\
\text { identification } \\
\text { number }\end{array}$ & Latitude & Longitude & $\begin{array}{c}\text { Well depth } \\
\text { (feet below } \\
\text { land surface) }\end{array}$ & $\begin{array}{l}\text { County and } \\
\text { State }\end{array}$ \\
\hline M1 & 50N05W07DABC01 & 474134117002201 & 474134.3 & 1170022.4 & 79.3 & Kootenai, Idaho \\
\hline M2 & 50N05W07DABC02 & 474134117002202 & 474134.6 & 1170022.3 & 45.8 & Kootenai, Idaho \\
\hline M3 & 26N46E31CBBC01 & 474226117024801 & 474225.6 & 1170248.1 & 151 & Spokane, Wash. \\
\hline M4 & 25N45E01ABDD01 & 474151117031101 & 474150.7 & 1170310.8 & 74.3 & Spokane, Wash. \\
\hline M5 & 25N45E01ABDD02 & 474151117031102 & 474150.7 & 1170310.8 & 60.7 & Spokane, Wash. \\
\hline M6 & 25N45E01ABDD03 & 474149117031101 & 474149.4 & 1170311.5 & 77.8 & Spokane, Wash. \\
\hline M7 & 25N45E01ACAD01 & 474144117031401 & 474144.2 & 1170314.1 & 86.9 & Spokane, Wash. \\
\hline M8 & 25N45E01CBBD02 & 474130117035902 & 474130.1 & 1170358.8 & 32.9 & Spokane, Wash. \\
\hline M9 & 25N45E01CBBD01 & 474130117035901 & 474130.1 & 1170358.8 & 76.1 & Spokane, Wash. \\
\hline M10 & $25 \mathrm{~N} 45 \mathrm{E} 01 \mathrm{CBBC} 01$ & 474131117040401 & 474130.9 & 1170403.5 & 71.0 & Spokane, Wash. \\
\hline M11 & 25N45E10BAAA01 & 474106117060501 & 474106.3 & 1170605.0 & 71.6 & Spokane, Wash. \\
\hline M12 & 25N45E10BAAA03 & 474107117060502 & 474106.6 & 1170605.2 & 136.7 & Spokane, Wash. \\
\hline M13 & 25N45E10BAAA02 & 474107117060501 & 474107.3 & 1170604.9 & 75.5 & Spokane, Wash. \\
\hline M14 & 25N45E03CDDD01 & 474110117060601 & 474109.6 & 1170605.8 & 88.8 & Spokane, Wash. \\
\hline M15 & 25N45E03CDDA01 & 474115117060301 & 474115.1 & 1170603.1 & 97.5 & Spokane, Wash. \\
\hline M16 & 25N45E03BDDA01 & 474140117060401 & 474140.2 & 1170604.3 & 117.3 & Spokane, Wash. \\
\hline M17 & 25N45E10CBDA01 & 474033117062501 & 474033.5 & 1170625.2 & 97.1 & Spokane, Wash. \\
\hline M18 & 25N45E09ADAD01 & 474050117064201 & 474050.4 & 1170641.7 & 77.4 & Spokane, Wash. \\
\hline M19 & 25N45E09ADAB01 & 474053117064701 & 474053.5 & 1170646.6 & 70.9 & Spokane, Wash. \\
\hline M20 & 25N45E17BBAA05 & 474016117085601 & 474015.5 & 1170855.8 & 113 & Spokane, Wash. \\
\hline M21 & $25 \mathrm{~N} 45 \mathrm{E} 08 \mathrm{CBBC} 02$ & 474037117091301 & 474037.2 & 1170913.1 & 98 & Spokane, Wash. \\
\hline M22 & $25 \mathrm{~N} 45 \mathrm{E} 08 \mathrm{CBBC} 01$ & 474038117091201 & 474038.2 & 1170912.0 & 97 & Spokane, Wash. \\
\hline M23 & 25N45E07ADDD01 & 474046117091501 & 474045.5 & 1170914.9 & 80 & Spokane, Wash. \\
\hline M24 & 25N45E07AAAA04 & 474109117091701 & 474109.3 & 1170917.1 & 100 & Spokane, Wash. \\
\hline M25 & 25N44E11DDAC01 & 474026117115301 & 474025.6 & 1171153.2 & 67 & Spokane, Wash. \\
\hline
\end{tabular}


data that were collected from the 86 wells (tables 23 and 26). Surface- and ground-water samples were analyzed for physical properties, major ions, nutrients, trace elements, organic carbon, volatile organic compounds (VOCs), dissolved pesticides, and selected isotopes. Selected ground-water samples also were analyzed for organic compounds that represent the breakdown products (degradates) of pesticides. Streambed-sediment and fish-tissue samples were analyzed for trace elements and organochlorine compounds.

\section{Description of Study Area}

The NROK study unit (fig. 1) covers about $31,500 \mathrm{mi}^{2}$ in western Montana, northern Idaho, and eastern Washington. The study area is composed of two major river basins: the Clark Fork-Pend Oreille River basin which encompasses about 24,900 $\mathrm{mi}^{2}$ within the United States, and the Spokane River basin which encompasses about 6,600 $\mathrm{mi}^{2}$ (Maret and Dutton, 1999). The population of the study unit in 1990 was about 725,000 with about 350,000 in Washington; 255,000 in Montana; and 120,000 in Idaho (Tornes, 1997). About 56 percent of the land in the study unit is public, 37 percent is private, and about 7 percent is owned by Tribes.

The NROK study unit includes four ecoregions: Northern Rockies (72 percent); Montana Valley and Foothill Prairies (16 percent); Canadian Rockies (9 percent); and Columbia Plateau (3 percent) (Maret and Dutton, 1999). The Northern and Canadian Rockies ecoregions are characterized by high-altitude mountains primarily covered by coniferous forests. The predominant land uses within these two ecoregions are timber harvesting, mining, and recreation. The Montana Valley and Foothill Prairies and Columbia Plateau ecoregions are characterized by lower-altitude flatlands and rolling rangeland hills primarily covered by grass and, in dryer areas, sagebrush. The predominant land use in these two ecoregions is nonirrigated and irrigated cropland and livestock grazing.

The climate in the NROK study unit is characterized by mild summers and cold winters with most precipitation occurring in the winter and spring months. Precipitation ranges from about 10 in. per year in some intermontane valleys of western Montana to more than 100 in. per year in parts of mountain ranges of northwestern Montana. Most of the valleys receive between 10 and $30 \mathrm{in.} \mathrm{of} \mathrm{precipitation} \mathrm{per} \mathrm{year,} \mathrm{much}$ of it as snow (Clark and Kendy, 1991).

Streams in the study unit are predominantly cold water. Snowmelt runoff generally represents the main source of surface water from April to July (Kendy and Tresch, 1996). Upland-forest and lower-altitude streams typically have coarse-grained substrates (gravel and cobble), high gradients, and well-defined riffle-pool habitats for aquatic life, with sparse macrophyte growth. Large rivers and streams at lower altitudes typically have fine-grained substrates and lower gradients.

The study unit has a complex geologic history of sedimentation, compressional deformation, igneous activity, and, most recently, extensional block faulting (Kendy and Tresch, 1996). The principal aquifers within the NROK study unit are composed of Cenozoic basin-fill deposits (Clark and Kendy, 1991). These deposits are present along most reaches of the large rivers and major tributaries within the study unit and are primarily composed of unconsolidated to consolidated gravel, sand, silt, and clay. In most areas, the basin-fill aquifers are underlain and laterally bounded by less-permeable bedrock of Quaternary to Middle Proterozoic age.

Two areas (subunits) were surveyed to assess the general quality of water in basin-fill aquifers of the NROK study unit (fig. 2). The first subunit survey (SUS1) was conducted in northern Idaho and northeastern Washington (fig. 3, table 2); the second subunit survey (SUS2) was conducted in western Montana (fig. 4, table 3). Both subunits contain U.S. Environmental Protection Agency (USEPA)designated sole-source aquifers as defined under section 1424(e) of the Safe Drinking Water Act of 1974 (U.S. Environmental Protection Agency, 2000). The USEPA defines such an aquifer as one that supplies at least 50 percent of the drinking water consumed in the area overlying the aquifer. People depending on a solesource aquifer generally do not have a viable alternative drinking-water source.

Part of the SUS1 study area between Post Falls, Idaho, and Spokane, Wash., was selected to better define the surface-water/ground-water interaction of the Spokane River and the adjacent basin-fill aquifer (fig. 5, table 4). Elevated trace-metal concentrations in the Spokane River have caused concerns about potential contamination of water in the sole-source aquifer. 


\section{Site-Identification Systems}

Surface-water sites are assigned a site number from 1 through 41 (table 1, fig. 1). In addition, most surface-water sites are assigned an eight digit stationidentification number that represents the standard USGS numbering system for streamflow-gaging stations. For miscellaneous or temporary surfacewater sites, a fifteen digit station-identification number is used; these numbers represent the approximate latitude and longitude of the site (first 13 digits), plus the sequence number (last 2 digits).

Ground-water sites (wells) used in water-quality assessments for the subunit surveys were assigned a well number from 1 through 61 (tables 2 and 3, figs. 3 and 4). Ground-water sites used in the surface-water/ ground-water interaction study along the Spokane River were assigned a well number from M1 through M25 (table 4; fig. 5). Fifteen digit site-identification numbers represent the approximate latitude and longitude (referenced to the horizontal coordinate system of North American Datum of 1983) of the well (first 13 digits), plus the sequence number (last 2 digits). However, some well-identification numbers differ from the latitude and longitude where wells were recently field checked with a global positioning system and locations were determined with greater accuracy. Last, ground-water sites were assigned a location number according to their geographic position within the rectangular grid system used for the subdivision of public lands (fig. 6). The location numbering consists of 14 characters. The first three characters specify the township and its position north $(\mathrm{N})$ of the Willamette Base Line in Washington, the Boise Base Line in Idaho, or the Montana Base Line in Montana. The next three characters specify the range and its position west (W) or east (E) of the Willamette Meridian in Washington, the Boise Meridian in Idaho, or the Montana Principal Meridian in Montana. The next two numbers represent the section number. The next four characters sequentially designate the quarter (160-acre tract), quarter-quarter (40-acre tract), quarter-quarter-quarter (10-acre tract), and the quarter-quarter-quarter-quarter section 2 1/2-acre tract). The locations of quarter subdivisions within a section are designated A, B, C, D in a counterclockwise direction, beginning in the northeast quadrant. The final two characters are a sequence number assigned to differentiate multiple wells within a single quarter-quarter-quarter-quarter section. For example, as shown in figure 6 ,
26N43E06ACBD01 (table 2) was the first well inventoried in the SE1/4NW1/4SW1/4NE1/4 sec. 6 , T. 26 N., R. 43 E., in Washington.

\section{Acknowledgments}

The authors wish to thank the following people for their efforts collecting and compiling the data included in this report: Fred A. Bailey, Rick L. Backsen, John C. Baker, Dorothy A. Barnett, C. Lee Chambers, Angela K. Frandsen, Keith L. Hein, Doug S. Ott, Karen L. Payne, Gregory J. Perry, Galen J. Schuster, Kenneth D. Skinner, Brett A. Smith, and Tricia R. Wotan. The authors also would like to thank the well owners who provided access to their wells.

\section{METHODS FOR THE COLLECTION OF WATER- QUALITY, STREAMBED-SEDIMENT, AND BIOLOGICAL SAMPLES}

\section{Surface Water}

Surface-water samples were collected and processed as described in Shelton (1994) and Horowitz and others (1994). Depth-integrated samples were collected at equidistant points along the stream cross section using either hand-held or cable-suspended samplers. Samples were collected in 3-L polyethylene or Teflon bottles and split into subsamples with a Teflon decaport cone splitter. At some surface-water sites, samples were composited and split using a plastic churn splitter. Table 5 summarizes filtration and preservation information for the various type of samples. Specific conductance and $\mathrm{pH}$ were measured onsite from an aliquot of the sample. Dissolved oxygen and temperature were measured directly in the stream channel as near to the center of the stream as possible. Prior to each sampling event, the equipment used to collect and process samples was cleaned with a nonphosphate detergent, rinsed with copious amounts of tap and deionized water, soaked for 30 minutes in 5-percent hydrochloric acid, rinsed again with copious amounts of deionized water, and air dried. Between sampling sites the field equipment was cleaned with deionized water, rinsed with 5-percent hydrochloric acid, rinsed again with deionized water, and last, rinsed with native stream water prior to sampling. 


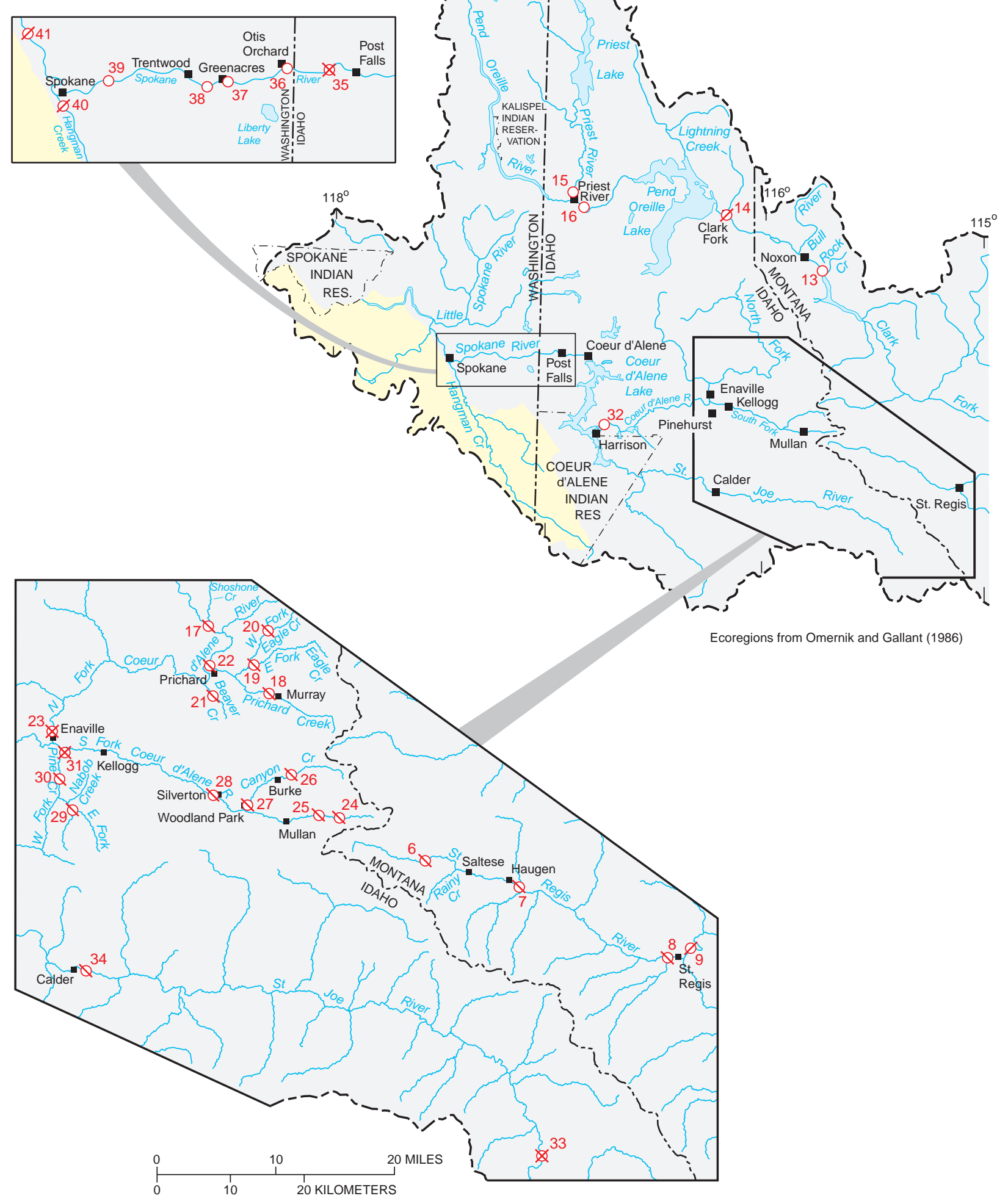

Figure 1. Surface-water-quality, streambed-sediment, and biological sampling sites 


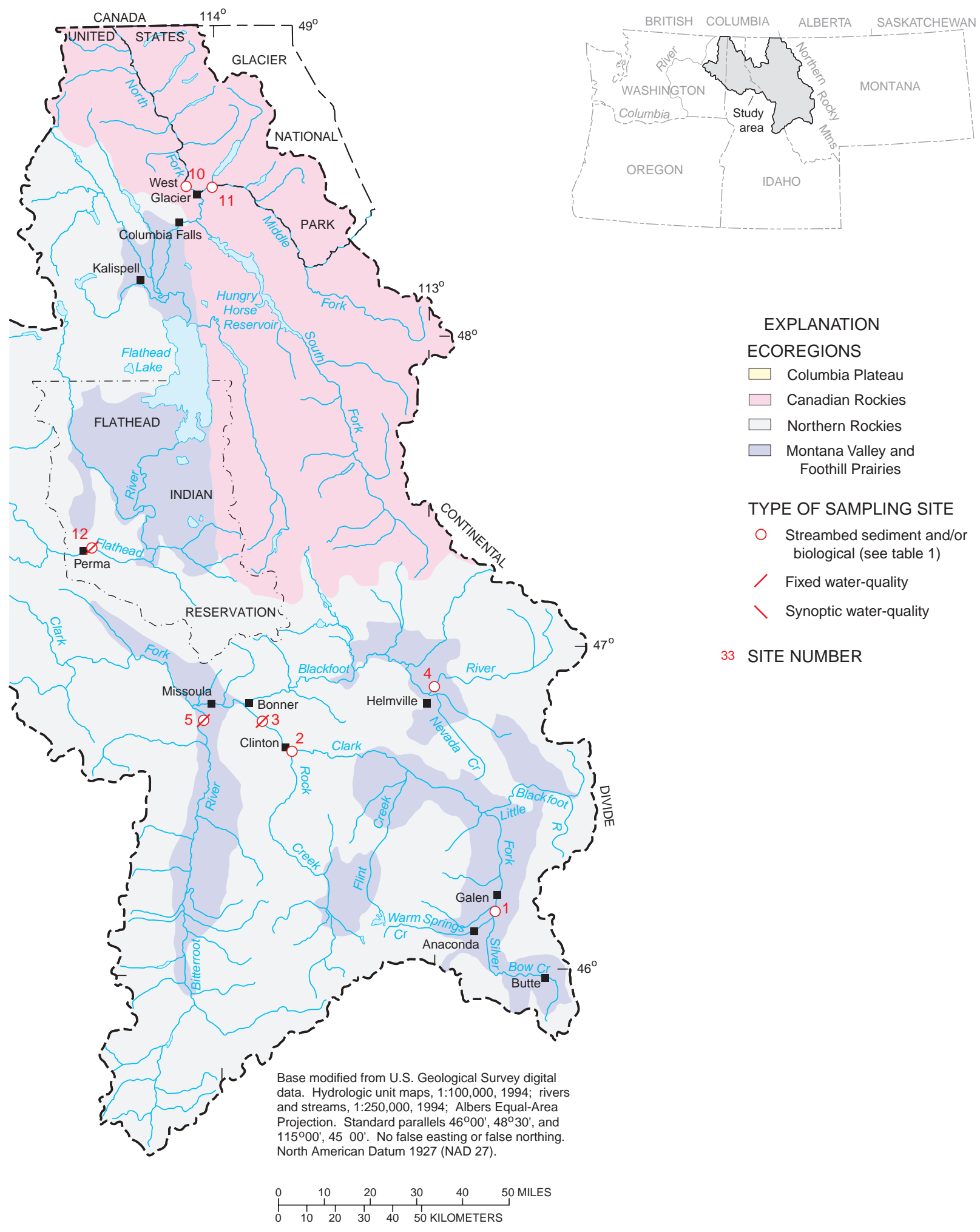

in the Northern Rockies Intermontane Basins study unit, Montana, Idaho, and Washington. 


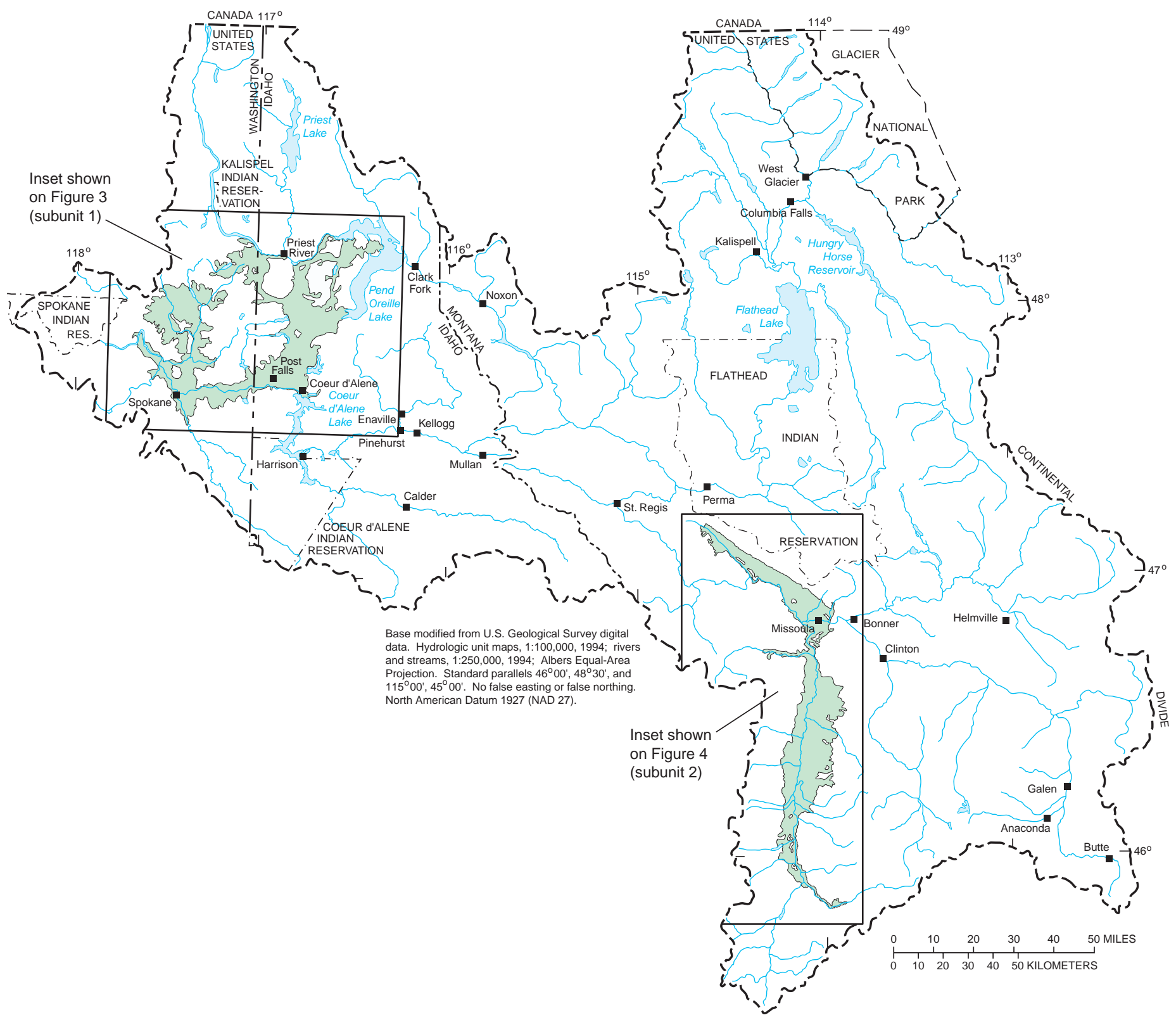

Figure 2. Ground-water study areas within the Northern Rockies Intermontane Basins study unit, Montana, Idaho, and Washington.

10 Water-Quality, Streambed-Sediment, and Biological Data from the Clark Fork-Pend Oreille and Spokane River Basins, Montana, Idaho, and Washington, 1998-2001 


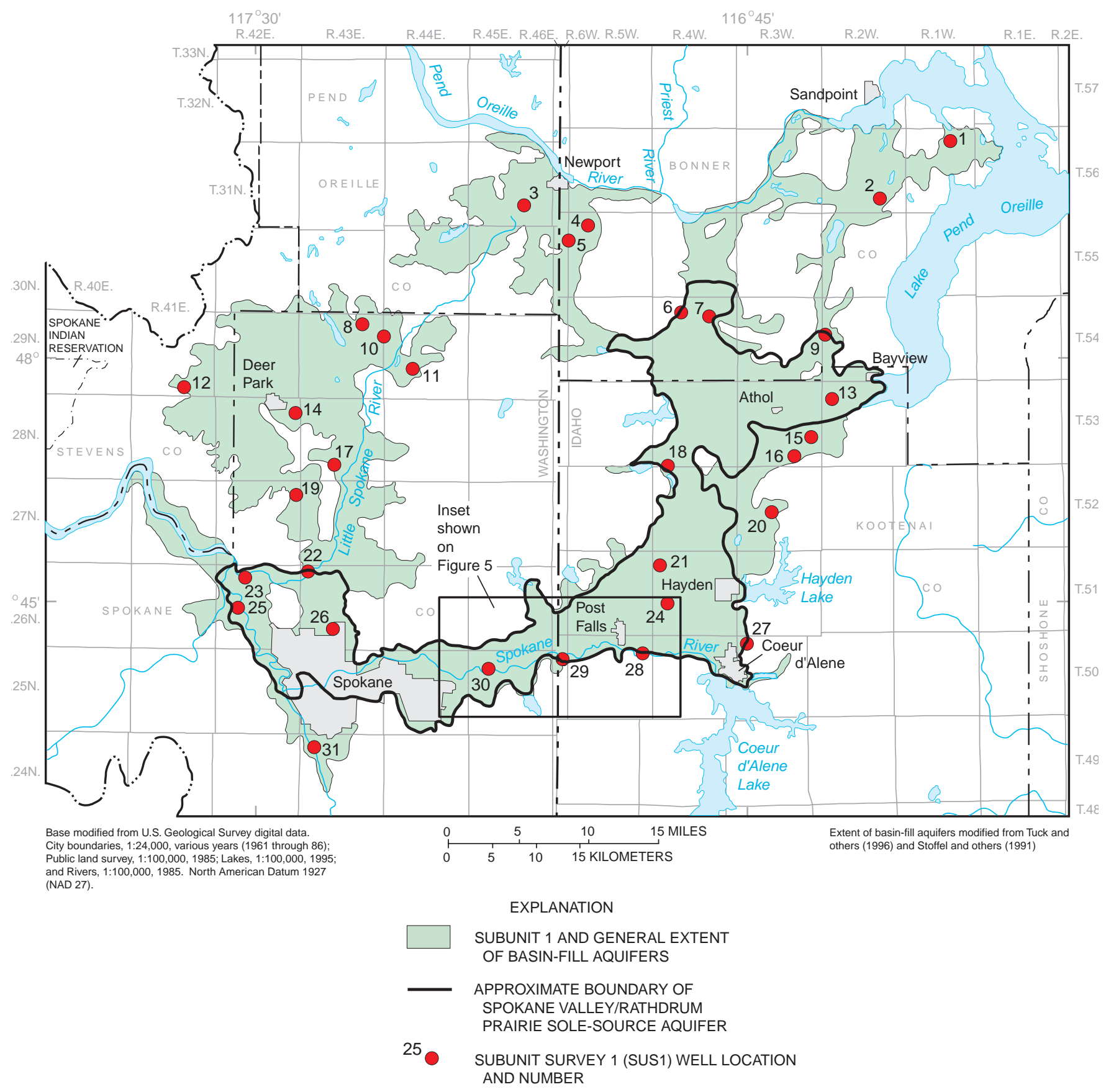

Figure 3. Locations of sampled wells in subunit survey 1, Idaho and Washington. 

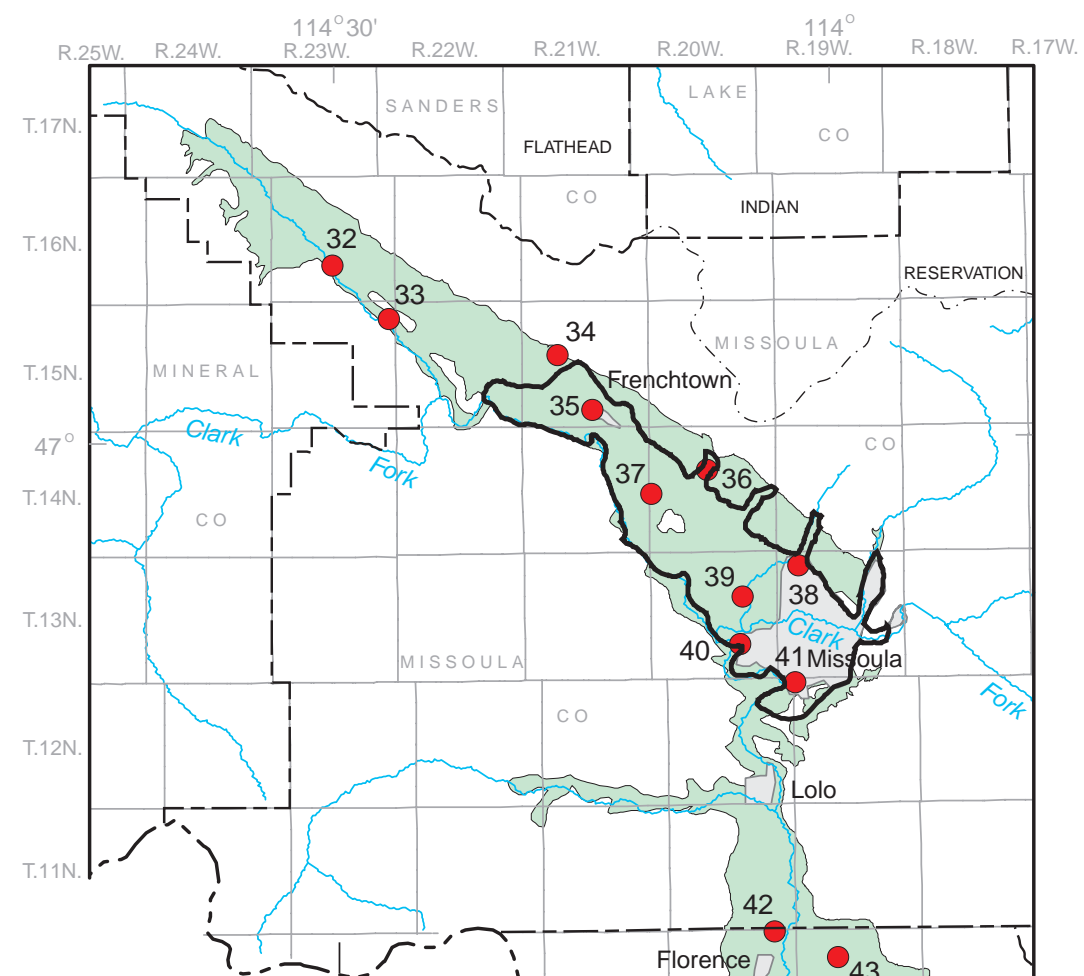
Base modified from U.S. Geological Survey digita data. City boundaries, $1: 24,000$, various years 1985: Lakes, 1:100,000, 1995; and Rivers, 1:100, 1985. North American Datum 1927 (NAD 27).

EXPLANATION

SUBUNIT 2 AND GENERAL EXTENT OF BASIN-FILL AQUIFERS

APPROXIMATE BOUNDARY OF MISSOULA SOLE-SOURCE AQUIFER

42

SUBUNIT SURVEY 2 (SUS2) WELL LOCATION AND NUMBER
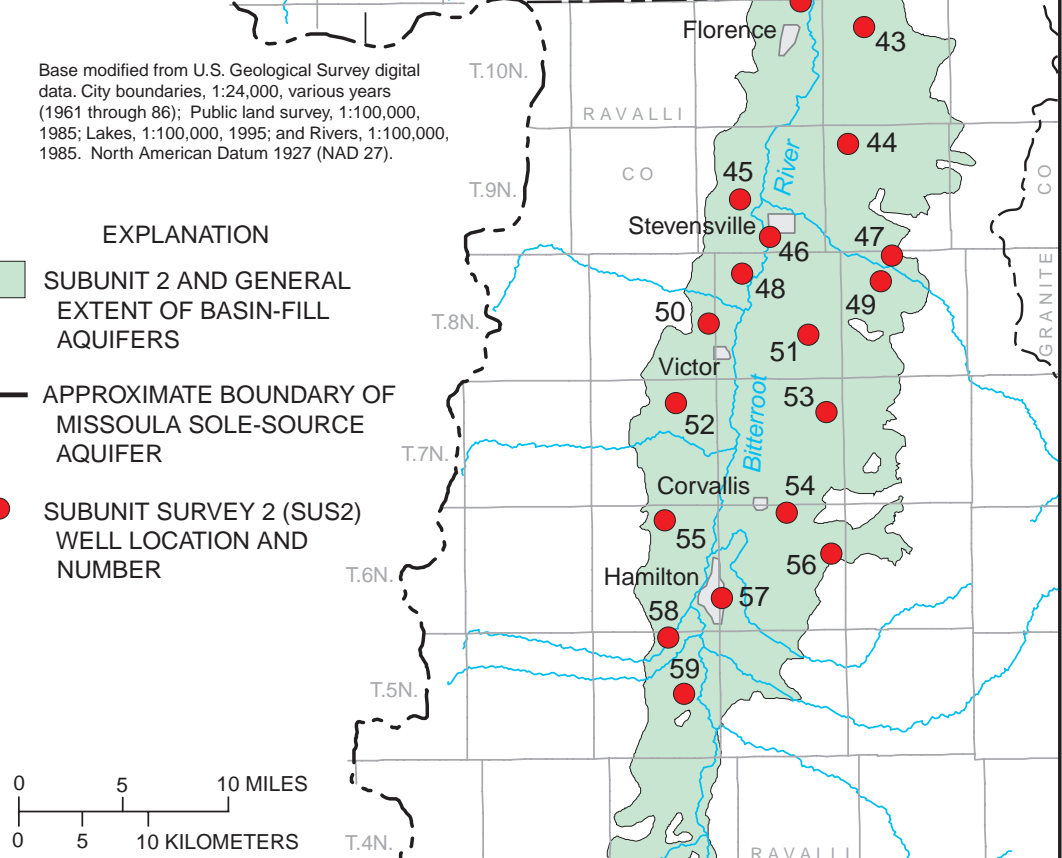


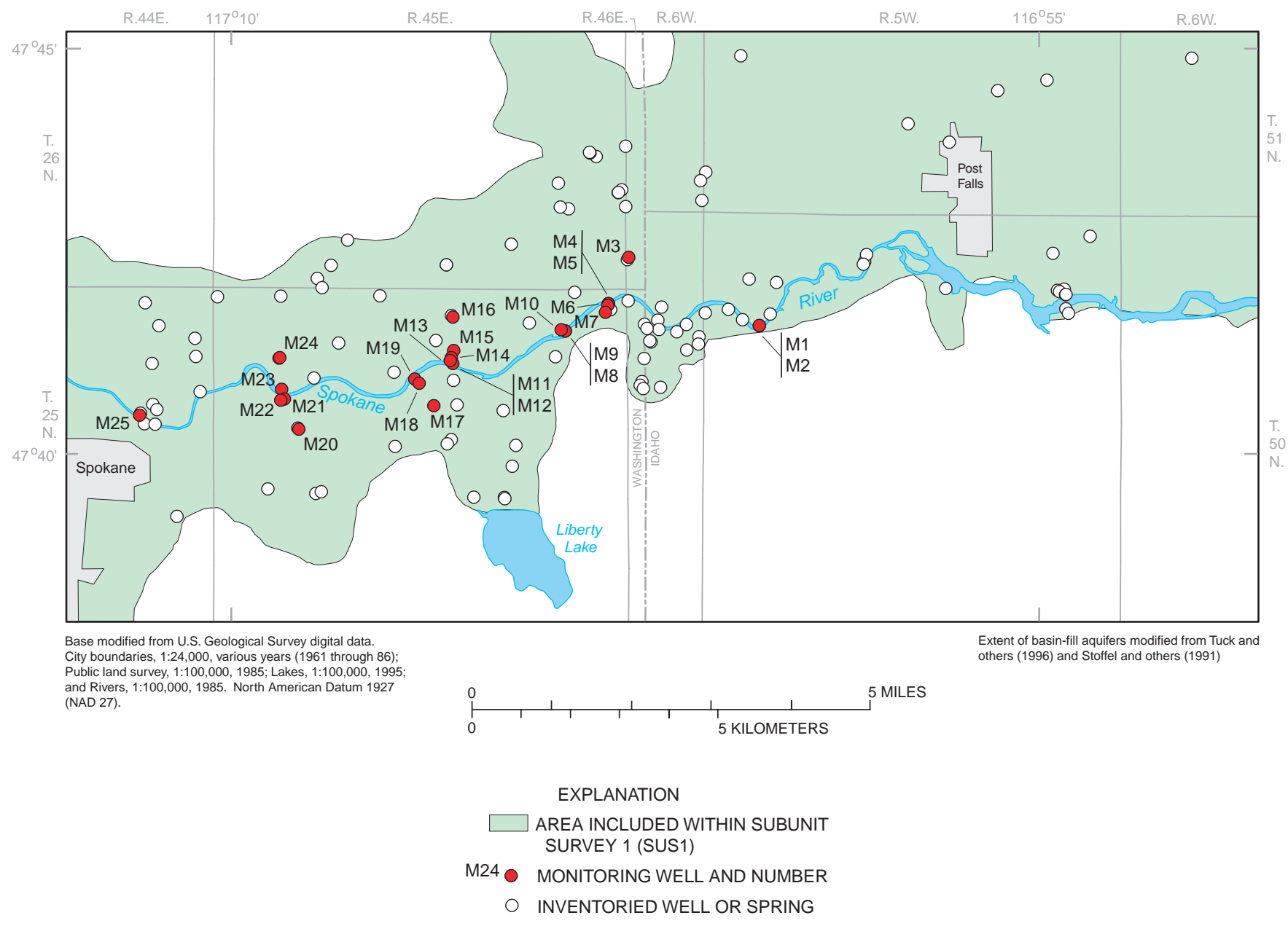

Figure 5. Locations of selected wells in the surface-water/ground-water interaction study area, Idaho and Washington. 

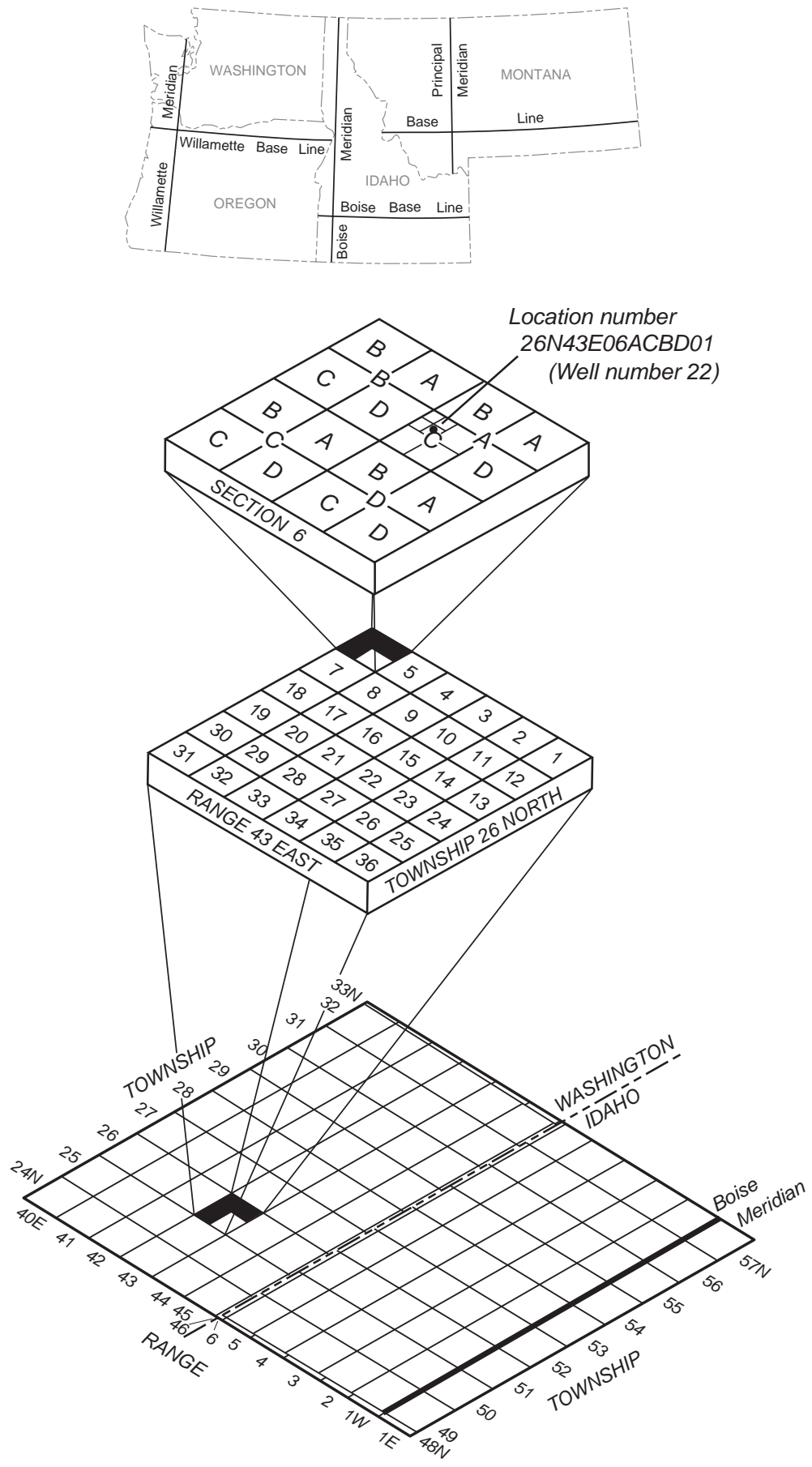

Figure 6. Location-numbering system for wells and springs.

14 Water-Quality, Streambed-Sediment, and Biological Data from the Clark Fork-Pend Oreille and Spokane River Basins, Montana, Idaho, and Washington, 1998-2001 
Filtered inorganic samples were passed through a $0.45-\mu \mathrm{m}$ disposable capsule filter that had been rinsed with $1 \mathrm{~L}$ of deionized water and native stream water. Samples for trace metals were preserved with tracemetal-grade nitric acid; samples for mercury were preserved with nitric acid and potassium dichromate. Whole-water (unfiltered) samples for nutrients were preserved with sulfuric acid. Nutrient, organic carbon, and pesticide samples were chilled and shipped on ice overnight to the USGS National Water Quality Laboratory (NWQL) in Denver, Colo.

Teflon sample bottles, nozzles, caps, and cone splitters were used for collecting pesticide samples and were rinsed with methanol in addition to the normal cleaning procedures. Pesticide samples were filtered onsite through a $0.7-\mu \mathrm{m}$ baked-glass fiber filter using a ceramic piston pump and Teflon tubing. Organic carbon samples were collected using baked-glass bottles and a weighted bottle sampler at a point near the middle of the stream. Samples were filtered through a $0.45-\mu \mathrm{m}$ silver membrane filter in a stainless-steel chamber pressurized with nitrogen gas.

Stream water was sampled for volatile organic compounds (VOCs) at two sites on the Spokane River using specific protocols detailed in Shelton (1997). The presence of chlorine was suspected at both sites since they were located downstream from municipalwastewater treatment facilities. Samples were collected at a single point near the middle of the stream using a VOC sampler designed to automatically flush seven volumes of water through $40-\mathrm{mL}$ baked-glass vials before sample collection. Because of the suspected presence of chlorine, VOC samples were preserved with ascorbic acid in addition to nitrogenpurged hydrochloric acid as per standard protocol (Wilde and others, 1999). VOC samples were shipped on ice overnight to NWQL for analysis.

\section{Ground Water}

Sixty-one wells sampled as part of the subunit surveys were typically pre-existing, low production water-supply wells, with submersible pumps, steel casing, and open intervals consisting of stainless-steel screens, perforated casing, or open-ended casing (figs. 3 and 4, tables 2 and 3). Samples from each of the subunit survey wells were analyzed for major ions, nutrients, trace elements, radon-222, pesticides, and VOCs. Samples from SUS1 were also analyzed for radium isotopes, dissolved organic carbon (DOC), and stable isotopes. A subset of samples from each subunit was analyzed for tritium. The subunit survey wells were typically sampled one time for this project.

Twenty-five monitoring wells were sampled as part of the surface-water/ground-water interaction study; eighteen of these wells (wells M1, M2, and M4 through M19) were installed as part of this project between June and September 2000 (fig. 5, table 4). All of the monitoring wells consisted of 2-in. PVC casing, with PVC screen lengths of 5 to $10 \mathrm{ft}$ (wells drilled for this project) or 30 to $40 \mathrm{ft}$ (pre-existing wells) at the bottom of the wells. Wells installed for this project were drilled with an air-rotary drill rig and 6-in. diameter steel casing driven to temporarily keep the hole open; after the 2-in. PVC casing and screen were in place, silica sand was placed from the bottom to about $5 \mathrm{ft}$ above the screen. About $1 \mathrm{ft}$ of bentonite pellets was placed immediately on top of the sand and the remainder of the annulus was filled with bentonite grout. The temporary steel casing was incrementally pulled out as the sand and bentonite material was placed in the annulus between the PVC and steel casing. Samples were collected from the monitoring wells using a 1.75-in. diameter stainless-steel submersible pump with Teflon impellers. Samples were analyzed for major ions and trace elements. Selected samples from the 25 monitoring wells as well as selected existing wells inventoried for this study also were analyzed for stable isotopes. The monitoring wells were sampled over a range of hydrologic conditions between June 2000 and August 2001 to assess effects of variation in streamflow, water-table elevation, and hydraulic gradient.

Sample water was collected using Teflon tubing, stainless-steel connections, and Teflon valves. Flow from the wells was controlled using a two-valve manifold that diverted a continuous stream of sample water to an enclosed collection chamber. All equipment was cleaned immediately after sampling using established NAWQA sampling procedures (Koterba and others, 1995).

Ground-water samples were collected and processed using established NAWQA sampling procedures (Koterba and others, 1995). Prior to sampling at each well, the water level was measured to the nearest $0.01 \mathrm{ft}$ with an electric or steel tape. The casing volume and pumping rate were calculated to estimate a purge time required to evacuate the water from the well. A minimum of three casing volumes 
were purged before sample collection in order to remove standing water and ensure that the well was replenished with water representative of the aquifer. Field measurements of specific conductance, $\mathrm{pH}$, temperature, turbidity, and dissolved oxygen were recorded at 3-5 minute intervals during purging. Samples were collected when field measurements had stabilized for three consecutive measurements.

All samples were processed in the field within 1 hour of sample collection. Alkalinity was determined in the field for all ground-water samples using the incremental titration method. Samples for analytically time-dependent constituents (radon, radium, pesticides, nutrients, DOC, and VOC) were shipped overnight on ice to the analyzing laboratories (table 5). Samples for the analysis of trace elements, major ions, nutrients, and radium were filtered onsite with a disposable 0.45 - $\mu \mathrm{m}$ pore-size capsule filter. Selected trace-element samples for the surface-water/groundwater interaction study were filtered onsite through a $0.10-\mu \mathrm{m}$ pore-size capsule filter, filtered onsite through a $0.001-\mu \mathrm{m}$ pore-size tangential-flow filtration system, or collected as whole-water (unfiltered) samples.

Pesticide samples were filtered through a $0.7-\mu \mathrm{m}$ poresize glass-fiber plate filter. DOC samples were filtered through a $0.45-\mu \mathrm{m}$ pore-size silver membrane filter. VOC, radon, and tritium samples were unfiltered. Major-ion (cation species), trace-element, VOC, and radium samples were acidified immediately following collection.

\section{Streambed Sediment}

Trace elements and organochlorine compounds in the bottom sediment of stream channels were sampled from sites throughout the study area during low-flow conditions in 1998 and 1999 (fig.1). Streambed-sediment samples were collected in accordance with USGS NAWQA procedures (Shelton and Capel, 1994). The upper $2 \mathrm{~cm}$ of bed sediment was collected from undisturbed, continuously wetted, lowvelocity depositional zones by using a plastic scoop. Five to 10 subsamples within a sampling reach were composited and wet sieved onsite (using native water) through a $63-\mu \mathrm{m}$ (silt and finer) nylon filter. Because trace-element sorption increases with surface area, the smaller size fraction was chosen to maximize the detection of trace elements. Streambed-sediment analyzed for organochlorine compounds was wet sieved onsite (using native water) through a $2-\mathrm{mm}$ (sand and finer) stainless-steel sieve into pre-cleaned 1-L glass jars. All samples were chilled and shipped on ice to the NWQL for laboratory analysis.

\section{Fish Tissue}

Fish were collected using electrofishing techniques from all habitats within a reach (Meador and others, 1993). At most sites, livers and fillet samples were collected for trace-element analysis from different individuals of similar size and of the same species and combined for a single composite sample. At some sites, it was necessary to use the liver and tissue samples from the same fish because of the low populations of adult fish of similar size. Whole-body samples were submitted for organochlorine analysis. Species collected include brook trout (Salvelinus fontinalis), brown trout (Salmo trutta), cutthroat trout (Oncorhynchus clarki), largescale sucker (Catostomus macrocheilus), mountain whitefish (Prosopium williamsoni), and rainbow trout (Oncorhynchus mykiss). All tissue samples were frozen onsite with dry ice and shipped overnight to the NWQL for analysis. Fish-tissue sampling and preparation methods used are detailed in Crawford and Luoma (1993).

\section{Benthic Algae}

Epilithic periphyton samples were collected within a riffle and processed using protocols developed by the USGS NAWQA Program to estimate and compare biomass (chlorophyll-a and ash-free dry weight) among sites (Porter and others, 1993). Epilithic periphyton samples were collected from 5 to 10 cobbles per riffle from 5 riffles per reach. A total of 25 to 50 subsamples were composited for a single sample. Periphyton samples were removed from cobbles by using the bottom portion of a 30 -mL syringe fitted with a neoprene O-ring to form a watertight seal against a rock surface. Periphyton within the syringe barrel were dislodged with a stiff-bristle brush and collected with a hand pipette. Samples were composited into a sample jar and mixed, and an aliquot of 5 to $10 \mathrm{~mL}$ was filtered through a $0.7-\mu \mathrm{m}$ glass-fiber filter. Filters then were wrapped in aluminum foil, placed in a glass vial, and frozen until they were

16 Water-Quality, Streambed-Sediment, and Biological Data from the Clark Fork-Pend Oreille and Spokane River Basins, Montana, Idaho, and Washington, 1998-2001 
processed for chlorophyll-a and ash-free dry weight by the Bureau of Reclamation laboratory in Boise, Idaho.

\section{Quality-Control Samples}

Quality-control samples were collected to examine potential contamination and analytical measurement variability and bias in the results of water, streambed-sediment, and biological samples analyses. Quality-control samples collected for this study included replicates, field blanks, source-solution blanks, trip blanks, and surrogate compounds (for pesticide recovery efficiency) in environmental water samples. Quality-control sampling design followed during data collection for this study is presented in Koterba and others (1995) and Mueller and others (1997). The analytical results for quality-control samples are presented along with environmental sample results in tables 6 through 32 .

Replicate samples were collected as a subset of all types of samples collected. Replicate samples consist of two or more samples considered to be identical in composition and, therefore, can be used to assess the precision of analytical results. Replicate samples can be obtained by either repeating the collection process to obtain two or more independent samples, or by splitting a single composite sample into two or more subsamples. The individual replicate samples are then processed and analyzed separately. In addition to quality-control samples submitted from the field, internal quality-assurance practices at the NWQL are performed systematically to provide quality control for analytical procedures (Pritt and Raese, 1995).

Blank samples of deionized water were routinely collected to determine if equipment decontamination procedures were adequate, and if sampling or analysis procedures resulted in contamination. Field blanks were processed through the same equipment used for environmental samples and analyzed for concentrations of major ions, trace elements, pesticides, VOCs, and dissolved organic compounds. Source-solution blanks (blank water placed directly in a sample container) were collected for volatile and dissolved organic compounds. Trip blanks were processed for VOCs. Trip blanks are prepared samples that have not been in contact with sampling equipment and that accompany the environmental sample to determine if a sample has been contaminated in shipping, handling, and storage.
A surrogate compound has physical and chemical properties similar to analytes being measured but typically is not present in the environmental sample. A surrogate compound is added to each pesticide sample that is processed at the NWQL as part of their quality-control protocols. The percentage recovery of the surrogate compounds allows a quality check on the amount of recovery relative to the theoretical 100 percent recovery, which can be used to evaluate compound stability and the reliability of environmental concentrations of similarly volatile pesticides.

\section{METHODS OF LABORATORY ANALYSIS}

Analytical results presented in this report are listed in reference to reporting levels (RL). The NWQL uses two reporting-level conventions: minimum reporting level (MRL) and laboratory reporting level (LRL). The MRL is defined as the minimum concentration an analyte can be reliably detected using a given analytical method (Timme, 1995). The NWQL collects quality-control data on a continuing basis to evaluate selected analytical methods in order to determine long-term method detection levels (LT-MDLs) and LRLs. These reporting and detection levels are evaluated each year on the basis of the most recent quality-control data, and consequently, might change from year to year. Accordingly, concentrations are reported as $<\mathrm{RL}$ for samples in which the constituent was either not detected or did not pass identification standards.

The USGS NWQL has developed a convention for reporting the presence of many constituents at low concentrations. These estimated values (indicated by a remark code "e" in this report) are smaller than a minimum amount reliably reported by a given analytical method (the minimum reporting level), but larger than the method detection limit which was established to keep the possible occurrence of false negative or false positive error to 1 percent or less. The remark code "e" also is used to indicate quantitative uncertainty intermittently introduced by chemical interference or variable recovery efficiency. An estimated value indicates that constituents have been identified in a sample, but the reported concentration has more uncertainty than concentrations that are reported without the " $\mathrm{e}$ " remark code (concentrations higher than the minimum reporting level [Childress and others, 1999]). A summary of the laboratory 
analytical methods and references to these methods is presented in table 5 .

\section{REFERENCES CITED}

American Public Health Association and others, 1998, Standard methods for the examination of water and wastewater (20th ed.): Washington, D.C., American Public Health Association, 1,220 p.

American Society for Testing and Materials, 2002, Annual book of ASTM standards 2002, volume 11.02: Philadelphia, Pa., p. 178-182.

Arbogast, B.F., ed., 1996, Analytical methods manual for the Mineral Resource Surveys Program, U.S. Geological Survey: U.S. Geological Survey Open-File Report 96$525,248 \mathrm{p}$.

Beckwith, M.A., 2002, Selected trace-element and synthetic-organic compound data for streambed sediment from the Clark Fork-Pend Oreille and Spokane River basins, Montana, Idaho, and Washington, 1998: U.S. Geological Survey Open-File Report 02-336, $26 \mathrm{p}$.

Brenton, R.W., and Arnett, T.L., 1993, Methods of analysis by the U.S. Geological Survey National Water Quality Laboratory-Determination of dissolved organic carbon by UV-promoted persulfate oxidation and infrared spectrometry: U.S. Geological Survey OpenFile Report 92-480, 12 p.

Briggs, P.H., and Meier, A.L., 1999, The determination of forty-two elements in geological materials by inductively coupled plasma-mass spectrometry: U.S. Geological Survey Open-File Report 99-166, 15 p.

Childress, C.J.O., Foreman, W.T., Connor, B.F., Maloney, T.J., 1999, New reporting procedures based on longterm method detection levels and some considerations for interpretations of water-quality data provided by the U.S. Geological Survey National Water Quality Laboratory: U.S. Geological Survey Open-File Report 99-193, $19 \mathrm{p}$.

Clark, D.W., and Kendy, Eloise, 1991, Regional analysis of the Northern Rocky Mountains Intermontane Basins, Montana and Idaho, in Prince, K.R., and Johnson, A.I., eds., Regional aquifer systems of the United States; aquifers of the Far West: American Water Resources Association Monograph Series 16, p. 55-64.

Connor, B.F., Rose, D.L., Noriega, M.C., Murtagh, L.K., and Abney, S.R., 1998, Methods of analysis by the U.S. Geological Survey National Water Quality Laboratory-Determination of 86 volatile organic compounds in water by gas chromatography/mass spectrometry, including detections less than the reporting limits: U.S. Geological Survey Open-File Report 97-829, 78 p.
Coplen, T.B., Wildman, J.D., and Chen, J., 1991, Improvements in gaseous hydrogen-water equilibration technique for hydrogen isotope ratio analysis: Analytical Chemistry, v. 63, p. 910-912.

Crawford, J.K., and Luoma, S.N., 1993, Guidelines for studies of contaminants in biological tissues for the National Water-Quality Assessment Program: U.S. Geological Survey Open-File Report 92-494, 69 p.

Epstein, S., and Mayeda, T., 1953, Variation of the O-18 content of water from natural sources: Geochimica et Cosmochimica Acta, v. 4, p. 213-224.

Faries, L.M., 1993, Methods of analysis by the U.S. Geological Survey National Water Quality Laboratory-Determination of metals in water by inductively coupled plasma-mass spectrometry: U.S. Geological Survey Open-File Report 93-634, 28 p.

Fishman, M.J., ed., 1993, Methods of analysis by the U.S. Geological Survey National Water Quality Laboratory-Determination of inorganic and organic constituents in water and fluvial sediments: U.S. Geological Survey Open-File Report 93-125, 217 p.

Fishman, M.J., and Friedman, L.C., eds., 1989, Methods for determination of inorganic substances in water and fluvial sediments: U.S. Geological Survey Techniques of Water-Resources Investigations, book 5, chap. A1, $545 \mathrm{p}$.

Foreman, W.T., Connor, B.F., Furlong, E.T., Vaught, D.G., and Merten, L.M., 1995, Methods of analysis by the U.S. Geological Survey National Water Quality Laboratory-Determination of organochlorine pesticides and polychlorinated biphenyls in bottom sediment by dual capillary-column gas chromatography with electron-capture detection: U.S. Geological Survey Open-File Report 95-140, 78 p.

Furlong, E.T., Vaught, D.G., Merten, L.M., Foreman, W.T., and Gates, P.M., 1996, Methods of analysis by the U.S. Geological Survey National Water Quality Laboratory-Determination of semivolatile organic compounds in bottom sediment by solvent extraction, gel permeation chromatographic fractionation, and capillary-column gas chromatography/mass spectrometry: U.S. Geological Survey Open-File Report 95-719, $67 \mathrm{p}$.

Furlong, E.T., Anderson, B.D., Werner, S.L., Soliven, P.P., Coffey, L.J., Burkhardt, M.R., 2001, Methods of analysis by the U. S. Geological Survey National Water Quality Laboratory-Determination of pesticides in water by graphitized carbon-based solid-phase extraction and high-performance liquid chromatography/mass spectrometry: U.S. Geological Survey Water-Resources Investigations Report 014134, $73 \mathrm{p}$.

18 Water-Quality, Streambed-Sediment, and Biological Data from the Clark Fork-Pend Oreille and Spokane River Basins, Montana, Idaho, and Washington, 1998-2001 
Garbarino, J.R., 1999, Methods of analysis by the U.S. Geological Survey National Water Quality Laboratory-Determination of dissolved arsenic, boron, lithium, selenium, strontium, thallium, and vanadium using inductively coupled plasma-mass spectrometry: U.S. Geological Survey Open-File Report 99-093, 31 p.

Garbarino, J.R., and Struzeski, T.M., 1998, Methods of analysis by the U.S. Geological Survey National Water Quality Laboratory-Determination of elements in whole-water digests using inductively coupled plasmaoptical emission spectrometry and inductively coupled plasma-mass spectrometry: U.S. Geological Survey Open-File Report 98-165, 101 p.

Gerstel, W.J., and Palmer, S.P., 1994, Geologic and geophysical mapping of the Spokane AquiferRelevance to growth management: Washington Geology, v. 22, p. 18-24.

Gurtz, M.E., 1994, Design of biological components of the National Water-Quality Assessment (NAWQA) Program, in Loeb, S.L., and Spacie, Anne, eds., Biological monitoring of aquatic systems: Boca Raton, Fla., Lewis Publishers, p. 323-354.

Hirsch, R.M., Alley, W.M., and Wilber, W.G., 1988, Concepts for a National Water-Quality Assessment Program: U.S. Geological Survey Circular 1021, 42 p.

Hoffman, G.L., 1996, Methods of analysis by the U.S. Geological Survey National Water Quality Laboratory-Preparation procedure for aquatic biological material determined for trace metals: U.S. Geological Survey Open-File Report 96-362, 42 p.

Hoffman, G.L., Fishman, M.J., and Garbarino, J.R., 1996, Methods of analysis by the U.S. Geological Survey National Water Quality Laboratory-In-bottle acid digestion of whole-water samples: U.S. Geological Survey Open-File Report 96-225, 28 p.

Horowitz, A.J., Demas, C.R., Fitzgerald, K.K., Miller, T.L., and Rickert, D.A., 1994, U.S. Geological Survey protocol for the collection and processing of surfacewater samples for the subsequent determination of inorganic constituents in filtered water: U.S. Geological Survey Open-File Report 94-539, 57 p.

Jones, S.R., and Garbarino, J.R., 1999, Methods of analysis by the U.S. Geological Survey National Water Quality Laboratory-Determination of arsenic and selenium in water and sediment by graphite furnace-atomic absorption spectrometry: U.S. Geological Survey Open-File Report 98-639, 39 p.

Jones, S.R., and McLain, B.J., 1997, Methods of analysis by the U.S. Geological Survey National Water Quality Laboratory-Determination of molybdenum in water by graphite furnace atomic absorption spectrophotometry: U.S. Geological Survey Open-File Report 97-198, 32 p.
Kendy, Eloise, and Tresch, R.E., 1996, Geographic, geologic, and hydrologic summaries of intermontane basins of the Northern Rocky Mountains, Montana: U.S. Geological Survey Water-Resources Investigations Report 96-4025, 233 p.

Koterba, M.T., Wilde, F.D., and Lapham, W.W., 1995, Ground-water data collection protocols and procedures for the National Water-Quality Assessment ProgramCollection and documentation of water-quality samples and related data: U.S. Geological Survey Open-File Report 95-399, 113 p.

Leahy, P.P., Rosenshein, J.S., and Knopman, D.S., 1990, Implementation plan for the National Water-Quality Assessment Program: U.S. Geological Survey OpenFile Report 90-174, 10 p.

Leiker, T.J., Madsen, J.E., Deacon, J.R., and Foreman, W.T., 1995, Methods of analysis by the U.S. Geological Survey National Water Quality LaboratoryDetermination of chlorinated pesticides in aquatic tissue by capillary-column gas chromatography with electron-capture detection: U.S. Geological Survey Open-File Report 94-710, 42 p.

Maret, T.R., and Dutton, D.M., 1999, Summary of information on synthetic organic compounds and trace elements in tissue of aquatic biota, Clark Fork-Pend Oreille and Spokane River basins, Montana, Idaho, and Washington, 1974-96: U.S. Geological Survey WaterResources Investigations Report 98-4254, 55 p.

Marti, P.B., and Garriques, R.S., 2001, Spokane River/ aquifer interaction project results, May-November 1999: Washington State Department of Ecology publication 01-03-024, $40 \mathrm{p}$.

McLain, B.J., 1993, Methods of analysis by the U.S. Geological Survey National Water Quality Laboratory-Determination of chromium in water by graphite furnace atomic absorption spectrophotometry: U.S. Geological Survey Open-File Report 93-449, 16 p.

Meador, M.R., Cuffney, T.F., and Gurtz, M.E., 1993, Methods for sampling fish communities as part of the National Water-Quality Assessment Program: U.S. Geological Survey Open-File Report 93-104, 40 p.

Mueller, D.K., Martin, J.D., and Lopes, T.J., 1997, Qualitycontrol design for surface-water sampling in the National Water-Quality Assessment Program: U.S. Geological Survey Open-File Report 97-223, 17 p.

Omernik, J.M., and Gallant, A.L., 1986, Ecoregions of the Pacific Northwest: Corvallis, Ore., Corvallis Environmental Research Laboratory, U.S. Environmental Protection Agency, EPA 600/3-86/033, $39 \mathrm{p}$. 
Ostlund, H.G., and Dorsey, H.G., 1975, Rapid electrolytic enrichment of hydrogen gas proportional counting of tritium: International Conference on Low Radioactivity Measurement and Applications, High Tatras, Czechoslovakia, October 1975 [Proceedings], $6 \mathrm{p}$.

Patton, C.J., and Truitt, E.P., 1992, Methods of analysis by the U.S. Geological Survey National Water Quality Laboratory-Determination of total phosphorus by a Kjeldahl digestion method and an automated colorimetric finish that includes dialysis: U.S. Geological Survey Open File Report 92-146, 39 p.

Patton, C.J., and Truitt, E.P., 2000, Methods of analysis by the U.S. Geological Survey National Water-Quality Laboratory-Determination of ammonium plus organic nitrogen by a Kjeldahl digestion method and an automated photometric finish that includes digest cleanup by gas diffusion: U.S. Geological Survey Open-File Report 00-170, 31 p.

Porter, S.D., Cuffney, T.F, Gurtz, M.E., and Meador, M.R., 1993, Methods for collecting algal samples as part of the National Water-Quality Assessment Program: U.S. Geological Survey Open-File Report 93-409, 39 p.

Pritt, J.W., and Raese, J.W., 1995, Quality assurance/quality control manual-National Water Quality Laboratory: U.S. Geological Survey Open-File Report 95-443, 35 p.

Rose, D.J., and Schroeder, M.P., 1995, Methods of analysis by the U.S. Geological Survey National Water Quality Laboratory-Determination of volatile organic compounds in water by purge and trap capillary gas chromatography/mass spectrometry: U.S. Geological Survey Open-File Report 94-708, 26 p.

Shelton, L.R., 1994, Field guide for collecting and processing stream-water samples for the National Water-Quality Assessment Program: U.S. Geological Survey Open-File Report 94-455, 42 p.

Shelton, L.R., 1997, Field guide for collecting samples for analysis of volatile organic compounds in stream water for the National Water-Quality Assessment Program: U.S. Geological Survey Open-File Report 97-401, 14 p.

Shelton, L.R., and Capel, P.D., 1994, Guidelines for collecting and processing samples of streambed sediment for the analysis of trace elements and organic contaminants for the National Water-Quality Assessment Program: U.S. Geological Survey OpenFile Report 94-458, $20 \mathrm{p}$.

Stoffel, K.L., Joseph, N.L., Waggoner, S.Z., Gulick, C.W., Korosec, M.A., and Bunning, B.B., 1991, Geologic map of Washington-northeast quadrant: Washington Division of Geology and Earth Resources Geologic Map GM-39, sheet 1, scale 1:250,000.
Thatcher, L.L., Janzer, V.J., and Edwards, K.W., 1977, Methods for determination of radioactive substances in water and fluvial substances: U.S. Geological Survey Techniques of Water-Resources Investigations, book 5, chap. A5, $95 \mathrm{p}$.

Timme, P.J., 1995, National Water Quality Laboratory 1995 services catalog: U.S. Geological Survey Open-File Report 95-352, $120 \mathrm{p}$.

Tornes, L.H., 1997, National Water-Quality Assessment Program, Northern Rockies Intermontane Basins: U.S. Geological Survey Fact Sheet 158-97, 4 p.

Tuck, L.K., Briar, D.W., and Clark, D.W., 1996, Geologic history and hydrogeologic units of intermontane basins of the northern Rocky Mountains, Montana and Idaho: U.S. Geological Survey Hydrologic Investigations Atlas HA-738-A, 2 sheets, scale 1:750,000.

U.S. Environmental Protection Agency, 1980a, Prescribed procedures for measurement of radioactivity in drinking water-Section 7 Radium-226 in drinking water radon emanation technique, method 903.1: EPA600/4-80-032, pages 38-48.

U.S. Environmental Protection Agency, 1980b, Prescribed procedures for measurement of radioactivity in drinking water-Section 8 radium-228 in drinking water radon emanation technique, method 904.0: EPA600/4-80-032, pages 49-57.

U.S. Environmental Protection Agency, 1993, Methods for the determination of inorganic substances in environmental samples: Cincinnati, Ohio, Environmental Monitoring and Support Laboratory, EPA/600/R-93/100, 79 p.

U.S. Environmental Protection Agency, 1997, Guidelines establishing test procedures for the analysis of pollutants (Appendix B, Part 136, Definitions and procedures for the determination of the method detection limit): U.S. Code of Federal Regulations, Title 40, revised July 1, 1997, p. 265-267.

U.S. Environmental Protection Agency, 2000, An overview of the Sole Source Aquifer Protection program in EPA Region 10, accessed February 14, 2001 at http:// yosemite.epa.gov/r10/WATER.NSF/

Wilde, F.D., Radtke, D.B., Gibs, Jacob, and Iwatsubo, R.T., 1999, Processing of water samples in National field manual for the collection of water-quality data: U.S. Geological Survey Techniques of Water-Resources Investigations, book 9, chap. A5 [variously paged].

Zaugg, S.D., Sandstrom, M.W., Smith, S.G., and Fehlberg, K.M., 1995, Methods of analysis by the U.S. Geological Survey National Water Quality Laboratory-Determination of pesticides in water by C-18 solid-phase extraction and capillary-column gas chromatography/mass spectrometry with selected-ion monitoring: U.S. Geological Survey Open-File Report 95-181, $60 \mathrm{p}$. 
DATA 
22 Water-Quality, Streambed-Sediment, and Biological Data from the Clark Fork-Pend Oreille and Spokane River Basins, Montana, Idaho, and Washington, 1998-2001 
Table 5. Summary of analytical method references, analyzing laboratories, and field processing of samples for chemical constituents in water, streambed sediment, fish tissue, and benthic algae, Northern Rockies Intermontane Basins study unit

[U.S. Geological Survey analytical schedule numbers are used for a suite of constituents, whereas laboratory codes (labcode) are used for individual constituents. Laboratory: BR, Bureau of Reclamation Laboratory, Boise, Idaho; Duke, Duke Engineering and Services, Westborough, Mass.; IL, U.S Geological Survey Isotope Laboratory, Reston, Va.; ITL, U.S. Geological Survey Isotope Tracers Laboratory, Menlo Park, Calif.; NWQL, U.S. Geological Survey National Water Quality Laboratory, Denver, Colo; Quanterra, Quanterra, Inc., Richland, Wash. Abbreviations: $\mathrm{C}-18$, carbon-18; DOC, dissolved organic carbon; $\mathrm{HCl}$, hydrochloric acid; $\mu$ m, micrometers; mm, millimeter; $\mathrm{HNO}_{3}$, nitric acid; $\mathrm{N}_{2}$, nitrogen gas; USEPA, U.S. Environmental Protection Agency; USGS, U.S. Geological Survey; UV, ultraviolet; VOC, volatile organic compounds]

\begin{tabular}{|c|c|c|c|c|c|}
\hline $\begin{array}{l}\text { Constituent or } \\
\text { constituent group }\end{array}$ & Analysis Method & Reference & $\begin{array}{l}\text { Labora- } \\
\text { tory }\end{array}$ & $\begin{array}{l}\text { Filtration } \\
\text { or sieve } \\
\text { size }\end{array}$ & $\begin{array}{l}\text { Preser- } \\
\text { vation }\end{array}$ \\
\hline \multicolumn{6}{|c|}{ Water } \\
\hline Major ions (USGS schedule 2750, 2701, 1256) & $\begin{array}{l}\text { Atomic absorption } \\
\text { spectrometry }\end{array}$ & $\begin{array}{l}\text { Fishman and Friedman (1989); } \\
\text { Fishman (1993) }\end{array}$ & NWQL & $0.45-\mu \mathrm{m}$ capsule & $\mathrm{HNO}_{3}$ \\
\hline Nutrients (USGS schedule 2752, 1119, 1257) & Various methods & $\begin{array}{l}\text { Patton and Truitt }(1992,2000) \\
\text { USEPA (1993) }\end{array}$ & NWQL & $0.45-\mu \mathrm{m}$ capsule & chilled $^{1}$ \\
\hline DOC (USGS schedule 2085) & $\begin{array}{l}\text { UV-promoted persulfate } \\
\text { oxidation and infared } \\
\text { spectrometry }\end{array}$ & Brenton and Arnett (1993) & NWQL & $0.45-\mu \mathrm{m}$ silver & chilled \\
\hline $\begin{array}{l}\text { Trace elements (USGS schedule 2703, } 2705 \text {, } \\
2710,1664 \text { ) }\end{array}$ & Various methods & $\begin{array}{l}\text { Fishman and Friedman (1989); } \\
\text { Faries (1993); Fishman (1993); } \\
\text { McLain (1993); Hoffman and } \\
\text { others (1996); Jones and McLain } \\
\text { (1997); Garbarino and Struzeski } \\
\text { (1998); Garbarino (1999); Jones } \\
\text { and Garbarino (1999) }\end{array}$ & NWQL & $\begin{array}{l}0.45-\mu \mathrm{m} \text { capsule } \\
0.10-\mu \mathrm{m} \text { capsule } \\
0.001-\mu \mathrm{m} \text { tangential }\end{array}$ & $\mathrm{HNO}_{3}$ \\
\hline Pesticides (USGS schedule 2001) & $\begin{array}{l}\text { Solid-phase extraction } \\
\text { technology using C-18 } \\
\text { cartridge and gas } \\
\text { chromotography/mass } \\
\text { spectromography }\end{array}$ & Zaugg and others (1995) & NWQL & $0.7-\mu \mathrm{m}$ glass fiber & chilled \\
\hline Pesticides (USGS schedule 2060) & $\begin{array}{l}\text { Graphitized carbon-based } \\
\text { solid-phase extraction and } \\
\text { high-performance liquid } \\
\text { chromatography/mass } \\
\text { spectrometry }\end{array}$ & Furlong and others (2001) & NWQL & $0.7-\mu \mathrm{m}$ glass fiber & chilled \\
\hline VOC (USGS schedule 2020) & $\begin{array}{l}\text { Purge and trap capillary gas } \\
\text { chromatography/mass } \\
\text { spectrometry }\end{array}$ & $\begin{array}{l}\text { Rose and Schroeder (1995); } \\
\text { Conner and others (1998) }\end{array}$ & NWQL & none & $\begin{array}{l}\mathrm{HCl}, \\
\text { chilled }\end{array}$ \\
\hline Radium-224 (USGS schedule 1263) & Alpha spectrometry & $\begin{array}{l}\text { American Society for Testing and } \\
\text { Materials (2002) }\end{array}$ & Duke & $0.45-\mu \mathrm{m}$ & $\mathrm{HNO}_{3}$ \\
\hline Radium-226 (USGS schedule 1263) & Radon emanation method & USEPA (1980a) & Quanterra & $0.45-\mu \mathrm{m}$ & $\mathrm{HNO}_{3}$ \\
\hline Radium-228 (USGS schedule 1263) & Gas proportional counting & USEP (1980b) & Quanterra & $0.45-\mu \mathrm{m}$ & $\mathrm{HNO}_{3}$ \\
\hline $\begin{array}{l}\text { Gross alpha and beta radioactivity (USGS schedule } \\
1263 \text { ) }\end{array}$ & Residue procedure & Thatcher and others (1977) & NWQL & $0.45-\mu \mathrm{m}$ & $\mathrm{HNO}_{3}$ \\
\hline Radon (USGS labcode 1369) & Liquid scintillation & Thatcher and others (1977) & NWQL & none & none \\
\hline Oxygen-18/Oxygen-16 & $\mathrm{CO}_{2}$ equilibrium technique & Epstein and Mayeda (1953) & IL & none & none \\
\hline Hydrogen-2/hydrogen-1 & $\begin{array}{l}\text { Hydrogen equilibrium } \\
\text { technique }\end{array}$ & Coplen and others (1991) & IL & none & none \\
\hline Tritium (USGS labcode 1565) & $\begin{array}{l}\text { Electrolytic enrichment } \\
\text { with gas counting }\end{array}$ & Ostlund and Dorsey (1975) & ITL & none & none \\
\hline \multicolumn{6}{|c|}{ Streambed Sediment } \\
\hline Trace elements (USGS schedule 2420) & Various methods & $\begin{array}{l}\text { Arbogast (1996) } \\
\text { Briggs and Meier (1999) }\end{array}$ & NWQL & $<63-\mu \mathrm{m}$ & none \\
\hline Organic compounds (USGS schedule 2500) & Various methods & $\begin{array}{l}\text { Forman and others (1995) } \\
\text { Furlong and others (1996) }\end{array}$ & NWQL & $<2-\mathrm{mm}$ & chilled \\
\hline \multicolumn{6}{|c|}{ Fish Tissue } \\
\hline Trace elements (USGS schedule 2200) & Various methods & Hoffman (1996) & NWQL & none & freeze \\
\hline Organic compounds (USGS schedule 2101) & $\begin{array}{l}\text { Capillary-column gas } \\
\text { chromatography with } \\
\text { electron-capture detection }\end{array}$ & Leiker and others (1995) & NWQL & none & freeze \\
\hline \multicolumn{6}{|c|}{ Benthic algae (periphyton) } \\
\hline Chlorophyll periphyton (Method 10200h) & Spectrophotometry & $\begin{array}{l}\text { American Public Health } \\
\text { Association and others (1998) }\end{array}$ & $\mathrm{BR}$ & $0.7-\mu \mathrm{m}$ glass fiber & freeze \\
\hline
\end{tabular}

\footnotetext{
${ }^{1}$ Unfiltered nutrient samples were preserved with $\mathrm{H}_{2} \mathrm{SO}_{4}$ (sulfuric acid).
} 
Table 6. Streamflow, physical, and major-ion concentration data for surface-water samples collected at fixed water-quality sites, Northern Rockies Intermontane Basins study unit, 1998-2001

[See figure 1 for site locations. Abbreviations: $\mathrm{ft}^{3} / \mathrm{s}$, cubic feet per second; ${ }^{\circ} \mathrm{C}$, degrees Celsius; e, estimated; Fb, field blank; $\mu \mathrm{S} / \mathrm{cm}, \mathrm{microsiemens} \mathrm{per}$ centimeter at $25^{\circ} \mathrm{C} ; \mathrm{mg} / \mathrm{L}$, milligrams per liter; mm, millimeters; R, replicate. Symbols: <, less than reporting level; --, no data]

\begin{tabular}{|c|c|c|c|c|c|c|c|c|c|c|c|c|c|c|}
\hline Date & Time & $\begin{array}{c}\text { Dis- } \\
\text { charge, } \\
\text { instan- } \\
\text { taneous } \\
\left(\mathrm{ft}^{3} / \mathrm{s}\right)\end{array}$ & $\begin{array}{c}\text { Sedi- } \\
\text { ment, } \\
\text { sus- } \\
\text { pended } \\
(\mathrm{mg} / \mathrm{L})\end{array}$ & $\begin{array}{c}\text { Sedi- } \\
\text { ment, } \\
\text { sus- } \\
\text { pended } \\
\text { (percent } \\
\text { finer } \\
\text { than } \\
0.062 \\
\text { mm) }\end{array}$ & $\begin{array}{c}\text { Specific } \\
\text { conduct- } \\
\text { ance, } \\
\text { field } \\
(\mu \mathrm{S} / \mathrm{cm})\end{array}$ & $\begin{array}{l}\text { pH, } \\
\text { field } \\
\text { (stan- } \\
\text { dard } \\
\text { units) }\end{array}$ & $\begin{array}{l}\text { Tem- } \\
\text { per- } \\
\text { ature, } \\
\text { water } \\
\left({ }^{\circ} \mathrm{C}\right)\end{array}$ & $\begin{array}{c}\text { Hard- } \\
\text { ness } \\
(\mathrm{mg} / \mathrm{L} \\
\text { as } \\
\left.\mathrm{CaCO}_{3}\right)\end{array}$ & $\begin{array}{l}\text { Calci- } \\
\text { um, } \\
\text { total } \\
(\mathrm{mg} / \mathrm{L})\end{array}$ & $\begin{array}{l}\text { Calci- } \\
\text { um, } \\
\text { dis- } \\
\text { solved } \\
(\mathrm{mg} / \mathrm{L})\end{array}$ & $\begin{array}{c}\text { Mag- } \\
\text { nesi- } \\
\text { um, } \\
\text { total } \\
(\mathrm{mg} / \mathrm{L})\end{array}$ & $\begin{array}{c}\text { Mag- } \\
\text { nesium, } \\
\text { dis- } \\
\text { solved } \\
(\mathrm{mg} / \mathrm{L})\end{array}$ & $\begin{array}{c}\text { Sodium } \\
\text { adsorp- } \\
\text { tion } \\
\text { ratio }\end{array}$ & $\begin{array}{c}\text { So- } \\
\text { dium, } \\
\text { total } \\
(\mathrm{mg} / \mathrm{L})\end{array}$ \\
\hline \multicolumn{15}{|c|}{ SITE 3 12334550-CLARK FORK AT TURAH BRIDGE, NEAR BONNER, MONT. } \\
\hline $11 / 05 / 98$ & 1000 & 988 & 4 & -- & 371 & 8.5 & 4.5 & 172 & 51 & 49 & 13 & 12 & 0.3 & 10 \\
\hline $11 / 05 / 98^{\mathbf{F b}}$ & 1005 & -- & -- & -- & -- & -- & -- & -- & -- & .003 & -- & $<.001$ & -- & -- \\
\hline $12 / 15 / 98$ & 1145 & 975 & 7 & -- & 376 & 7.5 & 1.5 & 177 & -- & 50 & -- & 13 & .3 & -- \\
\hline $01 / 27 / 99$ & 1245 & 831 & 8 & -- & 413 & 7.8 & 0.0 & 185 & -- & 52 & -- & 14 & .3 & -- \\
\hline $03 / 25 / 99$ & 1215 & 1,740 & 41 & 83 & 292 & 8.1 & 7.0 & 128 & -- & 36 & -- & 9.4 & .3 & -- \\
\hline 04/26/99 & 1115 & 1,800 & 21 & 80 & 248 & 8.4 & 11.0 & 117 & 32 & 32 & 8.4 & 8.8 & .3 & 6.0 \\
\hline $05 / 12 / 99$ & 0915 & 1,780 & 17 & 81 & 259 & 8.0 & 9.0 & 117 & -- & 33 & -- & 8.7 & .3 & -- \\
\hline $05 / 25 / 99$ & 1145 & 3,440 & 131 & 69 & 153 & 7.7 & 13.5 & 64 & 20 & 18 & 5.6 & 4.8 & .2 & 4.0 \\
\hline 06/07/99 & 1230 & 5,280 & 64 & 74 & 157 & 7.7 & 9.0 & 74 & 20 & 21 & 5.2 & 5.2 & .2 & 4.2 \\
\hline 06/19/99 & 1130 & 4,120 & 39 & 61 & 175 & 7.8 & 14.0 & 77 & 21 & 22 & 5.5 & 5.5 & .2 & 4.2 \\
\hline $07 / 21 / 99$ & 1015 & 1,060 & 4 & 48 & 305 & 7.9 & 15.0 & 133 & 37 & 37 & 9.8 & 9.9 & .3 & 7.2 \\
\hline 08/19/99 & 1000 & 750 & 9 & 90 & 352 & 7.8 & 17.0 & 160 & 45 & 44 & 12 & 12 & .3 & 9.7 \\
\hline $10 / 20 / 99$ & 1030 & 841 & 9 & 91 & 375 & 7.5 & 5.5 & 183 & 51 & 52 & 13 & 13 & .3 & 9.8 \\
\hline $12 / 03 / 99^{\mathbf{F b}}$ & 1030 & -- & -- & -- & -- & -- & -- & -- & -- & .007 & -- & $<.001$ & -- & -- \\
\hline $12 / 03 / 99$ & 1100 & 852 & 6 & 95 & 377 & 7.9 & 2.5 & 181 & 52 & 51 & 13 & 13 & .3 & 10 \\
\hline $01 / 26 / 00$ & 0945 & 770 & -- & -- & 390 & 8.0 & 2.0 & 179 & 52 & 50 & 14 & 13 & .3 & 11 \\
\hline 03/08/00 & 1045 & 929 & 14 & 82 & 355 & 8.2 & 5.0 & 170 & 49 & 47 & 12 & 12 & .3 & 9.4 \\
\hline $03 / 08 / 00^{\mathbf{R}}$ & 1046 & 929 & 13 & 85 & 356 & 8.2 & 4.5 & 161 & 48 & 45 & 12 & 12 & .3 & 9.4 \\
\hline $04 / 12 / 00$ & 0900 & 952 & 16 & 83 & 330 & 8.1 & 9.0 & 149 & 42 & 42 & 11 & 11 & .3 & 8.0 \\
\hline $05 / 25 / 00$ & 0830 & 1,230 & 13 & 63 & 198 & 8.0 & 11.0 & 93 & 24 & 26 & 6.5 & 7.0 & .2 & 4.4 \\
\hline $06 / 21 / 00$ & 1015 & 884 & 6 & 74 & 275 & 8.4 & 13.0 & 121 & 34 & 33 & 9.5 & 9.2 & .3 & 6.4 \\
\hline $07 / 31 / 00$ & 1045 & 287 & 7 & 67 & 301 & 8.4 & 19.0 & 146 & 36 & 39 & 11 & 12 & .3 & 6.6 \\
\hline $08 / 29 / 00$ & 1100 & 287 & 4 & 77 & 344 & 8.3 & 13.0 & 160 & 43 & 43 & 13 & 12 & .3 & 7.8 \\
\hline $11 / 07 / 00^{\mathbf{F b}}$ & 1300 & -- & -- & -- & -- & -- & -- & -- & -- & .006 & -- & $<.001$ & -- & -- \\
\hline $11 / 07 / 00$ & 1400 & 820 & 5 & 83 & 391 & 8.5 & 4.0 & 179 & -- & 52 & -- & 12 & .4 & -- \\
\hline $12 / 20 / 00$ & 1545 & 622 & 4 & 78 & 411 & 8.3 & .5 & 192 & -- & 54 & -- & 14 & .3 & -- \\
\hline 01/30/01 & 1100 & 487 & 3 & 88 & 411 & 8.5 & .5 & 190 & -- & 54 & -- & 14 & .3 & -- \\
\hline $03 / 12 / 01^{\mathbf{F b}}$ & 1040 & -- & -- & -- & -- & -- & -- & -- & -- & $<.002$ & -- & $<.001$ & -- & -- \\
\hline $03 / 12 / 01$ & 1100 & 830 & 17 & 91 & 410 & 8.3 & 3.0 & 187 & -- & 53 & -- & 13 & .3 & -- \\
\hline 04/10/01 & 1030 & 874 & 9 & 80 & 404 & 8.3 & 4.5 & 187 & -- & 53 & -- & 13 & .3 & -- \\
\hline $05 / 22 / 01$ & 1100 & 2,040 & 13 & 76 & 214 & 8.3 & 11.0 & 95 & -- & 27 & -- & 6.9 & .2 & -- \\
\hline \multicolumn{15}{|c|}{ SITE 5 12352500--BITTERROOT RIVER NEAR MISSOULA, MONT. } \\
\hline 03/04/99 & 1630 & 1,150 & 6 & 92 & 133 & 8.1 & 4.0 & 57 & -- & 16 & -- & 3.9 & .3 & -- \\
\hline 03/24/99 & 0930 & 2,150 & 31 & 71 & 96 & 7.8 & 8.5 & 40 & 11 & 11 & 2.8 & 2.8 & .2 & 3.5 \\
\hline 04/15/99 & 1300 & 1,530 & 8 & 85 & 102 & 7.8 & 7.0 & 44 & -- & 12 & -- & 3.1 & .3 & -- \\
\hline 05/13/99 & 0945 & 2,270 & 8 & 68 & 90 & 7.6 & 9.0 & 36 & 11 & 10 & 2.6 & 2.4 & .2 & 3.3 \\
\hline $05 / 25 / 99$ & 0915 & 10,600 & 159 & 59 & 39 & 7.4 & 11.0 & 15 & -- & 4.3 & -- & .96 & .2 & -- \\
\hline 06/07/99 & 1615 & 12,000 & 34 & 73 & 50 & 7.3 & 9.5 & 22 & 6.1 & 6.4 & 1.5 & 1.4 & .2 & 2.0 \\
\hline 06/19/99 & 1445 & 13,700 & 48 & 64 & 39 & 7.1 & 13.0 & 16 & 4.8 & 4.7 & 1.2 & 1.0 & .2 & 1.6 \\
\hline $07 / 21 / 99$ & 1530 & 2,010 & 2 & 74 & 89 & 8.1 & 18.0 & 37 & 11 & 11 & 2.5 & 2.5 & .2 & 3.2 \\
\hline 08/19/99 & 1500 & 1,180 & 3 & 86 & 138 & 7.8 & 20.5 & 57 & 17 & 17 & 3.8 & 3.8 & .3 & 5.0 \\
\hline $10 / 20 / 99$ & 1430 & 760 & 2 & 75 & 158 & 8.6 & 9.0 & 70 & 20 & 20 & 4.7 & 4.7 & .3 & 6.0 \\
\hline $12 / 02 / 99$ & 1600 & 980 & 3 & 80 & 127 & 7.9 & 4.0 & 57 & -- & 17 & -- & 3.8 & .3 & -- \\
\hline
\end{tabular}


Table 6. Streamflow, physical, and major-ion concentration data for surface-water samples collected at fixed water-quality sites, Northern Rockies Intermontane Basins study unit, 1998-2001 (Continued)

\begin{tabular}{|c|c|c|c|c|c|c|c|c|c|c|c|c|}
\hline Date & $\begin{array}{c}\text { Sodium, } \\
\text { dissolved } \\
(\mathrm{mg} / \mathrm{L})\end{array}$ & $\begin{array}{c}\text { Potas- } \\
\text { sium, } \\
\text { total } \\
(\mathrm{mg} / \mathrm{L})\end{array}$ & $\begin{array}{l}\text { Potas- } \\
\text { sium, } \\
\text { dissolved } \\
(\mathrm{mg} / \mathrm{L})\end{array}$ & $\begin{array}{c}\text { Alka- } \\
\text { linity, } \\
\text { field } \\
(\mathrm{mg} / \mathrm{L} \text { as } \\
\left.\mathrm{CaCO}_{3}\right)\end{array}$ & $\begin{array}{c}\text { Bicar- } \\
\text { bonate, } \\
\text { dissolved } \\
(\mathrm{mg} / \mathrm{L} \text { as } \\
\left.\mathrm{HCO}_{3}\right)\end{array}$ & $\begin{array}{c}\text { Carbo- } \\
\text { nate, } \\
\text { dissolved } \\
(\mathrm{mg} / \mathrm{L} \text { as } \\
\left.\mathrm{CO}_{3}\right)\end{array}$ & $\begin{array}{c}\text { Sulfate, } \\
\text { dissolved } \\
(\mathrm{mg} / \mathrm{L} \text { as } \\
\left.\mathrm{SO}_{4}\right)\end{array}$ & $\begin{array}{c}\text { Chloride, } \\
\text { dissolved } \\
(\mathrm{mg} / \mathrm{L})\end{array}$ & $\begin{array}{c}\text { Fluoride, } \\
\text { dissolved } \\
(\mathrm{mg} / \mathrm{L})\end{array}$ & $\begin{array}{c}\text { Silica, } \\
\text { dissolved } \\
(\mathrm{mg} / \mathrm{L} \text { as } \\
\left.\mathrm{SiO}_{2}\right)\end{array}$ & $\begin{array}{c}\text { Dissolved } \\
\text { solids, } \\
\text { sum of } \\
\text { constit- } \\
\text { uents } \\
(\mathrm{mg} / \mathrm{L})\end{array}$ & $\begin{array}{l}\text { Dissolved } \\
\text { solids, } \\
\text { residue at } \\
180^{\circ} \mathrm{C} \\
(\mathrm{mg} / \mathrm{L})\end{array}$ \\
\hline \multicolumn{13}{|c|}{ SITE 3 12334550--CLARK FORK AT TURAH BRIDGE, NEAR BONNER, MONT. } \\
\hline $11 / 05 / 98$ & 9.8 & 2.6 & 2.7 & 142 & 173 & 0 & 54 & 4.0 & 0.43 & 15 & 232 & 247 \\
\hline $11 / 05 / 98^{\mathbf{F b}}$ & $<.02$ & -- & -- & -- & -- & -- & -- & -- & -- & $<.02$ & -- & -- \\
\hline $12 / 15 / 98$ & 9.3 & -- & 2.6 & 131 & 160 & 0 & 54 & 4.0 & .40 & 16 & 228 & 242 \\
\hline 01/27/99 & 11 & -- & 2.7 & 142 & 173 & 0 & 64 & 4.5 & .45 & 17 & 251 & 269 \\
\hline 03/25/99 & 8.0 & -- & 2.7 & 102 & 124 & 0 & 38 & 4.6 & .24 & 15 & 174 & 193 \\
\hline $04 / 26 / 99$ & 6.7 & 1.9 & 1.9 & 85 & 104 & 0 & 33 & 3.4 & .22 & 14 & 151 & 166 \\
\hline $05 / 12 / 99$ & 6.7 & -- & 1.8 & 86 & 105 & 0 & 35 & 3.2 & .25 & 14 & 154 & 169 \\
\hline 05/25/99 & 3.9 & 1.7 & 1.6 & 46 & 56 & 0 & 18 & 2.0 & .15 & 12 & 88 & 109 \\
\hline 06/07/99 & 4.3 & 1.5 & 1.4 & 56 & 69 & 0 & 17 & 1.4 & .14 & 13 & 98 & 156 \\
\hline 06/19/99 & 4.2 & 1.5 & 1.5 & 63 & 77 & 0 & 17 & 1.9 & .16 & 12 & 102 & 113 \\
\hline $07 / 21 / 99$ & 7.2 & 2.3 & 2.3 & 104 & 129 & 0 & 36 & 3.2 & .25 & 15 & 174 & 190 \\
\hline 08/19/99 & 9.6 & 2.8 & 2.9 & 130 & 159 & 0 & 47 & 3.6 & .29 & 16 & 214 & 225 \\
\hline $10 / 20 / 99$ & 9.9 & 2.8 & 2.7 & 138 & 168 & 0 & 57 & 4.2 & .37 & 18 & 239 & 247 \\
\hline $12 / 03 / 99^{\mathbf{F b}}$ & $<.02$ & -- & -- & -- & -- & -- & -- & -- & -- & .02 & -- & -- \\
\hline $12 / 03 / 99$ & 10 & 2.6 & 2.5 & 130 & 159 & 0 & 60 & 7.1 & .38 & 16 & 240 & 244 \\
\hline $01 / 26 / 00$ & 10 & 2.7 & 2.3 & 148 & 180 & 0 & 62 & 4.4 & .41 & 16 & 247 & 249 \\
\hline $03 / 08 / 00$ & 9.7 & 2.8 & 2.6 & 133 & 162 & 0 & 54 & 3.6 & .35 & 14 & 224 & 227 \\
\hline $03 / 08 / 00^{\mathbf{R}}$ & 9.0 & 2.8 & 2.5 & 128 & 156 & 0 & 54 & 3.7 & .38 & 14 & 217 & 239 \\
\hline $04 / 12 / 00$ & 8.2 & 2.0 & 2.3 & 117 & 142 & 0 & 47 & 3.2 & .37 & 13 & 197 & 210 \\
\hline $05 / 25 / 00$ & 4.4 & 1.5 & 1.5 & 74 & 90 & 0 & 22 & 1.8 & .19 & 11 & 119 & 123 \\
\hline $06 / 21 / 00$ & 6.9 & 2.1 & 2.1 & 102 & 124 & 0 & 33 & 2.9 & .30 & 10 & 160 & 177 \\
\hline 07/31/00 & 7.0 & 2.1 & 2.2 & 110 & 134 & 0 & 43 & 3.4 & .31 & 13 & 186 & 195 \\
\hline $08 / 29 / 00$ & 8.1 & 2.5 & 2.4 & 123 & 150 & 0 & 51 & 3.3 & .32 & 13 & 208 & 216 \\
\hline $11 / 07 / 00^{\mathbf{F b}}$ & $<.02$ & -- & -- & -- & -- & -- & -- & -- & -- & $<.02$ & -- & -- \\
\hline $11 / 07 / 00$ & 11 & -- & 2.8 & 136 & 162 & 2 & 61 & 5.1 & .37 & 16 & 241 & 255 \\
\hline $12 / 20 / 00$ & 10 & -- & 2.7 & 142 & 174 & 0 & 67 & 5.8 & .41 & 16 & 257 & 267 \\
\hline 01/30/01 & 10 & -- & 2.6 & 141 & 172 & 0 & 68 & 4.9 & .40 & 15 & 252 & 283 \\
\hline $03 / 12 / 01^{\mathbf{F b}}$ & $<.02$ & -- & -- & -- & -- & -- & -- & -- & -- & $<.02$ & -- & -- \\
\hline 03/12/01 & 10 & -- & 3.4 & 129 & 157 & 0 & 66 & 5.1 & .38 & 15 & 244 & 263 \\
\hline 04/10/01 & 10 & -- & 2.7 & 132 & 161 & 0 & 64 & 5.2 & .39 & 14 & 242 & 264 \\
\hline $05 / 22 / 01$ & 4.5 & -- & 1.6 & 75 & 91 & 0 & 26 & 2.1 & .22 & 13 & 126 & 144 \\
\hline \multicolumn{13}{|c|}{ SITE 5 12352500--BITTERROOT RIVER NEAR MISSOULA, MONT. } \\
\hline 03/04/99 & 5.2 & -- & 1.4 & 61 & 74 & 0 & 3.2 & 2.4 & .13 & 13 & 82 & 92 \\
\hline 03/24/99 & 3.6 & 1.2 & 1.1 & 37 & 45 & 0 & 2.4 & 1.1 & .14 & 13 & 57 & 77 \\
\hline 04/15/99 & 4.1 & -- & 1.0 & 46 & 56 & 0 & 2.6 & 2.0 & .12 & 12 & 65 & 69 \\
\hline 05/13/99 & 3.3 & 1.1 & 1.1 & 40 & 49 & 0 & 2.2 & 1.4 & .12 & 11 & 56 & 62 \\
\hline 05/25/99 & 1.6 & -- & .89 & 14 & 17 & 0 & 1.3 & 1.3 & $<.10$ & 8.0 & 27 & 39 \\
\hline 06/07/99 & 2.0 & .9 & .78 & 21 & 26 & 0 & 1.5 & 1.2 & $<.10$ & 9.5 & 36 & 52 \\
\hline 06/19/99 & 1.5 & .8 & .69 & 16 & 20 & 0 & .99 & .4 & $<.10$ & 7.3 & 26 & 35 \\
\hline 07/21/99 & 3.2 & 1.2 & 1.2 & 40 & 49 & 0 & 2.1 & 1.2 & $<.10$ & 10 & 55 & 69 \\
\hline 08/19/99 & 5.0 & 1.7 & 1.7 & 65 & 80 & 0 & 2.8 & 1.4 & .12 & 14 & 85 & 88 \\
\hline $10 / 20 / 99$ & 6.2 & 2.0 & 2.0 & 78 & 93 & 0 & 3.6 & 2.0 & .16 & 14 & 99 & 101 \\
\hline $12 / 02 / 99$ & 5.1 & -- & 1.5 & 60 & 74 & 0 & 3.0 & 1.8 & .13 & 13 & 82 & 84 \\
\hline
\end{tabular}


Table 6. Streamflow, physical, and major-ion concentration data for surface-water samples collected at fixed water-quality sites, Northern Rockies Intermontane Basins study unit, 1998-2001 (Continued)

\begin{tabular}{|c|c|c|c|c|c|c|c|c|c|c|c|c|c|c|}
\hline Date & Time & $\begin{array}{c}\text { Dis- } \\
\text { charge, } \\
\text { instan- } \\
\text { taneous } \\
\left(\mathbf{f t}^{3} / \mathbf{s}\right)\end{array}$ & $\begin{array}{c}\text { Sedi- } \\
\text { ment, } \\
\text { sus- } \\
\text { pended } \\
(\mathrm{mg} / \mathrm{L})\end{array}$ & $\begin{array}{c}\text { Sedi- } \\
\text { ment, } \\
\text { sus- } \\
\text { pended } \\
\text { (percent } \\
\text { finer } \\
\text { than } \\
0.062 \\
\text { mm) }\end{array}$ & $\begin{array}{c}\text { Specific } \\
\text { conduct- } \\
\text { ance, } \\
\text { field } \\
(\mu \mathrm{S} / \mathrm{cm})\end{array}$ & $\begin{array}{l}\text { pH, } \\
\text { field } \\
\text { (stan- } \\
\text { dard } \\
\text { units) }\end{array}$ & $\begin{array}{c}\text { Tem- } \\
\text { per- } \\
\text { ature, } \\
\text { water } \\
\left({ }^{\circ} \mathrm{C}\right)\end{array}$ & $\begin{array}{c}\text { Hard- } \\
\text { ness } \\
(\mathrm{mg} / \mathrm{L} \\
\text { as } \\
\left.\mathrm{CaCO}_{3}\right)\end{array}$ & $\begin{array}{c}\text { Calci- } \\
\text { um, } \\
\text { total } \\
(\mathrm{mg} / \mathrm{L})\end{array}$ & $\begin{array}{c}\text { Calci- } \\
\text { um, } \\
\text { dis- } \\
\text { solved } \\
(\mathrm{mg} / \mathrm{L})\end{array}$ & $\begin{array}{c}\text { Mag- } \\
\text { nesi- } \\
\text { um, } \\
\text { total } \\
(\mathrm{mg} / \mathrm{L})\end{array}$ & $\begin{array}{c}\text { Mag- } \\
\text { nesium, } \\
\text { dis- } \\
\text { solved } \\
(\mathrm{mg} / \mathrm{L})\end{array}$ & $\begin{array}{c}\text { Sodium } \\
\text { adsorp- } \\
\text { tion } \\
\text { ratio }\end{array}$ & $\begin{array}{c}\text { So- } \\
\text { dium, } \\
\text { total } \\
(\mathrm{mg} / \mathrm{L})\end{array}$ \\
\hline $01 / 26 / 00$ & 1000 & 751 & -- & -- & 142 & 8.0 & 3.0 & 61 & -- & 18 & -- & 4.1 & .3 & -- \\
\hline $03 / 08 / 00$ & 1415 & $\mathrm{e} 1,050$ & 20 & 55 & 123 & 8.0 & 7.5 & 51 & 15 & 15 & 3.6 & 3.5 & .3 & 4.7 \\
\hline $03 / 08 / 00^{\mathbf{F b}}$ & 1500 & -- & -- & -- & -- & -- & -- & -- & -- & .003 & -- & $<.001$ & -- & -- \\
\hline $04 / 12 / 00$ & 1545 & 2,510 & 28 & 74 & 72 & 7.7 & 9.5 & 29 & 6.0 & 8.4 & 1.5 & 1.9 & .2 & 2.3 \\
\hline $05 / 02 / 00$ & 1300 & 3,980 & 18 & 62 & 52 & 7.5 & 11.0 & -- & 6.0 & -- & 1.5 & -- & -- & 2.3 \\
\hline $09 / 19 / 00$ & 1430 & 482 & 4 & 90 & 171 & 7.8 & 15.0 & 75 & -- & 22 & -- & 4.7 & .3 & -- \\
\hline $10 / 02 / 00$ & 1000 & 3,900 & 76 & 64 & 60 & 7.8 & 10.5 & -- & -- & -- & -- & -- & -- & -- \\
\hline $10 / 16 / 00$ & 1200 & 1,300 & 2 & 70 & 117 & 7.8 & 9.5 & 49 & -- & 14 & -- & 3.1 & .3 & -- \\
\hline $11 / 08 / 00$ & 1330 & 951 & 2 & 67 & 124 & 8.0 & 3.5 & 54 & -- & 16 & -- & 3.4 & .3 & -- \\
\hline $01 / 30 / 01$ & 1500 & 506 & 1 & 78 & 150 & 8.2 & 2.0 & 64 & -- & 19 & -- & 4.2 & .3 & -- \\
\hline $03 / 12 / 01$ & 1445 & 760 & 5 & 72 & 142 & 8.0 & 6.0 & 58 & -- & 17 & -- & 3.9 & .3 & -- \\
\hline $04 / 10 / 01$ & 1330 & 892 & 6 & 74 & 121 & 7.6 & 6.5 & 51 & -- & 15 & -- & 3.3 & .3 & -- \\
\hline $05 / 22 / 01$ & 1430 & 4,290 & 13 & 71 & 65 & 7.7 & 13.0 & 26 & -- & 7.9 & -- & 1.6 & .2 & -- \\
\hline \multicolumn{15}{|c|}{ SITE 9 12354500--CLARK FORK AT ST. REGIS, MONT. } \\
\hline $03 / 02 / 99$ & 1330 & 4,350 & 5 & 81 & -- & 8.0 & 5.0 & 106 & -- & 29 & -- & 8.3 & .4 & -- \\
\hline $04 / 13 / 99$ & 1500 & 5,430 & 5 & 91 & -- & 8.0 & 7.5 & 93 & 24 & 25 & 6.9 & 7.3 & .3 & 7.0 \\
\hline $11 / 30 / 99$ & 1300 & 3,970 & 4 & 76 & 218 & 8.1 & 4.0 & 101 & -- & 28 & -- & 7.8 & .3 & -- \\
\hline $01 / 24 / 00$ & 1400 & 2,730 & -- & -- & 267 & 8.0 & 2.0 & 117 & 32 & 32 & 9.2 & 9.1 & .4 & 9.6 \\
\hline $03 / 06 / 00$ & 1400 & 3,740 & 8 & 78 & 244 & 8.3 & 7.0 & 108 & 30 & 29 & 8.4 & 8.4 & .4 & 8.6 \\
\hline $04 / 10 / 00$ & 1400 & 6,470 & 11 & 87 & 184 & 8.2 & 9.0 & 78 & 22 & 21 & 6.0 & 6.1 & .3 & 6.5 \\
\hline $05 / 09 / 00$ & 1215 & 12,700 & 11 & 84 & 134 & 8.1 & 9.0 & 59 & 15 & 16 & 4.6 & 4.6 & .3 & 4.5 \\
\hline $05 / 24 / 00$ & 1230 & 18,700 & 53 & 63 & 95 & 7.8 & 12.5 & 45 & 12 & 13 & 3.4 & 3.4 & .1 & 2.5 \\
\hline $06 / 20 / 00$ & 0930 & 10,700 & 6 & 75 & 138 & 8.1 & 14.0 & 60 & 16 & 16 & 4.9 & 4.8 & .2 & 3.3 \\
\hline $08 / 01 / 00^{\mathbf{F b}}$ & 1330 & -- & -- & -- & -- & -- & -- & -- & -- & .003 & -- & $<.001$ & -- & -- \\
\hline 08/01/00 & 1430 & 2,100 & 1 & 83 & 218 & 8.4 & 22.0 & 107 & 26 & 29 & 7.9 & 8.6 & .2 & 5.5 \\
\hline $08 / 30 / 00$ & 1400 & 1,660 & 2 & 83 & 241 & 8.5 & 16.5 & 109 & 29 & 29 & 8.7 & 8.8 & .3 & 7.0 \\
\hline $11 / 07 / 00$ & 1000 & 3,020 & 3 & 67 & 252 & 8.0 & 4.0 & 111 & -- & 31 & -- & 8.4 & .4 & -- \\
\hline $12 / 19 / 00$ & 1645 & 2,380 & 2 & 70 & 264 & 8.2 & 0.0 & 120 & -- & 33 & -- & 9.2 & .3 & -- \\
\hline $01 / 31 / 01$ & 1500 & 1,920 & 2 & 76 & 274 & 8.5 & 1.5 & 123 & -- & 34 & -- & 9.4 & .3 & -- \\
\hline $03 / 13 / 01$ & 1500 & 2,410 & 9 & 94 & 277 & 8.4 & 5.5 & 117 & -- & 32 & -- & 8.8 & .4 & -- \\
\hline $04 / 11 / 01$ & 1400 & 3,310 & 9 & 84 & 249 & 8.3 & 6.5 & 109 & -- & 30 & -- & 8.3 & .4 & -- \\
\hline $05 / 23 / 01$ & 1430 & 11,600 & 12 & 79 & 140 & 8.2 & 14.5 & 59 & -- & 16 & -- & 4.4 & .3 & -- \\
\hline
\end{tabular}


Table 6. Streamflow, physical, and major-ion concentration data for surface-water samples collected at fixed water-quality sites, Northern Rockies Intermontane Basins study unit, 1998-2001 (Continued)

\begin{tabular}{|c|c|c|c|c|c|c|c|c|c|c|c|c|}
\hline Date & $\begin{array}{c}\text { Sodium, } \\
\text { dissolved } \\
(\mathrm{mg} / \mathrm{L})\end{array}$ & $\begin{array}{c}\text { Potas- } \\
\text { sium, } \\
\text { total } \\
(\mathrm{mg} / \mathrm{L})\end{array}$ & $\begin{array}{c}\text { Potas- } \\
\text { sium, } \\
\text { dissolved } \\
(\mathbf{m g} / \mathrm{L})\end{array}$ & $\begin{array}{c}\text { Alka- } \\
\text { linity, } \\
\text { field } \\
(\mathrm{mg} / \mathrm{L} \text { as } \\
\left.\mathrm{CaCO}_{3}\right)\end{array}$ & $\begin{array}{c}\text { Bicar- } \\
\text { bonate, } \\
\text { dissolved } \\
(\mathrm{mg} / \mathrm{L} \text { as } \\
\left.\mathrm{HCO}_{3}\right)\end{array}$ & $\begin{array}{c}\text { Carbo- } \\
\text { nate, } \\
\text { dissolved } \\
(\mathrm{mg} / \mathrm{L} \text { as } \\
\left.\mathrm{CO}_{3}\right)\end{array}$ & $\begin{array}{c}\text { Sulfate, } \\
\text { dissolved } \\
(\mathrm{mg} / \mathrm{L} \text { as } \\
\left.\mathrm{SO}_{4}\right)\end{array}$ & $\begin{array}{c}\text { Chloride, } \\
\text { dissolved } \\
(\mathrm{mg} / \mathrm{L})\end{array}$ & $\begin{array}{c}\text { Fluoride, } \\
\text { dissolved } \\
(\mathrm{mg} / \mathrm{L})\end{array}$ & $\begin{array}{c}\text { Silica, } \\
\text { dissolved } \\
(\mathrm{mg} / \mathrm{L} \text { as } \\
\left.\mathrm{SiO}_{2}\right)\end{array}$ & $\begin{array}{l}\text { Dissolved } \\
\text { solids, } \\
\text { sum of } \\
\text { constit- } \\
\text { uents } \\
\text { (mg/L) }\end{array}$ & $\begin{array}{l}\text { Dissolved } \\
\text { solids, } \\
\text { residue at } \\
180^{\circ} \mathrm{C} \\
(\mathrm{mg} / \mathrm{L})\end{array}$ \\
\hline \multicolumn{13}{|c|}{ SITE 5 12352500--BITTERROOT RIVER NEAR MISSOULA, MONT. (Continued) } \\
\hline $01 / 26 / 00$ & 5.2 & -- & 1.3 & 86 & 105 & 0 & 3.6 & 2.0 & .16 & 14 & 99 & 101 \\
\hline 03/08/00 & 4.7 & 1.3 & 1.2 & 59 & 72 & 0 & 2.9 & 1.6 & .16 & 12 & 76 & 86 \\
\hline $03 / 08 / 00^{\mathbf{F b}}$ & $<.02$ & -- & -- & -- & -- & -- & -- & -- & -- & $<.02$ & -- & -- \\
\hline $04 / 12 / 00$ & 2.9 & .8 & 1.0 & 33 & 40 & 0 & 1.9 & .9 & .14 & 10 & 47 & 52 \\
\hline $05 / 02 / 00$ & -- & .8 & .74 & 31 & 38 & 0 & 1.4 & .7 & $<.10$ & 8.4 & -- & 39 \\
\hline $05 / 23 / 00$ & 1.6 & .9 & .59 & 18 & 22 & 0 & 1.1 & .3 & $<.10$ & 7.5 & 28 & 34 \\
\hline $06 / 20 / 00$ & 2.3 & .8 & .73 & 27 & 33 & 0 & 1.1 & .6 & $<.10$ & 8.2 & 38 & 48 \\
\hline $07 / 31 / 00$ & 6.0 & -- & 1.9 & 78 & 95 & 0 & 3.1 & 2.1 & .15 & 13 & 99 & 101 \\
\hline $08 / 29 / 00$ & 6.7 & 2.1 & 2.1 & 86 & 105 & 0 & 3.2 & 2.2 & .17 & 13 & 107 & 109 \\
\hline 09/19/00 & 6.3 & -- & 2.1 & 84 & 102 & 0 & 3.3 & 2.1 & .16 & 13 & 104 & 103 \\
\hline $10 / 02 / 00$ & -- & -- & -- & -- & -- & -- & -- & -- & -- & -- & -- & -- \\
\hline $10 / 16 / 00$ & 4.5 & -- & 1.5 & -- & -- & -- & 2.8 & 1.6 & e. 14 & 11 & 73 & 77 \\
\hline $11 / 08 / 00$ & 4.8 & -- & 1.4 & 48 & 59 & 0 & 3.0 & 2.6 & e. 16 & 12 & 72 & 80 \\
\hline $01 / 30 / 01$ & 5.5 & -- & 1.5 & 71 & 87 & 0 & 4.5 & 2.1 & e. 16 & 11 & 91 & 106 \\
\hline 03/12/01 & 5.4 & -- & 1.7 & 62 & 76 & 0 & 3.8 & 2.2 & e. 14 & 11 & 83 & 92 \\
\hline $04 / 10 / 01$ & 4.5 & -- & 1.3 & 54 & 67 & 0 & 3.7 & 1.5 & e.16 & 11 & 74 & 81 \\
\hline $05 / 22 / 01$ & 2.6 & -- & .81 & 27 & 33 & 0 & 2.4 & .8 & $<.20$ & 9.8 & 42 & 46 \\
\hline \multicolumn{13}{|c|}{ SITE 9 12354500-CLARK FORK AT ST. REGIS, MONT. } \\
\hline 03/02/99 & 9.1 & -- & 1.7 & 96 & 114 & 1.9 & 20 & 4.1 & .14 & 11 & 141 & 153 \\
\hline 04/13/99 & 7.0 & 1.4 & 1.4 & 120 & 146 & 0 & 15 & 3.4 & .13 & 11 & 142 & 137 \\
\hline 05/10/99 & 3.2 & 1.0 & .64 & 63 & 77 & 0 & 12 & 2.2 & $<.10$ & 5.9 & 77 & 101 \\
\hline 05/26/99 & 2.4 & 1.2 & .90 & 41 & 50 & 0 & 4.9 & 1.5 & $<.10$ & 7.9 & 58 & 69 \\
\hline 06/08/99 & 2.7 & .9 & .85 & 47 & 57 & 0 & 5.5 & 1.3 & $<.10$ & 9.3 & 66 & 85 \\
\hline $06 / 21 / 99^{\mathbf{F b}}$ & $<.02$ & -- & -- & -- & -- & -- & -- & -- & -- & $<.02$ & -- & -- \\
\hline $06 / 21 / 99$ & 2.1 & .9 & .73 & 44 & 53 & 0 & 4.1 & 1.4 & $<.10$ & 7.6 & 58 & 65 \\
\hline 07/20/99 & 3.8 & 1.1 & 1.1 & 76 & 93 & 0 & 6.6 & 2.7 & $<.10$ & 8.8 & 96 & 92 \\
\hline 08/18/99 & 5.8 & 1.6 & 1.6 & 98 & 120 & 0 & 12 & 2.4 & .12 & 11 & 127 & 135 \\
\hline $10 / 18 / 99$ & 7.8 & 1.8 & 1.8 & 115 & 140 & 0 & 20 & 3.1 & .18 & 11 & 156 & 158 \\
\hline $11 / 30 / 99$ & 7.6 & -- & 1.5 & 94 & 115 & 0 & 16 & 2.9 & .14 & 12 & 133 & 140 \\
\hline $01 / 24 / 00$ & 9.3 & 1.7 & 1.5 & 116 & 141 & 0 & 23 & 3.5 & .21 & 11 & 160 & 161 \\
\hline 03/06/00 & 8.8 & 1.7 & 1.6 & 97 & 116 & 0 & 20 & 3.4 & .21 & 11 & 140 & 150 \\
\hline $04 / 10 / 00$ & 6.7 & 1.2 & 1.2 & 80 & 97 & 0 & 13 & 2.3 & .16 & 10 & 109 & 114 \\
\hline $05 / 09 / 00$ & 4.6 & .9 & .78 & 60 & 73 & 0 & 8.5 & 1.4 & .18 & 8.4 & 81 & 85 \\
\hline $05 / 24 / 00$ & 2.1 & .8 & .67 & 43 & 53 & 0 & 3.7 & 1.0 & $<.10$ & 7.5 & 57 & 60 \\
\hline $06 / 20 / 00$ & 3.5 & .9 & 1.0 & 64 & 78 & 0 & 6.2 & 1.2 & $<.10$ & 7.7 & 79 & 90 \\
\hline $08 / 01 / 00^{\mathbf{F b}}$ & $<.02$ & -- & -- & -- & -- & -- & -- & -- & -- & $<.02$ & -- & -- \\
\hline 08/01/00 & 5.8 & 1.4 & 1.3 & 102 & 122 & 0 & 10 & 2.7 & .13 & 9.6 & 128 & 132 \\
\hline 08/30/00 & 7.1 & 1.7 & 1.7 & 111 & 132 & 1.7 & 12 & 3.2 & .14 & 7.7 & 137 & 141 \\
\hline $11 / 07 / 00$ & 8.9 & -- & 1.7 & 102 & 124 & 0 & 21 & 3.0 & .18 & 10 & 145 & 154 \\
\hline $12 / 19 / 00$ & 7.8 & -- & 1.6 & 117 & 142 & 0 & 20 & 4.3 & .18 & 11 & 158 & 159 \\
\hline $01 / 31 / 01$ & 8.6 & -- & 1.7 & 116 & 141 & 0 & 24 & 4.0 & .18 & 8.7 & 160 & 179 \\
\hline 03/13/01 & 10 & -- & 2.0 & 105 & 128 & 0 & 26 & 4.2 & .18 & 9.3 & 156 & 164 \\
\hline $04 / 11 / 01$ & 8.8 & -- & 1.7 & 100 & 122 & 0 & 23 & 3.7 & .18 & 9.1 & 145 & 157 \\
\hline $05 / 23 / 01$ & 4.6 & -- & 1.0 & 57 & 69 & 0 & 8.4 & 1.7 & $<.20$ & 8.9 & 80 & 82 \\
\hline
\end{tabular}


Table 6. Streamflow, physical, and major-ion concentration data for surface-water samples collected at fixed water-quality sites, Northern Rockies Intermontane Basins study unit, 1998-2001 (Continued)

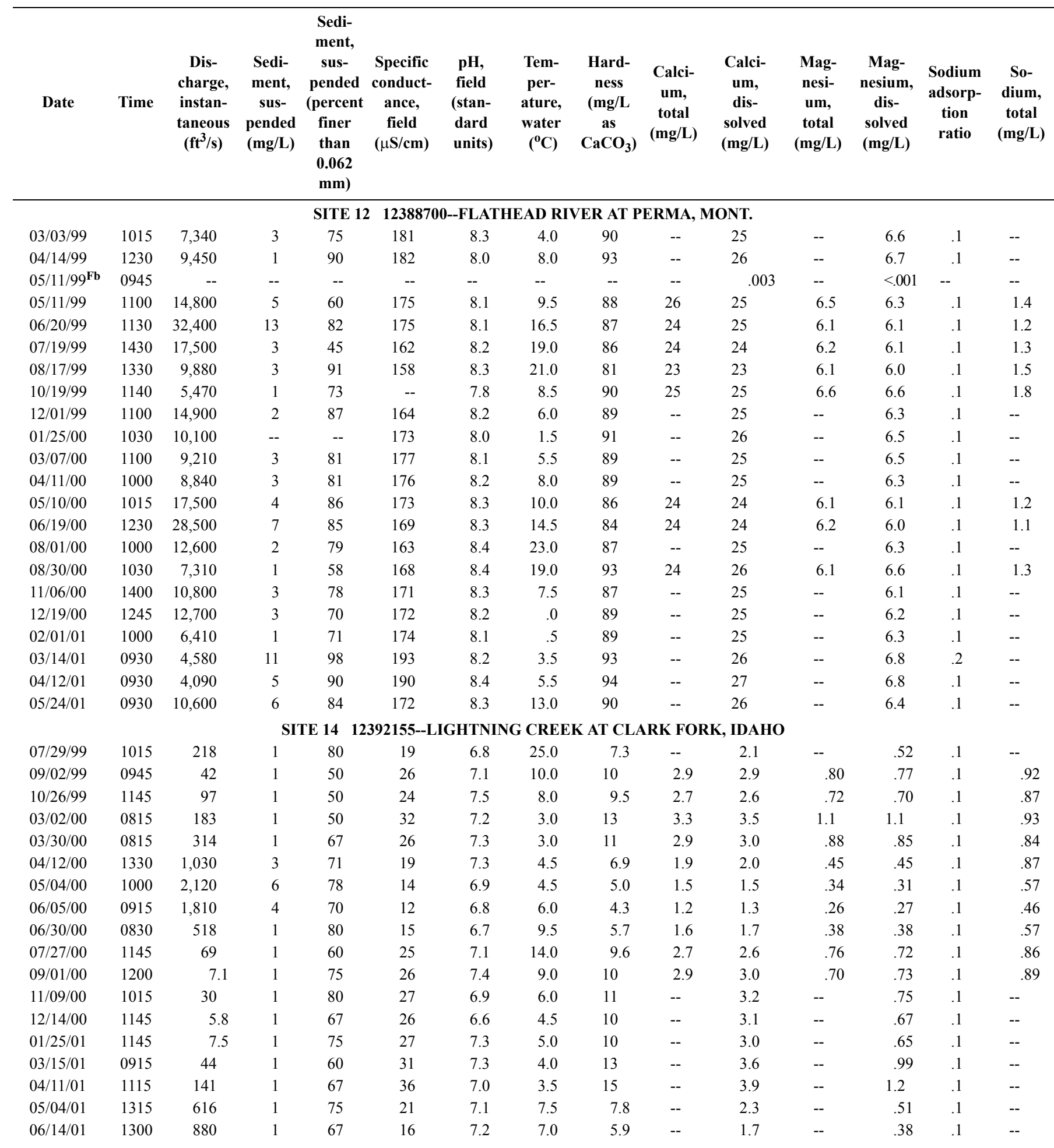


Table 6. Streamflow, physical, and major-ion concentration data for surface-water samples collected at fixed water-quality sites, Northern Rockies Intermontane Basins study unit, 1998-2001 (Continued)

\begin{tabular}{|c|c|c|c|c|c|c|c|c|c|c|c|c|}
\hline Date & $\begin{array}{c}\text { Sodium, } \\
\text { dissolved } \\
(\mathrm{mg} / \mathrm{L})\end{array}$ & $\begin{array}{c}\text { Potas- } \\
\text { sium, } \\
\text { total } \\
(\mathrm{mg} / \mathrm{L})\end{array}$ & $\begin{array}{c}\text { Potas- } \\
\text { sium, } \\
\text { dissolved } \\
(\mathrm{mg} / \mathrm{L})\end{array}$ & $\begin{array}{c}\text { Alka- } \\
\text { linity, } \\
\text { field } \\
(\mathrm{mg} / \mathrm{L} \text { as } \\
\left.\mathrm{CaCO}_{3}\right)\end{array}$ & $\begin{array}{c}\text { Bicar- } \\
\text { bonate, } \\
\text { dissolved } \\
(\mathrm{mg} / \mathrm{L} \text { as } \\
\left.\mathrm{HCO}_{3}\right)\end{array}$ & $\begin{array}{c}\text { Carbo- } \\
\text { nate, } \\
\text { dissolved } \\
(\mathrm{mg} / \mathrm{L} \text { as } \\
\left.\mathrm{CO}_{3}\right)\end{array}$ & $\begin{array}{c}\text { Sulfate, } \\
\text { dissolved } \\
(\mathrm{mg} / \mathrm{L} \text { as } \\
\left.\mathrm{SO}_{4}\right)\end{array}$ & $\begin{array}{c}\text { Chloride, } \\
\text { dissolved } \\
(\mathrm{mg} / \mathrm{L})\end{array}$ & $\begin{array}{c}\text { Fluoride, } \\
\text { dissolved } \\
(\mathrm{mg} / \mathrm{L})\end{array}$ & $\begin{array}{c}\text { Silica, } \\
\text { dissolved } \\
(\mathrm{mg} / \mathrm{L} \text { as } \\
\left.\mathrm{SiO}_{2}\right)\end{array}$ & $\begin{array}{l}\text { Dissolved } \\
\text { solids, } \\
\text { sum of } \\
\text { constit- } \\
\text { uents } \\
\text { (mg/L) }\end{array}$ & $\begin{array}{l}\text { Dissolved } \\
\text { solids, } \\
\text { residue at } \\
180^{\circ} \mathrm{C} \\
(\mathrm{mg} / \mathrm{L})\end{array}$ \\
\hline \multicolumn{13}{|c|}{ SITE 12 12388700--FLATHEAD RIVER AT PERMA, MONT. } \\
\hline 03/03/99 & 2.0 & -- & .49 & 89 & 109 & 0 & 2.9 & 2.5 & $<.10$ & 4.9 & 98 & 101 \\
\hline $04 / 14 / 99$ & 1.5 & -- & .41 & 85 & 104 & 0 & 2.6 & .63 & $<.10$ & 4.8 & 94 & 105 \\
\hline $05 / 11 / 99^{\mathbf{F b}}$ & $<.02$ & -- & -- & -- & -- & -- & -- & -- & -- & $<.02$ & -- & -- \\
\hline 05/11/99 & 1.5 & .43 & .42 & 83 & 101 & 0 & 2.8 & .87 & $<.10$ & 4.6 & 91 & 109 \\
\hline $06 / 20 / 99$ & 1.2 & .44 & .42 & 84 & 102 & 0 & 2.8 & .42 & $<.10$ & 4.5 & 91 & 97 \\
\hline 07/19/99 & 1.3 & .44 & .43 & 84 & 102 & 0 & 2.7 & .48 & $<.10$ & 4.6 & 91 & 94 \\
\hline 08/17/99 & 1.5 & .46 & .45 & 84 & 102 & .2 & 2.8 & .40 & $<.10$ & 4.6 & 89 & 96 \\
\hline $10 / 19 / 99$ & 1.8 & .48 & .48 & 89 & 109 & 0 & 3.0 & .48 & $<.10$ & 5.0 & 96 & 102 \\
\hline $12 / 01 / 99$ & 1.4 & -- & .41 & 87 & 106 & 0 & 3.0 & 1.6 & $<.10$ & 4.8 & 95 & 95 \\
\hline $01 / 25 / 00$ & 1.4 & -- & .45 & 86 & 105 & 0 & 3.2 & $<.29$ & $<.10$ & 4.9 & -- & 100 \\
\hline 03/07/00 & 1.8 & -- & .47 & 89 & 109 & 0 & 2.8 & $<.29$ & $<.10$ & 4.6 & -- & 105 \\
\hline $04 / 11 / 00$ & 1.5 & -- & .51 & 91 & 111 & 0 & 2.7 & .32 & $<.10$ & 4.7 & 96 & 99 \\
\hline $05 / 10 / 00$ & 1.3 & .48 & .38 & 87 & 106 & 0 & 2.7 & .30 & $<.10$ & 4.5 & 92 & 101 \\
\hline $06 / 19 / 00$ & 1.2 & .45 & .36 & 87 & 105 & .4 & 2.4 & .49 & $<.10$ & 4.4 & 91 & 105 \\
\hline $08 / 01 / 00$ & 1.2 & -- & .37 & 86 & 104 & .6 & 2.5 & .37 & $<.10$ & 4.7 & 92 & 94 \\
\hline $08 / 30 / 00$ & 1.4 & .43 & .45 & 86 & 105 & 0 & 2.5 & .39 & $<.10$ & 5.0 & 94 & 97 \\
\hline $11 / 06 / 00$ & 1.4 & -- & .48 & 88 & 104 & 1.4 & 2.8 & .62 & $<.16$ & 4.6 & 93 & 101 \\
\hline $12 / 19 / 00$ & 1.3 & -- & .42 & 88 & 108 & 0 & 2.8 & .60 & $<.16$ & 4.7 & 95 & 96 \\
\hline $02 / 01 / 01$ & 1.6 & -- & .44 & 88 & 107 & 0 & 4.0 & .71 & $<.16$ & 4.3 & 95 & 112 \\
\hline 03/14/01 & 3.6 & -- & 1.4 & 96 & 117 & 0 & 3.9 & 1.4 & $<.16$ & 4.5 & 106 & 112 \\
\hline $04 / 12 / 01$ & 3.1 & -- & .70 & 91 & 111 & 0 & 4.0 & .87 & $<.16$ & 3.7 & 100 & 112 \\
\hline $05 / 24 / 01$ & 1.2 & -- & .44 & 88 & 107 & 0 & 3.0 & .43 & $<.20$ & 4.4 & 94 & 111 \\
\hline \multicolumn{13}{|c|}{ SITE 14 12392155--LIGHTNING CREEK AT CLARK FORK, IDAHO } \\
\hline 07/29/99 & .73 & -- & .33 & 8.0 & 10 & 0 & 1.1 & .12 & $<.10$ & 5.8 & 16 & 21 \\
\hline 09/02/99 & .94 & .40 & .39 & 11 & 13 & 0 & 1.7 & $<.29$ & $<.10$ & 7.0 & -- & 28 \\
\hline $10 / 26 / 99$ & .81 & .39 & .41 & 9.0 & 11 & 0 & 2.0 & e. 24 & $<.10$ & 6.2 & -- & 21 \\
\hline $03 / 02 / 00$ & .96 & .34 & .33 & 11 & 14 & 0 & 2.2 & e. 22 & $<.10$ & 7.2 & -- & 27 \\
\hline 03/30/00 & .86 & .34 & .31 & 11 & 13 & 0 & 1.8 & e. 25 & $<.10$ & 7.0 & -- & 20 \\
\hline $04 / 12 / 00$ & .68 & $<.1$ & .29 & 6.6 & 8.0 & 0 & 1.1 & $<.29$ & $<.10$ & 5.9 & -- & 21 \\
\hline $05 / 04 / 00$ & .56 & .23 & .24 & 3.6 & 4.4 & 0 & .77 & $<.29$ & $<.10$ & 5.1 & -- & 16 \\
\hline $06 / 05 / 00$ & .51 & .20 & .24 & 4.2 & 5.1 & 0 & .80 & $<.29$ & $<.10$ & 4.6 & -- & 11 \\
\hline $06 / 30 / 00$ & .57 & .18 & .25 & 5.7 & 7.0 & 0 & .81 & $<.29$ & $<.10$ & 5.0 & -- & $<10$ \\
\hline $07 / 27 / 00$ & .83 & .36 & .36 & 11 & 13 & 0 & 1.4 & $<.29$ & $<.10$ & 6.4 & -- & 24 \\
\hline 09/01/00 & .96 & .37 & .40 & 12 & 14 & 0 & 1.6 & e. 15 & $<.10$ & 6.8 & -- & 19 \\
\hline $11 / 09 / 00$ & .94 & -- & .41 & 10 & 13 & 0 & 1.9 & .44 & $<.16$ & 6.8 & 21 & 23 \\
\hline $12 / 14 / 00$ & .96 & -- & .39 & 10 & 12 & 0 & 2.1 & .45 & $<.16$ & 7.0 & 21 & 18 \\
\hline $01 / 25 / 01$ & .91 & -- & .41 & 10 & 12 & 0 & 2.2 & .40 & $<.16$ & 6.6 & 21 & 19 \\
\hline 03/15/01 & .96 & -- & .40 & 11 & 14 & 0 & 2.8 & .54 & $<.16$ & 6.6 & 23 & 21 \\
\hline $04 / 11 / 01$ & .96 & -- & .45 & 13 & 16 & 0 & 2.5 & .48 & $<.16$ & 6.8 & 25 & 32 \\
\hline 05/04/01 & .75 & -- & .26 & 6.9 & 8.4 & 0 & 1.6 & .29 & $<.16$ & 6.0 & 17 & 16 \\
\hline $06 / 14 / 01$ & .60 & -- & .23 & 5.2 & 6.4 & 0 & 1.1 & .18 & $<.20$ & 5.1 & 13 & 18 \\
\hline
\end{tabular}


Table 6. Streamflow, physical, and major-ion concentration data for surface-water samples collected at fixed water-quality sites, Northern Rockies Intermontane Basins study unit, 1998-2001 (Continued)

\begin{tabular}{|c|c|c|c|c|c|c|c|c|c|c|c|c|c|c|}
\hline Date & Time & $\begin{array}{c}\text { Dis- } \\
\text { charge, } \\
\text { instan- } \\
\text { taneous } \\
\left(\mathbf{f t}^{3} / \mathbf{s}\right)\end{array}$ & $\begin{array}{c}\text { Sedi- } \\
\text { ment, } \\
\text { sus- } \\
\text { pended } \\
(\mathrm{mg} / \mathrm{L})\end{array}$ & $\begin{array}{c}\text { Sedi- } \\
\text { ment, } \\
\text { sus- } \\
\text { pended } \\
\text { (percent } \\
\text { finer } \\
\text { than } \\
\mathbf{0 . 0 6 2} \\
\text { mm) }\end{array}$ & $\begin{array}{c}\text { Specific } \\
\text { conduct- } \\
\text { ance, } \\
\text { field } \\
(\mu \mathrm{S} / \mathrm{cm})\end{array}$ & $\begin{array}{c}\text { pH, } \\
\text { field } \\
\text { (stan- } \\
\text { dard } \\
\text { units) }\end{array}$ & $\begin{array}{c}\text { Tem- } \\
\text { per- } \\
\text { ature, } \\
\text { water } \\
\left({ }^{\circ} \mathrm{C}\right)\end{array}$ & $\begin{array}{c}\text { Hard- } \\
\text { ness } \\
(\mathrm{mg} / \mathrm{L} \\
\text { as } \\
\left.\mathrm{CaCO}_{3}\right)\end{array}$ & $\begin{array}{c}\text { Calci- } \\
\text { um, } \\
\text { total } \\
(\mathrm{mg} / \mathrm{L})\end{array}$ & $\begin{array}{c}\text { Calci- } \\
\text { um, } \\
\text { dis- } \\
\text { solved } \\
(\mathrm{mg} / \mathrm{L})\end{array}$ & $\begin{array}{c}\text { Mag- } \\
\text { nesi- } \\
\text { um, } \\
\text { total } \\
(\mathrm{mg} / \mathrm{L})\end{array}$ & $\begin{array}{c}\text { Mag- } \\
\text { nesium, } \\
\text { dis- } \\
\text { solved } \\
(\mathrm{mg} / \mathrm{L})\end{array}$ & $\begin{array}{l}\text { Sodium } \\
\text { adsorp- } \\
\text { tion } \\
\text { ratio }\end{array}$ & $\begin{array}{c}\text { So- } \\
\text { dium, } \\
\text { total } \\
(\mathrm{mg} / \mathrm{L})\end{array}$ \\
\hline 03/02/99 & 1130 & 4,450 & 4 & 83 & 62 & 7.3 & 3.0 & 12 & -- & 2.9 & -- & 1.1 & .1 & -- \\
\hline $03 / 23 / 99$ & 1000 & 6,880 & 14 & 82 & 36 & 6.8 & 3.5 & 14 & 3.6 & 3.3 & 1.4 & 1.4 & .1 & .96 \\
\hline 05/20/99 & 1000 & 6,400 & 7 & 93 & 31 & 7.5 & 6.0 & 18 & 4.9 & 4.9 & 1.6 & 1.5 & .2 & 1.6 \\
\hline $05 / 25 / 99$ & 0930 & 11,100 & 84 & 76 & 27 & 6.7 & 8.0 & 11 & 2.8 & 2.7 & 1.3 & 1.0 & .1 & .75 \\
\hline $06 / 29 / 99$ & 1000 & 1,370 & 1 & 60 & 38 & 7.6 & 11.0 & 17 & 4.0 & 4.2 & 1.6 & 1.7 & .1 & .94 \\
\hline $01 / 11 / 00$ & 1100 & 931 & 1 & 75 & 41 & 7.3 & 1.5 & 19 & -- & 4.6 & -- & 1.8 & .1 & -- \\
\hline $02 / 29 / 00$ & 0800 & 2,020 & 2 & 69 & 38 & 7.0 & 3.0 & 17 & -- & 4.1 & -- & 1.7 & .1 & -- \\
\hline $03 / 27 / 00$ & 0945 & 3,290 & 2 & 84 & 37 & 7.2 & 4.0 & 16 & 4.0 & 4.0 & 1.6 & 1.5 & .1 & .98 \\
\hline $04 / 10 / 00$ & 0945 & 4,680 & 4 & 77 & 34 & 7.3 & 5.0 & 15 & 3.6 & 3.7 & 1.5 & 1.5 & .1 & 1.1 \\
\hline $04 / 14 / 00$ & 0945 & 18,200 & 207 & 90 & 25 & 7.0 & 4.5 & 10 & 2.9 & 2.5 & 1.6 & .94 & .1 & .89 \\
\hline $04 / 17 / 00$ & 0945 & 10,900 & 29 & 89 & 29 & 7.0 & 5.5 & 12 & 3.3 & 3.0 & 1.4 & 1.2 & .1 & .83 \\
\hline $05 / 02 / 00$ & 0900 & 5,080 & 6 & 87 & 32 & 7.1 & 7.5 & 14 & 3.3 & 3.3 & 1.4 & 1.4 & .1 & .86 \\
\hline $05 / 18 / 00$ & 0730 & 3,770 & 3 & 74 & 32 & 7.7 & 8.5 & 14 & -- & 3.4 & -- & 1.4 & .1 & -- \\
\hline $06 / 02 / 00$ & 0715 & 1,970 & 3 & 74 & 36 & 7.2 & 9.5 & 16 & 3.8 & 3.9 & 1.5 & 1.6 & .1 & .92 \\
\hline $06 / 29 / 00$ & 0800 & 954 & 1 & 80 & 43 & 7.3 & 13.5 & 19 & 4.5 & 4.7 & 1.8 & 1.8 & .1 & 1.0 \\
\hline $07 / 24 / 00$ & 1030 & 440 & 1 & 60 & 47 & 7.5 & 15.5 & 22 & -- & 5.2 & -- & 2.1 & .1 & -- \\
\hline $08 / 31 / 00$ & 0915 & 236 & 1 & 82 & 48 & 7.3 & 14.0 & 22 & 5.4 & 5.3 & 2.0 & 2.0 & .1 & 1.2 \\
\hline \multicolumn{15}{|c|}{ SITE 31 12413470--SOUTH FORK COEUR D'ALENE RIVER NEAR PINEHURST, IDAHO } \\
\hline $11 / 04 / 98$ & 1200 & 105 & 38 & -- & 260 & 7.5 & 7.5 & 93 & 24 & 24 & 8.3 & 8.0 & .4 & 8.7 \\
\hline $11 / 04 / 98^{\mathbf{F b}}$ & 1208 & -- & -- & -- & -- & -- & -- & -- & -- & .003 & -- & $<.001$ & -- & -- \\
\hline $12 / 09 / 98$ & 1210 & 275 & 3 & -- & 250 & 7.0 & 5.0 & 90 & 22 & 23 & 8.0 & 7.8 & .3 & 6.0 \\
\hline $02 / 03 / 99$ & 1100 & 322 & 1 & -- & 205 & 7.3 & 3.0 & 78 & -- & 20 & -- & 6.8 & .2 & -- \\
\hline $03 / 17 / 99$ & 1000 & 748 & 2 & 72 & 152 & 7.1 & 3.0 & 54 & 13 & 14 & 4.2 & 4.6 & .2 & 3.6 \\
\hline 03/23/99 & 1330 & 1,610 & 15 & 70 & 95 & 7.2 & 6.5 & 34 & 9.4 & 8.8 & 3.0 & 3.0 & .2 & 2.8 \\
\hline 04/20/99 & 1200 & 2,060 & 27 & 68 & 64 & 7.2 & 5.5 & 25 & 6.9 & 6.5 & 2.2 & 2.0 & .1 & 1.7 \\
\hline $05 / 25 / 99$ & 1245 & 4,000 & 207 & 80 & 48 & 7.1 & 8.0 & 18 & 5.4 & 4.9 & 1.8 & 1.3 & .1 & .98 \\
\hline $05 / 27 / 99$ & 1245 & 2,730 & 89 & 79 & 51 & 7.0 & 7.5 & 19 & 5.3 & 5.1 & 1.7 & 1.5 & .1 & 1.3 \\
\hline $06 / 29 / 99$ & 1300 & 983 & 7 & 70 & 100 & 7.2 & 11.0 & 42 & -- & 12 & -- & 3.0 & .1 & -- \\
\hline $07 / 26 / 99$ & 1315 & 326 & 2 & 69 & 147 & 7.1 & 15.0 & 65 & 15 & 17 & 4.6 & 5.4 & .3 & 4.2 \\
\hline 09/08/99 & 1300 & 146 & 2 & 82 & 242 & 7.4 & 13.5 & 94 & 25 & 24 & 8.2 & 8.0 & .3 & 7.0 \\
\hline $10 / 19 / 99$ & 1400 & 107 & 1 & 67 & 286 & 7.4 & 9.0 & 123 & 31 & 32 & 9.8 & 10 & .3 & 7.1 \\
\hline $12 / 02 / 99$ & 0850 & 413 & 2 & 69 & 169 & 7.1 & 5.5 & 68 & 18 & 18 & 5.3 & 5.4 & .2 & 4.6 \\
\hline $01 / 11 / 00$ & 1430 & 237 & 1 & 75 & 212 & 7.4 & 3.0 & 85 & 23 & 22 & 7.2 & 7.0 & .3 & 5.6 \\
\hline $02 / 28 / 00^{\mathbf{F b}}$ & 1020 & -- & -- & -- & -- & -- & -- & -- & -- & $<.002$ & -- & $<.001$ & -- & -- \\
\hline
\end{tabular}


Table 6. Streamflow, physical, and major-ion concentration data for surface-water samples collected at fixed water-quality sites, Northern Rockies Intermontane Basins study unit, 1998-2001 (Continued)

\begin{tabular}{|c|c|c|c|c|c|c|c|c|c|c|c|c|}
\hline Date & $\begin{array}{c}\text { Sodium, } \\
\text { dissolved } \\
(\mathrm{mg} / \mathrm{L})\end{array}$ & $\begin{array}{c}\text { Potas- } \\
\text { sium, } \\
\text { total } \\
(\mathrm{mg} / \mathrm{L})\end{array}$ & $\begin{array}{c}\text { Potas- } \\
\text { sium, } \\
\text { dissolved } \\
(\mathrm{mg} / \mathrm{L})\end{array}$ & $\begin{array}{c}\text { Alka- } \\
\text { linity, } \\
\text { field } \\
(\mathrm{mg} / \mathrm{L} \text { as } \\
\left.\mathrm{CaCO}_{3}\right)\end{array}$ & $\begin{array}{c}\text { Bicar- } \\
\text { bonate, } \\
\text { dissolved } \\
(\mathrm{mg} / \mathrm{L} \text { as } \\
\left.\mathrm{HCO}_{3}\right)\end{array}$ & $\begin{array}{l}\text { Carbo- } \\
\text { nate, } \\
\text { dissolved } \\
(\mathrm{mg} / \mathrm{L} \text { as } \\
\left.\mathrm{CO}_{3}\right)\end{array}$ & $\begin{array}{c}\text { Sulfate, } \\
\text { dissolved } \\
(\mathrm{mg} / \mathrm{L} \text { as } \\
\left.\mathrm{SO}_{4}\right)\end{array}$ & $\begin{array}{c}\text { Chloride, } \\
\text { dissolved } \\
(\mathrm{mg} / \mathrm{L})\end{array}$ & $\begin{array}{c}\text { Fluoride, } \\
\text { dissolved } \\
(\mathrm{mg} / \mathrm{L})\end{array}$ & $\begin{array}{c}\text { Silica, } \\
\text { dissolved } \\
(\mathrm{mg} / \mathrm{L} \text { as } \\
\left.\mathrm{SiO}_{2}\right)\end{array}$ & $\begin{array}{l}\text { Dissolved } \\
\text { solids, } \\
\text { sum of } \\
\text { constit- } \\
\text { uents } \\
(\mathrm{mg} / \mathrm{L})\end{array}$ & $\begin{array}{c}\text { Dissolved } \\
\text { solids, } \\
\text { residue at } \\
180^{\circ} \mathrm{C} \\
(\mathrm{mg} / \mathrm{L})\end{array}$ \\
\hline \multicolumn{13}{|c|}{ VILLE, IDAHO } \\
\hline 03/02/99 & .76 & -- & .26 & 16 & 19 & 0 & 1.8 & .33 & $<.10$ & 7.6 & 24 & 31 \\
\hline 03/23/99 & .96 & .36 & .40 & 14 & 17 & 0 & 2.0 & .30 & $<.10$ & 9.8 & 27 & 35 \\
\hline 05/20/99 & 1.7 & .63 & .63 & 14 & -- & 0 & 4.7 & .88 & $<.10$ & 11 & 34 & 39 \\
\hline $05 / 25 / 99$ & .81 & .45 & .40 & 11 & 13 & 0 & 1.3 & .16 & $<.10$ & 8.0 & 21 & 22 \\
\hline 06/29/99 & 1.0 & .31 & .36 & 18 & 21 & 0 & 1.6 & .22 & $<.10$ & 9.1 & 29 & 30 \\
\hline 07/26/99 & 1.2 & .42 & .43 & 22 & 27 & 0 & 1.1 & .22 & $<.10$ & 9.7 & 33 & 35 \\
\hline 09/08/99 & 1.3 & .44 & .51 & 23 & 28 & 0 & 1.9 & e. 23 & $<.10$ & 10 & -- & 25 \\
\hline $10 / 19 / 99$ & 1.1 & .43 & .39 & 22 & 27 & 0 & 2.0 & e. 28 & $<.10$ & 9.3 & -- & 36 \\
\hline $12 / 01 / 99$ & 1.1 & -- & .39 & 19 & 23 & 0 & 2.2 & .31 & $<.10$ & 9.8 & 32 & 36 \\
\hline 01/11/00 & 1.0 & -- & .33 & 17 & 20 & 0 & 2.4 & e. 26 & $<.10$ & 9.1 & -- & 34 \\
\hline $02 / 29 / 00$ & 1.1 & -- & .36 & 15 & 18 & 0 & 2.0 & .30 & $<.10$ & 9.5 & 28 & 34 \\
\hline $03 / 27 / 00$ & .99 & .38 & .35 & 17 & 20 & 0 & 1.9 & e. 25 & $<.10$ & 9.7 & -- & 25 \\
\hline $04 / 10 / 00$ & .92 & $<.1$ & .41 & 16 & 20 & 0 & 1.7 & e. 22 & $<.10$ & 9.5 & -- & 33 \\
\hline $04 / 14 / 00$ & .73 & .66 & .71 & 10 & 12 & 0 & 1.3 & e. 25 & $<.10$ & 9.0 & -- & 29 \\
\hline $04 / 17 / 00$ & .85 & .51 & .42 & 12 & 15 & 0 & 1.2 & e. 22 & $<.10$ & 9.5 & -- & 27 \\
\hline $05 / 02 / 00$ & 1.1 & .33 & .33 & 14 & 17 & 0 & 1.2 & e. 21 & $<.10$ & 8.9 & -- & 27 \\
\hline $05 / 18 / 00$ & .88 & -- & .33 & 15 & 19 & 0 & 1.4 & e. 25 & $<.10$ & 8.5 & -- & 23 \\
\hline 06/02/00 & .95 & .39 & .38 & 16 & 20 & 0 & 1.5 & e. 22 & $<.10$ & 8.6 & -- & 29 \\
\hline $06 / 29 / 00$ & 1.1 & .34 & .38 & -- & -- & 0 & 1.4 & e. 22 & $<.10$ & 9.1 & -- & 28 \\
\hline $07 / 24 / 00$ & 1.2 & -- & .45 & 22 & 26 & 0 & 1.5 & e. 18 & $<.10$ & 9.9 & -- & 35 \\
\hline $08 / 31 / 00$ & 1.2 & .43 & .43 & 30 & 37 & 0 & 1.8 & e. 19 & $<.10$ & 9.4 & -- & 33 \\
\hline $11 / 06 / 00$ & 1.1 & -- & .42 & 22 & 28 & 0 & 2.0 & .41 & $<.16$ & 8.8 & 34 & 37 \\
\hline $12 / 13 / 00$ & 1.1 & -- & .31 & 21 & 25 & 0 & 2.1 & .37 & $<.16$ & 8.7 & 32 & 33 \\
\hline $01 / 23 / 01$ & 1.1 & -- & .38 & 21 & 26 & 0 & 2.2 & .31 & $<.16$ & 8.1 & 32 & 31 \\
\hline $03 / 14 / 01$ & 1.1 & -- & .34 & 20 & 25 & 0 & 2.6 & .53 & $<.16$ & 8.1 & 32 & 30 \\
\hline 04/10/01 & 1.1 & -- & .37 & 19 & 23 & 0 & 2.4 & .49 & $<.16$ & 8.7 & 31 & 40 \\
\hline 05/03/01 & .92 & -- & .31 & 13 & 16 & 0 & 1.8 & .22 & $<.16$ & 8.9 & 25 & 24 \\
\hline $06 / 12 / 01$ & 1.1 & -- & .35 & 20 & 24 & 0 & 1.9 & .30 & $<.16$ & 9.0 & 31 & 34 \\
\hline \multicolumn{13}{|c|}{ SITE 31 12413470--SOUTH FORK COEUR D'ALENE RIVER NEAR PINEHURST, IDAHO } \\
\hline $11 / 04 / 98$ & 8.4 & 1.8 & 1.9 & 38 & 46 & 0 & 71 & 3.5 & .42 & 11 & 156 & 157 \\
\hline $11 / 04 / 98^{\mathbf{F b}}$ & $<.02$ & -- & -- & -- & -- & -- & -- & -- & -- & $<.02$ & -- & -- \\
\hline $12 / 09 / 98$ & 5.6 & 1.2 & 1.3 & 29 & 35 & 0 & 67 & 4.6 & .24 & 11 & 143 & 172 \\
\hline 02/03/99 & 4.9 & -- & 1.1 & 28 & 34 & 0 & 56 & 4.3 & .19 & 10 & 122 & 128 \\
\hline 03/17/99 & 3.8 & .84 & .82 & 23 & 28 & 0 & 32 & 4.0 & .11 & 11 & 87 & 93 \\
\hline 03/23/99 & 2.5 & .80 & .70 & 20 & 24 & 0 & 18 & 2.8 & $<.10$ & 11 & 59 & 68 \\
\hline 04/20/99 & 1.6 & .55 & .53 & 16 & 20 & 0 & 11 & 1.3 & $<.10$ & 8.9 & 42 & 48 \\
\hline $05 / 25 / 99$ & .99 & .69 & .45 & 12 & 15 & 0 & 7.8 & .4 & $<.10$ & 6.7 & 31 & 32 \\
\hline $05 / 27 / 99$ & 1.3 & .42 & .39 & 14 & 16 & 0 & 7.6 & .5 & $<.10$ & 7.0 & 32 & 35 \\
\hline 06/29/99 & 1.8 & -- & .52 & 20 & 24 & 0 & 25 & 1.6 & .12 & 7.6 & 64 & 67 \\
\hline $07 / 26 / 99$ & 4.8 & .92 & .17 & 28 & 34 & 0 & 29 & 1.3 & .14 & 9.9 & 86 & 130 \\
\hline 09/08/99 & 7.2 & 1.4 & 1.4 & 34 & 41 & 0 & 73 & 2.5 & .31 & 11 & 151 & 145 \\
\hline $10 / 19 / 99$ & 7.3 & 1.7 & 1.7 & 32 & 39 & 0 & 98 & 2.6 & .38 & 11 & 186 & 191 \\
\hline $12 / 02 / 99$ & 4.5 & 1.0 & 1.0 & 24 & 29 & 0 & 47 & 3.0 & .16 & 10 & 108 & 110 \\
\hline 01/11/00 & 5.4 & 1.2 & 1.2 & 26 & 32 & 0 & 66 & 3.8 & .24 & 9.7 & 135 & 147 \\
\hline $02 / 28 / 00^{\mathbf{F b}}$ & $<.02$ & -- & -- & -- & -- & -- & -- & -- & -- & .03 & -- & -- \\
\hline
\end{tabular}


Table 6. Streamflow, physical, and major-ion concentration data for surface-water samples collected at fixed water-quality sites, Northern Rockies Intermontane Basins study unit, 1998-2001 (Continued)

\begin{tabular}{|c|c|c|c|c|c|c|c|c|c|c|c|c|c|c|}
\hline Date & Time & $\begin{array}{c}\text { Dis- } \\
\text { charge, } \\
\text { instan- } \\
\text { taneous } \\
\left(\mathbf{f t}^{3} / \mathbf{s}\right)\end{array}$ & $\begin{array}{c}\text { Sedi- } \\
\text { ment, } \\
\text { sus- } \\
\text { pended } \\
(\mathrm{mg} / \mathrm{L})\end{array}$ & $\begin{array}{c}\text { Sedi- } \\
\text { ment, } \\
\text { sus- } \\
\text { pended } \\
\text { (percent } \\
\text { finer } \\
\text { than } \\
\mathbf{0 . 0 6 2} \\
\text { mm) }\end{array}$ & $\begin{array}{c}\text { Specific } \\
\text { conduct- } \\
\text { ance, } \\
\text { field } \\
(\mu \mathrm{S} / \mathrm{cm})\end{array}$ & $\begin{array}{l}\text { pH, } \\
\text { field } \\
\text { (stan- } \\
\text { dard } \\
\text { units) }\end{array}$ & $\begin{array}{c}\text { Tem- } \\
\text { per- } \\
\text { ature, } \\
\text { water } \\
\left({ }^{\circ} \mathrm{C}\right)\end{array}$ & $\begin{array}{c}\text { Hard- } \\
\text { ness } \\
(\mathrm{mg} / \mathrm{L} \\
\text { as } \\
\left.\mathrm{CaCO}_{3}\right)\end{array}$ & $\begin{array}{l}\text { Calci- } \\
\text { um, } \\
\text { total } \\
(\mathrm{mg} / \mathrm{L})\end{array}$ & $\begin{array}{c}\text { Calci- } \\
\text { um, } \\
\text { dis- } \\
\text { solved } \\
(\mathbf{m g} / \mathrm{L})\end{array}$ & $\begin{array}{c}\text { Mag- } \\
\text { nesi- } \\
\text { um, } \\
\text { total } \\
(\mathrm{mg} / \mathrm{L})\end{array}$ & $\begin{array}{c}\text { Mag- } \\
\text { nesium, } \\
\text { dis- } \\
\text { solved } \\
(\mathrm{mg} / \mathrm{L})\end{array}$ & $\begin{array}{c}\text { Sodium } \\
\text { adsorp- } \\
\text { tion } \\
\text { ratio }\end{array}$ & $\begin{array}{c}\text { So- } \\
\text { dium, } \\
\text { total } \\
(\mathrm{mg} / \mathrm{L})\end{array}$ \\
\hline $02 / 28 / 00$ & 1100 & 596 & 1 & 75 & 128 & 7.1 & 4.0 & 48 & 13 & 12 & 4.1 & 4.1 & .2 & 3.4 \\
\hline $03 / 27 / 00$ & 1320 & 700 & 2 & 55 & 124 & 7.5 & 8.0 & 49 & 13 & 12 & 4.5 & 4.3 & .2 & 3.2 \\
\hline $04 / 10 / 00$ & 1315 & 1,290 & 5 & 77 & 91 & 7.4 & 8.0 & 37 & 9.1 & 9.3 & 3.1 & 3.2 & .2 & 2.3 \\
\hline $04 / 14 / 00$ & 1315 & 4,890 & 302 & 74 & 46 & 7.1 & 5.5 & 18 & 4.9 & 4.8 & 2.0 & 1.5 & .1 & 1.3 \\
\hline $04 / 17 / 00$ & 1315 & 2,360 & 17 & 76 & 67 & 7.3 & 7.0 & 27 & 7.3 & 7.2 & 2.4 & 2.3 & .1 & 1.6 \\
\hline $05 / 02 / 00$ & 1145 & 1,890 & 11 & 68 & 73 & 7.1 & 6.5 & 29 & 7.6 & 7.7 & 2.4 & 2.4 & .1 & 1.5 \\
\hline $06 / 29 / 00$ & 1130 & 446 & 2 & 83 & 144 & 7.2 & 15.0 & 56 & 15 & 15 & 4.2 & 4.2 & .2 & 3.5 \\
\hline $07 / 24 / 00$ & 1300 & 241 & 1 & 67 & 224 & 7.1 & 18.0 & 94 & 23 & 26 & 6.7 & 7.1 & .2 & 4.5 \\
\hline $08 / 31 / 00$ & 1215 & 110 & 4 & 88 & 299 & 7.2 & 14.0 & 120 & -- & 30 & 11 & 11 & .3 & 6.2 \\
\hline $11 / 06 / 00$ & 1330 & 115 & 1 & 75 & 288 & 7.1 & 7.5 & 115 & -- & 29 & -- & 10 & .3 & -- \\
\hline $12 / 18 / 00$ & 1200 & 93 & 2 & 82 & 320 & 6.6 & 1.5 & 129 & -- & 32 & -- & 12 & .3 & -- \\
\hline $01 / 23 / 01$ & 1430 & 86 & 1 & 75 & 328 & 6.8 & 3.0 & 130 & -- & 32 & -- & 12 & .3 & -- \\
\hline 03/14/01 & 1145 & 229 & 5 & 81 & 195 & 7.3 & 5.0 & 73 & -- & 19 & -- & 5.9 & .3 & -- \\
\hline 04/10/01 & 1100 & 258 & 2 & 71 & 193 & 7.4 & 5.5 & 78 & -- & 20 & -- & 6.5 & .2 & -- \\
\hline $05 / 03 / 01$ & 1300 & 955 & 3 & 81 & 97 & 7.2 & 7.5 & 36 & -- & 9.6 & -- & 3.0 & .2 & -- \\
\hline $06 / 12 / 01$ & 1230 & 378 & 2 & 86 & 142 & 7.4 & 9.0 & 56 & -- & 15 & -- & 4.5 & .2 & -- \\
\hline 09/10/01 & 1130 & 82 & 2 & 75 & 325 & 7.2 & 13.0 & 136 & -- & 35 & -- & 12 & .2 & -- \\
\hline $09 / 10 / 01^{\mathbf{F b}}$ & 1345 & -- & -- & -- & -- & -- & -- & -- & -- & $<.01$ & -- & $<.008$ & -- & -- \\
\hline \multicolumn{15}{|c|}{ SITE 33 12413875--ST. JOE RIVER AT RED IVES RANGER STATION, IDAHO } \\
\hline $01 / 21 / 00$ & 1245 & 102 & 1 & 60 & 41 & 6.7 & 0.0 & 18 & 5.3 & 5.3 & 1.1 & 1.1 & .1 & 1.0 \\
\hline $05 / 17 / 00$ & 1215 & 1,040 & 2 & 92 & 29 & 8.0 & 6.0 & 13 & 3.8 & 3.8 & .88 & .83 & .1 & .75 \\
\hline $06 / 01 / 00$ & 1100 & 1,040 & 3 & 67 & 30 & 7.4 & 5.0 & 14 & 3.8 & 4.0 & .86 & .89 & .1 & .68 \\
\hline $06 / 28 / 00$ & 1100 & 430 & 2 & 83 & 34 & 7.3 & 9.0 & 16 & 4.4 & 4.8 & .99 & 1.0 & .1 & .77 \\
\hline $07 / 27 / 00$ & 1115 & 181 & 3 & 70 & 45 & 7.8 & 14.0 & 19 & 5.8 & 5.7 & 1.3 & 1.2 & .1 & 1.0 \\
\hline $09 / 07 / 00$ & 1330 & 101 & 1 & 83 & 47 & 7.8 & 10.5 & 22 & 6.4 & 6.5 & 1.4 & 1.4 & .1 & 1.0 \\
\hline $11 / 07 / 00$ & 1015 & 46 & 1 & 75 & 49 & 7.2 & 0.0 & 23 & -- & 6.9 & -- & 1.4 & .1 & -- \\
\hline $01 / 24 / 01$ & 1345 & 49 & 1 & 67 & 47 & 7.5 & 0.0 & 21 & -- & 6.4 & -- & 1.3 & .1 & -- \\
\hline 05/09/01 & 1200 & 488 & 2 & 60 & 33 & 7.9 & 6.0 & 14 & -- & 4.1 & -- & .90 & .1 & -- \\
\hline $06 / 13 / 01$ & 0930 & 408 & 1 & 75 & 37 & 7.6 & 3.5 & 17 & -- & 5.1 & -- & 1.1 & .1 & -- \\
\hline $09 / 13 / 01$ & 1045 & 64 & 1 & 71 & 51 & 7.7 & 9.5 & 24 & -- & 7.2 & -- & 1.5 & .1 & -- \\
\hline \multicolumn{15}{|c|}{ SITE 35 12419000--SPOKANE RIVER NEAR POST FALLS, IDAHO } \\
\hline 03/04/99 & 1230 & 1,3600 & 2 & 63 & 71 & 7.5 & 2.5 & 22 & -- & 5.9 & -- & 1.9 & .2 & -- \\
\hline $04 / 13 / 99$ & 1000 & 9,760 & 1 & 87 & 55 & 7.4 & 4.0 & 21 & 5.7 & 5.5 & 1.8 & 1.7 & .2 & 1.9 \\
\hline 05/19/99 & 1000 & 13,800 & 1 & 71 & 47 & 7.1 & 8.5 & -- & -- & -- & -- & -- & -- & -- \\
\hline $06 / 02 / 99$ & 0930 & 23,300 & 3 & 89 & 45 & 7.0 & 11.0 & 18 & 4.8 & 4.8 & 1.5 & 1.5 & .1 & 1.4 \\
\hline $06 / 23 / 99$ & 1030 & 12,500 & 2 & 77 & 41 & 7.6 & 15.0 & 16 & 4.6 & 4.4 & 1.3 & 1.3 & .1 & 1.2 \\
\hline
\end{tabular}


Table 6. Streamflow, physical, and major-ion concentration data for surface-water samples collected at fixed water-quality sites, Northern Rockies Intermontane Basins study unit, 1998-2001 (Continued)

\begin{tabular}{|c|c|c|c|c|c|c|c|c|c|c|c|c|}
\hline Date & $\begin{array}{c}\text { Sodium, } \\
\text { dissolved } \\
(\mathrm{mg} / \mathrm{L})\end{array}$ & $\begin{array}{c}\text { Potas- } \\
\text { sium, } \\
\text { total } \\
(\mathrm{mg} / \mathrm{L})\end{array}$ & $\begin{array}{l}\text { Potas- } \\
\text { sium, } \\
\text { dissolved } \\
(\mathrm{mg} / \mathrm{L})\end{array}$ & $\begin{array}{c}\text { Alka- } \\
\text { linity, } \\
\text { field } \\
(\mathrm{mg} / \mathrm{L} \text { as } \\
\left.\mathrm{CaCO}_{3}\right)\end{array}$ & $\begin{array}{c}\text { Bicar- } \\
\text { bonate, } \\
\text { dissolved } \\
(\mathrm{mg} / \mathrm{L} \text { as } \\
\left.\mathrm{HCO}_{3}\right)\end{array}$ & $\begin{array}{c}\text { Carbo- } \\
\text { nate, } \\
\text { dissolved } \\
(\mathrm{mg} / \mathrm{L} \text { as } \\
\left.\mathrm{CO}_{3}\right)\end{array}$ & $\begin{array}{c}\text { Sulfate, } \\
\text { dissolved } \\
(\mathrm{mg} / \mathrm{L} \text { as } \\
\left.\mathrm{SO}_{4}\right)\end{array}$ & $\begin{array}{c}\text { Chloride, } \\
\text { dissolved } \\
(\mathrm{mg} / \mathrm{L})\end{array}$ & $\begin{array}{c}\text { Fluoride, } \\
\text { dissolved } \\
(\mathrm{mg} / \mathrm{L})\end{array}$ & $\begin{array}{c}\text { Silica, } \\
\text { dissolved } \\
(\mathrm{mg} / \mathrm{L} \text { as } \\
\left.\mathrm{SiO}_{2}\right)\end{array}$ & $\begin{array}{l}\text { Dissolved } \\
\text { solids, } \\
\text { sum of } \\
\text { constit- } \\
\text { uents } \\
(\mathrm{mg} / \mathrm{L})\end{array}$ & $\begin{array}{c}\text { Dissolved } \\
\text { solids, } \\
\text { residue at } \\
180^{\circ} \mathrm{C} \\
(\mathrm{mg} / \mathrm{L})\end{array}$ \\
\hline \multicolumn{13}{|c|}{ ST, IDAHO (Continued) } \\
\hline $02 / 28 / 00$ & 3.5 & .82 & .77 & 20 & 25 & 0 & 28 & 3.2 & $<.10$ & 11 & 77 & 83 \\
\hline 03/27/00 & 3.2 & .87 & .81 & 25 & 30 & 0 & 30 & 2.8 & $<.10$ & 11 & 81 & 84 \\
\hline $04 / 10 / 00$ & 2.1 & .37 & .67 & 20 & 25 & 0 & 19 & 1.7 & $<.10$ & 9.9 & 59 & 66 \\
\hline $04 / 14 / 00$ & 1.1 & .70 & .61 & 12 & 15 & 0 & 6.9 & .8 & $<.10$ & 8.4 & 32 & 39 \\
\hline $04 / 17 / 00$ & 1.7 & .77 & .56 & 17 & 21 & 0 & 12 & 1.2 & $<.10$ & 9.4 & 45 & 49 \\
\hline 05/02/00 & 1.7 & .51 & .48 & 17 & 21 & 0 & 14 & .9 & $<.10$ & 7.9 & 46 & 50 \\
\hline 05/18/00 & 1.6 & .43 & .47 & 17 & 20 & 0 & 13 & .8 & $<.10$ & 7.4 & 43 & 45 \\
\hline $06 / 02 / 00$ & 2.7 & .56 & .57 & 20 & 24 & 0 & 21 & 1.2 & .10 & 7.8 & 58 & 58 \\
\hline $06 / 29 / 00^{\mathbf{F b}}$ & .02 & -- & -- & -- & -- & -- & -- & -- & -- & $<.02$ & -- & -- \\
\hline $06 / 29 / 00$ & 3.8 & .82 & .84 & 26 & 31 & 0 & 35 & 1.8 & .12 & 8.8 & 87 & 87 \\
\hline 07/24/00 & 4.6 & 1.1 & 1.2 & 30 & 37 & 0 & 65 & 2.4 & .24 & 10 & 137 & 147 \\
\hline 08/31/00 & 6.5 & 1.6 & 1.7 & 34 & 41 & 0 & 100 & 2.9 & .34 & 10 & 187 & 192 \\
\hline $11 / 06 / 00$ & 6.2 & -- & 1.8 & 33 & 40 & 0 & 93 & 3.8 & .35 & 10 & 180 & 195 \\
\hline $12 / 18 / 00$ & 6.6 & -- & 1.9 & 33 & 40 & 0 & 105 & 4.1 & .39 & 10 & 199 & 208 \\
\hline 01/23/01 & 7.0 & -- & 1.8 & 31 & 38 & 0 & 108 & 6.3 & .35 & 9.7 & 202 & 213 \\
\hline 03/14/01 & 5.4 & -- & 1.4 & 28 & 34 & 0 & 48 & 5.9 & .25 & 10 & 117 & 125 \\
\hline 04/10/01 & 4.5 & -- & 1.2 & 27 & 33 & 0 & 52 & 5.5 & .20 & 9.9 & 119 & 134 \\
\hline 05/03/01 & 2.3 & -- & .64 & 20 & 24 & 0 & 20 & 2.2 & $<.16$ & 9.2 & 60 & 61 \\
\hline 06/12/01 & 3.0 & -- & .76 & 25 & 31 & 0 & 32 & 2.6 & e. 12 & 8.3 & 83 & 88 \\
\hline 09/10/01 & 5.6 & -- & 1.7 & 36 & 44 & 0 & 107 & 4.7 & .40 & 11 & 203 & 206 \\
\hline $09 / 10 / 01^{\mathbf{F b}}$ & $<.06$ & -- & -- & -- & -- & -- & -- & -- & -- & $<.09$ & -- & -- \\
\hline \multicolumn{13}{|c|}{ SITE 33 12413875--ST. JOE RIVER AT RED IVES RANGER STATION, IDAHO } \\
\hline $02 / 18 / 99$ & 1.1 & .56 & .52 & 20 & 24 & 0 & 1.2 & .23 & $<.10$ & 7.5 & 29 & 32 \\
\hline 06/01/99 & .75 & .55 & .44 & 12 & 15 & 0 & .38 & $<.1$ & $<.10$ & 6.1 & -- & 37 \\
\hline $06 / 28 / 99$ & .71 & -- & .48 & 14 & 17 & 0 & .58 & $<.1$ & $<.10$ & 6.1 & -- & 22 \\
\hline 07/20/99 & .82 & .55 & .46 & 16 & 19 & 0 & .38 & .26 & $<.10$ & 6.5 & 23 & 29 \\
\hline 08/18/99 & 1.0 & .58 & .72 & 22 & 27 & 0 & .87 & .12 & $<.10$ & 7.5 & 31 & 33 \\
\hline $11 / 04 / 99$ & .92 & .63 & .59 & 19 & 23 & 0 & 1.0 & e. 26 & $<.10$ & 6.9 & -- & 33 \\
\hline $01 / 21 / 00$ & .95 & .64 & .57 & 18 & 23 & 0 & 1.3 & e. 25 & $<.10$ & 7.3 & -- & 30 \\
\hline $05 / 17 / 00$ & .75 & .42 & .43 & 14 & 17 & 0 & .61 & e. 21 & $<.10$ & 6.1 & -- & 22 \\
\hline 06/01/00 & .72 & .46 & .45 & 15 & 19 & 0 & .60 & $<.29$ & $<.10$ & 6.0 & -- & 21 \\
\hline $06 / 28 / 00$ & .79 & .37 & .42 & 18 & 22 & 0 & .53 & $<.29$ & $<.10$ & 6.4 & -- & 23 \\
\hline $07 / 27 / 00$ & .96 & .51 & .57 & 23 & 28 & 0 & .74 & $<.29$ & $<.10$ & 7.0 & -- & 35 \\
\hline 09/07/00 & 1.0 & .62 & .66 & 24 & 29 & 0 & .97 & e. 14 & $<.10$ & 7.3 & -- & 40 \\
\hline $11 / 07 / 00$ & 1.1 & -- & .63 & 24 & 29 & 0 & 1.3 & .26 & $<.16$ & 7.7 & 34 & 36 \\
\hline $01 / 24 / 01$ & 1.1 & -- & .66 & 23 & 28 & 0 & 1.2 & .20 & $<.16$ & 7.8 & 33 & 31 \\
\hline 05/09/01 & .79 & -- & .48 & 15 & 18 & 0 & .81 & .12 & $<.16$ & 5.9 & 22 & 36 \\
\hline 06/13/01 & .79 & -- & .43 & 19 & 24 & 0 & .76 & .12 & $<.16$ & 6.4 & 26 & 27 \\
\hline 09/13/01 & 1.1 & -- & .59 & 25 & 31 & 0 & 1.3 & .14 & $<.16$ & 6.0 & 33 & 28 \\
\hline \multicolumn{13}{|c|}{ SITE 35 12419000--SPOKANE RIVER NEAR POST FALLS, IDAHO } \\
\hline 03/04/99 & 1.7 & -- & .85 & 20 & 24 & 0 & 5.6 & 1.0 & $<.10$ & 10 & 39 & 45 \\
\hline 04/13/99 & 1.9 & .74 & .71 & 18 & 22 & 0 & 5.0 & 1.1 & $<.10$ & 10 & 37 & 48 \\
\hline 05/19/99 & -- & -- & -- & 17 & 21 & 0 & -- & -- & -- & -- & -- & -- \\
\hline 06/02/99 & 1.4 & .58 & .63 & 17 & 20 & 0 & 3.0 & .74 & $<.10$ & 10 & 32 & -- \\
\hline $06 / 23 / 99$ & 1.3 & .54 & .57 & 17 & 20 & 0 & 2.5 & .50 & $<.10$ & 8.8 & 29 & 42 \\
\hline
\end{tabular}


Table 6. Streamflow, physical, and major-ion concentration data for surface-water samples collected at fixed water-quality sites, Northern Rockies Intermontane Basins study unit, 1998-2001 (Continued)

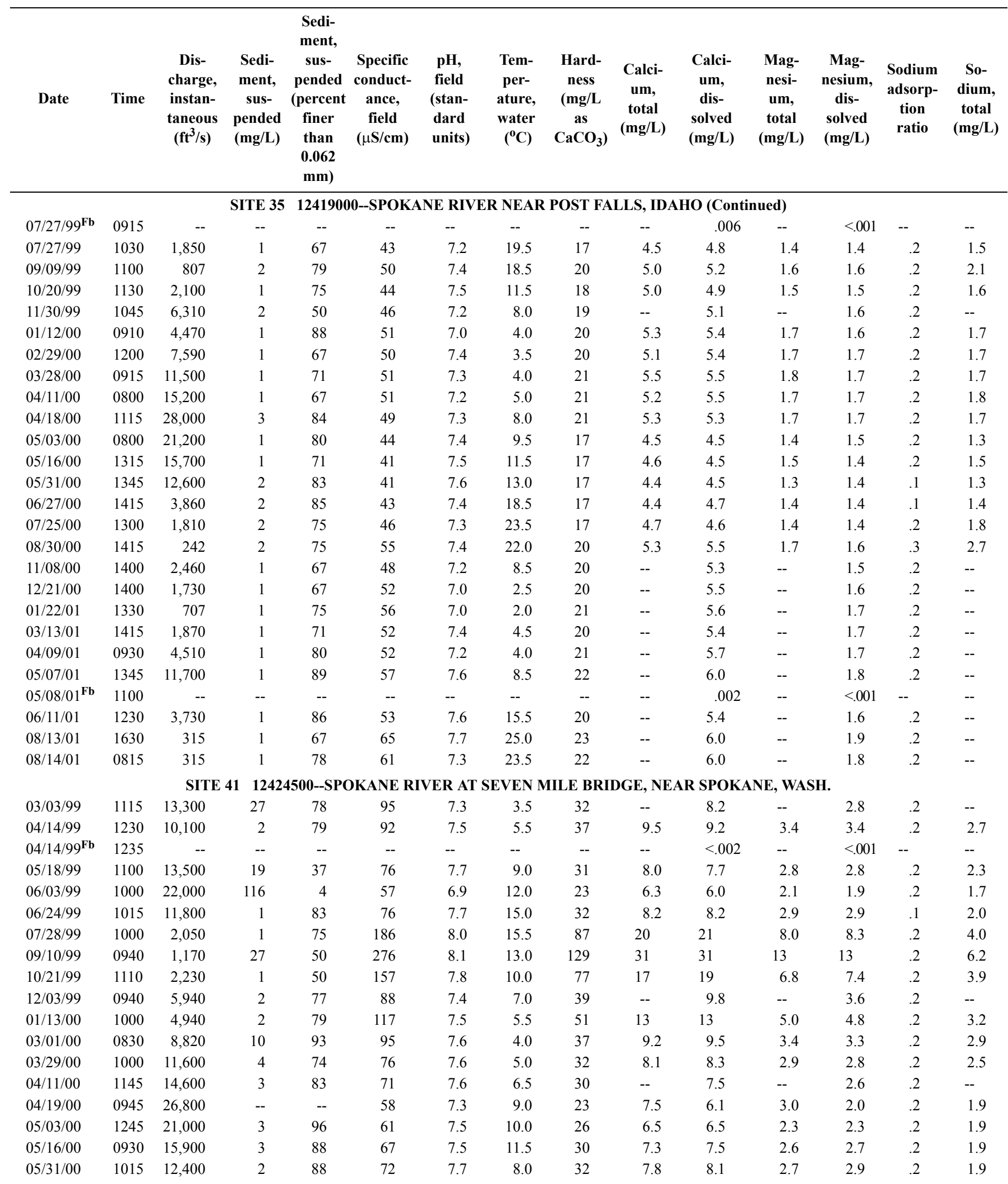


Table 6. Streamflow, physical, and major-ion concentration data for surface-water samples collected at fixed water-quality sites, Northern Rockies Intermontane Basins study unit, 1998-2001 (Continued)

\begin{tabular}{|c|c|c|c|c|c|c|c|c|c|c|c|c|}
\hline Date & $\begin{array}{c}\text { Sodium, } \\
\text { dissolved } \\
(\mathrm{mg} / \mathrm{L})\end{array}$ & $\begin{array}{c}\text { Potas- } \\
\text { sium, } \\
\text { total } \\
(\mathrm{mg} / \mathrm{L})\end{array}$ & $\begin{array}{l}\text { Potas- } \\
\text { sium, } \\
\text { dissolved } \\
(\mathrm{mg} / \mathrm{L})\end{array}$ & $\begin{array}{c}\text { Alka- } \\
\text { linity, } \\
\text { field } \\
(\mathrm{mg} / \mathrm{L} \text { as } \\
\left.\mathrm{CaCO}_{3}\right)\end{array}$ & $\begin{array}{c}\text { Bicar- } \\
\text { bonate, } \\
\text { dissolved } \\
(\mathrm{mg} / \mathrm{L} \text { as } \\
\left.\mathrm{HCO}_{3}\right)\end{array}$ & $\begin{array}{l}\text { Carbo- } \\
\text { nate, } \\
\text { dissolved } \\
(\mathrm{mg} / \mathrm{L} \text { as } \\
\left.\mathrm{CO}_{3}\right)\end{array}$ & $\begin{array}{c}\text { Sulfate, } \\
\text { dissolved } \\
(\mathrm{mg} / \mathrm{L} \text { as } \\
\left.\mathrm{SO}_{4}\right)\end{array}$ & $\begin{array}{c}\text { Chloride, } \\
\text { dissolved } \\
(\mathrm{mg} / \mathrm{L})\end{array}$ & $\begin{array}{c}\text { Fluoride, } \\
\text { dissolved } \\
(\mathrm{mg} / \mathrm{L})\end{array}$ & $\begin{array}{c}\text { Silica, } \\
\text { dissolved } \\
(\mathrm{mg} / \mathrm{L} \text { as } \\
\left.\mathrm{SiO}_{2}\right)\end{array}$ & $\begin{array}{l}\text { Dissolved } \\
\text { solids, } \\
\text { sum of } \\
\text { constit- } \\
\text { uents } \\
(\mathrm{mg} / \mathrm{L})\end{array}$ & $\begin{array}{c}\text { Dissolved } \\
\text { solids, } \\
\text { residue at } \\
180^{\circ} \mathrm{C} \\
(\mathrm{mg} / \mathrm{L})\end{array}$ \\
\hline \multicolumn{13}{|c|}{ SITE 35 12419000--SPOKANE RIVER NEAR POST FALLS, IDAHO (Continued) } \\
\hline $07 / 27 / 99^{\mathbf{F b}}$ & $<.02$ & -- & -- & -- & -- & -- & -- & -- & -- & .02 & -- & -- \\
\hline 07/27/99 & 1.5 & .57 & 1.0 & 17 & 20 & 0 & 2.5 & .71 & $<.10$ & 8.3 & 30 & 38 \\
\hline 09/09/99 & 2.2 & .69 & .74 & 18 & 22 & 0 & 3.8 & 1.4 & $<.10$ & 6.9 & 33 & 34 \\
\hline $10 / 20 / 99$ & 1.6 & .69 & .66 & 17 & 20 & 0 & 3.7 & .84 & $<.10$ & 7.6 & 32 & 34 \\
\hline $11 / 30 / 99$ & 1.5 & -- & .65 & 18 & 22 & 0 & 3.8 & .74 & $<.10$ & 9.2 & 34 & 38 \\
\hline $01 / 12 / 00$ & 1.7 & .66 & .68 & 16 & 20 & 0 & 4.7 & .86 & $<.10$ & 9.1 & 34 & 41 \\
\hline $02 / 29 / 00$ & 1.7 & .66 & .71 & 16 & 20 & 0 & 4.3 & .98 & $<.10$ & 9.5 & 35 & 41 \\
\hline 03/28/00 & 1.7 & .75 & .70 & 18 & 22 & 0 & 5.2 & .99 & $<.10$ & 9.8 & 37 & 39 \\
\hline $04 / 11 / 00$ & 1.7 & .41 & .74 & 19 & 23 & 0 & 4.8 & 1.0 & $<.10$ & 10 & 37 & 43 \\
\hline $04 / 18 / 00$ & 1.7 & .72 & .44 & 17 & 21 & 0 & 4.2 & .88 & $<.10$ & 12 & 37 & 42 \\
\hline 05/03/00 & 1.5 & .53 & .70 & 16 & 19 & 0 & 3.4 & .74 & $<.10$ & 11 & 33 & 38 \\
\hline $05 / 16 / 00$ & 1.5 & .64 & .56 & 16 & 20 & 0 & 3.4 & .64 & $<.10$ & 11 & 32 & 35 \\
\hline $05 / 31 / 00$ & 1.4 & .66 & .63 & 17 & 20 & 0 & 3.2 & .67 & $<.10$ & 9.7 & 32 & 35 \\
\hline $06 / 27 / 00$ & 1.4 & .53 & .57 & 16 & 20 & 0 & 2.8 & .66 & $<.10$ & 9.0 & 30 & 30 \\
\hline 07/25/00 & 1.7 & .59 & .63 & 18 & 22 & 0 & 3.1 & .91 & $<.10$ & 8.3 & 32 & 36 \\
\hline 08/30/00 & 2.9 & .81 & .74 & 20 & 25 & 0 & 4.1 & 1.8 & $<.10$ & 7.4 & 38 & 36 \\
\hline $11 / 08 / 00$ & 1.6 & -- & .68 & 20 & 24 & 0 & 3.8 & .93 & $<.16$ & 8.0 & 34 & 39 \\
\hline $12 / 21 / 00$ & 1.9 & -- & .73 & 20 & 24 & 0 & 4.2 & 1.1 & $<.16$ & 9.4 & 37 & 44 \\
\hline $01 / 22 / 01$ & 2.5 & -- & .86 & 19 & 23 & 0 & 4.4 & 1.6 & $<.16$ & 9.2 & 39 & 39 \\
\hline 03/13/01 & 2.0 & -- & .78 & 17 & 21 & 0 & 4.7 & 1.2 & $<.16$ & 8.9 & 35 & 46 \\
\hline 04/09/01 & 1.7 & -- & .72 & 19 & 23 & 0 & 4.9 & .94 & $<.16$ & 8.9 & 36 & 45 \\
\hline 05/07/01 & 1.7 & -- & .80 & 20 & 24 & 0 & 5.5 & .94 & $<.16$ & 8.6 & 38 & 36 \\
\hline $05 / 08 / 01^{\mathbf{F b}}$ & $<.02$ & -- & -- & -- & -- & -- & -- & -- & -- & $<.02$ & -- & -- \\
\hline $06 / 11 / 01$ & 1.7 & -- & .63 & 18 & 22 & 0 & 4.4 & .96 & $<.20$ & 8.6 & 34 & 40 \\
\hline 08/13/01 & 2.7 & -- & .84 & 21 & 25 & 0 & 5.1 & 1.7 & $<.20$ & 7.7 & 38 & 43 \\
\hline 08/14/01 & 2.6 & -- & .82 & 20 & 24 & 0 & 5.1 & 1.6 & $<.20$ & 7.7 & 37 & 38 \\
\hline \multicolumn{13}{|c|}{ SITE 41 12424500--SPOKANE RIVER AT SEVEN MILE BRIDGE, NEAR SPOKANE, WASH. } \\
\hline 03/03/99 & 2.6 & -- & .98 & 26 & 31 & 0 & 6.7 & 2.2 & $<.10$ & 12 & 53 & 60 \\
\hline 04/14/99 & 2.7 & 1.1 & 1.1 & 32 & 40 & 0 & 7.3 & 1.9 & $<.10$ & 10 & 58 & 66 \\
\hline $04 / 14 / 99^{\mathbf{F b}}$ & $<.02$ & -- & -- & -- & -- & -- & -- & -- & -- & $<.02$ & -- & -- \\
\hline 05/18/99 & 2.3 & .86 & .87 & 29 & 36 & 0 & 6.1 & 1.7 & $<.10$ & 11 & 52 & 53 \\
\hline 06/03/99 & 1.7 & .66 & .74 & 22 & 27 & 0 & 3.5 & 1.0 & $<.10$ & 10 & 38 & -- \\
\hline $06 / 24 / 99$ & 1.9 & .77 & .89 & 33 & 40 & 0 & 4.7 & 1.2 & $<.10$ & 9.3 & 50 & 60 \\
\hline 07/28/99 & 4.1 & 1.4 & 1.5 & 76 & 92 & 0 & 8.4 & 3.0 & $<.10$ & 11 & 107 & 112 \\
\hline 09/10/99 & 6.4 & 2.3 & 2.2 & 115 & 140 & 0 & 15 & 5.9 & $<.10$ & 12 & 163 & 170 \\
\hline $10 / 21 / 99$ & 4.1 & 1.4 & 1.4 & 66 & 80 & 0 & 8.7 & 2.8 & $<.10$ & 11 & 96 & 95 \\
\hline $12 / 03 / 99$ & 2.4 & -- & .91 & 35 & 43 & 0 & 5.8 & 1.6 & $<.10$ & 10 & 57 & 62 \\
\hline 01/13/00 & 3.0 & 1.2 & 1.1 & 43 & 52 & 0 & 7.5 & 2.7 & .12 & 10 & 71 & 77 \\
\hline 03/01/00 & 2.9 & 1.2 & 1.0 & 30 & 36 & 0 & 6.2 & 1.7 & $<.10$ & 12 & 59 & 67 \\
\hline 03/29/00 & 2.4 & .99 & .92 & 29 & 36 & 0 & 6.3 & 1.6 & $<.10$ & 11 & 52 & 53 \\
\hline 04/11/00 & 2.2 & -- & .88 & 27 & 33 & 0 & 5.6 & 1.4 & $<.10$ & 11 & 49 & 56 \\
\hline 04/19/00 & 2.0 & 1.0 & .82 & 21 & 26 & 0 & 4.6 & 1.2 & $<.10$ & 12 & 42 & 49 \\
\hline 05/03/00 & 1.9 & .87 & .77 & 24 & 29 & 0 & 4.0 & 1.2 & $<.10$ & 11 & 44 & 49 \\
\hline $05 / 16 / 00$ & 2.1 & .87 & .73 & 28 & 34 & 0 & 4.6 & 1.1 & $<.10$ & 11 & 48 & 51 \\
\hline $05 / 31 / 00$ & 2.0 & .86 & .85 & 30 & 36 & 0 & 4.5 & 1.2 & $<.10$ & 10 & 49 & 51 \\
\hline
\end{tabular}


Table 6. Streamflow, physical, and major-ion concentration data for surface-water samples collected at fixed water-quality sites, Northern Rockies Intermontane Basins study unit, 1998-2001 (Continued)

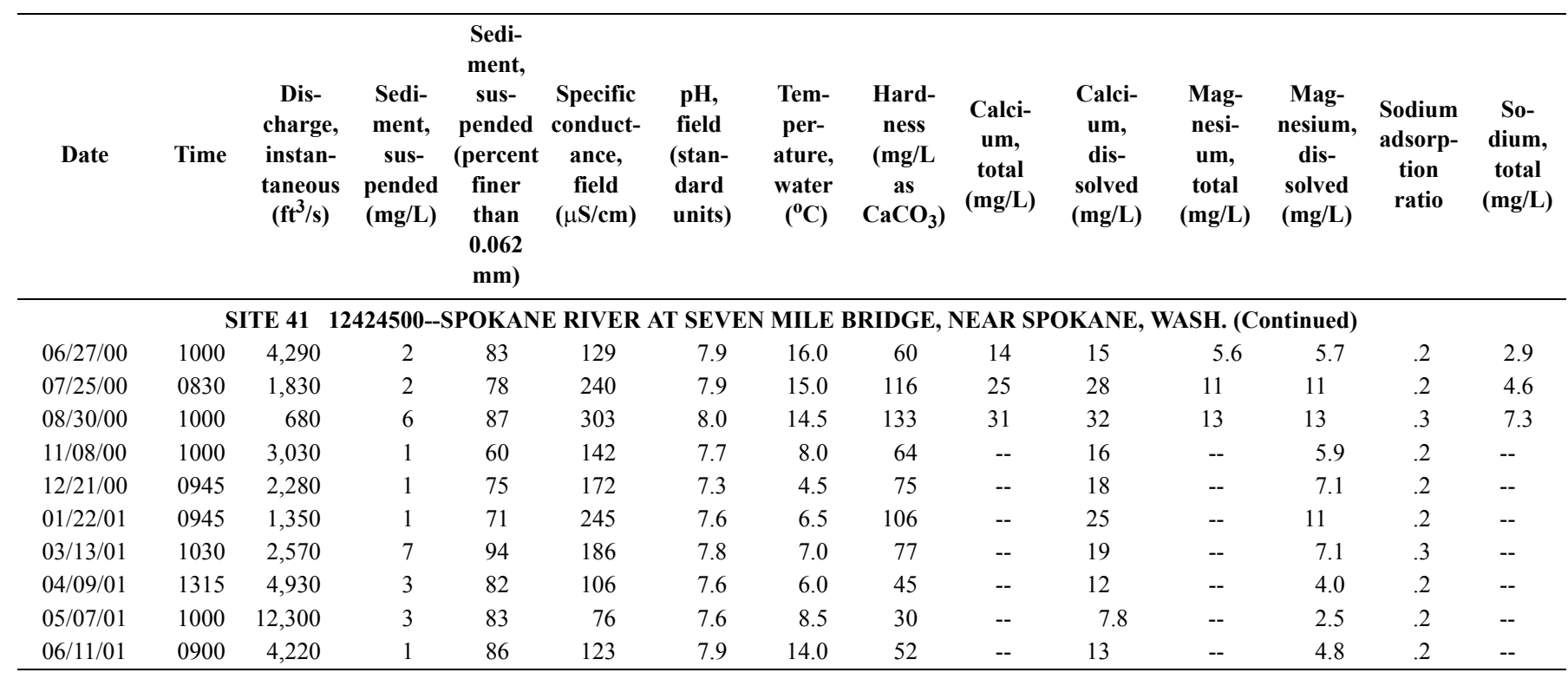


Table 6. Streamflow, physical, and major-ion concentration data for surface-water samples collected at fixed water-quality sites, Northern Rockies Intermontane Basins study unit, 1998-2001 (Continued)

\begin{tabular}{|c|c|c|c|c|c|c|c|c|c|c|c|c|}
\hline Date & $\begin{array}{c}\text { Sodium, } \\
\text { dissolved } \\
(\mathrm{mg} / \mathrm{L})\end{array}$ & $\begin{array}{l}\text { Potas- } \\
\text { sium, } \\
\text { total } \\
(\mathrm{mg} / \mathrm{L})\end{array}$ & $\begin{array}{l}\text { Potas- } \\
\text { sium, } \\
\text { dissolved } \\
(\mathrm{mg} / \mathrm{L})\end{array}$ & $\begin{array}{c}\text { Alka- } \\
\text { linity, } \\
\text { field } \\
(\mathrm{mg} / \mathrm{L} \text { as } \\
\left.\mathrm{CaCO}_{3}\right)\end{array}$ & $\begin{array}{c}\text { Bicar- } \\
\text { bonate, } \\
\text { dissolved } \\
(\mathrm{mg} / \mathrm{L} \text { as } \\
\left.\mathrm{HCO}_{3}\right)\end{array}$ & $\begin{array}{c}\text { Carbo- } \\
\text { nate, } \\
\text { dissolved } \\
(\mathrm{mg} / \mathrm{L} \text { as } \\
\left.\mathrm{CO}_{3}\right)\end{array}$ & $\begin{array}{c}\text { Sulfate, } \\
\text { dissolved } \\
(\mathrm{mg} / \mathrm{L} \text { as } \\
\left.\mathrm{SO}_{4}\right)\end{array}$ & $\begin{array}{c}\text { Chloride, } \\
\text { dissolved } \\
(\mathrm{mg} / \mathrm{L})\end{array}$ & $\begin{array}{c}\text { Fluoride, } \\
\text { dissolved } \\
(\mathrm{mg} / \mathrm{L})\end{array}$ & $\begin{array}{c}\text { Silica, } \\
\text { dissolved } \\
(\mathrm{mg} / \mathrm{L} \text { as } \\
\left.\mathrm{SiO}_{2}\right)\end{array}$ & $\begin{array}{c}\text { Dissolved } \\
\text { solids, } \\
\text { sum of } \\
\text { constit- } \\
\text { uents } \\
(\mathrm{mg} / \mathrm{L})\end{array}$ & $\begin{array}{l}\text { Dissolved } \\
\text { solids, } \\
\text { residue at } \\
180^{\circ} \mathrm{C} \\
(\mathrm{mg} / \mathrm{L})\end{array}$ \\
\hline \multicolumn{13}{|c|}{ IDGE, NEAR SPOKANE, WASH. (Continued) } \\
\hline $06 / 27 / 00$ & 3.1 & 1.1 & 1.1 & 52 & 64 & 0 & 6.5 & 2.2 & $<.10$ & 10 & 78 & 78 \\
\hline $07 / 25 / 00$ & 4.7 & 1.8 & 1.8 & 98 & 120 & 0 & 11 & 3.8 & $<.10$ & 12 & 137 & 140 \\
\hline $08 / 30 / 00$ & 7.8 & 2.4 & 2.5 & 114 & 139 & 0 & 17 & 7.3 & .12 & 12 & 170 & 175 \\
\hline $11 / 08 / 00$ & 3.3 & -- & 1.2 & 58 & 71 & 0 & 7.5 & 2.5 & $<.16$ & 9.4 & 84 & 90 \\
\hline $12 / 21 / 00$ & 4.2 & -- & 1.5 & 66 & 80 & 0 & 8.9 & 3.2 & $<.16$ & 11 & 98 & 103 \\
\hline $01 / 22 / 01$ & 5.7 & -- & 2.0 & 92 & 113 & 0 & 11 & 7.6 & $<.16$ & 11 & 136 & 139 \\
\hline 03/13/01 & 5.1 & -- & 2.1 & 64 & 79 & 0 & 10 & 3.6 & $<.16$ & 13 & 112 & 120 \\
\hline 04/09/01 & 3.2 & -- & 1.1 & 42 & 51 & 0 & 7.5 & 2.3 & $<.16$ & 10 & 68 & 77 \\
\hline 05/07/01 & 2.3 & -- & .84 & 26 & 32 & 0 & 6.3 & 1.4 & $<.16$ & 8.9 & 47 & 49 \\
\hline $06 / 11 / 01$ & 3.0 & -- & 1.1 & 46 & 56 & 0 & 7.4 & 2.2 & $<.20$ & 9.5 & 71 & 76 \\
\hline
\end{tabular}


Table 7. Nutrient and organic-carbon concentration data for surface-water samples collected at fixed water-quality sites, Northern Rockies Intermontane Basins study unit, 1998-2001

[See figure 1 for site locations. Abbreviations: e, estimated; Fb, field blank; mg/L, milligrams per liter; R, replicate. Symbols: <, less than reporting level; ,-- no data]

\begin{tabular}{|c|c|c|c|c|c|c|c|c|c|c|c|}
\hline Date & Time & $\begin{array}{c}\text { Nitrite, } \\
\text { dissolved } \\
\text { (mg/L } \\
\text { as N) }\end{array}$ & $\begin{array}{l}\text { Nitrite plus } \\
\text { nitrate, } \\
\text { dissolved } \\
\text { (mg/L } \\
\text { as N) }\end{array}$ & $\begin{array}{c}\text { Ammonia, } \\
\text { dissolved } \\
(\mathrm{mg} / \mathrm{L} \\
\text { as } \mathrm{N})\end{array}$ & $\begin{array}{c}\text { Ammonia } \\
\text { plus } \\
\text { organic } \\
\text { nitrogen, } \\
\text { dissolved } \\
\text { (mg/L } \\
\text { as } \mathrm{N} \text { ) }\end{array}$ & $\begin{array}{c}\text { Ammonia } \\
\text { plus organic } \\
\text { nitrogen, } \\
\text { total } \\
(\mathrm{mg} / \mathrm{L} \\
\text { as N) }\end{array}$ & $\begin{array}{c}\text { Phos- } \\
\text { phorus, } \\
\text { dissolved } \\
(\mathbf{m g} / \mathrm{L})\end{array}$ & $\begin{array}{c}\text { Phos- } \\
\text { phorus, } \\
\text { ortho- } \\
\text { phosphate } \\
\text { dissolved } \\
\text { (mg/L } \\
\text { as P) }\end{array}$ & $\begin{array}{l}\text { Phos- } \\
\text { pho- } \\
\text { rus, } \\
\text { total } \\
(\mathrm{mg} / \mathrm{L})\end{array}$ & $\begin{array}{c}\text { Organic } \\
\text { carbon, } \\
\text { suspended } \\
(\mathbf{m g} / \mathrm{L} \\
\text { as C) }\end{array}$ & $\begin{array}{c}\text { Organic } \\
\text { carbon, } \\
\text { dissolved } \\
(\mathbf{m g} / \mathrm{L} \\
\text { as C) }\end{array}$ \\
\hline \multicolumn{12}{|c|}{ SITE 3 12334550--CLARK FORK AT TURAH BRIDGE, NEAR BONNER, MONT. } \\
\hline $11 / 05 / 98$ & 1000 & $<0.001$ & 0.006 & $<0.002$ & $<0.10$ & 0.14 & 0.007 & 0.006 & 0.016 & 0.4 & 1.4 \\
\hline $12 / 15 / 98^{\mathbf{F b}}$ & 1000 & $<.001$ & $<.005$ & .002 & $<.10$ & $<.10$ & $<.001$ & .001 & .001 & -- & -- \\
\hline $12 / 15 / 98$ & 1145 & .002 & .078 & .006 & $<.10$ & .16 & .006 & .006 & .019 & .8 & 1.3 \\
\hline $01 / 27 / 99$ & 1245 & .002 & .20 & .011 & .11 & .13 & .019 & .018 & .033 & .5 & 1.2 \\
\hline $01 / 27 / 99^{\mathbf{F b}}$ & 1445 & -- & -- & -- & -- & -- & -- & -- & -- & $<.2$ & $<.1$ \\
\hline $03 / 25 / 99$ & 1215 & .001 & .063 & .006 & .17 & .55 & .029 & .023 & .108 & 2.1 & 3.3 \\
\hline $04 / 26 / 99$ & 1115 & $<.001$ & .011 & .006 & .12 & .39 & .015 & .011 & .053 & .4 & 3.3 \\
\hline 05/12/99 & 0915 & $<.001$ & .007 & .005 & .16 & .29 & .013 & .007 & .037 & .4 & 3.0 \\
\hline $05 / 25 / 99$ & 1145 & .001 & .018 & .005 & .20 & .86 & .025 & .017 & .172 & 4.5 & 5.1 \\
\hline 06/07/99 & 1230 & .003 & .034 & .002 & .14 & .25 & .023 & .015 & .087 & 1.0 & -- \\
\hline 06/19/99 & 1130 & .001 & .017 & .002 & .20 & .22 & .021 & .018 & .056 & 1.0 & 3.6 \\
\hline 07/21/99 & 1015 & $<.001$ & .008 & .005 & e.09 & .14 & .014 & .010 & .025 & .2 & 2.0 \\
\hline 08/19/99 & 1000 & .001 & $<.005$ & $<.002$ & .23 & .26 & .011 & .004 & .033 & -- & 2.0 \\
\hline 10/20/99 & 1030 & .001 & .009 & $<.002$ & e. 10 & .21 & .012 & .008 & .029 & .8 & 1.7 \\
\hline $12 / 03 / 99$ & 1100 & .001 & .103 & $<.002$ & e. 06 & .15 & .012 & .005 & .023 & .3 & 1.3 \\
\hline $01 / 26 / 00$ & 0945 & .002 & .144 & .002 & e. 06 & e. 08 & .008 & .005 & .023 & .4 & 1.1 \\
\hline 03/08/00 & 1045 & .001 & .028 & $<.002$ & e. 10 & .28 & .015 & .013 & .043 & -- & -- \\
\hline $03 / 08 / 00^{\mathbf{R}}$ & 1046 & $<.001$ & .025 & $<.002$ & .12 & .28 & .016 & .013 & .043 & -- & -- \\
\hline $04 / 12 / 00$ & 0900 & $<.001$ & .013 & .005 & .12 & .34 & .013 & .006 & .038 & -- & -- \\
\hline $05 / 25 / 00$ & 0830 & $<.001$ & $<.005$ & .003 & e. 07 & .23 & .009 & .010 & .023 & -- & -- \\
\hline $06 / 21 / 00$ & 1015 & .001 & .008 & .003 & .11 & .26 & .008 & $<.001$ & .017 & -- & -- \\
\hline $07 / 31 / 00$ & 1045 & $<.001$ & .021 & .010 & .10 & .22 & e. 005 & .004 & .014 & -- & -- \\
\hline 08/29/00 & 1100 & $<.001$ & .014 & .007 & $<.10$ & .15 & e. 004 & .001 & .012 & -- & -- \\
\hline $11 / 07 / 00$ & 1400 & $<.001$ & .009 & $<.002$ & .12 & .16 & e. 004 & e.004 & .016 & -- & -- \\
\hline $12 / 20 / 00$ & 1545 & .002 & .150 & $<.002$ & e. 07 & e. 08 & .006 & e. 005 & .012 & -- & -- \\
\hline 01/30/01 & 1100 & .001 & .055 & $<.002$ & .11 & .09 & e. 005 & $<.007$ & .011 & -- & -- \\
\hline 03/12/01 & 1100 & .002 & .167 & .008 & .15 & .39 & .009 & $<.007$ & .049 & -- & -- \\
\hline 04/10/01 & 1030 & .001 & .010 & .008 & e. 10 & .25 & .008 & e.004 & .025 & -- & -- \\
\hline $05 / 22 / 01$ & 1100 & .001 & .013 & .003 & .13 & .26 & .012 & e. 005 & .036 & -- & -- \\
\hline \multicolumn{12}{|c|}{ SITE 5 12352500--BITTERROOT RIVER NEAR MISSOULA, MONT. } \\
\hline 03/04/99 & 1630 & .001 & .061 & .012 & e.07 & .28 & .006 & .004 & .025 & .2 & 2.1 \\
\hline 03/24/99 & 0930 & .020 & .018 & $<.002$ & .15 & .40 & .007 & .001 & .040 & .3 & 3.2 \\
\hline $04 / 15 / 99$ & 1300 & .001 & .017 & .005 & e. 07 & .22 & $<.004$ & .004 & .023 & 1.0 & 2.5 \\
\hline 05/13/99 & 0945 & $<.001$ & $<.005$ & .002 & .12 & .20 & .005 & .001 & .020 & .2 & 2.9 \\
\hline 05/25/99 & 0915 & .001 & .072 & .004 & .20 & .57 & .015 & .008 & .105 & 2.8 & 4.6 \\
\hline 06/07/99 & 1615 & .001 & $<.005$ & .013 & .14 & e. 05 & .011 & $<.001$ & .031 & .6 & 3.8 \\
\hline 06/19/99 & 1445 & .001 & $<.005$ & .006 & .14 & .22 & .009 & .001 & .031 & .8 & 3.1 \\
\hline 07/21/99 & 1530 & $<.001$ & $<.005$ & $<.002$ & .16 & .15 & .006 & .001 & .016 & .2 & 2.1 \\
\hline 08/19/99 & 1500 & .001 & $<.005$ & $<.002$ & .14 & .17 & .020 & .001 & .019 & -- & 2.2 \\
\hline $10 / 20 / 99$ & 1430 & .002 & .013 & $<.002$ & .10 & .14 & e. 005 & .001 & e. 006 & .2 & 1.8 \\
\hline $12 / 02 / 99$ & 1600 & .001 & .078 & $<.002$ & $<.10$ & .16 & e. 005 & $<.001$ & .018 & .2 & 1.8 \\
\hline $01 / 26 / 00$ & 1000 & .001 & .102 & .005 & e. 07 & e. 09 & .009 & .004 & .014 & .2 & 1.3 \\
\hline 03/08/00 & 1415 & .001 & .054 & .004 & .11 & .28 & .006 & .003 & .033 & -- & -- \\
\hline $04 / 12 / 00$ & 1545 & $<.001$ & .033 & .005 & .12 & .31 & .008 & .002 & .040 & -- & -- \\
\hline $05 / 02 / 00$ & 1300 & $<.001$ & .035 & .004 & e. 08 & .17 & e. 005 & .002 & .020 & -- & -- \\
\hline
\end{tabular}


Table 7. Nutrient and organic-carbon concentration data for surface-water samples collected at fixed water-quality sites, Northern Rockies Intermontane Basins study unit, 1998-2001 (Continued)

\begin{tabular}{|c|c|c|c|c|c|c|c|c|c|c|c|}
\hline Date & Time & $\begin{array}{c}\text { Nitrite, } \\
\text { dissolved } \\
(\mathrm{mg} / \mathrm{L} \\
\text { as N) }\end{array}$ & $\begin{array}{l}\text { Nitrite plus } \\
\text { nitrate, } \\
\text { dissolved } \\
\text { (mg/L } \\
\text { as N) }\end{array}$ & $\begin{array}{c}\text { Ammonia, } \\
\text { dissolved } \\
(\mathrm{mg} / \mathrm{L} \\
\text { as } \mathrm{N})\end{array}$ & $\begin{array}{c}\text { Ammonia } \\
\text { plus } \\
\text { organic } \\
\text { nitrogen, } \\
\text { dissolved } \\
\text { (mg/L } \\
\text { as } \mathrm{N})\end{array}$ & $\begin{array}{c}\text { Ammonia } \\
\text { plus organic } \\
\text { nitrogen, } \\
\text { total } \\
(\mathrm{mg} / \mathrm{L} \\
\text { as } \mathrm{N})\end{array}$ & $\begin{array}{c}\text { Phos- } \\
\text { phorus, } \\
\text { dissolved } \\
(\mathrm{mg} / \mathrm{L})\end{array}$ & $\begin{array}{l}\text { Phos- } \\
\text { phorus, } \\
\text { ortho- } \\
\text { phosphate } \\
\text { dissolved } \\
\text { (mg/L } \\
\text { as P) }\end{array}$ & $\begin{array}{l}\text { Phos- } \\
\text { pho- } \\
\text { rus, } \\
\text { total } \\
(\mathrm{mg} / \mathrm{L})\end{array}$ & $\begin{array}{c}\text { Organic } \\
\text { carbon, } \\
\text { suspended } \\
(\mathrm{mg} / \mathrm{L} \\
\text { as C) }\end{array}$ & $\begin{array}{c}\text { Organic } \\
\text { carbon, } \\
\text { dissolved } \\
(\mathbf{m g} / \mathbf{L} \\
\text { as C) }\end{array}$ \\
\hline \multicolumn{12}{|c|}{ SITE 5 12352500--BITTERROOT RIVER NEAR MISSOULA, MONT. (Continued) } \\
\hline $05 / 23 / 00$ & 1015 & .001 & .039 & .005 & .18 & .36 & .007 & .004 & .056 & -- & -- \\
\hline $06 / 20 / 00$ & 1130 & .002 & .017 & $<.002$ & e. 10 & .16 & .006 & $<.001$ & .012 & -- & -- \\
\hline $07 / 31 / 00$ & 1430 & $<.001$ & .016 & .003 & .12 & .18 & e. 005 & .003 & .011 & -- & -- \\
\hline 08/29/00 & 1430 & $<.001$ & .019 & .004 & .13 & .14 & .006 & .002 & .012 & -- & -- \\
\hline 09/19/00 & 1430 & .001 & .017 & .006 & e. 10 & .20 & e. 005 & .003 & .016 & -- & -- \\
\hline $10 / 02 / 00$ & 1000 & .001 & .086 & .006 & .21 & .62 & .012 & .007 & .117 & -- & -- \\
\hline $10 / 16 / 00$ & 1200 & .001 & .012 & $<.002$ & e. 10 & .16 & $<.006$ & $<.007$ & .012 & -- & -- \\
\hline $11 / 08 / 00$ & 1330 & $<.001$ & .013 & $<.002$ & .12 & .09 & $<.006$ & $<.007$ & .004 & -- & -- \\
\hline 01/30/01 & 1500 & .001 & .052 & .002 & .10 & e. 07 & $<.006$ & $<.007$ & .009 & -- & -- \\
\hline 03/12/01 & 1445 & .001 & .047 & .017 & .14 & .24 & .012 & e. 005 & .032 & -- & -- \\
\hline $04 / 10 / 01$ & 1330 & .001 & .031 & .007 & e.09 & .17 & .006 & $<.007$ & .017 & -- & -- \\
\hline $05 / 22 / 01$ & 1430 & $<.001$ & .032 & .006 & .12 & .17 & .007 & $<.007$ & .020 & -- & -- \\
\hline \multicolumn{12}{|c|}{ SITE 9 12354500-CLARK FORK AT ST. REGIS, MONT. } \\
\hline 03/02/99 & 1330 & .002 & .041 & .006 & e.06 & .23 & .004 & .002 & .027 & -- & 1.6 \\
\hline 04/13/99 & 1500 & .001 & .016 & .018 & e.06 & .25 & $<.004$ & .005 & .021 & .4 & 2.5 \\
\hline $05 / 10 / 99$ & 1500 & .001 & .019 & .005 & .11 & .17 & .005 & .002 & .017 & $<.2$ & 2.9 \\
\hline $05 / 26 / 99$ & 0930 & .001 & .074 & .013 & .20 & .66 & .013 & .010 & .183 & 3.4 & 2.0 \\
\hline 06/08/99 & 1045 & .001 & .040 & .002 & $<.10$ & .25 & .011 & .006 & .050 & .8 & 3.0 \\
\hline $06 / 21 / 99^{\mathbf{F b}}$ & 0840 & -- & -- & -- & -- & -- & -- & -- & -- & -- & .2 \\
\hline $06 / 21 / 99$ & 1000 & .002 & .027 & .008 & .14 & .25 & .008 & .007 & .048 & .6 & 1.8 \\
\hline 07/20/99 & 1615 & $<.001$ & $<.005$ & .002 & e. 09 & .14 & .006 & .001 & .012 & .2 & 1.4 \\
\hline 08/18/99 & 0930 & $<.001$ & $<.005$ & $<.002$ & e. 08 & .14 & .011 & $<.001$ & .016 & .2 & 1.8 \\
\hline $10 / 18 / 99$ & 1415 & .003 & .021 & .009 & .12 & .20 & $<.006$ & .002 & e. 005 & .2 & 1.6 \\
\hline $11 / 30 / 99$ & 1300 & .003 & .090 & $<.002$ & $<.10$ & .17 & e. 004 & .001 & .014 & .2 & 2.1 \\
\hline $01 / 24 / 00$ & 1400 & .002 & .093 & .002 & e.06 & .12 & e. 003 & .001 & .009 & .2 & 1.4 \\
\hline $03 / 06 / 00$ & 1400 & .003 & .067 & $<.002$ & .14 & .31 & .009 & .006 & .030 & -- & -- \\
\hline $04 / 10 / 00$ & 1400 & .001 & .041 & .004 & e. 08 & .27 & .012 & .006 & .032 & -- & -- \\
\hline 05/09/00 & 1215 & $<.001$ & .047 & $<.002$ & e.09 & .20 & e. 004 & .002 & .020 & -- & -- \\
\hline $05 / 24 / 00$ & 1230 & $<.001$ & .034 & .006 & .11 & .27 & .007 & .008 & .038 & -- & -- \\
\hline $06 / 20 / 00$ & 0930 & .001 & .024 & .002 & e. 07 & .19 & e. 005 & $<.001$ & .013 & -- & -- \\
\hline $08 / 01 / 00$ & 1430 & $<.001$ & $<.005$ & .005 & e. 08 & .40 & $<.006$ & .002 & .009 & -- & -- \\
\hline $08 / 30 / 00$ & 1400 & $<.001$ & $<.005$ & .003 & .10 & .15 & e. 003 & $<.001$ & e. 007 & -- & -- \\
\hline $11 / 07 / 00$ & 1000 & .001 & .028 & $<.002$ & .10 & .11 & $<.006$ & $<.007$ & .005 & -- & -- \\
\hline $12 / 19 / 00$ & 1645 & .003 & .159 & .007 & e.07 & e. 06 & e.004 & $<.007$ & .004 & -- & -- \\
\hline 01/31/01 & 1500 & .003 & .060 & .002 & e. 10 & .17 & $<.006$ & $<.007$ & .004 & -- & -- \\
\hline 03/13/01 & 1500 & .003 & .128 & .010 & .13 & .35 & .006 & $<.007$ & .030 & -- & -- \\
\hline $04 / 11 / 01$ & 1400 & .002 & .037 & .008 & e. 10 & .26 & .009 & .008 & .028 & -- & -- \\
\hline $05 / 23 / 01$ & 1430 & .001 & .044 & .006 & .12 & .16 & .009 & e.004 & .028 & -- & -- \\
\hline \multicolumn{12}{|c|}{ SITE 12 12388700--FLATHEAD RIVER AT PERMA, MONT. } \\
\hline 03/03/99 & 1015 & $<.001$ & .031 & .002 & $<.10$ & .11 & $<.004$ & .001 & .005 & .2 & 1.3 \\
\hline 04/14/99 & 1230 & .001 & .026 & .006 & $<.10$ & .11 & $<.004$ & .002 & .005 & .2 & 2.6 \\
\hline 05/11/99 Fb & 0945 & $<.001$ & .005 & .004 & e.06 & $<.10$ & $<.004$ & .001 & $<.004$ & -- & -- \\
\hline 05/11/99 & 1100 & .001 & .020 & .007 & e.09 & .14 & $<.004$ & .001 & .006 & $<.2$ & 1.5 \\
\hline $06 / 20 / 99$ & 1130 & .002 & .019 & .006 & .11 & .13 & $<.004$ & .001 & .008 & .3 & 2.1 \\
\hline 07/19/99 & 1430 & .001 & .012 & .002 & e. 08 & e. 08 & $<.004$ & .001 & .004 & $<.2$ & 1.7 \\
\hline
\end{tabular}


Table 7. Nutrient and organic-carbon concentration data for surface-water samples collected at fixed water-quality sites, Northern Rockies Intermontane Basins study unit, 1998-2001 (Continued)

\begin{tabular}{|c|c|c|c|c|c|c|c|c|c|c|c|}
\hline Date & Time & $\begin{array}{c}\text { Nitrite, } \\
\text { dissolved } \\
\text { (mg/L } \\
\text { as } \mathbf{N})\end{array}$ & $\begin{array}{c}\text { Nitrite plus } \\
\text { nitrate, } \\
\text { dissolved } \\
\text { (mg/L } \\
\text { as N) }\end{array}$ & $\begin{array}{c}\text { Ammonia, } \\
\text { dissolved } \\
(\mathrm{mg} / \mathrm{L} \\
\text { as } \mathrm{N})\end{array}$ & $\begin{array}{c}\text { Ammonia } \\
\text { plus } \\
\text { organic } \\
\text { nitrogen, } \\
\text { dissolved } \\
\text { (mg/L } \\
\text { as } \mathrm{N})\end{array}$ & $\begin{array}{c}\text { Ammonia } \\
\text { plus organic } \\
\text { nitrogen, } \\
\text { total } \\
(\mathrm{mg} / \mathrm{L} \\
\text { as } \mathrm{N})\end{array}$ & $\begin{array}{c}\text { Phos- } \\
\text { phorus, } \\
\text { dissolved } \\
(\mathrm{mg} / \mathrm{L})\end{array}$ & $\begin{array}{c}\text { Phos- } \\
\text { phorus, } \\
\text { ortho- } \\
\text { phosphate } \\
\text { dissolved } \\
\text { (mg/L } \\
\text { as P) }\end{array}$ & $\begin{array}{l}\text { Phos- } \\
\text { pho- } \\
\text { rus, } \\
\text { total } \\
\text { (mg/L) }\end{array}$ & $\begin{array}{c}\text { Organic } \\
\text { carbon, } \\
\text { suspended } \\
(\mathrm{mg} / \mathrm{L} \\
\text { as C) }\end{array}$ & $\begin{array}{c}\text { Organic } \\
\text { carbon, } \\
\text { dissolved } \\
(\mathbf{m g} / \mathbf{L} \\
\text { as C) }\end{array}$ \\
\hline \multicolumn{12}{|c|}{ SITE 12 12388700--FLATHEAD RIVER AT PERMA, MONT. (Continued) } \\
\hline 08/17/99 & 1330 & .001 & .007 & .002 & e.07 & e.08 & $<.004$ & $<.001$ & .004 & $<.2$ & 1.6 \\
\hline $10 / 19 / 99$ & 1140 & .002 & $<.005$ & $<.002$ & .18 & .14 & e. 004 & .001 & $<.008$ & $<.2$ & 1.6 \\
\hline $12 / 01 / 99$ & 1100 & $<.001$ & .027 & $<.002$ & e.06 & e. 08 & $<.006$ & $<.001$ & $<.008$ & $<.2$ & 1.5 \\
\hline $01 / 25 / 00$ & 1030 & $<.001$ & .050 & $<.002$ & e.06 & e. 09 & $<.006$ & $<.001$ & $<.008$ & .2 & 1.3 \\
\hline $03 / 07 / 00$ & 1100 & $<.001$ & .029 & $<.002$ & e. 08 & .12 & $<.006$ & $<.001$ & e. 004 & -- & -- \\
\hline $04 / 11 / 00$ & 1000 & $<.001$ & .024 & $<.002$ & $<.10$ & .12 & $<.006$ & $<.001$ & $<.008$ & -- & -- \\
\hline $05 / 10 / 00$ & 1015 & $<.001$ & .022 & $<.002$ & e. 08 & .15 & $<.006$ & $<.001$ & $<.008$ & -- & -- \\
\hline $06 / 19 / 00$ & 1230 & .001 & .019 & .006 & e.06 & .13 & $<.006$ & $<.001$ & e. 004 & -- & -- \\
\hline $08 / 01 / 00$ & 1000 & $<.001$ & .006 & .002 & e.09 & .21 & $<.006$ & .002 & $<.008$ & -- & -- \\
\hline $08 / 30 / 00$ & 1030 & $<.001$ & .011 & .005 & e.09 & .11 & $<.006$ & $<.001$ & $<.008$ & -- & -- \\
\hline $11 / 06 / 00$ & 1400 & $<.001$ & .013 & $<.002$ & .12 & .08 & $<.006$ & $<.007$ & e.002 & -- & -- \\
\hline $12 / 19 / 00$ & 1245 & $<.001$ & .042 & $<.002$ & $<.10$ & $<.08$ & $<.006$ & $<.007$ & e.002 & -- & -- \\
\hline $02 / 01 / 01$ & 1000 & $<.001$ & .033 & $<.002$ & e. 05 & e. 07 & $<.006$ & $<.007$ & e. 002 & -- & -- \\
\hline $03 / 14 / 01$ & 0930 & .002 & .064 & .045 & .25 & .30 & .013 & .007 & .038 & -- & -- \\
\hline $04 / 12 / 01$ & 0930 & $<.001$ & $<.005$ & .006 & e. 08 & .17 & $<.006$ & $<.007$ & .011 & -- & -- \\
\hline $05 / 24 / 01$ & 0930 & $<.001$ & .018 & .003 & e.06 & .10 & $<.006$ & $<.007$ & .008 & -- & -- \\
\hline \multicolumn{12}{|c|}{ SITE 14 12392155--LIGHTNING CREEK AT CLARK FORK, IDAHO } \\
\hline $07 / 29 / 99$ & 1015 & .001 & .028 & .002 & $<.10$ & e. 10 & $<.004$ & .002 & $<.004$ & $<.2$ & .5 \\
\hline $09 / 02 / 99$ & 0945 & $<.001$ & .050 & $<.002$ & $<.10$ & e. 10 & $<.004$ & $<.001$ & .004 & $<.2$ & .5 \\
\hline $10 / 26 / 99$ & 1145 & $<.001$ & .086 & .005 & .10 & e. 06 & $<.006$ & $<.001$ & $<.008$ & $<.2$ & .6 \\
\hline $03 / 02 / 00$ & 0815 & $<.001$ & .094 & .004 & $<.10$ & $<.10$ & $<.006$ & .003 & $<.008$ & -- & .7 \\
\hline $03 / 30 / 00$ & 0815 & $<.001$ & .112 & $<.002$ & $<.10$ & $<.10$ & $<.006$ & .003 & $<.008$ & -- & -- \\
\hline $04 / 12 / 00$ & 1330 & $<.001$ & .207 & .005 & $<.10$ & e. 07 & $<.006$ & $<.001$ & $<.008$ & -- & -- \\
\hline $05 / 04 / 00$ & 1000 & $<.001$ & .211 & $<.002$ & $<.10$ & e. 06 & $<.006$ & $<.001$ & e.004 & -- & -- \\
\hline $06 / 05 / 00$ & 0915 & $<.001$ & .107 & $<.002$ & $<.10$ & .19 & $<.006$ & $<.001$ & $<.008$ & -- & -- \\
\hline $06 / 30 / 00$ & 0830 & $<.001$ & .042 & $<.002$ & e. 07 & e. 07 & $<.006$ & .001 & $<.008$ & -- & -- \\
\hline $07 / 27 / 00$ & 1145 & .001 & .029 & .008 & $<.10$ & .13 & $<.006$ & .001 & $<.008$ & -- & -- \\
\hline $09 / 01 / 00$ & 1200 & $<.001$ & .025 & .006 & $<.10$ & $<.10$ & $<.006$ & $<.001$ & $<.008$ & -- & -- \\
\hline $11 / 09 / 00$ & 1015 & $<.001$ & .083 & $<.002$ & $<.10$ & $<.08$ & $<.006$ & $<.007$ & $<.004$ & -- & -- \\
\hline $12 / 14 / 00$ & 1145 & $<.001$ & .114 & .024 & $<.10$ & $<.08$ & $<.006$ & $<.007$ & $<.004$ & -- & -- \\
\hline $01 / 25 / 01$ & 1145 & .001 & .156 & $<.002$ & $<.10$ & $<.08$ & .009 & $<.007$ & e. 002 & -- & -- \\
\hline $03 / 15 / 01$ & 0915 & $<.001$ & .162 & .003 & $<.10$ & $<.08$ & $<.006$ & $<.007$ & e. 003 & -- & -- \\
\hline $04 / 11 / 01$ & 1115 & .001 & .140 & .002 & $<.10$ & e. 06 & $<.006$ & $<.007$ & e. 002 & -- & -- \\
\hline $05 / 04 / 01$ & 1315 & $<.001$ & .247 & $<.002$ & $<.10$ & e. 08 & $<.006$ & $<.007$ & $<.004$ & -- & -- \\
\hline $06 / 14 / 01$ & 1300 & $<.001$ & .072 & .003 & $<.10$ & e. 06 & $<.006$ & $<.007$ & .004 & -- & -- \\
\hline \multicolumn{12}{|c|}{ SITE 23 12413000--NORTH FORK COEUR D'ALENE RIVER AT ENAVILLE, IDAHO } \\
\hline 03/02/99 & 1130 & $<.001$ & .020 & .003 & $<.10$ & e.06 & $<.004$ & .005 & .010 & $<.2$ & 1.0 \\
\hline $03 / 23 / 99$ & 1000 & .001 & .017 & $<.002$ & $<.10$ & .11 & .009 & .005 & .021 & .2 & 1.1 \\
\hline $05 / 20 / 99$ & 1000 & $<.001$ & .007 & .003 & e. 05 & e. 06 & $<.004$ & .003 & .013 & .2 & .8 \\
\hline $05 / 25 / 99$ & 0930 & .001 & .036 & .004 & .11 & .29 & .006 & .005 & .068 & 1.5 & 1.3 \\
\hline $06 / 29 / 99$ & 1000 & $<.001$ & .006 & $<.002$ & $<.10$ & e. 08 & $<.004$ & .002 & $<.004$ & $<.2$ & .5 \\
\hline $07 / 26 / 99$ & 1000 & $<.001$ & $<.005$ & $<.002$ & $<.10$ & $<.10$ & $<.004$ & $<.001$ & .004 & $<.2$ & .5 \\
\hline 09/08/99 & 0930 & .001 & .009 & .002 & .10 & e. 08 & $<.004$ & $<.001$ & .004 & $<.2$ & .4 \\
\hline $10 / 19 / 99$ & 1000 & .001 & .013 & $<.002$ & e. 05 & .13 & $<.006$ & .002 & $<.008$ & $<.2$ & .36 \\
\hline $12 / 01 / 99$ & 1145 & $<.001$ & .045 & $<.002$ & e. 10 & $<.10$ & e. 004 & $<.001$ & .009 & $<.2$ & 1.1 \\
\hline
\end{tabular}


Table 7. Nutrient and organic-carbon concentration data for surface-water samples collected at fixed water-quality sites, Northern Rockies Intermontane Basins study unit, 1998-2001 (Continued)

\begin{tabular}{|c|c|c|c|c|c|c|c|c|c|c|c|}
\hline Date & Time & $\begin{array}{c}\text { Nitrite, } \\
\text { dissolved } \\
(\mathrm{mg} / \mathrm{L} \\
\text { as } \mathrm{N})\end{array}$ & $\begin{array}{l}\text { Nitrite plus } \\
\text { nitrate, } \\
\text { dissolved } \\
\text { (mg/L } \\
\text { as N) }\end{array}$ & $\begin{array}{c}\text { Ammonia, } \\
\text { dissolved } \\
(\mathrm{mg} / \mathrm{L} \\
\text { as } \mathrm{N})\end{array}$ & $\begin{array}{c}\text { Ammonia } \\
\text { plus } \\
\text { organic } \\
\text { nitrogen, } \\
\text { dissolved } \\
\text { (mg/L } \\
\text { as } \mathrm{N})\end{array}$ & $\begin{array}{c}\text { Ammonia } \\
\text { plus organic } \\
\text { nitrogen, } \\
\text { total } \\
(\mathrm{mg} / \mathrm{L} \\
\text { as } \mathrm{N})\end{array}$ & $\begin{array}{l}\text { Phos- } \\
\text { phorus, } \\
\text { dissolved } \\
(\mathbf{m g} / \mathrm{L})\end{array}$ & $\begin{array}{c}\text { Phos- } \\
\text { phorus, } \\
\text { ortho- } \\
\text { phosphate } \\
\text { dissolved } \\
\text { (mg/L } \\
\text { as P) }\end{array}$ & $\begin{array}{l}\text { Phos- } \\
\text { pho- } \\
\text { rus, } \\
\text { total } \\
(\mathrm{mg} / \mathrm{L})\end{array}$ & $\begin{array}{c}\text { Organic } \\
\text { carbon, } \\
\text { suspended } \\
(\mathbf{m g} / \mathbf{L} \\
\text { as C) }\end{array}$ & $\begin{array}{c}\text { Organic } \\
\text { carbon, } \\
\text { dissolved } \\
\text { (mg/L } \\
\text { as C) }\end{array}$ \\
\hline \multicolumn{12}{|c|}{ SITE 23 12413000--NORTH FORK COEUR D'ALENE RIVER AT ENAVILLE, IDAHO (Continued) } \\
\hline $01 / 11 / 00$ & 1100 & $<.001$ & .038 & .014 & $<.10$ & $<.10$ & $<.006$ & .003 & $<.008$ & $<.2$ & .53 \\
\hline $02 / 29 / 00$ & 0800 & $<.001$ & .010 & $<.002$ & $<.10$ & e. 09 & $<.006$ & .002 & e. 006 & $<.2$ & .74 \\
\hline $03 / 27 / 00$ & 0945 & $<.001$ & .005 & $<.002$ & $<.10$ & e. 06 & $<.006$ & .004 & $<.008$ & -- & -- \\
\hline $04 / 10 / 00$ & 0945 & $<.001$ & .009 & $<.002$ & $<.10$ & e. 06 & e. 005 & .007 & e. 007 & -- & -- \\
\hline $04 / 14 / 00$ & 0945 & $<.001$ & .048 & $<.002$ & e. 07 & .29 & .016 & .008 & .189 & -- & -- \\
\hline $04 / 17 / 00$ & 0945 & $<.001$ & .031 & .003 & $<.10$ & .10 & .007 & .006 & .010 & -- & -- \\
\hline $05 / 02 / 00$ & 0900 & $<.001$ & .019 & .007 & $<.10$ & .36 & e. 004 & .004 & .009 & -- & -- \\
\hline 05/18/00 & 0730 & $<.001$ & $<.005$ & .002 & $<.10$ & e. 06 & e. 003 & .002 & e. 007 & -- & -- \\
\hline $06 / 02 / 00$ & 0715 & $<.001$ & $<.005$ & .005 & e. 05 & .11 & e. 004 & $<.001$ & e. 004 & -- & -- \\
\hline $06 / 29 / 00$ & 0800 & $<.001$ & $<.005$ & .003 & e. 05 & $<.10$ & e. 004 & .001 & e. 005 & -- & -- \\
\hline $07 / 24 / 00$ & 1030 & $<.001$ & $<.005$ & $<.002$ & $<.10$ & e. 07 & $<.006$ & .003 & e. 004 & -- & -- \\
\hline $08 / 31 / 00$ & 0915 & $<.001$ & .017 & .006 & $<.10$ & $<.10$ & $<.006$ & .002 & $<.008$ & -- & -- \\
\hline $11 / 06 / 00$ & 1030 & $<.001$ & .013 & .009 & e. 07 & $<.08$ & $<.006$ & $<.007$ & e. 003 & -- & -- \\
\hline $12 / 13 / 00$ & 1100 & $<.001$ & .016 & $<.002$ & $<.10$ & $<.08$ & $<.006$ & $<.007$ & e. 003 & -- & -- \\
\hline $01 / 23 / 01$ & 1000 & .001 & .022 & $<.002$ & $<.10$ & $<.08$ & e.004 & $<.007$ & e. 003 & -- & -- \\
\hline 03/14/01 & 0845 & $<.001$ & .023 & .008 & $<.10$ & $<.08$ & e.003 & $<.007$ & e. 003 & -- & -- \\
\hline 04/10/01 & 0815 & $<.001$ & .013 & .004 & $<.10$ & e. 06 & $<.006$ & $<.007$ & .004 & -- & -- \\
\hline 05/03/01 & 1015 & $<.001$ & .017 & .002 & $<.10$ & $<.08$ & e. 005 & $<.007$ & .011 & -- & -- \\
\hline $06 / 12 / 01$ & 0930 & $<.001$ & .005 & .002 & $<.10$ & $<.08$ & e.004 & $<.007$ & .005 & -- & -- \\
\hline \multicolumn{12}{|c|}{ SITE 31 12413470--SOUTH FORK COEUR D'ALENE RIVER NEAR PINEHURST, IDAHO } \\
\hline $11 / 04 / 98$ & 1200 & .015 & .326 & .387 & .42 & .58 & .029 & .027 & .096 & .3 & .6 \\
\hline $11 / 04 / 98^{\mathbf{F b}}$ & 1208 & $<.001$ & $<.005$ & $<.002$ & $<.10$ & $<.10$ & .001 & .001 & $<.001$ & $<.2$ & .2 \\
\hline $12 / 09 / 98$ & 1210 & .006 & .408 & .184 & .23 & .26 & .012 & .014 & .023 & -- & -- \\
\hline 02/03/99 & 1100 & -- & .252 & .070 & -- & .15 & .016 & -- & .027 & $<.2$ & .5 \\
\hline 03/17/99 & 1000 & .002 & .133 & .034 & $<.10$ & e. 09 & .005 & .005 & .018 & .2 & .7 \\
\hline 03/23/99 & 1330 & .001 & .092 & .018 & e.06 & .11 & .005 & .005 & .028 & .2 & .9 \\
\hline 04/20/99 & 1200 & .001 & .055 & .011 & $<.10$ & e. 09 & .006 & .005 & .040 & .3 & .8 \\
\hline 05/25/99 & 1245 & $<.001$ & .042 & .006 & .11 & .39 & .005 & .004 & .153 & 1.5 & .7 \\
\hline $05 / 27 / 99$ & 1245 & .001 & .038 & .016 & e.09 & .24 & .004 & .003 & .060 & .5 & .6 \\
\hline 06/29/99 & 1300 & .001 & .025 & .043 & .11 & .11 & .009 & .007 & .016 & $<.2$ & .4 \\
\hline 07/26/99 & 1315 & .005 & .088 & .031 & e. 07 & e. 10 & .012 & .009 & .028 & $<.2$ & .4 \\
\hline 09/08/99 & 1300 & .012 & .223 & .246 & .27 & .34 & .026 & .016 & .056 & .2 & .6 \\
\hline $10 / 19 / 99$ & 1400 & .006 & .195 & .214 & .26 & .40 & .021 & .017 & .056 & .2 & .6 \\
\hline $12 / 02 / 99$ & 0850 & .013 & .250 & .120 & .17 & .21 & .016 & .011 & .032 & $<.2$ & .7 \\
\hline $01 / 11 / 00$ & 1430 & .007 & .253 & .122 & .19 & .16 & .012 & .006 & .033 & -- & .5 \\
\hline $02 / 28 / 00$ & 1100 & $<.001$ & .155 & .060 & e. 08 & .22 & .009 & .008 & .023 & $<.2$ & .8 \\
\hline $03 / 27 / 00$ & 1320 & $<.001$ & .119 & .046 & e.09 & .16 & .007 & .009 & .020 & -- & -- \\
\hline $04 / 10 / 00$ & 1315 & $<.001$ & .080 & .027 & e.06 & e. 09 & .006 & .008 & .017 & -- & -- \\
\hline $04 / 14 / 00$ & 1315 & $<.001$ & .064 & .011 & $<.10$ & .62 & .008 & .002 & .171 & -- & -- \\
\hline $04 / 17 / 00$ & 1315 & .001 & .067 & .026 & $<.10$ & .11 & .007 & .005 & .108 & -- & -- \\
\hline $05 / 02 / 00$ & 1145 & .001 & .060 & .015 & $<.10$ & e. 06 & e. 005 & .004 & .017 & -- & -- \\
\hline 05/18/00 & 1000 & $<.001$ & .035 & .019 & $<.10$ & e. 08 & e.005 & .003 & .014 & -- & -- \\
\hline $06 / 02 / 00$ & 0930 & .001 & .054 & .042 & e.08 & .13 & .007 & .005 & .012 & -- & -- \\
\hline $06 / 29 / 00$ & 1130 & .004 & .086 & .089 & .16 & .18 & .014 & .009 & .026 & -- & -- \\
\hline $07 / 24 / 00$ & 1300 & .006 & .128 & .129 & .21 & .28 & .016 & .015 & .022 & -- & -- \\
\hline
\end{tabular}


Table 7. Nutrient and organic-carbon concentration data for surface-water samples collected at fixed water-quality sites, Northern Rockies Intermontane Basins study unit, 1998-2001 (Continued)

\begin{tabular}{|c|c|c|c|c|c|c|c|c|c|c|c|}
\hline Date & Time & $\begin{array}{c}\text { Nitrite, } \\
\text { dissolved } \\
\text { (mg/L } \\
\text { as N) }\end{array}$ & $\begin{array}{l}\text { Nitrite plus } \\
\text { nitrate, } \\
\text { dissolved } \\
\text { (mg/L } \\
\text { as N) }\end{array}$ & $\begin{array}{c}\text { Ammonia, } \\
\text { dissolved } \\
(\mathrm{mg} / \mathrm{L} \\
\text { as } \mathrm{N})\end{array}$ & $\begin{array}{c}\text { Ammonia } \\
\text { plus } \\
\text { organic } \\
\text { nitrogen, } \\
\text { dissolved } \\
\text { (mg/L } \\
\text { as } \mathrm{N})\end{array}$ & $\begin{array}{c}\text { Ammonia } \\
\text { plus organic } \\
\text { nitrogen, } \\
\text { total } \\
(\mathrm{mg} / \mathrm{L} \\
\text { as } \mathrm{N})\end{array}$ & $\begin{array}{c}\text { Phos- } \\
\text { phorus, } \\
\text { dissolved } \\
(\mathrm{mg} / \mathrm{L})\end{array}$ & $\begin{array}{l}\text { Phos- } \\
\text { phorus, } \\
\text { ortho- } \\
\text { phosphate } \\
\text { dissolved } \\
\text { (mg/L } \\
\text { as P) }\end{array}$ & $\begin{array}{l}\text { Phos- } \\
\text { pho- } \\
\text { rus, } \\
\text { total } \\
(\mathrm{mg} / \mathrm{L})\end{array}$ & $\begin{array}{c}\text { Organic } \\
\text { carbon, } \\
\text { suspended } \\
(\mathrm{mg} / \mathrm{L} \\
\text { as C) }\end{array}$ & $\begin{array}{c}\text { Organic } \\
\text { carbon, } \\
\text { dissolved } \\
(\mathbf{m g} / \mathbf{L} \\
\text { as C) }\end{array}$ \\
\hline \multicolumn{12}{|c|}{ SITE 31 12413470--SOUTH FORK COEUR D'ALENE RIVER NEAR PINEHURST, IDAHO (Continued) } \\
\hline $08 / 31 / 00$ & 1215 & .008 & .222 & .236 & .29 & .38 & .030 & .024 & .053 & -- & -- \\
\hline $11 / 06 / 00$ & 1330 & .004 & .333 & .270 & .34 & .34 & .023 & .020 & .035 & -- & -- \\
\hline $12 / 18 / 00$ & 1200 & .003 & .406 & .408 & .41 & .40 & .020 & .020 & .061 & -- & -- \\
\hline $01 / 23 / 01$ & 1430 & .003 & .422 & .316 & .39 & .43 & .022 & .016 & .050 & -- & -- \\
\hline 03/14/01 & 1145 & .002 & .448 & .212 & .29 & .34 & .018 & .014 & .079 & -- & -- \\
\hline 04/10/01 & 1100 & .002 & .275 & .142 & .17 & .21 & .015 & .013 & .037 & -- & -- \\
\hline 05/03/01 & 1300 & .001 & .125 & .048 & e. 09 & e. 07 & .009 & e. 005 & .023 & -- & -- \\
\hline $06 / 12 / 01$ & 1230 & .001 & .072 & .081 & e.09 & .28 & .014 & .011 & .023 & -- & -- \\
\hline 09/10/01 & 1130 & .010 & .244 & .372 & .46 & .56 & .032 & .026 & .068 & -- & -- \\
\hline \multicolumn{12}{|c|}{ SITE 33 12413875--ST. JOE RIVER AT RED IVES RANGER STATION, IDAHO } \\
\hline $02 / 18 / 99$ & 1545 & $<.001$ & .024 & $<.002$ & $<.10$ & $<.10$ & $<.004$ & .004 & .004 & $<.2$ & .7 \\
\hline 06/01/99 & 1415 & .001 & .028 & .003 & $<.10$ & $<.10$ & .006 & .004 & .012 & .2 & 8.5 \\
\hline 06/28/99 & 1415 & $<.001$ & .009 & $<.002$ & $<.10$ & e. 08 & $<.004$ & .004 & .007 & .2 & .7 \\
\hline 07/20/99 & 0945 & $<.001$ & $<.005$ & $<.002$ & e.06 & $<.10$ & .005 & .002 & .007 & .2 & .7 \\
\hline 08/18/99 & 1500 & $<.001$ & $<.005$ & $<.002$ & $<.10$ & e. 06 & $<.004$ & $<.001$ & .005 & $<.2$ & 1.7 \\
\hline $11 / 04 / 99$ & 1300 & $<.001$ & .053 & $<.002$ & $<.10$ & $<.10$ & e. 003 & $<.001$ & $<.008$ & $<.2$ & 1.2 \\
\hline $01 / 21 / 00$ & 1245 & $<.001$ & .037 & $<.002$ & $<.10$ & $<.10$ & e. 003 & .001 & $<.008$ & $<.2$ & .6 \\
\hline $05 / 17 / 00$ & 1215 & $<.001$ & .007 & $<.002$ & $<.10$ & e.07 & e. 003 & .002 & e.007 & -- & -- \\
\hline 06/01/00 & 1100 & $<.001$ & .011 & .009 & e. 05 & .12 & e. 003 & .001 & e.004 & -- & -- \\
\hline $06 / 28 / 00$ & 1100 & $<.001$ & $<.005$ & .003 & $<.10$ & e. 07 & e. 005 & .001 & e. 007 & -- & -- \\
\hline $07 / 27 / 00$ & 1115 & .001 & .005 & .011 & $<.10$ & .15 & e. 003 & .003 & e. 007 & -- & -- \\
\hline 09/07/00 & 1330 & $<.001$ & $<.005$ & $<.002$ & $<.10$ & e. 05 & e. 003 & .001 & $<.008$ & -- & -- \\
\hline $11 / 07 / 00$ & 1015 & $<.001$ & .010 & $<.002$ & $<.10$ & .16 & $<.006$ & $<.007$ & e. 003 & -- & -- \\
\hline $01 / 24 / 01$ & 1345 & $<.001$ & .045 & $<.002$ & $<.10$ & $<.08$ & .006 & $<.007$ & .005 & -- & -- \\
\hline 05/09/01 & 1200 & .003 & .028 & $<.002$ & $<.10$ & .15 & $<.006$ & $<.007$ & .005 & -- & -- \\
\hline 06/13/01 & 0930 & $<.001$ & .005 & $<.002$ & $<.10$ & $<.08$ & $<.006$ & $<.007$ & .004 & -- & -- \\
\hline $09 / 13 / 01$ & 1045 & $<.001$ & .005 & $<.002$ & $<.10$ & e.06 & $<.006$ & $<.007$ & e.003 & -- & -- \\
\hline \multicolumn{12}{|c|}{ SITE 35 12419000--SPOKANE RIVER NEAR POST FALLS, IDAHO } \\
\hline 03/04/99 & 1230 & $<.001$ & .054 & .009 & $<.10$ & .13 & .005 & .003 & .012 & .2 & 1.6 \\
\hline 04/13/99 & 1000 & .001 & .073 & .006 & e.09 & .13 & .005 & .003 & .011 & .5 & 1.7 \\
\hline 05/19/99 & 0900 & .001 & .028 & .004 & $<.10$ & .13 & $<.004$ & .001 & .011 & .2 & 1.6 \\
\hline 06/02/99 & 0930 & $<.001$ & .013 & .002 & $<.10$ & e. 07 & $<.004$ & .001 & .009 & .2 & 1.5 \\
\hline 06/23/99 & 1030 & .001 & .006 & .002 & .10 & .11 & $<.004$ & .001 & .057 & .2 & 1.3 \\
\hline 07/27/99 & 1030 & .002 & .033 & .002 & e. 07 & e. 06 & $<.004$ & $<.001$ & .005 & -- & 3.7 \\
\hline 09/09/99 & 1100 & .003 & .123 & $<.002$ & .12 & .18 & $<.004$ & $<.001$ & .009 & .2 & 1.4 \\
\hline $10 / 20 / 99$ & 1130 & .002 & .063 & $<.002$ & e.06 & .23 & .009 & .133 & .016 & .2 & 1.3 \\
\hline $11 / 30 / 99$ & 1045 & .001 & .031 & $<.002$ & $<.10$ & e. 06 & .007 & $<.001$ & .013 & .2 & 1.3 \\
\hline $01 / 12 / 00$ & 0910 & .001 & .087 & .007 & e.06 & e. 08 & .010 & .005 & .012 & $<.2$ & 1.4 \\
\hline $02 / 29 / 00$ & 1200 & $<.001$ & .073 & .006 & $<.10$ & e. 05 & e. 004 & .002 & .010 & .2 & 1.4 \\
\hline 03/28/00 & 0915 & $<.001$ & .071 & $<.002$ & e.07 & .12 & e. 003 & .006 & .009 & -- & -- \\
\hline $04 / 11 / 00$ & 0800 & $<.001$ & .082 & .004 & e.05 & .14 & $<.006$ & .001 & .009 & -- & -- \\
\hline $04 / 18 / 00$ & 1115 & $<.001$ & .071 & .002 & $<.10$ & .18 & $<.006$ & .001 & .029 & -- & -- \\
\hline $05 / 03 / 00$ & 0800 & $<.001$ & .030 & .014 & e.07 & .65 & $<.006$ & $<.001$ & .009 & -- & -- \\
\hline $05 / 16 / 00$ & 1315 & $<.001$ & .009 & .004 & e.09 & .17 & e. 003 & $<.001$ & .008 & -- & -- \\
\hline $05 / 31 / 00$ & 1345 & $<.001$ & $<.005$ & $<.002$ & $<.10$ & .15 & $<.006$ & $<.001$ & e.005 & -- & -- \\
\hline
\end{tabular}


Table 7. Nutrient and organic-carbon concentration data for surface-water samples collected at fixed water-quality sites, Northern Rockies Intermontane Basins study unit, 1998-2001 (Continued)

\begin{tabular}{|c|c|c|c|c|c|c|c|c|c|c|c|}
\hline Date & Time & $\begin{array}{c}\text { Nitrite, } \\
\text { dissolved } \\
(\mathrm{mg} / \mathrm{L} \\
\text { as N) }\end{array}$ & $\begin{array}{l}\text { Nitrite plus } \\
\text { nitrate, } \\
\text { dissolved } \\
\text { (mg/L } \\
\text { as N) }\end{array}$ & $\begin{array}{c}\text { Ammonia, } \\
\text { dissolved } \\
(\mathrm{mg} / \mathrm{L} \\
\text { as } \mathrm{N})\end{array}$ & $\begin{array}{c}\text { Ammonia } \\
\text { plus } \\
\text { organic } \\
\text { nitrogen, } \\
\text { dissolved } \\
\text { (mg/L } \\
\text { as N) }\end{array}$ & $\begin{array}{c}\text { Ammonia } \\
\text { plus organic } \\
\text { nitrogen, } \\
\text { total } \\
(\mathrm{mg} / \mathrm{L} \\
\text { as } \mathrm{N})\end{array}$ & $\begin{array}{c}\text { Phos- } \\
\text { phorus, } \\
\text { dissolved } \\
(\mathrm{mg} / \mathrm{L})\end{array}$ & $\begin{array}{c}\text { Phos- } \\
\text { phorus, } \\
\text { ortho- } \\
\text { phosphate } \\
\text { dissolved } \\
\text { (mg/L } \\
\text { as P) }\end{array}$ & $\begin{array}{l}\text { Phos- } \\
\text { pho- } \\
\text { rus, } \\
\text { total } \\
(\mathrm{mg} / \mathrm{L})\end{array}$ & $\begin{array}{c}\text { Organic } \\
\text { carbon, } \\
\text { suspended } \\
(\mathrm{mg} / \mathrm{L} \\
\text { as C) }\end{array}$ & $\begin{array}{c}\text { Organic } \\
\text { carbon, } \\
\text { dissolved } \\
(\mathbf{m g} / \mathbf{L} \\
\text { as } \mathrm{C})\end{array}$ \\
\hline \multicolumn{12}{|c|}{ SITE 35 12419000--SPOKANE RIVER NEAR POST FALLS, IDAHO (Continued) } \\
\hline $06 / 27 / 00$ & 1415 & .001 & .026 & .003 & e.09 & .15 & $<.006$ & .002 & $<.008$ & -- & -- \\
\hline $07 / 25 / 00$ & 1300 & .002 & .072 & .005 & e.07 & .12 & $<.006$ & .002 & $<.008$ & -- & -- \\
\hline $08 / 30 / 00$ & 1415 & .002 & .257 & .011 & .11 & .17 & e.004 & .001 & .009 & -- & -- \\
\hline $11 / 08 / 00$ & 1400 & .001 & .045 & $<.002$ & e.07 & .10 & .007 & e. 004 & .011 & -- & -- \\
\hline $12 / 21 / 00$ & 1400 & .003 & .125 & .032 & e. 10 & .10 & .018 & .014 & .022 & -- & -- \\
\hline $01 / 22 / 01$ & 1330 & .006 & .236 & .052 & .19 & .15 & .036 & .031 & .044 & -- & -- \\
\hline $03 / 13 / 01$ & 1415 & .002 & .103 & .020 & .10 & .14 & .016 & .013 & .025 & -- & -- \\
\hline $04 / 09 / 01$ & 0930 & $<.001$ & .051 & .008 & $<.10$ & .12 & $<.006$ & $<.007$ & .006 & -- & -- \\
\hline $05 / 07 / 01$ & 1345 & .001 & .027 & .005 & $<.10$ & .35 & e.003 & $<.007$ & .007 & -- & -- \\
\hline $06 / 11 / 01$ & 1230 & $<.001$ & .021 & .005 & e. 07 & .14 & $<.006$ & $<.007$ & .011 & -- & -- \\
\hline \multicolumn{12}{|c|}{ SITE 41 12424500--SPOKANE RIVER AT SEVEN MILE BRIDGE, NEAR SPOKANE, WASH. } \\
\hline 03/03/99 & 1115 & .002 & .591 & .067 & .14 & .26 & .016 & .015 & .062 & .4 & 1.9 \\
\hline 04/14/99 & 1230 & .001 & .438 & .008 & e.09 & .12 & .012 & .011 & .024 & .3 & 1.7 \\
\hline $05 / 18 / 99$ & 1100 & .001 & .235 & .003 & $<.10$ & e. 06 & $<.004$ & .002 & .027 & .2 & 1.6 \\
\hline $06 / 03 / 99$ & 1000 & .011 & .101 & .020 & e. 09 & .14 & $<.004$ & .002 & .026 & .3 & 1.3 \\
\hline $06 / 24 / 99$ & 1015 & .009 & .227 & $<.002$ & e. 10 & .11 & $<.004$ & .001 & .006 & .3 & 1.3 \\
\hline $07 / 28 / 99$ & 1000 & .002 & .997 & .003 & .12 & e. 10 & .016 & .011 & .022 & .2 & 1.0 \\
\hline 09/10/99 & 0940 & .008 & 2.02 & .030 & e. 08 & .24 & .017 & .012 & .048 & .3 & 1.0 \\
\hline $10 / 21 / 99$ & 1110 & .001 & .766 & $<.002$ & e. 09 & .12 & .036 & .023 & .036 & .2 & 1.1 \\
\hline $12 / 03 / 99$ & 0940 & .001 & .36 & $<.002$ & e. 08 & e. 09 & .026 & .019 & .032 & .2 & 1.4 \\
\hline $01 / 13 / 00$ & 1000 & .003 & .628 & .004 & e.05 & e.08 & .034 & .029 & .040 & $<.2$ & 1.3 \\
\hline $03 / 01 / 00$ & 0830 & .001 & 1.09 & .005 & e. 10 & .17 & .027 & .026 & .053 & .2 & 2.0 \\
\hline $03 / 29 / 00$ & 1000 & $<.001$ & .369 & $<.002$ & .15 & .15 & .019 & .018 & .031 & -- & -- \\
\hline $04 / 11 / 00$ & 1145 & $<.001$ & .294 & .003 & $<.10$ & .15 & .016 & .014 & .032 & -- & -- \\
\hline $04 / 19 / 00$ & 0945 & .001 & .170 & .005 & .10 & .23 & .011 & .008 & .081 & -- & -- \\
\hline $05 / 03 / 00$ & 1245 & $<.001$ & .214 & .005 & e. 07 & .18 & .009 & .008 & .021 & -- & -- \\
\hline $05 / 16 / 00$ & 0930 & .001 & .241 & .011 & e. 07 & .20 & $<.006$ & $<.001$ & .011 & -- & -- \\
\hline $05 / 31 / 00$ & 1015 & $<.001$ & .223 & .003 & $<.10$ & .14 & e.003 & .001 & .008 & -- & -- \\
\hline $06 / 27 / 00$ & 1000 & .001 & .606 & $<.002$ & e. 08 & .16 & e.005 & .002 & .010 & -- & -- \\
\hline $07 / 25 / 00$ & 0830 & .002 & 1.26 & .003 & e. 07 & .15 & .013 & .012 & .011 & -- & -- \\
\hline $08 / 30 / 00$ & 1000 & .006 & 2.46 & .022 & .12 & .26 & .016 & .014 & .036 & -- & -- \\
\hline $11 / 08 / 00$ & 1000 & .001 & .714 & $<.002$ & e.06 & .10 & .034 & .029 & .040 & -- & -- \\
\hline $12 / 21 / 00$ & 0945 & .002 & 1.04 & $<.002$ & e. 05 & $<.08$ & .064 & .053 & .071 & -- & -- \\
\hline $01 / 22 / 01$ & 0945 & .003 & 1.65 & .010 & .15 & .16 & .117 & .108 & .118 & -- & -- \\
\hline $03 / 13 / 01$ & 1030 & .009 & 2.86 & .024 & .20 & .31 & .068 & .061 & .100 & -- & -- \\
\hline $04 / 09 / 01$ & 1315 & .002 & .626 & .007 & $<.10$ & .16 & .007 & e. 005 & .020 & -- & -- \\
\hline $05 / 07 / 01$ & 1000 & .001 & .202 & $<.002$ & e.06 & .17 & e.003 & $<.007$ & .013 & -- & -- \\
\hline $06 / 11 / 01$ & 0900 & $<.001$ & .461 & .006 & e. 08 & .14 & e.004 & $<.007$ & .015 & -- & -- \\
\hline
\end{tabular}


Table 8. Trace-element concentration data for surface-water samples collected at fixed water-quality sites, Northern Rockies Intermontane Basins study unit, 1998-2001

[See figure 1 for site location. Abbreviations: e, estimated; Fb, Field blank; $\mu \mathrm{g} / \mathrm{L}$, micrograms per liter; R, replicate. Symbols: <, less than reporting level; ,-- no data]

\begin{tabular}{|c|c|c|c|c|c|c|c|c|c|c|}
\hline Date & Time & $\begin{array}{l}\text { Alumi- } \\
\text { num, } \\
\text { total } \\
\text { recov- } \\
\text { erable } \\
(\mu \mathrm{g} / \mathrm{L})\end{array}$ & $\begin{array}{l}\text { Alumi- } \\
\text { num, } \\
\text { dis- } \\
\text { solved } \\
(\mu \mathrm{g} / \mathrm{L})\end{array}$ & $\begin{array}{c}\text { Anti- } \\
\text { mony, } \\
\text { total } \\
\text { recov- } \\
\text { erable } \\
(\mu \mathrm{g} / \mathrm{L})\end{array}$ & $\begin{array}{c}\text { Anti- } \\
\text { mony, } \\
\text { dis- } \\
\text { solved } \\
(\mu \mathrm{g} / \mathrm{L})\end{array}$ & $\begin{array}{c}\text { Arsenic, } \\
\text { total } \\
\text { recov- } \\
\text { erable } \\
(\mu \mathrm{g} / \mathrm{L})\end{array}$ & $\begin{array}{c}\text { Arsenic, } \\
\text { dis- } \\
\text { solved } \\
(\mu \mathrm{g} / \mathrm{L})\end{array}$ & $\begin{array}{c}\text { Barium, } \\
\text { total } \\
\text { recov- } \\
\text { erable } \\
(\mu \mathrm{g} / \mathrm{L})\end{array}$ & $\begin{array}{c}\text { Barium, } \\
\text { dis- } \\
\text { solved } \\
(\mu \mathrm{g} / \mathbf{L})\end{array}$ & $\begin{array}{l}\text { Beryl- } \\
\text { lium, } \\
\text { total } \\
\text { recov- } \\
\text { erable } \\
(\mu \mathrm{g} / \mathrm{L})\end{array}$ \\
\hline \multicolumn{11}{|c|}{ 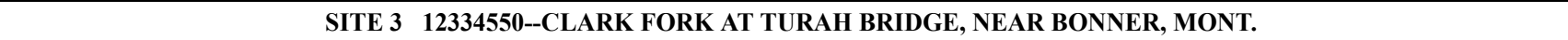 } \\
\hline $11 / 05 / 98$ & 1000 & 71 & $<1.0$ & $<1.0$ & $<1.0$ & 6.6 & 6.1 & $<100$ & 75 & $<10$ \\
\hline $11 / 05 / 98^{\mathbf{F b}}$ & 1005 & -- & $<.3$ & -- & $<.2$ & -- & -- & -- & $<.2$ & -- \\
\hline $12 / 15 / 98$ & 1145 & -- & -- & -- & -- & -- & -- & -- & -- & -- \\
\hline $01 / 27 / 99$ & 1245 & -- & -- & -- & -- & -- & -- & -- & -- & -- \\
\hline $03 / 25 / 99$ & 1215 & -- & -- & -- & -- & -- & -- & -- & -- & -- \\
\hline 04/26/99 & 1115 & 383 & 2.6 & $<1.0$ & $<1.0$ & 6.6 & 4.7 & 78 & 72 & $<4.0$ \\
\hline $05 / 12 / 99$ & 0915 & -- & 2.9 & -- & $<1.0$ & -- & 4.5 & -- & 68 & -- \\
\hline $05 / 25 / 99$ & 1145 & 1,400 & 11 & $<1.0$ & $<1.0$ & 11 & 4.7 & 89 & 55 & $<4.0$ \\
\hline 06/07/99 & 1230 & 769 & 12 & $<1.0$ & $<1.0$ & 9.6 & 5.6 & 62 & 44 & $<4.0$ \\
\hline 06/19/99 & 1130 & 490 & 12 & $<1.0$ & $<1.0$ & 7.6 & 6.5 & 62 & 54 & $<4.0$ \\
\hline $07 / 21 / 99$ & 1015 & 28 & 6.3 & $<1.0$ & $<1.0$ & 5.7 & 6.0 & 79 & 84 & $<4.0$ \\
\hline 08/19/99 & 1000 & 133 & 1.7 & $<1.0$ & $<1.0$ & 8.2 & 7.9 & 92 & 92 & $<4.0$ \\
\hline $10 / 20 / 99$ & 1030 & 107 & 6.2 & $<1.0$ & $<1.0$ & 6.9 & 6.2 & 80 & 85 & $<5.0$ \\
\hline $12 / 03 / 99^{\mathbf{F b}}$ & 1030 & -- & .84 & -- & $<.2$ & -- & -- & -- & $<.2$ & -- \\
\hline $12 / 03 / 99$ & 1100 & 75 & $<1.0$ & $<1.0$ & $<1.0$ & 6.3 & 6.6 & 75 & 76 & $<5.0$ \\
\hline $01 / 26 / 00$ & 0945 & 97 & 5.3 & $<1.0$ & $<1.0$ & 5.3 & 5.5 & 78 & 78 & $<5.0$ \\
\hline 03/08/00 & 1045 & 196 & -- & $<1.0$ & $<1.0$ & 6.4 & 5.1 & 77 & 72 & $<5.0$ \\
\hline $03 / 08 / 00^{\mathbf{R}}$ & 1046 & 168 & -- & $<1.0$ & $<1.0$ & 6.2 & 5.8 & 76 & 77 & $<5.0$ \\
\hline $04 / 12 / 00$ & 0900 & 218 & 1.3 & $<1.0$ & $<1.0$ & 6.4 & 5.5 & 79 & 79 & $<5.0$ \\
\hline $05 / 25 / 00$ & 0830 & 209 & e11 & $<1.0$ & $<1.0$ & 2.9 & 3.1 & 71 & 67 & $<5.0$ \\
\hline $06 / 21 / 00$ & 1015 & 57 & 1.4 & $<1.0$ & $<1.0$ & 3.7 & 4.0 & 79 & 81 & $<5.0$ \\
\hline $07 / 31 / 00$ & 1045 & 55 & $<1.0$ & $<1.0$ & $<1.0$ & 4.1 & 4.1 & 94 & 95 & $<5.0$ \\
\hline $08 / 29 / 00$ & 1100 & 36 & $<1.0$ & $<1.0$ & $<1.0$ & 4.6 & 4.2 & 94 & 94 & $<5.0$ \\
\hline $11 / 07 / 00^{\mathbf{F b}}$ & 1300 & -- & $<.3$ & -- & $<.2$ & -- & -- & -- & $<.2$ & -- \\
\hline $11 / 07 / 00$ & 1400 & -- & -- & -- & -- & 6.1 & 5.6 & -- & -- & -- \\
\hline $12 / 20 / 00$ & 1545 & -- & -- & -- & -- & 5.2 & 5.4 & -- & -- & -- \\
\hline $01 / 30 / 01$ & 1100 & -- & -- & -- & -- & 4.2 & 4.7 & -- & -- & -- \\
\hline $03 / 12 / 01^{\mathbf{F b}}$ & 1040 & -- & $<.3$ & -- & $<.2$ & -- & -- & -- & $<.2$ & -- \\
\hline 03/12/01 & 1100 & -- & -- & -- & -- & 7.5 & 5.8 & -- & -- & -- \\
\hline 04/10/01 & 1030 & -- & -- & -- & -- & 6.6 & 5.8 & -- & -- & -- \\
\hline $05 / 22 / 01$ & 1100 & -- & -- & -- & -- & 4.4 & 3.9 & -- & -- & -- \\
\hline \multicolumn{11}{|c|}{ SITE 5 12352500--BITTERROOT RIVER NEAR MISSOULA, MONT. } \\
\hline 03/04/99 & 1630 & -- & -- & -- & -- & -- & -- & -- & -- & -- \\
\hline $03 / 24 / 99$ & 0930 & 462 & 5.1 & $<1.0$ & $<1.0$ & $<1.0$ & $<1.0$ & 33 & 27 & $<4.0$ \\
\hline $04 / 15 / 99$ & 1300 & -- & -- & -- & -- & -- & -- & -- & -- & -- \\
\hline 05/13/99 & 0945 & 161 & 9.0 & $<1.0$ & $<1.0$ & $<1.0$ & $<1.0$ & 28 & 25 & $<4.0$ \\
\hline $05 / 25 / 99$ & 0915 & -- & 33 & -- & $<1.0$ & -- & $<1.0$ & -- & 13 & -- \\
\hline 06/07/99 & 1615 & 480 & 28 & $<1.0$ & $<1.0$ & $<1.0$ & $<1.0$ & 21 & 13 & $<4.0$ \\
\hline $06 / 19 / 99$ & 1445 & 591 & 30 & $<1.0$ & $<1.0$ & $<1.0$ & $<1.0$ & 19 & 11 & $<4.0$ \\
\hline $07 / 21 / 99$ & 1530 & 42 & 9.8 & $<1.0$ & $<1.0$ & $<1.0$ & $<1.0$ & 24 & 25 & $<4.0$ \\
\hline 08/19/99 & 1500 & 55 & 4.4 & $<1.0$ & $<1.0$ & $<1.0$ & 1.5 & 37 & 38 & $<4.0$ \\
\hline $10 / 20 / 99$ & 1430 & $<28$ & 5.6 & $<1.0$ & $<1.0$ & $<2.6$ & $<2.0$ & 40 & 45 & $<5.0$ \\
\hline $12 / 02 / 99$ & 1600 & -- & -- & -- & -- & -- & -- & -- & -- & -- \\
\hline $01 / 26 / 00$ & 1000 & -- & -- & -- & -- & -- & -- & -- & -- & -- \\
\hline 03/08/00 & 1415 & 216 & 2.7 & $<1.0$ & $<1.0$ & $<2.6$ & $<2.0$ & 34 & 30 & $<5.0$ \\
\hline $03 / 08 / 00^{\mathbf{F b}}$ & 1500 & -- & $<.3$ & -- & $<.2$ & -- & -- & -- & $<.2$ & -- \\
\hline
\end{tabular}


Table 8. Trace-element concentration data for surface-water samples collected at fixed wter-quality sites, Northern Rockies Intermontane Basins study unit, 1998-2001 (Continued)

\begin{tabular}{|c|c|c|c|c|c|c|c|c|c|c|c|}
\hline Date & $\begin{array}{c}\text { Beryl- } \\
\text { lium, } \\
\text { dis- } \\
\text { solved } \\
(\mu \mathrm{g} / \mathrm{L})\end{array}$ & $\begin{array}{c}\text { Boron, } \\
\text { dis- } \\
\text { solved } \\
(\mu \mathrm{g} / \mathrm{L})\end{array}$ & $\begin{array}{c}\text { Cad- } \\
\text { mium, } \\
\text { total } \\
\text { recov- } \\
\text { erable } \\
(\mu \mathrm{g} / \mathrm{L})\end{array}$ & $\begin{array}{c}\text { Cad- } \\
\text { mium, } \\
\text { dis- } \\
\text { solved } \\
(\mu \mathrm{g} / \mathrm{L})\end{array}$ & $\begin{array}{c}\text { Chro- } \\
\text { mium, } \\
\text { total } \\
\text { recov- } \\
\text { erable } \\
(\mu \mathrm{g} / \mathrm{L})\end{array}$ & $\begin{array}{c}\text { Chro- } \\
\text { mium, } \\
\text { dis- } \\
\text { solved } \\
(\mu \mathrm{g} / \mathrm{L})\end{array}$ & $\begin{array}{c}\text { Cobalt, } \\
\text { total } \\
\text { recov- } \\
\text { erable } \\
(\mu \mathrm{g} / \mathrm{L})\end{array}$ & $\begin{array}{c}\text { Cobalt, } \\
\text { dis- } \\
\text { solved } \\
(\mu \mathrm{g} / \mathrm{L})\end{array}$ & $\begin{array}{c}\text { Copper, } \\
\text { total } \\
\text { recov- } \\
\text { erable } \\
(\mu \mathrm{g} / \mathrm{L})\end{array}$ & $\begin{array}{c}\text { Copper, } \\
\text { dis- } \\
\text { solved } \\
(\mu \mathrm{g} / \mathrm{L})\end{array}$ & $\begin{array}{l}\text { Iron, } \\
\text { total } \\
\text { recov- } \\
\text { erable } \\
(\mu \mathrm{g} / \mathrm{L})\end{array}$ \\
\hline \multicolumn{12}{|c|}{ SITE 3 12334550--CLARK FORK AT TURAH BRIDGE, NEAR BONNER, MONT. } \\
\hline $11 / 05 / 98$ & $<1.0$ & -- & $<1.0$ & $<1.0$ & $<1.0$ & 1.6 & $<1.0$ & $<1.0$ & $<10$ & 2.0 & -- \\
\hline $11 / 05 / 98^{\mathbf{F b}}$ & $<.2$ & $<2$ & -- & $<.3$ & -- & $<.2$ & -- & $<.2$ & -- & $<.2$ & -- \\
\hline $12 / 15 / 98$ & -- & -- & -- & -- & -- & -- & -- & -- & -- & -- & -- \\
\hline $01 / 27 / 99$ & -- & -- & -- & -- & -- & -- & -- & -- & -- & -- & -- \\
\hline $03 / 25 / 99$ & -- & -- & -- & -- & -- & -- & -- & -- & -- & -- & -- \\
\hline $04 / 26 / 99$ & $<1.0$ & -- & $<1.0$ & $<1.0$ & $<1.0$ & $<1.0$ & $<1.0$ & $<1.0$ & e9.9 & 2.8 & 430 \\
\hline $05 / 12 / 99$ & $<1.0$ & -- & -- & $<1.0$ & -- & $<1.0$ & -- & $<1.0$ & -- & 2.7 & -- \\
\hline $05 / 25 / 99$ & $<1.0$ & -- & $<1.0$ & $<1.0$ & 1.5 & $<1.0$ & 2.0 & $<1.0$ & 48 & 3.5 & 1,770 \\
\hline 06/07/99 & $<1.0$ & -- & $<1.0$ & $<1.0$ & $<1.0$ & & $<1.0$ & $<1.0$ & 35 & 4.8 & 970 \\
\hline $06 / 19 / 99$ & $<1.0$ & -- & $<1.0$ & $<1.0$ & $<1.0$ & $<1.0$ & $<1.0$ & $<1.0$ & 21 & 4.1 & 600 \\
\hline $07 / 21 / 99$ & $<1.0$ & -- & $<1.0$ & $<1.0$ & $<1.0$ & $<1.0$ & $<1.0$ & $<1.0$ & e8.1 & 2.5 & 40 \\
\hline 08/19/99 & $<1.0$ & -- & $<1.0$ & $<1.0$ & $<1.0$ & $<1.0$ & 1.0 & $<1.0$ & e6.9 & 2.9 & 150 \\
\hline $10 / 20 / 99$ & $<1.0$ & -- & $<.11$ & $<1.0$ & $<1.0$ & $<.80$ & $<1.8$ & $<1.0$ & $<20$ & 2.5 & 140 \\
\hline $12 / 03 / 99^{\mathbf{F b}}$ & $<.2$ & $<2$ & -- & $<.3$ & -- & $<.01$ & -- & $<.2$ & -- & .23 & -- \\
\hline $12 / 03 / 99$ & $<1.0$ & -- & $<.11$ & $<1.0$ & $<1.0$ & $<1.0$ & $<2.0$ & $<1.0$ & $<20$ & 2.2 & 120 \\
\hline $01 / 26 / 00$ & $<1.0$ & -- & $<.11$ & $<1.0$ & $<1.0$ & $<.80$ & $<2.0$ & $<1.0$ & $<20$ & 2.5 & 130 \\
\hline $03 / 08 / 00$ & $<1.0$ & -- & $<.11$ & $<1.0$ & $<1.0$ & $<1.0$ & $<2.0$ & $<1.0$ & $<20$ & 6.5 & 220 \\
\hline $03 / 08 / 00^{\mathbf{R}}$ & $<1.0$ & -- & $<.11$ & $<1.0$ & $<1.0$ & $<1.0$ & $<2.0$ & $<1.0$ & e14 & 3.2 & 210 \\
\hline $04 / 12 / 00$ & $<1.0$ & -- & e. 07 & $<1.0$ & $<1.0$ & $<.80$ & $<2.0$ & $<1.0$ & e13 & 2.9 & 260 \\
\hline $05 / 25 / 00$ & $<1.0$ & e10 & $<.11$ & $<1.0$ & $<1.0$ & $<.80$ & $<2.0$ & $<1.0$ & $<20$ & 3.5 & 220 \\
\hline $06 / 21 / 00$ & $<1.0$ & 15 & $<.11$ & $<1.0$ & $<1.0$ & $<.80$ & $<2.0$ & $<1.0$ & $<20$ & 2.3 & 90 \\
\hline $07 / 31 / 00$ & $<1.0$ & 16 & $<.11$ & $<1.0$ & $<1.0$ & $<.80$ & $<2.0$ & $<1.0$ & $<20$ & 2.1 & 80 \\
\hline $08 / 29 / 00$ & $<1.0$ & 21 & $<.11$ & $<1.0$ & $<1.0$ & $<.80$ & $<1.8$ & $<1.0$ & $<20$ & 1.9 & 40 \\
\hline $11 / 07 / 00^{\mathbf{F b}}$ & $<.2$ & $<2$ & -- & $<.3$ & -- & $<.2$ & -- & $<.2$ & -- & $<.2$ & -- \\
\hline $11 / 07 / 00$ & -- & -- & .05 & $<.04$ & -- & -- & -- & -- & 5.3 & 2.2 & -- \\
\hline $12 / 20 / 00$ & -- & -- & .04 & e.02 & -- & -- & -- & -- & 3.5 & 2.0 & -- \\
\hline $01 / 30 / 01$ & -- & -- & .04 & .04 & -- & -- & -- & -- & 3.6 & 2.2 & -- \\
\hline $03 / 12 / 01^{\mathbf{F b}}$ & $<.2$ & $<2$ & -- & $<.3$ & -- & $<.2$ & -- & $<.2$ & -- & $<.2$ & -- \\
\hline 03/12/01 & -- & -- & .13 & e. 04 & -- & -- & -- & -- & 17 & 5.4 & -- \\
\hline $04 / 10 / 01$ & -- & -- & .07 & e. 02 & -- & -- & -- & -- & 8.8 & 3.4 & -- \\
\hline $05 / 22 / 01$ & -- & -- & .07 & $<.04$ & -- & -- & -- & -- & 8.9 & 2.6 & -- \\
\hline \multicolumn{12}{|c|}{ SITE 5 12352500--BITTERROOT RIVER NEAR MISSOULA, MONT. } \\
\hline 03/04/99 & -- & -- & -- & -- & -- & -- & -- & -- & -- & -- & -- \\
\hline $03 / 24 / 99$ & $<1.0$ & -- & $<1.0$ & $<1.0$ & $<1.0$ & $<1.0$ & $<1$ & $<1.0$ & $<12$ & $<1.0$ & 540 \\
\hline $04 / 15 / 99$ & -- & -- & -- & -- & -- & -- & -- & -- & -- & -- & -- \\
\hline 05/13/99 & $<1.0$ & -- & $<1.0$ & $<1.0$ & $<1.0$ & $<1.0$ & $<1$ & $<1.0$ & $<12$ & $<1.0$ & 190 \\
\hline $05 / 25 / 99$ & $<1.0$ & -- & -- & $<1.0$ & -- & $<1.0$ & -- & $<1.0$ & -- & $<1.0$ & -- \\
\hline $06 / 07 / 99$ & $<1.0$ & -- & $<1.0$ & $<1.0$ & $<1.0$ & $<1.0$ & $<1$ & $<1.0$ & $<20$ & $<1.0$ & 470 \\
\hline 06/19/99 & $<1.0$ & -- & $<1.0$ & $<1.0$ & $<1.0$ & $<1.0$ & $<1$ & $<1.0$ & $<12$ & $<1.0$ & 540 \\
\hline $07 / 21 / 99$ & $<1.0$ & -- & $<1.0$ & $<1.0$ & $<1.0$ & $<1.0$ & $<1$ & $<1.0$ & $<12$ & $<1.0$ & 80 \\
\hline 08/19/99 & $<1.0$ & -- & 1.9 & $<1.0$ & $<1.0$ & $<1.0$ & $<1$ & $<1.0$ & $<12$ & $<1.0$ & 110 \\
\hline $10 / 20 / 99$ & $<1.0$ & -- & $<.11$ & $<1.0$ & $<1.0$ & $<.80$ & $<1.8$ & $<1.0$ & $<20$ & $<1.0$ & 50 \\
\hline $12 / 02 / 99$ & -- & -- & -- & -- & -- & -- & -- & -- & -- & -- & -- \\
\hline $01 / 26 / 00$ & -- & -- & -- & -- & -- & -- & -- & -- & -- & -- & -- \\
\hline 03/08/00 & $<1.0$ & -- & $<.11$ & $<1.0$ & $<1.0$ & $<1.0$ & $<1.8$ & $<1.0$ & $<20$ & $<1.0$ & 340 \\
\hline $03 / 08 / 00^{\mathbf{F b}}$ & $<.2$ & 3.0 & -- & $<.3$ & -- & $<.2$ & -- & $<.2$ & -- & $<.2$ & -- \\
\hline
\end{tabular}


Table 8. Trace-element concentration data for surface-water samples collected at fixed water-quality sites, Northern Rockies Intermontane Basins study unit, 1998-2001 (Continued)

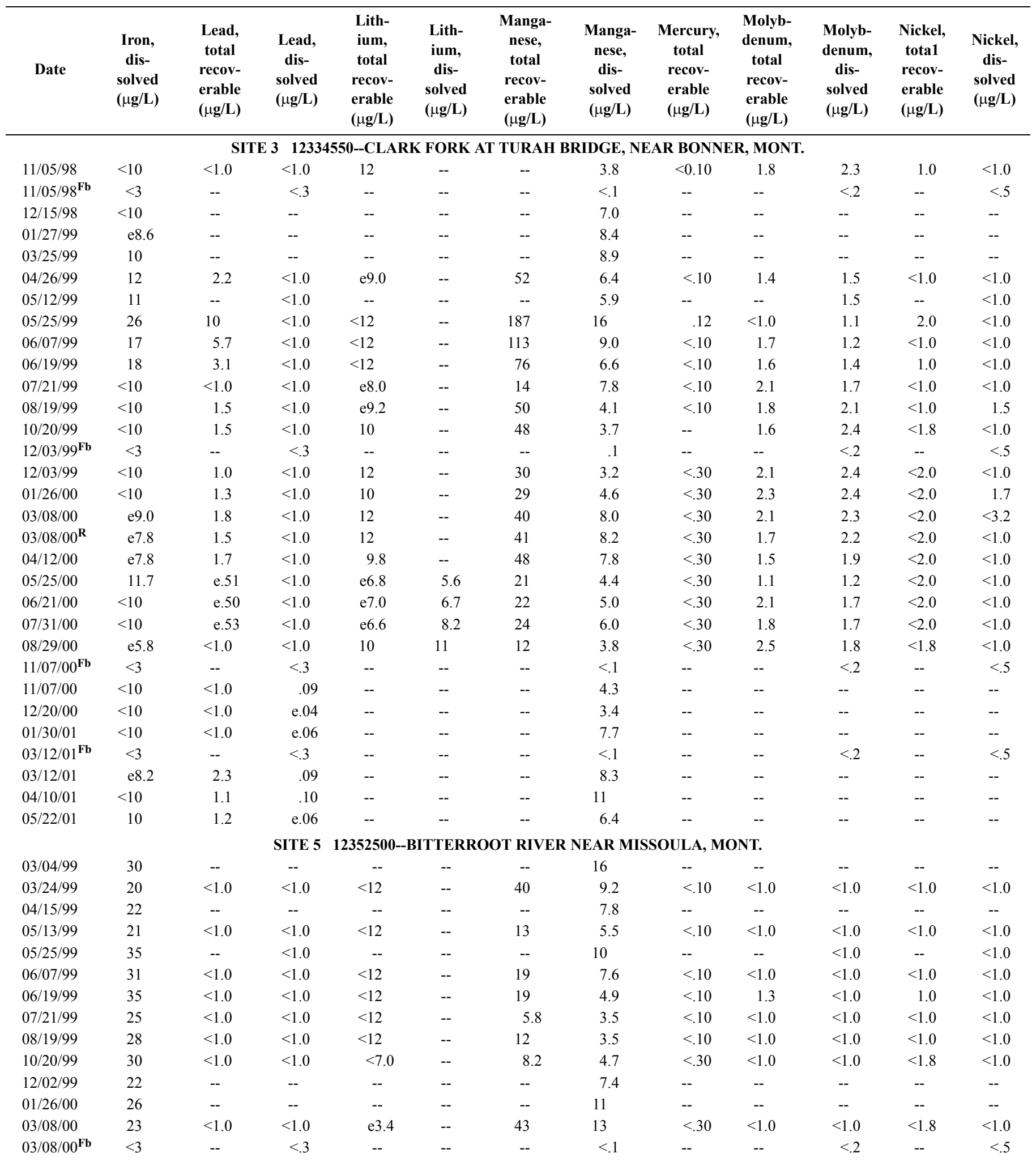


Table 8. Trace-element concentration data for surface-water samples collected at fixed water-quality sites, Northern Rockies Intermontane Basins study unit, 1998-2001(Continued)

\begin{tabular}{|c|c|c|c|c|c|c|c|c|c|c|c|}
\hline Date & $\begin{array}{c}\text { Selenium, } \\
\text { total } \\
\text { recov- } \\
\text { erable } \\
(\mu \mathrm{g} / \mathrm{L})\end{array}$ & $\begin{array}{c}\text { Selenium, } \\
\text { dis- } \\
\text { solved } \\
(\mu \mathrm{g} / \mathbf{L})\end{array}$ & $\begin{array}{c}\text { Silver, } \\
\text { total } \\
\text { recov- } \\
\text { erable } \\
(\mu \mathrm{g} / \mathrm{L})\end{array}$ & $\begin{array}{c}\text { Silver, } \\
\text { dis- } \\
\text { solved } \\
(\mu \mathrm{g} / \mathrm{L})\end{array}$ & $\begin{array}{c}\text { Stron- } \\
\text { tium, } \\
\text { total } \\
\text { recov- } \\
\text { erable } \\
(\mu \mathrm{g} / \mathrm{L})\end{array}$ & $\begin{array}{c}\text { Stron- } \\
\text { tium, } \\
\text { dis- } \\
\text { solved } \\
(\mu \mathrm{g} / \mathrm{L})\end{array}$ & $\begin{array}{l}\text { Thal- } \\
\text { lium, } \\
\text { dis- } \\
\text { solved } \\
(\mu \mathrm{g} / \mathrm{L})\end{array}$ & $\begin{array}{c}\text { Uranium, } \\
\text { natural } \\
(\mu \mathrm{g} / \mathrm{L})\end{array}$ & $\begin{array}{c}\text { Vana- } \\
\text { dium, } \\
\text { dis- } \\
\text { solved } \\
(\mu \mathrm{g} / \mathrm{L})\end{array}$ & $\begin{array}{l}\text { Zinc, } \\
\text { total } \\
\text { recov- } \\
\text { erable } \\
(\mu \mathrm{g} / \mathrm{L})\end{array}$ & $\begin{array}{c}\text { Zinc, } \\
\text { dis- } \\
\text { solved } \\
(\mu \mathrm{g} / \mathrm{L})\end{array}$ \\
\hline \multicolumn{12}{|c|}{ SITE 3 12334550-CLARK FORK AT TURAH BRIDGE, NEAR BONNER, MONT. } \\
\hline $11 / 05 / 98$ & $<1.0$ & $<1.0$ & $<1.0$ & $<1.0$ & 264 & -- & -- & 4.3 & -- & -- & 3.3 \\
\hline $11 / 05 / 98^{\mathbf{F b}}$ & -- & -- & -- & $<.2$ & -- & $<.1$ & $<.1$ & $<.2$ & -- & -- & 1.2 \\
\hline $12 / 15 / 98$ & -- & -- & -- & -- & -- & -- & -- & -- & -- & -- & -- \\
\hline $01 / 27 / 99$ & -- & -- & -- & -- & -- & -- & -- & -- & -- & -- & -- \\
\hline $03 / 25 / 99$ & -- & -- & -- & -- & -- & -- & -- & -- & -- & -- & -- \\
\hline $04 / 26 / 99$ & $<1.0$ & $<1.0$ & $<1.0$ & $<1.0$ & 146 & -- & -- & 2.1 & -- & $<40$ & 2.5 \\
\hline $05 / 12 / 99$ & -- & $<1.0$ & -- & $<1.0$ & -- & -- & -- & 2.3 & -- & & 3.0 \\
\hline $05 / 25 / 99$ & $<1.0$ & $<1.0$ & $<1.0$ & $<1.0$ & 91 & -- & -- & 1.1 & -- & 90 & 4.0 \\
\hline $06 / 07 / 99$ & $<1.0$ & $<1.0$ & $<1.0$ & $<1.0$ & 91 & -- & -- & 1.2 & -- & 62 & 5.3 \\
\hline $06 / 19 / 99$ & $<1.0$ & $<1.0$ & $<1.0$ & $<1.0$ & 97 & -- & -- & 1.3 & -- & e34 & 4.0 \\
\hline $07 / 21 / 99$ & $<1.0$ & $<1.0$ & $<1.0$ & $<1.0$ & 179 & -- & -- & 2.0 & -- & $<40$ & 3.1 \\
\hline 08/19/99 & $<1.0$ & $<1.0$ & $<1.0$ & $<1.0$ & 239 & -- & -- & 2.6 & -- & $<40$ & 2.7 \\
\hline 10/20/99 & $<2.6$ & $<2.4$ & $<1.0$ & $<1.0$ & 246 & -- & -- & 4.0 & -- & $<31$ & 4.1 \\
\hline $12 / 03 / 99^{\mathbf{F b}}$ & -- & -- & -- & $<.2$ & -- & $<.1$ & $<.1$ & $<.2$ & -- & -- & $<.5$ \\
\hline $12 / 03 / 99$ & $<2.6$ & $<2.4$ & $<1.0$ & $<1.0$ & 277 & -- & -- & 5.1 & -- & $<31$ & 3.8 \\
\hline $01 / 26 / 00$ & $<2.6$ & $<2.4$ & $<1.0$ & $<1.0$ & 260 & -- & -- & 5.9 & -- & $<31$ & 5.6 \\
\hline $03 / 08 / 00$ & $<2.6$ & $<2.4$ & $<1.0$ & $<1.0$ & 237 & -- & -- & 4.5 & -- & e22 & 6.9 \\
\hline $03 / 08 / 00^{\mathbf{R}}$ & $<2.6$ & $<2.4$ & $<1.0$ & $<1.0$ & 237 & -- & -- & 4.5 & -- & $\mathrm{e} 28$ & 8.1 \\
\hline $04 / 12 / 00$ & $<2.6$ & $<2.4$ & $<1.0$ & $<1.0$ & 202 & -- & -- & 3.4 & -- & $\mathrm{e} 28$ & 3.8 \\
\hline $05 / 25 / 00$ & $<2.6$ & $<.7$ & $<1.0$ & $<1.0$ & 117 & 120 & $<.90$ & 1.3 & $<1.0$ & $<31$ & 3.8 \\
\hline $06 / 21 / 00$ & $<2.6$ & $<.7$ & $<1.0$ & $<1.0$ & 165 & 182 & $<.90$ & 1.9 & 1.9 & $<31$ & 3.8 \\
\hline $07 / 31 / 00$ & $<2.6$ & $<.7$ & $<1.0$ & $<1.0$ & 189 & 201 & $<.90$ & 1.7 & $<1.0$ & e17 & 6.1 \\
\hline $08 / 29 / 00$ & $<2.6$ & $<.7$ & $<1.0$ & $<1.0$ & 225 & 233 & $<.90$ & 2.1 & $<1.0$ & $<31$ & 2.2 \\
\hline $11 / 07 / 00^{\mathbf{F b}}$ & -- & -- & -- & $<.2$ & -- & $<.1$ & $<.1$ & $<.2$ & -- & -- & $<.5$ \\
\hline $11 / 07 / 00$ & -- & -- & -- & -- & -- & -- & -- & -- & -- & 11 & 3.6 \\
\hline $12 / 20 / 00$ & -- & -- & -- & -- & -- & -- & -- & -- & -- & 8 & 4.7 \\
\hline $01 / 30 / 01$ & -- & -- & -- & -- & -- & -- & -- & -- & -- & 8 & 4.9 \\
\hline $03 / 12 / 01^{\mathbf{F b}}$ & -- & -- & -- & $<.2$ & -- & $<.1$ & $<.1$ & $<.2$ & -- & -- & $<.5$ \\
\hline 03/12/01 & -- & -- & -- & -- & -- & -- & -- & -- & -- & 27 & 7.3 \\
\hline $04 / 10 / 01$ & -- & -- & -- & -- & -- & -- & -- & -- & -- & 14 & 4.5 \\
\hline $05 / 22 / 01$ & -- & -- & -- & -- & -- & -- & -- & -- & -- & 13 & 2.4 \\
\hline \multicolumn{12}{|c|}{ SITE 5 12352500--BITTERROOT RIVER NEAR MISSOULA, MONT. } \\
\hline $03 / 04 / 99$ & -- & -- & -- & -- & -- & -- & -- & -- & -- & -- & -- \\
\hline $03 / 24 / 99$ & $<1.0$ & $<1.0$ & $<1.0$ & $<1.0$ & 54 & -- & -- & $<1.0$ & -- & $<40$ & $<1.0$ \\
\hline $04 / 15 / 99$ & -- & -- & -- & -- & -- & -- & -- & -- & -- & -- & -- \\
\hline 05/13/99 & $<1.0$ & $<1.0$ & $<1.0$ & $<1.0$ & 53 & -- & -- & $<1.0$ & -- & $<40$ & 1.0 \\
\hline $05 / 25 / 99$ & -- & $<1.0$ & -- & $<1.0$ & -- & -- & -- & $<1.0$ & -- & -- & $<1.0$ \\
\hline $06 / 07 / 99$ & $<1.0$ & $<1.0$ & $<1.0$ & $<1.0$ & 32 & -- & -- & $<1.0$ & -- & $<31$ & 1.7 \\
\hline $06 / 19 / 99$ & $<1.0$ & $<1.0$ & $<1.0$ & $<1.0$ & 28 & -- & -- & $<1.0$ & -- & $<40$ & $<1.0$ \\
\hline $07 / 21 / 99$ & $<1.0$ & $<1.0$ & $<1.0$ & $<1.0$ & 48 & -- & -- & $<1.0$ & -- & $<40$ & $<1.0$ \\
\hline 08/19/99 & $<1.0$ & $<1.0$ & $<1.0$ & $<1.0$ & 70 & -- & -- & 1.1 & -- & $<40$ & $<1.0$ \\
\hline $10 / 20 / 99$ & $<2.6$ & $<2.4$ & $<1.0$ & $<1.0$ & 81 & -- & -- & 1.7 & -- & $<31$ & $<1.0$ \\
\hline $12 / 02 / 99$ & -- & -- & -- & -- & -- & -- & -- & -- & -- & -- & -- \\
\hline $01 / 26 / 00$ & -- & -- & -- & -- & -- & -- & -- & -- & -- & -- & -- \\
\hline 03/08/00 & $<2.6$ & $<2.4$ & $<1.0$ & $<1.0$ & 68 & -- & -- & 1.3 & -- & $<31$ & 1.8 \\
\hline $03 / 08 / 00^{\mathbf{F b}}$ & -- & -- & -- & $<.2$ & -- & $<.1$ & $<.1$ & $<.2$ & -- & -- & .8 \\
\hline
\end{tabular}


Table 8. Trace-element concentration data for surface-water samples collected at fixed water-quality sites, Northern Rockies Intermontane Basins study unit, 1998-2001 (Continued)

\begin{tabular}{|c|c|c|c|c|c|c|c|c|c|c|}
\hline Date & Time & $\begin{array}{l}\text { Alumi- } \\
\text { num, } \\
\text { total } \\
\text { recov- } \\
\text { erable } \\
(\mu \mathrm{g} / \mathrm{L})\end{array}$ & $\begin{array}{c}\text { Alumi- } \\
\text { num, } \\
\text { dis- } \\
\text { solved } \\
(\mu \mathrm{g} / \mathrm{L})\end{array}$ & $\begin{array}{c}\text { Anti- } \\
\text { mony, } \\
\text { total } \\
\text { recov- } \\
\text { erable } \\
(\mu \mathrm{g} / \mathrm{L})\end{array}$ & $\begin{array}{c}\text { Anti- } \\
\text { mony, } \\
\text { dis- } \\
\text { solved } \\
(\mu \mathrm{g} / \mathrm{L})\end{array}$ & $\begin{array}{c}\text { Arsenic, } \\
\text { total } \\
\text { recov- } \\
\text { erable } \\
(\mu \mathrm{g} / \mathrm{L})\end{array}$ & $\begin{array}{c}\text { Arsenic, } \\
\text { dis- } \\
\text { solved } \\
(\mu \mathrm{g} / \mathrm{L})\end{array}$ & $\begin{array}{c}\text { Barium, } \\
\text { total } \\
\text { recov- } \\
\text { erable } \\
(\mu \mathrm{g} / \mathrm{L})\end{array}$ & $\begin{array}{c}\text { Barium, } \\
\text { dis- } \\
\text { solved } \\
(\mu \mathrm{g} / \mathrm{L})\end{array}$ & $\begin{array}{l}\text { Beryl- } \\
\text { lium, } \\
\text { total } \\
\text { recov- } \\
\text { erable } \\
(\mu \mathrm{g} / \mathrm{L})\end{array}$ \\
\hline \multicolumn{11}{|c|}{ SITE 5 12352500--BITTERROOT RIVER NEAR MISSOULA, MONT. (Continued) } \\
\hline $04 / 12 / 00$ & 1545 & 262 & 17 & $<1.0$ & $<1.0$ & $<2.6$ & $<2.0$ & 18 & 15 & $<5.0$ \\
\hline $05 / 02 / 00$ & 1300 & 262 & 17 & $<1.0$ & $<1.0$ & $<2.6$ & $<2.0$ & 18 & 15 & $<5.0$ \\
\hline $05 / 23 / 00$ & 1130 & 1,280 & $<37$ & $<1.0$ & $<1.0$ & $<2.6$ & $<.9$ & 26 & 11 & $<5.0$ \\
\hline $06 / 20 / 00$ & 1130 & 123 & 19 & $<1.0$ & $<1.0$ & $<2.6$ & $<.9$ & 17 & 16 & $<5.0$ \\
\hline $07 / 31 / 00$ & 1430 & -- & -- & -- & -- & -- & -- & -- & -- & -- \\
\hline 08/29/00 & 1430 & $\mathrm{e} 23$ & $<1.0$ & $<1.0$ & $<1.0$ & $<2.6$ & e. 7 & 46 & 46 & $<5.0$ \\
\hline 09/19/00 & 1430 & -- & -- & -- & -- & -- & -- & -- & -- & -- \\
\hline $10 / 02 / 00$ & 1000 & -- & -- & -- & -- & -- & -- & -- & -- & -- \\
\hline $10 / 16 / 00$ & 1200 & -- & -- & -- & -- & -- & -- & -- & -- & -- \\
\hline $11 / 08 / 00$ & 1330 & -- & -- & -- & -- & $<1.9$ & .37 & -- & -- & -- \\
\hline $01 / 30 / 01$ & 1500 & -- & -- & -- & -- & $<1.9$ & .41 & -- & -- & -- \\
\hline $03 / 12 / 01$ & 1445 & -- & -- & -- & -- & $<1.9$ & .37 & -- & -- & -- \\
\hline $04 / 10 / 01$ & 1330 & -- & -- & -- & -- & $<1.9$ & .32 & -- & -- & -- \\
\hline $05 / 22 / 01$ & 1430 & -- & -- & -- & -- & $<1.9$ & .24 & -- & -- & -- \\
\hline \multicolumn{11}{|c|}{ SITE 9 12354500-CLARK FORK AT ST. REGIS, MONT. } \\
\hline 03/02/99 & 1330 & -- & -- & -- & -- & -- & -- & -- & -- & -- \\
\hline 04/13/99 & 1500 & 82 & 3.0 & $<1.0$ & $<1.0$ & $<1.0$ & 1.2 & 74 & 82 & $<4.0$ \\
\hline 05/10/99 & 1500 & 149 & 4.1 & $<1.0$ & $<1.0$ & 1.6 & 1.1 & 74 & 43 & $<4.0$ \\
\hline $05 / 26 / 99$ & 0930 & 1,980 & 14 & $<1.0$ & $<1.0$ & 3.1 & 1.3 & 90 & 42 & $<4.0$ \\
\hline $06 / 08 / 99$ & 1045 & 647 & 14 & $<1.0$ & $<1.0$ & 2.1 & 1.5 & 60 & 48 & $<4.0$ \\
\hline $06 / 21 / 99^{\mathbf{F b}}$ & 0835 & -- & $<.3$ & -- & $<.2$ & -- & -- & -- & $<.2$ & -- \\
\hline $06 / 21 / 99$ & 1000 & 615 & 19 & $<1.0$ & $<1.0$ & 2.1 & $<1.0$ & 56 & 42 & $<4.0$ \\
\hline 07/20/99 & 1615 & e25 & 5.9 & $<1.0$ & $<1.0$ & $<1.0$ & 1.3 & 74 & 81 & $<4.0$ \\
\hline 08/18/99 & 0930 & 46 & 2.5 & $<1.0$ & $<1.0$ & 2.8 & 2.3 & 98 & 100 & $<4.0$ \\
\hline $10 / 18 / 99$ & 1415 & $\mathrm{e} 15$ & $<1.0$ & $<1.0$ & $<1.0$ & $\mathrm{e} 2.5$ & 2.4 & 103 & 110 & $<5.0$ \\
\hline $11 / 30 / 99$ & 1300 & -- & -- & -- & -- & -- & -- & -- & -- & -- \\
\hline $01 / 24 / 00$ & 1400 & e24 & 6.6 & $<1.0$ & $<1.0$ & $\mathrm{e} 1.5$ & 2.1 & 98 & 103 & $<5.0$ \\
\hline $03 / 06 / 00$ & 1400 & 82 & 1.4 & $<1.0$ & $<1.0$ & $\mathrm{e} 2.3$ & 2.3 & 87 & 85 & $<5.0$ \\
\hline $04 / 10 / 00$ & 1400 & 185 & 6.9 & $<1.0$ & $<1.0$ & $\mathrm{e} 1.4$ & e1.6 & 68 & 70 & $<5.0$ \\
\hline $05 / 09 / 00$ & 1215 & 127 & $<23$ & $<1.0$ & $<1.0$ & $<2.6$ & e. 8 & 61 & 63 & $<5.0$ \\
\hline $05 / 24 / 00$ & 1230 & 646 & $<18$ & $<1.0$ & $<1.0$ & $<2.6$ & e. 7 & 53 & 42 & $<5.0$ \\
\hline $06 / 20 / 00$ & 0930 & 84 & $<14$ & $<1.0$ & $<1.0$ & $<2.6$ & e. 9 & 63 & 64 & $<5.0$ \\
\hline $08 / 01 / 00^{\mathbf{F b}}$ & 1330 & -- & $<.3$ & -- & $<.2$ & -- & -- & -- & $<.2$ & -- \\
\hline $08 / 01 / 00$ & 1430 & $<28$ & 1.2 & $<1.0$ & $<1.0$ & $<2.6$ & 1.6 & 102 & 105 & $<5.0$ \\
\hline $08 / 30 / 00$ & 1400 & e20 & $<1.0$ & $<1.0$ & $<1.0$ & $\mathrm{e} 2.4$ & 1.7 & 107 & 108 & $<5.0$ \\
\hline $11 / 07 / 00$ & 1000 & -- & -- & -- & -- & 2.0 & 1.8 & -- & -- & -- \\
\hline $12 / 19 / 00$ & 1645 & -- & -- & -- & -- & e1.9 & 1.6 & -- & -- & -- \\
\hline $01 / 31 / 01$ & 1500 & -- & -- & -- & -- & e1.1 & 1.8 & -- & -- & -- \\
\hline 03/13/01 & 1500 & -- & -- & -- & -- & 2.3 & 2.0 & -- & -- & -- \\
\hline $04 / 11 / 01$ & 1400 & -- & -- & -- & -- & 2.5 & 2.0 & -- & -- & -- \\
\hline 05/23/01 & 1430 & -- & -- & -- & -- & $\mathrm{e} 1.3$ & 1.1 & -- & -- & -- \\
\hline \multicolumn{11}{|c|}{ SITE 12 12388700--FLATHEAD RIVER AT PERMA, MONT. } \\
\hline 03/03/99 & 1015 & -- & -- & -- & -- & -- & -- & -- & -- & -- \\
\hline 04/14/99 & 1230 & -- & -- & -- & -- & -- & -- & -- & -- & -- \\
\hline $05 / 11 / 99^{\mathbf{F b}}$ & 0945 & -- & $<.3$ & -- & $<.2$ & -- & -- & -- & $<.2$ & -- \\
\hline 05/11/99 & 1100 & 61 & 1.1 & $<1.0$ & $<1.0$ & $<1.0$ & $<1.0$ & 92 & 91 & $<4.0$ \\
\hline $06 / 20 / 99$ & 1130 & 135 & 3.0 & $<1.0$ & $<1.0$ & $<1.0$ & $<1.0$ & 87 & 89 & $<4.0$ \\
\hline $07 / 19 / 99$ & 1430 & 37 & 6.4 & $<1.0$ & $<1.0$ & $<1.0$ & $<1.0$ & 82 & 87 & $<4.0$ \\
\hline
\end{tabular}


Table 8. Trace-element concentration data for surface-water samples collected at fixed water-quality sites, Northern Rockies Intermontane Basins study unit, 1998-2001 (Continued)

\begin{tabular}{|c|c|c|c|c|c|c|c|c|c|c|c|}
\hline Date & $\begin{array}{c}\text { Beryl- } \\
\text { lium, } \\
\text { dis- } \\
\text { solved } \\
(\mu \mathrm{g} / \mathrm{L})\end{array}$ & $\begin{array}{c}\text { Boron, } \\
\text { dis- } \\
\text { solved } \\
(\mu \mathrm{g} / \mathrm{L})\end{array}$ & $\begin{array}{l}\text { Cad- } \\
\text { mium, } \\
\text { total } \\
\text { recov- } \\
\text { erable } \\
(\mu \mathrm{g} / \mathrm{L})\end{array}$ & $\begin{array}{c}\text { Cad- } \\
\text { mium, } \\
\text { dis- } \\
\text { solved } \\
(\mu \mathrm{g} / \mathrm{L})\end{array}$ & $\begin{array}{c}\text { Chro- } \\
\text { mium, } \\
\text { total } \\
\text { recov- } \\
\text { erable } \\
(\mu \mathrm{g} / \mathrm{L})\end{array}$ & $\begin{array}{c}\text { Chro- } \\
\text { mium, } \\
\text { dis- } \\
\text { solved } \\
(\mu \mathrm{g} / \mathrm{L})\end{array}$ & $\begin{array}{c}\text { Cobalt, } \\
\text { total } \\
\text { recov- } \\
\text { erable } \\
(\mu \mathrm{g} / \mathrm{L})\end{array}$ & $\begin{array}{c}\text { Cobalt, } \\
\text { dis- } \\
\text { solved } \\
(\mu \mathrm{g} / \mathrm{L})\end{array}$ & $\begin{array}{c}\text { Copper, } \\
\text { total } \\
\text { recov- } \\
\text { erable } \\
(\mu \mathrm{g} / \mathrm{L})\end{array}$ & $\begin{array}{c}\text { Copper, } \\
\text { dis- } \\
\text { solved } \\
(\mu \mathrm{g} / \mathrm{L})\end{array}$ & $\begin{array}{c}\text { Iron, } \\
\text { total } \\
\text { recov- } \\
\text { erable } \\
(\mu \mathrm{g} / \mathrm{L})\end{array}$ \\
\hline \multicolumn{12}{|c|}{ 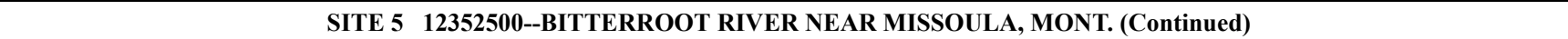 } \\
\hline $04 / 12 / 00$ & $<1.0$ & -- & $<.11$ & $<1.0$ & $<1.0$ & $<.80$ & $<1.8$ & $<1.0$ & $<20$ & $<1.0$ & 270 \\
\hline $05 / 02 / 00$ & $<1.0$ & -- & $<.11$ & $<1.0$ & $<1.0$ & $<.80$ & $<1.8$ & $<1.0$ & $<20$ & $<1.0$ & 270 \\
\hline $05 / 23 / 00$ & $<1.0$ & $<12$ & $<.11$ & $<1.0$ & e. 84 & $<.80$ & $<1.8$ & $<1.0$ & $<20$ & $<1.0$ & 1,140 \\
\hline $06 / 20 / 00$ & $<1.0$ & $<12$ & $<.11$ & $<1.0$ & $<1.0$ & $<.80$ & $<1.8$ & $<1.0$ & $<20$ & $<1.0$ & 170 \\
\hline $07 / 31 / 00$ & -- & -- & -- & -- & -- & -- & -- & -- & -- & -- & -- \\
\hline $08 / 29 / 00$ & $<1.0$ & e11 & $<.11$ & $<1.0$ & $<1.0$ & $<.80$ & $<1.8$ & $<1.0$ & $<20$ & $<1.0$ & 90 \\
\hline $09 / 19 / 00$ & -- & -- & -- & -- & -- & -- & -- & -- & -- & -- & -- \\
\hline $10 / 02 / 00$ & -- & -- & -- & -- & -- & -- & -- & -- & -- & -- & -- \\
\hline $10 / 16 / 00$ & -- & -- & -- & -- & -- & -- & -- & -- & -- & -- & -- \\
\hline $11 / 08 / 00$ & -- & -- & $<.04$ & $<.04$ & -- & -- & -- & -- & e. 52 & .52 & -- \\
\hline $01 / 30 / 01$ & -- & -- & $<.04$ & e. 02 & -- & -- & -- & -- & e. 52 & .62 & -- \\
\hline 03/12/01 & -- & -- & $<.04$ & $<.04$ & -- & -- & -- & -- & .76 & .54 & -- \\
\hline 04/10/01 & -- & -- & $<.04$ & $<.04$ & -- & -- & -- & -- & .78 & .86 & -- \\
\hline $05 / 22 / 01$ & -- & -- & $<.04$ & $<.04$ & -- & -- & -- & -- & 1.2 & .79 & -- \\
\hline \multicolumn{12}{|c|}{ SITE 912354500 -CLARK FORK AT ST. REGIS, MONT. } \\
\hline 03/02/99 & -- & -- & -- & -- & -- & -- & -- & -- & -- & -- & -- \\
\hline 04/13/99 & $<1.0$ & -- & $<1.0$ & $<1.0$ & $<1.0$ & $<1.0$ & $<1.0$ & $<1.0$ & $<12$ & 1.2 & 114 \\
\hline 05/10/99 & $<1.0$ & -- & $<1.0$ & $<1.0$ & $<1.0$ & $<1.0$ & $<1.0$ & $<1.0$ & $<12$ & $<1.0$ & 179 \\
\hline 05/26/99 & $<1.0$ & -- & $<1.0$ & $<1.0$ & 1.7 & $<1.0$ & 1.0 & $<1.0$ & 21 & 1.6 & 2,660 \\
\hline 06/08/99 & $<1.0$ & -- & $<1.0$ & $<1.0$ & $<1.0$ & -- & $<1.0$ & $<1.0$ & $\mathrm{e} 12$ & 2.0 & 755 \\
\hline $06 / 21 / 99^{\mathbf{F b}}$ & $<.2$ & $<2.0$ & -- & $<.3$ & -- & $<.2$ & -- & $<.2$ & -- & $<.2$ & -- \\
\hline $06 / 21 / 99$ & $<1.0$ & -- & $<1.0$ & $<1.0$ & $<1.0$ & $<1.0$ & $<1.0$ & $<1.0$ & e9.0 & 2.4 & 704 \\
\hline 07/20/99 & $<1.0$ & -- & $<1.0$ & $<1.0$ & $<1.0$ & $<1.0$ & $<1.0$ & $<1.0$ & $<12$ & 1.3 & 42 \\
\hline 08/18/99 & $<1.0$ & -- & $<1.0$ & $<1.0$ & $<1.0$ & $<1.0$ & $<1.0$ & $<1.0$ & $<12$ & 1.3 & 77 \\
\hline $10 / 18 / 99$ & $<1.0$ & -- & $<.11$ & $<1.0$ & $<1.0$ & $<.8$ & $<1.8$ & $<1.0$ & $<20$ & 1.2 & 27 \\
\hline $11 / 30 / 99$ & -- & -- & -- & -- & -- & -- & -- & -- & -- & -- & -- \\
\hline $01 / 24 / 00$ & $<1.0$ & -- & $<.11$ & $<1.0$ & $<1.0$ & $<.8$ & $<1.8$ & $<1.0$ & $<20$ & 1.1 & 32 \\
\hline 03/06/00 & $<1.0$ & -- & $<.11$ & $<1.0$ & $<1.0$ & $<1.0$ & $<1.8$ & $<1.0$ & $<20$ & 1.4 & 111 \\
\hline $04 / 10 / 00$ & $<1.0$ & -- & $<.11$ & $<1.0$ & $<1.0$ & $<.8$ & $<1.8$ & $<1.0$ & $<20$ & $<1.0$ & 199 \\
\hline 05/09/00 & $<1.0$ & e7.3 & $<.11$ & $<1.0$ & $<1.0$ & $<.8$ & $<1.8$ & $<1.0$ & $<20$ & $<1.0$ & 161 \\
\hline $05 / 24 / 00$ & $<1.0$ & $<12$ & $<.11$ & $<1.0$ & $<1.0$ & $<.8$ & $\mathrm{e} 1.0$ & $<1.0$ & $<20$ & $<1.0$ & 640 \\
\hline $06 / 20 / 00$ & $<1.0$ & $<12$ & $<.11$ & $<1.0$ & $<1.0$ & $<.8$ & $<1.8$ & $<1.0$ & $<20$ & $<1.0$ & 104 \\
\hline $08 / 01 / 00^{\mathbf{F b}}$ & $<.2$ & 2.5 & -- & $<.3$ & -- & $<.2$ & -- & $<.2$ & -- & $<.2$ & -- \\
\hline 08/01/00 & $<1.0$ & e8.7 & $<.11$ & $<1.0$ & $<1.0$ & $<.8$ & $<1.8$ & $<1.0$ & $<20$ & 1.1 & 29 \\
\hline $08 / 30 / 00$ & $<1.0$ & $\mathrm{e} 12$ & $<.11$ & $<1.0$ & $<1.0$ & $<.8$ & $<1.8$ & $<1.0$ & $<20$ & 1.1 & 35 \\
\hline $11 / 07 / 00$ & -- & -- & $<.04$ & $<.04$ & -- & -- & -- & -- & 1.2 & 1.0 & -- \\
\hline $12 / 19 / 00$ & -- & -- & $<.04$ & $<.04$ & -- & -- & -- & -- & 1.0 & 1.0 & -- \\
\hline $01 / 31 / 01$ & -- & -- & $<.04$ & e. 02 & -- & -- & -- & -- & 1.0 & 1.2 & -- \\
\hline 03/13/01 & -- & -- & e.02 & $<.04$ & -- & -- & -- & -- & 2.8 & 1.6 & -- \\
\hline 04/11/01 & -- & -- & e.02 & $<.04$ & -- & -- & -- & -- & 2.7 & 2.0 & -- \\
\hline $05 / 23 / 01$ & -- & -- & e. 02 & $<.04$ & -- & -- & -- & -- & 3.0 & 1.4 & -- \\
\hline \multicolumn{12}{|c|}{ SITE 12 12388700--FLATHEAD RIVER AT PERMA, MONT. } \\
\hline 03/03/99 & -- & -- & -- & -- & -- & -- & -- & -- & -- & -- & -- \\
\hline $04 / 14 / 99$ & -- & -- & -- & -- & -- & -- & -- & -- & -- & -- & -- \\
\hline $05 / 11 / 99^{\mathbf{F b}}$ & $<.2$ & $<2$ & -- & $<.3$ & -- & $<.2$ & -- & $<.2$ & -- & $<.2$ & -- \\
\hline 05/11/99 & $<1.0$ & -- & $<1.0$ & $<1.0$ & $<1.0$ & $<1.0$ & $<1.0$ & $<1.0$ & $<12$ & $<1.0$ & 81 \\
\hline 06/20/99 & $<1.0$ & -- & $<1.0$ & $<1.0$ & $<1.0$ & $<1.0$ & $<1.0$ & $<1.0$ & $<12$ & $<1.0$ & 146 \\
\hline 07/19/99 & $<1.0$ & -- & $<1.0$ & $<1.0$ & $<1.0$ & $<1.0$ & $<1.0$ & $<1.0$ & $<12$ & $<1.0$ & 36 \\
\hline
\end{tabular}


Table 8. Trace-element concentration data for surface-water samples collected at fixed water-quality sites, Northern Rockies Intermontane Basins study unit, 1998-2001 (Continued)

\begin{tabular}{|c|c|c|c|c|c|c|c|c|c|c|c|c|}
\hline Date & $\begin{array}{c}\text { Iron, } \\
\text { dis- } \\
\text { solved } \\
(\mu \mathrm{g} / \mathbf{L})\end{array}$ & $\begin{array}{l}\text { Lead, } \\
\text { total } \\
\text { recov- } \\
\text { erable } \\
(\mu \mathrm{g} / \mathrm{L})\end{array}$ & $\begin{array}{c}\text { Lead, } \\
\text { dis- } \\
\text { solved } \\
(\mu \mathrm{g} / \mathrm{L})\end{array}$ & $\begin{array}{l}\text { Lith- } \\
\text { ium, } \\
\text { total } \\
\text { recov- } \\
\text { erable } \\
(\mu \mathrm{g} / \mathrm{L})\end{array}$ & $\begin{array}{l}\text { Lith- } \\
\text { ium, } \\
\text { dis- } \\
\text { solved } \\
(\mu \mathrm{g} / \mathrm{L})\end{array}$ & $\begin{array}{l}\text { Manga- } \\
\text { nese, } \\
\text { total } \\
\text { recov- } \\
\text { erable } \\
(\mu \mathrm{g} / \mathrm{L})\end{array}$ & $\begin{array}{c}\text { Manga- } \\
\text { nese, } \\
\text { dis- } \\
\text { solved } \\
(\mu \mathrm{g} / \mathrm{L})\end{array}$ & $\begin{array}{c}\text { Mercury, } \\
\text { total } \\
\text { recov- } \\
\text { erable } \\
(\mu \mathrm{g} / \mathrm{L})\end{array}$ & $\begin{array}{c}\text { Molyb- } \\
\text { denum, } \\
\text { total } \\
\text { recov- } \\
\text { erable } \\
(\mu \mathrm{g} / \mathrm{L})\end{array}$ & $\begin{array}{c}\text { Molyb- } \\
\text { denum, } \\
\text { dis- } \\
\text { solved } \\
(\mu \mathrm{g} / \mathrm{L})\end{array}$ & $\begin{array}{c}\text { Nickel, } \\
\text { tota1 } \\
\text { recov- } \\
\text { erable } \\
(\mu \mathrm{g} / \mathrm{L})\end{array}$ & $\begin{array}{c}\text { Nickel, } \\
\text { dis- } \\
\text { solved } \\
(\mu \mathrm{g} / \mathrm{L})\end{array}$ \\
\hline \multicolumn{13}{|c|}{ SITE 5 12352500--BITTERROOT RIVER NEAR MISSOULA, MONT. (Continued) } \\
\hline $04 / 12 / 00$ & 20 & $<1.0$ & $<1.0$ & $<7.0$ & -- & 14 & 4.9 & $<.30$ & $<1.0$ & $<1.0$ & $<1.8$ & $<1.0$ \\
\hline $05 / 02 / 00$ & 36 & $<1.0$ & $<1.0$ & $<7.0$ & -- & 14 & 3.6 & $<.30$ & $<1.0$ & $<1.0$ & $<1.8$ & $<1.0$ \\
\hline $05 / 23 / 00$ & 22 & $\mathrm{e} 1.0$ & $<1.0$ & e3.8 & 1.1 & 44 & 4.5 & $<.30$ & $<1.0$ & $<1.0$ & $<1.8$ & $<1.0$ \\
\hline $06 / 20 / 00$ & 22 & $<1.0$ & $<1.0$ & $<7.0$ & 1.1 & 8.4 & 3.8 & $<.30$ & 1.4 & $<1.0$ & $<1.8$ & $<1.0$ \\
\hline $07 / 31 / 00$ & 63 & -- & -- & -- & -- & -- & 6.1 & -- & -- & -- & -- & -- \\
\hline 08/29/00 & 40 & $<1.0$ & $<1.0$ & e 3.8 & 2.7 & 14 & 5.8 & $<.30$ & $<1.0$ & $<1.0$ & $<1.8$ & $<1.0$ \\
\hline 09/19/00 & 40 & -- & -- & -- & -- & -- & 4.0 & -- & -- & -- & -- & -- \\
\hline $10 / 02 / 00$ & -- & -- & -- & -- & -- & -- & -- & -- & -- & -- & -- & -- \\
\hline $10 / 16 / 00$ & 30 & -- & -- & -- & -- & -- & 4.8 & -- & -- & -- & -- & -- \\
\hline $11 / 08 / 00$ & 27 & $<1.0$ & $<.08$ & -- & -- & -- & 8.3 & -- & -- & -- & -- & -- \\
\hline $01 / 30 / 01$ & 37 & $<1.0$ & $<.08$ & -- & -- & -- & 24 & -- & -- & -- & -- & -- \\
\hline 03/12/01 & 40 & $<1.0$ & $<.08$ & -- & -- & -- & 17 & -- & -- & -- & -- & -- \\
\hline $04 / 10 / 01$ & 32 & $<1.0$ & $<.08$ & -- & -- & -- & 10 & -- & -- & -- & -- & -- \\
\hline $05 / 22 / 01$ & 24 & $<1.0$ & $<.08$ & -- & -- & -- & 5.0 & -- & -- & -- & -- & -- \\
\hline \multicolumn{13}{|c|}{ SITE 9 12354500-CLARK FORK AT ST. REGIS, MONT. } \\
\hline 03/02/99 & $\mathrm{e} 10$ & -- & -- & -- & -- & -- & 7.4 & -- & -- & -- & -- & -- \\
\hline 04/13/99 & e5.1 & $<1.0$ & $<1.0$ & $<12$ & -- & 18 & 4.6 & $<.10$ & 1.1 & $<1.0$ & $<1.0$ & $<1.0$ \\
\hline 05/10/99 & e7.1 & $<1.0$ & $<1.0$ & $<12$ & -- & 17 & 1.9 & $<.10$ & $<1.0$ & $<1.0$ & $<1.0$ & $<1.0$ \\
\hline $05 / 26 / 99$ & 19 & 6.7 & $<1.0$ & $<12$ & -- & 176 & 2.7 & $<.10$ & $<1.0$ & $<1.0$ & 2.1 & $<1.0$ \\
\hline $06 / 08 / 99$ & 17 & 1.9 & $<1.0$ & $<12$ & -- & 55 & 3.9 & $<.10$ & $<1.0$ & $<1.0$ & $<1.0$ & $<1.0$ \\
\hline $06 / 21 / 99^{\mathbf{F b}}$ & $<3$ & -- & $<.3$ & -- & -- & -- & $<.1$ & -- & -- & $<.2$ & -- & $<.5$ \\
\hline 06/21/99 & 17 & 1.6 & $<1.0$ & $<12$ & -- & 43 & 1.9 & $<.10$ & $<1.0$ & $<1.0$ & $<1.0$ & $<1.0$ \\
\hline 07/20/99 & e 8.5 & $<1.0$ & $<1.0$ & $<12$ & -- & 7.6 & 2.3 & $<.10$ & 1.1 & $<1.0$ & $<1.0$ & $<1.0$ \\
\hline 08/18/99 & e6.6 & $<1.0$ & $<1.0$ & $<12$ & -- & 17 & 1.8 & $<.10$ & $<1.0$ & $<1.0$ & 2.2 & $<1.0$ \\
\hline $10 / 18 / 99$ & e7.1 & $<1.0$ & $<1.0$ & e5.3 & -- & 7.0 & 2.0 & $<.30$ & $<1.0$ & 1.0 & $<1.8$ & $<1.0$ \\
\hline $11 / 30 / 99$ & e6.6 & -- & -- & -- & -- & -- & $\mathrm{e} 1.3$ & -- & -- & -- & -- & -- \\
\hline $01 / 24 / 00$ & $<10$ & $<1.0$ & $<1.0$ & e 4.4 & -- & 7.9 & 4.0 & $<.30$ & 1.8 & 1.1 & $<1.8$ & 1.1 \\
\hline 03/06/00 & 13 & $<1.0$ & $<1.0$ & $<7.0$ & -- & 28 & 4.7 & $<.30$ & $<1.0$ & $<1.0$ & $\mathrm{e} 1.2$ & $<1.0$ \\
\hline $04 / 10 / 00$ & 11 & e. 64 & $<1.0$ & $<7.0$ & -- & 28 & 3.3 & $<.30$ & $<1.0$ & $<1.0$ & $<1.8$ & $<1.0$ \\
\hline 05/09/00 & 11 & $<1.0$ & $<1.0$ & $<7.0$ & 1.8 & 16 & 2.5 & $<.30$ & $<1.0$ & $<1.0$ & $<1.8$ & $<1.0$ \\
\hline $05 / 24 / 00$ & e9.9 & e. 70 & $<1.0$ & $<7.0$ & 1.4 & 43 & 2.4 & $<.30$ & $<1.0$ & $<1.0$ & $<1.8$ & $<1.0$ \\
\hline $06 / 20 / 00$ & e8.0 & $<1.0$ & $<1.0$ & $<7.0$ & 1.8 & 10 & 2.1 & $<.30$ & 1.5 & $<1.0$ & $<1.8$ & $<1.0$ \\
\hline $08 / 01 / 00^{\mathbf{F b}}$ & $<3$ & -- & $<.3$ & -- & -- & -- & $<.1$ & -- & -- & $<.2$ & -- & $<.5$ \\
\hline 08/01/00 & $<10$ & e. 62 & $<1.0$ & $<7.0$ & 3.2 & 17 & 2.7 & $<.30$ & 1.4 & $<1.0$ & $<1.8$ & $<1.0$ \\
\hline 08/30/00 & e6.3 & $<1.0$ & $<1.0$ & $\mathrm{e} 4.3$ & 4.1 & 18 & 3.2 & $<.30$ & $<1.0$ & $<1.0$ & $<1.8$ & $<1.0$ \\
\hline $11 / 07 / 00$ & e6.0 & $<1.0$ & e. 04 & -- & -- & -- & e2.4 & -- & -- & -- & -- & -- \\
\hline $12 / 19 / 00$ & $<10$ & $<1.0$ & e. 04 & -- & -- & -- & e3.1 & -- & -- & -- & -- & -- \\
\hline $01 / 31 / 01$ & e8.4 & $<1.0$ & e. 05 & -- & -- & -- & 6.1 & -- & -- & -- & -- & -- \\
\hline 03/13/01 & 13 & $<1.0$ & e. 04 & -- & -- & -- & 6.2 & -- & -- & -- & -- & -- \\
\hline 04/11/01 & e9.2 & $<1.0$ & .10 & -- & -- & -- & 6.2 & -- & -- & -- & -- & -- \\
\hline $05 / 23 / 01$ & 13 & $<1.0$ & $<.08$ & -- & -- & -- & $\mathrm{e} 2.2$ & -- & -- & -- & -- & -- \\
\hline \multicolumn{13}{|c|}{ SITE 12 12388700-FLATHEAD RIVER AT PERMA, MONT. } \\
\hline 03/03/99 & $<10$ & -- & -- & -- & -- & -- & $\mathrm{e} 2.0$ & -- & -- & -- & -- & -- \\
\hline 04/14/99 & $<10$ & -- & -- & -- & -- & -- & $\mathrm{e} 2.0$ & -- & -- & -- & -- & -- \\
\hline $05 / 11 / 99^{\mathbf{F b}}$ & $<3$ & -- & $<.3$ & -- & -- & -- & $<.1$ & -- & -- & $<.2$ & -- & $<.5$ \\
\hline 05/11/99 & $<10$ & $<1.0$ & $<1.0$ & $<12$ & -- & 5.9 & 1.1 & $<.10$ & $<1.0$ & $<1.0$ & $<1.0$ & $<1.0$ \\
\hline 06/20/99 & $<10$ & $<1.0$ & $<1.0$ & $<12$ & -- & 10 & 1.1 & $<.10$ & $<1.0$ & $<1.0$ & $<1.0$ & $<1.0$ \\
\hline 07/19/99 & $<10$ & $<1.0$ & $<1.0$ & $<12$ & -- & 3.5 & 1.1 & $<.10$ & $<1.0$ & $<1.0$ & $<1.0$ & $<1.0$ \\
\hline
\end{tabular}


Table 8. Trace-element concentration data for surface-water samples collected at fixed water-quality sites, Northern Rockies Intermontane Basins study unit, 1998-2001 (Continued)

\begin{tabular}{|c|c|c|c|c|c|c|c|c|c|c|c|}
\hline Date & $\begin{array}{c}\text { Selenium, } \\
\text { total } \\
\text { recov- } \\
\text { erable } \\
(\mu \mathrm{g} / \mathrm{L})\end{array}$ & $\begin{array}{c}\text { Selenium, } \\
\text { dis- } \\
\text { solved } \\
(\mu \mathrm{g} / \mathrm{L})\end{array}$ & $\begin{array}{c}\text { Silver, } \\
\text { total } \\
\text { recov- } \\
\text { erable } \\
(\mu \mathrm{g} / \mathrm{L})\end{array}$ & $\begin{array}{c}\text { Silver, } \\
\text { dis- } \\
\text { solved } \\
(\mu \mathrm{g} / \mathrm{L})\end{array}$ & $\begin{array}{l}\text { Stron- } \\
\text { tium, } \\
\text { total } \\
\text { recov- } \\
\text { erable } \\
(\mu \mathrm{g} / \mathrm{L})\end{array}$ & $\begin{array}{c}\text { Stron- } \\
\text { tium, } \\
\text { dis- } \\
\text { solved } \\
(\mu \mathrm{g} / \mathrm{L})\end{array}$ & $\begin{array}{c}\text { Thal- } \\
\text { lium, } \\
\text { dis- } \\
\text { solved } \\
(\mu \mathrm{g} / \mathrm{L})\end{array}$ & $\begin{array}{c}\text { Uranium, } \\
\text { natural } \\
(\mu \mathrm{g} / \mathrm{L})\end{array}$ & $\begin{array}{c}\text { Vana- } \\
\text { dium, } \\
\text { dis- } \\
\text { solved } \\
(\mu \mathrm{g} / \mathrm{L})\end{array}$ & $\begin{array}{l}\text { Zinc, } \\
\text { total } \\
\text { recov- } \\
\text { erable } \\
(\mu \mathrm{g} / \mathrm{L})\end{array}$ & $\begin{array}{c}\text { Zinc, } \\
\text { dis- } \\
\text { solved } \\
(\mu \mathrm{g} / \mathrm{L})\end{array}$ \\
\hline \multicolumn{12}{|c|}{ 12352500--BITTERROOT RIVER NEAR MISSOULA, MONT. (Continued) } \\
\hline $04 / 12 / 00$ & $<2.6$ & $<2.4$ & $<1.0$ & $<1.0$ & 34 & -- & -- & $<1.0$ & -- & $<31$ & $<1.0$ \\
\hline $05 / 02 / 00$ & $<2.6$ & $<2.4$ & $<1.0$ & $<1.0$ & 34 & -- & -- & $<1.0$ & -- & $<31$ & $<1.0$ \\
\hline $05 / 23 / 00$ & $<2.6$ & $<.7$ & $<1.0$ & $<1.0$ & 32 & 28 & $<.90$ & $<1.0$ & $<1.0$ & $<31$ & $<2.5$ \\
\hline $06 / 20 / 00$ & $<2.6$ & $<.7$ & $<1.0$ & $<1.0$ & 35 & 38 & $<.90$ & $<1.0$ & $<1.0$ & $<31$ & $<1.0$ \\
\hline $07 / 31 / 00$ & -- & -- & -- & -- & -- & -- & -- & -- & -- & -- & -- \\
\hline $08 / 29 / 00$ & $<2.6$ & $<.7$ & $<1.0$ & $<1.0$ & 83 & 86 & $<.90$ & 1.7 & $<1.0$ & $<31$ & $<1.0$ \\
\hline 09/19/00 & -- & -- & -- & -- & -- & -- & -- & -- & -- & -- & -- \\
\hline $10 / 02 / 00$ & -- & -- & -- & -- & -- & -- & -- & -- & -- & -- & -- \\
\hline $10 / 16 / 00$ & -- & -- & -- & -- & -- & -- & -- & -- & -- & -- & -- \\
\hline $11 / 08 / 00$ & -- & -- & -- & -- & -- & -- & -- & -- & -- & $<1.0$ & $<1.0$ \\
\hline $01 / 30 / 01$ & -- & -- & -- & -- & -- & -- & -- & -- & -- & $<1.0$ & $<1.0$ \\
\hline $03 / 12 / 01$ & -- & -- & -- & -- & -- & -- & -- & -- & -- & 1.8 & $<1.0$ \\
\hline 04/10/01 & -- & -- & -- & -- & -- & -- & -- & -- & -- & 1.6 & 1.8 \\
\hline $05 / 22 / 01$ & -- & -- & -- & -- & -- & -- & -- & -- & -- & 1.6 & $<1.0$ \\
\hline \multicolumn{12}{|c|}{ SITE 9 12354500-CLARK FORK AT ST. REGIS, MONT. } \\
\hline 03/02/99 & -- & -- & -- & -- & -- & -- & -- & -- & -- & -- & -- \\
\hline 04/13/99 & $<1.0$ & $<1.0$ & $<1.0$ & $<1.0$ & 85 & -- & -- & 1.4 & -- & $<40$ & 1.1 \\
\hline $05 / 10 / 99$ & $<1.0$ & $<1.0$ & $<1.0$ & $<1.0$ & 66 & -- & -- & $<1.0$ & -- & $<40$ & $<1.0$ \\
\hline 05/26/99 & $<1.0$ & $<1.0$ & $<1.0$ & $<1.0$ & 40 & -- & -- & $<1.0$ & -- & e36 & 1.3 \\
\hline 06/08/99 & $<1.0$ & $<1.0$ & $<1.0$ & $<1.0$ & 45 & -- & -- & $<1.0$ & -- & $<40$ & 2.1 \\
\hline $06 / 21 / 99^{\mathbf{F b}}$ & -- & -- & -- & $<.2$ & -- & $<.1$ & $<.1$ & $<.2$ & -- & -- & .83 \\
\hline $06 / 21 / 99$ & $<1.0$ & $<1.0$ & $<1.0$ & $<1.0$ & 41 & -- & -- & $<1.0$ & -- & $<40$ & 1.8 \\
\hline 07/20/99 & $<1.0$ & $<1.0$ & $<1.0$ & $<1.0$ & 66 & -- & -- & $<1.0$ & -- & $<40$ & 1.3 \\
\hline 08/18/99 & $<1.0$ & $<1.0$ & $<1.0$ & $<1.0$ & 95 & -- & -- & 1.1 & -- & $<40$ & 1.4 \\
\hline $10 / 18 / 99$ & $<2.6$ & $<2.4$ & $<1.0$ & $<1.0$ & 121 & -- & -- & 1.7 & -- & $<31$ & $<1.0$ \\
\hline $11 / 30 / 99$ & -- & -- & -- & -- & -- & -- & -- & -- & -- & -- & -- \\
\hline $01 / 24 / 00$ & $<2.6$ & $<2.4$ & $<1.0$ & $<1.0$ & 121 & -- & -- & 2.5 & -- & $<31$ & 1.9 \\
\hline 03/06/00 & $<2.6$ & $<2.4$ & $<1.0$ & $<1.0$ & 111 & -- & -- & 1.9 & -- & $\mathrm{e} 18$ & 2.9 \\
\hline $04 / 10 / 00$ & $<2.6$ & $<2.4$ & $<1.0$ & $<1.0$ & 72 & -- & -- & 1.1 & -- & $\mathrm{e} 20$ & $<1.0$ \\
\hline 05/09/00 & $<2.6$ & $<.70$ & $<1.0$ & $<1.0$ & 47 & 50 & $<.90$ & $<1.0$ & $<1.0$ & $<31$ & $<3.9$ \\
\hline $05 / 24 / 00$ & $<2.6$ & $<.70$ & $<1.0$ & $<1.0$ & 37 & 37 & $<.90$ & $<1.0$ & $<1.0$ & $<31$ & $<2.6$ \\
\hline $06 / 20 / 00$ & $<2.6$ & $<.70$ & $<1.0$ & $<1.0$ & 52 & 57 & $<.90$ & $<1.0$ & $<1.0$ & $<31$ & $<1.0$ \\
\hline $08 / 01 / 00^{\mathbf{F b}}$ & -- & -- & -- & $<.2$ & -- & $<.1$ & $<.1$ & $<.2$ & -- & -- & $<.5$ \\
\hline 08/01/00 & $<2.6$ & $<.70$ & $<1.0$ & $<1.0$ & 83 & 90 & $<.90$ & $<1.0$ & $<1.0$ & $<31$ & $<1.0$ \\
\hline $08 / 30 / 00$ & $<2.6$ & $<.70$ & $<1.0$ & $<1.0$ & 94 & 98 & $<.90$ & 1.2 & $<1.0$ & $<31$ & 1.2 \\
\hline $11 / 07 / 00$ & -- & -- & -- & -- & -- & -- & -- & -- & -- & 2.4 & 1.2 \\
\hline $12 / 19 / 00$ & -- & -- & -- & -- & -- & -- & -- & -- & -- & 2.1 & 1.4 \\
\hline $01 / 31 / 01$ & -- & -- & -- & -- & -- & -- & -- & -- & -- & 1.5 & 1.5 \\
\hline 03/13/01 & -- & -- & -- & -- & -- & -- & -- & -- & -- & 5.4 & 1.3 \\
\hline 04/11/01 & -- & -- & -- & -- & -- & -- & -- & -- & -- & 4.6 & 1.6 \\
\hline $05 / 23 / 01$ & -- & -- & -- & -- & -- & -- & -- & -- & -- & 4.2 & 1.1 \\
\hline \multicolumn{12}{|c|}{ SITE 12 12388700--FLATHEAD RIVER AT PERMA, MONT. } \\
\hline 03/03/99 & -- & -- & -- & -- & -- & -- & -- & -- & -- & -- & -- \\
\hline $04 / 14 / 99$ & -- & -- & -- & -- & -- & -- & -- & -- & -- & -- & -- \\
\hline $05 / 11 / 99^{\mathbf{F b}}$ & -- & -- & -- & $<.2$ & -- & $<.1$ & $<.1$ & $<.2$ & -- & -- & $<.5$ \\
\hline 05/11/99 & $<1.0$ & $<1.0$ & $<1.0$ & $<1.0$ & 44 & -- & -- & $<1.0$ & -- & $<40$ & $<1.0$ \\
\hline $06 / 20 / 99$ & $<1.0$ & $<1.0$ & $<1.0$ & $<1.0$ & 42 & -- & -- & $<1.0$ & -- & $<40$ & 1.0 \\
\hline 07/19/99 & $<1.0$ & $<1.0$ & $<1.0$ & $<1.0$ & 42 & -- & -- & $<1.0$ & -- & $<40$ & $<1.0$ \\
\hline
\end{tabular}


Table 8. Trace-element concentration data for surface-water samples collected at fixed water-quality sites, Northern Rockies Intermontane Basins study unit, 1998-2001 (Continued)

\begin{tabular}{|c|c|c|c|c|c|c|c|c|c|c|}
\hline Date & Time & $\begin{array}{l}\text { Alumi- } \\
\text { num, } \\
\text { total } \\
\text { recov- } \\
\text { erable } \\
(\mu \mathrm{g} / \mathrm{L})\end{array}$ & $\begin{array}{c}\text { Alumi- } \\
\text { num, } \\
\text { dis- } \\
\text { solved } \\
(\mu \mathrm{g} / \mathrm{L})\end{array}$ & $\begin{array}{c}\text { Anti- } \\
\text { mony, } \\
\text { total } \\
\text { recov- } \\
\text { erable } \\
(\mu \mathrm{g} / \mathrm{L})\end{array}$ & $\begin{array}{c}\text { Anti- } \\
\text { mony, } \\
\text { dis- } \\
\text { solved } \\
(\mu \mathrm{g} / \mathrm{L})\end{array}$ & $\begin{array}{c}\text { Arsenic, } \\
\text { total } \\
\text { recov- } \\
\text { erable } \\
(\mu \mathrm{g} / \mathrm{L})\end{array}$ & $\begin{array}{c}\text { Arsenic, } \\
\text { dis- } \\
\text { solved } \\
(\mu \mathrm{g} / \mathrm{L})\end{array}$ & $\begin{array}{c}\text { Barium, } \\
\text { total } \\
\text { recov- } \\
\text { erable } \\
(\mu \mathrm{g} / \mathrm{L})\end{array}$ & $\begin{array}{c}\text { Barium, } \\
\text { dis- } \\
\text { solved } \\
(\mu \mathrm{g} / \mathrm{L})\end{array}$ & $\begin{array}{c}\text { Beryl- } \\
\text { lium, } \\
\text { total } \\
\text { recov- } \\
\text { erable } \\
(\mu \mathrm{g} / \mathrm{L})\end{array}$ \\
\hline \multicolumn{11}{|c|}{ SITE 12 12388700--FLATHEAD RIVER AT PERMA, MONT. (Continued) } \\
\hline 08/17/99 & 1330 & e19 & 2.4 & $<1.0$ & $<1.0$ & 1.1 & $<1.0$ & 82 & 85 & $<4.0$ \\
\hline $10 / 19 / 99$ & 1140 & $<28$ & 1.1 & $<1.0$ & $<1.0$ & $<2.6$ & $<2.0$ & 87 & 93 & $<5.0$ \\
\hline $12 / 01 / 99$ & 1100 & -- & -- & -- & -- & -- & -- & -- & -- & -- \\
\hline $01 / 25 / 00$ & 1030 & -- & -- & -- & -- & -- & -- & -- & -- & -- \\
\hline 03/07/00 & 1100 & -- & -- & -- & -- & -- & -- & -- & -- & -- \\
\hline $04 / 11 / 00$ & 1000 & -- & -- & -- & -- & -- & -- & -- & -- & -- \\
\hline 05/10/00 & 1015 & e23 & $<10$ & $<1.0$ & $<1.0$ & $<2.6$ & $<.90$ & 86 & 92 & $<5.0$ \\
\hline 06/19/00 & 1230 & 75 & 1.5 & $<1.0$ & $<1.0$ & $<2.6$ & $<.90$ & 83 & 86 & $<5.0$ \\
\hline 08/01/00 & 1000 & -- & -- & -- & -- & -- & -- & -- & -- & -- \\
\hline $08 / 30 / 00$ & 1030 & e18 & 1.5 & $<1.0$ & $<1.0$ & $<2.6$ & e.49 & 83 & 83 & $<5.0$ \\
\hline $11 / 06 / 00$ & 1400 & -- & -- & -- & -- & $<1.9$ & .39 & -- & -- & -- \\
\hline $12 / 19 / 00$ & 1245 & -- & -- & -- & -- & $<2.6$ & .37 & -- & -- & -- \\
\hline 02/01/01 & 1000 & -- & -- & -- & -- & $<1.9$ & .46 & -- & -- & -- \\
\hline 03/14/01 & 0930 & -- & -- & -- & -- & $<1.9$ & .56 & -- & -- & -- \\
\hline $04 / 12 / 01$ & 0930 & -- & -- & -- & -- & $<1.9$ & .49 & -- & -- & -- \\
\hline 05/24/01 & 0930 & -- & -- & -- & -- & $<2.0$ & .39 & -- & -- & -- \\
\hline \multicolumn{11}{|c|}{ SITE 14 12392155--LIGHTNING CREEK AT CLARK FORK, IDAHO } \\
\hline 07/29/99 & 1015 & -- & -- & -- & -- & -- & -- & -- & -- & -- \\
\hline 09/02/99 & 0945 & $<28$ & 4.2 & $<1.0$ & $<1.0$ & $<2.6$ & $<2.0$ & 3.8 & 3.8 & $<5.0$ \\
\hline $10 / 26 / 99$ & 1145 & $<28$ & 11 & $<1.0$ & $<1.0$ & $<2.6$ & $<2.0$ & 3.6 & 3.9 & $<5.0$ \\
\hline 03/02/00 & 0815 & $<28$ & 6.0 & $<1.0$ & $<1.0$ & $<2.6$ & $<2.0$ & 3.9 & 4.0 & $<5.0$ \\
\hline 03/30/00 & 0815 & $<28$ & 14 & $<1.0$ & $<1.0$ & $<2.6$ & $<2.0$ & 3.2 & 3.5 & $<5.0$ \\
\hline $04 / 12 / 00$ & 1330 & 63 & 15 & $<1.0$ & $<1.0$ & $<2.6$ & $<2.0$ & 2.6 & 3.0 & $<5.0$ \\
\hline 05/04/00 & 1000 & 87 & $<38$ & $<1.0$ & $<1.0$ & $<2.6$ & $<2.0$ & 2.9 & 2.8 & $<5.0$ \\
\hline $06 / 05 / 00$ & 0915 & 65 & $<28$ & $<1.0$ & $<1.0$ & $<2.6$ & $<.90$ & 2.7 & 2.6 & $<5.0$ \\
\hline $06 / 30 / 00$ & 0830 & 35 & 13 & $<1.0$ & $<1.0$ & $<2.6$ & $<.90$ & 2.7 & 2.9 & $<5.0$ \\
\hline $07 / 27 / 00$ & 1145 & $<28$ & 4.1 & $<1.0$ & $<1.0$ & $<2.6$ & $<.90$ & 3.0 & 3.8 & $<5.0$ \\
\hline 09/01/00 & 1200 & $<28$ & 3.7 & $<1.0$ & $<1.0$ & $<2.6$ & $<.90$ & 3.5 & 3.6 & $<5.0$ \\
\hline $11 / 09 / 00$ & 1015 & -- & -- & -- & -- & $<1.9$ & e. 13 & -- & -- & -- \\
\hline $12 / 14 / 00$ & 1145 & -- & -- & -- & -- & $<2.6$ & $<.18$ & -- & -- & -- \\
\hline $01 / 25 / 01$ & 1145 & -- & -- & -- & -- & $<1.9$ & e. 12 & -- & -- & -- \\
\hline 03/15/01 & 0915 & -- & -- & -- & -- & $<1.9$ & e. 18 & -- & -- & -- \\
\hline 04/11/01 & 1115 & -- & -- & -- & -- & $<1.9$ & e. 16 & -- & -- & -- \\
\hline 05/04/01 & 1315 & -- & -- & -- & -- & $<1.9$ & e. 16 & -- & -- & -- \\
\hline $06 / 14 / 01$ & 1300 & -- & -- & -- & -- & $<2.0$ & e.11 & -- & -- & -- \\
\hline \multicolumn{11}{|c|}{ SITE 23 12413000--NORTH FORK COEUR D'ALENE RIVER AT ENAVILLE, IDAHO } \\
\hline 03/02/99 & 1130 & -- & -- & -- & -- & -- & -- & -- & -- & -- \\
\hline 03/23/99 & 1000 & 232 & 4.6 & $<1.0$ & $<1.0$ & $<1.0$ & $<1.0$ & 15 & 13 & $<4.0$ \\
\hline 05/20/99 & 1000 & 75 & 3.4 & $<1.0$ & $<1.0$ & $<1.0$ & $<1.0$ & 13 & 12 & $<4.0$ \\
\hline 05/25/99 & 0930 & 811 & 9.1 & $<1.0$ & $<1.0$ & 1.7 & $<1.0$ & 23 & 11 & $<4.0$ \\
\hline 06/29/99 & 1000 & $<28$ & 2.9 & $<1.0$ & $<1.0$ & $<1.0$ & $<1.0$ & 14 & 15 & $<4.0$ \\
\hline 07/26/99 & 1000 & $\mathrm{e} 27$ & 2.6 & $<1.0$ & $<1.0$ & $<1.0$ & $<1.0$ & 17 & 17 & $<4.0$ \\
\hline 09/08/99 & 0930 & $<28$ & 1.9 & $<1.0$ & $<1.0$ & $<1.0$ & $<1.0$ & 19 & 20 & $<5.0$ \\
\hline $10 / 19 / 99$ & 1000 & $<28$ & 6.1 & $<1.0$ & $<1.0$ & $<2.6$ & $<2.0$ & 18 & 19 & $<5.0$ \\
\hline $12 / 01 / 99$ & 1145 & -- & -- & -- & -- & -- & -- & -- & -- & -- \\
\hline 01/11/00 & 1100 & -- & -- & -- & -- & -- & -- & -- & -- & -- \\
\hline $02 / 29 / 00$ & 0800 & -- & -- & -- & -- & -- & -- & -- & -- & -- \\
\hline $03 / 27 / 00$ & 0945 & 30 & 3.0 & $<1.0$ & $<1.0$ & $<2.6$ & $<2.0$ & 13 & 13 & $<5.0$ \\
\hline
\end{tabular}


Table 8. Trace-element concentration data for surface-water samples collected at fixed water-quality sites, Northern Rockies Intermontane Basins study unit, 1998-2001 (Continued)

\begin{tabular}{|c|c|c|c|c|c|c|c|c|c|c|c|}
\hline Date & $\begin{array}{c}\text { Beryl- } \\
\text { lium, } \\
\text { dis- } \\
\text { solved } \\
(\mu \mathrm{g} / \mathrm{L})\end{array}$ & $\begin{array}{c}\text { Boron, } \\
\text { dis- } \\
\text { solved } \\
(\mu \mathrm{g} / \mathrm{L})\end{array}$ & $\begin{array}{c}\text { Cad- } \\
\text { mium, } \\
\text { total } \\
\text { recov- } \\
\text { erable } \\
(\mu \mathrm{g} / \mathrm{L})\end{array}$ & $\begin{array}{c}\text { Cad- } \\
\text { mium, } \\
\text { dis- } \\
\text { solved } \\
(\mu \mathrm{g} / \mathrm{L})\end{array}$ & $\begin{array}{c}\text { Chro- } \\
\text { mium, } \\
\text { total } \\
\text { recov- } \\
\text { erable } \\
(\mu \mathrm{g} / \mathrm{L})\end{array}$ & $\begin{array}{c}\text { Chro- } \\
\text { mium, } \\
\text { dis- } \\
\text { solved } \\
(\mu \mathrm{g} / \mathrm{L})\end{array}$ & $\begin{array}{c}\text { Cobalt, } \\
\text { total } \\
\text { recov- } \\
\text { erable } \\
(\mu \mathrm{g} / \mathrm{L})\end{array}$ & $\begin{array}{c}\text { Cobalt, } \\
\text { dis- } \\
\text { solved } \\
(\mu \mathrm{g} / \mathrm{L})\end{array}$ & $\begin{array}{c}\text { Copper, } \\
\text { total } \\
\text { recov- } \\
\text { erable } \\
(\mu \mathrm{g} / \mathrm{L})\end{array}$ & $\begin{array}{c}\text { Copper, } \\
\text { dis- } \\
\text { solved } \\
(\mu \mathrm{g} / \mathrm{L})\end{array}$ & $\begin{array}{c}\text { Iron, } \\
\text { total } \\
\text { recov- } \\
\text { erable } \\
(\mu \mathrm{g} / \mathrm{L})\end{array}$ \\
\hline \multicolumn{12}{|c|}{ SITE 12 12388700--FLATHEAD RIVER AT PERMA, MONT. (Continued) } \\
\hline $08 / 17 / 99$ & $<1.0$ & -- & $<1.0$ & $<1.0$ & $<1.0$ & $<1.0$ & 1.0 & $<1.0$ & $<12$ & $<1.0$ & 25 \\
\hline $01 / 25 / 00$ & -- & -- & -- & -- & -- & -- & -- & -- & -- & -- & -- \\
\hline 03/07/00 & -- & -- & -- & -- & -- & -- & -- & -- & -- & -- & -- \\
\hline $04 / 11 / 00$ & -- & -- & -- & -- & -- & -- & -- & -- & -- & -- & -- \\
\hline $05 / 10 / 00$ & $<1.0$ & $<12$ & $<.11$ & $<1.0$ & $<1.0$ & $<.8$ & $<1.8$ & $<1.0$ & $<20$ & $<1.0$ & 43 \\
\hline $06 / 19 / 00$ & $<1.0$ & $<12$ & $<.11$ & $<1.0$ & $<1.0$ & e.6 & $<1.8$ & $<1.0$ & $<20$ & $<1.0$ & 80 \\
\hline $02 / 01 / 01$ & -- & -- & $<.04$ & e. 02 & -- & -- & -- & -- & e. 57 & .5 & -- \\
\hline 03/14/01 & -- & -- & $<.04$ & $<.04$ & -- & -- & -- & -- & 1.1 & .8 & -- \\
\hline $04 / 12 / 01$ & -- & -- & $<.04$ & $<.04$ & -- & -- & -- & -- & .77 & .6 & -- \\
\hline $05 / 24 / 01$ & -- & -- & .11 & $<.04$ & -- & -- & -- & -- & e. 47 & .5 & -- \\
\hline \multicolumn{12}{|c|}{ SITE 14 12392155--LIGHTNING CREEK AT CLARK FORK, IDAHO } \\
\hline 07/29/99 & -- & -- & -- & -- & -- & -- & -- & -- & -- & -- & -- \\
\hline $09 / 02 / 99$ & $<1.0$ & -- & $<.11$ & $<1.0$ & $<1.0$ & $<.80$ & $<1.8$ & $<1.0$ & $<20$ & $<1.0$ & 22 \\
\hline $10 / 26 / 99$ & $<1.0$ & -- & $<.11$ & $<1.0$ & $<1.0$ & $<.80$ & $<1.8$ & $<1.0$ & $<20$ & $<1.0$ & 26 \\
\hline $03 / 02 / 00$ & $<1.0$ & -- & $<.11$ & $<1.0$ & $<1.0$ & $<1.0$ & $<1.8$ & $<1.0$ & $<20$ & $<1.0$ & $<21$ \\
\hline 03/30/00 & $<1.0$ & -- & $<.11$ & $<1.0$ & $<1.0$ & $<1.0$ & $<1.8$ & $<1.0$ & $<20$ & $<1.0$ & $<21$ \\
\hline $04 / 12 / 00$ & $<1.0$ & -- & $<.11$ & $<1.0$ & $<1.0$ & $<.80$ & $<1.8$ & $<1.0$ & $<20$ & $<1.0$ & 73 \\
\hline 03/15/01 & -- & -- & $<.04$ & $<.04$ & -- & -- & -- & -- & $<.60$ & e. 22 & -- \\
\hline 04/11/01 & -- & -- & $<.04$ & $<.04$ & -- & -- & -- & -- & e. 42 & .60 & -- \\
\hline 05/04/01 & -- & -- & $<.04$ & $<.04$ & -- & -- & -- & -- & $<.60$ & .29 & -- \\
\hline $06 / 14 / 01$ & -- & -- & $<.04$ & $<.04$ & -- & -- & -- & -- & e. 41 & .43 & -- \\
\hline \multicolumn{12}{|c|}{ SITE 23 12413000--NORTH FORK COEUR D'ALENE RIVER AT ENAVILLE, IDAHO } \\
\hline 03/02/99 & -- & -- & -- & -- & -- & -- & -- & -- & -- & -- & -- \\
\hline 03/23/99 & $<1.0$ & -- & $<1.0$ & $<1.0$ & $<1.0$ & -- & -- & $<1.0$ & $<10$ & $<1.0$ & 292 \\
\hline 05/20/99 & $<1.0$ & -- & $<1.0$ & $<1.0$ & $<1.0$ & $<1.0$ & $<1.0$ & $<1.0$ & e6.6 & $<1.0$ & 109 \\
\hline 05/25/99 & $<1.0$ & -- & $<1.0$ & $<1.0$ & $<1.0$ & $<1.0$ & 1.1 & $<1.0$ & e8.5 & $<1.0$ & 1,130 \\
\hline 06/29/99 & $<1.0$ & -- & $<1.0$ & $<1.0$ & $<1.0$ & $<1.0$ & $<1.0$ & $<1.0$ & $<12$ & $<1.0$ & 28 \\
\hline $07 / 26 / 99$ & $<1.0$ & -- & $<1.0$ & $<1.0$ & $<1.0$ & $<1.0$ & $<1.0$ & $<1.0$ & $<12$ & $<1.0$ & 26 \\
\hline 09/08/99 & $<1.0$ & -- & $<1.0$ & $<1.0$ & $<1.0$ & $<1.0$ & $<1.0$ & $<1.0$ & $<20$ & $<1.0$ & e19 \\
\hline $10 / 19 / 99$ & $<1.0$ & -- & $<.11$ & $<1.0$ & $<1.0$ & $<.80$ & $<1.8$ & $<1.0$ & $<20$ & $<1.0$ & $<21$ \\
\hline $12 / 01 / 99$ & -- & -- & -- & -- & -- & -- & -- & -- & -- & -- & -- \\
\hline 01/11/00 & -- & -- & -- & -- & -- & -- & -- & -- & -- & -- & -- \\
\hline $02 / 29 / 00$ & -- & -- & -- & -- & -- & -- & -- & -- & -- & -- & -- \\
\hline $03 / 27 / 00$ & $<1.0$ & -- & $<.11$ & $<1.0$ & $<1.0$ & $<1.0$ & $<1.8$ & $<1.0$ & $\mathrm{e} 11$ & $<1.0$ & 35 \\
\hline
\end{tabular}


Table 8. Trace-element concentration data for surface-water samples collected at fixed water-quality sites, Northern Rockies Intermontane Basins study unit, 1998-2001 (Continued)

\begin{tabular}{|c|c|c|c|c|c|c|c|c|c|c|c|c|}
\hline Date & $\begin{array}{c}\text { Iron, } \\
\text { dis- } \\
\text { solved } \\
(\mu \mathrm{g} / \mathrm{L})\end{array}$ & $\begin{array}{l}\text { Lead, } \\
\text { total } \\
\text { recov- } \\
\text { erable } \\
(\mu \mathrm{g} / \mathrm{L})\end{array}$ & $\begin{array}{c}\text { Lead, } \\
\text { dis- } \\
\text { solved } \\
(\mu \mathrm{g} / \mathrm{L})\end{array}$ & $\begin{array}{l}\text { Lith- } \\
\text { ium, } \\
\text { total } \\
\text { recov- } \\
\text { erable } \\
(\mu \mathrm{g} / \mathrm{L})\end{array}$ & $\begin{array}{l}\text { Lith- } \\
\text { ium, } \\
\text { dis- } \\
\text { solved } \\
(\mu \mathrm{g} / \mathrm{L})\end{array}$ & $\begin{array}{c}\text { Manga- } \\
\text { nese, } \\
\text { total } \\
\text { recov- } \\
\text { erable } \\
(\mu \mathrm{g} / \mathrm{L})\end{array}$ & $\begin{array}{c}\text { Manga- } \\
\text { nese, } \\
\text { dis- } \\
\text { solved } \\
(\mu \mathrm{g} / \mathrm{L})\end{array}$ & $\begin{array}{c}\text { Mercury, } \\
\text { total } \\
\text { recov- } \\
\text { erable } \\
(\mu \mathrm{g} / \mathrm{L})\end{array}$ & $\begin{array}{c}\text { Molyb- } \\
\text { denum, } \\
\text { total } \\
\text { recov- } \\
\text { erable } \\
(\mu \mathrm{g} / \mathrm{L})\end{array}$ & $\begin{array}{c}\text { Molyb- } \\
\text { denum, } \\
\text { dis- } \\
\text { solved } \\
(\mu \mathrm{g} / \mathrm{L})\end{array}$ & $\begin{array}{c}\text { Nickel, } \\
\text { tota1 } \\
\text { recov- } \\
\text { erable } \\
(\mu \mathrm{g} / \mathrm{L})\end{array}$ & $\begin{array}{c}\text { Nickel, } \\
\text { dis- } \\
\text { solved } \\
(\mu \mathrm{g} / \mathrm{L})\end{array}$ \\
\hline \multicolumn{13}{|c|}{ SITE 12 12388700--FLATHEAD RIVER AT PERMA, MONT. (Continued) } \\
\hline $08 / 17 / 99$ & $<10$ & $<1.0$ & $<1.0$ & $<12$ & -- & 4.1 & $<1.0$ & $<.10$ & $<1.0$ & $<1.0$ & $<1.0$ & $<1.0$ \\
\hline $10 / 19 / 99$ & $<10$ & $<1.0$ & $<1.0$ & $<7.0$ & -- & 3.3 & $<1.0$ & $<.30$ & $<1.0$ & $<1.0$ & $<1.8$ & $<1.0$ \\
\hline $12 / 01 / 99$ & $<10$ & -- & -- & -- & -- & -- & $<2.2$ & -- & -- & -- & -- & -- \\
\hline $01 / 25 / 00$ & $<10$ & -- & -- & -- & -- & -- & e 1.8 & -- & -- & -- & -- & -- \\
\hline $03 / 07 / 00$ & $<10$ & -- & -- & -- & -- & -- & $\mathrm{e} 1.5$ & -- & -- & -- & -- & -- \\
\hline $04 / 11 / 00$ & e5.6 & -- & -- & -- & -- & -- & $\mathrm{e} 1.4$ & -- & -- & -- & -- & -- \\
\hline 05/10/00 & $<10$ & $<1.0$ & $<1.0$ & $<7.0$ & 1.0 & 4.0 & $<1.0$ & $<.30$ & $<1.0$ & $<1.0$ & $<1.8$ & $<1.0$ \\
\hline $06 / 19 / 00$ & $<10$ & $<1.0$ & $<1.0$ & $<7.0$ & .81 & 5.3 & $<1.0$ & $<.30$ & $<1.0$ & $<1.0$ & $<1.8$ & $<1.0$ \\
\hline 08/01/00 & e5.7 & -- & -- & -- & -- & -- & $<2.2$ & -- & -- & -- & -- & -- \\
\hline $08 / 30 / 00$ & $<10$ & $<1.0$ & $<1.0$ & $<7.0$ & 1.4 & 4.7 & $<1.0$ & $<.30$ & 1.0 & $<1.0$ & $<1.8$ & $<1.0$ \\
\hline $11 / 06 / 00$ & $<10$ & $<1.0$ & $<.08$ & -- & -- & -- & $<3.2$ & -- & -- & -- & -- & -- \\
\hline $12 / 19 / 00$ & $<10$ & $<1.0$ & $<.08$ & -- & -- & -- & $<3.2$ & -- & -- & -- & -- & -- \\
\hline $02 / 01 / 01$ & $<10$ & $<1.0$ & $<.08$ & -- & -- & -- & $<3.2$ & -- & -- & -- & -- & -- \\
\hline 03/14/01 & 14 & $<1.0$ & e. 04 & -- & -- & -- & 7.8 & -- & -- & -- & -- & -- \\
\hline $04 / 12 / 01$ & $<10$ & $<1.0$ & $<.08$ & -- & -- & -- & e2.7 & -- & -- & -- & -- & -- \\
\hline $05 / 24 / 01$ & $<10$ & $<1.0$ & $<.08$ & -- & -- & -- & e1.6 & -- & -- & -- & -- & -- \\
\hline \multicolumn{13}{|c|}{ SITE 14 12392155--LIGHTNING CREEK AT CLARK FORK, IDAHO } \\
\hline 07/29/99 & $\mathrm{e} 8.3$ & -- & -- & -- & -- & -- & $<3.0$ & -- & -- & -- & -- & -- \\
\hline 09/02/99 & e8.1 & $<1.0$ & $<1.0$ & $<7.0$ & -- & $<2.8$ & $<1.0$ & $<.30$ & $<1.0$ & $<1.0$ & $<1.8$ & $<1.0$ \\
\hline $10 / 26 / 99$ & e8.4 & e. .58 & $<1.0$ & $<7.0$ & -- & $\mathrm{e} 2.1$ & $<1.0$ & $<.30$ & $<1.0$ & $<1.0$ & $<1.8$ & $<1.0$ \\
\hline $03 / 02 / 00$ & e6.6 & $<1.0$ & $<1.0$ & e 3.1 & -- & $<2.8$ & $<1.0$ & $<.30$ & $<1.0$ & $<1.0$ & $<1.8$ & $<1.0$ \\
\hline 03/30/00 & $<10$ & $<1.0$ & $<1.0$ & $<7.0$ & -- & $<2.8$ & $<1.0$ & $<.30$ & $<1.0$ & $<1.0$ & $<1.8$ & $<1.0$ \\
\hline $04 / 12 / 00$ & $<10$ & e. 66 & $<1.0$ & $<7.0$ & -- & $\mathrm{e} 1.8$ & $<1.0$ & $<.30$ & $<1.0$ & $<1.0$ & $<1.8$ & $<1.0$ \\
\hline 05/04/00 & $<10$ & $<1.0$ & $<1.0$ & $<7.0$ & -- & $\mathrm{e} 2.5$ & $<1.0$ & $<.30$ & $<1.0$ & $<1.0$ & $<1.8$ & $<1.0$ \\
\hline $06 / 05 / 00$ & e5.2 & $<1.0$ & $<1.0$ & $<7.0$ & $<.3$ & $\mathrm{e} 1.8$ & $<1.0$ & $<.30$ & $<1.0$ & $<1.0$ & $<1.8$ & $<1.0$ \\
\hline $06 / 30 / 00$ & e5.1 & $<1.0$ & $<1.0$ & $<7.0$ & $<.3$ & $<2.8$ & $<1.0$ & $<.30$ & $<1.0$ & $<1.0$ & $<1.8$ & $<1.0$ \\
\hline $07 / 27 / 00$ & e5.1 & e. 51 & $<1.0$ & $<7.0$ & e. 16 & $<2.8$ & $<1.0$ & $<.30$ & 1.2 & $<1.0$ & $<1.8$ & $<1.0$ \\
\hline 09/01/00 & e 8.0 & $<1.0$ & $<1.0$ & $<7.0$ & $<.3$ & $<2.8$ & $<1.0$ & $<.30$ & $<1.0$ & $<1.0$ & $<1.8$ & $<1.0$ \\
\hline $11 / 09 / 00$ & e6.1 & $<1.0$ & .18 & -- & -- & -- & $<3.2$ & -- & -- & -- & -- & -- \\
\hline $12 / 14 / 00$ & $<10$ & $<1.0$ & e. 05 & -- & -- & -- & $<3.2$ & -- & -- & -- & -- & -- \\
\hline $01 / 25 / 01$ & $<10$ & $<1.0$ & .12 & -- & -- & -- & $<3.2$ & -- & -- & -- & -- & -- \\
\hline 03/15/01 & $<10$ & $<1.0$ & .15 & -- & -- & -- & $<3.2$ & -- & -- & -- & -- & -- \\
\hline $04 / 11 / 01$ & $<10$ & $<1.0$ & .25 & -- & -- & -- & $\mathrm{e} 2.5$ & -- & -- & -- & -- & -- \\
\hline 05/04/01 & e6.1 & $<1.0$ & .20 & -- & -- & -- & $<3.2$ & -- & -- & -- & -- & -- \\
\hline $06 / 14 / 01$ & e7.1 & $<1.0$ & .26 & -- & -- & -- & $<3.0$ & -- & -- & -- & -- & -- \\
\hline \multicolumn{13}{|c|}{ SITE 23 12413000--NORTH FORK COEUR D'ALENE RIVER AT ENAVILLE, IDAHO } \\
\hline 03/02/99 & $\mathrm{e} 6.3$ & -- & -- & -- & -- & -- & e1.6 & -- & -- & -- & -- & -- \\
\hline $03 / 23 / 99$ & e6.5 & 2.0 & $<1.0$ & $<16$ & -- & 11 & 1.4 & $<.10$ & $<1.0$ & $<1.0$ & $<1.0$ & $<1.0$ \\
\hline $05 / 20 / 99$ & 17 & 4.0 & $<1.0$ & $<12$ & -- & 7.5 & 1.1 & $<.10$ & $<1.0$ & $<1.0$ & $<1.0$ & $<1.0$ \\
\hline $05 / 25 / 99$ & 15 & 11 & $<1.0$ & $<12$ & -- & 49 & 4.8 & $<.10$ & $<1.0$ & $<1.0$ & 1.5 & $<1.0$ \\
\hline 06/29/99 & e7.9 & $<1.0$ & $<1.0$ & $<12$ & -- & $\mathrm{e} 2.3$ & 1.6 & $<.10$ & $<1.0$ & $<1.0$ & $<1.0$ & $<1.0$ \\
\hline 07/26/99 & e5.5 & $<1.0$ & $<1.0$ & $<12$ & -- & e1.9 & 1.4 & $<.10$ & $<1.0$ & $<1.0$ & $<1.0$ & $<1.0$ \\
\hline 09/08/99 & e6.0 & $<1.0$ & $<1.0$ & $<7.0$ & -- & e1.8 & 1.4 & $<.10$ & $<1.0$ & $<1.0$ & $<1.0$ & $<1.0$ \\
\hline $10 / 19 / 99$ & $<10$ & $<1.0$ & $<1.0$ & $<7.0$ & -- & $\mathrm{e} 1.8$ & 1.2 & $<.30$ & $<1.0$ & $<1.0$ & $<1.8$ & $<1.0$ \\
\hline $12 / 01 / 99$ & e8.1 & -- & -- & -- & -- & -- & $<2.2$ & -- & -- & -- & -- & -- \\
\hline $01 / 11 / 00$ & $<10$ & -- & -- & -- & -- & -- & $<2.2$ & -- & -- & -- & -- & -- \\
\hline $02 / 29 / 00$ & e6.0 & -- & -- & -- & -- & -- & $<2.2$ & -- & -- & -- & -- & -- \\
\hline $03 / 27 / 00$ & e7.9 & $<1.0$ & $<1.0$ & $<7.0$ & -- & $\mathrm{e} 2.0$ & $<1.0$ & $<.30$ & $<1.0$ & $<1.0$ & $<1.8$ & $<1.0$ \\
\hline
\end{tabular}


Table 8. Trace-element concentration data for surface-water samples collected at fixed water-quality sites, Northern Rockies Intermontane Basins study unit, 1998-2001 (Continued)

\begin{tabular}{|c|c|c|c|c|c|c|c|c|c|c|c|}
\hline Date & $\begin{array}{c}\text { Selenium, } \\
\text { total } \\
\text { recov- } \\
\text { erable } \\
(\mu \mathrm{g} / \mathrm{L})\end{array}$ & $\begin{array}{c}\text { Selenium, } \\
\text { dis- } \\
\text { solved } \\
(\mu \mathrm{g} / \mathrm{L})\end{array}$ & $\begin{array}{c}\text { Silver, } \\
\text { total } \\
\text { recov- } \\
\text { erable } \\
(\mu \mathrm{g} / \mathrm{L})\end{array}$ & $\begin{array}{c}\text { Silver, } \\
\text { dis- } \\
\text { solved } \\
(\mu \mathrm{g} / \mathrm{L})\end{array}$ & $\begin{array}{c}\text { Stron- } \\
\text { tium, } \\
\text { total } \\
\text { recov- } \\
\text { erable } \\
(\mu \mathrm{g} / \mathrm{L})\end{array}$ & $\begin{array}{c}\text { Stron- } \\
\text { tium, } \\
\text { dis- } \\
\text { solved } \\
(\mu \mathrm{g} / \mathrm{L})\end{array}$ & $\begin{array}{l}\text { Thal- } \\
\text { lium, } \\
\text { dis- } \\
\text { solved } \\
(\mu \mathrm{g} / \mathrm{L})\end{array}$ & $\begin{array}{c}\text { Uranium, } \\
\text { natural } \\
(\mu \mathrm{g} / \mathrm{L})\end{array}$ & $\begin{array}{c}\text { Vana- } \\
\text { dium, } \\
\text { dis- } \\
\text { solved } \\
(\mu \mathrm{g} / L)\end{array}$ & $\begin{array}{l}\text { Zinc, } \\
\text { total } \\
\text { recov- } \\
\text { erable } \\
(\mu \mathrm{g} / \mathrm{L})\end{array}$ & $\begin{array}{c}\text { Zinc, } \\
\text { dis- } \\
\text { solved } \\
(\mu \mathrm{g} / \mathrm{L})\end{array}$ \\
\hline \multicolumn{12}{|c|}{ SITE 12 12388700--FLATHEAD RIVER AT PERMA, MONT. (Continued) } \\
\hline $08 / 17 / 99$ & $<1.0$ & $<1.0$ & $<1.0$ & $<1.0$ & 43 & -- & -- & $<1.0$ & -- & $<40$ & $<1.0$ \\
\hline $10 / 19 / 99$ & $<2.6$ & $<2.4$ & $<1.0$ & $<1.0$ & 44 & -- & -- & $<1.0$ & -- & $<31$ & $<1.0$ \\
\hline $12 / 01 / 99$ & -- & -- & -- & -- & -- & -- & -- & -- & -- & -- & -- \\
\hline $01 / 25 / 00$ & -- & -- & -- & -- & -- & -- & -- & -- & -- & -- & -- \\
\hline 03/07/00 & -- & -- & -- & -- & -- & -- & -- & -- & -- & -- & -- \\
\hline 04/11/00 & -- & -- & -- & -- & -- & -- & -- & -- & -- & -- & -- \\
\hline $05 / 10 / 00$ & $<2.6$ & $<.70$ & $<1.0$ & $<1.0$ & 42 & 44 & $<.90$ & $<1.0$ & $<1.0$ & $<31$ & $<3.0$ \\
\hline 06/19/00 & $<2.6$ & $<.70$ & $<1.0$ & $<1.0$ & 40 & 44 & $<.90$ & $<1.0$ & $<1.0$ & $\mathrm{e} 17$ & $<1.0$ \\
\hline $08 / 01 / 00$ & -- & -- & -- & -- & -- & -- & -- & -- & -- & -- & -- \\
\hline 08/30/00 & $<2.6$ & $<.70$ & $<1.0$ & $<1.0$ & 41 & 40 & $<.90$ & $<1.0$ & $<1.0$ & $<31$ & $<1.0$ \\
\hline $11 / 06 / 00$ & -- & -- & -- & -- & -- & -- & -- & -- & -- & $<1.0$ & $<1.0$ \\
\hline $12 / 19 / 00$ & -- & -- & -- & -- & -- & -- & -- & -- & -- & $<1.0$ & $<1.0$ \\
\hline $02 / 01 / 01$ & -- & -- & -- & -- & -- & -- & -- & -- & -- & $<1.0$ & $<1.0$ \\
\hline 03/14/01 & -- & -- & -- & -- & -- & -- & -- & -- & -- & 2.7 & $<1.0$ \\
\hline $04 / 12 / 01$ & -- & -- & -- & -- & -- & -- & -- & -- & -- & 1.0 & $<1.0$ \\
\hline $05 / 24 / 01$ & -- & -- & -- & -- & -- & -- & -- & -- & -- & 1.2 & $<1.0$ \\
\hline \multicolumn{12}{|c|}{ SITE 14 12392155--LIGHTNING CREEK AT CLARK FORK, IDAHO } \\
\hline $07 / 29 / 99$ & -- & -- & -- & -- & -- & -- & -- & -- & -- & -- & -- \\
\hline $09 / 02 / 99$ & $<2.6$ & $<2.4$ & $<1.0$ & $<1.0$ & 18 & -- & -- & $<1.0$ & -- & $<31$ & 1.1 \\
\hline $10 / 26 / 99$ & $<2.6$ & $<2.4$ & $<1.0$ & $<1.0$ & 16 & -- & -- & $<1.0$ & -- & $<31$ & $<1.0$ \\
\hline $03 / 02 / 00$ & $<2.6$ & $<2.4$ & $<1.0$ & $<1.0$ & 17 & -- & -- & $<1.0$ & -- & $<31$ & $<1.0$ \\
\hline 03/30/00 & $<2.6$ & $<2.4$ & $<1.0$ & $<1.0$ & 17 & -- & -- & $<1.0$ & -- & $<31$ & 3.0 \\
\hline $04 / 12 / 00$ & $<2.6$ & $<2.4$ & $<1.0$ & $<1.0$ & 12 & -- & -- & $<1.0$ & -- & $<31$ & $<1.0$ \\
\hline $05 / 04 / 00$ & $<2.6$ & $<2.4$ & $<1.0$ & $<1.0$ & 10 & -- & -- & $<1.0$ & -- & $<31$ & $<3.6$ \\
\hline $06 / 05 / 00$ & $<2.6$ & $<.70$ & $<1.0$ & $<1.0$ & 9.2 & 9.2 & $<.90$ & $<1.0$ & $<1.0$ & $<31$ & $<3.4$ \\
\hline $06 / 30 / 00$ & $<2.6$ & $<.70$ & $<1.0$ & $<1.0$ & 10 & 10 & $<.90$ & $<1.0$ & $<1.0$ & $<31$ & $<1.0$ \\
\hline $07 / 27 / 00$ & $<2.6$ & $<.70$ & $<1.0$ & $<1.0$ & 16 & 17 & $<.90$ & $<1.0$ & $<1.0$ & $<31$ & $<1.0$ \\
\hline 09/01/00 & $<2.6$ & $<.70$ & $<1.0$ & $<1.0$ & 18 & 18 & $<.90$ & $<1.0$ & $<1.0$ & $<31$ & $<1.0$ \\
\hline $11 / 09 / 00$ & -- & -- & -- & -- & -- & -- & -- & -- & -- & $<1$ & 1.0 \\
\hline $12 / 14 / 00$ & -- & -- & -- & -- & -- & -- & -- & -- & -- & $<1$ & $<1.0$ \\
\hline $01 / 25 / 01$ & -- & -- & -- & -- & -- & -- & -- & -- & -- & $<1$ & $<1.0$ \\
\hline 03/15/01 & -- & -- & -- & -- & -- & -- & -- & -- & -- & $<1$ & $<1.0$ \\
\hline $04 / 11 / 01$ & -- & -- & -- & -- & -- & -- & -- & -- & -- & 2.0 & 1.2 \\
\hline $05 / 04 / 01$ & -- & -- & -- & -- & -- & -- & -- & -- & -- & $<1$ & $<1.0$ \\
\hline $06 / 14 / 01$ & -- & -- & -- & -- & -- & -- & -- & -- & -- & $<1$ & $<1.0$ \\
\hline \multicolumn{12}{|c|}{ SITE 23 12413000--NORTH FORK COEUR D'ALENE RIVER AT ENAVILLE, IDAHO } \\
\hline 03/02/99 & -- & -- & -- & -- & -- & -- & -- & -- & -- & -- & -- \\
\hline $03 / 23 / 99$ & $<1.0$ & $<1.0$ & $<1.0$ & $<1.0$ & 12 & -- & -- & $<1.0$ & -- & e29 & 6.4 \\
\hline 05/20/99 & $<1.0$ & $<1.0$ & $<1.0$ & $<1.0$ & 17 & -- & -- & $<1.0$ & -- & 84 & 66 \\
\hline 05/25/99 & $<1.0$ & $<1.0$ & $<1.0$ & $<1.0$ & 9.2 & -- & -- & $<1.0$ & -- & $<40$ & 4.9 \\
\hline 06/29/99 & $<1.0$ & $<1.0$ & $<1.0$ & $<1.0$ & 13 & -- & -- & $<1.0$ & -- & $<40$ & 5.5 \\
\hline $07 / 26 / 99$ & $<1.0$ & $<1.0$ & $<1.0$ & $<1.0$ & 15 & -- & -- & $<1.0$ & -- & $<40$ & 3.6 \\
\hline 09/08/99 & $<1.0$ & $<1.0$ & $<1.0$ & $<1.0$ & 16 & -- & -- & $<1.0$ & -- & $<31$ & 2.7 \\
\hline 10/19/99 & $<2.6$ & $\mathrm{e} 1.2$ & $<1.0$ & $<1.0$ & 16 & -- & -- & $<1.0$ & -- & $<31$ & 2.8 \\
\hline $12 / 01 / 99$ & -- & -- & -- & -- & -- & -- & -- & -- & -- & -- & -- \\
\hline $01 / 11 / 00$ & -- & -- & -- & -- & -- & -- & -- & -- & -- & -- & -- \\
\hline $02 / 29 / 00$ & -- & -- & -- & -- & -- & -- & -- & -- & -- & -- & -- \\
\hline $03 / 27 / 00$ & $<2.6$ & $<2.4$ & $<1.0$ & $<1.0$ & 13 & -- & -- & $<1.0$ & -- & $<31$ & 5.0 \\
\hline
\end{tabular}


Table 8. Trace-element concentration data for surface-water samples collected at fixed water-quality sites, Northern Rockies Intermontane Basins study unit, 1998-2001 (Continued)

\begin{tabular}{|c|c|c|c|c|c|c|c|c|c|c|}
\hline Date & Time & $\begin{array}{l}\text { Alumi- } \\
\text { num, } \\
\text { total } \\
\text { recov- } \\
\text { erable } \\
(\mu \mathrm{g} / \mathrm{L})\end{array}$ & $\begin{array}{l}\text { Alumi- } \\
\text { num, } \\
\text { dis- } \\
\text { solved } \\
(\mu \mathrm{g} / \mathrm{L})\end{array}$ & $\begin{array}{c}\text { Anti- } \\
\text { mony, } \\
\text { total } \\
\text { recov- } \\
\text { erable } \\
(\mu \mathrm{g} / \mathrm{L})\end{array}$ & $\begin{array}{c}\text { Anti- } \\
\text { mony, } \\
\text { dis- } \\
\text { solved } \\
(\mu \mathrm{g} / \mathrm{L})\end{array}$ & $\begin{array}{c}\text { Arsenic, } \\
\text { total } \\
\text { recov- } \\
\text { erable } \\
(\mu \mathrm{g} / \mathrm{L})\end{array}$ & $\begin{array}{c}\text { Arsenic, } \\
\text { dis- } \\
\text { solved } \\
(\mu \mathrm{g} / \mathrm{L})\end{array}$ & $\begin{array}{c}\text { Barium, } \\
\text { total } \\
\text { recov- } \\
\text { erable } \\
(\mu \mathrm{g} / \mathrm{L})\end{array}$ & $\begin{array}{c}\text { Barium, } \\
\text { dis- } \\
\text { solved } \\
(\mu \mathrm{g} / \mathrm{L})\end{array}$ & $\begin{array}{l}\text { Beryl- } \\
\text { lium, } \\
\text { total } \\
\text { recov- } \\
\text { erable } \\
(\mu \mathrm{g} / \mathrm{L})\end{array}$ \\
\hline \multicolumn{11}{|c|}{ SITE 23 12413000--NORTH FORK COEUR D'ALENE RIVER AT ENAVILLE, IDAHO (Continued) } \\
\hline $04 / 10 / 00$ & 0945 & 56 & -- & $<1.0$ & -- & $<2.6$ & -- & 13 & -- & $<5.0$ \\
\hline $04 / 14 / 00$ & 0945 & 2,160 & 22 & $<1.0$ & $<1.0$ & 3.0 & $<2.0$ & 46 & 10 & $<5.0$ \\
\hline $04 / 17 / 00$ & 0945 & 309 & $<10$ & $<1.0$ & $<1.0$ & $<2.6$ & $<2.0$ & 18 & 10 & $<5.0$ \\
\hline $05 / 02 / 00$ & 0900 & 81 & $<15$ & $<1.0$ & $<1.0$ & $<2.6$ & $<2.0$ & 13 & 12 & $<5.0$ \\
\hline $05 / 18 / 00$ & 0730 & -- & -- & -- & -- & -- & -- & -- & -- & -- \\
\hline $06 / 02 / 00$ & 0715 & 34 & $<9.7$ & $<1.0$ & $<1.0$ & $<2.6$ & $<.90$ & 14 & 14 & $<5.0$ \\
\hline $06 / 29 / 00$ & 0800 & e22 & 1.9 & $<1.0$ & $<1.0$ & $<2.6$ & $<.90$ & 16 & 16 & $<5.0$ \\
\hline $07 / 24 / 00$ & 1030 & -- & -- & -- & -- & -- & -- & -- & -- & -- \\
\hline $08 / 31 / 00$ & 0915 & e16 & 2.0 & $<1.0$ & $<1.0$ & $<2.6$ & $<.90$ & 19 & 19 & $<5.0$ \\
\hline $11 / 06 / 00$ & 1030 & -- & -- & -- & -- & $<1.9$ & e. 13 & -- & -- & -- \\
\hline $12 / 13 / 00$ & 1100 & -- & -- & -- & -- & $<2.6$ & e.09 & -- & -- & -- \\
\hline $01 / 23 / 01$ & 1000 & -- & -- & -- & -- & $<1.9$ & e. 13 & -- & -- & -- \\
\hline 03/14/01 & 0845 & -- & -- & -- & -- & $<1.9$ & e. 16 & -- & -- & -- \\
\hline $04 / 10 / 01$ & 0815 & -- & -- & -- & -- & $<1.9$ & e. 16 & -- & -- & -- \\
\hline $05 / 03 / 01$ & 1015 & -- & -- & -- & -- & $<1.9$ & e. 16 & -- & -- & -- \\
\hline $06 / 12 / 01$ & 0930 & -- & -- & -- & -- & $<1.9$ & .22 & -- & -- & -- \\
\hline \multicolumn{11}{|c|}{ SITE 31 12413470--SOUTH FORK COEUR D'ALENE RIVER NEAR PINEHURST, IDAHO } \\
\hline $11 / 04 / 98$ & 1200 & 455 & 20 & 11 & 7.7 & 5.9 & 1.0 & $<100$ & 47 & $<10$ \\
\hline $11 / 04 / 98^{\mathbf{F b}}$ & 1208 & -- & $<.3$ & -- & $<.2$ & -- & -- & -- & $<.2$ & -- \\
\hline $12 / 09 / 98$ & 1210 & 44 & 8.2 & 8.3 & 8.0 & 1.2 & $<1.0$ & $<100$ & 41 & $<10$ \\
\hline 02/03/99 & 1100 & -- & -- & -- & -- & -- & -- & -- & -- & -- \\
\hline 03/17/99 & 1000 & 43 & 6.9 & 3.1 & 3.2 & $<1.0$ & $<1.0$ & 25 & 27 & $<4.0$ \\
\hline 03/23/99 & 1330 & 172 & 6.9 & 1.9 & 1.7 & 2.5 & $<1.0$ & 25 & 23 & $<4.0$ \\
\hline $04 / 20 / 99$ & 1200 & 192 & 6.6 & 3.3 & 1.6 & 4.3 & $<1.0$ & 23 & 18 & 6.9 \\
\hline $05 / 25 / 99$ & 1245 & 1,390 & 6.6 & 8.2 & 1.1 & 13 & $<1.0$ & 50 & 14 & $<4.0$ \\
\hline 05/27/99 & 1245 & 622 & 7.2 & 5.1 & 2.6 & 4.7 & $<1.0$ & 29 & 15 & $<4.0$ \\
\hline $06 / 29 / 99$ & 1300 & -- & 11 & -- & 2.4 & -- & $<1.0$ & -- & 25 & -- \\
\hline 07/26/99 & 1315 & 52 & 19 & 5.5 & 5.2 & 1.6 & 1.0 & 35 & 36 & $<4.0$ \\
\hline 09/08/99 & 1300 & 63 & 19 & 6.8 & 6.3 & 1.2 & $<1.0$ & 45 & 44 & $<5.0$ \\
\hline $10 / 19 / 99$ & 1400 & 52 & 21 & 6.4 & 6.1 & $<2.6$ & $<2.0$ & 45 & 47 & $<5.0$ \\
\hline $12 / 02 / 99$ & 0850 & 38 & 9.5 & 6.8 & 6.0 & $<2.6$ & $<2.0$ & 32 & 34 & $<5.0$ \\
\hline $01 / 11 / 00$ & 1430 & 54 & 21 & 5.0 & 5.4 & $<2.6$ & $<2.0$ & 36 & 35 & $<5.0$ \\
\hline $02 / 28 / 00^{\mathbf{F b}}$ & 1020 & -- & 7.0 & -- & $<.2$ & -- & -- & -- & $<.2$ & -- \\
\hline $02 / 28 / 00$ & 1100 & 65 & 13 & 3.1 & 2.5 & $<2.6$ & $<2.0$ & 25 & 27 & $<5.0$ \\
\hline $03 / 27 / 00$ & 1320 & 92 & 11 & 2.7 & 2.2 & $<2.6$ & $<2.0$ & 25 & 25 & $<5.0$ \\
\hline $04 / 10 / 00$ & 1315 & 58 & 8.1 & 2.4 & 2.2 & $<2.6$ & $<2.0$ & 21 & 22 & $<5.0$ \\
\hline $04 / 14 / 00$ & 1315 & 2,030 & 12 & 7.7 & 1.2 & 12 & $<2.0$ & 55 & 13 & $<5.0$ \\
\hline $04 / 17 / 00$ & 1315 & 126 & 6.1 & 1.9 & 1.4 & $\mathrm{e} 1.5$ & $<2.0$ & 22 & 19 & $<5.0$ \\
\hline $05 / 02 / 00$ & 1145 & 98 & 20 & 2.2 & 1.4 & $<2.6$ & $<2.0$ & 19 & 19 & $<5.0$ \\
\hline $05 / 18 / 00$ & 1000 & 74 & 22 & 2.2 & 1.4 & $<2.6$ & $<.90$ & 22 & 18 & $<5.0$ \\
\hline $06 / 02 / 00$ & 0930 & 48 & 9.8 & 1.3 & 1.9 & $<2.6$ & $<.90$ & 24 & 23 & $<5.0$ \\
\hline $06 / 29 / 00^{\mathbf{F b}}$ & 1045 & -- & $<.3$ & -- & $<.2$ & -- & -- & -- & $<.2$ & -- \\
\hline $06 / 29 / 00$ & 1130 & 39 & 13 & 4.4 & 3.5 & $<2.6$ & e.55 & 32 & 32 & $<5.0$ \\
\hline $07 / 24 / 00$ & 1300 & 31 & 19 & 5.7 & 5.5 & $<2.6$ & e. 58 & 40 & 44 & $<5.0$ \\
\hline $08 / 31 / 00$ & 1215 & 50 & 19 & 7.0 & 6.6 & $<2.6$ & e. 67 & 47 & 48 & $<5.0$ \\
\hline $11 / 06 / 00$ & 1330 & -- & -- & -- & -- & $<1.9$ & .59 & -- & -- & -- \\
\hline $12 / 18 / 00$ & 1200 & -- & -- & -- & -- & $<2.6$ & .40 & -- & -- & -- \\
\hline $01 / 23 / 01$ & 1430 & -- & -- & -- & -- & $<1.9$ & .44 & -- & -- & -- \\
\hline
\end{tabular}


Table 8. Trace-element concentration data for surface-water samples collected at fixed water-quality sites, Northern Rockies Intermontane Basins study unit, 1998-2001 (Continued)

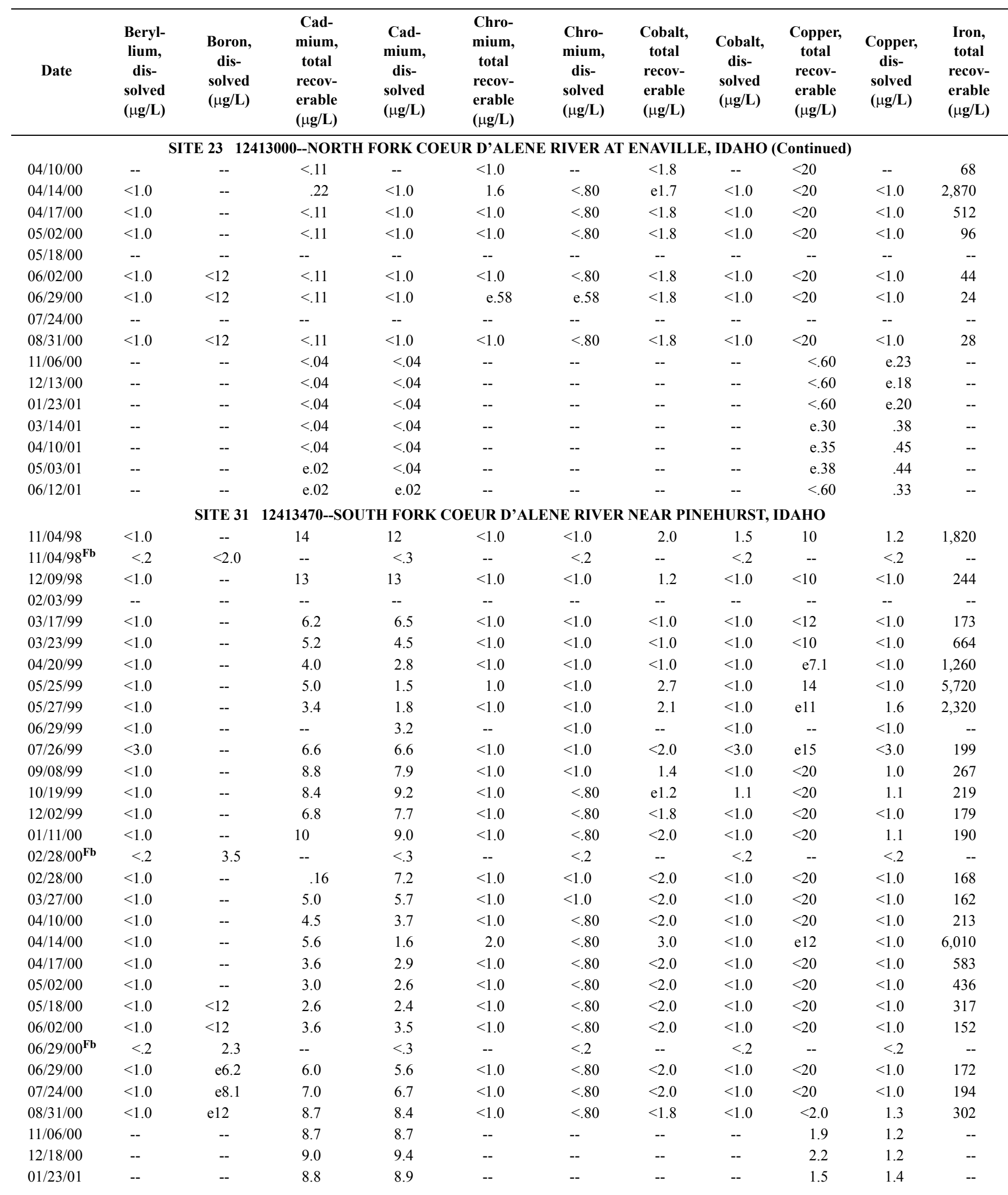


Table 8. Trace-element concentration data for surface-water samples collected at fixed water-quality sites, Northern Rockies Intermontane Basins study unit, 1998-2001 (Continued)

\begin{tabular}{|c|c|c|c|c|c|c|c|c|c|c|c|c|}
\hline Date & $\begin{array}{c}\text { Iron, } \\
\text { dis- } \\
\text { solved } \\
(\mu \mathrm{g} / \mathrm{L})\end{array}$ & $\begin{array}{c}\text { Lead, } \\
\text { total } \\
\text { recov- } \\
\text { erable } \\
(\mu \mathrm{g} / \mathrm{L})\end{array}$ & $\begin{array}{c}\text { Lead, } \\
\text { dis- } \\
\text { solved } \\
(\mu \mathrm{g} / \mathrm{L})\end{array}$ & $\begin{array}{l}\text { Lith- } \\
\text { ium, } \\
\text { total } \\
\text { recov- } \\
\text { erable } \\
(\mu \mathrm{g} / \mathrm{L})\end{array}$ & $\begin{array}{l}\text { Lith- } \\
\text { ium, } \\
\text { dis- } \\
\text { solved } \\
(\mu \mathrm{g} / \mathrm{L})\end{array}$ & $\begin{array}{l}\text { Manga- } \\
\text { nese, } \\
\text { total } \\
\text { recov- } \\
\text { erable } \\
(\mu \mathrm{g} / \mathrm{L})\end{array}$ & $\begin{array}{c}\text { Manga- } \\
\text { nese, } \\
\text { dis- } \\
\text { solved } \\
(\mu \mathrm{g} / \mathrm{L})\end{array}$ & $\begin{array}{c}\text { Mercury, } \\
\text { total } \\
\text { recov- } \\
\text { erable } \\
(\mu \mathrm{g} / \mathrm{L})\end{array}$ & $\begin{array}{c}\text { Molyb- } \\
\text { denum, } \\
\text { total } \\
\text { recov- } \\
\text { erable } \\
(\mu \mathrm{g} / \mathrm{L})\end{array}$ & $\begin{array}{c}\text { Molyb- } \\
\text { denum, } \\
\text { dis- } \\
\text { solved } \\
(\mu \mathrm{g} / \mathrm{L})\end{array}$ & $\begin{array}{c}\text { Nickel, } \\
\text { tota1 } \\
\text { recov- } \\
\text { erable } \\
(\mu \mathrm{g} / \mathrm{L})\end{array}$ & $\begin{array}{c}\text { Nickel, } \\
\text { dis- } \\
\text { solved } \\
(\mu \mathrm{g} / \mathrm{L})\end{array}$ \\
\hline \multicolumn{13}{|c|}{ SITE 23 12413000--NORTH FORK COEUR D'ALENE RIVER AT ENAVILLE, IDAHO (Continued) } \\
\hline $04 / 10 / 00$ & $<10$ & $<1.0$ & -- & $<7.0$ & -- & $\mathrm{e} 2.0$ & $\mathrm{e} 1.4$ & $<.30$ & $<1.0$ & - & $<1.8$ & -- \\
\hline $04 / 14 / 00$ & 32 & 24 & $<1.0$ & $<7.0$ & -- & 140 & 5.2 & $<.30$ & $<1.0$ & $<1.0$ & 2.1 & $<1.0$ \\
\hline $04 / 17 / 00$ & 16 & 2.5 & $<1.0$ & $<7.0$ & -- & 15 & 2.5 & $<.30$ & $<1.0$ & $<1.0$ & $<1.8$ & $<1.0$ \\
\hline 05/02/00 & $<10$ & $<1.0$ & $<1.0$ & $<7.0$ & -- & 3.0 & 1.4 & $<.30$ & $<1.0$ & $<1.0$ & $<1.8$ & $<1.0$ \\
\hline $05 / 18 / 00$ & 30 & -- & -- & -- & -- & -- & $<2.2$ & -- & -- & -- & -- & -- \\
\hline 06/02/00 & 11 & $<1.0$ & $<1.0$ & $<7.0$ & $<.3$ & e2.0 & 1.4 & $<.30$ & $<1.0$ & $<1.0$ & $<1.8$ & $<1.0$ \\
\hline 06/29/00 & e8.3 & $<1.0$ & $<1.0$ & $<7.0$ & .70 & $\mathrm{e} 2.0$ & 1.1 & $<.30$ & $<1.0$ & $<1.0$ & $<1.8$ & $<1.0$ \\
\hline $07 / 24 / 00$ & $<10$ & -- & -- & -- & -- & -- & e1.4 & -- & -- & -- & -- & -- \\
\hline $08 / 31 / 00$ & $<10$ & $<1.0$ & $<1.0$ & $<7.0$ & .38 & $\mathrm{e} 1.8$ & 1.4 & $<.30$ & $<1.0$ & $<1.0$ & $<1.8$ & $<1.0$ \\
\hline $11 / 06 / 00$ & $<10$ & $<1.0$ & $<.08$ & -- & -- & -- & $<3.2$ & -- & -- & -- & -- & -- \\
\hline $12 / 13 / 00$ & $<10$ & $<1.0$ & $<.08$ & -- & -- & -- & $<3.2$ & -- & -- & -- & -- & -- \\
\hline $01 / 23 / 01$ & $<10$ & $<1.0$ & e. 05 & -- & -- & -- & $<3.2$ & -- & -- & -- & -- & -- \\
\hline 03/14/01 & $<10$ & $<1.0$ & $<.08$ & -- & -- & -- & $<3.2$ & -- & -- & -- & -- & -- \\
\hline 04/10/01 & $<10$ & $<1.0$ & $<.08$ & -- & -- & -- & e1.9 & -- & -- & -- & -- & -- \\
\hline 05/03/01 & e8.1 & $<1.0$ & .09 & -- & -- & -- & $<3.2$ & -- & -- & -- & -- & -- \\
\hline 06/12/01 & e9.2 & $<1.0$ & e. 04 & -- & -- & -- & $<3.0$ & -- & -- & -- & -- & -- \\
\hline \multicolumn{13}{|c|}{ SITE 31 12413470--SOUTH FORK COEUR D'ALENE RIVER NEAR PINEHURST, IDAHO } \\
\hline $11 / 04 / 98$ & 56 & 267 & 12 & $<10$ & -- & 727 & 666 & .15 & $<1.0$ & $<1.0$ & 4.7 & 3.6 \\
\hline $11 / 04 / 98^{\mathbf{F b}}$ & $<3$ & -- & $<.3$ & -- & -- & -- & $<.1$ & -- & -- & $<.2$ & -- & $<.5$ \\
\hline $12 / 09 / 98$ & 79 & 32 & 3.1 & $<10$ & -- & 358 & 392 & $<.10$ & $<1.0$ & $<1.0$ & 2.5 & 2.6 \\
\hline 02/03/99 & 85 & -- & -- & -- & -- & -- & 310 & -- & -- & -- & -- & -- \\
\hline 03/17/99 & 23 & 20 & 2.5 & $<12$ & -- & 139 & 138 & $<.10$ & $<1.0$ & $<1.0$ & 1.1 & 1.4 \\
\hline 03/23/99 & 12 & 85 & 3.4 & $<16$ & -- & 154 & 80 & $<.10$ & $<1.0$ & $<1.0$ & 1.2 & 1.0 \\
\hline 04/20/99 & 13 & 164 & 5.3 & $<16$ & -- & -- & 50 & $<.10$ & $<1.0$ & $<1.0$ & $<1.0$ & $<1.0$ \\
\hline 05/25/99 & 13 & 790 & 4.6 & $<12$ & -- & 654 & 42 & .66 & $<1.0$ & $<1.0$ & 2.6 & $<1.0$ \\
\hline 05/27/99 & e8.0 & 350 & 2.8 & $<12$ & -- & 294 & 49 & .30 & $<1.0$ & $<1.0$ & 2.0 & $<1.0$ \\
\hline 06/29/99 & 24 & -- & 3.6 & -- & -- & -- & 102 & -- & -- & $<1.0$ & -- & 1.2 \\
\hline 07/26/99 & 103 & 22 & 8.7 & $<12$ & -- & 224 & 218 & $<.10$ & $<1.0$ & $<3.0$ & $<2.0$ & $<3.0$ \\
\hline 09/08/99 & 72 & 22 & 4.8 & $<7.0$ & -- & 413 & 388 & $<.10$ & $<1.0$ & $<1.0$ & 2.5 & 3.0 \\
\hline $10 / 19 / 99$ & 74 & 19 & 6.3 & $\mathrm{e} 4.3$ & -- & 481 & 473 & $<.30$ & $<1.0$ & $<1.0$ & 2.4 & 3.1 \\
\hline $12 / 02 / 99$ & 75 & 16 & 2.1 & $<7.0$ & -- & 211 & 204 & $<.30$ & $<1.0$ & $<1.0$ & $\mathrm{e} 1.2$ & 1.6 \\
\hline $01 / 11 / 00$ & 94 & 13 & 3.8 & $<7.0$ & -- & 300 & 295 & $<.30$ & $<1.0$ & $<1.0$ & $\mathrm{e} 1.8$ & 3.2 \\
\hline $02 / 28 / 00^{\mathbf{F b}}$ & $<3$ & -- & $<.3$ & -- & -- & -- & $<.1$ & -- & -- & $<.2$ & -- & $<.5$ \\
\hline $02 / 28 / 00$ & 48 & 13 & 2.7 & e3.1 & -- & 227 & 233 & $<.30$ & $<1.0$ & $<1.0$ & 2.8 & 1.2 \\
\hline 03/27/00 & 42 & 19 & 3.7 & $<7.0$ & -- & 319 & 320 & $<.30$ & $<1.0$ & $<1.0$ & e. 97 & 1.1 \\
\hline $04 / 10 / 00$ & 19 & 26 & 2.6 & $<7.0$ & -- & 431 & 424 & $<.30$ & $<1.0$ & $<1.0$ & $<1.8$ & $<1.0$ \\
\hline $04 / 14 / 00$ & 19 & 630 & 3.1 & $<7.0$ & -- & 705 & 99 & .40 & $<1.0$ & $<1.0$ & 2.4 & $<1.0$ \\
\hline $04 / 17 / 00$ & 17 & 73 & 3.2 & $<7.0$ & -- & 124 & 85 & $<.30$ & $<1.0$ & $<1.0$ & $<1.8$ & $<1.0$ \\
\hline $05 / 02 / 00$ & 18 & 54 & 3.8 & $<7.0$ & -- & 126 & 106 & $<.30$ & $<1.0$ & $<1.0$ & $<1.8$ & $<1.0$ \\
\hline 05/18/00 & 23 & 40 & 3.8 & $<7.0$ & -- & 138 & 117 & $<.30$ & $<1.0$ & $<1.0$ & $<1.8$ & $<1.0$ \\
\hline $06 / 02 / 00$ & 34 & 18 & 4.4 & $<7.0$ & -- & 260 & 263 & $<.30$ & $<1.0$ & $<1.0$ & $<1.8$ & $<1.0$ \\
\hline $06 / 29 / 00^{\mathbf{F b}}$ & $<3$ & -- & $<.3$ & -- & -- & -- & $<.1$ & -- & -- & $<.2$ & -- & $<.5$ \\
\hline $06 / 29 / 00$ & 65 & 17 & 5.6 & e4.1 & -- & 175 & 174 & $<.30$ & $<1.0$ & $<1.0$ & $<1.8$ & 1.5 \\
\hline $07 / 24 / 00$ & 83 & 15 & 6.8 & e3.2 & -- & 270 & 288 & $<.30$ & 1.6 & $<1.0$ & e1.6 & 2.0 \\
\hline 08/31/00 & 92 & 17 & 6.2 & e 5.3 & 4.6 & 940 & 878 & $<.30$ & $<1.0$ & $<1.0$ & 2.7 & 1.9 \\
\hline $11 / 06 / 00$ & 83 & 16 & 5.6 & -- & -- & -- & 1,400 & -- & -- & -- & -- & -- \\
\hline $12 / 18 / 00$ & 86 & 17 & 4.5 & -- & -- & -- & 2,550 & -- & -- & -- & -- & -- \\
\hline $01 / 23 / 01$ & 69 & 13 & 5.2 & -- & -- & -- & 1,870 & -- & -- & -- & -- & -- \\
\hline
\end{tabular}


Table 8. Trace-element concentration data for surface-water samples collected at fixed water-quality sites, Northern Rockies Intermontane Basins study unit, 1998-2001 (Continued)

\begin{tabular}{|c|c|c|c|c|c|c|c|c|c|c|c|}
\hline Date & $\begin{array}{c}\text { Selenium, } \\
\text { total } \\
\text { recov- } \\
\text { erable } \\
(\mu \mathrm{g} / \mathrm{L})\end{array}$ & $\begin{array}{c}\text { Selenium, } \\
\text { dis- } \\
\text { solved } \\
(\mu \mathrm{g} / \mathrm{L})\end{array}$ & $\begin{array}{c}\text { Silver, } \\
\text { total } \\
\text { recov- } \\
\text { erable } \\
(\mu \mathrm{g} / \mathrm{L})\end{array}$ & $\begin{array}{l}\text { Silver, } \\
\text { dis- } \\
\text { solved } \\
(\mu \mathrm{g} / \mathrm{L})\end{array}$ & $\begin{array}{l}\text { Stron- } \\
\text { tium, } \\
\text { total } \\
\text { recov- } \\
\text { erable } \\
(\mu \mathrm{g} / \mathrm{L})\end{array}$ & $\begin{array}{c}\text { Stron- } \\
\text { tium, } \\
\text { dis- } \\
\text { solved } \\
(\mu \mathrm{g} / \mathrm{L})\end{array}$ & $\begin{array}{c}\text { Thal- } \\
\text { lium, } \\
\text { dis- } \\
\text { solved } \\
(\mu \mathrm{g} / \mathrm{L})\end{array}$ & $\begin{array}{c}\text { Uranium, } \\
\text { natural } \\
(\mu \mathrm{g} / \mathrm{L})\end{array}$ & $\begin{array}{c}\text { Vana- } \\
\text { dium, } \\
\text { dis- } \\
\text { solved } \\
(\mu \mathrm{g} / \mathrm{L})\end{array}$ & $\begin{array}{l}\text { Zinc, } \\
\text { total } \\
\text { recov- } \\
\text { erable } \\
(\mu \mathrm{g} / \mathrm{L})\end{array}$ & $\begin{array}{c}\text { Zinc, } \\
\text { dis- } \\
\text { solved } \\
(\mu \mathrm{g} / \mathrm{L})\end{array}$ \\
\hline \multicolumn{12}{|c|}{ SITE 23 12413000--NORTH FORK COEUR D'ALENE RIVER AT ENAVILLE, IDAHO (Continued) } \\
\hline $04 / 10 / 00$ & $<2.6$ & -- & $<1.0$ & -- & 12 & -- & -- & -- & -- & e16 & -- \\
\hline $04 / 14 / 00$ & $<2.6$ & $<2.4$ & $<1.0$ & $<1.0$ & 11 & -- & -- & $<1.0$ & -- & 44 & 5.4 \\
\hline $04 / 17 / 00$ & $<2.6$ & $<2.4$ & $<1.0$ & $<1.0$ & 9.4 & -- & -- & $<1.0$ & -- & $<31$ & $<5.5$ \\
\hline $05 / 02 / 00$ & $<2.6$ & $<2.4$ & $<1.0$ & $<1.0$ & 10 & -- & -- & $<1.0$ & -- & $<31$ & $<6.5$ \\
\hline $05 / 18 / 00$ & -- & -- & -- & -- & -- & -- & -- & -- & -- & -- & -- \\
\hline $06 / 02 / 00$ & $<2.6$ & $<.70$ & $<1.0$ & $<1.0$ & 13 & 13 & $<.90$ & $<1.0$ & $<1.0$ & $<31$ & $<8.1$ \\
\hline $06 / 29 / 00$ & $<2.6$ & $<.70$ & $<1.0$ & $<1.0$ & 14 & 14 & $<.90$ & $<1.0$ & $<1.0$ & $<31$ & 4.3 \\
\hline $07 / 24 / 00$ & -- & -- & -- & -- & -- & -- & -- & -- & -- & -- & -- \\
\hline $08 / 31 / 00$ & $<2.6$ & $<.70$ & $<1.0$ & $<1.0$ & 17 & 17 & $<.90$ & $<1.0$ & $<1.0$ & $<31$ & 2.7 \\
\hline $11 / 06 / 00$ & -- & -- & -- & -- & -- & -- & -- & -- & -- & 2.6 & 2.5 \\
\hline $12 / 13 / 00$ & -- & -- & -- & -- & -- & -- & -- & -- & -- & 2.1 & 2.3 \\
\hline $01 / 23 / 01$ & -- & -- & -- & -- & -- & -- & -- & -- & -- & 2.7 & 1.8 \\
\hline 03/14/01 & -- & -- & -- & -- & -- & -- & -- & -- & -- & 2.8 & 3.1 \\
\hline 04/10/01 & -- & -- & -- & -- & -- & -- & -- & -- & -- & 4.4 & 4.3 \\
\hline 05/03/01 & -- & -- & -- & -- & -- & -- & -- & -- & -- & 5.5 & 4.5 \\
\hline 06/12/01 & -- & -- & -- & -- & -- & -- & -- & -- & -- & 4.3 & 5.8 \\
\hline \multicolumn{12}{|c|}{ SITE 31 12413470--SOUTH FORK COEUR D'ALENE RIVER NEAR PINEHURST, IDAHO } \\
\hline $11 / 04 / 98$ & $<1.0$ & $<1.0$ & $<1.0$ & $<1.0$ & 106 & -- & -- & $<1.0$ & -- & 2,190 & 2,120 \\
\hline $11 / 04 / 98^{\mathbf{F b}}$ & -- & -- & -- & $<.2$ & -- & $<.1$ & $<.1$ & $<.2$ & -- & -- & .96 \\
\hline $12 / 09 / 98$ & $<1.0$ & $<1.0$ & $<1.0$ & $<1.0$ & 88 & -- & -- & $<1.0$ & -- & 1,760 & 1,900 \\
\hline 02/03/99 & -- & -- & -- & -- & -- & -- & -- & -- & -- & -- & -- \\
\hline 03/17/99 & $<1.0$ & $<1.0$ & $<1.0$ & $<1.0$ & 44 & -- & -- & $<1.0$ & -- & 906 & 936 \\
\hline 03/23/99 & $<1.0$ & $<1.0$ & $<1.0$ & $<1.0$ & 35 & -- & -- & $<1.0$ & -- & 738 & 331 \\
\hline 04/20/99 & $<1.0$ & $<1.0$ & $<1.0$ & $<1.0$ & 26 & -- & -- & $<1.0$ & -- & 573 & 432 \\
\hline 05/25/99 & $<1.0$ & $<1.0$ & 2.5 & $<1.0$ & 18 & -- & -- & $<1.0$ & -- & 667 & 227 \\
\hline 05/27/99 & $<1.0$ & $<1.0$ & $<1.0$ & $<1.0$ & 19 & -- & -- & $<1.0$ & -- & 446 & 274 \\
\hline 06/29/99 & -- & $<1.0$ & -- & $<1.0$ & -- & -- & -- & $<1.0$ & -- & -- & 482 \\
\hline 07/26/99 & $<1.0$ & $<1.0$ & $<2.0$ & $<3.0$ & 48 & -- & -- & $<3.0$ & -- & 927 & 911 \\
\hline 09/08/99 & $<1.0$ & $<1.0$ & $<1.0$ & $<1.0$ & 72 & -- & -- & $<1.0$ & -- & 1,710 & 1,440 \\
\hline $10 / 19 / 99$ & $<2.6$ & $<2.4$ & $<1.0$ & $<1.0$ & 84 & -- & -- & $<1.0$ & -- & 1,640 & 1,690 \\
\hline $12 / 02 / 99$ & $<2.6$ & $<2.4$ & $<1.0$ & $<1.0$ & 54 & -- & -- & $<1.0$ & -- & 1,140 & 1,110 \\
\hline $01 / 11 / 00$ & $<2.6$ & $<2.4$ & $<1.0$ & $<1.0$ & 66 & -- & -- & $<1.0$ & -- & 1,310 & 1,400 \\
\hline $02 / 28 / 00^{\mathbf{F b}}$ & -- & -- & -- & $<.2$ & -- & $<.1$ & $<.1$ & $<.2$ & -- & -- & $<.5$ \\
\hline $02 / 28 / 00$ & $<2.6$ & $<2.4$ & $<1.0$ & $<1.0$ & 44 & -- & -- & $<1.0$ & -- & 973 & 915 \\
\hline $03 / 27 / 00$ & $<2.6$ & $<2.4$ & $<1.0$ & $<1.0$ & 45 & -- & -- & $<1.0$ & -- & 750 & 740 \\
\hline 04/10/00 & $<2.6$ & $<2.4$ & $<1.0$ & $<1.0$ & 32 & -- & -- & $<1.0$ & -- & 628 & 551 \\
\hline $04 / 14 / 00$ & $<2.6$ & $<2.4$ & 1.6 & $<1.0$ & 21 & -- & -- & $<1.0$ & -- & 719 & 242 \\
\hline $04 / 17 / 00$ & $<2.6$ & $<2.4$ & $<1.0$ & $<1.0$ & 25 & -- & -- & $<1.0$ & -- & 422 & 435 \\
\hline 05/02/00 & $<2.6$ & $<2.4$ & $<1.0$ & $<1.0$ & 24 & -- & -- & $<1.0$ & -- & 383 & 380 \\
\hline $05 / 18 / 00$ & $<2.6$ & $<.7$ & $<1.0$ & $<1.0$ & 23 & 22 & $<.90$ & $<1.0$ & $<1.0$ & 363 & 324 \\
\hline $06 / 02 / 00$ & $<2.6$ & $<.7$ & $<1.0$ & $<1.0$ & 30 & 32 & $<.90$ & $<1.0$ & $<1.0$ & 513 & 515 \\
\hline $06 / 29 / 02^{\mathbf{F b}}$ & -- & -- & -- & $<.2$ & -- & $<.1$ & $<.1$ & $<.2$ & -- & -- & $<.5$ \\
\hline 06/29/00 & $<2.6$ & $<.7$ & $<1.0$ & $<1.0$ & 43 & 43 & $<.90$ & $<1.0$ & $<1.0$ & 836 & 693 \\
\hline 07/24/00 & $<2.6$ & $<.7$ & $<1.0$ & $<1.0$ & 62 & 65 & $<.90$ & $<1.0$ & $<1.0$ & 1,060 & 1,080 \\
\hline $08 / 31 / 00$ & $<2.6$ & $<.7$ & $<1.0$ & $<1.0$ & 78 & 79 & $<.90$ & $<1.0$ & $<1.0$ & 1,460 & 1,360 \\
\hline $11 / 06 / 00$ & -- & -- & -- & -- & -- & -- & -- & -- & -- & 1,390 & 1,520 \\
\hline $12 / 18 / 00$ & -- & -- & -- & -- & -- & -- & -- & -- & -- & 1,690 & 1,710 \\
\hline $01 / 23 / 01$ & -- & -- & -- & -- & -- & -- & -- & -- & -- & 1,600 & 1,610 \\
\hline
\end{tabular}


Table 8. Trace-element concentration data for surface-water samples collected at fixed water-quality sites, Northern Rockies Intermontane Basins study unit, 1998-2001 (Continued)

\begin{tabular}{|c|c|c|c|c|c|c|c|c|c|c|}
\hline Date & Time & $\begin{array}{l}\text { Alumi- } \\
\text { num, } \\
\text { total } \\
\text { recov- } \\
\text { erable } \\
(\mu \mathrm{g} / \mathrm{L})\end{array}$ & $\begin{array}{l}\text { Alumi- } \\
\text { num, } \\
\text { dis- } \\
\text { solved } \\
(\mu \mathrm{g} / \mathrm{L})\end{array}$ & $\begin{array}{c}\text { Anti- } \\
\text { mony, } \\
\text { total } \\
\text { recov- } \\
\text { erable } \\
(\mu \mathrm{g} / \mathrm{L})\end{array}$ & $\begin{array}{c}\text { Anti- } \\
\text { mony, } \\
\text { dis- } \\
\text { solved } \\
(\mu \mathrm{g} / \mathrm{L})\end{array}$ & $\begin{array}{c}\text { Arsenic, } \\
\text { total } \\
\text { recov- } \\
\text { erable } \\
(\mu \mathrm{g} / \mathrm{L})\end{array}$ & $\begin{array}{c}\text { Arsenic, } \\
\text { dis- } \\
\text { solved } \\
(\mu \mathrm{g} / \mathrm{L})\end{array}$ & $\begin{array}{c}\text { Barium, } \\
\text { total } \\
\text { recov- } \\
\text { erable } \\
(\mu \mathrm{g} / \mathrm{L})\end{array}$ & $\begin{array}{c}\text { Barium, } \\
\text { dis- } \\
\text { solved } \\
(\mu \mathrm{g} / \mathrm{L})\end{array}$ & $\begin{array}{l}\text { Beryl- } \\
\text { lium, } \\
\text { total } \\
\text { recov- } \\
\text { erable } \\
(\mu \mathrm{g} / \mathrm{L})\end{array}$ \\
\hline \multicolumn{11}{|c|}{ SITE 31 12413470--SOUTH FORK COEUR D'ALENE RIVER NEAR PINEHURST, IDAHO (Continued) } \\
\hline 03/14/01 & 1145 & -- & -- & -- & -- & $\mathrm{e} 1.4$ & .53 & -- & -- & -- \\
\hline 04/10/01 & 1100 & -- & -- & -- & -- & $\mathrm{e} 1.0$ & .44 & -- & -- & -- \\
\hline 05/03/01 & 1300 & -- & -- & -- & -- & $<1.9$ & .30 & -- & -- & -- \\
\hline 06/12/01 & 1230 & -- & -- & -- & -- & $<2.0$ & .47 & -- & -- & -- \\
\hline 09/10/01 & 1130 & -- & -- & -- & -- & $<2.0$ & .68 & -- & -- & -- \\
\hline $09 / 10 / 01^{\mathbf{F b}}$ & 1345 & -- & $<1.0$ & -- & $<.05$ & -- & e. 12 & -- & $<1.0$ & -- \\
\hline \multicolumn{11}{|c|}{ SITE 33 12413875--ST. JOE RIVER AT RED IVES RANGER STATION, IDAHO } \\
\hline 02/18/99 & 1545 & 33 & 5.1 & $<1.0$ & $<1.0$ & $<1.0$ & $<1.0$ & $<100$ & 4.7 & $<10$ \\
\hline 06/01/99 & 1415 & 165 & 16 & $<1.0$ & $<1.0$ & $<1.0$ & $<1.0$ & 4.1 & 3.4 & $<4.0$ \\
\hline 06/28/99 & 1415 & -- & 13 & 2.4 & $<1.0$ & -- & $<1.0$ & -- & 3.7 & -- \\
\hline 07/20/99 & 0945 & $\mathrm{e} 23$ & 13 & $<1.0$ & $<1.0$ & $<1.0$ & $<1.0$ & 4.3 & 4.7 & $<4.0$ \\
\hline 08/18/99 & 1500 & e23 & 11 & $<1.0$ & $<1.0$ & $<1.0$ & $<1.0$ & 5.4 & 5.3 & $<4.0$ \\
\hline $11 / 04 / 99$ & 1300 & e17 & 16 & $<1.0$ & $<1.0$ & $<2.6$ & $<2.0$ & 4.7 & 5.2 & $<5.0$ \\
\hline $01 / 21 / 00$ & 1245 & e18 & 6.1 & $<1.0$ & $<1.0$ & $<2.6$ & $<2.0$ & 5.0 & 5.2 & $<5.0$ \\
\hline $05 / 17 / 00$ & 1215 & 56 & $<26$ & $<1.0$ & $<1.0$ & $<2.6$ & $<.90$ & 3.7 & 3.6 & $<5.0$ \\
\hline $06 / 01 / 00$ & 1100 & 50 & $<13$ & $<1.0$ & $<1.0$ & $<2.6$ & $<.90$ & 3.9 & 4.0 & $<5.0$ \\
\hline $06 / 28 / 00$ & 1100 & $<28$ & 24 & $<1.0$ & $<1.0$ & $<2.6$ & $<.90$ & 4.3 & 4.5 & $<5.0$ \\
\hline $07 / 27 / 00$ & 1115 & $\mathrm{e} 22$ & 11 & $<1.0$ & $<1.0$ & $<2.6$ & $<.90$ & 5.2 & 5.7 & $<5.0$ \\
\hline 09/07/00 & 1330 & $<28$ & 7.9 & $<1.0$ & $<1.0$ & $<2.6$ & $<.90$ & 5.5 & 5.8 & $<5.0$ \\
\hline $11 / 07 / 00$ & 1015 & -- & -- & -- & -- & $<1.9$ & e. 17 & -- & -- & -- \\
\hline $01 / 24 / 01$ & 1345 & -- & -- & -- & -- & $<1.9$ & .24 & -- & -- & -- \\
\hline 05/09/01 & 1200 & -- & -- & -- & -- & $<1.9$ & e. 13 & -- & -- & -- \\
\hline $06 / 13 / 01$ & 0930 & -- & -- & -- & -- & $<1.9$ & e. 11 & -- & -- & -- \\
\hline 09/13/01 & 1045 & -- & -- & -- & -- & $<1.9$ & .33 & -- & -- & -- \\
\hline \multicolumn{11}{|c|}{ SITE 35 12419000--SPOKANE RIVER NEAR POST FALLS, IDAHO } \\
\hline 03/04/99 & 1000 & -- & -- & -- & -- & -- & -- & -- & -- & -- \\
\hline 04/13/99 & 1230 & 128 & 4.4 & $<1.0$ & $<1.0$ & $<1.0$ & $<1.0$ & 14 & 13 & $<4.0$ \\
\hline 05/19/99 & 1000 & 121 & 3.6 & -- & $<1.0$ & $<1.0$ & $<1.0$ & 13 & 14 & $<4.0$ \\
\hline $06 / 02 / 99$ & 0900 & 58 & 2.8 & $<1.0$ & $<1.0$ & 1.6 & $<1.0$ & 12 & 11 & $<4.0$ \\
\hline 06/23/99 & 0930 & 31 & 4.9 & $<1.0$ & $<1.0$ & $<1.0$ & $<1.0$ & 10 & 9.9 & $<4.0$ \\
\hline $07 / 27 / 99^{\mathbf{F b}}$ & 0915 & -- & .33 & -- & $<.2$ & -- & -- & -- & $<.2$ & -- \\
\hline 07/27/99 & 1030 & e19 & 3.7 & $<1.0$ & $<1.0$ & $<1.0$ & $<1.0$ & 10 & 10 & $<4.0$ \\
\hline 09/09/99 & 1100 & e21 & 3.8 & $<1.0$ & $<1.0$ & $<1.0$ & $<1.0$ & 11 & 11 & $<5.0$ \\
\hline $10 / 20 / 99$ & 1130 & $<28$ & 2.3 & $<1.0$ & $<1.0$ & $<2.6$ & $<2.0$ & 11 & 11 & $<5.0$ \\
\hline $11 / 30 / 99$ & 1045 & -- & -- & -- & -- & -- & -- & -- & -- & -- \\
\hline $01 / 12 / 00$ & 0910 & 36 & 3.6 & $<1.0$ & $<1.0$ & $<2.6$ & $<2.0$ & 12 & 12 & $<5.0$ \\
\hline $02 / 29 / 00$ & 1200 & 54 & 2.8 & $<1.0$ & $<1.0$ & $<2.6$ & $<.90$ & 13 & 13 & $<5.0$ \\
\hline 03/28/00 & 0915 & 50 & 2.7 & $<1.0$ & $<1.0$ & $<2.6$ & $<.90$ & 13 & 12 & $<5.0$ \\
\hline $04 / 11 / 00$ & 0800 & 67 & 2.5 & $<1.0$ & $<1.0$ & $<2.6$ & $<.90$ & 13 & 13 & $<5.0$ \\
\hline $04 / 18 / 00$ & 1115 & 142 & 16 & $<1.0$ & $<1.0$ & $<2.6$ & $<.90$ & 14 & 12 & $<5.0$ \\
\hline 05/03/00 & 0800 & 93 & 8.0 & $<1.0$ & $<1.0$ & $<2.6$ & $<2.0$ & 12 & 12 & $<5.0$ \\
\hline 05/16/00 & 1315 & 50 & 18 & $<1.0$ & $<1.0$ & $<2.6$ & $<.90$ & 12 & 11 & $<5.0$ \\
\hline $05 / 31 / 00$ & 1345 & 30 & 10 & $<1.0$ & $<1.0$ & $<2.6$ & $<.90$ & 11 & 11 & $<5.0$ \\
\hline $06 / 27 / 00$ & 1415 & e24 & 3.0 & $<1.0$ & $<1.0$ & $<2.6$ & $<.90$ & 9.9 & 9.7 & $<5.0$ \\
\hline $07 / 25 / 00$ & 1300 & $<28$ & 3.1 & $<1.0$ & $<1.0$ & $<2.6$ & $<.90$ & 10 & 11 & $<5.0$ \\
\hline 08/30/00 & 1415 & e21 & 4.5 & $<1.0$ & $<1.0$ & $<2.6$ & e. 50 & 11 & 11 & $<5.0$ \\
\hline $11 / 08 / 00$ & 1400 & -- & -- & -- & -- & $<1.9$ & .44 & -- & -- & -- \\
\hline $12 / 21 / 00$ & 1400 & -- & -- & -- & -- & $<2.6$ & .47 & -- & -- & -- \\
\hline
\end{tabular}


Table 8. Trace-element concentration data for surface-water samples collected at fixed water-quality sites, Northern Rockies Intermontane Basins study unit, 1998-2001 (Continued)

\begin{tabular}{|c|c|c|c|c|c|c|c|c|c|c|c|}
\hline Date & $\begin{array}{c}\text { Beryl- } \\
\text { lium, } \\
\text { dis- } \\
\text { solved } \\
(\mu \mathrm{g} / \mathrm{L})\end{array}$ & $\begin{array}{c}\text { Boron, } \\
\text { dis- } \\
\text { solved } \\
(\mu \mathrm{g} / \mathrm{L})\end{array}$ & $\begin{array}{c}\text { Cad- } \\
\text { mium, } \\
\text { total } \\
\text { recov- } \\
\text { erable } \\
(\mu \mathrm{g} / \mathrm{L})\end{array}$ & $\begin{array}{c}\text { Cad- } \\
\text { mium, } \\
\text { dis- } \\
\text { solved } \\
(\mu \mathrm{g} / \mathrm{L})\end{array}$ & $\begin{array}{c}\text { Chro- } \\
\text { mium, } \\
\text { total } \\
\text { recov- } \\
\text { erable } \\
(\mu \mathrm{g} / \mathrm{L})\end{array}$ & $\begin{array}{c}\text { Chro- } \\
\text { mium, } \\
\text { dis- } \\
\text { solved } \\
(\mu \mathrm{g} / \mathrm{L})\end{array}$ & $\begin{array}{c}\text { Cobalt, } \\
\text { total } \\
\text { recov- } \\
\text { erable } \\
(\mu \mathrm{g} / \mathrm{L})\end{array}$ & $\begin{array}{c}\text { Cobalt, } \\
\text { dis- } \\
\text { solved } \\
(\mu \mathrm{g} / \mathrm{L})\end{array}$ & $\begin{array}{c}\text { Copper, } \\
\text { total } \\
\text { recov- } \\
\text { erable } \\
(\mu \mathrm{g} / \mathrm{L})\end{array}$ & $\begin{array}{c}\text { Copper, } \\
\text { dis- } \\
\text { solved } \\
(\mu \mathrm{g} / \mathrm{L})\end{array}$ & $\begin{array}{c}\text { Iron, } \\
\text { total } \\
\text { recov- } \\
\text { erable } \\
(\mu \mathrm{g} / \mathrm{L})\end{array}$ \\
\hline \multicolumn{12}{|c|}{ SITE 31 12413470--SOUTH FORK COEUR D'ALENE RIVER NEAR PINEHURST, IDAHO (Continued) } \\
\hline 03/14/01 & -- & -- & 19 & 18 & -- & -- & -- & -- & 2.5 & 1.4 & -- \\
\hline $06 / 12 / 01$ & -- & -- & 5.0 & 5.0 & -- & -- & -- & -- & 1.2 & .84 & -- \\
\hline 09/10/01 & -- & -- & 8.9 & 8.6 & -- & -- & -- & -- & 1.7 & 1.9 & -- \\
\hline $09 / 10 / 01^{\mathbf{F b}}$ & $<.06$ & $<7.0$ & -- & $<.04$ & -- & $<.80$ & -- & $<.02$ & -- & .35 & -- \\
\hline \multicolumn{12}{|c|}{ SITE 33 12413875--ST. JOE RIVER AT RED IVES RANGER STATION, IDAHO } \\
\hline $02 / 18 / 99$ & $<1.0$ & -- & $<1.0$ & $<1.0$ & $<1.0$ & $<1.0$ & $<1.0$ & $<1.0$ & $<10$ & $<1.0$ & 18 \\
\hline $11 / 04 / 99$ & $<1.0$ & -- & $<.11$ & $<1.0$ & $<1.0$ & $<.80$ & $<1.8$ & $<1.0$ & $<20$ & $<1.0$ & 23 \\
\hline $01 / 21 / 00$ & $<1.0$ & -- & $<.11$ & $<1.0$ & $<1.0$ & $<.80$ & $<1.8$ & $<1.0$ & $<20$ & $<1.0$ & $<21$ \\
\hline $05 / 17 / 00$ & $<1.0$ & $<12$ & $<.11$ & $<1.0$ & $<1.0$ & $<.80$ & $<1.8$ & $<1.0$ & $<20$ & $<1.0$ & 39 \\
\hline $06 / 01 / 00$ & $<1.0$ & $<12$ & $<.11$ & $<1.0$ & $<1.0$ & $<.80$ & $<1.8$ & $<1.0$ & $<20$ & $<1.0$ & 50 \\
\hline $06 / 28 / 00$ & $<1.0$ & $<12$ & $<.11$ & $<1.0$ & $<1.0$ & .90 & $<1.8$ & $<1.0$ & $<20$ & $<1.0$ & $<21$ \\
\hline $07 / 27 / 00$ & $<1.0$ & $<12$ & $<.11$ & $<1.0$ & $<1.0$ & $<.80$ & $<1.8$ & $<1.0$ & $<20$ & $<1.0$ & 32 \\
\hline $09 / 07 / 00$ & $<1.0$ & $<12$ & $<.11$ & $<1.0$ & $<1.0$ & $<.80$ & $<1.8$ & $<1.0$ & $<20$ & $<1.0$ & $<21$ \\
\hline $11 / 07 / 00$ & -- & -- & $<.04$ & $<.04$ & -- & -- & -- & -- & $<.60$ & e. 16 & -- \\
\hline $01 / 24 / 01$ & -- & -- & $<.04$ & $<.04$ & -- & -- & -- & -- & $<.60$ & .42 & -- \\
\hline 05/09/01 & -- & -- & $<.04$ & e.02 & -- & -- & -- & -- & e. 31 & .30 & -- \\
\hline $06 / 13 / 01$ & -- & -- & $<.04$ & $<.04$ & -- & -- & -- & -- & $<.60$ & e. 16 & -- \\
\hline \multicolumn{12}{|c|}{ SITE 35 12419000--SPOKANE RIVER NEAR POST FALLS, IDAHO } \\
\hline $07 / 27 / 99^{\mathbf{F b}}$ & $<.2$ & $<2.0$ & -- & $<.3$ & -- & .24 & -- & $<.2$ & -- & $<2.0$ & -- \\
\hline 07/27/99 & $<1.0$ & -- & $<1.0$ & $<1.0$ & $<1.0$ & $<1.0$ & $<1.0$ & $<1.0$ & $<12$ & 1.2 & 55 \\
\hline 09/09/99 & $<1.0$ & -- & $<1.0$ & $<1.0$ & $<1.0$ & $<1.0$ & $<1.0$ & $<1.0$ & $<20$ & $<1.0$ & 33 \\
\hline $10 / 20 / 99$ & $<1.0$ & -- & .15 & $<1.0$ & $<1.0$ & $<.80$ & $<1.8$ & $<1.0$ & $<20$ & $<1.0$ & 21 \\
\hline $11 / 30 / 99$ & -- & -- & -- & -- & -- & -- & -- & -- & -- & -- & -- \\
\hline $01 / 12 / 00$ & $<1.0$ & -- & .30 & $<1.0$ & $<1.0$ & $<.80$ & $<1.8$ & $<1.0$ & $<20$ & $<1.0$ & 38 \\
\hline $02 / 29 / 00$ & $<1.0$ & $<12$ & .40 & $<1.0$ & $<1.0$ & $<1.0$ & $<1.8$ & $<1.0$ & $<20$ & $<1.0$ & 63 \\
\hline $03 / 28 / 00$ & $<1.0$ & $<12$ & .32 & $<1.0$ & $<1.0$ & $<1.0$ & $<1.8$ & $<1.0$ & e11 & $<1.0$ & 75 \\
\hline $04 / 11 / 00$ & $<1.0$ & $<12$ & .37 & $<1.0$ & $<1.0$ & $<.80$ & $<1.8$ & $<1.0$ & $<20$ & $<1.0$ & 94 \\
\hline $04 / 18 / 00$ & $<1.0$ & $<12$ & .44 & $<1.0$ & $<1.0$ & $<.80$ & $<1.8$ & $<1.0$ & $<20$ & $<1.0$ & 195 \\
\hline $05 / 03 / 00$ & $<1.0$ & $<12$ & .29 & $<1.0$ & $<1.0$ & $<.80$ & $<1.8$ & $<1.0$ & $<20$ & $<1.0$ & 144 \\
\hline $05 / 16 / 00$ & $<1.0$ & $<12$ & .26 & $<1.0$ & $<1.0$ & $<.80$ & $<1.8$ & $<1.0$ & $<20$ & $<1.0$ & 83 \\
\hline $05 / 31 / 00$ & $<1.0$ & $<12$ & .21 & $<1.0$ & e. 58 & $<.80$ & $<1.8$ & $<1.0$ & e14 & $<1.0$ & 131 \\
\hline $06 / 27 / 00$ & $<1.0$ & $<12$ & .15 & $<1.0$ & $<1.0$ & e. 40 & $<1.8$ & $<1.0$ & $<20$ & $<1.0$ & 31 \\
\hline $07 / 25 / 00$ & $<1.0$ & $<12$ & .13 & $<1.0$ & $<1.0$ & $<.80$ & $<1.8$ & $<1.0$ & $<20$ & $<1.0$ & 27 \\
\hline $08 / 30 / 00$ & $<1.0$ & $\mathrm{e} 8.2$ & $<.11$ & $<1.0$ & $<1.0$ & $<.80$ & $<1.8$ & $<1.0$ & $<20$ & $<1.0$ & 28 \\
\hline $11 / 08 / 00$ & -- & -- & .18 & .14 & -- & -- & -- & -- & .60 & .54 & -- \\
\hline $12 / 21 / 00$ & -- & -- & .23 & .20 & -- & -- & -- & -- & .69 & .63 & -- \\
\hline
\end{tabular}


Table 8. Trace-element concentration data for surface-water samples collected at fixed water-quality sites, Northern Rockies Intermontane Basins study unit, 1998-2001 (Continued)

\begin{tabular}{|c|c|c|c|c|c|c|c|c|c|c|c|c|}
\hline Date & $\begin{array}{c}\text { Iron, } \\
\text { dis- } \\
\text { solved } \\
(\mu \mathrm{g} / \mathrm{L})\end{array}$ & $\begin{array}{c}\text { Lead, } \\
\text { total } \\
\text { recov- } \\
\text { erable } \\
(\mu \mathrm{g} / \mathrm{L})\end{array}$ & $\begin{array}{c}\text { Lead, } \\
\text { dis- } \\
\text { solved } \\
(\mu \mathrm{g} / \mathrm{L})\end{array}$ & $\begin{array}{l}\text { Lith- } \\
\text { ium, } \\
\text { total } \\
\text { recov- } \\
\text { erable } \\
(\mu \mathrm{g} / \mathrm{L})\end{array}$ & $\begin{array}{l}\text { Lith- } \\
\text { ium, } \\
\text { dis- } \\
\text { solved } \\
(\mu \mathrm{g} / \mathrm{L})\end{array}$ & $\begin{array}{l}\text { Manga- } \\
\text { nese, } \\
\text { total } \\
\text { recov- } \\
\text { erable } \\
(\mu \mathrm{g} / \mathrm{L})\end{array}$ & $\begin{array}{c}\text { Manga- } \\
\text { nese, } \\
\text { dis- } \\
\text { solved } \\
(\mu \mathrm{g} / \mathrm{L})\end{array}$ & $\begin{array}{c}\text { Mercury, } \\
\text { total } \\
\text { recov- } \\
\text { erable } \\
(\mu \mathrm{g} / \mathrm{L})\end{array}$ & $\begin{array}{c}\text { Molyb- } \\
\text { denum, } \\
\text { total } \\
\text { recov- } \\
\text { erable } \\
(\mu \mathrm{g} / \mathrm{L})\end{array}$ & $\begin{array}{c}\text { Molyb- } \\
\text { denum, } \\
\text { dis- } \\
\text { solved } \\
(\mu \mathrm{g} / \mathrm{L})\end{array}$ & $\begin{array}{c}\text { Nickel, } \\
\text { tota1 } \\
\text { recov- } \\
\text { erable } \\
(\mu \mathrm{g} / \mathrm{L})\end{array}$ & $\begin{array}{c}\text { Nickel, } \\
\text { dis- } \\
\text { solved } \\
(\mu \mathrm{g} / \mathrm{L})\end{array}$ \\
\hline \multicolumn{13}{|c|}{ SITE 31 12413470--SOUTH FORK COEUR D'ALENE RIVER NEAR PINEHURST, IDAHO (Continued) } \\
\hline 03/14/01 & 85 & 32 & 4.4 & -- & -- & -- & 317 & -- & -- & -- & -- & -- \\
\hline 04/10/01 & 90 & 15 & 5.1 & -- & -- & -- & 316 & -- & -- & -- & -- & -- \\
\hline 05/03/01 & 32 & 30 & 3.2 & -- & -- & -- & 130 & -- & -- & -- & -- & -- \\
\hline $06 / 12 / 01$ & 73 & 13 & 4.9 & -- & -- & -- & 187 & -- & -- & -- & -- & -- \\
\hline 09/10/01 & 129 & 15 & 5.4 & -- & -- & -- & 488 & -- & -- & -- & -- & -- \\
\hline $09 / 10 / 01^{\mathbf{F b}}$ & $<10$ & -- & $<.08$ & -- & $<.3$ & -- & $<.1$ & -- & -- & $<.2$ & -- & $<.06$ \\
\hline \multicolumn{13}{|c|}{ SITE 33 12413875--ST. JOE RIVER AT RED IVES RANGER STATION, IDAHO } \\
\hline $02 / 18 / 99$ & e5.4 & $<1.0$ & $<1.0$ & $<10$ & -- & $<10$ & $<1.0$ & $<.10$ & $<1.0$ & $<1.0$ & $<1.0$ & $<1.0$ \\
\hline 06/01/99 & e5.1 & $<1.0$ & $<1.0$ & $<12$ & -- & 4.6 & $<1.0$ & $<.10$ & $<1.0$ & $<1.0$ & $<1.0$ & $<1.0$ \\
\hline 06/28/99 & $<10$ & -- & $<1.0$ & $<12$ & -- & -- & $<1.0$ & $<.10$ & -- & $<1.0$ & -- & $<1.0$ \\
\hline 07/20/99 & $\mathrm{e} 8.0$ & $<1.0$ & $<1.0$ & $<12$ & -- & $<3.0$ & $<1.0$ & $<.10$ & $<1.0$ & $<1.0$ & $<1.0$ & $<1.0$ \\
\hline 08/18/99 & e7.2 & $<1.0$ & $<1.0$ & $<12$ & -- & e2.0 & $<1.0$ & $<.10$ & $<1.0$ & $<1.0$ & $<1.0$ & $<1.0$ \\
\hline $11 / 04 / 99$ & e9.1 & $<1.0$ & $<1.0$ & $<7.0$ & -- & $<2.8$ & $<1.0$ & $<.30$ & $<1.0$ & $<1.0$ & $<1.8$ & $<1.0$ \\
\hline $01 / 21 / 00$ & $\mathrm{e} 6.3$ & $<1.0$ & $<1.0$ & $<7.0$ & -- & $<2.8$ & $<1.0$ & $<.30$ & $<1.0$ & $<1.0$ & $<1.8$ & $<1.0$ \\
\hline $05 / 17 / 00$ & $<10$ & $<1.0$ & $<1.0$ & $<7.0$ & e. 21 & 3.0 & $<1.0$ & $<.30$ & $<1.0$ & $<1.0$ & $<1.8$ & $<1.0$ \\
\hline 06/01/00 & e5.7 & $<1.0$ & $<1.0$ & $<7.0$ & $<.3$ & $\mathrm{e} 1.5$ & $<1.0$ & $<.30$ & $<1.0$ & $<1.0$ & $<1.8$ & $<1.0$ \\
\hline $06 / 28 / 00$ & e6.4 & $<1.0$ & $<1.0$ & $<7.0$ & $<.3$ & $<2.8$ & 1.2 & $<.30$ & $<1.0$ & $<1.0$ & $<1.8$ & $<1.0$ \\
\hline $07 / 27 / 00$ & e6.6 & $<1.0$ & $<1.0$ & $<7.0$ & e. 17 & $\mathrm{e} 1.5$ & $<1.0$ & $<.30$ & $<1.0$ & $<1.0$ & $<1.8$ & $<1.0$ \\
\hline 09/07/00 & 10 & $<1.0$ & $<1.0$ & $<7.0$ & e. 20 & $<2.8$ & $<1.0$ & $<.30$ & $<1.0$ & $<1.0$ & $<1.8$ & $<1.0$ \\
\hline $11 / 07 / 00$ & e6.9 & $<1.0$ & $<.08$ & -- & -- & -- & $\mathrm{e} 2.0$ & -- & -- & -- & -- & -- \\
\hline $01 / 24 / 01$ & $<10$ & $<1.0$ & .09 & -- & -- & -- & $<3.2$ & -- & -- & -- & -- & -- \\
\hline 05/09/01 & $<10$ & $<1.0$ & $<.08$ & -- & -- & -- & $<3.2$ & -- & -- & -- & -- & -- \\
\hline 06/13/01 & e9.7 & $<1.0$ & $<.08$ & -- & -- & -- & $<3.0$ & -- & -- & -- & -- & -- \\
\hline 09/13/01 & $<10$ & $<1.0$ & $<.08$ & -- & -- & -- & $<3.0$ & -- & -- & -- & -- & -- \\
\hline \multicolumn{13}{|c|}{ SITE 35 12419000--SPOKANE RIVER NEAR POST FALLS, IDAHO } \\
\hline 03/04/99 & 20 & -- & -- & -- & -- & -- & -- & -- & -- & -- & -- & -- \\
\hline 04/13/99 & 23 & 2.1 & $<1.0$ & $<16$ & -- & 5.1 & 1.2 & $<.10$ & $<1.0$ & $<1.0$ & $<1.0$ & $<1.0$ \\
\hline 05/19/99 & 18 & $<1.0$ & $<1.0$ & $<12$ & -- & 6.3 & 1.6 & $<.10$ & $<1.0$ & $<1.0$ & $<1.0$ & $<1.0$ \\
\hline 06/02/99 & 14 & 7.7 & $<1.0$ & $<12$ & -- & 11 & 1.8 & $<.10$ & $<1.0$ & $<1.0$ & $<1.0$ & $<1.0$ \\
\hline 06/23/99 & 13 & 2.8 & $<1.0$ & $<12$ & -- & 10 & 1.3 & $<.10$ & $<1.0$ & $<1.0$ & $<1.0$ & $<1.0$ \\
\hline $07 / 27 / 99^{\mathbf{F b}}$ & $<3.0$ & -- & $<.3$ & -- & -- & -- & $<.1$ & -- & -- & $<.2$ & -- & $<.5$ \\
\hline 07/27/99 & e5.5 & 1.1 & $<1.0$ & $<12$ & -- & 10 & 1.5 & $<.10$ & $<1.0$ & $<1.0$ & $<1.0$ & $<1.0$ \\
\hline 09/09/99 & $\mathrm{e} 8.5$ & 1.2 & $<1.0$ & $<7.0$ & -- & 4.5 & 1.6 & $<.10$ & $<1.0$ & $<1.0$ & $<1.0$ & $<1.0$ \\
\hline $10 / 20 / 99$ & e7.8 & $<1.0$ & $<1.0$ & $<7.0$ & -- & 4.2 & 1.8 & $<.30$ & $<1.0$ & $<1.0$ & $<1.8$ & $<1.0$ \\
\hline $11 / 30 / 99$ & $<10$ & -- & -- & -- & -- & -- & $\mathrm{e} 1.4$ & -- & -- & -- & -- & -- \\
\hline $01 / 12 / 00$ & $<10$ & e. 59 & $<1.0$ & $<7.0$ & -- & 5.6 & 1.4 & $<.30$ & $<1.0$ & $<1.0$ & $<1.8$ & $<1.0$ \\
\hline $02 / 29 / 00$ & 10 & 1.4 & $<1.0$ & $\mathrm{e} 4.0$ & .49 & 5.2 & 1.3 & $<.30$ & $<1.0$ & $<1.0$ & $<1.8$ & $<1.0$ \\
\hline 03/28/00 & 17 & e.74 & $<1.0$ & $<7.0$ & .49 & 4.8 & 1.0 & $<.30$ & $<1.0$ & $<1.0$ & $<1.8$ & $<1.0$ \\
\hline $04 / 11 / 00$ & 19 & 2.1 & $<1.0$ & $<7.0$ & .50 & 2.9 & 1.0 & $<.30$ & $<1.0$ & $<1.0$ & $<1.8$ & $<1.0$ \\
\hline $04 / 18 / 00$ & 26 & 5.6 & $<1.0$ & $<7.0$ & .62 & 17 & 4.5 & $<.30$ & $<1.0$ & $<1.0$ & $<1.8$ & $<1.0$ \\
\hline 05/03/00 & 27 & 7.8 & 1.6 & $<7.0$ & .53 & 17 & 3.1 & $<.30$ & $<1.0$ & $<1.0$ & $<1.8$ & $<1.0$ \\
\hline $05 / 16 / 00$ & 17 & 3.6 & $<1.0$ & $<7.0$ & .53 & 13 & 1.6 & $<.30$ & $<1.0$ & $<1.0$ & $<1.8$ & $<1.0$ \\
\hline $05 / 31 / 00$ & 17 & 2.1 & $<1.0$ & $<7.0$ & e. 20 & 11 & 1.0 & $<.30$ & $<1.0$ & $<1.0$ & $<1.8$ & $<1.0$ \\
\hline $06 / 27 / 00$ & $<10$ & 1.1 & $<1.0$ & $<7.0$ & .34 & 6.2 & $<1.0$ & $<.30$ & 1.8 & $<1.0$ & $<1.8$ & $<1.0$ \\
\hline $07 / 25 / 00$ & $<10$ & 1.1 & $<1.0$ & $<7.0$ & .57 & 5.7 & 1.8 & $<.30$ & 1.1 & $<1.0$ & $<1.8$ & $<1.0$ \\
\hline 08/30/00 & $<10$ & 1.3 & $<1.0$ & $<7.0$ & .54 & 6.3 & 2.1 & $<.30$ & $<1.0$ & $<1.0$ & $<1.8$ & $<1.0$ \\
\hline $11 / 08 / 00$ & $<10$ & $<1.0$ & .13 & -- & -- & -- & $<3.2$ & -- & -- & -- & -- & -- \\
\hline $12 / 21 / 00$ & $<10$ & $<1.0$ & .17 & -- & -- & -- & e1.7 & -- & -- & -- & -- & -- \\
\hline
\end{tabular}


Table 8. Trace-element concentration data for surface-water samples collected at fixed water-quality sites, Northern Rockies Intermontane Basins study unit, 1998-2001 (Continued)

\begin{tabular}{|c|c|c|c|c|c|c|c|c|c|c|c|}
\hline Date & $\begin{array}{c}\text { Selenium, } \\
\text { total } \\
\text { recov- } \\
\text { erable } \\
(\mu \mathrm{g} / \mathrm{L})\end{array}$ & $\begin{array}{c}\text { Selenium, } \\
\text { dis- } \\
\text { solved } \\
(\mu \mathrm{g} / \mathrm{L})\end{array}$ & $\begin{array}{c}\text { Silver, } \\
\text { total } \\
\text { recov- } \\
\text { erable } \\
(\mu \mathrm{g} / \mathrm{L})\end{array}$ & $\begin{array}{c}\text { Silver, } \\
\text { dis- } \\
\text { solved } \\
(\mu \mathrm{g} / \mathrm{L})\end{array}$ & $\begin{array}{l}\text { Stron- } \\
\text { tium, } \\
\text { total } \\
\text { recov- } \\
\text { erable } \\
(\mu \mathrm{g} / \mathrm{L})\end{array}$ & $\begin{array}{c}\text { Stron- } \\
\text { tium, } \\
\text { dis- } \\
\text { solved } \\
(\mu \mathrm{g} / \mathrm{L})\end{array}$ & $\begin{array}{c}\text { Thal- } \\
\text { lium, } \\
\text { dis- } \\
\text { solved } \\
(\mu \mathrm{g} / \mathrm{L})\end{array}$ & $\begin{array}{c}\text { Uranium, } \\
\text { natural } \\
(\mu \mathrm{g} / \mathrm{L})\end{array}$ & $\begin{array}{c}\text { Vana- } \\
\text { dium, } \\
\text { dis- } \\
\text { solved } \\
(\mu \mathrm{g} / \mathrm{L})\end{array}$ & $\begin{array}{l}\text { Zinc, } \\
\text { total } \\
\text { recov- } \\
\text { erable } \\
(\mu \mathrm{g} / \mathrm{L})\end{array}$ & $\begin{array}{c}\text { Zinc, } \\
\text { dis- } \\
\text { solved } \\
(\mu \mathrm{g} / \mathrm{L})\end{array}$ \\
\hline \multicolumn{12}{|c|}{ SITE 31 12413470--SOUTH FORK COEUR D'ALENE RIVER NEAR PINEHURST, IDAHO (Continued) } \\
\hline 03/14/01 & -- & -- & -- & -- & -- & -- & -- & -- & -- & 1,780 & 1,650 \\
\hline 04/10/01 & -- & -- & -- & -- & -- & -- & -- & -- & -- & 1,270 & 1,340 \\
\hline 05/03/01 & -- & -- & -- & -- & -- & -- & -- & -- & -- & 661 & 632 \\
\hline $06 / 12 / 01$ & -- & -- & -- & -- & -- & -- & -- & -- & -- & 805 & 810 \\
\hline 09/10/01 & -- & -- & -- & -- & -- & -- & -- & -- & -- & 1,630 & 1,560 \\
\hline $09 / 10 / 01^{\mathbf{F b}}$ & -- & .48 & -- & $<1.0$ & -- & $<.08$ & $<.04$ & $<.02$ & $<.2$ & -- & 1.3 \\
\hline \multicolumn{12}{|c|}{ SITE 33 12413875--ST. JOE RIVER AT RED IVES RANGER STATION, IDAHO } \\
\hline $02 / 18 / 99$ & $<1.0$ & $<1.0$ & $<1.0$ & $<1.0$ & 30 & -- & -- & $<1.0$ & -- & $<10$ & $<1.0$ \\
\hline $06 / 01 / 99$ & 1.6 & $<1.0$ & $<1.0$ & $<1.0$ & 5.6 & -- & -- & $<1.0$ & -- & $<40$ & 1.0 \\
\hline $06 / 28 / 99$ & -- & $<1.0$ & -- & $<1.0$ & -- & -- & -- & $<1.0$ & -- & -- & $<1.0$ \\
\hline $07 / 20 / 99$ & $<1.0$ & $<1.0$ & $<1.0$ & $<1.0$ & 7.4 & -- & -- & $<1.0$ & -- & $<40$ & $<1.0$ \\
\hline 08/18/99 & $<1.0$ & $<1.0$ & $<1.0$ & $<1.0$ & 9.8 & -- & -- & $<1.0$ & -- & $<40$ & $<1.0$ \\
\hline $11 / 04 / 99$ & $<2.6$ & $<2.4$ & $<1.0$ & $<1.0$ & 8.8 & -- & -- & $<1.0$ & -- & $<31$ & $<1.0$ \\
\hline $01 / 21 / 00$ & $<2.6$ & $<2.4$ & $<1.0$ & $<1.0$ & 8.9 & -- & -- & $<1.0$ & -- & $<31$ & $<1.0$ \\
\hline $05 / 17 / 00$ & $<2.6$ & $<.70$ & $<1.0$ & $<1.0$ & 6.5 & 6.4 & $<.90$ & $<1.0$ & $<1.0$ & $<31$ & $<3.5$ \\
\hline $06 / 01 / 00$ & $<2.6$ & $<.70$ & $<1.0$ & $<1.0$ & 6.7 & 6.8 & $<.90$ & $<1.0$ & $<1.0$ & $<31$ & $<3.5$ \\
\hline $06 / 28 / 00$ & $<2.6$ & $<.70$ & $<1.0$ & $<1.0$ & 7.4 & 7.6 & $<.90$ & $<1.0$ & $<1.0$ & $<31$ & $<1.0$ \\
\hline $07 / 27 / 00$ & $<2.6$ & $<.70$ & $<1.0$ & $<1.0$ & 9.5 & 9.7 & $<.90$ & $<1.0$ & $<1.0$ & $<31$ & $<1.0$ \\
\hline $09 / 07 / 00$ & $<2.6$ & $<.70$ & $<1.0$ & $<1.0$ & 10 & 11 & $<.90$ & $<1.0$ & $<1.0$ & $<31$ & $<1.0$ \\
\hline $11 / 07 / 00$ & -- & -- & -- & -- & -- & -- & -- & -- & -- & $<1.0$ & $<1.1$ \\
\hline $01 / 24 / 01$ & -- & -- & -- & -- & -- & -- & -- & -- & -- & $<1.0$ & $<1.0$ \\
\hline $05 / 09 / 01$ & -- & -- & -- & -- & -- & -- & -- & -- & -- & $<1.0$ & $<1.0$ \\
\hline $06 / 13 / 01$ & -- & -- & -- & -- & -- & -- & -- & -- & -- & $<1.0$ & $<1.0$ \\
\hline 09/13/01 & -- & -- & -- & -- & -- & -- & -- & -- & -- & $<1.0$ & $<1.0$ \\
\hline \multicolumn{12}{|c|}{ SITE 35 12419000--SPOKANE RIVER NEAR POST FALLS, IDAHO } \\
\hline 03/04/99 & -- & -- & -- & -- & -- & -- & -- & -- & -- & -- & -- \\
\hline 04/13/99 & $<1.0$ & $<1.0$ & $<1.0$ & $<1.0$ & 21 & -- & -- & $<1.0$ & -- & 82 & 90 \\
\hline 05/19/99 & $<1.0$ & $<1.0$ & $<1.0$ & $<1.0$ & 9.6 & -- & -- & $<1.0$ & -- & $<40$ & 60 \\
\hline $06 / 02 / 99$ & $<1.0$ & $<1.0$ & $<1.0$ & $<1.0$ & 15 & -- & -- & $<1.0$ & -- & 69 & 58 \\
\hline 06/23/99 & $<1.0$ & $<1.0$ & $<1.0$ & $<1.0$ & 14 & -- & -- & $<1.0$ & -- & 63 & 46 \\
\hline $07 / 27 / 99^{\mathbf{F b}}$ & -- & -- & -- & $<.2$ & -- & $<.1$ & $<.1$ & $<.2$ & -- & -- & .52 \\
\hline 07/27/99 & $<1.0$ & $<1.0$ & $<1.0$ & $<1.0$ & 14 & -- & -- & $<1.0$ & -- & 43 & 44 \\
\hline 09/09/99 & $<1.0$ & $<1.0$ & $<1.0$ & $<1.0$ & 16 & -- & -- & $<1.0$ & -- & 36 & 31 \\
\hline $10 / 20 / 99$ & $<2.6$ & $\mathrm{e} 1.5$ & $<1.0$ & $<1.0$ & 15 & -- & -- & $<1.0$ & -- & 37 & 43 \\
\hline $11 / 30 / 99$ & -- & -- & -- & -- & -- & -- & -- & -- & -- & -- & -- \\
\hline $01 / 12 / 00$ & $<2.6$ & $<2.4$ & $<1.0$ & $<1.0$ & 18 & -- & -- & $<1.0$ & -- & 65 & 76 \\
\hline $02 / 29 / 00$ & $<2.6$ & $<.70$ & $<1.0$ & $<1.0$ & 18 & 21 & $<.90$ & $<1.0$ & $<1.0$ & 69 & 75 \\
\hline $03 / 28 / 00$ & $<2.6$ & $<.70$ & $<1.0$ & $<1.0$ & 20 & 20 & $<.90$ & $<1.0$ & $<1.0$ & 81 & 80 \\
\hline $04 / 11 / 00$ & $<2.6$ & $<.70$ & $<1.0$ & $<1.0$ & 20 & 20 & $<.90$ & $<1.0$ & $<1.0$ & 96 & 81 \\
\hline $04 / 18 / 00$ & $<2.6$ & $<.70$ & $<1.0$ & $<1.0$ & 20 & 19 & $<.90$ & $<1.0$ & $<1.0$ & 92 & 71 \\
\hline $05 / 03 / 00$ & $<2.6$ & $<2.4$ & $<1.0$ & $<1.0$ & 16 & 18 & $<.90$ & $<1.0$ & $<1.0$ & 67 & 56 \\
\hline $05 / 16 / 00$ & $<2.6$ & $<.70$ & $<1.0$ & $<1.0$ & 16 & 16 & $<.90$ & $<1.0$ & $<1.0$ & 62 & 46 \\
\hline $05 / 31 / 00$ & $<2.6$ & $<.70$ & $<1.0$ & $<1.0$ & 15 & 16 & $<.90$ & $<1.0$ & $<1.0$ & 63 & 51 \\
\hline $06 / 27 / 00$ & $<2.6$ & $<.70$ & $<1.0$ & $<1.0$ & 14 & 14 & $<.90$ & $<1.0$ & $<1.0$ & 47 & 38 \\
\hline $07 / 25 / 00$ & $<2.6$ & $<.70$ & $<1.0$ & $<1.0$ & 15 & 16 & $<.90$ & $<1.0$ & $<1.0$ & e29 & 38 \\
\hline $08 / 30 / 00$ & $<2.6$ & $<.70$ & $<1.0$ & $<1.0$ & 17 & 17 & $<.90$ & $<1.0$ & $<1.0$ & e26 & 22 \\
\hline $11 / 08 / 00$ & -- & -- & -- & -- & -- & -- & -- & -- & -- & 46 & 45 \\
\hline $12 / 21 / 00$ & -- & -- & -- & -- & -- & -- & -- & -- & -- & 68 & 66 \\
\hline
\end{tabular}


Table 8. Trace-element concentration data for surface-water samples collected at fixed water-quality sites, Northern Rockies Intermontane Basins study unit, 1998-2001 (Continued)

\begin{tabular}{|c|c|c|c|c|c|c|c|c|c|c|}
\hline Date & Time & $\begin{array}{l}\text { Alumi- } \\
\text { num, } \\
\text { total } \\
\text { recov- } \\
\text { erable } \\
(\mu \mathrm{g} / \mathrm{L})\end{array}$ & $\begin{array}{c}\text { Alumi- } \\
\text { num, } \\
\text { dis- } \\
\text { solved } \\
(\mu \mathrm{g} / \mathrm{L})\end{array}$ & $\begin{array}{c}\text { Anti- } \\
\text { mony, } \\
\text { total } \\
\text { recov- } \\
\text { erable } \\
(\mu \mathrm{g} / \mathrm{L})\end{array}$ & $\begin{array}{c}\text { Anti- } \\
\text { mony, } \\
\text { dis- } \\
\text { solved } \\
(\mu \mathrm{g} / \mathrm{L})\end{array}$ & $\begin{array}{c}\text { Arsenic, } \\
\text { total } \\
\text { recov- } \\
\text { erable } \\
(\mu \mathrm{g} / \mathrm{L})\end{array}$ & $\begin{array}{c}\text { Arsenic, } \\
\text { dis- } \\
\text { solved } \\
(\mu \mathrm{g} / \mathrm{L})\end{array}$ & $\begin{array}{c}\text { Barium, } \\
\text { total } \\
\text { recov- } \\
\text { erable } \\
(\mu \mathrm{g} / \mathrm{L})\end{array}$ & $\begin{array}{c}\text { Barium, } \\
\text { dis- } \\
\text { solved } \\
(\mu \mathrm{g} / \mathrm{L})\end{array}$ & $\begin{array}{c}\text { Beryl- } \\
\text { lium, } \\
\text { total } \\
\text { recov- } \\
\text { erable } \\
(\mu \mathrm{g} / \mathrm{L})\end{array}$ \\
\hline \multicolumn{11}{|c|}{ SITE 35 12419000--SPOKANE RIVER NEAR POST FALLS, IDAHO (Continued) } \\
\hline $01 / 22 / 01$ & 1330 & -- & -- & -- & -- & $<1.9$ & .53 & -- & -- & -- \\
\hline 03/13/01 & 1415 & -- & -- & -- & -- & $<1.9$ & .45 & -- & -- & -- \\
\hline $04 / 09 / 01$ & 0930 & -- & -- & -- & -- & $<1.9$ & .40 & -- & -- & -- \\
\hline 05/07/01 & 1345 & -- & -- & -- & -- & $<1.9$ & .38 & -- & -- & -- \\
\hline $05 / 08 / 01^{\mathbf{F b}}$ & 1100 & -- & $<.3$ & -- & $<.2$ & -- & -- & -- & $<.2$ & -- \\
\hline $06 / 11 / 01$ & 1230 & -- & -- & -- & -- & $<1.9$ & .39 & -- & -- & -- \\
\hline 08/13/01 & 1630 & -- & -- & -- & -- & $<1.9$ & .57 & -- & -- & -- \\
\hline $08 / 14 / 01$ & 0815 & -- & -- & -- & -- & $<1.9$ & .59 & -- & -- & -- \\
\hline \multicolumn{11}{|c|}{ SITE 41 12424500--SPOKANE RIVER AT SEVEN MILE BRIDGE, NEAR SPOKANE, WASH. } \\
\hline 03/03/99 & 1115 & -- & -- & -- & -- & -- & -- & -- & -- & -- \\
\hline $04 / 14 / 99$ & 1230 & 137 & 6.3 & $<1.0$ & $<1.0$ & $<1.0$ & $<1.0$ & 16 & 16 & $<4.0$ \\
\hline $04 / 14 / 99^{\mathbf{F b}}$ & 1235 & -- & $<.3$ & -- & $<.2$ & -- & -- & -- & $<.2$ & -- \\
\hline 05/18/99 & 1100 & 208 & 3.9 & $<1.0$ & $<1.0$ & $<1.0$ & $<1.0$ & 17 & 12 & $<4.0$ \\
\hline 06/03/99 & 1000 & 125 & 8.7 & $<1.0$ & $<1.0$ & 1.7 & $<1.0$ & 13 & 12 & $<4.0$ \\
\hline $06 / 24 / 99$ & 1015 & e23 & 5.7 & $<1.0$ & $<1.0$ & $<1.0$ & $<1.0$ & 13 & 13 & $<4.0$ \\
\hline 07/28/99 & 1000 & e16 & 4.7 & $<1.0$ & $<1.0$ & 1.9 & 1.5 & 20 & 20 & $<4.0$ \\
\hline 09/10/99 & 0940 & 240 & 16 & $<1.0$ & $<1.0$ & 2.7 & 3.2 & 27 & 26 & $<5.0$ \\
\hline $10 / 21 / 99$ & 1110 & $<28$ & 7.9 & $<1.0$ & $<1.0$ & e 1.4 & $\mathrm{e} 1.7$ & 18 & 19 & $<5.0$ \\
\hline $12 / 03 / 99$ & 0940 & -- & -- & -- & -- & -- & -- & -- & -- & -- \\
\hline $01 / 13 / 00$ & 1000 & 66 & 2.5 & $<1.0$ & $<1.0$ & $<2.6$ & $<2.0$ & 17 & 17 & $<5.0$ \\
\hline 03/01/00 & 0830 & 320 & 4.1 & $<1.0$ & $<1.0$ & $<2.6$ & e. 66 & 23 & 20 & $<5.0$ \\
\hline $03 / 29 / 00$ & 1000 & 113 & 2.5 & $<1.0$ & $<1.0$ & $<2.6$ & e. 62 & 16 & 15 & $<5.0$ \\
\hline $04 / 11 / 00$ & 1145 & -- & 2.6 & $<1.0$ & $<1.0$ & -- & e. 51 & -- & 15 & -- \\
\hline $04 / 19 / 00$ & 0945 & 1,140 & 15 & $<1.0$ & $<1.0$ & $<2.6$ & $<.90$ & 26 & 13 & $<5.0$ \\
\hline $05 / 03 / 00$ & 1245 & 109 & 6.6 & $<1.0$ & $<1.0$ & $<2.6$ & e.61 & 14 & 14 & $<5.0$ \\
\hline $05 / 16 / 00$ & 0930 & 80 & 8.8 & $<1.0$ & $<1.0$ & $<2.6$ & e. 61 & 13 & 13 & $<5.0$ \\
\hline $05 / 31 / 00$ & 1015 & 37 & 17 & $<1.0$ & $<1.0$ & $<2.6$ & e. 65 & 13 & 13 & $<5.0$ \\
\hline $06 / 27 / 00$ & 1000 & 34 & 5.3 & $<1.0$ & $<1.0$ & $<2.6$ & 1.2 & 16 & 16 & $<5.0$ \\
\hline $07 / 25 / 00$ & 0830 & $<28$ & 7.4 & $<1.0$ & $<1.0$ & e2.0 & 2.0 & 22 & 24 & $<5.0$ \\
\hline $08 / 30 / 00$ & 1000 & 76 & 14 & $<1.0$ & $<1.0$ & $\mathrm{e} 2.0$ & 2.6 & 26 & 26 & $<5.0$ \\
\hline $11 / 08 / 00$ & 1000 & -- & -- & -- & -- & $\mathrm{e} 1.2$ & 1.3 & -- & -- & -- \\
\hline $12 / 21 / 00$ & 0945 & -- & -- & -- & -- & e1.6 & 1.5 & -- & -- & -- \\
\hline $01 / 22 / 01$ & 0945 & -- & -- & -- & -- & 2.2 & 2.3 & -- & -- & -- \\
\hline 03/13/01 & 1030 & -- & -- & -- & -- & e1.7 & 1.5 & -- & -- & -- \\
\hline $04 / 09 / 01$ & 1315 & -- & -- & -- & -- & $\mathrm{e} 1.2$ & .77 & -- & -- & -- \\
\hline $05 / 07 / 01$ & 1000 & -- & -- & -- & -- & $<1.9$ & .51 & -- & -- & -- \\
\hline $06 / 11 / 01$ & 0900 & -- & -- & -- & -- & e1.4 & 1.0 & -- & -- & -- \\
\hline
\end{tabular}


Table 8. Trace-element concentration data for surface-water samples collected at fixed water-quality sites, Northern Rockies Intermontane Basins study unit, 1998-2001 (Continued)

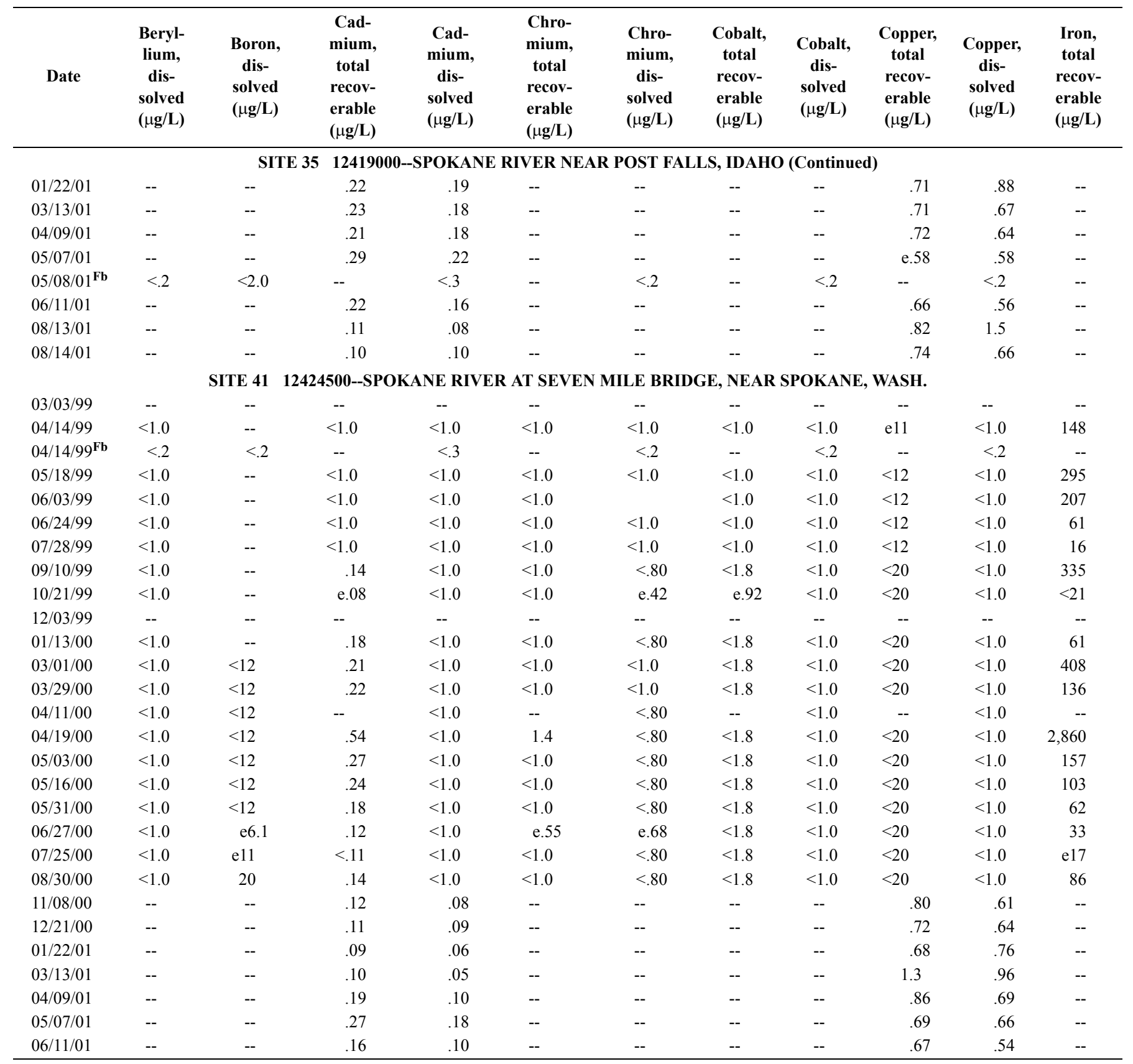


Table 8. Trace-element concentration data for surface-water samples collected at fixed water-quality sites, Northern Rockies Intermontane Basins study unit, 1998-2001 (Continued)

\begin{tabular}{|c|c|c|c|c|c|c|c|c|c|c|c|c|}
\hline Date & $\begin{array}{c}\text { Iron, } \\
\text { dis- } \\
\text { solved } \\
(\mu \mathrm{g} / \mathrm{L})\end{array}$ & $\begin{array}{c}\text { Lead, } \\
\text { total } \\
\text { recov- } \\
\text { erable } \\
(\mu \mathrm{g} / \mathrm{L})\end{array}$ & $\begin{array}{c}\text { Lead, } \\
\text { dis- } \\
\text { solved } \\
(\mu \mathrm{g} / \mathrm{L})\end{array}$ & $\begin{array}{l}\text { Lith- } \\
\text { ium, } \\
\text { total } \\
\text { recov- } \\
\text { erable } \\
(\mu \mathrm{g} / \mathrm{L})\end{array}$ & $\begin{array}{c}\text { Lith- } \\
\text { ium, } \\
\text { dis- } \\
\text { solved } \\
(\mu \mathrm{g} / \mathrm{L})\end{array}$ & $\begin{array}{c}\text { Manga- } \\
\text { nese, } \\
\text { total } \\
\text { recov- } \\
\text { erable } \\
(\mu \mathrm{g} / \mathrm{L})\end{array}$ & $\begin{array}{c}\text { Manga- } \\
\text { nese, } \\
\text { dis- } \\
\text { solved } \\
(\mu \mathrm{g} / \mathrm{L})\end{array}$ & $\begin{array}{c}\text { Mercury, } \\
\text { total } \\
\text { recov- } \\
\text { erable } \\
(\mu \mathrm{g} / \mathrm{L})\end{array}$ & $\begin{array}{c}\text { Molyb- } \\
\text { denum, } \\
\text { total } \\
\text { recov- } \\
\text { erable } \\
(\mu \mathrm{g} / \mathrm{L})\end{array}$ & $\begin{array}{c}\text { Molyb- } \\
\text { denum, } \\
\text { dis- } \\
\text { solved } \\
(\mu \mathrm{g} / \mathrm{L})\end{array}$ & $\begin{array}{c}\text { Nickel, } \\
\text { tota1 } \\
\text { recov- } \\
\text { erable } \\
(\mu \mathrm{g} / \mathrm{L})\end{array}$ & $\begin{array}{c}\text { Nickel } \\
\text { dis- } \\
\text { solved } \\
(\mu \mathrm{g} / \mathrm{L})\end{array}$ \\
\hline \multicolumn{13}{|c|}{ SITE 35 12419000--SPOKANE RIVER NEAR POST FALLS, IDAHO (Continued) } \\
\hline $01 / 22 / 01$ & $<10$ & $<1.0$ & .15 & -- & -- & -- & e2.0 & -- & -- & -- & -- & -- \\
\hline $03 / 13 / 01$ & e8.8 & $<1.0$ & .16 & -- & -- & -- & e2.1 & -- & -- & -- & -- & -- \\
\hline $04 / 09 / 01$ & $<10$ & $<1.0$ & e.04 & -- & -- & -- & $\mathrm{e} 2.5$ & -- & -- & -- & -- & -- \\
\hline $05 / 07 / 01$ & 20 & 1.9 & .41 & -- & -- & -- & e2.3 & -- & -- & -- & -- & -- \\
\hline $05 / 08 / 01^{\mathbf{F b}}$ & $<3.0$ & -- & $<.3$ & -- & -- & -- & $<.1$ & -- & -- & $<.2$ & -- & $<.5$ \\
\hline $06 / 11 / 01$ & $<10$ & 1.5 & .17 & -- & -- & -- & $<3.0$ & -- & -- & -- & -- & -- \\
\hline $08 / 13 / 01$ & $<10$ & 1.0 & .12 & -- & -- & -- & $\mathrm{e} 2.4$ & -- & -- & -- & -- & -- \\
\hline $08 / 14 / 01$ & e5.8 & 1.1 & .13 & -- & -- & -- & e1.7 & -- & -- & -- & -- & -- \\
\hline \multicolumn{13}{|c|}{ SITE 41 12424500--SPOKANE RIVER AT SEVEN MILE BRIDGE, NEAR SPOKANE, WASH. } \\
\hline 03/03/99 & 10 & -- & -- & -- & -- & -- & 3.6 & -- & -- & -- & -- & -- \\
\hline $04 / 14 / 99$ & 16 & 2.0 & $<1.0$ & $<16$ & -- & 6.4 & 2.2 & $<.10$ & $<1.0$ & $<1.0$ & $<1.0$ & $<1.0$ \\
\hline $04 / 14 / 99^{\mathbf{F b}}$ & $<3$ & -- & $<.3$ & -- & -- & -- & $<.1$ & -- & -- & $<.2$ & -- & $<.5$ \\
\hline 05/18/99 & 15 & 5.6 & $<1.0$ & $<12$ & -- & 13 & 1.3 & $<.10$ & $<1.0$ & $<1.0$ & $<1.0$ & $<1.0$ \\
\hline 06/03/99 & 14 & 8.1 & $<1.0$ & $<12$ & -- & 16 & 1.5 & $<.10$ & $<1.0$ & $<1.0$ & $<1.0$ & $<1.0$ \\
\hline $06 / 24 / 99$ & 13 & 2.4 & $<1.0$ & $<12$ & -- & 9.5 & 1.4 & $<.10$ & $<1.0$ & $<1.0$ & $<1.0$ & $<1.0$ \\
\hline $07 / 28 / 99$ & $<10$ & $<1.0$ & $<1.0$ & $<12$ & -- & 3.6 & 1.5 & $<.10$ & $<1.0$ & $<1.0$ & $<1.0$ & $<1.0$ \\
\hline 09/10/99 & $<10$ & 1.4 & $<1.0$ & e 3.6 & -- & 16 & 2.6 & $<.30$ & 1.4 & 1.2 & $<1.8$ & $<1.0$ \\
\hline $10 / 21 / 99$ & $<10$ & $<1.0$ & $<1.0$ & $<7.0$ & -- & 3.0 & 1.7 & $<.30$ & $<1.0$ & $<1.0$ & $<1.8$ & $<1.0$ \\
\hline $12 / 03 / 99$ & $<10$ & -- & -- & -- & -- & -- & $<2.2$ & -- & -- & -- & -- & -- \\
\hline $01 / 13 / 00$ & $<10$ & $<1.0$ & $<1.0$ & $<7.0$ & -- & 4.9 & 1.5 & $<.30$ & $<1.0$ & $<1.0$ & $<1.8$ & $<1.0$ \\
\hline $03 / 01 / 00$ & 14 & 1.3 & $<1.0$ & $<7.0$ & .88 & 13 & 2.3 & $<.30$ & $<1.0$ & $<1.0$ & $<1.8$ & $<1.0$ \\
\hline $03 / 29 / 00$ & 14 & 1.8 & $<1.0$ & $<7.0$ & .76 & 6.3 & 1.5 & $<.30$ & $<1.0$ & $<1.0$ & $<1.8$ & $<1.0$ \\
\hline $04 / 11 / 00$ & 15 & $<1.0$ & $<1.0$ & 27 & .69 & -- & 1.4 & $<.30$ & -- & $<1.0$ & -- & $<1.0$ \\
\hline $04 / 19 / 00$ & 30 & 16 & $<1.0$ & $<7.0$ & .63 & 56 & 2.6 & $<.30$ & $<1.0$ & $<1.0$ & 1.8 & $<1.0$ \\
\hline $05 / 03 / 00$ & 21 & 7.9 & 1.4 & $<7.0$ & .76 & 14 & 3.0 & $<.30$ & $<1.0$ & $<1.0$ & $<1.8$ & $<1.0$ \\
\hline $05 / 16 / 00$ & 15 & 3.3 & $<1.0$ & $<7.0$ & .87 & 13 & 1.6 & $<.30$ & $<1.0$ & $<1.0$ & $<1.8$ & $<1.0$ \\
\hline $05 / 31 / 00$ & 15 & 2.2 & $<1.0$ & $<7.0$ & 1.0 & 8.6 & 1.2 & $<.30$ & $<1.0$ & $<1.0$ & $<1.8$ & $<1.0$ \\
\hline $06 / 27 / 00$ & $<10$ & e. 88 & $<1.0$ & $<7.0$ & 1.2 & 5.0 & 1.3 & $<.30$ & $<1.0$ & $<1.0$ & $<1.8$ & $<1.0$ \\
\hline $07 / 25 / 00$ & $<10$ & 2.1 & $<1.0$ & $<7.0$ & 2.7 & 4.1 & 1.2 & $<.30$ & 1.5 & $<1.0$ & $<1.8$ & $<1.0$ \\
\hline $08 / 30 / 00$ & $<10$ & 1.6 & $<1.0$ & $<7.0$ & 3.5 & 11 & 1.9 & $<.30$ & 1.8 & 1.4 & $<1.8$ & $<1.0$ \\
\hline $11 / 08 / 00$ & $<10$ & $<1.0$ & .11 & -- & -- & -- & $\mathrm{e} 2.0$ & -- & -- & -- & -- & -- \\
\hline $12 / 21 / 00$ & $<10$ & $<1.0$ & .10 & -- & -- & -- & $<3.2$ & -- & -- & -- & -- & -- \\
\hline $01 / 22 / 01$ & $<10$ & $<1.0$ & .10 & -- & -- & -- & $\mathrm{e} 2.0$ & -- & -- & -- & -- & -- \\
\hline 03/13/01 & e8.2 & $<1.0$ & e.06 & -- & -- & -- & e3.1 & -- & -- & -- & -- & -- \\
\hline $04 / 09 / 01$ & $<10$ & $<1.0$ & e.06 & -- & -- & -- & $\mathrm{e} 2.5$ & -- & -- & -- & -- & -- \\
\hline 05/07/01 & 12 & 2.5 & .32 & -- & -- & -- & $\mathrm{e} 2.9$ & -- & -- & -- & -- & -- \\
\hline $06 / 11 / 01$ & $<10$ & $<1.0$ & .11 & -- & -- & -- & $\mathrm{e} 1.7$ & -- & -- & -- & -- & -- \\
\hline
\end{tabular}


Table 8. Trace-element concentration data for surface-water samples collected at fixed water-quality sites, Northern Rockies Intermontane Basins study unit, 1998-2001 (Continued)

\begin{tabular}{|c|c|c|c|c|c|c|c|c|c|c|c|}
\hline Date & $\begin{array}{c}\text { Selenium, } \\
\text { total } \\
\text { recov- } \\
\text { erable } \\
(\mu \mathrm{g} / \mathrm{L})\end{array}$ & $\begin{array}{c}\text { Selenium, } \\
\text { dis- } \\
\text { solved } \\
(\mu \mathrm{g} / \mathrm{L})\end{array}$ & $\begin{array}{c}\text { Silver, } \\
\text { total } \\
\text { recov- } \\
\text { erable } \\
(\mu \mathrm{g} / \mathrm{L})\end{array}$ & $\begin{array}{c}\text { Silver, } \\
\text { dis- } \\
\text { solved } \\
(\mu \mathrm{g} / \mathrm{L})\end{array}$ & $\begin{array}{l}\text { Stron- } \\
\text { tium, } \\
\text { total } \\
\text { recov- } \\
\text { erable } \\
(\mu \mathrm{g} / \mathrm{L})\end{array}$ & $\begin{array}{c}\text { Stron- } \\
\text { tium, } \\
\text { dis- } \\
\text { solved } \\
(\mu \mathrm{g} / \mathrm{L})\end{array}$ & $\begin{array}{c}\text { Thal- } \\
\text { lium, } \\
\text { dis- } \\
\text { solved } \\
(\mu \mathrm{g} / \mathrm{L})\end{array}$ & $\begin{array}{c}\text { Uranium, } \\
\text { natural } \\
(\mu \mathrm{g} / \mathrm{L})\end{array}$ & $\begin{array}{c}\text { Vana- } \\
\text { dium, } \\
\text { dis- } \\
\text { solved } \\
(\mu \mathrm{g} / \mathrm{L})\end{array}$ & $\begin{array}{l}\text { Zinc, } \\
\text { total } \\
\text { recov- } \\
\text { erable } \\
(\mu \mathrm{g} / \mathrm{L})\end{array}$ & $\begin{array}{c}\text { Zinc, } \\
\text { dis- } \\
\text { solved } \\
(\mu \mathrm{g} / \mathrm{L})\end{array}$ \\
\hline \multicolumn{12}{|c|}{ SITE 35 12419000--SPOKANE RIVER NEAR POST FALLS, IDAHO (Continued) } \\
\hline $01 / 22 / 01$ & -- & -- & -- & -- & -- & -- & -- & -- & -- & 60 & 66 \\
\hline 03/13/01 & -- & -- & -- & -- & -- & -- & -- & -- & -- & 66 & 65 \\
\hline $04 / 09 / 01$ & -- & -- & -- & -- & -- & -- & -- & -- & -- & 65 & 61 \\
\hline $05 / 07 / 01$ & -- & -- & -- & -- & -- & -- & -- & -- & -- & 71 & 60 \\
\hline $05 / 08 / 01^{\mathbf{F b}}$ & -- & -- & -- & $<.2$ & -- & $<.1$ & $<.1$ & $<.2$ & -- & -- & $<.5$ \\
\hline $06 / 11 / 01$ & -- & -- & -- & -- & -- & -- & -- & -- & -- & 51 & 46 \\
\hline $08 / 13 / 01$ & -- & -- & -- & -- & -- & -- & -- & -- & -- & 30 & 28 \\
\hline $08 / 14 / 01$ & -- & -- & -- & -- & -- & -- & -- & -- & -- & 33 & 30 \\
\hline \multicolumn{12}{|c|}{ SITE 41 12424500--SPOKANE RIVER AT SEVEN MILE BRIDGE, NEAR SPOKANE, WASH. } \\
\hline 03/03/99 & -- & -- & -- & -- & -- & -- & -- & -- & -- & -- & -- \\
\hline 04/14/99 & $<1.0$ & $<1.0$ & $<1.0$ & $<1.0$ & 32 & -- & -- & $<1.0$ & -- & 70 & 76 \\
\hline $04 / 14 / 99^{\mathbf{F b}}$ & -- & -- & -- & $<.2$ & -- & $<.1$ & $<.1$ & $<.2$ & -- & -- & $<.5$ \\
\hline 05/18/99 & $<1.0$ & $<1.0$ & $<1.0$ & $<1.0$ & 27 & -- & -- & $<1.0$ & -- & 73 & -- \\
\hline 06/03/99 & $<1.0$ & $<1.0$ & $<1.0$ & $<1.0$ & 19 & -- & -- & $<1.0$ & -- & 68 & 51 \\
\hline $06 / 24 / 99$ & $<1.0$ & $<1.0$ & $<1.0$ & $<1.0$ & 24 & -- & -- & $<1.0$ & -- & 52 & 39 \\
\hline 07/28/99 & $<1.0$ & $<1.0$ & $<1.0$ & $<1.0$ & 60 & -- & -- & 1.3 & -- & 46 & 24 \\
\hline 09/10/99 & $<2.6$ & $<2.4$ & $<1.0$ & $<1.0$ & 90 & -- & -- & 2.3 & -- & $<31$ & 10 \\
\hline $10 / 21 / 99$ & $<2.6$ & $<2.4$ & $<1.0$ & $<1.0$ & 52 & -- & -- & $<1.0$ & -- & $\mathrm{e} 24$ & 27 \\
\hline $12 / 03 / 99$ & -- & -- & -- & -- & -- & -- & -- & -- & -- & -- & -- \\
\hline $01 / 13 / 00$ & $<2.6$ & $<2.4$ & $<1.0$ & $<1.0$ & 42 & -- & -- & $<1.0$ & -- & 48 & 49 \\
\hline $03 / 01 / 00$ & $<2.6$ & $<.70$ & $<1.0$ & $<1.0$ & 37 & 40 & $<.90$ & $<1.0$ & $<1.0$ & 58 & 49 \\
\hline 03/29/00 & $<2.6$ & $<.70$ & $<1.0$ & $<1.0$ & 30 & 31 & $<.90$ & $<1.0$ & $<1.0$ & 71 & 66 \\
\hline $04 / 11 / 00$ & -- & $<.70$ & -- & $<1.0$ & -- & 26 & $<.90$ & $<1.0$ & $<1.0$ & -- & 71 \\
\hline 04/19/00 & $<2.6$ & $<.70$ & $<1.0$ & $<1.0$ & 24 & 22 & $<.90$ & $<1.0$ & $<1.0$ & 114 & 64 \\
\hline $05 / 03 / 00$ & $<2.6$ & $<.70$ & $<1.0$ & $<1.0$ & 22 & 25 & $<.90$ & $<1.0$ & $<1.0$ & 61 & 51 \\
\hline $05 / 16 / 00$ & $<2.6$ & $<.70$ & $<1.0$ & $<1.0$ & 25 & 25 & $<.90$ & $<1.0$ & $<1.0$ & 46 & 40 \\
\hline $05 / 31 / 00$ & $<2.6$ & $<.70$ & $<1.0$ & $<1.0$ & 26 & 28 & $<.90$ & $<1.0$ & $<1.0$ & 46 & 41 \\
\hline $06 / 27 / 00$ & $<2.6$ & $<.70$ & $<1.0$ & $<1.0$ & 42 & 40 & $<.90$ & $<1.0$ & $<1.0$ & 37 & 24 \\
\hline $07 / 25 / 00$ & $<2.6$ & $<.70$ & $<1.0$ & $<1.0$ & 75 & 79 & $<.90$ & 2.0 & $<1.0$ & $<31$ & 14 \\
\hline $08 / 30 / 00$ & $<2.6$ & $<.70$ & $<1.0$ & $<1.0$ & 93 & 95 & $<.90$ & 2.2 & $<1.0$ & $\mathrm{e} 20$ & 8.5 \\
\hline $11 / 08 / 00$ & -- & -- & -- & -- & -- & -- & -- & -- & -- & 35 & 32 \\
\hline $12 / 21 / 00$ & -- & -- & -- & -- & -- & -- & -- & -- & -- & 40 & 38 \\
\hline $01 / 22 / 01$ & -- & -- & -- & -- & -- & -- & -- & -- & -- & 22 & 22 \\
\hline 03/13/01 & -- & -- & -- & -- & -- & -- & -- & -- & -- & 28 & 20 \\
\hline $04 / 09 / 01$ & -- & -- & -- & -- & -- & -- & -- & -- & -- & 48 & 40 \\
\hline $05 / 07 / 01$ & -- & -- & -- & -- & -- & -- & -- & -- & -- & 69 & 56 \\
\hline $06 / 11 / 01$ & -- & -- & -- & -- & -- & -- & -- & -- & -- & 36 & 31 \\
\hline
\end{tabular}


Table 9. Pesticide concentration data for filtered surface-water samples collected at selected fixed water-quality sites, Northern Rockies Intermontane Basins study unit, 1999-2000

[See figure 1 for site locations. Bold text denotes detected concentrations. Abbreviations: $\mathrm{ft}^{3} / \mathrm{s}$, cubic feet per second; e, estimated; Fb, field blank; $\mu \mathrm{g} / \mathrm{L}$, micrograms per liter. Symbols: <, less than reporting level; --, no data]

\begin{tabular}{|c|c|c|c|c|c|c|c|c|c|c|c|c|}
\hline Date & Time & $\begin{array}{c}\text { Discharge, } \\
\text { instanta- } \\
\text { neous } \\
\left(\mathbf{f t}^{3} / \mathbf{s}\right)\end{array}$ & $\begin{array}{c}2,6- \\
\text { Diethyl- } \\
\text { aniline } \\
(\mu \mathrm{g} / \mathrm{L})\end{array}$ & $\begin{array}{c}\text { Aceto- } \\
\text { chlor } \\
(\mu \mathrm{g} / \mathrm{L})\end{array}$ & $\begin{array}{c}\text { Alachlor } \\
(\mu \mathrm{g} / \mathrm{L})\end{array}$ & $\begin{array}{c}\text { alpha- } \\
\text { HCH } \\
(\mu \mathrm{g} / \mathrm{L})\end{array}$ & $\begin{array}{l}\text { alpha- } \\
\text { HCH-d }_{6} \text {, } \\
\text { surrogate } \\
\text { (percent) }\end{array}$ & $\begin{array}{c}\text { Atra- } \\
\text { zine } \\
(\mu \mathrm{g} / \mathrm{L})\end{array}$ & $\begin{array}{l}\text { Azin- } \\
\text { phos- } \\
\text { methyl } \\
(\mu \mathrm{g} / \mathrm{L})\end{array}$ & $\begin{array}{c}\text { Ben- } \\
\text { fluralin } \\
(\mu \mathrm{g} / \mathrm{L})\end{array}$ & $\begin{array}{c}\text { Butyl- } \\
\text { ate } \\
(\mu \mathrm{g} / \mathrm{L})\end{array}$ & $\begin{array}{c}\text { Carb- } \\
\text { aryl } \\
(\mu \mathrm{g} / \mathrm{L})\end{array}$ \\
\hline 03/04/99 & 1630 & 1,150 & $<0.003$ & $<0.002$ & $<0.002$ & $<0.002$ & 84 & $<0.001$ & $<0.001$ & $<0.002$ & $<0.002$ & $<0.003$ \\
\hline $03 / 24 / 99$ & 0930 & 2,150 & $<.003$ & $<.002$ & $<.002$ & $<.002$ & 84 & $<.001$ & $<.001$ & $<.002$ & $<.002$ & $<.003$ \\
\hline $05 / 13 / 99$ & 0945 & 2,270 & $<.003$ & $<.002$ & $<.002$ & $<.002$ & 85 & $<.001$ & $<.001$ & $<.002$ & $<.002$ & $<.003$ \\
\hline $07 / 21 / 99$ & 1530 & 2,010 & $<.003$ & $<.002$ & $<.002$ & $<.002$ & 83 & $<.001$ & $<.001$ & $<.002$ & $<.002$ & $<.003$ \\
\hline 08/19/99 & 1500 & 1,180 & $<.003$ & $<.002$ & $<.002$ & $<.002$ & 92 & .006 & $<.001$ & $<.002$ & $<.002$ & $<.003$ \\
\hline $10 / 20 / 99$ & 1430 & 760 & $<.003$ & $<.002$ & $<.002$ & $<.002$ & 104 & $<.001$ & $<.001$ & $<.002$ & $<.002$ & $<.003$ \\
\hline $03 / 08 / 00$ & 1415 & $\mathrm{e} 1,050$ & $<.003$ & $<.002$ & $<.002$ & $<.002$ & 99 & $<.001$ & $<.001$ & $<.002$ & $<.002$ & $<.003$ \\
\hline $05 / 23 / 00$ & 1015 & 7,960 & $<.003$ & $<.002$ & $<.002$ & $<.002$ & 98 & $<.006$ & $<.001$ & $<.002$ & $<.002$ & $<.003$ \\
\hline $08 / 18 / 99$ & 0930 & 3,960 & $<.003$ & $<.002$ & $<.002$ & $<.002$ & 107 & $<.001$ & $<.001$ & $<.002$ & $<.002$ & $<.003$ \\
\hline $10 / 18 / 99$ & 1415 & 2,790 & $<.003$ & $<.002$ & $<.002$ & $<.002$ & 98 & $<.001$ & $<.001$ & $<.002$ & $<.002$ & $<.003$ \\
\hline \multicolumn{13}{|c|}{ SITE 12 12388700--FLATHEAD RIVER AT PERMA, MONT. } \\
\hline $05 / 11 / 99$ & 1100 & 14,800 & $<.003$ & $<.002$ & $<.002$ & $<.002$ & 96 & $<.001$ & $<.001$ & $<.002$ & $<.002$ & $<.003$ \\
\hline $07 / 19 / 99$ & 1430 & 17,500 & $<.003$ & $<.002$ & $<.002$ & $<.002$ & 86 & $<.001$ & $<.001$ & $<.002$ & $<.002$ & $<.003$ \\
\hline 08/17/99 & 1330 & 9,880 & $<.003$ & $<.002$ & $<.002$ & $<.002$ & 104 & $<.001$ & $<.001$ & $<.002$ & $<.002$ & $<.003$ \\
\hline $10 / 19 / 99$ & 1140 & 5,470 & $<.003$ & $<.002$ & $<.002$ & $<.002$ & 98 & $<.001$ & $<.001$ & $<.002$ & $<.002$ & $<.003$ \\
\hline \multicolumn{13}{|c|}{ SITE 35 12419000--SPOKANE RIVER NEAR POST FALLS, IDAHO } \\
\hline $04 / 13 / 99$ & 1230 & 9,760 & $<.003$ & $<.002$ & $<.002$ & $<.002$ & 69 & $<.001$ & $<.001$ & $<.002$ & $<.002$ & $<.003$ \\
\hline $05 / 03 / 00$ & 0800 & 21,200 & $<.003$ & $<.002$ & $<.002$ & $<.002$ & 94 & $<.001$ & $<.001$ & $<.002$ & $<.002$ & $<.006$ \\
\hline $05 / 31 / 00$ & 1345 & 12,600 & $<.003$ & $<.002$ & $<.002$ & $<.002$ & 107 & $<.001$ & $<.001$ & $<.002$ & $<.002$ & e.004 \\
\hline $06 / 27 / 00$ & 1415 & 3,860 & $<.003$ & $<.002$ & $<.002$ & $<.002$ & 102 & $<.001$ & $<.001$ & $<.002$ & $<.002$ & $<.003$ \\
\hline 08/30/00 & 1415 & 242 & $<.003$ & $<.002$ & $<.002$ & $<.002$ & 106 & $<.001$ & $<.001$ & $<.002$ & $<.002$ & $<.003$ \\
\hline \multicolumn{13}{|c|}{ SITE 40 12424000--HANGMAN CREEK AT SPOKANE, WASH. } \\
\hline $02 / 16 / 00$ & 1110 & 762 & $<.003$ & $<.002$ & $<.002$ & $<.002$ & 105 & e.004 & $<.001$ & $<.002$ & $<.002$ & $<.003$ \\
\hline $03 / 20 / 00$ & 1100 & 433 & $<.003$ & $<.002$ & $<.002$ & $<.002$ & 90 & .005 & $<.001$ & $<.002$ & $<.002$ & $<.003$ \\
\hline $03 / 20 / 00^{\mathbf{F b}}$ & 1108 & -- & $<.003$ & $<.002$ & $<.002$ & $<.002$ & 91 & $<.001$ & $<.001$ & $<.002$ & $<.002$ & $<.003$ \\
\hline $04 / 14 / 00$ & 1230 & 1,240 & $<.003$ & $<.002$ & $<.005$ & $<.002$ & 89 & .036 & $<.001$ & $<.002$ & .009 & $<.003$ \\
\hline $05 / 04 / 00$ & 1045 & 249 & $<.003$ & $<.002$ & $<.002$ & $<.002$ & 94 & $<.074$ & $<.001$ & $<.002$ & $<.002$ & e.007 \\
\hline $06 / 01 / 00$ & 1000 & 124 & $<.003$ & $<.002$ & $<.002$ & $<.002$ & 85 & $<.001$ & $<.001$ & $<.002$ & $<.002$ & $<.003$ \\
\hline $06 / 29 / 00$ & 1315 & 47.6 & $<.003$ & $<.002$ & $<.002$ & $<.002$ & 89 & $<.006$ & $<.001$ & $<.002$ & $<.002$ & $<.003$ \\
\hline $08 / 30 / 00$ & 1130 & 21.0 & $<.003$ & $<.002$ & $<.002$ & $<.002$ & 88 & $<.001$ & $<.001$ & $<.002$ & $<.002$ & $<.003$ \\
\hline
\end{tabular}


Table 9. Pesticide concentration data for filtered surface-water samples collected at selected fixed water-quality sites, Northern Rockies Intermontane Basins study unit, 1999-2000 (Continued)

\begin{tabular}{|c|c|c|c|c|c|c|c|c|c|c|}
\hline Date & $\begin{array}{c}\text { Carbo- } \\
\text { furan } \\
(\mu \mathrm{g} / \mathrm{L})\end{array}$ & $\begin{array}{l}\text { Chlor- } \\
\text { pyrifos } \\
(\mu \mathrm{g} / \mathrm{L})\end{array}$ & $\begin{array}{c}\text { cis-Per- } \\
\text { methrin } \\
(\mu \mathrm{g} / \mathrm{L})\end{array}$ & $\begin{array}{l}\text { Cyan- } \\
\text { azine } \\
(\mu \mathrm{g} / \mathrm{L})\end{array}$ & $\begin{array}{c}\text { Dacthal } \\
(\mu \mathrm{g} / \mathrm{L})\end{array}$ & $\begin{array}{c}\text { Deethyl- } \\
\text { atra- } \\
\text { zine } \\
(\mu \mathrm{g} / \mathrm{L})\end{array}$ & $\begin{array}{c}\text { Diaz- } \\
\text { inon } \\
(\mu \mathrm{g} / \mathrm{L})\end{array}$ & $\begin{array}{l}\text { Diazinon- } \\
\quad d_{10}, \\
\text { surrogate } \\
\text { (percent) }\end{array}$ & $\begin{array}{c}\text { Dieldrin } \\
(\mu \mathrm{g} / \mathrm{L})\end{array}$ & $\begin{array}{c}\text { Disul- } \\
\text { foton } \\
(\mu \mathrm{g} / \mathrm{L})\end{array}$ \\
\hline \multicolumn{11}{|c|}{ SITE 5 12352500--BITTERROOT RIVER NEAR MISSOULA, MONT. } \\
\hline 03/04/99 & $<0.003$ & $<0.004$ & $<0.005$ & $<0.004$ & $<0.002$ & $<0.002$ & $<0.002$ & 103 & $<0.001$ & $<0.017$ \\
\hline 03/24/99 & $<.003$ & $<.004$ & $<.005$ & $<.004$ & $<.002$ & $<.002$ & $<.002$ & 81 & $<.001$ & $<.017$ \\
\hline 05/13/99 & $<.003$ & $<.004$ & $<.005$ & $<.004$ & $<.002$ & $<.002$ & $<.002$ & 106 & $<.001$ & $<.017$ \\
\hline 06/07/99 & $<.003$ & $<.004$ & $<.005$ & $<.004$ & $<.002$ & $<.002$ & $<.002$ & 122 & $<.001$ & $<.017$ \\
\hline $06 / 19 / 99$ & $<.003$ & $<.004$ & $<.005$ & $<.004$ & $<.002$ & $<.002$ & $<.002$ & 94 & $<.001$ & $<.017$ \\
\hline 07/21/99 & $<.003$ & $<.004$ & $<.005$ & $<.004$ & $<.002$ & $<.002$ & $<.002$ & 93 & $<.001$ & $<.017$ \\
\hline 08/19/99 & $<.003$ & $<.004$ & $<.005$ & $<.004$ & $<.002$ & $<.002$ & $<.002$ & 112 & $<.001$ & $<.017$ \\
\hline $10 / 20 / 99$ & $<.003$ & $<.004$ & $<.005$ & $<.004$ & $<.002$ & $<.002$ & $<.002$ & 91 & $<.001$ & $<.017$ \\
\hline $03 / 08 / 00$ & $<.003$ & $<.004$ & $<.005$ & $<.004$ & $<.002$ & $<.002$ & $<.002$ & 101 & $<.001$ & $<.017$ \\
\hline $05 / 23 / 00$ & $<.003$ & $<.004$ & $<.005$ & $<.004$ & $<.002$ & $<.002$ & $<.002$ & 103 & $<.001$ & $<.017$ \\
\hline $06 / 20 / 00$ & $<.003$ & $<.004$ & $<.005$ & $<.004$ & $<.002$ & $<.002$ & e.003 & 99 & $<.001$ & $<.017$ \\
\hline \multicolumn{11}{|c|}{ SITE 9 12354500-CLARK FORK AT ST. REGIS, MONT. } \\
\hline 05/10/99 & $<.003$ & $<.004$ & $<.005$ & $<.004$ & $<.002$ & $<.002$ & $<.002$ & 92 & $<.001$ & $<.017$ \\
\hline 08/18/99 & $<.003$ & $<.004$ & $<.005$ & $<.004$ & $<.002$ & $<.002$ & $<.002$ & 103 & $<.001$ & $<.017$ \\
\hline $10 / 18 / 99$ & $<.003$ & $<.004$ & $<.005$ & $<.004$ & $<.002$ & $<.002$ & $<.002$ & 107 & $<.001$ & $<.017$ \\
\hline \multicolumn{11}{|c|}{ SITE 12 12388700--FLATHEAD RIVER AT PERMA, MONT. } \\
\hline 05/11/99 & $<.003$ & $<.004$ & $<.005$ & $<.004$ & $<.002$ & $<.002$ & $<.002$ & 97 & $<.001$ & $<.017$ \\
\hline 07/19/99 & $<.003$ & $<.004$ & $<.005$ & $<.004$ & $<.002$ & $<.002$ & $<.002$ & 104 & $<.001$ & $<.017$ \\
\hline $08 / 17 / 99$ & $<.003$ & $<.004$ & $<.005$ & $<.004$ & $<.002$ & $<.002$ & $<.002$ & 107 & $<.001$ & $<.017$ \\
\hline $10 / 19 / 99$ & $<.003$ & $<.004$ & $<.005$ & $<.004$ & $<.002$ & $<.002$ & $<.002$ & 109 & $<.001$ & $<.017$ \\
\hline \multicolumn{11}{|c|}{ SITE 35 12419000--SPOKANE RIVER NEAR POST FALLS, IDAHO } \\
\hline 04/13/99 & $<.003$ & $<.004$ & $<.005$ & $<.004$ & $<.002$ & $<.002$ & $<.002$ & 99 & $<.001$ & $<.017$ \\
\hline $04 / 13 / 99^{\mathbf{F b}}$ & $<.003$ & $<.004$ & $<.005$ & $<.004$ & $<.002$ & $<.002$ & $<.002$ & 96 & $<.001$ & $<.017$ \\
\hline 05/19/99 & $<.003$ & $<.004$ & $<.005$ & $<.004$ & $<.002$ & $<.002$ & $<.002$ & 101 & $<.001$ & $<.017$ \\
\hline $06 / 23 / 99$ & $<.003$ & $<.004$ & $<.005$ & $<.004$ & $<.002$ & $<.002$ & $<.002$ & 91 & $<.001$ & $<.017$ \\
\hline 07/27/99 & $<.003$ & $<.004$ & $<.005$ & $<.004$ & $<.002$ & $<.002$ & $<.002$ & 112 & $<.001$ & $<.017$ \\
\hline 09/09/99 & $<.003$ & $<.004$ & $<.005$ & $<.004$ & $<.002$ & $<.002$ & $<.002$ & 96 & $<.001$ & $<.017$ \\
\hline $10 / 20 / 99$ & $<.003$ & $<.004$ & $<.005$ & $<.004$ & e.003 & $<.002$ & $<.002$ & 101 & $<.001$ & $<.017$ \\
\hline $03 / 28 / 00$ & $<.003$ & $<.004$ & $<.005$ & $<.004$ & $<.002$ & $<.002$ & $<.002$ & 115 & $<.001$ & $<.017$ \\
\hline $05 / 03 / 00$ & $<.003$ & $<.004$ & $<.005$ & $<.004$ & $<.002$ & $<.002$ & $<.002$ & 108 & $<.001$ & $<.017$ \\
\hline $05 / 31 / 00$ & $<.003$ & $<.004$ & $<.005$ & $<.004$ & $<.002$ & $<.002$ & $<.002$ & 112 & $<.001$ & $<.017$ \\
\hline $06 / 27 / 00$ & $<.003$ & $<.004$ & $<.005$ & $<.004$ & $<.002$ & $<.002$ & $<.002$ & 111 & $<.001$ & $<.017$ \\
\hline $08 / 30 / 00$ & $<.003$ & $<.004$ & $<.005$ & $<.004$ & $<.002$ & $<.002$ & e.002 & 108 & $<.001$ & $<.017$ \\
\hline \multicolumn{11}{|c|}{ SITE 40 12424000--HANGMAN CREEK AT SPOKANE, WASH. } \\
\hline $02 / 16 / 00$ & $<.003$ & $<.004$ & $<.005$ & $<.004$ & $<.002$ & $<.002$ & $<.002$ & 115 & $<.001$ & $<.017$ \\
\hline $03 / 20 / 00$ & $<.003$ & $<.004$ & $<.005$ & $<.004$ & $<.002$ & $<.002$ & $<.002$ & 87 & $<.001$ & $<.017$ \\
\hline $03 / 20 / 00^{\mathbf{F b}}$ & $<.003$ & $<.004$ & $<.005$ & $<.004$ & $<.002$ & $<.002$ & $<.002$ & 85 & $<.001$ & $<.017$ \\
\hline $04 / 14 / 00$ & $<.003$ & $<.004$ & $<.005$ & $<.004$ & e.003 & e. 005 & $<.005$ & 107 & $<.001$ & $<.017$ \\
\hline $05 / 04 / 00$ & $<.003$ & $<.004$ & $<.005$ & $<.004$ & $<.002$ & $<.087$ & $<.002$ & 112 & $<.001$ & $<.017$ \\
\hline $06 / 01 / 00$ & $<.003$ & $<.004$ & $<.005$ & $<.004$ & $<.002$ & $<.002$ & $<.002$ & 92 & $<.001$ & $<.017$ \\
\hline $06 / 29 / 00$ & $<.003$ & $<.004$ & $<.005$ & $<.004$ & $<.002$ & $<.002$ & $<.002$ & 98 & $<.001$ & $<.017$ \\
\hline $08 / 30 / 00$ & $<.003$ & $<.004$ & $<.005$ & $<.004$ & $<.002$ & $<.002$ & $<.002$ & 120 & $<.001$ & $<.017$ \\
\hline
\end{tabular}


Table 9. Pesticide concentration data for filtered surface-water samples collected at selected fixed water-quality sites, Northern Rockies Intermontane Basins study unit, 1999-2000 (Continued)

\begin{tabular}{|c|c|c|c|c|c|c|c|c|c|c|}
\hline Date & $\begin{array}{l}\text { EPTC } \\
(\mu \mathrm{g} / \mathrm{L})\end{array}$ & $\begin{array}{c}\text { Ethal- } \\
\text { fluralin } \\
(\mu \mathrm{g} / \mathbf{L})\end{array}$ & $\begin{array}{c}\text { Etho- } \\
\text { pro- } \\
\text { phos } \\
(\mu \mathrm{g} / \mathrm{L})\end{array}$ & $\begin{array}{c}\text { Fonofos } \\
(\mu \mathbf{g} / \mathbf{L})\end{array}$ & $\begin{array}{c}\text { Lindane } \\
(\mu \mathrm{g} / \mathrm{L})\end{array}$ & $\begin{array}{c}\text { Linuron } \\
(\mu \mathbf{g} / \mathbf{L})\end{array}$ & $\begin{array}{c}\text { Mala- } \\
\text { thion } \\
(\mu \mathrm{g} / \mathrm{L})\end{array}$ & $\begin{array}{l}\text { Metol- } \\
\text { achlor } \\
(\mu \mathrm{g} / \mathrm{L})\end{array}$ & $\begin{array}{c}\text { Metri- } \\
\text { buzin } \\
(\mu \mathrm{g} / \mathrm{L})\end{array}$ & $\begin{array}{c}\text { Molin- } \\
\text { ate } \\
(\mu \mathrm{g} / \mathrm{L})\end{array}$ \\
\hline \multicolumn{11}{|c|}{ 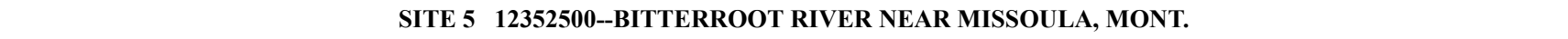 } \\
\hline 03/04/99 & $<0.002$ & $<0.004$ & $<0.003$ & $<0.003$ & $<0.004$ & $<0.002$ & $<0.005$ & $<0.002$ & $<0.004$ & $<0.004$ \\
\hline $03 / 24 / 99$ & $<.002$ & $<.004$ & $<.003$ & $<.003$ & $<.004$ & $<.002$ & $<.005$ & $<.002$ & $<.004$ & $<.004$ \\
\hline 05/13/99 & $<.002$ & $<.004$ & $<.003$ & $<.003$ & $<.004$ & $<.002$ & $<.005$ & $<.002$ & $<.004$ & $<.004$ \\
\hline $06 / 07 / 99$ & $<.002$ & $<.004$ & $<.003$ & $<.003$ & $<.004$ & $<.002$ & $<.005$ & $<.002$ & $<.004$ & $<.004$ \\
\hline 06/19/99 & $<.002$ & $<.004$ & $<.003$ & $<.003$ & $<.004$ & $<.002$ & $<.005$ & $<.002$ & $<.004$ & $<.004$ \\
\hline 07/21/99 & $<.002$ & $<.004$ & $<.003$ & $<.003$ & $<.004$ & $<.002$ & $<.005$ & $<.002$ & $<.004$ & $<.004$ \\
\hline 08/19/99 & $<.002$ & $<.004$ & $<.003$ & $<.003$ & $<.004$ & $<.002$ & $<.005$ & $<.002$ & $<.004$ & $<.004$ \\
\hline $10 / 20 / 99$ & $<.002$ & $<.004$ & $<.003$ & $<.003$ & $<.004$ & $<.002$ & $<.005$ & $<.002$ & $<.004$ & $<.004$ \\
\hline $03 / 08 / 00$ & $<.002$ & $<.004$ & $<.003$ & $<.003$ & $<.004$ & $<.002$ & $<.005$ & $<.002$ & $<.004$ & $<.004$ \\
\hline $05 / 23 / 00$ & $<.002$ & $<.004$ & $<.003$ & $<.003$ & $<.004$ & $<.002$ & $<.005$ & $<.002$ & $<.004$ & $<.004$ \\
\hline $06 / 20 / 00$ & $<.002$ & $<.004$ & $<.003$ & $<.003$ & $<.004$ & $<.002$ & $<.005$ & $<.002$ & $<.004$ & $<.004$ \\
\hline \multicolumn{11}{|c|}{ SITE 9 12354500-CLARK FORK AT ST. REGIS, MONT. } \\
\hline 05/10/99 & $<.002$ & $<.004$ & $<.003$ & $<.003$ & $<.004$ & $<.002$ & $<.005$ & $<.002$ & $<.004$ & $<.004$ \\
\hline 08/18/99 & $<.002$ & $<.004$ & $<.003$ & $<.003$ & $<.004$ & $<.002$ & $<.005$ & $<.002$ & $<.004$ & $<.004$ \\
\hline $10 / 18 / 99$ & $<.002$ & $<.004$ & $<.003$ & $<.003$ & $<.004$ & $<.002$ & $<.005$ & $<.002$ & $<.004$ & $<.004$ \\
\hline \multicolumn{11}{|c|}{ SITE 12 12388700--FLATHEAD RIVER AT PERMA, MONT. } \\
\hline 05/11/99 & $<.002$ & $<.004$ & $<.003$ & $<.003$ & $<.004$ & $<.002$ & $<.005$ & $<.002$ & $<.004$ & $<.004$ \\
\hline 07/19/99 & $<.002$ & $<.004$ & $<.003$ & $<.003$ & $<.004$ & $<.002$ & $<.005$ & $<.002$ & $<.004$ & $<.004$ \\
\hline 08/17/99 & $<.002$ & $<.004$ & $<.003$ & $<.003$ & $<.004$ & $<.002$ & $<.005$ & $<.002$ & $<.004$ & $<.004$ \\
\hline $10 / 19 / 99$ & $<.002$ & $<.004$ & $<.003$ & $<.003$ & $<.004$ & $<.002$ & $<.005$ & $<.002$ & $<.004$ & $<.004$ \\
\hline \multicolumn{11}{|c|}{ SITE 35 12419000--SPOKANE RIVER NEAR POST FALLS, IDAHO } \\
\hline 04/13/99 & $<.002$ & $<.004$ & $<.003$ & $<.003$ & $<.004$ & $<.002$ & $<.005$ & $<.002$ & $<.004$ & $<.004$ \\
\hline $04 / 13 / 99^{\mathbf{F b}}$ & $<.002$ & $<.004$ & $<.003$ & $<.003$ & $<.004$ & $<.002$ & $<.005$ & $<.002$ & $<.004$ & $<.004$ \\
\hline 05/19/99 & $<.002$ & $<.004$ & $<.003$ & $<.003$ & $<.004$ & $<.002$ & $<.005$ & $<.002$ & $<.004$ & $<.004$ \\
\hline $06 / 23 / 99$ & $<.002$ & $<.004$ & $<.003$ & $<.003$ & $<.004$ & $<.002$ & $<.005$ & $<.002$ & $<.004$ & $<.004$ \\
\hline $07 / 27 / 99$ & $<.002$ & $<.004$ & $<.003$ & $<.003$ & $<.004$ & $<.002$ & $<.005$ & $<.002$ & $<.004$ & $<.004$ \\
\hline 09/09/99 & $<.002$ & $<.004$ & $<.003$ & $<.003$ & $<.004$ & $<.002$ & $<.005$ & $<.002$ & $<.004$ & $<.004$ \\
\hline $10 / 20 / 99$ & .019 & $<.004$ & $<.003$ & $<.003$ & $<.004$ & $<.002$ & $<.005$ & $<.002$ & $<.004$ & $<.004$ \\
\hline $03 / 28 / 00$ & $<.002$ & $<.004$ & $<.003$ & $<.003$ & $<.004$ & $<.002$ & $<.005$ & $<.002$ & $<.004$ & $<.004$ \\
\hline $05 / 03 / 00$ & $<.002$ & $<.004$ & $<.003$ & $<.003$ & $<.004$ & $<.002$ & $<.005$ & $<.002$ & $<.004$ & $<.004$ \\
\hline $05 / 31 / 00$ & e.002 & $<.004$ & $<.003$ & $<.003$ & $<.004$ & $<.002$ & $<.005$ & $<.002$ & $<.004$ & $<.004$ \\
\hline $06 / 27 / 00$ & $<.005$ & $<.004$ & $<.003$ & $<.003$ & $<.004$ & $<.002$ & $<.005$ & $<.002$ & $<.004$ & $<.004$ \\
\hline $08 / 30 / 00$ & $<.002$ & $<.004$ & $<.003$ & $<.003$ & $<.004$ & $<.002$ & $<.005$ & $<.002$ & $<.004$ & $<.004$ \\
\hline \multicolumn{11}{|c|}{ SITE 40 12424000--HANGMAN CREEK AT SPOKANE, WASH. } \\
\hline $02 / 16 / 00$ & $<.002$ & $<.004$ & $<.003$ & $<.003$ & .005 & $<.002$ & $<.005$ & $<.002$ & .015 & $<.004$ \\
\hline $03 / 20 / 00$ & $<.002$ & $<.004$ & $<.003$ & $<.003$ & e.003 & $<.002$ & $<.005$ & $<.002$ & .008 & $<.004$ \\
\hline $03 / 20 / 00^{\mathbf{F b}}$ & $<.002$ & $<.004$ & $<.003$ & $<.003$ & $<.004$ & $<.002$ & $<.005$ & $<.002$ & $<.004$ & $<.004$ \\
\hline $04 / 14 / 00$ & .008 & $<.004$ & .006 & $<.003$ & .008 & $<.002$ & $<.005$ & .005 & .200 & $<.004$ \\
\hline 05/04/00 & e.002 & $<.004$ & $<.003$ & $<.003$ & $<.004$ & $<.002$ & $<.005$ & $<.002$ & .138 & $<.004$ \\
\hline $06 / 01 / 00$ & $<.002$ & $<.004$ & $<.003$ & $<.003$ & $<.004$ & $<.002$ & $<.005$ & $<.002$ & $<.004$ & $<.004$ \\
\hline $06 / 29 / 00$ & $<.002$ & $<.004$ & $<.003$ & $<.003$ & $<.004$ & $<.002$ & $<.005$ & $<.002$ & $<.004$ & $<.004$ \\
\hline $08 / 30 / 00$ & $<.002$ & $<.004$ & $<.003$ & $<.003$ & $<.004$ & $<.002$ & $<.005$ & $<.002$ & $<.004$ & $<.004$ \\
\hline
\end{tabular}


Table 9. Pesticide concentration data for filtered surface-water samples collected at selected fixed water-quality sites, Northern Rockies Intermontane Basins study unit, 1999-2000 (Continued)

\begin{tabular}{|c|c|c|c|c|c|c|c|c|c|}
\hline Date & $\begin{array}{c}\text { Naprop- } \\
\text { amide } \\
(\mu \mathrm{g} / \mathbf{L})\end{array}$ & $\begin{array}{l}\text { Para- } \\
\text { thion } \\
(\mu \mathrm{g} / \mathrm{L})\end{array}$ & $\begin{array}{l}\text { Para- } \\
\text { thion- } \\
\text { methyl } \\
(\mu \mathrm{g} / \mathrm{L})\end{array}$ & $\begin{array}{c}\text { Pebulate } \\
(\mu \mathrm{g} / \mathrm{L})\end{array}$ & $\begin{array}{c}\text { Pendi- } \\
\text { methalin } \\
(\mu \mathrm{g} / \mathrm{L})\end{array}$ & $\begin{array}{c}\text { Phorate } \\
(\mu \mathrm{g} / \mathrm{L})\end{array}$ & $\begin{array}{c}\mathbf{p}, \mathbf{p}^{\prime}-\mathbf{D D} \mathbf{E} \\
(\mu \mathrm{g} / \mathrm{L})\end{array}$ & $\begin{array}{c}\text { Pro- } \\
\text { meton } \\
(\mu \mathrm{g} / \mathrm{L})\end{array}$ & $\begin{array}{l}\text { Prop- } \\
\text { achlor } \\
(\mu \mathrm{g} / \mathrm{L})\end{array}$ \\
\hline \multicolumn{10}{|c|}{ SITE 5 12352500--BITTERROOT RIVER NEAR MISSOULA, MONT. } \\
\hline 03/04/99 & $<0.003$ & $<0.004$ & $<0.006$ & $<0.004$ & $<0.004$ & $<0.002$ & $<0.006$ & $<0.018$ & $<0.007$ \\
\hline $03 / 24 / 99$ & $<.003$ & $<.004$ & $<.006$ & $<.004$ & $<.004$ & $<.002$ & $<.006$ & e.002 & $<.007$ \\
\hline $05 / 13 / 99$ & $<.003$ & $<.004$ & $<.006$ & $<.004$ & $<.004$ & $<.002$ & $<.006$ & $<.018$ & $<.007$ \\
\hline 06/07/99 & $<.003$ & $<.004$ & $<.006$ & $<.004$ & $<.004$ & $<.002$ & $<.006$ & $<.018$ & $<.007$ \\
\hline $06 / 19 / 99$ & $<.003$ & $<.004$ & $<.006$ & $<.004$ & $<.004$ & $<.002$ & $<.006$ & $<.018$ & $<.007$ \\
\hline $07 / 21 / 99$ & $<.003$ & $<.004$ & $<.006$ & $<.004$ & $<.004$ & $<.002$ & $<.006$ & $<.018$ & $<.007$ \\
\hline 08/19/99 & $<.003$ & $<.004$ & $<.006$ & $<.004$ & $<.004$ & $<.002$ & $<.006$ & e.004 & $<.007$ \\
\hline $10 / 20 / 99$ & $<.003$ & $<.004$ & $<.006$ & $<.004$ & $<.004$ & $<.002$ & e.001 & e.005 & $<.007$ \\
\hline $03 / 08 / 00$ & $<.003$ & $<.004$ & $<.006$ & $<.004$ & $<.004$ & $<.002$ & $<.006$ & e.002 & $<.007$ \\
\hline $05 / 23 / 00$ & $<.003$ & $<.004$ & $<.006$ & $<.004$ & $<.004$ & $<.002$ & $<.006$ & $<.018$ & $<.007$ \\
\hline $06 / 20 / 00$ & $<.003$ & $<.004$ & $<.006$ & $<.004$ & $<.004$ & $<.002$ & $<.006$ & $<.018$ & $<.007$ \\
\hline \multicolumn{10}{|c|}{ SITE 9 12354500--CLARK FORK AT ST. REGIS, MONT. } \\
\hline 05/10/99 & $<.003$ & $<.004$ & $<.006$ & $<.004$ & $<.004$ & $<.002$ & $<.006$ & $<.018$ & $<.007$ \\
\hline 08/18/99 & $<.003$ & $<.004$ & $<.006$ & $<.004$ & $<.004$ & $<.002$ & $<.006$ & $<.018$ & $<.007$ \\
\hline $10 / 18 / 99$ & $<.003$ & $<.004$ & $<.006$ & $<.004$ & $<.004$ & $<.002$ & $<.006$ & $<.018$ & $<.007$ \\
\hline \multicolumn{10}{|c|}{ SITE 12 12388700--FLATHEAD RIVER AT PERMA, MONT. } \\
\hline 05/11/99 & $<.003$ & $<.004$ & $<.006$ & $<.004$ & $<.004$ & $<.002$ & $<.006$ & $<.018$ & $<.007$ \\
\hline 07/19/99 & $<.003$ & $<.004$ & $<.006$ & $<.004$ & $<.004$ & $<.002$ & $<.006$ & $<.018$ & $<.007$ \\
\hline $08 / 17 / 99$ & $<.003$ & $<.004$ & $<.006$ & $<.004$ & $<.004$ & $<.002$ & $<.006$ & $<.018$ & $<.007$ \\
\hline $10 / 19 / 99$ & $<.003$ & $<.004$ & $<.006$ & $<.004$ & $<.004$ & $<.002$ & $<.006$ & $<.018$ & $<.007$ \\
\hline \multicolumn{10}{|c|}{ SITE 35 12419000--SPOKANE RIVER NEAR POST FALLS, IDAHO } \\
\hline $04 / 13 / 99$ & $<.003$ & $<.004$ & $<.006$ & $<.004$ & $<.004$ & $<.002$ & $<.006$ & $<.018$ & $<.007$ \\
\hline $04 / 13 / 99^{\mathbf{F b}}$ & $<.003$ & $<.004$ & $<.006$ & $<.004$ & $<.004$ & $<.002$ & $<.006$ & $<.018$ & $<.007$ \\
\hline $05 / 19 / 99$ & $<.003$ & $<.004$ & $<.006$ & $<.004$ & $<.004$ & $<.002$ & $<.006$ & $<.018$ & $<.007$ \\
\hline $06 / 23 / 99$ & $<.003$ & $<.004$ & $<.006$ & $<.004$ & $<.004$ & $<.002$ & $<.006$ & $<.018$ & $<.007$ \\
\hline $07 / 27 / 99$ & $<.003$ & $<.004$ & $<.006$ & $<.004$ & $<.004$ & $<.002$ & $<.006$ & $<.018$ & $<.007$ \\
\hline 09/09/99 & $<.003$ & $<.004$ & $<.006$ & $<.004$ & $<.004$ & $<.002$ & e.001 & $<.018$ & $<.007$ \\
\hline $10 / 20 / 99$ & $<.003$ & $<.004$ & $<.006$ & $<.004$ & .019 & $<.002$ & $<.006$ & $<.018$ & $<.007$ \\
\hline $03 / 28 / 00$ & $<.003$ & $<.004$ & $<.006$ & $<.004$ & $<.004$ & $<.002$ & $<.006$ & $<.018$ & $<.007$ \\
\hline $05 / 03 / 00$ & $<.003$ & $<.004$ & $<.006$ & $<.004$ & $<.004$ & $<.002$ & $<.006$ & $<.018$ & $<.007$ \\
\hline $05 / 31 / 00$ & $<.003$ & $<.004$ & $<.006$ & $<.004$ & $<.004$ & $<.002$ & $<.006$ & $<.018$ & $<.007$ \\
\hline $06 / 27 / 00$ & $<.003$ & $<.004$ & $<.006$ & $<.004$ & $<.004$ & $<.002$ & $<.006$ & $<.018$ & $<.007$ \\
\hline $08 / 30 / 00$ & $<.003$ & $<.004$ & $<.006$ & $<.004$ & $<.004$ & $<.002$ & $<.006$ & $<.018$ & $<.007$ \\
\hline \multicolumn{10}{|c|}{ SITE 40 12424000--HANGMAN CREEK AT SPOKANE, WASH. } \\
\hline $02 / 16 / 00$ & $<.003$ & $<.004$ & $<.006$ & $<.004$ & $<.004$ & $<.002$ & $<.006$ & e.003 & $<.007$ \\
\hline $03 / 20 / 00$ & $<.003$ & $<.004$ & $<.006$ & $<.004$ & $<.004$ & $<.002$ & e.002 & e.005 & $<.007$ \\
\hline $03 / 20 / 00^{\mathbf{F b}}$ & $<.003$ & $<.004$ & $<.006$ & $<.004$ & $<.004$ & $<.002$ & $<.006$ & $<.018$ & $<.007$ \\
\hline $04 / 14 / 00$ & $<.003$ & $<.004$ & $<.020$ & $<.004$ & .017 & $<.002$ & $<.006$ & e.009 & $<.007$ \\
\hline $05 / 04 / 00$ & $<.003$ & $<.004$ & $<.006$ & $<.004$ & $<.004$ & $<.002$ & $<.006$ & e.013 & $<.007$ \\
\hline $06 / 01 / 00$ & $<.003$ & $<.004$ & $<.006$ & $<.004$ & $<.004$ & $<.002$ & $<.006$ & $<.018$ & $<.007$ \\
\hline $06 / 29 / 00$ & $<.003$ & $<.004$ & $<.006$ & $<.004$ & $<.004$ & $<.002$ & $<.006$ & e.005 & $<.007$ \\
\hline $08 / 30 / 00$ & $<.003$ & $<.004$ & $<.006$ & $<.004$ & $<.004$ & $<.002$ & $<.006$ & $<.018$ & $<.007$ \\
\hline
\end{tabular}


Table 9. Pesticide concentration data for filtered surface-water samples collected at selected fixed water-quality sites, Northern Rockies Intermontane Basins study unit, 1999-2000 (Continued)

\begin{tabular}{|c|c|c|c|c|c|c|c|c|c|c|}
\hline Date & $\begin{array}{c}\text { Prop- } \\
\text { anil } \\
(\mu \mathrm{g} / \mathrm{L})\end{array}$ & $\begin{array}{l}\text { Prop- } \\
\text { argite } \\
(\mu \mathrm{g} / \mathrm{L})\end{array}$ & $\begin{array}{c}\text { Propyz- } \\
\text { amide } \\
(\mu \mathrm{g} / \mathrm{L})\end{array}$ & $\begin{array}{c}\text { Sima- } \\
\text { zine } \\
(\mu \mathrm{g} / \mathrm{L})\end{array}$ & $\begin{array}{c}\text { Tebu- } \\
\text { thiuron } \\
(\mu \mathrm{g} / \mathrm{L})\end{array}$ & $\begin{array}{c}\text { Terbacil } \\
(\mu \mathrm{g} / \mathrm{L})\end{array}$ & $\begin{array}{c}\text { Terbufos } \\
(\mu \mathrm{g} / \mathbf{L})\end{array}$ & $\begin{array}{l}\text { Thio- } \\
\text { ben- } \\
\text { carb } \\
(\mu \mathrm{g} / \mathrm{L})\end{array}$ & $\begin{array}{c}\text { Tri- } \\
\text { allate } \\
(\mu \mathrm{g} / \mathrm{L})\end{array}$ & $\begin{array}{c}\text { Tri- } \\
\text { fluralin } \\
(\mu \mathrm{g} / \mathrm{L})\end{array}$ \\
\hline \multicolumn{11}{|c|}{ 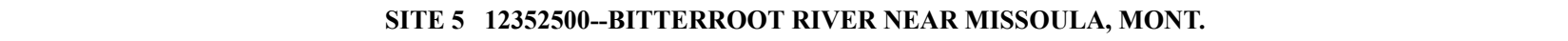 } \\
\hline 03/04/99 & $<0.004$ & $<0.013$ & $<0.003$ & $<0.005$ & $<0.010$ & $<0.007$ & $<0.013$ & $<0.002$ & $<0.001$ & $<0.002$ \\
\hline $03 / 24 / 99$ & $<.004$ & $<.013$ & $<.003$ & $<.005$ & $<.010$ & $<.007$ & $<.013$ & $<.002$ & $<.001$ & $<.002$ \\
\hline $05 / 13 / 99$ & $<.004$ & $<.013$ & $<.003$ & $<.005$ & $<.010$ & $<.007$ & $<.013$ & $<.002$ & $<.001$ & $<.002$ \\
\hline 06/07/99 & $<.004$ & $<.013$ & $<.003$ & $<.005$ & $<.010$ & $<.007$ & $<.013$ & $<.002$ & $<.001$ & $<.002$ \\
\hline 06/19/99 & $<.004$ & $<.013$ & $<.003$ & $<.005$ & $<.010$ & $<.007$ & $<.013$ & $<.002$ & $<.001$ & $<.002$ \\
\hline $07 / 21 / 99$ & $<.004$ & $<.013$ & $<.003$ & $<.005$ & $<.010$ & $<.007$ & $<.013$ & $<.002$ & $<.001$ & $<.002$ \\
\hline 08/19/99 & $<.004$ & $<.013$ & $<.003$ & $<.005$ & $<.010$ & $<.007$ & $<.013$ & $<.002$ & $<.001$ & $<.002$ \\
\hline $10 / 20 / 99$ & $<.004$ & $<.090$ & $<.003$ & $<.005$ & $<.010$ & $<.007$ & $<.013$ & $<.002$ & e.002 & e.004 \\
\hline $03 / 08 / 00$ & $<.004$ & $<.013$ & $<.003$ & $<.005$ & $<.010$ & $<.007$ & $<.013$ & $<.002$ & $<.001$ & $<.002$ \\
\hline $05 / 23 / 00$ & $<.004$ & $<.013$ & $<.003$ & $<.005$ & $<.010$ & $<.007$ & $<.013$ & $<.002$ & $<.001$ & $<.002$ \\
\hline $06 / 20 / 00$ & $<.004$ & $<.013$ & $<.003$ & $<.005$ & $<.010$ & $<.007$ & $<.013$ & $<.002$ & $<.001$ & $<.002$ \\
\hline \multicolumn{11}{|c|}{ SITE 9 12354500--CLARK FORK AT ST. REGIS, MONT. } \\
\hline 05/10/99 & $<.004$ & $<.013$ & $<.003$ & $<.005$ & $<.010$ & $<.007$ & $<.013$ & $<.002$ & $<.001$ & $<.002$ \\
\hline 08/18/99 & $<.004$ & $<.013$ & $<.003$ & $<.005$ & $<.010$ & $<.007$ & $<.013$ & $<.002$ & $<.001$ & $<.002$ \\
\hline $10 / 18 / 99$ & $<.004$ & $<.013$ & $<.003$ & $<.005$ & $<.010$ & $<.007$ & $<.013$ & $<.002$ & $<.001$ & $<.002$ \\
\hline \multicolumn{11}{|c|}{ SITE 12 12388700--FLATHEAD RIVER AT PERMA, MONT. } \\
\hline $05 / 11 / 99$ & $<.004$ & $<.013$ & $<.003$ & $<.005$ & $<.010$ & $<.007$ & $<.013$ & $<.002$ & $<.001$ & $<.002$ \\
\hline $07 / 19 / 99$ & $<.004$ & $<.013$ & $<.003$ & $<.005$ & $<.010$ & $<.007$ & $<.013$ & $<.002$ & $<.001$ & $<.002$ \\
\hline $08 / 17 / 99$ & $<.004$ & -- & $<.003$ & $<.005$ & $<.010$ & $<.007$ & $<.013$ & $<.002$ & $<.001$ & $<.002$ \\
\hline $10 / 19 / 99$ & $<.004$ & $<.013$ & $<.003$ & $<.005$ & $<.010$ & $<.007$ & $<.013$ & $<.002$ & $<.001$ & $<.002$ \\
\hline \multicolumn{11}{|c|}{35 12419000--SPOKANE RIVER NEAR POST FALLS, IDAHO } \\
\hline 04/13/99 & $<.004$ & $<.013$ & $<.003$ & $<.005$ & $<.010$ & $<.007$ & $<.013$ & $<.002$ & $<.001$ & $<.002$ \\
\hline $04 / 13 / 99^{\mathbf{F b}}$ & $<.004$ & $<.013$ & $<.003$ & $<.005$ & $<.077$ & $<.007$ & $<.013$ & $<.002$ & $<.001$ & $<.002$ \\
\hline 05/19/99 & $<.004$ & $<.013$ & $<.003$ & $<.005$ & $<.010$ & $<.007$ & $<.013$ & $<.002$ & e.002 & $<.002$ \\
\hline $06 / 23 / 99$ & $<.004$ & $<.013$ & $<.003$ & $<.005$ & $<.010$ & $<.007$ & $<.013$ & $<.002$ & $<.001$ & $<.002$ \\
\hline $07 / 27 / 99$ & $<.004$ & $<.013$ & $<.003$ & $<.005$ & $<.010$ & $<.007$ & $<.013$ & $<.002$ & $<.001$ & $<.002$ \\
\hline 09/09/99 & $<.004$ & $<.013$ & $<.003$ & $<.005$ & $<.010$ & $<.007$ & $<.013$ & $<.002$ & $<.001$ & $<.002$ \\
\hline $10 / 20 / 99$ & $<.004$ & $<.013$ & $<.003$ & $<.005$ & $<.010$ & $<.007$ & $<.013$ & $<.002$ & $<.001$ & .031 \\
\hline $03 / 28 / 00$ & $<.004$ & $<.013$ & $<.003$ & $<.005$ & $<.010$ & $<.007$ & $<.013$ & $<.002$ & e.001 & $<.002$ \\
\hline $05 / 03 / 00$ & $<.004$ & $<.013$ & $<.003$ & $<.005$ & $<.010$ & $<.007$ & $<.013$ & $<.002$ & e.004 & $<.002$ \\
\hline $05 / 31 / 00$ & $<.004$ & $<.013$ & $<.003$ & $<.005$ & $<.010$ & $<.007$ & $<.013$ & $<.002$ & e.003 & $<.002$ \\
\hline $06 / 27 / 00$ & $<.004$ & $<.013$ & $<.003$ & $<.005$ & $<.010$ & $<.007$ & $<.013$ & $<.002$ & e.002 & $<.002$ \\
\hline $08 / 30 / 00$ & $<.004$ & $<.013$ & $<.003$ & $<.005$ & $<.010$ & $<.007$ & $<.013$ & $<.002$ & $<.001$ & $<.002$ \\
\hline \multicolumn{11}{|c|}{ SITE 40 12424000--HANGMAN CREEK AT SPOKANE, WASH. } \\
\hline $02 / 16 / 00$ & $<.004$ & $<.013$ & $<.003$ & e.004 & $<.010$ & e.019 & $<.013$ & $<.002$ & .026 & $<.002$ \\
\hline $03 / 20 / 00$ & $<.004$ & $<.013$ & $<.003$ & e.005 & $<.010$ & e.021 & $<.013$ & $<.002$ & .008 & $<.002$ \\
\hline $03 / 20 / 00^{\mathbf{F b}}$ & $<.004$ & $<.013$ & $<.003$ & $<.005$ & $<.010$ & $<.007$ & $<.013$ & $<.002$ & $<.001$ & $<.002$ \\
\hline $04 / 14 / 00$ & $<.004$ & $<.013$ & $<.003$ & .008 & $<.010$ & e.009 & $<.013$ & $<.002$ & .350 & $<.002$ \\
\hline $05 / 04 / 00$ & $<.004$ & $<.013$ & $<.003$ & .085 & e.007 & e.007 & $<.013$ & $<.002$ & .020 & $<.002$ \\
\hline $06 / 01 / 00$ & $<.004$ & $<.013$ & $<.003$ & .066 & e.004 & $<.020$ & $<.013$ & $<.002$ & $<.001$ & $<.002$ \\
\hline $06 / 29 / 00$ & $<.004$ & $<.013$ & $<.003$ & .019 & $<.010$ & $<.007$ & $<.013$ & $<.002$ & $<.001$ & $<.002$ \\
\hline $08 / 30 / 00$ & $<.004$ & $<.013$ & $<.003$ & $<.010$ & $<.010$ & $<.007$ & $<.013$ & $<.002$ & $<.001$ & $<.002$ \\
\hline
\end{tabular}


Table 9. Pesticide concentration data for filtered surface-water samples collected at selected fixed water-quality sites, Northern Rockies Intermontane Basins study unit, 1999-2000 (Continued)

\begin{tabular}{|c|c|c|c|c|c|c|c|c|c|c|c|c|}
\hline Date & Time & $\begin{array}{c}\text { Discharge, } \\
\text { instanta- } \\
\text { neous } \\
\left(\mathbf{f t}^{3} / \mathbf{s}\right)\end{array}$ & $\begin{array}{c}\text { 2,6- } \\
\text { Diethyl- } \\
\text { aniline } \\
(\mu \mathrm{g} / \mathrm{L})\end{array}$ & $\begin{array}{c}\text { Aceto- } \\
\text { chlor } \\
(\mu \mathrm{g} / \mathrm{L})\end{array}$ & $\begin{array}{c}\text { Alachlor } \\
(\mu \mathrm{g} / \mathrm{L})\end{array}$ & $\begin{array}{c}\text { alpha- } \\
\mathbf{H C H} \\
(\mu \mathrm{g} / \mathrm{L})\end{array}$ & $\begin{array}{l}\text { alpha- } \\
\text { HCH-d }_{6} \text {, } \\
\text { surrogate } \\
\text { (percent) }\end{array}$ & $\begin{array}{c}\text { Atra- } \\
\text { zine } \\
(\mu \mathrm{g} / \mathrm{L})\end{array}$ & $\begin{array}{l}\text { Azin- } \\
\text { phos- } \\
\text { methyl } \\
(\mu \mathrm{g} / \mathrm{L})\end{array}$ & $\begin{array}{c}\text { Ben- } \\
\text { fluralin } \\
(\mu \mathrm{g} / \mathrm{L})\end{array}$ & $\begin{array}{c}\text { Butyl- } \\
\text { ate } \\
(\mu \mathrm{g} / \mathrm{L})\end{array}$ & $\begin{array}{c}\text { Carb- } \\
\text { aryl } \\
(\mu \mathrm{g} / \mathrm{L})\end{array}$ \\
\hline $04 / 14 / 99$ & 1230 & 10,100 & $<.003$ & $<.002$ & $<.002$ & $<.002$ & 106 & $<.001$ & $<.001$ & $<.002$ & $<.002$ & $<.003$ \\
\hline $05 / 18 / 99$ & 1100 & 13,500 & $<.003$ & $<.002$ & $<.002$ & $<.002$ & 91 & $<.001$ & $<.001$ & $<.002$ & $<.002$ & $<.003$ \\
\hline $06 / 24 / 99$ & 1015 & 11,800 & $<.003$ & $<.002$ & $<.002$ & $<.002$ & 100 & $<.001$ & $<.001$ & $<.002$ & $<.002$ & $<.003$ \\
\hline 09/10/99 & 0940 & 1,170 & $<.003$ & $<.002$ & $<.002$ & $<.002$ & 106 & e.003 & $<.001$ & $<.002$ & $<.002$ & $<.003$ \\
\hline $10 / 21 / 99$ & 1110 & 2,230 & $<.003$ & $<.002$ & $<.002$ & $<.002$ & 100 & $<.001$ & $<.001$ & $<.002$ & $<.002$ & $<.003$ \\
\hline $03 / 29 / 00$ & 1000 & 11,600 & $<.003$ & $<.002$ & $<.002$ & $<.002$ & 91 & e.002 & $<.001$ & $<.002$ & $<.002$ & $<.003$ \\
\hline $05 / 03 / 00$ & 1245 & 21,000 & $<.003$ & $<.002$ & $<.002$ & $<.002$ & 97 & e.004 & $<.001$ & $<.002$ & $<.002$ & $<.006$ \\
\hline $05 / 31 / 00$ & 1015 & 12,400 & $<.003$ & $<.002$ & $<.002$ & $<.002$ & 104 & $<.001$ & $<.001$ & $<.002$ & $<.002$ & e.004 \\
\hline $06 / 27 / 00$ & 1000 & 4,290 & $<.003$ & $<.002$ & $<.002$ & $<.002$ & 98 & e.002 & $<.001$ & $<.002$ & $<.002$ & $<.003$ \\
\hline
\end{tabular}


Table 9. Pesticide concentration data for filtered surface-water samples collected at selected fixed water-quality sites, Northern Rockies Intermontane Basins study unit, 1999-2000 (Continued)

\begin{tabular}{|c|c|c|c|c|c|c|c|c|c|c|}
\hline Date & $\begin{array}{c}\text { Carbo- } \\
\text { furan } \\
(\mu \mathrm{g} / \mathrm{L})\end{array}$ & $\begin{array}{c}\text { Chlor- } \\
\text { pyrifos } \\
(\mu \mathrm{g} / \mathrm{L})\end{array}$ & $\begin{array}{c}\text { cis-Per- } \\
\text { methrin } \\
(\mu \mathrm{g} / \mathrm{L})\end{array}$ & $\begin{array}{l}\text { Cyan- } \\
\text { azine } \\
(\mu \mathrm{g} / \mathrm{L})\end{array}$ & $\begin{array}{c}\text { Dacthal } \\
(\mu \mathrm{g} / \mathrm{L})\end{array}$ & $\begin{array}{c}\text { Deethyl- } \\
\text { atra- } \\
\text { zine } \\
(\mu \mathrm{g} / \mathrm{L})\end{array}$ & $\begin{array}{c}\text { Diaz- } \\
\text { inon } \\
(\mu \mathrm{g} / \mathrm{L})\end{array}$ & $\begin{array}{l}\text { Diazinon- } \\
\quad d_{10}, \\
\text { surrogate } \\
\text { (percent) }\end{array}$ & $\begin{array}{c}\text { Dieldrin } \\
(\mu \mathrm{g} / \mathrm{L})\end{array}$ & $\begin{array}{c}\text { Disul- } \\
\text { foton } \\
(\mu \mathrm{g} / \mathrm{L})\end{array}$ \\
\hline \multicolumn{11}{|c|}{ SITE 41 12424500--SPOKANE RIVER AT SEVEN MILE BRIDGE, NEAR SPOKANE, WASH. } \\
\hline 04/14/99 & $<.003$ & $<.004$ & $<.005$ & $<.004$ & $<.002$ & $<.002$ & $<.002$ & 132 & $<.001$ & $<.017$ \\
\hline 05/18/99 & $<.003$ & $<.004$ & $<.005$ & $<.004$ & $<.002$ & $<.002$ & $<.002$ & 101 & $<.001$ & $<.017$ \\
\hline $06 / 24 / 99$ & $<.003$ & $<.004$ & $<.005$ & $<.004$ & $<.002$ & $<.002$ & $<.002$ & 99 & $<.001$ & $<.017$ \\
\hline 07/28/99 & $<.003$ & $<.004$ & $<.005$ & $<.004$ & $<.002$ & $<.087$ & $<.002$ & 117 & $<.001$ & $<.017$ \\
\hline 09/10/99 & $<.003$ & $<.004$ & $<.005$ & $<.004$ & $<.002$ & e.004 & $<.002$ & 122 & $<.001$ & $<.017$ \\
\hline $10 / 21 / 99$ & $<.003$ & $<.004$ & $<.005$ & $<.004$ & $<.002$ & $<.002$ & $<.002$ & 87 & $<.001$ & $<.017$ \\
\hline 03/29/00 & $<.003$ & $<.004$ & $<.005$ & $<.004$ & $<.002$ & $<.002$ & $<.002$ & 96 & $<.001$ & $<.017$ \\
\hline 05/03/00 & $<.003$ & $<.004$ & $<.005$ & $<.004$ & $<.002$ & $<.002$ & $<.002$ & 112 & $<.001$ & $<.017$ \\
\hline $05 / 31 / 00$ & $<.003$ & $<.004$ & $<.005$ & $<.004$ & e.001 & $<.002$ & $<.002$ & 107 & $<.001$ & $<.017$ \\
\hline $06 / 27 / 00$ & $<.003$ & $<.004$ & $<.005$ & $<.004$ & $<.002$ & $<.002$ & e.003 & 104 & $<.001$ & $<.017$ \\
\hline $08 / 30 / 00$ & $<.003$ & $<.004$ & $<.005$ & $<.004$ & $<.002$ & $<.002$ & $<.002$ & 104 & $<.001$ & $<.017$ \\
\hline
\end{tabular}


Table 9. Pesticide concentration data for filtered surface-water samples collected at selected fixed water-quality sites, Northern Rockies Intermontane Basins study unit, 1999-2000 (Continued)

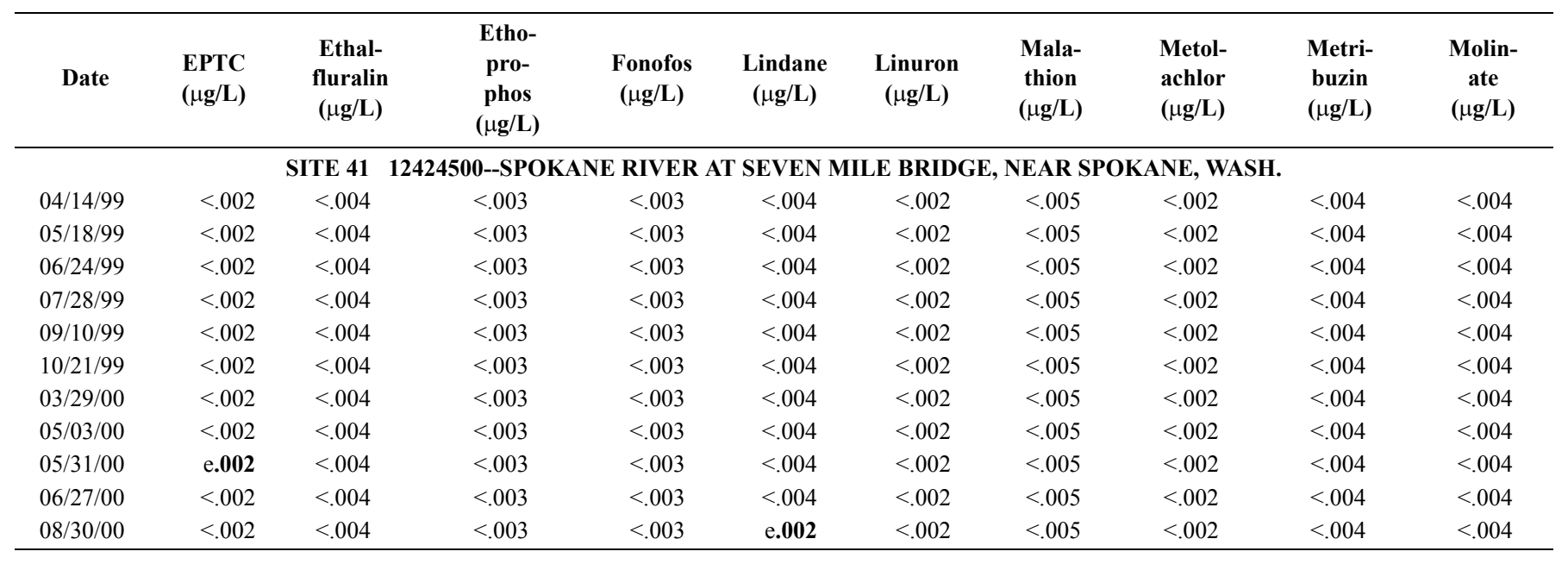


Table 9. Pesticide concentration data for filtered surface-water samples collected at selected fixed water-quality sites, Northern Rockies Intermontane Basins study unit, 1999-2000 (Continued)

\begin{tabular}{|c|c|c|c|c|c|c|c|c|c|}
\hline Date & $\begin{array}{c}\text { Naprop- } \\
\text { amide } \\
(\mu \mathrm{g} / \mathrm{L})\end{array}$ & $\begin{array}{c}\text { Para- } \\
\text { thion } \\
(\mu \mathrm{g} / \mathrm{L})\end{array}$ & $\begin{array}{l}\text { Para- } \\
\text { thion- } \\
\text { methyl } \\
(\mu \mathrm{g} / \mathrm{L})\end{array}$ & $\begin{array}{c}\text { Pebulate } \\
(\mu \mathbf{g} / \mathbf{L})\end{array}$ & $\begin{array}{c}\text { Pendi- } \\
\text { methalin } \\
(\mu \mathrm{g} / \mathrm{L})\end{array}$ & $\begin{array}{c}\text { Phorate } \\
(\mu \mathrm{g} / \mathrm{L})\end{array}$ & $\begin{array}{c}\mathbf{p}, \mathbf{p}^{\prime}-\mathbf{D D E} \\
(\mu \mathbf{g} / \mathbf{L})\end{array}$ & $\begin{array}{c}\text { Pro- } \\
\text { meton } \\
(\mu \mathrm{g} / \mathrm{L})\end{array}$ & $\begin{array}{l}\text { Prop- } \\
\text { achlor } \\
(\mu \mathrm{g} / \mathrm{L})\end{array}$ \\
\hline \multicolumn{10}{|c|}{ SITE 4} \\
\hline $04 / 14 / 99$ & $<.003$ & -- & $<.006$ & $<.004$ & $<.004$ & $<.002$ & $<.006$ & $<.018$ & $<.007$ \\
\hline 05/18/99 & $<.003$ & $<.004$ & $<.006$ & $<.004$ & $<.004$ & $<.002$ & $<.006$ & $<.018$ & $<.007$ \\
\hline $06 / 24 / 99$ & $<.003$ & $<.004$ & $<.006$ & $<.004$ & $<.004$ & $<.002$ & $<.006$ & $<.018$ & $<.007$ \\
\hline $07 / 28 / 99$ & $<.003$ & $<.004$ & $<.006$ & $<.004$ & $<.004$ & $<.002$ & $<.006$ & $<.018$ & $<.007$ \\
\hline 09/10/99 & $<.003$ & $<.004$ & $<.006$ & $<.004$ & $<.004$ & $<.002$ & e.002 & e.004 & $<.007$ \\
\hline $10 / 21 / 99$ & $<.003$ & $<.004$ & $<.006$ & $<.004$ & $<.004$ & $<.002$ & $<.006$ & $<.018$ & $<.007$ \\
\hline $03 / 29 / 00$ & $<.003$ & $<.004$ & $<.006$ & $<.004$ & $<.004$ & $<.002$ & e.001 & $<.018$ & $<.007$ \\
\hline $05 / 03 / 00$ & $<.003$ & $<.004$ & $<.006$ & $<.004$ & $<.004$ & $<.002$ & $<.006$ & $<.018$ & $<.007$ \\
\hline $05 / 31 / 00$ & $<.003$ & $<.004$ & $<.006$ & $<.004$ & $<.004$ & $<.002$ & $<.006$ & $<.018$ & $<.007$ \\
\hline $06 / 27 / 00$ & $<.003$ & $<.004$ & $<.006$ & $<.004$ & $<.004$ & $<.002$ & $<.006$ & $<.018$ & $<.007$ \\
\hline $08 / 30 / 00$ & $<.003$ & $<.004$ & $<.006$ & $<.004$ & $<.004$ & $<.002$ & $<.006$ & e.004 & $<.007$ \\
\hline
\end{tabular}


Table 9. Pesticide concentration data for filtered surface-water samples collected at selected fixed water-quality sites, Northern Rockies Intermontane Basins study unit, 1999-2000 (Continued)

\begin{tabular}{|c|c|c|c|c|c|c|c|c|c|c|}
\hline Date & $\begin{array}{c}\text { Prop- } \\
\text { anil } \\
(\mu \mathrm{g} / \mathrm{L})\end{array}$ & $\begin{array}{l}\text { Prop- } \\
\text { argite } \\
(\mu \mathrm{g} / \mathrm{L})\end{array}$ & $\begin{array}{c}\text { Propyz- } \\
\text { amide } \\
(\mu \mathrm{g} / \mathrm{L})\end{array}$ & $\begin{array}{c}\text { Sima- } \\
\text { zine } \\
(\mu \mathrm{g} / \mathrm{L})\end{array}$ & $\begin{array}{c}\text { Tebu- } \\
\text { thiuron } \\
(\mu \mathrm{g} / \mathrm{L})\end{array}$ & $\begin{array}{c}\text { Terbacil } \\
(\mu \mathrm{g} / \mathrm{L})\end{array}$ & $\begin{array}{c}\text { Terbufos } \\
(\mu \mathrm{g} / \mathrm{L})\end{array}$ & $\begin{array}{l}\text { Thio- } \\
\text { ben- } \\
\text { carb } \\
(\mu \mathrm{g} / \mathrm{L})\end{array}$ & $\begin{array}{c}\text { Tri- } \\
\text { allate } \\
(\mu \mathrm{g} / \mathrm{L})\end{array}$ & $\begin{array}{c}\text { Tri- } \\
\text { fluralin } \\
(\mu \mathbf{g} / \mathbf{L})\end{array}$ \\
\hline \multicolumn{11}{|c|}{ SITE 41 12424500--SPOKANE RIVER AT SEVEN MILE BRIDGE, NEAR SPOKANE, WASH. } \\
\hline 04/14/99 & $<.004$ & $<.013$ & $<.003$ & $<.005$ & $<.010$ & $<.007$ & $<.013$ & $<.002$ & $<.001$ & $<.002$ \\
\hline $05 / 18 / 99$ & $<.004$ & $<.013$ & $<.003$ & $<.005$ & $<.010$ & $<.007$ & $<.013$ & $<.002$ & $<.001$ & $<.002$ \\
\hline 06/24/99 & $<.004$ & $<.013$ & $<.003$ & $<.005$ & $<.010$ & $<.007$ & $<.013$ & $<.002$ & $<.001$ & $<.002$ \\
\hline 07/28/99 & $<.004$ & $<.013$ & $<.003$ & $<.005$ & $<.010$ & $<.007$ & $<.013$ & $<.002$ & $<.001$ & $<.002$ \\
\hline 09/10/99 & $<.004$ & $<.013$ & $<.003$ & e.003 & $<.010$ & $<.007$ & $<.013$ & $<.002$ & $<.001$ & $<.002$ \\
\hline $10 / 21 / 99$ & $<.004$ & $<.013$ & $<.003$ & $<.005$ & $<.010$ & $<.007$ & $<.013$ & $<.002$ & $<.001$ & $<.002$ \\
\hline 03/29/00 & $<.004$ & $<.013$ & $<.003$ & $<.005$ & $<.010$ & $<.007$ & $<.013$ & $<.002$ & e.002 & $<.002$ \\
\hline 05/03/00 & $<.004$ & $<.013$ & $<.003$ & $<.007$ & $<.010$ & $<.007$ & $<.013$ & $<.002$ & e.004 & $<.002$ \\
\hline $05 / 31 / 00$ & $<.004$ & $<.013$ & $<.003$ & $<.005$ & $<.010$ & $<.007$ & $<.013$ & $<.002$ & e.003 & $<.002$ \\
\hline $06 / 27 / 00$ & $<.004$ & $<.013$ & $<.003$ & $<.005$ & $<.010$ & $<.007$ & $<.013$ & $<.002$ & e.002 & $<.002$ \\
\hline $08 / 30 / 00$ & $<.004$ & $<.013$ & $<.003$ & $<.005$ & $<.010$ & $<.007$ & $<.013$ & $<.002$ & $<.001$ & $<.002$ \\
\hline
\end{tabular}


Table 10. Volatile organic-compound concentration data for surface-water samples collected at fixed water-quality sites along the Spokane River, Idaho and Washington, 1999-2000

[See figure 1 for site locations. Bold text denotes detected concentrations. Abbreviations: $\mathrm{ft}^{3} / \mathrm{s}$, cubic feet per second; e, estimated; Fb, field blank; $\mu \mathrm{g} / \mathrm{L}$, micrograms per liter; Sb, source-solution blank; Tb, trip blank. Symbols: <, less than reporting level; --, no data]

\begin{tabular}{|c|c|c|c|c|c|c|c|c|c|c|c|c|}
\hline Date & Time & $\begin{array}{c}\text { Discharge, } \\
\text { instanta- } \\
\text { neous } \\
\left(\mathbf{f t}^{3} / \mathbf{s}\right)\end{array}$ & $\begin{array}{c}\text { 1,1,1,2- } \\
\text { Tetra- } \\
\text { chloro- } \\
\text { ethane } \\
(\mu \mathrm{g} / \mathrm{L})\end{array}$ & $\begin{array}{c}\text { 1,1,1- } \\
\text { Trichloro- } \\
\text { ethane } \\
(\mu \mathrm{g} / \mathbf{L})\end{array}$ & $\begin{array}{l}\text { 1,1,2,2- } \\
\text { Tetra- } \\
\text { chloro- } \\
\text { ethane } \\
(\mu \mathrm{g} / \mathrm{L})\end{array}$ & $\begin{array}{c}\text { 1,1,2- } \\
\text { Trichloro- } \\
\text { ethane } \\
(\mu \mathrm{g} / \mathrm{L})\end{array}$ & $\begin{array}{c}1,1,2- \\
\text { Trichloro- } \\
\text { trifluoro- } \\
\text { ethane } \\
(\mu \mathrm{g} / \mathrm{L})\end{array}$ & $\begin{array}{c}\text { 1,1- } \\
\text { Dichloro- } \\
\text { ethane } \\
(\mu \mathrm{g} / \mathrm{L})\end{array}$ & $\begin{array}{c}\text { 1,1- } \\
\text { Dichloro- } \\
\text { ethylene } \\
(\mu \mathrm{g} / \mathrm{L})\end{array}$ & $\begin{array}{c}\text { 1,1- } \\
\text { Dichloro- } \\
\text { propene } \\
(\mu \mathrm{g} / \mathrm{L})\end{array}$ & $\begin{array}{c}1,2,3,4- \\
\text { Tetra- } \\
\text { methyl- } \\
\text { benzene } \\
(\mu \mathrm{g} / \mathrm{L})\end{array}$ & $\begin{array}{c}1,2,3,5- \\
\text { Tetra- } \\
\text { methyl- } \\
\text { benzene } \\
(\mu \mathrm{g} / \mathrm{L})\end{array}$ \\
\hline \multicolumn{13}{|c|}{ SITE 35 12419000--SPOKANE RIVER NEAR POST FALLS, IDAHO } \\
\hline $02 / 09 / 99$ & 1020 & 6,380 & $<0.044$ & $<0.032$ & $<0.130$ & $<0.064$ & $<0.032$ & $<0.066$ & $<0.044$ & $<0.026$ & $<0.230$ & $<0.200$ \\
\hline $02 / 09 / 99^{\mathbf{F b}}$ & 1200 & -- & $<.044$ & $<.032$ & $<.130$ & $<.064$ & $<.032$ & $<.066$ & $<.044$ & $<.026$ & $<.230$ & $<.200$ \\
\hline $05 / 19 / 99^{\mathbf{F b}}$ & 0959 & -- & $<.044$ & $<.032$ & $<.130$ & $<.064$ & $<.032$ & $<.066$ & $<.044$ & $<.026$ & $<.230$ & $<.200$ \\
\hline 05/19/99 & 1000 & 13,800 & $<.044$ & $<.032$ & $<.130$ & $<.064$ & $<.032$ & $<.066$ & $<.044$ & $<.026$ & $<.230$ & $<.200$ \\
\hline $06 / 23 / 99$ & 0930 & 12,500 & $<.044$ & $<.032$ & $<.130$ & $<.064$ & $<.032$ & $<.066$ & $<.044$ & $<.026$ & $<.230$ & $<.200$ \\
\hline $06 / 23 / 99^{\mathbf{T b}}$ & 0931 & -- & $<.044$ & $<.032$ & $<.130$ & $<.064$ & $<.032$ & $<.066$ & $<.044$ & $<.026$ & $<.230$ & $<.200$ \\
\hline 07/27/99 & 1030 & 1,850 & $<.044$ & $<.032$ & $<.130$ & $<.064$ & $<.032$ & $<.066$ & $<.044$ & $<.026$ & $<.230$ & $<.200$ \\
\hline 09/09/99 & 1100 & 807 & $<.044$ & $<.032$ & $<.130$ & $<.064$ & $<.032$ & $<.066$ & $<.044$ & $<.026$ & $<.230$ & $<.200$ \\
\hline $10 / 20 / 99^{\mathbf{F b}}$ & 0958 & -- & $<.030$ & $<.032$ & $<.090$ & $<.060$ & $<.060$ & $<.066$ & $<.040$ & $<.026$ & $<.230$ & $<.200$ \\
\hline $10 / 20 / 99^{\mathbf{S b}}$ & 1000 & -- & $<.030$ & $<.032$ & $<.090$ & $<.060$ & $<.060$ & $<.066$ & $<.040$ & $<.026$ & $<.230$ & $<.200$ \\
\hline $10 / 20 / 99$ & 1130 & 2,100 & $<.030$ & $<.032$ & $<.090$ & $<.060$ & $<.060$ & $<.066$ & $<.040$ & $<.026$ & $<.230$ & $<.200$ \\
\hline $07 / 25 / 00$ & 1300 & 1,810 & $<.030$ & $<.032$ & $<.090$ & $<.060$ & $<.060$ & $<.066$ & $<.040$ & $<.026$ & $<.230$ & $<.200$ \\
\hline $07 / 25 / 00^{\mathbf{F b}}$ & 1301 & -- & $<.030$ & $<.032$ & $<.090$ & $<.060$ & $<.060$ & $<.066$ & $<.040$ & $<.026$ & $<.230$ & $<.200$ \\
\hline $08 / 30 / 00$ & 1415 & 242 & $<.030$ & $<.032$ & $<.090$ & $<.060$ & $<.060$ & $<.066$ & $<.040$ & $<.026$ & $<.230$ & $<.200$ \\
\hline $08 / 30 / 00^{\mathbf{T b}}$ & 1416 & -- & $<.030$ & $<.032$ & $<.090$ & $<.060$ & $<.060$ & $<.066$ & $<.040$ & $<.026$ & $<.230$ & $<.200$ \\
\hline \multicolumn{13}{|c|}{ SITE 4112424500 SPOKANE RIVER AT SEVEN MILE BRIDGE, NEAR SPOKANE, WASH. } \\
\hline $02 / 09 / 99$ & 1400 & 7,550 & $<.044$ & e.006 & $<.130$ & $<.064$ & $<.032$ & $<.066$ & $<.044$ & $<.026$ & $<.230$ & $<.200$ \\
\hline $05 / 18 / 99$ & 1100 & 13,600 & $<.044$ & $<.032$ & $<.130$ & $<.064$ & $<.032$ & $<.066$ & $<.044$ & $<.026$ & $<.230$ & $<.200$ \\
\hline $05 / 18 / 99^{\mathbf{F b}}$ & 1101 & -- & $<.044$ & $<.032$ & $<.130$ & $<.064$ & $<.032$ & $<.066$ & $<.044$ & $<.026$ & $<.230$ & $<.200$ \\
\hline $06 / 24 / 99$ & 1015 & 11,900 & $<.044$ & $<.032$ & $<.130$ & $<.064$ & $<.032$ & $<.066$ & $<.044$ & $<.026$ & $<.230$ & $<.200$ \\
\hline $06 / 24 / 99^{\mathbf{T b}}$ & 1016 & -- & $<.044$ & $<.032$ & $<.130$ & $<.064$ & $<.032$ & $<.066$ & $<.044$ & $<.026$ & $<.230$ & $<.200$ \\
\hline $07 / 28 / 99$ & 1000 & 2,110 & $<.044$ & e.012 & $<.130$ & $<.064$ & $<.032$ & $<.066$ & $<.044$ & $<.026$ & $<.230$ & $<.200$ \\
\hline $07 / 28 / 99^{\mathbf{T b}}$ & 1001 & -- & $<.044$ & $<.032$ & $<.130$ & $<.064$ & $<.032$ & $<.066$ & $<.044$ & $<.026$ & $<.230$ & $<.200$ \\
\hline 09/10/99 & 0940 & 1,230 & $<.044$ & e.014 & $<.130$ & $<.064$ & $<.032$ & $<.066$ & $<.044$ & $<.026$ & $<.230$ & $<.200$ \\
\hline $10 / 21 / 99$ & 1110 & 2,230 & $<.030$ & e.012 & $<.090$ & $<.060$ & $<.060$ & $<.066$ & $<.040$ & $<.026$ & $<.230$ & $<.200$ \\
\hline
\end{tabular}


Table 10. Volatile organic-compound concentration data for surface-water samples collected at fixed water-quality sites along the Spokane River, Idaho and Washington, 1999-2001 (Continued)

\begin{tabular}{|c|c|c|c|c|c|c|c|c|c|c|}
\hline Date & $\begin{array}{c}1,2,3- \\
\text { Trichloro- } \\
\text { benzene } \\
(\mu \mathrm{g} / \mathrm{L})\end{array}$ & $\begin{array}{c}\text { 1,2,3- } \\
\text { Trichloro- } \\
\text { propane } \\
(\mu \mathrm{g} / \mathrm{L})\end{array}$ & $\begin{array}{c}1,2,3- \\
\text { Trimethyl- } \\
\text { benzene } \\
(\mu \mathrm{g} / \mathrm{L})\end{array}$ & $\begin{array}{c}1,2,4- \\
\text { Trichloro- } \\
\text { benzene } \\
(\mu \mathrm{g} / \mathrm{L})\end{array}$ & $\begin{array}{c}1,2,4- \\
\text { Trimethyl-1- } \\
\text { benzene } \\
(\mu \mathrm{g} / \mathrm{L})\end{array}$ & $\begin{array}{c}\text { 1,2,- } \\
\text { Dibromo- } \\
\text { 3-chloro- } \\
\text { propane } \\
(\mu \mathrm{g} / \mathrm{L})\end{array}$ & $\begin{array}{c}\text { 1,2- } \\
\text { Dibromo- } \\
\text { ethane } \\
(\mu \mathrm{g} / \mathrm{L})\end{array}$ & $\begin{array}{c}\text { 1,2- } \\
\text { Dichloro- } \\
\text { benzene } \\
(\mu \mathrm{g} / \mathrm{L})\end{array}$ & $\begin{array}{c}\text { 1,2- } \\
\text { Dichloro- } \\
\text { ethane } \\
(\mu \mathrm{g} / \mathrm{L})\end{array}$ & $\begin{array}{c}1,2,- \\
\text { Dichloro- } \\
\text { ethane-d } \\
\text { surrogate } \\
\text { (percent) }\end{array}$ \\
\hline \multicolumn{11}{|c|}{ SITE 35 12419000--SPOKANE RIVER NEAR POST FALLS, IDAHO } \\
\hline 02/09/99 & $<0.270$ & $<0.160$ & $<0.120$ & $<0.190$ & $<0.056$ & $<0.210$ & $<0.036$ & $<0.048$ & $<0.130$ & 104 \\
\hline $02 / 09 / 99^{\mathbf{F b}}$ & $<.270$ & $<.160$ & $<.120$ & $<.190$ & $<.056$ & $<.210$ & $<.036$ & $<.048$ & $<.130$ & 102 \\
\hline $05 / 19 / 99^{\mathbf{F b}}$ & $<.270$ & $<.160$ & $<.120$ & $<.190$ & $<.056$ & $<.210$ & $<.036$ & $<.048$ & $<.130$ & 103 \\
\hline 05/19/99 & $<.270$ & $<.160$ & $<.120$ & $<.190$ & e. 005 & $<.210$ & $<.036$ & $<.048$ & $<.130$ & 98 \\
\hline $06 / 23 / 99$ & $<.270$ & $<.160$ & $<.120$ & $<.190$ & $<.056$ & $<.210$ & $<.036$ & $<.048$ & $<.130$ & 106 \\
\hline $06 / 23 / 99^{\mathbf{T b}}$ & $<.270$ & $<.160$ & $<.120$ & $<.190$ & $<.056$ & $<.210$ & $<.036$ & $<.048$ & $<.130$ & 105 \\
\hline $07 / 27 / 99$ & $<.270$ & $<.160$ & $<.120$ & $<.190$ & $<.056$ & $<.210$ & $<.036$ & $<.048$ & $<.130$ & 105 \\
\hline 09/09/99 & $<.270$ & $<.160$ & $<.120$ & $<.190$ & $<.056$ & $<.210$ & $<.036$ & $<.048$ & $<.130$ & 107 \\
\hline $10 / 20 / 99^{\mathbf{F b}}$ & $<.270$ & $<.160$ & $<.120$ & $<.190$ & $<.056$ & $<.210$ & $<.036$ & $<.048$ & $<.103$ & 101 \\
\hline $10 / 20 / 99^{\mathbf{S b}}$ & $<.270$ & $<.160$ & $<.120$ & $<.190$ & $<.056$ & $<.210$ & $<.036$ & $<.048$ & $<.130$ & 108 \\
\hline $10 / 20 / 99$ & $<.270$ & $<.160$ & $<.120$ & $<.190$ & $<.056$ & $<.210$ & $<.036$ & $<.048$ & $<.130$ & 113 \\
\hline $07 / 25 / 00$ & $<.270$ & $<.160$ & $<.120$ & $<.190$ & $<.056$ & $<.210$ & $<.036$ & $<.048$ & $<.130$ & 114 \\
\hline $07 / 25 / 00^{\mathbf{F b}}$ & $<.270$ & $<.160$ & $<.120$ & $<.190$ & $<.056$ & $<.210$ & $<.036$ & $<.048$ & $<.130$ & 108 \\
\hline 08/30/00 & $<.270$ & $<.160$ & $<.120$ & $<.190$ & $<.056$ & $<.210$ & $<.036$ & $<.048$ & $<.130$ & 91 \\
\hline $08 / 30 / 00^{\mathbf{T b}}$ & $<.270$ & $<.160$ & $<.120$ & $<.190$ & $<.056$ & $<.210$ & $<.036$ & $<.048$ & $<.130$ & 104 \\
\hline \multicolumn{11}{|c|}{ SITE 4112424500 SPOKANE RIVER AT SEVEN MILE BRIDGE, NEAR SPOKANE, WASH. } \\
\hline 02/09/99 & $<.270$ & $<.160$ & $<.120$ & $<.190$ & $<.056$ & $<.210$ & $<.036$ & $<.048$ & $<.130$ & 104 \\
\hline 05/18/99 & $<.270$ & $<.160$ & $<.120$ & $<.190$ & e.008 & $<.210$ & $<.036$ & $<.048$ & $<.130$ & 99 \\
\hline $05 / 18 / 99^{\mathbf{F b}}$ & $<.270$ & $<.160$ & $<.120$ & $<.190$ & $<.056$ & $<.210$ & $<.036$ & $<.048$ & $<.130$ & 100 \\
\hline $06 / 24 / 99$ & $<.270$ & $<.160$ & $<.120$ & $<.190$ & $<.056$ & $<.210$ & $<.036$ & $<.048$ & $<.130$ & 109 \\
\hline $06 / 24 / 99^{\mathbf{T b}}$ & $<.270$ & $<.160$ & $<.120$ & $<.190$ & $<.056$ & $<.210$ & $<.036$ & $<.048$ & $<.130$ & 103 \\
\hline 07/28/99 & $<.270$ & $<.160$ & $<.120$ & $<.190$ & $<.056$ & $<.210$ & $<.036$ & $<.048$ & $<.130$ & 102 \\
\hline $07 / 28 / 99^{\mathbf{T b}}$ & $<.270$ & $<.160$ & $<.120$ & $<.190$ & $<.056$ & $<.210$ & $<.036$ & $<.048$ & $<.130$ & 103 \\
\hline 09/10/99 & $<.270$ & $<.160$ & $<.120$ & $<.190$ & e.006 & $<.210$ & $<.036$ & $<.048$ & $<.130$ & 93 \\
\hline $10 / 21 / 99$ & $<.270$ & $<.160$ & $<.120$ & $<.190$ & $<.056$ & $<.210$ & $<.036$ & $<.048$ & $<.130$ & 113 \\
\hline
\end{tabular}


Table 10. Volatile organic-compound concentration data for surface-water samples collected at fixed water-quality sites along the Spokane River, Idaho and Washington, 1999-2001 (Continued)

\begin{tabular}{|c|c|c|c|c|c|c|c|c|c|c|c|}
\hline Date & $\begin{array}{c}\text { 1,2- } \\
\text { Dichloro- } \\
\text { propane } \\
(\mu \mathrm{g} / \mathrm{L})\end{array}$ & $\begin{array}{c}1,3,5- \\
\text { Trimethyl- } \\
\text { benzene } \\
(\mu \mathbf{g} / \mathbf{L})\end{array}$ & $\begin{array}{c}\text { 1,3- } \\
\text { Dichloro- } \\
\text { benzene } \\
(\mu \mathrm{g} / \mathrm{L})\end{array}$ & $\begin{array}{c}\text { 1,3- } \\
\text { Dichloro- } \\
\text { propane } \\
(\mu \mathrm{g} / \mathrm{L})\end{array}$ & $\begin{array}{l}\text { 1,4-Bromo- } \\
\text { fluoro- } \\
\text { benzene, } \\
\text { surrogate } \\
\text { (percent) }\end{array}$ & $\begin{array}{c}1,4- \\
\text { Dichloro- } \\
\text { benzene } \\
(\mu \mathrm{g} / \mathrm{L})\end{array}$ & $\begin{array}{c}2,2- \\
\text { Dichloro- } \\
\text { propane } \\
(\mu \mathrm{g} / \mathrm{L})\end{array}$ & $\begin{array}{c}\text { 2-Butanone } \\
(\mu \mathrm{g} / \mathbf{L})\end{array}$ & $\begin{array}{c}\text { 2-Chloro- } \\
\text { toluene } \\
(\mu \mathrm{g} / \mathrm{L})\end{array}$ & $\begin{array}{c}2- \\
\text { Hexanone } \\
(\mu \mathrm{g} / \mathrm{L})\end{array}$ & $\begin{array}{c}\text { 3-Chloro- } \\
\text { propene } \\
(\mu \mathrm{g} / \mathrm{L})\end{array}$ \\
\hline \multicolumn{12}{|c|}{ SITE 35 12419000--SPOKANE RIVER NEAR POST FALLS, IDAHO } \\
\hline $02 / 09 / 99$ & $<0.068$ & $<0.044$ & $<0.054$ & $<0.120$ & 87 & $<0.050$ & $<0.078$ & $<1.6$ & $<0.042$ & $<0.700$ & $<0.200$ \\
\hline $02 / 09 / 99^{\mathbf{F b}}$ & $<.068$ & $<.044$ & $<.054$ & $<.120$ & 90 & $<.050$ & $<.078$ & $<1.6$ & $<.042$ & $<.700$ & $<.200$ \\
\hline $05 / 19 / 99^{\mathbf{F b}}$ & $<.068$ & $<.044$ & $<.054$ & $<.120$ & 109 & $<.050$ & $<.078$ & $<1.6$ & $<.042$ & $<.700$ & $<.200$ \\
\hline 05/19/99 & $<.068$ & $<.044$ & $<.054$ & $<.120$ & 99 & $<.050$ & $<.078$ & $<1.6$ & $<.042$ & $<.700$ & $<.200$ \\
\hline $06 / 23 / 99$ & $<.068$ & $<.044$ & $<.054$ & $<.120$ & 102 & $<.050$ & $<.078$ & $<1.6$ & $<.042$ & $<.700$ & $<.200$ \\
\hline $06 / 23 / 99^{\mathbf{T b}}$ & $<.068$ & $<.044$ & $<.054$ & $<.120$ & 99 & $<.050$ & $<.078$ & $<1.6$ & $<.042$ & $<.700$ & $<.200$ \\
\hline 07/27/99 & $<.068$ & $<.044$ & $<.054$ & $<.120$ & 97 & $<.050$ & $<.078$ & $<1.6$ & $<.042$ & $<.700$ & $<.200$ \\
\hline 09/09/99 & $<.068$ & $<.044$ & $<.054$ & $<.120$ & 94 & $<.050$ & $<.078$ & $<1.6$ & $<.042$ & $<.700$ & $<.200$ \\
\hline $10 / 20 / 99^{\mathbf{F b}}$ & $<.068$ & $<.044$ & $<.054$ & $<.120$ & 100 & $<.050$ & $<.050$ & $<1.6$ & $<.042$ & $<.700$ & $<.200$ \\
\hline $10 / 20 / 99^{\mathbf{S b}}$ & $<.068$ & $<.044$ & $<.054$ & $<.120$ & 106 & $<.050$ & $<.050$ & $<1.6$ & $<.042$ & $<.700$ & $<.200$ \\
\hline $10 / 20 / 99$ & $<.068$ & $<.044$ & $<.054$ & $<.120$ & 76 & $<.050$ & $<.050$ & $<1.6$ & $<.042$ & $<.700$ & $<.200$ \\
\hline $07 / 25 / 00$ & $<.068$ & $<.044$ & $<.054$ & $<.120$ & 66 & $<.050$ & $<.050$ & $<1.6$ & $<.042$ & $<.700$ & $<.200$ \\
\hline $07 / 25 / 00^{\mathbf{F b}}$ & $<.068$ & $<.044$ & $<.054$ & $<.120$ & 73 & $<.050$ & $<.050$ & $<1.6$ & $<.042$ & $<.700$ & $<.200$ \\
\hline $08 / 30 / 00$ & $<.068$ & $<.044$ & $<.054$ & $<.120$ & 117 & $<.050$ & $<.050$ & $<1.6$ & $<.042$ & $<.700$ & $<.200$ \\
\hline $08 / 30 / 00^{\mathbf{T b}}$ & $<.068$ & $<.044$ & $<.054$ & $<.120$ & 111 & $<.050$ & $<.050$ & $<1.6$ & $<.042$ & $<.700$ & $<.200$ \\
\hline \multicolumn{12}{|c|}{ SITE 4112424500 SPOKANE RIVER AT SEVEN MILE BRIDGE, NEAR SPOKANE, WASH. } \\
\hline 02/09/99 & $<.068$ & $<.044$ & $<.054$ & $<.120$ & 88 & $<.050$ & $<.078$ & $<1.6$ & $<.042$ & $<.700$ & $<.200$ \\
\hline 05/18/99 & $<.068$ & $<.044$ & $<.054$ & $<.120$ & 103 & $<.050$ & $<.078$ & $<1.6$ & $<.042$ & $<.700$ & $<.200$ \\
\hline $05 / 18 / 99^{\mathbf{F b}}$ & $<.068$ & $<.044$ & $<.054$ & $<.120$ & 108 & $<.050$ & $<.078$ & $<1.6$ & $<.042$ & $<.700$ & $<.200$ \\
\hline $06 / 24 / 99$ & $<.068$ & $<.044$ & $<.054$ & $<.120$ & 98 & $<.050$ & $<.078$ & $<1.6$ & $<.042$ & $<.700$ & $<.200$ \\
\hline $06 / 24 / 99^{\mathbf{T b}}$ & $<.068$ & $<.044$ & $<.054$ & $<.120$ & 96 & $<.050$ & $<.078$ & $<1.6$ & $<.042$ & $<.700$ & $<.200$ \\
\hline 07/28/99 & $<.068$ & $<.044$ & $<.054$ & $<.120$ & 85 & $<.050$ & $<.078$ & $<1.6$ & $<.042$ & $<.700$ & $<.200$ \\
\hline $07 / 28 / 99^{\mathbf{T b}}$ & $<.068$ & $<.044$ & $<.054$ & $<.120$ & 87 & $<.050$ & $<.078$ & $<1.6$ & $<.042$ & $<.700$ & $<.200$ \\
\hline 09/10/99 & $<.068$ & $<.044$ & $<.054$ & $<.120$ & 103 & e.036 & $<.078$ & $<1.6$ & $<.042$ & $<.700$ & $<.200$ \\
\hline $10 / 21 / 99$ & $<.068$ & $<.044$ & $<.054$ & $<.120$ & 103 & $<.050$ & $<.050$ & $<1.6$ & $<.042$ & $<.700$ & $<.200$ \\
\hline
\end{tabular}


Table 10. Volatile organic-compound concentration data for surface-water samples collected at fixed water-quality sites along the Spokane River, Idaho and Washington, 1999-2001 (Continued)

\begin{tabular}{|c|c|c|c|c|c|c|c|c|c|c|c|c|}
\hline Date & $\begin{array}{c}\text { 4-Chloro- } \\
\text { toluene } \\
(\mu \mathrm{g} / \mathrm{L})\end{array}$ & $\begin{array}{c}\text { 4-Isopro- } \\
\text { pyl- } \\
\text { 1-methyl- } \\
\text { benzene } \\
(\mu \mathrm{g} / \mathrm{L})\end{array}$ & $\begin{array}{l}\text { 4-Methyl-2- } \\
\text { pentanone } \\
(\mu \mathrm{g} / \mathrm{L})\end{array}$ & $\begin{array}{c}\text { Ace- } \\
\text { tone } \\
(\mu \mathrm{g} / \mathrm{L})\end{array}$ & $\begin{array}{c}\text { Acrylo- } \\
\text { nitrile } \\
(\mu \mathrm{g} / \mathrm{L})\end{array}$ & $\begin{array}{c}\text { Benzene } \\
(\mu \mathrm{g} / \mathbf{L})\end{array}$ & $\begin{array}{c}\text { Bromo- } \\
\text { benzene } \\
(\mu \mathrm{g} / \mathrm{L})\end{array}$ & $\begin{array}{c}\text { Bromo- } \\
\text { chloro- } \\
\text { methane } \\
(\mu \mathrm{g} / \mathrm{L})\end{array}$ & $\begin{array}{c}\text { Bromo- } \\
\text { dichloro- } \\
\text { methane } \\
(\mu \mathrm{g} / \mathrm{L})\end{array}$ & $\begin{array}{c}\text { Bromo- } \\
\text { ethane } \\
(\mu \mathrm{g} / \mathrm{L})\end{array}$ & $\begin{array}{c}\text { Bromo- } \\
\text { form } \\
(\mu \mathrm{g} / \mathrm{L})\end{array}$ & $\begin{array}{c}\text { Bromo- } \\
\text { methane } \\
(\mu \mathrm{g} / \mathrm{L})\end{array}$ \\
\hline \multicolumn{13}{|c|}{ SITE 35 12419000--SPOKANE RIVER NEAR POST FALLS, IDAHO } \\
\hline $02 / 09 / 99$ & $<0.056$ & e0.008 & $<0.370$ & e4.8 & $<1.2$ & $<0.100$ & $<0.036$ & $<0.044$ & $<0.048$ & $<0.100$ & $<0.100$ & $<0.150$ \\
\hline $02 / 09 / 99^{\mathbf{F b}}$ & $<.056$ & $<.110$ & $<.370$ & $<5.0$ & $<1.2$ & $<.100$ & $<.036$ & $<.044$ & $<.048$ & $<.100$ & $<.100$ & $<.150$ \\
\hline $05 / 19 / 99^{\mathbf{F b}}$ & $<.056$ & $<.110$ & $<.370$ & $<5.0$ & $<1.2$ & $<.100$ & $<.036$ & $<.044$ & $<.048$ & $<.100$ & $<.100$ & $<.150$ \\
\hline 05/19/99 & $<.056$ & $<.110$ & $<.370$ & $<5.0$ & $<1.2$ & e.015 & $<.036$ & $<.044$ & $<.048$ & $<.100$ & $<.100$ & $<.150$ \\
\hline $06 / 23 / 99$ & $<.056$ & $<.110$ & $<.370$ & e1.9 & $<1.2$ & e.022 & $<.036$ & $<.044$ & $<.048$ & $<.100$ & $<.100$ & $<.150$ \\
\hline $06 / 23 / 99^{\mathbf{T b}}$ & $<.056$ & $<.110$ & $<.370$ & $<5.0$ & $<1.2$ & $<.100$ & $<.036$ & $<.044$ & $<.048$ & $<.100$ & $<.100$ & $<.150$ \\
\hline $07 / 27 / 99$ & $<.056$ & $<.110$ & $<.370$ & 5.6 & $<1.2$ & .181 & $<.036$ & $<.044$ & e.024 & $<.100$ & $<.100$ & $<.150$ \\
\hline 09/09/99 & $<.056$ & $<.110$ & $<.370$ & e4.9 & $<1.2$ & e.031 & $<.036$ & $<.044$ & $<.048$ & $<.100$ & $<.100$ & $<.150$ \\
\hline $10 / 20 / 99^{\mathbf{F b}}$ & $<.060$ & $<.070$ & $<.370$ & $<7.0$ & $<1.2$ & $<.035$ & $<.036$ & $<.044$ & $<.048$ & $<.100$ & $<.060$ & $<.260$ \\
\hline $10 / 20 / 99^{S b}$ & $<.060$ & $<.070$ & $<.370$ & e3.7 & $<1.2$ & $<.035$ & $<.036$ & $<.044$ & $<.048$ & $<.100$ & $<.060$ & $<.260$ \\
\hline $10 / 20 / 99$ & $<.060$ & $<.070$ & $<.370$ & e5.8 & $<1.2$ & $<.035$ & $<.036$ & $<.044$ & $<.048$ & $<.100$ & $<.100$ & $<.260$ \\
\hline $07 / 25 / 00$ & $<.060$ & $<.070$ & $<.370$ & $<7.0$ & $<1.2$ & .115 & $<.036$ & $<.044$ & $<.048$ & $<.100$ & $<.060$ & $<.260$ \\
\hline $07 / 25 / 00^{\mathbf{F b}}$ & $<.060$ & $<.070$ & $<.370$ & $<7.0$ & $<1.2$ & $<.035$ & $<.036$ & $<.044$ & $<.048$ & $<.100$ & $<.060$ & $<.260$ \\
\hline $08 / 30 / 00$ & $<.060$ & $<.070$ & $<.370$ & $<7.0$ & $<1.2$ & e.012 & $<.036$ & $<.044$ & e.022 & $<.100$ & $<.060$ & $<.260$ \\
\hline $08 / 30 / 00^{\mathbf{T b}}$ & $<.060$ & $<.070$ & $<.370$ & $<7.0$ & $<1.2$ & $<.035$ & $<.036$ & $<.044$ & $<.048$ & $<.100$ & $<.060$ & $<.260$ \\
\hline \multicolumn{13}{|c|}{ SITE 41 12424500--SPOKANE RIVER AT SEVEN MILE BRIDGE, NEAR SPOKANE, WASH. } \\
\hline 02/09/99 & $<.056$ & $<.110$ & $<.370$ & e2.9 & $<1.2$ & e.015 & $<.036$ & $<.044$ & $<.048$ & $<.100$ & $<.100$ & $<.150$ \\
\hline 05/18/99 & $<.056$ & $<.110$ & $<.370$ & $<5.0$ & $<1.2$ & e.011 & $<.036$ & $<.044$ & $<.048$ & $<.100$ & $<.100$ & $<.150$ \\
\hline $05 / 18 / 99^{\mathbf{F b}}$ & $<.056$ & $<.110$ & $<.370$ & $<5.0$ & $<1.2$ & $<.100$ & $<.036$ & $<.044$ & $<.048$ & $<.100$ & $<.100$ & $<.150$ \\
\hline $06 / 24 / 99$ & $<.056$ & $<.110$ & $<.370$ & e2.1 & $<1.2$ & e.009 & $<.036$ & $<.044$ & $<.048$ & $<.100$ & $<.100$ & $<.150$ \\
\hline $06 / 24 / 99^{\mathbf{T b}}$ & $<.056$ & $<.110$ & $<.370$ & $<5.0$ & $<1.2$ & $<.100$ & $<.036$ & $<.044$ & $<.048$ & $<.100$ & $<.100$ & $<.150$ \\
\hline $07 / 28 / 99$ & $<.056$ & $<.110$ & $<.370$ & e3.2 & $<1.2$ & e.007 & $<.036$ & $<.044$ & e. 044 & $<.100$ & $<.100$ & $<.150$ \\
\hline $07 / 28 / 99^{\mathbf{T b}}$ & $<.056$ & $<.110$ & $<.370$ & $<5.0$ & $<1.2$ & $<.100$ & $<.036$ & $<.044$ & $<.048$ & $<.100$ & $<.100$ & $<.150$ \\
\hline 09/10/99 & $<.056$ & $<.110$ & $<.370$ & $<5.0$ & $<1.2$ & e.007 & $<.036$ & $<.044$ & $<.048$ & $<.100$ & $<.100$ & $<.150$ \\
\hline $10 / 21 / 99$ & $<.060$ & $<.070$ & $<.370$ & 15 & $<1.2$ & e.013 & $<.036$ & $<.044$ & e.024 & $<.100$ & $<.060$ & $<.260$ \\
\hline
\end{tabular}


Table 10. Volatile organic-compound concentration data for surface-water samples collected at fixed water-quality sites along the Spokane River, Idaho and Washington, 1999-2001 (Continued)

\begin{tabular}{|c|c|c|c|c|c|c|c|c|c|c|c|}
\hline Date & $\begin{array}{c}\text { Butyl- } \\
\text { benzene } \\
(\mu \mathrm{g} / \mathrm{L})\end{array}$ & $\begin{array}{c}\text { Carbon } \\
\text { disulfide } \\
(\mu \mathrm{g} / \mathrm{L})\end{array}$ & $\begin{array}{c}\text { Chloro- } \\
\text { benzene } \\
(\mu \mathrm{g} / \mathrm{L})\end{array}$ & $\begin{array}{c}\text { Chloro- } \\
\text { ethane } \\
(\mu \mathrm{g} / \mathrm{L})\end{array}$ & $\begin{array}{c}\text { Chloro- } \\
\text { form } \\
(\mu \mathrm{g} / \mathrm{L})\end{array}$ & $\begin{array}{c}\text { Chloro- } \\
\text { methane } \\
(\mu \mathrm{g} / \mathrm{L})\end{array}$ & $\begin{array}{c}\text { cis- } \\
1,2,- \\
\text { Dichloro- } \\
\text { ethylene } \\
(\mu \mathrm{g} / \mathrm{L})\end{array}$ & $\begin{array}{c}\text { cis- } \\
1,3- \\
\text { Dichloro- } \\
\text { propene } \\
(\mu \mathrm{g} / \mathrm{L})\end{array}$ & $\begin{array}{c}\text { Dibromo- } \\
\text { chloro- } \\
\text { methane } \\
(\mu \mathrm{g} / \mathrm{L})\end{array}$ & $\begin{array}{c}\text { Dibromo- } \\
\text { methane } \\
(\mu \mathrm{g} / \mathrm{L})\end{array}$ & $\begin{array}{c}\text { Dichloro- } \\
\text { difluoro- } \\
\text { methane } \\
(\mu \mathrm{g} / \mathrm{L})\end{array}$ \\
\hline \multicolumn{12}{|c|}{$\begin{array}{ll}\text { SITE } 35 \text { 12419000--SPOKANE RIVER NEAR POST FALLS, IDAHO } \\
\end{array}$} \\
\hline 02/09/99 & $<0.190$ & $<0.370$ & $<0.028$ & $<0.120$ & $\mathrm{e} 0.011$ & $<0.250$ & $<0.038$ & $<0.090$ & $<0.180$ & $<0.050$ & $<0.140$ \\
\hline $02 / 09 / 99^{\mathbf{F b}}$ & $<.190$ & $<.370$ & $<.028$ & $<.120$ & $<.052$ & $<.250$ & $<.038$ & $<.090$ & $<.180$ & $<.050$ & $<.140$ \\
\hline $05 / 19 / 99$ & $<.190$ & $<.370$ & $<.028$ & $<.120$ & $<.052$ & $<.250$ & $<.038$ & $<.090$ & $<.180$ & $<.050$ & $<.140$ \\
\hline $06 / 23 / 99$ & $<.190$ & $<.370$ & $<.028$ & $<.120$ & e.008 & $<.250$ & $<.038$ & $<.090$ & $<.180$ & $<.050$ & $<.140$ \\
\hline $06 / 23 / 99^{\mathbf{T b}}$ & $<.190$ & $<.370$ & $<.028$ & $<.120$ & $<.052$ & $<.250$ & $<.038$ & $<.090$ & $<.180$ & $<.050$ & $<.140$ \\
\hline $07 / 27 / 99$ & $<.190$ & $<.370$ & $<.028$ & $<.120$ & e.045 & $<.250$ & $<.038$ & $<.090$ & $<.180$ & $<.050$ & $<.140$ \\
\hline 09/09/99 & $<.190$ & $<.370$ & $<.028$ & $<.120$ & e.064 & $<.250$ & $<.038$ & $<.090$ & $<.180$ & $<.050$ & $<.140$ \\
\hline $10 / 20 / 99^{\mathbf{F b}}$ & $<.190$ & $<.070$ & $<.028$ & $<.120$ & $<.052$ & $<.250$ & $<.038$ & $<.090$ & $<.180$ & $<.050$ & $<.270$ \\
\hline $08 / 30 / 00$ & $<.190$ & $<.070$ & $<.028$ & $<.120$ & e. 068 & $<.250$ & $<.038$ & $<.090$ & $<.180$ & $<.050$ & $<.270$ \\
\hline $08 / 30 / 00^{\mathbf{T b}}$ & $<.190$ & $<.070$ & $<.028$ & $<.120$ & $<.052$ & $<.250$ & $<.038$ & $<.090$ & $<.180$ & $<.050$ & $<.270$ \\
\hline \multicolumn{12}{|c|}{ SITE 41 12424500--SPOKANE RIVER AT SEVEN MILE BRIDGE, NEAR SPOKANE, WASH. } \\
\hline 02/09/99 & $<.190$ & $<.370$ & $<.028$ & $<.120$ & e.011 & $<.250$ & $<.038$ & $<.090$ & $<.180$ & $<.050$ & $<.140$ \\
\hline $05 / 18 / 99$ & $<.190$ & $<.370$ & $<.028$ & $<.120$ & e.019 & $<.250$ & $<.038$ & $<.090$ & $<.180$ & $<.050$ & $<.140$ \\
\hline $05 / 18 / 99^{\mathbf{F b}}$ & $<.190$ & $<.370$ & $<.028$ & $<.120$ & $<.052$ & $<.250$ & $<.038$ & $<.090$ & $<.180$ & $<.050$ & $<.140$ \\
\hline $06 / 24 / 99$ & $<.190$ & $<.370$ & $<.028$ & $<.120$ & $<.052$ & $<.250$ & $<.038$ & $<.090$ & $<.180$ & $<.050$ & $<.140$ \\
\hline $06 / 24 / 99^{\mathbf{T b}}$ & $<.190$ & $<.370$ & $<.028$ & $<.120$ & $<.052$ & $<.250$ & $<.038$ & $<.090$ & $<.180$ & $<.050$ & $<.140$ \\
\hline 07/28/99 & $<.190$ & $<.370$ & $<.028$ & $<.120$ & .131 & $<.250$ & $<.038$ & $<.090$ & $<.180$ & $<.050$ & $<.140$ \\
\hline $07 / 28 / 99^{\mathbf{T b}}$ & $<.190$ & $<.370$ & $<.028$ & $<.120$ & $<.052$ & $<.250$ & $<.038$ & $<.090$ & $<.180$ & $<.050$ & $<.140$ \\
\hline
\end{tabular}


Table 10. Volatile organic-compound concentration data for surface-water samples collected at fixed water-quality sites along the Spokane River, Idaho and Washington, 1999-2001 (Continued)

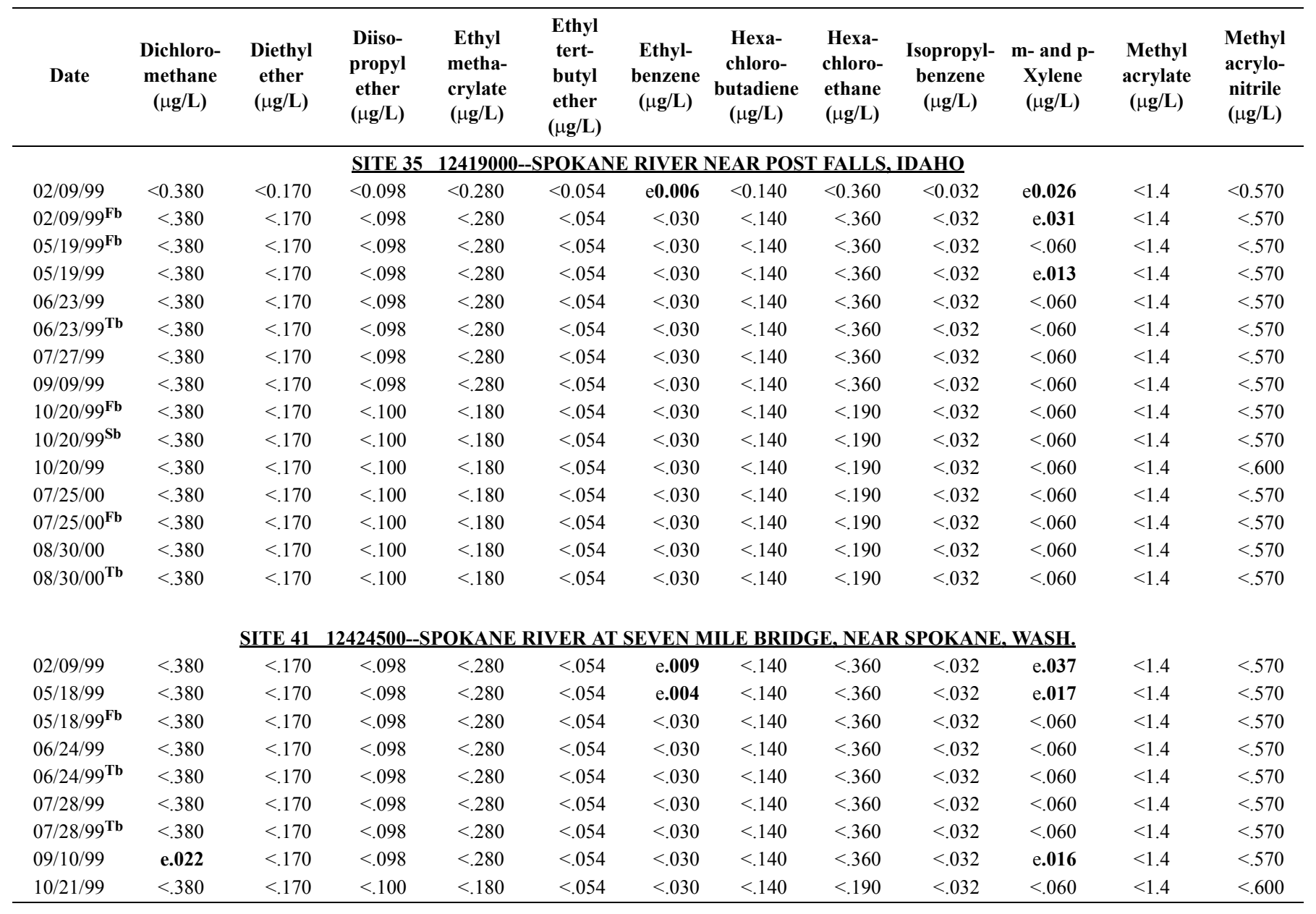


Table 10. Volatile organic-compound concentration data for surface-water samples collected at fixed water-quality sites along the Spokane River, Idaho and Washington, 1999-2001 (Continued)

\begin{tabular}{|c|c|c|c|c|c|c|c|c|c|c|c|}
\hline Date & $\begin{array}{c}\text { Methyl } \\
\text { iodide } \\
(\mu \mathrm{g} / \mathrm{L})\end{array}$ & $\begin{array}{l}\text { Methyl } \\
\text { metha- } \\
\text { crylate } \\
(\mu \mathrm{g} / \mathrm{L})\end{array}$ & $\begin{array}{l}\text { Naphtha- } \\
\text { lene } \\
(\mu \mathrm{g} / \mathrm{L})\end{array}$ & $\begin{array}{c}\text { n-Propyl- } \\
\text { benzene } \\
(\mu \mathrm{g} / \mathrm{L})\end{array}$ & $\begin{array}{c}\text { o-Ethyl } \\
\text { toluene } \\
(\mu \mathrm{g} / \mathrm{L})\end{array}$ & $\begin{array}{c}\text { o-Xylene } \\
(\mu \mathrm{g} / \mathrm{L})\end{array}$ & $\begin{array}{c}\text { sec-Butyl- } \\
\text { benzene } \\
(\mu \mathrm{g} / \mathrm{L})\end{array}$ & $\begin{array}{l}\text { Styrene } \\
(\mu \mathrm{g} / \mathrm{L})\end{array}$ & $\begin{array}{c}\text { tert- } \\
\text { Butyl } \\
\text { methyl } \\
\text { ether } \\
(\mu \mathrm{g} / \mathrm{L})\end{array}$ & $\begin{array}{c}\text { tert- } \\
\text { Butyl- } \\
\text { benzene } \\
(\mu \mathrm{g} / \mathrm{L})\end{array}$ & $\begin{array}{c}\text { tert-Pentyl } \\
\text { methyl } \\
\text { ether } \\
(\mu \mathrm{g} / \mathrm{L})\end{array}$ \\
\hline \multicolumn{12}{|c|}{ SITE 35 12419000--SPOKANE RIVER NEAR POST FALLS, IDAHO } \\
\hline $02 / 09 / 99$ & $<0.210$ & $<0.350$ & $<0.250$ & $<0.042$ & $<0.100$ & $<0.060$ & $<0.048$ & $<0.042$ & $<0.170$ & $<0.100$ & $<0.110$ \\
\hline $02 / 09 / 99^{\mathbf{F b}}$ & $<.210$ & $<.350$ & $<.250$ & $<.042$ & $<.100$ & $<.060$ & $<.048$ & $<.042$ & $<.170$ & $<.100$ & $<.110$ \\
\hline $05 / 19 / 99^{\mathbf{F b}}$ & $<.210$ & $<.350$ & $<.250$ & $<.042$ & $<.100$ & $<.060$ & $<.048$ & $<.042$ & $<.170$ & $<.100$ & $<.110$ \\
\hline 05/19/99 & $<.210$ & $<.350$ & $<.250$ & $<.042$ & $<.100$ & $<.060$ & $<.048$ & $<.042$ & $<.170$ & $<.100$ & $<.110$ \\
\hline $06 / 23 / 99$ & $<.210$ & $<.350$ & $<.250$ & $<.042$ & $<.100$ & $<.060$ & $<.048$ & $<.042$ & $<.170$ & $<.100$ & $<.110$ \\
\hline $06 / 23 / 99^{\mathbf{T b}}$ & $<.210$ & $<.350$ & $<.250$ & $<.042$ & $<.100$ & $<.060$ & $<.048$ & $<.042$ & $<.170$ & $<.100$ & $<.110$ \\
\hline 07/27/99 & $<.210$ & $<.350$ & $<.250$ & $<.042$ & $<.100$ & $<.060$ & $<.048$ & $<.042$ & .292 & $<.100$ & $<.110$ \\
\hline 09/09/99 & $<.210$ & $<.350$ & $<.250$ & $<.042$ & $<.100$ & $<.060$ & $<.048$ & $<.042$ & .285 & $<.100$ & $<.110$ \\
\hline $10 / 20 / 99^{\mathbf{F b}}$ & $<.120$ & $<.350$ & $<.250$ & $<.042$ & $<.060$ & $<.038$ & $<.032$ & $<.042$ & $<.170$ & $<.060$ & $<.110$ \\
\hline $10 / 20 / 99^{\mathbf{S b}}$ & $<.120$ & $<.350$ & $<.250$ & $<.042$ & $<.060$ & $<.038$ & $<.032$ & $<.042$ & $<.170$ & $<.060$ & $<.110$ \\
\hline $10 / 20 / 99$ & $<.120$ & $<.350$ & $<.250$ & $<.042$ & $<.060$ & $<.038$ & $<.032$ & $<.042$ & $<.170$ & $<.060$ & $<.110$ \\
\hline $07 / 25 / 00$ & $<.120$ & $<.350$ & $<.250$ & $<.042$ & $<.060$ & $<.038$ & $<.032$ & $<.042$ & e.108 & $<.060$ & $<.110$ \\
\hline $07 / 25 / 00^{\mathbf{F b}}$ & $<.120$ & $<.350$ & $<.250$ & $<.042$ & $<.060$ & $<.038$ & $<.032$ & $<.042$ & $<.170$ & $<.060$ & $<.110$ \\
\hline 08/30/00 & $<.120$ & $<.350$ & $<.250$ & $<.042$ & $<.060$ & $<.038$ & $<.032$ & $<.042$ & $<.170$ & $<.060$ & $<.110$ \\
\hline $08 / 30 / 00^{\mathbf{T b}}$ & $<.120$ & $<.350$ & $<.250$ & $<.042$ & $<.060$ & $<.038$ & $<.032$ & $<.042$ & $<.170$ & $<.060$ & $<.110$ \\
\hline \multicolumn{12}{|c|}{ SITE 41 12424500--SPOKANE RIVER AT SEVEN MILE BRIDGE, NEAR SPOKANE, WASH. } \\
\hline 02/09/99 & $<.210$ & $<.350$ & $<.250$ & $<.042$ & $<.100$ & $<.060$ & $<.048$ & $<.042$ & $<.170$ & $<.100$ & $<.110$ \\
\hline $05 / 18 / 99$ & $<.210$ & $<.350$ & $<.250$ & $<.042$ & $<.100$ & $<.060$ & $<.048$ & $<.042$ & $<.170$ & $<.100$ & $<.110$ \\
\hline $05 / 18 / 99^{\mathbf{F b}}$ & $<.210$ & $<.350$ & $<.250$ & $<.042$ & $<.100$ & $<.060$ & $<.048$ & $<.042$ & $<.170$ & $<.100$ & $<.110$ \\
\hline $06 / 24 / 99$ & $<.210$ & $<.350$ & $<.250$ & $<.042$ & $<.100$ & $<.060$ & $<.048$ & $<.042$ & $<.170$ & $<.100$ & $<.110$ \\
\hline $06 / 24 / 99^{\mathbf{T b}}$ & $<.210$ & $<.350$ & $<.250$ & $<.042$ & $<.100$ & $<.060$ & $<.048$ & $<.042$ & $<.170$ & $<.100$ & $<.110$ \\
\hline 07/28/99 & $<.210$ & $<.350$ & $<.250$ & $<.042$ & $<.100$ & $<.060$ & $<.048$ & $<.042$ & $<.170$ & $<.100$ & $<.110$ \\
\hline $07 / 28 / 99^{\mathbf{T b}}$ & $<.210$ & $<.350$ & $<.250$ & $<.042$ & $<.100$ & $<.060$ & $<.048$ & $<.042$ & $<.170$ & $<.100$ & $<.110$ \\
\hline 09/10/99 & $<.210$ & $<.350$ & $<.250$ & $<.042$ & $<.100$ & $<.060$ & $<.048$ & e.007 & $<.170$ & $<.100$ & $<.110$ \\
\hline $10 / 21 / 99$ & $<.120$ & $<.350$ & $<.250$ & $<.042$ & $<.060$ & $<.038$ & $<.032$ & $<.042$ & $<.170$ & $<.060$ & $<.110$ \\
\hline
\end{tabular}


Table 10. Volatile organic-compound concentration data for surface-water samples collected at fixed water-quality sites along the Spokane River, Idaho and Washington, 1999-2001 (Continued)

\begin{tabular}{|c|c|c|c|c|c|c|c|c|c|c|c|}
\hline Date & $\begin{array}{c}\text { Tetra- } \\
\text { chloro- } \\
\text { ethylene } \\
(\mu \mathrm{g} / \mathrm{L})\end{array}$ & $\begin{array}{c}\text { Tetra- } \\
\text { chloro- } \\
\text { methane } \\
(\mu \mathrm{g} / \mathrm{L})\end{array}$ & $\begin{array}{c}\text { Tetra- } \\
\text { hydro- } \\
\text { furan } \\
(\mu \mathrm{g} / \mathrm{L})\end{array}$ & $\begin{array}{c}\text { Toluene } \\
(\mu \mathrm{g} / \mathrm{L})\end{array}$ & $\begin{array}{c}\text { Toluene-d }_{8}, \\
\text { surrogate } \\
\text { (percent) }\end{array}$ & $\begin{array}{c}\text { trans-1,2- } \\
\text { Dichloro- } \\
\text { ethylene } \\
(\mu \mathrm{g} / \mathrm{L})\end{array}$ & $\begin{array}{c}\text { trans-1,3- } \\
\text { Dichloro- } \\
\text { propene } \\
(\mu \mathrm{g} / \mathrm{L})\end{array}$ & $\begin{array}{c}\text { trans-1,4,- } \\
\text { Dichloro- } \\
\text { 2-butene } \\
(\mu \mathrm{g} / \mathrm{L})\end{array}$ & $\begin{array}{c}\text { Trichloro- } \\
\text { ethylene } \\
(\mu \mathbf{g} / \mathbf{L})\end{array}$ & $\begin{array}{c}\text { Trichloro- } \\
\text { fluoro- } \\
\text { methane } \\
(\mu \mathrm{g} / \mathrm{L})\end{array}$ & $\begin{array}{c}\text { Vinyl } \\
\text { chloride } \\
(\mu \mathrm{g} / \mathrm{L})\end{array}$ \\
\hline \multicolumn{12}{|c|}{ SITE 35 12419000--SPOKANE RIVER NEAR POST FALLS, IDAHO } \\
\hline $02 / 09 / 99$ & $<0.100$ & $<0.088$ & $<9.0$ & 0.226 & 97 & $<0.032$ & $<0.130$ & $<0.700$ & $<0.038$ & $<0.090$ & $<0.110$ \\
\hline $02 / 09 / 99^{\mathbf{F b}}$ & $<.100$ & $<.088$ & $<9.0$ & .247 & 97 & $<.032$ & $<.130$ & $<.700$ & $<.038$ & $<.090$ & $<.110$ \\
\hline $05 / 19 / 99^{\mathbf{F b}}$ & $<.100$ & $<.088$ & $<9.0$ & e.021 & 105 & $<.032$ & $<.130$ & $<.700$ & $<.038$ & $<.090$ & $<.110$ \\
\hline $05 / 19 / 99$ & $<.100$ & $<.088$ & $<9.0$ & e.050 & 103 & $<.032$ & $<.130$ & $<.700$ & $<.038$ & $<.090$ & $<.110$ \\
\hline $06 / 23 / 99$ & $<.100$ & $<.088$ & $<9.0$ & e.034 & 100 & $<.032$ & $<.130$ & $<.700$ & $<.038$ & $<.090$ & $<.110$ \\
\hline $06 / 23 / 99^{\mathbf{T b}}$ & $<.100$ & $<.088$ & $<9.0$ & e.013 & 99 & $<.032$ & $<.130$ & $<.700$ & $<.038$ & $<.090$ & $<.110$ \\
\hline $07 / 27 / 99$ & $<.100$ & $<.088$ & $<9.0$ & e.011 & 101 & $<.032$ & $<.130$ & $<.700$ & $<.038$ & $<.090$ & $<.110$ \\
\hline 09/09/99 & $<.100$ & $<.088$ & $<9.0$ & e.022 & 91 & $<.032$ & $<.130$ & $<.700$ & $<.038$ & $<.090$ & $<.110$ \\
\hline $10 / 20 / 99^{\mathbf{F b}}$ & $<.100$ & $<.060$ & $<2.2$ & $<.050$ & 102 & $<.032$ & $<.090$ & $<.700$ & $<.038$ & $<.090$ & $<.110$ \\
\hline $10 / 20 / 99^{\mathbf{S b}}$ & $<.100$ & $<.060$ & $<2.2$ & e.005 & 96 & $<.032$ & $<.090$ & $<.700$ & $<.038$ & $<.090$ & $<.110$ \\
\hline $10 / 20 / 99$ & $<.100$ & $<.060$ & $<2.2$ & $<.050$ & 93 & $<.032$ & $<.090$ & $<.700$ & $<.038$ & $<.090$ & $<.110$ \\
\hline $07 / 25 / 00$ & $<.100$ & $<.060$ & $<2.2$ & $<.050$ & 92 & $<.032$ & $<.090$ & $<.700$ & $<.038$ & $<.090$ & $<.110$ \\
\hline $07 / 25 / 00^{\mathbf{F b}}$ & $<.100$ & $<.060$ & $<2.2$ & $<.050$ & 95 & $<.032$ & $<.090$ & $<.700$ & $<.038$ & $<.090$ & $<.110$ \\
\hline $08 / 30 / 00$ & $<.100$ & $<.060$ & $<2.2$ & $<.050$ & 100 & $<.032$ & $<.090$ & $<.700$ & $<.038$ & $<.090$ & $<.110$ \\
\hline $08 / 30 / 00^{\mathbf{T b}}$ & $<.100$ & $<.060$ & $<2.2$ & e.029 & 107 & $<.032$ & $<.090$ & $<.700$ & $<.038$ & $<.090$ & $<.110$ \\
\hline \multicolumn{12}{|c|}{ SITE 41 12424500--SPOKANE RIVER AT SEVEN MILE BRIDGE, NEAR SPOKANE, WASH. } \\
\hline 02/09/99 & e.010 & $<.088$ & $<9.0$ & .258 & 97 & $<.032$ & $<.130$ & $<.700$ & $<.038$ & $<.090$ & $<.110$ \\
\hline $05 / 18 / 99$ & $<.100$ & $<.088$ & $<9.0$ & e.049 & 102 & $<.032$ & $<.130$ & $<.700$ & $<.038$ & $<.090$ & $<.110$ \\
\hline $05 / 18 / 99^{\mathbf{F b}}$ & e.010 & $<.088$ & $<9.0$ & .050 & 97 & $<.032$ & $<.130$ & $<.700$ & $<.038$ & $<.090$ & $<.110$ \\
\hline $06 / 24 / 99$ & $<.100$ & $<.088$ & $<9.0$ & e.020 & 102 & $<.032$ & $<.130$ & $<.700$ & $<.038$ & $<.090$ & $<.110$ \\
\hline $06 / 24 / 99^{\mathbf{T b}}$ & e.010 & $<.088$ & $<9.0$ & e.014 & 97 & $<.032$ & $<.130$ & $<.700$ & $<.038$ & $<.090$ & $<.110$ \\
\hline $07 / 28 / 99$ & $<.100$ & $<.088$ & $<9.0$ & e.015 & 102 & $<.032$ & $<.130$ & $<.700$ & $<.038$ & $<.090$ & $<.110$ \\
\hline $07 / 28 / 99^{\mathbf{T b}}$ & e.010 & $<.088$ & $<9.0$ & e.023 & 97 & $<.032$ & $<.130$ & $<.700$ & $<.038$ & $<.090$ & $<.110$ \\
\hline 09/10/99 & $<.100$ & $<.088$ & $<9.0$ & e.034 & 102 & $<.032$ & $<.130$ & $<.700$ & $<.038$ & $<.090$ & $<.110$ \\
\hline $10 / 21 / 99$ & e.010 & $<.088$ & $<9.0$ & e.021 & 97 & $<.032$ & $<.130$ & $<.700$ & $<.038$ & $<.090$ & $<.110$ \\
\hline
\end{tabular}


Table 11. Streamflow, physical, and major-ion concentration data for surface-water samples collected for the synoptic study of miningaffected areas, Northern Rockies Intermontane Basins study unit, August-September 2000

[Abbreviations: $\mathrm{ft}^{3} / \mathrm{s}$, cubic feet per second; ${ }^{\circ} \mathrm{C}$, degrees Celsius; e, estimated; Fb, field blank; $\mu \mathrm{S} / \mathrm{cm}$, microsiemens per centimeter at 25 degrees Celsius; mg/L, milligrams per liter. Symbols: --, no data; $<$, less than reporting level]

\begin{tabular}{|c|c|c|c|c|c|c|}
\hline $\begin{array}{c}\text { Site } \\
\text { number } \\
\text { (fig. 1) }\end{array}$ & Site name & Date & Time & $\begin{array}{c}\text { Discharge, } \\
\text { instanta- } \\
\text { neous } \\
\left(\mathbf{f t}^{3} / \mathbf{s}\right)\end{array}$ & $\begin{array}{c}\text { pH, } \\
\text { field } \\
\text { (standard } \\
\text { units) }\end{array}$ & $\begin{array}{c}\text { Specific } \\
\text { conduc- } \\
\text { tance, } \\
\text { field } \\
(\mu \mathrm{S} / \mathrm{cm})\end{array}$ \\
\hline 6 & St. Regis River above Rainy Creek, near Saltese, Mont. & $08 / 02 / 00$ & 0930 & 15 & 7.6 & 69 \\
\hline 7 & St. Regis River near Haugan, Mont. & $08 / 02 / 00$ & 1330 & 46 & 7.3 & 59 \\
\hline 8 & St. Regis River near St. Regis, Mont. & $08 / 03 / 00$ & 0930 & 84 & 7.7 & 93 \\
\hline 17 & North Fork Coeur d'Alene River above Shoshone Creek, near Prichard, Idaho & $09 / 06 / 00$ & 1000 & 108 & 7.7 & 55 \\
\hline 18 & Prichard Creek near Murray, Idaho & $08 / 16 / 00$ & 1230 & 1.6 & 6.9 & 31 \\
\hline 19 & East Fork Eagle Creek near mouth, near Prichard, Idaho & $08 / 16 / 00$ & 1100 & 5.8 & 6.9 & 34 \\
\hline 20 & West Fork Eagle Creek below Settlers Grove, Idaho & $08 / 16 / 00$ & 0920 & 5.0 & 6.8 & 30 \\
\hline 21 & Beaver Creek near mouth, near Murray, Idaho & $08 / 16 / 00$ & 1530 & 4.3 & 7.3 & 96 \\
\hline 22 & Prichard Creek at mouth, at Prichard, Idaho & $08 / 16 / 00$ & 1400 & 28 & 6.9 & 32 \\
\hline 23 & North Fork Coeur d'Alene River at Enaville, Idaho & $09 / 07 / 00$ & 1530 & 283 & 7.5 & 46 \\
\hline 24 & South Fork Coeur d'Alene River at Shoshone Park, near Mullan, Idaho & $08 / 24 / 00$ & 1000 & 6.7 & 7.3 & 40 \\
\hline 25 & South Fork Coeur d'Alene River above Deadman Gulch, near Mullan, Idaho & $08 / 24 / 00$ & 1430 & 13 & 7.7 & 148 \\
\hline 26 & Canyon Creek near Burke, Idaho & $08 / 23 / 00$ & 1030 & 4.5 & 7.0 & 15 \\
\hline 27 & Canyon Creek at Woodland Park, Idaho & $08 / 23 / 00$ & 1500 & 16 & 7.8 & 105 \\
\hline 28 & South Fork Coeur d'Alene River at Silverton, Idaho & $08 / 25 / 00$ & 1000 & 56 & 7.7 & 149 \\
\hline 29 & East Fork Pine Creek above Nabob Creek, near Pinehurst, Idaho & $08 / 16 / 00$ & 1730 & 9.0 & 7.0 & 50 \\
\hline 30 & Pine Creek below Amy Gulch, near Pinehurst, Idaho & $08 / 16 / 00$ & 1840 & 21 & 6.6 & 30 \\
\hline $31^{\mathbf{F b}}$ & South Fork Coeur d'Alene River near Pinehurst, Idaho & $09 / 06 / 00$ & 1300 & -- & -- & -- \\
\hline 31 & South Fork Coeur d'Alene River near Pinehurst, Idaho & $09 / 06 / 00$ & 1400 & 123 & 7.2 & 272 \\
\hline 33 & St. Joe River at Red Ives Ranger Station, Idaho & $09 / 05 / 00$ & 1230 & 100 & 7.6 & 52 \\
\hline 34 & St. Joe River at Calder, Idaho & $09 / 07 / 00$ & 1100 & 398 & 7.5 & 57 \\
\hline
\end{tabular}


Table 11. Streamflow, physical, and major-ion concentration data for surface-water samples collected for the synoptic study of miningaffected areas, Northern Rockies Intermontane Basins study unit, August-September 2000 (Continued)

\begin{tabular}{|c|c|c|c|c|c|c|c|c|c|c|c|c|}
\hline $\begin{array}{c}\text { Temper- } \\
\text { ature, } \\
\text { water } \\
\left({ }^{\circ} \mathrm{C}\right)\end{array}$ & 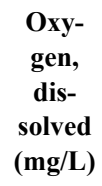 & $\begin{array}{c}\text { Dissolved } \\
\text { oxygen } \\
\text { (percent } \\
\text { saturation) }\end{array}$ & $\begin{array}{c}\text { Hard- } \\
\text { ness, } \\
\text { total } \\
(\mathrm{mg} / \mathrm{L} \text { as } \\
\left.\mathrm{CaCO}_{3}\right)\end{array}$ & $\begin{array}{c}\text { Cal- } \\
\text { cium, } \\
\text { dis- } \\
\text { solved } \\
(\mathrm{mg} / \mathrm{L})\end{array}$ & $\begin{array}{c}\text { Magne- } \\
\text { sium, } \\
\text { dis- } \\
\text { solved } \\
(\mathrm{mg} / \mathrm{L})\end{array}$ & $\begin{array}{c}\text { Alka- } \\
\text { linity, } \\
\text { field } \\
(\mathrm{mg} / \mathrm{L} \text { as } \\
\left.\mathrm{CaCO}_{3}\right)\end{array}$ & $\begin{array}{c}\text { Sodi- } \\
\text { um, } \\
\text { dis- } \\
\text { solved } \\
(\mathrm{mg} / \mathrm{L})\end{array}$ & $\begin{array}{c}\text { Sulfate, } \\
\text { dissolved } \\
(\mathrm{mg} / \mathrm{L} \\
\left.\text { as } \mathrm{SO}_{4}\right)\end{array}$ & $\begin{array}{c}\text { Chlo- } \\
\text { ride, } \\
\text { dis- } \\
\text { solved } \\
(\mathrm{mg} / \mathrm{L})\end{array}$ & $\begin{array}{l}\text { Fluo- } \\
\text { ride, } \\
\text { dis- } \\
\text { solved } \\
(\mathrm{mg} / \mathrm{L})\end{array}$ & $\begin{array}{c}\text { Silica, } \\
\text { dissolved } \\
(\mathrm{mg} / \mathrm{L} \text { as } \\
\left.\mathrm{SiO}_{2}\right)\end{array}$ & $\begin{array}{c}\begin{array}{c}\text { Site } \\
\text { number } \\
\text { (fig. 1) }\end{array} \\
\text { (fing }\end{array}$ \\
\hline 9.5 & 10.2 & 101 & 33 & 9.9 & 2.0 & 34 & 1.8 & .85 & 1.6 & $<0.10$ & 8.1 & 6 \\
\hline 15.0 & 8.8 & 98 & 27 & 8.0 & 1.6 & 30 & 2.1 & .85 & 1.6 & $<.10$ & 9.2 & 7 \\
\hline 13.5 & 9.5 & 101 & 41 & 12 & 2.6 & 46 & 1.9 & 1.0 & 1.2 & $<.10$ & 11 & 8 \\
\hline 12.0 & 9.8 & 98 & 28 & 6.3 & 2.9 & 29 & 1.0 & 1.4 & $<.29$ & $<.10$ & 9.7 & 17 \\
\hline 17.0 & -- & -- & 9.8 & 2.7 & .76 & 10 & 1.7 & 5.0 & e. 14 & $<.10$ & 13 & 18 \\
\hline 15.0 & -- & -- & 12 & 2.9 & 1.0 & 10 & 1.5 & 5.9 & $<.29$ & $<.10$ & 11 & 19 \\
\hline 10.5 & -- & -- & 12 & 3.1 & 1.1 & 14 & 1.1 & 1.6 & $<.29$ & $<.10$ & 8.5 & 20 \\
\hline 15.0 & -- & -- & 44 & 11 & 4.0 & 42 & 1.5 & 6.5 & .30 & $<.10$ & 15 & 21 \\
\hline 15.0 & -- & -- & 12 & 3.0 & 1.0 & 14 & 1.4 & 3.5 & e. 14 & $<.10$ & 12 & 22 \\
\hline 15.0 & 9.6 & 104 & 22 & 5.4 & 2.0 & 24 & 1.2 & 1.8 & e. 20 & $<.10$ & 11 & 23 \\
\hline 11.5 & 9.2 & 96 & 28 & 7.2 & 2.5 & 28 & 1.5 & 2.0 & 1.8 & $<.10$ & 9.9 & 24 \\
\hline 13.0 & 9.2 & 99 & 63 & 18 & 4.7 & 46 & 2.9 & 21 & 2.3 & $<.10$ & 9.1 & 25 \\
\hline 8.0 & 10.3 & 100 & 5.6 & 1.7 & .32 & 7.0 & .86 & .83 & $<.29$ & $<.10$ & 7.9 & 26 \\
\hline 19.0 & 8.8 & 101 & 46 & 13 & 3.3 & 36 & 1.6 & 16 & .40 & $<.10$ & 8.9 & 27 \\
\hline 12.5 & 9.5 & 98 & 68 & 18 & 5.3 & 50 & 3.4 & 21 & 3.1 & $<.10$ & 9.6 & 28 \\
\hline 17.0 & -- & -- & 19 & 4.7 & 1.8 & 14 & 1.4 & 9.8 & e. 20 & $<.10$ & 11 & 29 \\
\hline 13.0 & -- & -- & 11 & 2.9 & 1.0 & 10 & 1.1 & 4.0 & e. 19 & $<.10$ & 10 & 30 \\
\hline -- & -- & -- & -- & .003 & $<.001$ & -- & $<.025$ & -- & -- & -- & $<.02$ & $31^{\mathbf{F b}}$ \\
\hline 13.0 & 10.0 & 102 & 118 & 30 & 10 & 34 & 6.3 & 94 & 2.8 & .34 & 12 & 31 \\
\hline 8.5 & 10.2 & 100 & 22 & 6.4 & 1.3 & 24 & 1.0 & .91 & $<.29$ & $<.10$ & 7.8 & 33 \\
\hline 12.5 & 10.1 & 102 & 27 & 8.1 & 1.7 & 30 & 1.3 & .94 & e. 22 & $<.10$ & 10 & 34 \\
\hline
\end{tabular}


Table 12. Nutrient data for surface-water samples collected for the synoptic study of mining-affected areas, Northern Rockies Intermontane Basins study unit, August-September 2000

[All constituents are dissolved except phosphorus. Abbreviations: e, estimated; mg/L, milligrams per liter. Symbol: <, less than reporting level]

\begin{tabular}{|c|c|c|c|c|c|c|c|c|}
\hline $\begin{array}{l}\text { Site no. } \\
\text { (fig. 1) }\end{array}$ & Site name & Date & Time & $\begin{array}{c}\text { Nitrite } \\
\text { plus } \\
\text { nitrate } \\
(\mathrm{mg} / \mathrm{L} \\
\text { as N) }\end{array}$ & $\begin{array}{c}\text { Ammonia } \\
\text { plus } \\
\text { organic } \\
\text { nitrogen } \\
\text { (mg/L } \\
\text { as N) }\end{array}$ & $\begin{array}{c}\text { Ammonia } \\
(\mathrm{mg} / \mathrm{L} \\
\text { as N) }\end{array}$ & $\begin{array}{c}\text { Phos- } \\
\text { phorus, } \\
\text { ortho } \\
\text { phos- } \\
\text { phate } \\
\text { (mg/L } \\
\text { as P) }\end{array}$ & $\begin{array}{c}\text { Phos- } \\
\text { phorus, } \\
\text { total } \\
(\mathrm{mg} / \mathrm{L})\end{array}$ \\
\hline 6 & St. Regis River above Rainy Creek, near Saltese, Mont. & $08 / 02 / 00$ & 0930 & 0.016 & $\mathrm{e} 0.007$ & 0.003 & 0.003 & $\mathrm{e} 0.004$ \\
\hline 7 & St. Regis River near Haugan, Mont. & $08 / 02 / 00$ & 1330 & .012 & $<.10$ & $<.002$ & .003 & $<.008$ \\
\hline 8 & St. Regis River near St. Regis, Mont. & 08/03/00 & 0930 & .008 & $<.10$ & .002 & .003 & $<.008$ \\
\hline 17 & $\begin{array}{l}\text { North Fork Coeur d'Alene River above Shoshone Creek, near } \\
\text { Prichard, Idaho }\end{array}$ & 09/06/00 & 1000 & $<.005$ & $<.10$ & $<.002$ & .001 & $<.008$ \\
\hline 18 & Prichard Creek near Murray, Idaho & $08 / 16 / 00$ & 1230 & .013 & e. 07 & .003 & .005 & $<.008$ \\
\hline 19 & East Fork Eagle Creek near mouth, near Prichard, Idaho & $08 / 16 / 00$ & 1100 & .013 & $<.10$ & $<.002$ & .002 & $<.008$ \\
\hline 20 & West Fork Eagle Creek below Settlers Grove, Idaho & $08 / 16 / 00$ & 0920 & .021 & $<.10$ & $<.002$ & .004 & $<.008$ \\
\hline 21 & Beaver Creek near mouth, near Murray, Idaho & $08 / 16 / 00$ & 1530 & .005 & $<.10$ & .002 & .003 & $<.008$ \\
\hline 22 & Prichard Creek at mouth, at Prichard, Idaho & $08 / 16 / 00$ & 1400 & $<.005$ & $<.10$ & $<.002$ & .002 & $<.008$ \\
\hline 23 & North Fork Coeur d'Alene River at Enaville, Idaho & 09/07/00 & 1530 & .012 & e. 06 & .003 & .002 & e. 004 \\
\hline 24 & $\begin{array}{l}\text { South Fork Coeur d'Alene River at Shoshone Park, near Mullan, } \\
\text { Idaho }\end{array}$ & $08 / 24 / 00$ & 1000 & .009 & e. 05 & $<.002$ & .003 & e. 007 \\
\hline 25 & $\begin{array}{l}\text { South Fork Coeur d'Alene River above Deadman Gulch, near } \\
\text { Mullan, Idaho }\end{array}$ & $08 / 24 / 00$ & 1430 & .036 & e. 07 & .015 & .002 & e. 005 \\
\hline 26 & Canyon Creek near Burke, Idaho & $08 / 23 / 00$ & 1030 & .006 & $<.10$ & .004 & .002 & e. 005 \\
\hline 27 & Canyon Creek at Woodland Park, Idaho & $08 / 23 / 00$ & 1500 & .005 & e. 05 & $<.002$ & $<.001$ & $<.008$ \\
\hline 28 & South Fork Coeur d'Alene River at Silverton, Idaho & $08 / 25 / 00$ & 1000 & .080 & e. 05 & .004 & $<.001$ & $<.008$ \\
\hline 29 & East Fork Pine Creek above Nabob Creek, near Pinehurst, Idaho & $08 / 16 / 00$ & 1730 & .023 & e. 06 & $<.002$ & .002 & $<.008$ \\
\hline 30 & Pine Creek below Amy Gulch, near Pinehurst, Idaho & $08 / 16 / 00$ & 1840 & .039 & $<.10$ & $<.002$ & .002 & $<.008$ \\
\hline 31 & South Fork Coeur d'Alene River near Pinehurst, Idaho & 09/06/00 & 1400 & .208 & .35 & .254 & .026 & .071 \\
\hline 33 & St. Joe River at Red Ives Ranger Station, Idaho & 09/05/00 & 1230 & $<.005$ & $<.10$ & .003 & .002 & $<.008$ \\
\hline 34 & St. Joe River at Calder, Idaho & 09/07/00 & 1100 & $<.005$ & e. 06 & .003 & $<.001$ & $<.008$ \\
\hline
\end{tabular}


Table 13. Trace-element concentration data for surface-water samples collected for the synoptic study of mining-affected areas, Northern Rockies Intermontane Basins study unit, August-September 2000

[Abbreviations: e, estimated; Fb, field blank; $\mu \mathrm{g} / \mathrm{L}$, micrograms per liter. Symbols: <, less than reporting level; --, no data]

\begin{tabular}{|c|c|c|c|c|c|}
\hline $\begin{array}{c}\text { Site } \\
\text { number } \\
\text { (fig. 1) }\end{array}$ & Site name & Date & Time & $\begin{array}{c}\text { Cadmium, } \\
\text { total } \\
\text { recov- } \\
\text { erable } \\
(\mu \mathrm{g} / \mathrm{L})\end{array}$ & $\begin{array}{c}\text { Cad- } \\
\text { mium, } \\
\text { dissolved } \\
(\mu \mathrm{g} / \mathrm{L})\end{array}$ \\
\hline 6 & St. Regis River above Rainy Creek, near Saltese, Mont. & $08 / 02 / 00$ & 0930 & $<1.0$ & $<1.0$ \\
\hline 7 & St. Regis River near Haugan, Mont. & $08 / 02 / 00$ & 1330 & $<1.0$ & $<1.0$ \\
\hline 8 & St. Regis River near St. Regis, Mont. & $08 / 03 / 00$ & 0930 & $<1.0$ & $<1.0$ \\
\hline 17 & North Fork Coeur d'Alene River above Shoshone Creek, near Prichard, Idaho & $09 / 06 / 00$ & 1000 & $<1.0$ & $<1.0$ \\
\hline 18 & Prichard Creek near Murray, Idaho & $08 / 16 / 00$ & 1230 & $<1.0$ & $<1.0$ \\
\hline 19 & East Fork Eagle Creek near mouth, near Prichard, Idaho & $08 / 16 / 00$ & 1100 & $<1.0$ & $<1.0$ \\
\hline 20 & West Fork Eagle Creek below Settlers Grove, Idaho & $08 / 16 / 00$ & 0920 & $<1.0$ & $<1.0$ \\
\hline 21 & Beaver Creek near mouth, near Murray, Idaho & $08 / 16 / 00$ & 1530 & $<1.0$ & $<1.0$ \\
\hline 22 & Prichard Creek at mouth, at Prichard, Idaho & $08 / 16 / 00$ & 1400 & $<1.0$ & $<1.0$ \\
\hline 23 & North Fork Coeur d'Alene River at Enaville, Idaho & $09 / 07 / 00$ & 1530 & $<1.0$ & $<1.0$ \\
\hline 24 & South Fork Coeur d'Alene River at Shoshone Park, near Mullan, Idaho & $08 / 24 / 00$ & 1000 & $<1.0$ & $<1.0$ \\
\hline 25 & South Fork Coeur d'Alene River above Deadman Gulch, near Mullan, Idaho & $08 / 24 / 00$ & 1430 & $<1.0$ & $<1.0$ \\
\hline 26 & Canyon Creek near Burke, Idaho & $08 / 23 / 00$ & 1030 & $<1.0$ & $<1.0$ \\
\hline 27 & Canyon Creek at Woodland Park, Idaho & $08 / 23 / 00$ & 1500 & 13 & 13 \\
\hline 28 & South Fork Coeur d'Alene River at Silverton, Idaho & $08 / 25 / 00$ & 1000 & 7.0 & 7.0 \\
\hline 29 & East Fork Pine Creek above Nabob Creek, near Pinehurst, Idaho & $08 / 16 / 00$ & 1730 & 1.2 & 1.3 \\
\hline 30 & Pine Creek below Amy Gulch, near Pinehurst, Idaho & $08 / 16 / 00$ & 1840 & $<1.0$ & $<1.0$ \\
\hline $31^{\mathbf{F b}}$ & South Fork Coeur d'Alene River near Pinehurst, Idaho & $09 / 06 / 00$ & 1300 & -- & $<.3$ \\
\hline 31 & South Fork Coeur d'Alene River, near Pinehurst, Idaho & $09 / 06 / 00$ & 1400 & 9.0 & 8.7 \\
\hline 33 & St. Joe River at Red Ives Ranger Station, Idaho & $09 / 05 / 00$ & 1230 & $<1.0$ & $<1.0$ \\
\hline 34 & St. Joe River at Calder, Idaho & $09 / 07 / 00$ & 1100 & $<1.0$ & $<1.0$ \\
\hline
\end{tabular}


Table 13. Trace-element concentration data for surface-water samples collected for the synoptic study of miningaffected areas, Northern Rockies Intermontane Basins study unit, August-September 2000 (Continued)

\begin{tabular}{|c|c|c|c|c|c|c|c|c|}
\hline $\begin{array}{l}\text { Iron, } \\
\text { total } \\
\text { recov- } \\
\text { erable } \\
(\mu \mathrm{g} / \mathrm{L})\end{array}$ & $\begin{array}{c}\text { Iron, } \\
\text { dissolved } \\
(\mu \mathrm{g} / \mathrm{L})\end{array}$ & $\begin{array}{l}\text { Lead, } \\
\text { total } \\
\text { recov- } \\
\text { erable } \\
(\mu \mathrm{g} / \mathrm{L})\end{array}$ & $\begin{array}{c}\text { Lead, } \\
\text { dissolved } \\
(\mu \mathrm{g} / \mathrm{L})\end{array}$ & $\begin{array}{c}\text { Manganese, } \\
\text { total } \\
\text { recov- } \\
\text { erable } \\
(\mu \mathrm{g} / \mathrm{L})\end{array}$ & $\begin{array}{c}\text { Manganese, } \\
\text { dissolved } \\
(\mu \mathrm{g} / \mathrm{L})\end{array}$ & $\begin{array}{l}\text { Zinc, } \\
\text { total } \\
\text { recov- } \\
\text { erable } \\
(\mu \mathrm{g} / \mathrm{L})\end{array}$ & $\begin{array}{c}\text { Zinc, } \\
\text { dissolved } \\
(\mu \mathrm{g} / \mathrm{L})\end{array}$ & $\begin{array}{c}\text { Site } \\
\text { number } \\
\text { (fig. 1) }\end{array}$ \\
\hline$<21$ & $<10$ & $<1.0$ & $<1.0$ & $<1.0$ & $<1.0$ & $<8.0$ & 6.2 & 6 \\
\hline$<21$ & $<10$ & $<1.0$ & $<1.0$ & $<1.0$ & $<1.0$ & $<1.2$ & $<1.0$ & 7 \\
\hline$<21$ & $<10$ & $<1.0$ & $<1.0$ & 2.2 & 2.0 & $<1.0$ & $<1.0$ & 8 \\
\hline$<21$ & $<10$ & $<1.0$ & $<1.0$ & $<1.0$ & $<1.0$ & $<1.0$ & $<1.0$ & 17 \\
\hline$<21$ & $<10$ & $<1.0$ & $<1.0$ & $<1.0$ & $<1.0$ & 44 & 49 & 18 \\
\hline$<21$ & $<10$ & $<1.0$ & $<1.0$ & $<1.0$ & $<1.0$ & 61 & 67 & 19 \\
\hline$<21$ & $<10$ & $<1.0$ & $<1.0$ & $<1.0$ & $<1.0$ & 1.1 & 3.1 & 20 \\
\hline$<21$ & $<10$ & $<1.0$ & $<1.0$ & $<1.0$ & $<1.0$ & 13 & 14 & 21 \\
\hline e18 & e5.0 & $<1.0$ & $<1.0$ & 2.1 & 2.2 & 21 & 24 & 22 \\
\hline 30 & e7.6 & $<1.0$ & $<1.0$ & 1.5 & 1.2 & 1.4 & 1.6 & 23 \\
\hline 100 & 33 & $<1.0$ & $<1.0$ & 13 & 12 & 3.2 & 3.4 & 24 \\
\hline 176 & 52 & 4.3 & $<1.0$ & 272 & 280 & 21 & 16 & 25 \\
\hline$<21$ & $<10$ & $<1.0$ & $<1.0$ & $<1.0$ & $<1.0$ & 2.8 & 2.8 & 26 \\
\hline 37 & 14 & 38 & 30 & 59 & 67 & 1,390 & 1,510 & 27 \\
\hline 26 & e5.3 & 15 & 11 & 30 & 31 & 899 & 986 & 28 \\
\hline$<21$ & $<10$ & 1.3 & $<1.0$ & $<1.0$ & $<1.0$ & 284 & 324 & 29 \\
\hline 162 & $<10$ & $<1.0$ & $<1.0$ & $<1.0$ & $<1.0$ & 96 & 112 & 30 \\
\hline -- & $<3$ & -- & $<.3$ & -- & $<.1$ & -- & $<.5$ & $31^{\mathbf{F b}}$ \\
\hline 357 & 90 & 23 & 5.6 & 1,470 & 1,450 & 1,480 & 1,440 & 31 \\
\hline 22 & e8.8 & $<1.0$ & $<1.0$ & 1.1 & $<1.0$ & $<1.0$ & $<1.0$ & 33 \\
\hline 23 & e9.6 & $<1.0$ & $<1.0$ & 2.2 & 1.9 & $<1.0$ & $<1.0$ & 34 \\
\hline
\end{tabular}


Table 14. Stable-isotope data for surface-water samples along the Spokane River, Idaho and Washington, 1999-2001

[See figure 1 for site locations. Stable-isotope ratios are reported relative to the Vienna Standard Mean Ocean Water (VSMOW). Abbreviation: per mil, parts per thousand]

\begin{tabular}{|c|c|c|c|c|c|}
\hline \multirow{2}{*}{ Date } & \multicolumn{2}{|c|}{$\begin{array}{c}\text { Stable-isotope ratio } \\
\text { (per mil) }\end{array}$} & \multirow{2}{*}{ Date } & \multicolumn{2}{|c|}{$\begin{array}{c}\text { Stable-isotope ratio } \\
\text { (per mil) }\end{array}$} \\
\hline & $\begin{array}{l}\text { Hydrogen-2/ } \\
\text { Hydrogen-1 }\end{array}$ & $\begin{array}{r}\text { Oxygen-18/ } \\
\text { Oxygen-16 }\end{array}$ & & $\begin{array}{r}\text { Hydrogen-2/ } \\
\text { Hydrogen-1 }\end{array}$ & $\begin{array}{l}\text { Oxygen-18/ } \\
\text { Oxygen-16 }\end{array}$ \\
\hline \multicolumn{6}{|c|}{ Site 35 12419000--Spokane River near Post Falls, Idaho } \\
\hline 04/01/99 & -111.53 & -14.89 & $06 / 12 / 00$ & -113.31 & -15.21 \\
\hline 09/09/99 & -113.09 & -14.92 & $06 / 27 / 00$ & -112.70 & -15.21 \\
\hline $10 / 20 / 99$ & -114.04 & -15.28 & $07 / 05 / 00$ & -112.81 & -15.10 \\
\hline $11 / 30 / 99$ & -112.67 & -15.03 & $07 / 25 / 00$ & -110.52 & -14.92 \\
\hline $01 / 13 / 00$ & -113.13 & -15.19 & $08 / 30 / 00$ & -109.87 & -14.50 \\
\hline $02 / 29 / 00$ & -111.68 & -15.04 & $09 / 06 / 00$ & -109.86 & -14.46 \\
\hline $04 / 11 / 00$ & -112.53 & -15.07 & $12 / 19 / 00$ & -111.34 & -14.86 \\
\hline $04 / 18 / 00$ & -112.03 & -15.04 & $01 / 23 / 01$ & -110.66 & -14.79 \\
\hline 05/03/00 & -113.02 & -15.10 & 03/13/01 & -111.39 & -14.83 \\
\hline 05/11/00 & -112.19 & -15.13 & 05/03/01 & -110.60 & -14.81 \\
\hline $05 / 16 / 00$ & -112.30 & -15.15 & 06/11/01 & -111.36 & -14.79 \\
\hline $05 / 31 / 00$ & -112.66 & -15.18 & $08 / 14 / 01$ & -113.78 & -15.02 \\
\hline \multicolumn{6}{|c|}{ Site 37 12420500--Spokane River at Greenacres, Wash. } \\
\hline 04/01/99 & -111.80 & -14.83 & 11/01/99 & -112.74 & -15.07 \\
\hline 06/01/99 & -113.50 & -15.30 & $01 / 06 / 00$ & -112.91 & -15.03 \\
\hline 07/01/99 & -114.06 & -15.38 & $02 / 17 / 00$ & -113.88 & -15.00 \\
\hline 08/01/99 & -113.71 & -15.17 & $03 / 09 / 00$ & -113.53 & -15.04 \\
\hline 09/01/99 & -113.17 & -14.84 & & & \\
\hline
\end{tabular}


Table 15. Physical and major-ion concentration data for ground-water samples collected from basin-fill aquifers, Northern Rockies Intermontane Basins study unit, 1999 and 2001

[Site identification number described in text. Constituents are reported as dissolved, except as noted. Abbreviations: ${ }^{\circ} \mathrm{C}$, degrees Celsius; e, estimated; Fb, field blank; $\mu \mathrm{S} / \mathrm{cm}$, microsiemens per centimeter at 25 degrees Celsius; $\mathrm{mg} / \mathrm{L}$, milligrams per liter; NTU, nephelometric turbidity units; R, replicate. Symbols: --, no data; $<$, less than reporting level]

\begin{tabular}{|c|c|c|c|c|c|c|c|c|c|c|c|c|}
\hline $\begin{array}{c}\text { Well } \\
\text { number } \\
\text { (figs. } 3 \\
\text { and 4) }\end{array}$ & $\begin{array}{c}\text { Site } \\
\text { identification } \\
\text { number }\end{array}$ & Date & Time & $\begin{array}{c}\text { Spe- } \\
\text { cific con- } \\
\text { duc- } \\
\text { tance, } \\
\text { field } \\
(\mu \mathrm{S} / \mathrm{cm})\end{array}$ & $\begin{array}{c}\text { pH, } \\
\text { field } \\
\text { (stan- } \\
\text { dard } \\
\text { units) }\end{array}$ & $\begin{array}{l}\text { Water } \\
\text { tem- } \\
\text { pera- } \\
\text { ture } \\
\left({ }^{\circ} \mathrm{C}\right)\end{array}$ & $\begin{array}{c}\text { Tur- } \\
\text { bidity } \\
\text { (NTU) }\end{array}$ & $\begin{array}{c}\text { Oxy- } \\
\text { gen, } \\
\text { dis- } \\
\text { solved } \\
(\mathrm{mg} / \mathrm{L})\end{array}$ & $\begin{array}{c}\text { Hard- } \\
\text { ness, } \\
\text { total } \\
(\mathrm{mg} / \mathrm{L} \text { as } \\
\left.\mathrm{CaCO}_{3}\right)\end{array}$ & $\begin{array}{c}\text { Cal- } \\
\text { cium, } \\
\text { dis- } \\
\text { solved } \\
(\mathrm{mg} / \mathrm{L})\end{array}$ & $\begin{array}{l}\text { Magne- } \\
\text { sium, } \\
\text { dis- } \\
\text { solved } \\
(\mathrm{mg} / \mathrm{L})\end{array}$ & $\begin{array}{c}\text { Sodium } \\
\text { adsorp- } \\
\text { tion } \\
\text { ratio }\end{array}$ \\
\hline \multicolumn{13}{|c|}{ Subunit Survev 1, Idaho and Washington } \\
\hline 1 & 481320116261101 & $07 / 14 / 99$ & 1700 & 360 & 8.1 & 9.0 & 2.2 & 0.0 & 131 & 36 & 10 & 1.0 \\
\hline 2 & 480950116324401 & $07 / 14 / 99$ & 1100 & 67 & 6.8 & 9.0 & 2.2 & 9.1 & 25 & 5.4 & 2.8 & .4 \\
\hline 3 & 480928117053201 & 07/07/99 & 1000 & 253 & 8.1 & 9.0 & .18 & 9.9 & 130 & 38 & 8.7 & .1 \\
\hline 4 & 480813116593701 & 07/07/99 & 1600 & 153 & 7.0 & 8.5 & .06 & 9.2 & 63 & 18 & 4.3 & .3 \\
\hline 5 & 480718117012501 & $07 / 06 / 99$ & 1800 & 432 & 8.0 & 7.5 & .28 & 0.0 & 229 & 51 & 25 & .2 \\
\hline 6 & 480249116510001 & $07 / 08 / 99$ & 1000 & 279 & 8.0 & 8.0 & .14 & 9.4 & 119 & 31 & 10 & .2 \\
\hline 7 & 480235116483001 & $07 / 13 / 99$ & 1700 & 426 & 7.6 & 7.5 & .40 & 7.6 & 235 & 56 & 23 & .1 \\
\hline 8 & 480203117200601 & $06 / 08 / 99$ & 0800 & 125 & 6.5 & 9.0 & .04 & 9.4 & 49 & 13 & 3.9 & .3 \\
\hline 9 & 480128116374601 & $08 / 04 / 99$ & 0900 & 116 & 7.0 & 7.0 & .60 & 11 & 53 & 15 & 3.6 & .2 \\
\hline 10 & 480119117182101 & $06 / 08 / 99$ & 1600 & 312 & 8.0 & 9.5 & 4.3 & 4.3 & 152 & 44 & 10 & .3 \\
\hline 11 & 475925117153001 & $07 / 06 / 99$ & 1100 & 179 & 7.4 & 9.5 & 1.1 & 3.9 & 79 & 25 & 4.1 & .3 \\
\hline 12 & 475825117364401 & $06 / 09 / 99$ & 1000 & 114 & 6.5 & 8.5 & 2.9 & 4.0 & 48 & 12 & 4.3 & .4 \\
\hline 13 & 475731116371301 & $06 / 23 / 99$ & 1200 & 228 & 8.1 & -- & .32 & 5.7 & 114 & 29 & 10 & .1 \\
\hline 13 & & $07 / 27 / 99$ & 2000 & 223 & 8.2 & 8.0 & -- & -- & -- & -- & -- & -- \\
\hline 14 & 475637117262501 & 06/09/99 & 1700 & 162 & 7.5 & 11.0 & .13 & 9.1 & 76 & 21 & 5.8 & .2 \\
\hline 15 & 475510116391201 & 08/03/99 & 1400 & 125 & 8.3 & 7.5 & 2.7 & 11 & 61 & 19 & 3.4 & .1 \\
\hline $15^{\mathrm{Fb}}$ & & $08 / 03 / 99$ & 1411 & - & -- & -- & -- & -- & - & $<.002$ & $<.001$ & -- \\
\hline 16 & 475400116404201 & $06 / 22 / 99$ & 1700 & 125 & 7.1 & 8.0 & 3.2 & 11 & 58 & 18 & 3.3 & .1 \\
\hline 17 & 475343117225201 & $06 / 10 / 99$ & 0900 & 331 & 7.7 & 11.0 & .80 & 6.7 & 165 & 48 & 11 & .2 \\
\hline 18 & 475322116522201 & $07 / 12 / 99$ & 1200 & 199 & 8.1 & 9.5 & .79 & 7.2 & 94 & 24 & 8.5 & .1 \\
\hline $18^{\mathbf{R}}$ & & $07 / 12 / 99$ & 1201 & -- & -- & -- & -- & -- & 98 & 25 & 8.9 & .1 \\
\hline $18^{\mathbf{F b}}$ & & $07 / 12 / 99$ & 1206 & -- & -- & -- & -- & -- & -- & $<.02$ & $<.004$ & -- \\
\hline 19 & 475130117262201 & 06/07/99 & 0900 & 444 & 7.5 & 10.0 & .53 & 9.9 & 228 & 71 & 12 & .2 \\
\hline 20 & 475035116424801 & $06 / 22 / 99$ & 1100 & 57 & 6.2 & 11.5 & 18 & 8.4 & 19 & 3.6 & 2.5 & .3 \\
\hline 21 & 474718116530201 & $06 / 24 / 99$ & 0900 & 185 & 8.1 & 9.0 & 2.6 & 9.6 & 90 & 23 & 8.3 & .1 \\
\hline $21^{R}$ & & $06 / 24 / 99$ & 0901 & -- & -- & -- & -- & -- & 90 & 22 & 8.2 & .1 \\
\hline $21^{\mathrm{Fb}}$ & & $06 / 24 / 99$ & 0906 & -- & -- & -- & -- & -- & -- & $<.02$ & $<.004$ & -- \\
\hline 22 & 474708117250501 & 06/07/99 & 1600 & 381 & 7.6 & 10.5 & .08 & 7.8 & 186 & 54 & 13 & .3 \\
\hline 23 & 474629117305101 & $05 / 25 / 99$ & 1100 & 276 & 8.2 & 11.5 & .22 & .1 & 133 & 31 & 14 & .1 \\
\hline $23^{R}$ & & $05 / 25 / 99$ & 1101 & -- & -- & -- & -- & -- & 132 & 30 & 14 & .1 \\
\hline $23^{\mathbf{F b}}$ & & $05 / 26 / 99$ & 0906 & -- & -- & -- & -- & -- & -- & $<.002$ & $<.001$ & -- \\
\hline 24 & 474456116522001 & $06 / 21 / 99$ & 1700 & 389 & 7.9 & 10.0 & .20 & 9.9 & 210 & 33 & 31 & .1 \\
\hline 25 & 474427117312101 & $05 / 24 / 99$ & 1400 & 410 & 7.8 & 9.5 & .02 & .3 & 195 & 46 & 19 & .2 \\
\hline 26 & 474317117225301 & 05/27/99 & 0900 & 251 & 8.1 & 10.5 & .04 & 5.4 & 121 & 26 & 13 & .1 \\
\hline 27 & 474218116445601 & $07 / 15 / 99$ & 1000 & 210 & 6.8 & 9.0 & .96 & 9.4 & 105 & 28 & 8.2 & .2 \\
\hline 28 & 474147116544001 & $07 / 26 / 99$ & 1300 & 121 & 7.2 & 14.0 & 8.0 & 5.6 & 59 & 17 & 3.8 & .1 \\
\hline 29 & 474130117015401 & $07 / 29 / 99$ & 1000 & 55 & 7.0 & 10.5 & .41 & 8.2 & 23 & 6.3 & 1.9 & .2 \\
\hline 30 & 474050117084101 & $07 / 28 / 99$ & 1700 & 50 & 7.0 & 16.5 & 1.6 & 5.2 & 20 & 5.3 & 1.6 & .2 \\
\hline 31 & 473612117243601 & $05 / 26 / 99$ & 1100 & 406 & 7.8 & 10.0 & .03 & .1 & 187 & 51 & 14 & .3 \\
\hline
\end{tabular}


Table 15. Physical and major-ion concentration data for ground-water samples collected from basin-fill aquifers, Northern Rockies Intermontane Basins study unit, 1999 and 2001

\begin{tabular}{|c|c|c|c|c|c|c|c|c|c|c|c|c|}
\hline $\begin{array}{l}\text { Sodium, } \\
\text { dis- } \\
\text { solved } \\
(\mathbf{m g} / \mathbf{L})\end{array}$ & $\begin{array}{c}\text { Potas- } \\
\text { sium, } \\
\text { dis- } \\
\text { solved } \\
(\mathrm{mg} / \mathrm{L})\end{array}$ & $\begin{array}{c}\text { Bicar- } \\
\text { bonate, } \\
\text { dis- } \\
\text { solved } \\
(\mathrm{mg} / \mathrm{L})\end{array}$ & $\begin{array}{c}\text { Carbo- } \\
\text { nate, } \\
\text { dis- } \\
\text { solved } \\
(\mathrm{mg} / \mathrm{L} \text { as } \\
\left.\mathrm{HCO}_{3}\right)\end{array}$ & $\begin{array}{c}\text { Alka- } \\
\text { linity, } \\
\text { dis- } \\
\text { solved } \\
(\mathrm{mg} / \mathrm{L} \\
\left.\text { as } \mathrm{CaCO}_{3}\right)\end{array}$ & $\begin{array}{c}\text { Sulfate, } \\
\text { dis- } \\
\text { solved } \\
(\mathrm{mg} / \mathrm{L} \\
\left.\text { as } \mathrm{SO}_{4}\right)\end{array}$ & $\begin{array}{c}\text { Chlo- } \\
\text { ride, } \\
\text { dis- } \\
\text { solved } \\
(\mathrm{mg} / \mathrm{L})\end{array}$ & $\begin{array}{c}\text { Fluo- } \\
\text { ride, } \\
\text { dis- } \\
\text { solved } \\
(\mathrm{mg} / \mathrm{L})\end{array}$ & $\begin{array}{c}\text { Bromide, } \\
\text { dis- } \\
\text { solved } \\
(\mathrm{mg} / \mathrm{L})\end{array}$ & $\begin{array}{c}\text { Silica, } \\
\text { dis- } \\
\text { solved } \\
(\mathrm{mg} / \mathrm{L} \text { as } \\
\left.\mathrm{SiO}_{2}\right)\end{array}$ & $\begin{array}{c}\text { Dis- } \\
\text { solved } \\
\text { solids, } \\
\text { sum of } \\
\text { constit- } \\
\text { uents }\end{array}$ & $\begin{array}{l}\text { Dissolved } \\
\text { solids, } \\
\text { residue at } \\
180^{\circ} \mathrm{C} \\
(\mathrm{mg} / \mathrm{L})\end{array}$ & $\begin{array}{c}\begin{array}{c}\text { Well } \\
\text { number } \\
\text { (figs. } 3 \\
\text { and 4) }\end{array}\end{array}$ \\
\hline
\end{tabular}

\begin{tabular}{|c|c|c|c|c|c|c|c|c|c|c|c|c|}
\hline \multicolumn{13}{|c|}{ Subunit Survev 1, Idaho and Washington } \\
\hline 25 & 1.3 & 120 & 0 & 99 & 74 & 0.79 & 0.16 & 0.02 & 13 & 219 & 226 & 1 \\
\hline 5.2 & .18 & 38 & 0 & 31 & .61 & .61 & $<.10$ & $<.01$ & 33 & 68 & 68 & 2 \\
\hline 3.6 & 1.5 & 152 & 0 & 125 & 8.0 & 1.4 & $<.10$ & .01 & 14 & 152 & 177 & 3 \\
\hline 5.7 & 1.6 & 83 & 0 & 68 & 5.0 & 1.4 & $<.10$ & .01 & 33 & 111 & 121 & 4 \\
\hline 5.1 & 3.3 & 278 & 0 & 228 & 12 & 1.2 & $<.10$ & .02 & 12 & 246 & 213 & 5 \\
\hline 3.8 & 1.8 & 140 & 0 & 115 & 7.5 & 1.3 & .11 & .02 & 22 & 147 & 167 & 6 \\
\hline 3.4 & 2.9 & 251 & 0 & 206 & 16 & 2.9 & .12 & .04 & 14 & 242 & 245 & 7 \\
\hline 4.4 & 1.5 & 43 & 0 & 35 & 3.6 & 4.1 & .10 & $<.01$ & 27 & 94 & 108 & 8 \\
\hline 3.0 & 1.4 & 60 & 0 & 49 & 6.0 & 1.2 & .12 & $<.01$ & 27 & 88 & 92 & 9 \\
\hline 7.6 & 1.2 & 170 & 0 & 139 & 5.6 & 2.4 & .13 & .02 & 29 & 200 & 205 & 10 \\
\hline 5.3 & .90 & 87 & 0 & 72 & 5.8 & 2.4 & .12 & .01 & 35 & 131 & 147 & 11 \\
\hline 5.7 & 1.1 & 66 & 0 & 54 & 2.1 & 1.6 & .20 & $<.01$ & 36 & 96 & 99 & 12 \\
\hline 2.9 & 1.2 & 130 & 0 & 107 & 11 & 1.4 & $<.10$ & .01 & 7.4 & 128 & 130 & 13 \\
\hline -- & -- & -- & -- & -- & -- & -- & -- & -- & -- & -- & -- & 13 \\
\hline 4.2 & 1.1 & 91 & 0 & 74 & 1.0 & .70 & .17 & $<.01$ & 39 & 125 & 136 & 14 \\
\hline 2.1 & .94 & 75 & 0 & 62 & 1.6 & .34 & $<.10$ & $<.01$ & 17 & 81 & 81 & 15 \\
\hline$<.02$ & -- & -- & -- & -- & -- & -- & -- & -- & $<.02$ & -- & -- & $15^{\mathbf{F b}}$ \\
\hline 2.2 & .91 & 71 & 0 & 58 & 2.2 & 1.2 & $<.10$ & .01 & 16 & 81 & 84 & 16 \\
\hline 4.4 & 2.1 & 174 & 0 & 143 & 10 & 2.7 & .17 & .02 & 19 & 198 & 221 & 17 \\
\hline 2.3 & 1.1 & 107 & 0 & 88 & 7.3 & .89 & $<.10$ & .01 & 10 & 108 & 111 & 18 \\
\hline 2.4 & 1.2 & -- & -- & -- & 7.5 & .82 & $<.10$ & .01 & 11 & 110 & 111 & $18^{\mathbf{R}}$ \\
\hline$<.06$ & $<.10$ & -- & -- & -- & $<.10$ & $<.10$ & $<.10$ & $<.01$ & $<.05$ & -- & -- & $18^{\mathbf{F b}}$ \\
\hline 5.5 & 2.2 & 226 & 0 & 185 & 8.1 & 3.7 & $<.10$ & .02 & 30 & 277 & 284 & 19 \\
\hline 3.4 & 1.6 & 30 & 0 & 24 & 1.1 & 1.4 & $<.10$ & .02 & 15 & 44 & 42 & 20 \\
\hline 2.2 & 1.4 & 104 & 0 & 86 & 5.2 & 1.5 & $<.10$ & .01 & 12 & 108 & 109 & 21 \\
\hline 2.3 & 1.4 & -- & -- & -- & 5.2 & 1.5 & $<.10$ & .02 & 12 & -- & -- & $21^{\mathbf{R}}$ \\
\hline$<.06$ & $<.10$ & -- & -- & -- & $<.10$ & $<.10$ & $<.10$ & $<.01$ & $<.05$ & -- & -- & $21^{\mathrm{Fb}}$ \\
\hline 8.1 & 2.2 & 204 & 0 & 167 & 9.9 & 4.9 & .24 & .02 & 28 & 238 & 245 & 22 \\
\hline 3.5 & 2.0 & 144 & 0 & 118 & 21 & 2.3 & $<.10$ & $<.01$ & 11 & 156 & 159 & 23 \\
\hline 3.5 & 2.0 & -- & -- & -- & 22 & 2.3 & $<.10$ & $<.01$ & 11 & 155 & 159 & $23^{\mathbf{R}}$ \\
\hline$<.02$ & $<.10$ & -- & -- & -- & $<.10$ & $<.10$ & $<.10$ & $<.01$ & $<.02$ & -- & -- & $23^{\mathbf{F b}}$ \\
\hline 2.9 & 2.8 & 215 & 0 & 176 & 23 & 1.1 & $<.10$ & .02 & 10 & 227 & 234 & 24 \\
\hline 8.0 & 4.1 & 210 & 0 & 172 & 39 & 6.6 & .18 & .04 & 13 & 240 & 245 & 25 \\
\hline 3.2 & 1.7 & 135 & 0 & 111 & 12 & 2.1 & $<.10$ & $<.01$ & 11 & 141 & 139 & 26 \\
\hline 5.5 & 1.5 & 106 & 0 & 87 & 7.8 & 5.5 & $<.10$ & .04 & 25 & 141 & 158 & 27 \\
\hline 2.3 & 1.3 & 63 & 0 & 52 & 3.5 & 1.4 & $<.10$ & $<.01$ & 17 & 79 & 85 & 28 \\
\hline 1.6 & .66 & 26 & 0 & 22 & 3.9 & .77 & $<.10$ & $<.01$ & 9.1 & 38 & 40 & 29 \\
\hline 1.6 & .75 & 23 & 0 & 19 & 2.7 & .95 & $<.10$ & $<.01$ & 10 & 36 & 40 & 30 \\
\hline 9.2 & 3.4 & 234 & 0 & 192 & 15 & 5.9 & .17 & .02 & 21 & 238 & 240 & 31 \\
\hline
\end{tabular}


Table 15. Physical and major-ion concentration data for ground-water samples collected from basin-fill aquifers, Northern Rockies Intermontane Basins study unit, 1999 and 2001 (Continued)

\begin{tabular}{|c|c|c|c|c|c|c|c|c|c|c|c|c|}
\hline $\begin{array}{c}\text { Well } \\
\text { number } \\
\text { (figs. } 3 \\
\text { and 4) }\end{array}$ & $\begin{array}{c}\text { Site } \\
\text { identification } \\
\text { number }\end{array}$ & Date & Time & $\begin{array}{c}\text { Spe- } \\
\text { cific con- } \\
\text { duc- } \\
\text { tance, } \\
\text { field } \\
(\mu \mathrm{S} / \mathrm{cm})\end{array}$ & $\begin{array}{c}\text { pH, } \\
\text { field } \\
\text { (stan- } \\
\text { dard } \\
\text { units) }\end{array}$ & $\begin{array}{l}\text { Water } \\
\text { tem- } \\
\text { pera- } \\
\text { ture } \\
\left({ }^{\circ} \mathrm{C}\right)\end{array}$ & $\begin{array}{c}\text { Tur- } \\
\text { bidity } \\
\text { (NTU) }\end{array}$ & $\begin{array}{c}\text { Oxy- } \\
\text { gen, } \\
\text { dis- } \\
\text { solved } \\
(\mathrm{mg} / \mathrm{L})\end{array}$ & $\begin{array}{c}\text { Hard- } \\
\text { ness, } \\
\text { total } \\
(\mathrm{mg} / \mathrm{L} \text { as } \\
\left.\mathrm{CaCO}_{3}\right)\end{array}$ & $\begin{array}{c}\text { Cal- } \\
\text { cium, } \\
\text { dis- } \\
\text { solved } \\
(\mathrm{mg} / \mathrm{L})\end{array}$ & $\begin{array}{l}\text { Magne- } \\
\text { sium, } \\
\text { dis- } \\
\text { solved } \\
\text { (mg/L) }\end{array}$ & $\begin{array}{c}\text { Sodium } \\
\text { adsorp- } \\
\text { tion } \\
\text { ratio }\end{array}$ \\
\hline \multicolumn{13}{|c|}{ Subunit Survey 2, Montana } \\
\hline 32 & 470719114301401 & $06 / 12 / 01$ & 1300 & 75 & 6.8 & 5.5 & 5.2 & 5.7 & 34 & 7.9 & 3.3 & .2 \\
\hline 33 & 470502114265301 & $06 / 12 / 01$ & 0900 & 81 & 6.6 & 8.5 & 1.8 & 3.6 & 34 & 8.0 & 3.5 & .2 \\
\hline 34 & 470328114164301 & $06 / 28 / 01$ & 0900 & 420 & 7.6 & 8.0 & .39 & 11 & 226 & 55 & 22 & .1 \\
\hline 35 & 470112114144001 & $06 / 07 / 01$ & 0900 & 337 & 8.1 & 11.0 & .42 & 3.5 & 171 & 44 & 15 & .2 \\
\hline 36 & 465838114074501 & $06 / 27 / 01$ & 1200 & 236 & 6.9 & 10.0 & .89 & 7.0 & 99 & 21 & 11 & .5 \\
\hline $36^{\mathbf{R}}$ & & $06 / 27 / 01$ & 1201 & -- & -- & -- & -- & -- & 100 & 21 & 11 & .5 \\
\hline $36^{\mathbf{F b}}$ & & $06 / 27 / 01$ & 1206 & -- & -- & -- & -- & -- & -- & $<.01$ & $<.008$ & -- \\
\hline 37 & 465741114110601 & $06 / 27 / 01$ & 1700 & 332 & 7.2 & 10.0 & .54 & 6.2 & 149 & 37 & 14 & .4 \\
\hline 38 & 465440114022101 & $06 / 19 / 01$ & 1100 & 141 & 6.7 & 8.5 & 1.2 & 4.1 & 65 & 15 & 6.8 & .2 \\
\hline $38^{\mathbf{R}}$ & & $06 / 19 / 01$ & 1101 & -- & -- & -- & -- & -- & 65 & 15 & 6.8 & .2 \\
\hline $38^{\mathrm{Fb}}$ & & $06 / 19 / 01$ & 1106 & -- & -- & -- & -- & -- & -- & $<.01$ & $<.008$ & -- \\
\hline 39 & 465323114054301 & $06 / 20 / 01$ & 1300 & 412 & 7.8 & 11.0 & .09 & 4.8 & 196 & 52 & 16 & .3 \\
\hline 40 & 465127114055401 & $06 / 20 / 01$ & 1000 & 379 & 7.6 & 11.0 & .11 & 5.5 & 180 & 49 & 14 & .2 \\
\hline 41 & 464951114023701 & $06 / 19 / 01$ & 1600 & 428 & 7.7 & 10.5 & .17 & 6.4 & 201 & 53 & 16 & .3 \\
\hline 42 & 463932114035901 & $05 / 29 / 01$ & 1300 & 102 & 6.6 & 10.5 & .10 & .4 & 32 & 9.1 & 2.3 & .7 \\
\hline 43 & 463827114001201 & $05 / 29 / 01$ & 1600 & 316 & 7.9 & 13.0 & 3.6 & 1.0 & 126 & 32 & 11 & .8 \\
\hline 44 & 463335114011701 & $05 / 30 / 01$ & 1600 & 197 & 6.7 & 10.5 & 3.8 & .2 & 80 & 23 & 5.2 & .4 \\
\hline $44^{\mathbf{F b}}$ & & $05 / 30 / 01$ & 1611 & -- & -- & -- & -- & -- & -- & $<.01$ & $<.008$ & -- \\
\hline 45 & 463122114074701 & $05 / 30 / 01$ & 0900 & 139 & 6.6 & 10.0 & .80 & 3.9 & 60 & 16 & 4.8 & .4 \\
\hline $45^{\mathbf{R}}$ & & $05 / 30 / 01$ & 0901 & -- & -- & -- & -- & -- & 59 & 16 & 4.8 & .4 \\
\hline 46 & 462948114060101 & $05 / 30 / 01$ & 1200 & 226 & 6.9 & 12.0 & .90 & 5.6 & 95 & 29 & 5.5 & .4 \\
\hline 47 & 462859113574401 & $06 / 11 / 01$ & 1200 & 137 & 7.1 & 5.5 & 1.1 & 7.2 & 65 & 19 & 4.0 & .1 \\
\hline 48 & 462818114074101 & $06 / 04 / 01$ & 1200 & 161 & 6.6 & 10.5 & .09 & 3.1 & 71 & 20 & 5.3 & .2 \\
\hline $48^{\mathbf{F b}}$ & & $06 / 04 / 01$ & 1206 & -- & -- & -- & -- & -- & -- & $<.01$ & $<.008$ & -- \\
\hline 49 & 462754113592701 & $06 / 05 / 01$ & 1500 & 309 & 8.0 & 9.5 & .47 & 9.6 & 116 & 25 & 13 & 1.0 \\
\hline 50 & 462616114094301 & $06 / 05 / 01$ & 1300 & 132 & 6.5 & 9.5 & .30 & 5.5 & 65 & 16 & 6.0 & .2 \\
\hline 51 & 462545114034301 & $06 / 05 / 01$ & 1800 & 373 & 7.5 & 14.0 & 1.6 & 8.3 & 124 & 36 & 8.2 & 1.2 \\
\hline 52 & 462256114114501 & $06 / 04 / 01$ & 1700 & 29 & 6.1 & 8.0 & 12 & 9.1 & 8.5 & 2.3 & .68 & .3 \\
\hline 53 & 462228114030301 & $06 / 06 / 01$ & 1100 & 318 & 7.6 & 10.5 & .22 & 8.8 & 127 & 40 & 6.9 & .9 \\
\hline 54 & 461823114050901 & $06 / 06 / 01$ & 1300 & 432 & 7.1 & 10.0 & .20 & 5.5 & 212 & 71 & 8.2 & .6 \\
\hline 55 & 461807114123001 & $06 / 11 / 01$ & 1600 & 66 & 6.5 & 10.0 & 3.7 & 4.4 & 20 & 5.8 & 1.3 & .7 \\
\hline 56 & 461638114023401 & $06 / 06 / 01$ & 0800 & 580 & 7.4 & 11.0 & .64 & 7.5 & 210 & 63 & 13 & 1.3 \\
\hline 57 & 461451114090801 & $06 / 06 / 01$ & 1600 & 301 & 7.2 & 12.0 & .51 & 6.6 & 172 & 49 & 12 & .2 \\
\hline 58 & 461320114121501 & $06 / 05 / 01$ & 0900 & 97 & 6.1 & 9.5 & 80 & 4.4 & 32 & 9.2 & 2.2 & .6 \\
\hline 59 & 461055114112601 & $06 / 21 / 01$ & 0900 & 27 & 5.8 & 8.5 & 31 & 5.0 & 9.3 & 2.6 & .65 & .3 \\
\hline 60 & 460110114110901 & $06 / 20 / 01$ & 1800 & 59 & 6.7 & 11.5 & 1.1 & 8.7 & 24 & 6.7 & 1.7 & .2 \\
\hline 61 & 455521114074801 & $06 / 20 / 01$ & 2000 & 143 & 6.3 & 10.0 & 2.3 & .6 & 59 & 17 & 4.0 & .4 \\
\hline
\end{tabular}


Table 15. Physical and major-ion concentration data for ground-water samples collected from basin-fill aquifers, Northern Rockies Intermontane Basins study unit, 1999 and 2001 (Continued)

\begin{tabular}{|c|c|c|c|c|c|c|c|c|c|c|c|c|}
\hline $\begin{array}{l}\text { Sodium, } \\
\text { dis- } \\
\text { solved } \\
(\mathrm{mg} / \mathrm{L})\end{array}$ & $\begin{array}{l}\text { Potas- } \\
\text { sium, } \\
\text { dis- } \\
\text { solved } \\
(\mathrm{mg} / \mathrm{L})\end{array}$ & $\begin{array}{c}\text { Bicar- } \\
\text { bonate, } \\
\text { dis- } \\
\text { solved } \\
(\mathrm{mg} / \mathrm{L})\end{array}$ & $\begin{array}{c}\text { Carbo- } \\
\text { nate, } \\
\text { dis- } \\
\text { solved } \\
(\mathrm{mg} / \mathrm{L} \text { as } \\
\left.\mathrm{HCO}_{3}\right)\end{array}$ & $\begin{array}{c}\text { Alka- } \\
\text { linity, } \\
\text { dis- } \\
\text { solved } \\
(\mathrm{mg} / \mathrm{L} \\
\left.\text { as } \mathrm{CaCO}_{3}\right)\end{array}$ & $\begin{array}{c}\begin{array}{c}\text { Sulfate, } \\
\text { dis- } \\
\text { solved } \\
(\mathrm{mg} / \mathrm{L} \\
\left.\text { as } \mathrm{SO}_{4}\right)\end{array} \\
\end{array}$ & $\begin{array}{c}\text { Chlo- } \\
\text { ride, } \\
\text { dis- } \\
\text { solved } \\
(\mathrm{mg} / \mathrm{L})\end{array}$ & $\begin{array}{l}\text { Fluo- } \\
\text { ride, } \\
\text { dis- } \\
\text { solved } \\
(\mathrm{mg} / \mathrm{L})\end{array}$ & $\begin{array}{c}\text { Bromide, } \\
\text { dis- } \\
\text { solved } \\
(\mathrm{mg} / \mathrm{L})\end{array}$ & $\begin{array}{c}\text { Silica, } \\
\text { dis- } \\
\text { solved } \\
(\mathrm{mg} / \mathrm{L} \text { as } \\
\left.\mathrm{SiO}_{2}\right)\end{array}$ & $\begin{array}{c}\text { Dis- } \\
\text { solved } \\
\text { solids, } \\
\text { sum of } \\
\text { constit- } \\
\text { uents }\end{array}$ & $\begin{array}{l}\text { Dissolved } \\
\text { solids, } \\
\text { residue at } \\
180^{\circ} \mathrm{C} \\
(\mathrm{mg} / \mathrm{L})\end{array}$ & $\begin{array}{c}\begin{array}{c}\text { Well } \\
\text { number } \\
\text { (figs. } 3 \\
\text { and 4) }\end{array}\end{array}$ \\
\hline \multicolumn{13}{|c|}{ Subunit Survev 2, Montana } \\
\hline 2.1 & .43 & 38 & 0 & 32 & 3.7 & .46 & $<.20$ & $<.01$ & 12 & 49 & 49 & 32 \\
\hline 2.9 & .55 & 42 & 0 & 34 & 3.0 & .63 & e.09 & $<.01$ & 15 & 55 & 54 & 33 \\
\hline 3.2 & 3.3 & 257 & 0 & 211 & 7.9 & 1.5 & e.13 & .02 & 15 & 234 & 243 & 34 \\
\hline 7.4 & 1.7 & 195 & 0 & 160 & 14 & $<.08$ & .20 & .03 & 20 & 198 & 250 & 35 \\
\hline 11 & 1.1 & 107 & 0 & 88 & 5.0 & 12 & .17 & .02 & 19 & 136 & 129 & 36 \\
\hline 11 & 1.1 & -- & -- & -- & 5.0 & 12 & .16 & .02 & 19 & -- & 136 & $36^{\mathbf{R}}$ \\
\hline$<.06$ & $<.09$ & -- & -- & -- & $<.10$ & $<.08$ & $<.20$ & $<.01$ & $<.09$ & -- & $<10$ & $36^{\mathbf{F b}}$ \\
\hline 12 & 1.2 & 167 & 0 & 137 & 9.8 & 7.0 & .18 & .02 & 20 & 190 & 194 & 37 \\
\hline 3.4 & .77 & 70 & 0 & 57 & 3.4 & 4.1 & $<.20$ & .01 & 12 & 82 & 90 & 38 \\
\hline 3.4 & .74 & -- & -- & -- & 3.4 & 4.1 & $<.20$ & .02 & 12 & 82 & 91 & $38^{\mathbf{R}}$ \\
\hline$<.06$ & $<.09$ & -- & -- & -- & $<.10$ & $<.08$ & $<.20$ & $<.01$ & $<.09$ & -- & 10 & $38^{\mathbf{F b}}$ \\
\hline 9.8 & 1.4 & 205 & 0 & 168 & 16 & 10 & $<.20$ & .04 & 14 & 227 & 251 & 39 \\
\hline 6.9 & 1.7 & 195 & 0 & 160 & 17 & 5.4 & .20 & .02 & 14 & 209 & 229 & 40 \\
\hline 10 & 1.9 & 218 & 0 & 179 & 16 & 8.2 & e.11 & .04 & 17 & 237 & 263 & 41 \\
\hline 9.2 & 1.1 & 51 & 0 & 42 & 4.0 & 4.2 & .24 & .06 & 23 & 79 & 85 & 42 \\
\hline 20 & 3.3 & 178 & 0 & 146 & 19 & 4.8 & .55 & .07 & 22 & 200 & 205 & 43 \\
\hline 7.3 & 3.9 & 96 & 0 & 79 & 8.2 & 3.9 & .18 & .02 & 41 & 146 & 142 & 44 \\
\hline$<.06$ & -- & -- & -- & -- & $<.10$ & $<.08$ & -- & -- & $<.09$ & -- & -- & $44^{\mathbf{F b}}$ \\
\hline 6.9 & .54 & 82 & 0 & 67 & 2.5 & 1.4 & .18 & $<.01$ & 36 & 110 & 103 & 45 \\
\hline 6.9 & .55 & -- & -- & -- & 2.5 & 1.4 & .20 & .02 & 36 & 109 & 103 & $45^{\mathbf{R}}$ \\
\hline 10 & 2.8 & 133 & 0 & 109 & 5.2 & 2.6 & .16 & .02 & 17 & 141 & 146 & 46 \\
\hline 2.5 & 1.6 & 77 & 0 & 63 & 3.6 & .68 & e.12 & $<.01$ & 13 & 83 & 85 & 47 \\
\hline 4.7 & 1.0 & 88 & 0 & 72 & 3.2 & 3.2 & .19 & .01 & 29 & 114 & 127 & 48 \\
\hline$<.06$ & e. 08 & -- & -- & -- & $<.10$ & $<.08$ & $<.20$ & $<.01$ & $<.09$ & -- & -- & $48^{\mathbf{F b}}$ \\
\hline 23 & 6.0 & 189 & 0 & 155 & 3.0 & .50 & 1.7 & $<.01$ & 13 & 180 & 176 & 49 \\
\hline 2.8 & 2.0 & 78 & 0 & 64 & 2.7 & 2.2 & .17 & $<.01$ & 29 & 102 & 109 & 50 \\
\hline 32 & 9.2 & 166 & 0 & 136 & 22 & 20 & .46 & .17 & 55 & 270 & 269 & 51 \\
\hline 2.2 & .70 & 13 & 0 & 11 & 2.2 & .77 & $<.20$ & $<.01$ & 16 & 32 & 47 & 52 \\
\hline 23 & 8.4 & 213 & 0 & 175 & 6.0 & 2.8 & .45 & .03 & 22 & 216 & 212 & 53 \\
\hline 22 & 3.7 & 287 & 0 & 237 & 9.0 & 2.9 & .36 & .05 & 31 & 291 & 294 & 54 \\
\hline 7.0 & 1.0 & 41 & 0 & 33 & .29 & .57 & .22 & .01 & 35 & 72 & 69 & 55 \\
\hline 43 & 4.0 & 300 & 0 & 246 & 24 & 16 & .36 & .09 & 50 & 369 & 372 & 56 \\
\hline 5.9 & 3.4 & 206 & 0 & 169 & 4.3 & $<.08$ & .20 & .02 & 17 & 194 & 200 & 57 \\
\hline 8.4 & .41 & 56 & 0 & 46 & 2.1 & 1.4 & e. 12 & .02 & 36 & 89 & 103 & 58 \\
\hline 2.2 & .26 & 14 & 0 & 11 & 1.1 & .50 & $<.20$ & $<.01$ & 17 & 32 & 46 & 59 \\
\hline 2.8 & .27 & 31 & 0 & 25 & 1.2 & 1.4 & $<.20$ & $<.01$ & 36 & 67 & 79 & 60 \\
\hline 7.3 & 1.6 & 80 & 0 & 65 & 3.5 & 3.3 & e. 10 & $<.01$ & 29 & 106 & 120 & 61 \\
\hline
\end{tabular}


Table 16. Nutrient and dissolved organic-carbon concentration data for ground-water samples collected from basin-fill aquifers, Northern Rockies Intermontane Basins study unit, 1999 and 2001

[Site identification number described in text. Abbreviations: e, estimated; Fb, field blank; mg/L, milligrams per liter; R, replicate; Sb, source-solution blank. Symbols: <, less than reporting level; --, no data]

\begin{tabular}{|c|c|c|c|c|c|c|c|c|c|c|}
\hline $\begin{array}{c}\text { Well } \\
\text { number } \\
\text { (figs. } 3 \\
\text { and 4) }\end{array}$ & $\begin{array}{c}\text { Site } \\
\text { identification } \\
\text { number }\end{array}$ & Date & Time & $\begin{array}{c}\text { Nitrite, } \\
\text { dissolved } \\
(\mathrm{mg} / \mathbf{L} \\
\text { as N) }\end{array}$ & $\begin{array}{c}\text { Nitrite plus } \\
\text { nitrate, } \\
\text { dissolved } \\
\text { (mg/L } \\
\text { as N) }\end{array}$ & $\begin{array}{c}\text { Ammonia, } \\
\text { dissolved } \\
(\mathrm{mg} / \mathbf{L} \\
\text { as } \mathbf{N})\end{array}$ & $\begin{array}{c}\text { Ammonia } \\
\text { plus } \\
\text { organic } \\
\text { nitrogen, } \\
\text { dissolved } \\
\text { (mg/L } \\
\text { as N) }\end{array}$ & $\begin{array}{l}\text { Phos- } \\
\text { phorus, } \\
\text { dissolved } \\
(\mathrm{mg} / \mathrm{L})\end{array}$ & $\begin{array}{c}\text { Phos- } \\
\text { phorus, } \\
\text { ortho- } \\
\text { phos- } \\
\text { phate, } \\
\text { dissolved } \\
\text { (mg/L as } \mathbf{P})\end{array}$ & $\begin{array}{c}\text { Organic } \\
\text { carbon, } \\
\text { dissolved } \\
\text { (mg/L } \\
\text { as C) }\end{array}$ \\
\hline \multicolumn{11}{|c|}{ Subunit Survey 1, Idaho and Washington } \\
\hline 1 & 481320116261101 & $07 / 14 / 99$ & 1700 & $<0.01$ & $<0.05$ & $<0.02$ & $\mathrm{e} 0.06$ & 0.012 & 0.02 & 0.5 \\
\hline 2 & 480950116324401 & $07 / 14 / 99$ & 1100 & $<.01$ & .21 & $<.02$ & e.07 & .051 & .05 & .7 \\
\hline 3 & 480928117053201 & 07/07/99 & 1000 & $<.01$ & .34 & $<.02$ & e.06 & .009 & .02 & .4 \\
\hline 4 & 480813116593701 & 07/07/99 & 1600 & $<.01$ & .22 & .02 & e.09 & .025 & .03 & 1.2 \\
\hline 5 & 480718117012501 & 07/06/99 & 1800 & $<.01$ & $<.05$ & $<.02$ & e. 08 & .028 & .03 & .6 \\
\hline 6 & 480249116510001 & 07/08/99 & 1000 & $<.01$ & .19 & .02 & e.09 & .006 & .01 & .6 \\
\hline 7 & 480235116483001 & 07/13/99 & 1700 & $<.01$ & .22 & $<.02$ & e. 07 & $<.004$ & $<.01$ & .7 \\
\hline 8 & 480203117200601 & 06/08/99 & 0800 & $<.01$ & 3.6 & .04 & e.06 & .012 & .02 & .5 \\
\hline 9 & 480128116374601 & 08/04/99 & 0900 & $<.01$ & .12 & $<.02$ & e.09 & .021 & .02 & 1.7 \\
\hline 10 & 480119117182101 & $06 / 08 / 99$ & 1600 & $<.01$ & 3.7 & $<.02$ & $<.10$ & .021 & .03 & .3 \\
\hline 11 & 475925117153001 & $07 / 06 / 99$ & 1100 & $<.01$ & 2.0 & $<.02$ & e.06 & .027 & .03 & .5 \\
\hline 12 & 475825117364401 & 06/09/99 & 1000 & $<.01$ & .26 & $<.02$ & e. 05 & .033 & .03 & .6 \\
\hline 13 & 475731116371301 & $06 / 23 / 99$ & 1200 & $<.01$ & .14 & $<.02$ & $<.10$ & $<.004$ & .01 & .4 \\
\hline 14 & 475637117262501 & 06/09/99 & 1700 & $<.01$ & 1.6 & $<.02$ & $<.10$ & .093 & .08 & .3 \\
\hline 15 & 475510116391201 & 08/03/99 & 1400 & $<.01$ & .07 & $<.02$ & e.05 & .006 & .01 & .4 \\
\hline $15^{\mathbf{R}}$ & & $08 / 03 / 99$ & 1401 & -- & -- & -- & -- & -- & -- & .6 \\
\hline 16 & 475400116404201 & $06 / 22 / 99$ & 1700 & $<.01$ & .44 & $<.02$ & $<.10$ & .008 & .02 & .4 \\
\hline 17 & 475343117225201 & 06/10/99 & 0900 & $<.01$ & 3.3 & $<.02$ & $<.10$ & .020 & .02 & .7 \\
\hline 18 & 475322116522201 & $07 / 12 / 99$ & 1200 & $<.01$ & .19 & $<.02$ & e. 08 & $<.004$ & $<.01$ & .4 \\
\hline $18^{\mathbf{R}}$ & & $07 / 12 / 99$ & 1201 & $<.01$ & .18 & $<.02$ & e.09 & $<.004$ & $<.01$ & .3 \\
\hline $18^{\mathbf{F b}}$ & & $07 / 12 / 99$ & 1205 & $<.01$ & $<.05$ & $<.02$ & $<.10$ & $<.004$ & $<.01$ & 1.8 \\
\hline${ }_{18} \mathbf{S b}$ & & $07 / 12 / 99$ & 1207 & -- & -- & -- & -- & -- & -- & $<.1$ \\
\hline 19 & 475130117262201 & 06/07/99 & 0900 & $<.01$ & 7.6 & .04 & $<.10$ & .018 & .03 & .5 \\
\hline 20 & 475035116424801 & $06 / 22 / 99$ & 1100 & $<.01$ & .16 & $<.02$ & e.07 & .005 & .02 & .2 \\
\hline 21 & 474718116530201 & $06 / 24 / 99$ & 0900 & $<.01$ & .85 & $<.02$ & $<.10$ & .005 & .02 & .4 \\
\hline $21^{\mathbf{R}}$ & & $06 / 24 / 99$ & 0901 & $<.01$ & .83 & $<.02$ & e. 06 & $<.004$ & .01 & .4 \\
\hline $21^{\mathbf{F b}}$ & & $06 / 24 / 99$ & 0905 & $<.01$ & $<.05$ & $<.02$ & e. 08 & $<.004$ & .01 & 4.0 \\
\hline 22 & 474708117250501 & 06/07/99 & 1600 & $<.01$ & 4.0 & .03 & e.09 & .044 & .04 & .8 \\
\hline 23 & 474629117305101 & $05 / 25 / 99$ & 1100 & $<.01$ & $<.05$ & .04 & e. 08 & .026 & .03 & .2 \\
\hline $23^{\mathbf{R}}$ & & $05 / 25 / 99$ & 1101 & $<.01$ & $<.05$ & .04 & e. 10 & .025 & .03 & .2 \\
\hline $23^{\mathbf{F b}}$ & & $05 / 26 / 99$ & 0905 & $<.01$ & $<.05$ & $<.02$ & e. 06 & $<.004$ & .01 & .5 \\
\hline 24 & 474456116522001 & $06 / 21 / 99$ & 1700 & $<.01$ & 3.6 & $<.02$ & e.06 & $<.004$ & .01 & .4 \\
\hline 25 & 474427117312101 & $05 / 24 / 99$ & 1400 & $<.01$ & $<.05$ & .03 & $<.10$ & .007 & .04 & .5 \\
\hline 26 & 474317117225301 & $05 / 27 / 99$ & 0900 & $<.01$ & .92 & .03 & e. 05 & $<.004$ & .01 & .2 \\
\hline 27 & 474218116445601 & $07 / 15 / 99$ & 1000 & $<.01$ & 1.6 & $<.02$ & e.07 & .012 & .01 & .9 \\
\hline 28 & 474147116544001 & $07 / 26 / 99$ & 1300 & $<.01$ & .46 & $<.02$ & $<.10$ & $<.004$ & $<.01$ & .3 \\
\hline 29 & 474130117015401 & $07 / 29 / 99$ & 1000 & $<.01$ & .15 & $<.02$ & $<.10$ & $<.004$ & $<.01$ & .6 \\
\hline 30 & 474050117084101 & $07 / 28 / 99$ & 1700 & $<.01$ & .16 & $<.02$ & e.05 & .006 & $<.01$ & .4 \\
\hline 31 & 473612117243601 & $05 / 26 / 99$ & 1100 & $<.01$ & .64 & $<.02$ & e. 10 & .054 & .05 & 1.0 \\
\hline
\end{tabular}


Table 16. Nutrient and dissolved organic-carbon concentration data for ground-water samples collected from basin-fill aquifers, Northern Rockies Intermontane Basins study unit, 1999 and 2001 (Continued)

\begin{tabular}{|c|c|c|c|c|c|c|c|c|c|c|}
\hline $\begin{array}{c}\text { Well } \\
\text { number } \\
\text { (figs. } 3 \\
\text { and 4) }\end{array}$ & $\begin{array}{c}\text { Site } \\
\text { identification } \\
\text { number }\end{array}$ & Date & Time & $\begin{array}{c}\text { Nitrite, } \\
\text { dissolved } \\
(\mathrm{mg} / \mathrm{L} \\
\text { as } \mathrm{N})\end{array}$ & $\begin{array}{c}\text { Nitrite plus } \\
\text { nitrate, } \\
\text { dissolved } \\
\text { (mg/L } \\
\text { as N) }\end{array}$ & $\begin{array}{c}\text { Ammonia, } \\
\text { dissolved } \\
(\mathrm{mg} / \mathrm{L} \\
\text { as } \mathrm{N})\end{array}$ & $\begin{array}{c}\text { Ammonia } \\
\text { plus } \\
\text { organic } \\
\text { nitrogen, } \\
\text { dissolved } \\
\text { (mg/L } \\
\text { as N) }\end{array}$ & $\begin{array}{c}\text { Phos- } \\
\text { phorus, } \\
\text { dissolved } \\
(\mathrm{mg} / \mathrm{L})\end{array}$ & $\begin{array}{c}\text { Phos- } \\
\text { phorus, } \\
\text { ortho- } \\
\text { phos- } \\
\text { phate, } \\
\text { dissolved } \\
\text { (mg/L as } P \text { ) }\end{array}$ & $\begin{array}{c}\text { Organic } \\
\text { carbon, } \\
\text { dissolved } \\
(\mathrm{mg} / \mathrm{L} \\
\text { as C) }\end{array}$ \\
\hline \multicolumn{11}{|c|}{ Subunit Survey 2, Montana } \\
\hline 32 & 470719114301401 & $06 / 12 / 01$ & 1300 & $<.006$ & .07 & $<.04$ & $<.10$ & e. 004 & $<.02$ & -- \\
\hline 33 & 470502114265301 & $06 / 12 / 01$ & 0900 & $<.006$ & .18 & $<.04$ & $<.10$ & $<.006$ & $<.02$ & -- \\
\hline 34 & 470328114164301 & $06 / 28 / 01$ & 0900 & .006 & .05 & $<.04$ & $<.10$ & $<.006$ & $<.02$ & -- \\
\hline 35 & 470112114144001 & $06 / 07 / 01$ & 0900 & $<.006$ & .93 & $<.04$ & $<.10$ & .058 & .05 & -- \\
\hline 36 & 465838114074501 & $06 / 27 / 01$ & 1200 & $<.006$ & .55 & $<.04$ & $<.10$ & .019 & .02 & -- \\
\hline $36^{\mathbf{R}}$ & & $06 / 27 / 01$ & 1201 & $<.006$ & .55 & $<.04$ & $<.10$ & .020 & e. 02 & -- \\
\hline $36^{\mathbf{F b}}$ & & $06 / 27 / 01$ & 1206 & $<.006$ & $<.005$ & $<.04$ & $<.10$ & $<.006$ & $<.02$ & -- \\
\hline 37 & 465741114110601 & $06 / 27 / 01$ & 1700 & e. 005 & 1.4 & $<.04$ & $<.10$ & e. 005 & e. 01 & -- \\
\hline 38 & 465440114022101 & $06 / 19 / 01$ & 1100 & $<.006$ & .45 & $<.04$ & $<.10$ & $<.006$ & $<.02$ & -- \\
\hline $38^{\mathbf{R}}$ & & $06 / 19 / 01$ & 1101 & $<.006$ & .44 & $<.04$ & $<.10$ & e. 004 & $<.02$ & -- \\
\hline $38^{\mathbf{F b}}$ & & 06/19/01 & 1106 & $<.006$ & $<.05$ & $<.04$ & $<.10$ & $<.006$ & $<.02$ & -- \\
\hline 39 & 465323114054301 & $06 / 20 / 01$ & 1300 & $<.006$ & 1.4 & $<.04$ & e. 12 & e. 005 & $<.02$ & -- \\
\hline 40 & 465127114055401 & $06 / 20 / 01$ & 1000 & $<.006$ & .96 & $<.04$ & $<.10$ & .009 & $<.02$ & -- \\
\hline 41 & 464951114023701 & $06 / 19 / 01$ & 1600 & $<.006$ & 1.3 & $<.04$ & $<.10$ & .017 & $<.02$ & -- \\
\hline 42 & 463932114035901 & $05 / 29 / 01$ & 1300 & e. 004 & e. 04 & $<.04$ & $<.10$ & .009 & $<.02$ & -- \\
\hline 43 & 463827114001201 & $05 / 29 / 01$ & 1600 & $<.006$ & $<.05$ & $<.04$ & .25 & .046 & .04 & -- \\
\hline 44 & 463335114011701 & 05/30/01 & 1600 & $<.006$ & 1.3 & $<.04$ & $<.10$ & .084 & .07 & -- \\
\hline 45 & 463122114074701 & $05 / 30 / 01$ & 0900 & $<.006$ & .28 & $<.04$ & $<.10$ & .018 & e. 01 & -- \\
\hline $45^{\mathbf{R}}$ & & $05 / 30 / 01$ & 0901 & $<.006$ & .29 & $<.04$ & $<.10$ & .017 & e. 01 & -- \\
\hline 46 & 462948114060101 & $05 / 30 / 01$ & 1200 & $<.006$ & .72 & $<.04$ & $<.10$ & .015 & e. 01 & -- \\
\hline 47 & 462859113574401 & $06 / 11 / 01$ & 1200 & e. 003 & .07 & $<.04$ & $<.10$ & .022 & .02 & -- \\
\hline 48 & 462818114074101 & $06 / 04 / 01$ & 1200 & $<.006$ & .80 & $<.04$ & $<.10$ & .026 & .02 & -- \\
\hline $48^{\mathbf{F b}}$ & & $06 / 04 / 01$ & 1206 & $<.006$ & $<.05$ & $<.04$ & $<.10$ & $<.006$ & $<.02$ & -- \\
\hline 49 & 462754113592701 & $06 / 05 / 01$ & 1500 & $<.006$ & .27 & $<.04$ & $<.10$ & .036 & .03 & -- \\
\hline 50 & 462616114094301 & $06 / 05 / 01$ & 1300 & $<.006$ & .57 & $<.04$ & $<.10$ & .018 & e. 02 & -- \\
\hline 51 & 462545114034301 & $06 / 05 / 01$ & 1800 & $<.006$ & 1.2 & $<.04$ & $<.10$ & .021 & e. 02 & -- \\
\hline 52 & 462256114114501 & $06 / 04 / 01$ & 1700 & e. 003 & .10 & .04 & $<.10$ & .021 & .07 & -- \\
\hline 53 & 462228114030301 & $06 / 06 / 01$ & 1100 & $<.006$ & .29 & $<.04$ & $<.10$ & .015 & e. 01 & -- \\
\hline 54 & 461823114050901 & $06 / 06 / 01$ & 1300 & $<.006$ & .45 & $<.04$ & e. 05 & .013 & e. 01 & -- \\
\hline 55 & 461807114123001 & $06 / 11 / 01$ & 1600 & e.003 & .20 & $<.04$ & $<.10$ & .040 & .04 & -- \\
\hline 56 & 461638114023401 & $06 / 06 / 01$ & 0800 & $<.006$ & 1.8 & $<.04$ & e. 05 & .040 & .03 & -- \\
\hline 57 & 461451114090801 & $06 / 06 / 01$ & 1600 & $<.006$ & .84 & $<.04$ & $<.10$ & .013 & $<.02$ & -- \\
\hline 58 & 461320114121501 & $06 / 05 / 01$ & 0900 & $<.006$ & .22 & $<.04$ & $<.10$ & .041 & .04 & -- \\
\hline 59 & 461055114112601 & $06 / 21 / 01$ & 0900 & $<.006$ & .14 & e. 02 & e. 06 & .018 & .02 & -- \\
\hline 60 & 460110114110901 & $06 / 20 / 01$ & 1800 & $<.006$ & .36 & $<.04$ & $<.10$ & .040 & .04 & -- \\
\hline 61 & 455521114074801 & $06 / 20 / 01$ & 2000 & $<.006$ & .36 & e. 03 & $<.10$ & .027 & .03 & -- \\
\hline
\end{tabular}


Table 17. Trace-element concentration data for ground-water samples collected from basin-fill aquifers, Northern Rockies Intermontane Basins study unit, 1999 and 2001

[Site identification number described in text. Abbreviations: e, estimated; Fb, field blank; $\mu \mathrm{g} / \mathrm{L}$, micrograms per liter; R, replicate. Symbols: <, less than reporting level; --, no data]

\begin{tabular}{|c|c|c|c|c|c|c|c|c|c|c|c|c|c|c|}
\hline $\begin{array}{c}\text { Well } \\
\text { number } \\
\text { (figs. } 3 \\
\text { and 4) }\end{array}$ & $\begin{array}{c}\text { Site } \\
\text { identification } \\
\text { number }\end{array}$ & Date & Time & $\begin{array}{c}\text { Alumi- } \\
\text { num, } \\
\text { dis- } \\
\text { solved } \\
(\mu \mathrm{g} / \mathrm{L})\end{array}$ & $\begin{array}{c}\text { Anti- } \\
\text { mony, } \\
\text { dis- } \\
\text { solved } \\
(\mu \mathrm{g} / \mathrm{L})\end{array}$ & $\begin{array}{c}\text { Arse- } \\
\text { nic, } \\
\text { dis- } \\
\text { solved } \\
(\mu \mathrm{g} / \mathrm{L})\end{array}$ & $\begin{array}{c}\text { Bari- } \\
\text { um, } \\
\text { dis- } \\
\text { solved } \\
(\mu \mathrm{g} / \mathrm{L})\end{array}$ & $\begin{array}{l}\text { Beryl- } \\
\text { lium, } \\
\text { dis- } \\
\text { solved } \\
(\mu \mathrm{g} / \mathrm{L})\end{array}$ & $\begin{array}{c}\text { Boron, } \\
\text { dis- } \\
\text { solved } \\
(\mu \mathrm{g} / \mathrm{L})\end{array}$ & $\begin{array}{c}\text { Cad- } \\
\text { mium, } \\
\text { dis- } \\
\text { solved } \\
(\mu \mathrm{g} / \mathrm{L})\end{array}$ & $\begin{array}{c}\text { Chro- } \\
\text { mium, } \\
\text { dis- } \\
\text { solved } \\
(\mu \mathrm{g} / \mathrm{L})\end{array}$ & $\begin{array}{c}\text { Cobalt, } \\
\text { dis- } \\
\text { solved } \\
(\mu \mathrm{g} / \mathrm{L})\end{array}$ & $\begin{array}{c}\text { Cop- } \\
\text { per, } \\
\text { dis- } \\
\text { solved } \\
(\mu \mathrm{g} / \mathrm{L})\end{array}$ & $\begin{array}{c}\text { Iron, } \\
\text { dis- } \\
\text { solved } \\
(\mu \mathrm{g} / \mathrm{L})\end{array}$ \\
\hline \multicolumn{15}{|c|}{ Subunit Survev 1, Idaho and Washington } \\
\hline 1 & 481320116261101 & $07 / 14 / 99$ & 1700 & 1.6 & $<1.0$ & 1.4 & 11 & $<1.0$ & -- & $<1.0$ & $<1.0$ & $<1.0$ & $<1.0$ & 71 \\
\hline 2 & 480950116324401 & $07 / 14 / 99$ & 1100 & $<1.0$ & $<1.0$ & $<1.0$ & 5.9 & $<1.0$ & -- & $<1.0$ & $<1.0$ & $<1.0$ & $<1.0$ & $<10$ \\
\hline 3 & 480928117053201 & 07/07/99 & 1000 & 1.2 & $<1.0$ & 2.4 & 79 & $<1.0$ & -- & $<1.0$ & $<1.0$ & $<1.0$ & $<1.0$ & $<10$ \\
\hline 4 & 480813116593701 & $07 / 07 / 99$ & 1600 & $<1.0$ & $<1.0$ & $<1.0$ & 19 & $<1.0$ & -- & $<1.0$ & $<1.0$ & $<1.0$ & $<1.0$ & $<10$ \\
\hline 5 & 480718117012501 & 07/06/99 & 1800 & $<1.0$ & $<1.0$ & 31 & 107 & $<1.0$ & -- & $<1.0$ & $<1.0$ & $<1.0$ & $<1.0$ & 397 \\
\hline 6 & 480249116510001 & $07 / 08 / 99$ & 1000 & $<1.0$ & $<1.0$ & 1.4 & 18 & $<1.0$ & -- & $<1.0$ & $<1.0$ & $<1.0$ & $<1.0$ & $<10$ \\
\hline 7 & 480235116483001 & $07 / 13 / 99$ & 1700 & 1.1 & $<1.0$ & $<1.0$ & 67 & $<1.0$ & -- & $<1.0$ & $<1.0$ & $<1.0$ & $<1.0$ & $<10$ \\
\hline 8 & 480203117200601 & $06 / 08 / 99$ & 0800 & $<1.0$ & $<1.0$ & $<1.0$ & 31 & $<1.0$ & -- & $<1.0$ & $<1.0$ & $<1.0$ & $<1.0$ & 20 \\
\hline 9 & 480128116374601 & 08/04/99 & 0900 & $<1.0$ & $<1.0$ & 2.0 & 11 & $<1.0$ & -- & $<1.0$ & $<1.0$ & $<1.0$ & 1.2 & 49 \\
\hline 10 & 480119117182101 & $06 / 08 / 99$ & 1600 & 1.9 & $<1.0$ & 3.1 & 74 & $<1.0$ & -- & $<1.0$ & 1.1 & $<1.0$ & $<1.0$ & $<10$ \\
\hline 11 & 475925117153001 & 07/06/99 & 1100 & $<1.0$ & $<1.0$ & $<1.0$ & 33 & $<1.0$ & -- & $<1.0$ & $<1.0$ & $<1.0$ & $<1.0$ & e9.0 \\
\hline 12 & 475825117364401 & $06 / 09 / 99$ & 1000 & $<1.0$ & $<1.0$ & $<1.0$ & 33 & $<1.0$ & -- & $<1.0$ & $<1.0$ & $<1.0$ & $<1.0$ & 23 \\
\hline 13 & 475731116371301 & $06 / 23 / 99$ & 1200 & 6.0 & $<1.0$ & 4.5 & 12 & $<1.0$ & -- & $<1.0$ & $<1.0$ & $<1.0$ & $<1.0$ & $\mathrm{e} 7.4$ \\
\hline 14 & 475637117262501 & $06 / 09 / 99$ & 1700 & $<1.0$ & $<1.0$ & $<1.0$ & 18 & $<1.0$ & -- & $<1.0$ & $<1.0$ & $<1.0$ & $<1.0$ & $<10$ \\
\hline 15 & 475510116391201 & 08/03/99 & 1400 & 1.8 & $<1.0$ & 5.6 & 7.4 & $<1.0$ & -- & $<1.0$ & $<1.0$ & $<1.0$ & $<1.0$ & 51 \\
\hline $15^{\mathrm{Fb}}$ & & 08/03/99 & 1411 & $<.30$ & $<.20$ & $<1.0$ & $<.20$ & $<.20$ & $<2.0$ & $<.30$ & $<.20$ & $<.20$ & .22 & $<3.0$ \\
\hline 16 & 475400116404201 & $06 / 22 / 99$ & 1700 & 4.5 & $<1.0$ & 1.4 & 14 & $<1.0$ & -- & $<1.0$ & $<1.0$ & $<1.0$ & $<1.0$ & e6.3 \\
\hline 17 & 475343117225201 & 06/10/99 & 0900 & 2.8 & $<1.0$ & 5.5 & 58 & $<1.0$ & -- & $<1.0$ & 1.2 & $<1.0$ & $<1.0$ & $<10$ \\
\hline 18 & 475322116522201 & $07 / 12 / 99$ & 1200 & 2.7 & $<1.0$ & 13 & 65 & $<1.0$ & -- & $<1.0$ & $<1.0$ & $<1.0$ & $<1.0$ & $<10$ \\
\hline $18^{\mathbf{R}}$ & & $07 / 12 / 99$ & 1201 & 1.4 & $<1.0$ & 15 & 64 & $<1.0$ & -- & $<1.0$ & $<1.0$ & $<1.0$ & $<1.0$ & $<10$ \\
\hline $18^{\mathbf{F b}}$ & & 07/12/99 & 1211 & $<.30$ & $<.20$ & $<1.0$ & $<.20$ & $<.20$ & $<2.0$ & $<.30$ & $<.20$ & $<.20$ & $<.20$ & $<3.0$ \\
\hline 19 & 475130117262201 & $06 / 07 / 99$ & 0900 & $<1.0$ & $<1.0$ & 4.1 & 106 & $<1.0$ & -- & $<1.0$ & 1.4 & $<1.0$ & $<1.0$ & $<10$ \\
\hline 20 & 475035116424801 & $06 / 22 / 99$ & 1100 & 1.9 & $<1.0$ & $<1.0$ & 39 & $<1.0$ & -- & $<1.0$ & $<1.0$ & $<1.0$ & $<1.0$ & 59 \\
\hline 21 & 474718116530201 & $06 / 24 / 99$ & 0900 & 1.2 & $<1.0$ & 2.6 & 16 & $<1.0$ & -- & $<1.0$ & $<1.0$ & $<1.0$ & $<1.0$ & $<10$ \\
\hline $21^{\mathbf{R}}$ & & $06 / 24 / 99$ & 0901 & 1.0 & $<1.0$ & 2.3 & 16 & $<1.0$ & -- & $<1.0$ & $<1.0$ & $<1.0$ & $<1.0$ & $<10$ \\
\hline $21^{\mathbf{F b}}$ & & $06 / 24 / 99$ & 0906 & $<.30$ & $<.20$ & $<1.0$ & $<.20$ & $<.20$ & $<2.0$ & $<.30$ & $<.20$ & $<.20$ & $<1.0$ & $<10$ \\
\hline 22 & 474708117250501 & 06/07/99 & 1600 & 1.2 & $<1.0$ & 5.5 & 80 & $<1.0$ & -- & $<1.0$ & 1.4 & $<1.0$ & $<1.0$ & $<10$ \\
\hline 23 & 474629117305101 & $05 / 25 / 99$ & 1100 & 3.1 & $<1.0$ & 12 & 129 & $<1.0$ & -- & $<1.0$ & $<1.0$ & $<1.0$ & $<1.0$ & 87 \\
\hline $23^{\mathbf{R}}$ & & $05 / 25 / 99$ & 1101 & 3.3 & $<1.0$ & 14 & 130 & $<1.0$ & -- & $<1.0$ & $<1.0$ & $<1.0$ & $<1.0$ & 88 \\
\hline $23^{\mathrm{Fb}}$ & & 05/26/99 & 0906 & $<.30$ & $<.20$ & $<1.0$ & $<.20$ & $<.20$ & $<2.0$ & $<.30$ & $<.20$ & $<.20$ & .32 & $<3.0$ \\
\hline 24 & 474456116522001 & $06 / 21 / 99$ & 1700 & 1.3 & $<1.0$ & 3.4 & 37 & $<1.0$ & -- & $<1.0$ & $<1.0$ & $<1.0$ & $<1.0$ & $<10$ \\
\hline 25 & 474427117312101 & $05 / 24 / 99$ & 1400 & 2.6 & $<1.0$ & 5.0 & 117 & $<1.0$ & -- & $<1.0$ & $<1.0$ & $<1.0$ & $<1.0$ & 604 \\
\hline 26 & 474317117225301 & $05 / 27 / 99$ & 0900 & 4.7 & $<1.0$ & 2.2 & 28 & $<1.0$ & -- & $<1.0$ & $<1.0$ & $<1.0$ & $<1.0$ & $<10$ \\
\hline 27 & 474218116445601 & $07 / 15 / 99$ & 1000 & 4.6 & $<1.0$ & $<1.0$ & 27 & $<1.0$ & -- & $<1.0$ & $<1.0$ & $<1.0$ & $<1.0$ & $<10$ \\
\hline 28 & 474147116544001 & $07 / 26 / 99$ & 1300 & $<1.0$ & $<1.0$ & $<1.0$ & 29 & $<1.0$ & -- & $<1.0$ & $<1.0$ & $<1.0$ & $<1.0$ & e6.2 \\
\hline 29 & 474130117015401 & $07 / 29 / 99$ & 1000 & $<1.0$ & $<1.0$ & $<1.0$ & 11 & $<1.0$ & -- & $<1.0$ & $<1.0$ & $<1.0$ & $<1.0$ & $<10$ \\
\hline 30 & 474050117084101 & $07 / 28 / 99$ & 1700 & $<1.0$ & $<1.0$ & $<1.0$ & 13 & $<1.0$ & -- & $<1.0$ & $<1.0$ & $<1.0$ & 1.5 & e6.2 \\
\hline 31 & 473612117243601 & $05 / 26 / 99$ & 1100 & 2.5 & $<1.0$ & 8.0 & 105 & $<1.0$ & -- & $<1.0$ & $<1.0$ & $<1.0$ & $<1.0$ & $\mathrm{e} 7.3$ \\
\hline
\end{tabular}


Table 17. Trace-element concentration data for ground-water samples collected from basin-fill aquifers, Northern Rockies Intermontane Basins study unit, 1999 and 2001 (Continued)

\begin{tabular}{|c|c|c|c|c|c|c|c|c|c|c|c|c|}
\hline $\begin{array}{c}\text { Lead, } \\
\text { dis- } \\
\text { solved } \\
(\mu \mathrm{g} / \mathrm{L})\end{array}$ & $\begin{array}{l}\text { Lith- } \\
\text { ium, } \\
\text { dis- } \\
\text { solved } \\
(\mu \mathrm{g} / \mathrm{L})\end{array}$ & $\begin{array}{c}\text { Man- } \\
\text { ganese, } \\
\text { dis- } \\
\text { solved } \\
(\mu \mathrm{g} / \mathrm{L})\end{array}$ & $\begin{array}{c}\text { Molyb- } \\
\text { denum, } \\
\text { dis- } \\
\text { solved } \\
(\mu \mathrm{g} / \mathrm{L})\end{array}$ & $\begin{array}{c}\text { Nickel, } \\
\text { dis- } \\
\text { solved } \\
(\mu \mathrm{g} / \mathrm{L})\end{array}$ & $\begin{array}{c}\text { Sele- } \\
\text { nium, } \\
\text { dis- } \\
\text { solved } \\
(\mu \mathrm{g} / \mathrm{L})\end{array}$ & $\begin{array}{c}\text { Silver, } \\
\text { dis- } \\
\text { solved } \\
(\mu \mathrm{g} / \mathrm{L})\end{array}$ & $\begin{array}{c}\text { Stron- } \\
\text { tium, } \\
\text { dis- } \\
\text { solved } \\
(\mu \mathrm{g} / \mathrm{L})\end{array}$ & $\begin{array}{c}\text { Thal- } \\
\text { lium, } \\
\text { dis- } \\
\text { solved } \\
(\mu \mathrm{g} / \mathrm{L})\end{array}$ & $\begin{array}{l}\text { Uran- } \\
\text { ium, } \\
\text { dis- } \\
\text { solved } \\
(\mu \mathrm{g} / \mathrm{L})\end{array}$ & $\begin{array}{c}\text { Vana- } \\
\text { dium, } \\
\text { dis- } \\
\text { solved } \\
(\mu \mathrm{g} / \mathrm{L})\end{array}$ & $\begin{array}{c}\text { Zinc, } \\
\text { dis- } \\
\text { solved } \\
(\mu \mathrm{g} / \mathrm{L})\end{array}$ & $\begin{array}{c}\text { Well } \\
\text { number } \\
\text { (figs. } 3 \\
\text { and 4) }\end{array}$ \\
\hline \multicolumn{13}{|c|}{ Subunit Survey 1, Idaho and Washington } \\
\hline$<1.0$ & -- & 3.6 & 4.7 & $<1.0$ & $<1.0$ & $<1.0$ & -- & -- & 1.4 & -- & 4.1 & 1 \\
\hline$<1.0$ & -- & 3.2 & $<1.0$ & $<1.0$ & $<1.0$ & $<1.0$ & -- & -- & $<1.0$ & -- & 9.9 & 2 \\
\hline$<1.0$ & -- & 2.0 & 1.7 & $<1.0$ & $<1.0$ & $<1.0$ & -- & -- & 1.7 & -- & 4.6 & 3 \\
\hline$<1.0$ & -- & $<1.0$ & $<1.0$ & $<1.0$ & $<1.0$ & $<1.0$ & -- & -- & $<1.0$ & -- & 81 & 4 \\
\hline$<1.0$ & -- & 129 & 1.7 & $<1.0$ & $<1.0$ & $<1.0$ & -- & -- & 4.0 & -- & 22 & 5 \\
\hline$<1.0$ & -- & $<1.0$ & $<1.0$ & $<1.0$ & $<1.0$ & $<1.0$ & -- & -- & 1.1 & -- & 37 & 6 \\
\hline$<1.0$ & -- & $<1.0$ & 2.0 & 1.2 & $<1.0$ & $<1.0$ & -- & -- & 3.1 & -- & 3.5 & 7 \\
\hline$<1.0$ & -- & $<1.0$ & $<1.0$ & $<1.0$ & $<1.0$ & $<1.0$ & -- & -- & $<1.0$ & -- & 20 & 8 \\
\hline$<1.0$ & -- & $<1.0$ & 2.0 & $<1.0$ & $<1.0$ & $<1.0$ & -- & -- & 2.8 & -- & 47 & 9 \\
\hline$<1.0$ & -- & $<1.0$ & $<1.0$ & $<1.0$ & $<1.0$ & $<1.0$ & -- & -- & 1.3 & -- & 8.3 & 10 \\
\hline$<1.0$ & -- & 2.3 & $<1.0$ & $<1.0$ & $<1.0$ & $<1.0$ & -- & -- & 1.3 & -- & 14 & 11 \\
\hline$<1.0$ & -- & 1.1 & 1.1 & $<1.0$ & $<1.0$ & $<1.0$ & -- & -- & $<1.0$ & -- & 36 & 12 \\
\hline$<1.0$ & -- & $<1.0$ & 1.1 & $<1.0$ & $<1.0$ & $<1.0$ & -- & -- & 1.2 & -- & 123 & 13 \\
\hline$<1.0$ & -- & $<1.0$ & $<1.0$ & $<1.0$ & $<1.0$ & $<1.0$ & -- & -- & $<1.0$ & -- & 8.0 & 14 \\
\hline$<1.0$ & -- & $<1.0$ & $<1.0$ & $<1.0$ & $<1.0$ & $<1.0$ & -- & -- & $<1.0$ & -- & 25 & 15 \\
\hline$<.30$ & -- & $<.10$ & $<.20$ & $<.50$ & $<1.0$ & $<.20$ & $<1.0$ & $<1.0$ & $<.20$ & -- & $<.50$ & $15^{\mathbf{F b}}$ \\
\hline$<1.0$ & -- & $<1.0$ & $<1.0$ & $<1.0$ & $<1.0$ & $<1.0$ & -- & -- & $<1.0$ & -- & 349 & 16 \\
\hline$<1.0$ & -- & $<1.0$ & $<1.0$ & $<1.0$ & $<1.0$ & $<1.0$ & -- & -- & 2.7 & -- & 28 & 17 \\
\hline$<1.0$ & -- & $<1.0$ & 1.3 & $<1.0$ & $<1.0$ & $<1.0$ & -- & -- & 2.0 & -- & 55 & 18 \\
\hline$<1.0$ & -- & $<1.0$ & 1.2 & $<1.0$ & $<1.0$ & $<1.0$ & -- & -- & 2.1 & -- & 54 & $18^{\mathbf{R}}$ \\
\hline$<.30$ & -- & $<.10$ & $<.20$ & $<.50$ & $<1.0$ & $<.20$ & $<1.0$ & $<1.0$ & $<.20$ & -- & $<.50$ & $18^{\mathbf{F b}}$ \\
\hline$<1.0$ & -- & $<1.0$ & $<1.0$ & 1.1 & $<1.0$ & $<1.0$ & -- & -- & 1.6 & -- & 2.6 & 19 \\
\hline$<1.0$ & -- & 2.3 & $<1.0$ & $<1.0$ & $<1.0$ & $<1.0$ & -- & -- & $<1.0$ & -- & 34 & 20 \\
\hline$<1.0$ & -- & $<1.0$ & $<1.0$ & $<1.0$ & $<1.0$ & $<1.0$ & -- & -- & 1.3 & -- & 75 & 21 \\
\hline$<1.0$ & -- & $<1.0$ & $<1.0$ & $<1.0$ & $<1.0$ & $<1.0$ & -- & -- & 1.3 & -- & 66 & $21^{R}$ \\
\hline$<.30$ & -- & $<1.0$ & $<1.0$ & $<1.0$ & $<1.0$ & $<1.0$ & -- & -- & $<1.0$ & -- & $<1.0$ & $21^{\mathbf{F b}}$ \\
\hline$<1.0$ & -- & $<1.0$ & $<1.0$ & $<1.0$ & $<1.0$ & $<1.0$ & -- & -- & 4.2 & -- & 4.4 & 22 \\
\hline$<1.0$ & -- & 121 & 2.1 & $<1.0$ & $<1.0$ & $<1.0$ & -- & -- & $<1.0$ & -- & 33 & 23 \\
\hline$<1.0$ & -- & 122 & 2.1 & $<1.0$ & $<1.0$ & $<1.0$ & -- & -- & $<1.0$ & -- & 34 & $23^{R}$ \\
\hline$<.30$ & -- & $<.10$ & $<.20$ & $<.50$ & $<1.0$ & $<.20$ & $<.10$ & $<.10$ & $<.20$ & -- & 1.5 & $23^{\mathbf{F b}}$ \\
\hline$<1.0$ & -- & $<1.0$ & 1.9 & $<1.0$ & $<1.0$ & $<1.0$ & -- & -- & 5.8 & -- & 51 & 24 \\
\hline$<1.0$ & -- & 82 & 3.2 & $<1.0$ & $<1.0$ & $<1.0$ & -- & -- & 4.8 & -- & 7.2 & 25 \\
\hline$<1.0$ & -- & $<1.0$ & 1.5 & $<1.0$ & $<1.0$ & $<1.0$ & -- & -- & 3.0 & -- & 19 & 26 \\
\hline$<1.0$ & -- & $<1.0$ & $<1.0$ & $<1.0$ & $<1.0$ & $<1.0$ & -- & -- & $<1.0$ & -- & 8.9 & 27 \\
\hline$<1.0$ & -- & $<1.0$ & $<1.0$ & $<1.0$ & $<1.0$ & $<1.0$ & -- & -- & $<1.0$ & -- & 75 & 28 \\
\hline$<1.0$ & -- & $<1.0$ & $<1.0$ & $<1.0$ & $<1.0$ & $<1.0$ & -- & -- & $<1.0$ & -- & 53 & 29 \\
\hline$<1.0$ & -- & $<1.0$ & $<1.0$ & $<1.0$ & $<1.0$ & $<1.0$ & -- & -- & $<1.0$ & -- & 208 & 30 \\
\hline$<1.0$ & -- & 21 & 1.5 & $<1.0$ & $<1.0$ & $<1.0$ & -- & -- & 1.8 & -- & 1.9 & 31 \\
\hline
\end{tabular}


Table 17. Trace-element concentration data for ground-water samples collected from basin-fill aquifers, Northern Rockies Intermontane Basins study unit, 1999 and 2001 (Continued)

\begin{tabular}{|c|c|c|c|c|c|c|c|c|c|c|c|c|c|c|}
\hline $\begin{array}{c}\text { Well } \\
\text { number } \\
\text { (figs. } 3 \\
\text { and 4) }\end{array}$ & $\begin{array}{c}\text { Site } \\
\text { identification } \\
\text { number }\end{array}$ & Date & Time & $\begin{array}{c}\text { Alumi- } \\
\text { num, } \\
\text { dis- } \\
\text { solved } \\
(\mu \mathrm{g} / \mathrm{L})\end{array}$ & $\begin{array}{c}\text { Anti- } \\
\text { mony, } \\
\text { dis- } \\
\text { solved } \\
(\mu \mathrm{g} / \mathrm{L})\end{array}$ & $\begin{array}{c}\text { Arse- } \\
\text { nic, } \\
\text { dis- } \\
\text { solved } \\
(\mu \mathrm{g} / \mathrm{L})\end{array}$ & $\begin{array}{c}\text { Bari- } \\
\text { um, } \\
\text { dis- } \\
\text { solved } \\
(\mu \mathrm{g} / \mathrm{L})\end{array}$ & $\begin{array}{c}\text { Beryl- } \\
\text { lium, } \\
\text { dis- } \\
\text { solved } \\
(\mu \mathrm{g} / \mathrm{L})\end{array}$ & $\begin{array}{c}\text { Boron, } \\
\text { dis- } \\
\text { solved } \\
(\mu \mathrm{g} / \mathrm{L})\end{array}$ & $\begin{array}{c}\text { Cad- } \\
\text { mium, } \\
\text { dis- } \\
\text { solved } \\
(\mu \mathrm{g} / \mathrm{L})\end{array}$ & $\begin{array}{c}\text { Chro- } \\
\text { mium, } \\
\text { dis- } \\
\text { solved } \\
(\mu \mathrm{g} / \mathrm{L})\end{array}$ & $\begin{array}{c}\text { Cobalt, } \\
\text { dis- } \\
\text { solved } \\
(\mu \mathrm{g} / \mathrm{L})\end{array}$ & $\begin{array}{c}\text { Cop- } \\
\text { per, } \\
\text { dis- } \\
\text { solved } \\
(\mu \mathrm{g} / \mathrm{L})\end{array}$ & $\begin{array}{c}\text { Iron, } \\
\text { dis- } \\
\text { solved } \\
(\mu \mathrm{g} / \mathrm{L})\end{array}$ \\
\hline \multicolumn{15}{|c|}{ Subunit Survev 2, Montana } \\
\hline 32 & 470719114301401 & $06 / 12 / 01$ & 1300 & $<1.0$ & .06 & .18 & 37 & $<.06$ & $<7.0$ & $<.04$ & $<.80$ & .02 & 1.7 & e9.7 \\
\hline 33 & 470502114265301 & $06 / 12 / 01$ & 0900 & $<1.0$ & e. 03 & $<.20$ & 42 & $<.06$ & $<7.0$ & $<.04$ & $<.80$ & e. 01 & .46 & $\mathrm{e} 8.0$ \\
\hline 34 & 470328114164301 & $06 / 28 / 01$ & 0900 & $<1.0$ & .07 & .31 & 21 & $<.06$ & $<7.0$ & $<.04$ & e. 60 & .09 & .35 & $<10$ \\
\hline 35 & 470112114144001 & $06 / 07 / 01$ & 0900 & $<1.0$ & .11 & 4.4 & 353 & $<.06$ & 8.6 & $<.04$ & .83 & .06 & .31 & $\mathrm{e} 7.3$ \\
\hline 36 & 465838114074501 & $06 / 27 / 01$ & 1200 & $<1.0$ & .07 & .78 & 160 & $<.06$ & 9.2 & $<.04$ & $<.80$ & .04 & .81 & $<10$ \\
\hline $36^{\mathbf{R}}$ & & $06 / 27 / 01$ & 1201 & $<1.0$ & .07 & .80 & 162 & $<.06$ & 9.0 & $<.04$ & $<.80$ & .04 & .50 & $<10$ \\
\hline $36^{\mathbf{F b}}$ & & $06 / 27 / 01$ & 1211 & $<1.0$ & $<.05$ & $<.20$ & $<1.0$ & $<.06$ & $<7.0$ & $<.04$ & $<.80$ & $<.02$ & $<.20$ & $<10$ \\
\hline 37 & 465741114110601 & $06 / 27 / 01$ & 1700 & $<1.0$ & e. 05 & e. 11 & 194 & $<.06$ & 8.0 & $<.04$ & e. 45 & .06 & .33 & $<10$ \\
\hline 38 & 465440114022101 & $06 / 19 / 01$ & 1100 & $<1.0$ & $<.05$ & $<.20$ & 146 & $<.06$ & $<7.0$ & e. 03 & $<.80$ & .03 & .88 & 10 \\
\hline $38^{\mathbf{R}}$ & 465440114022101 & $06 / 19 / 01$ & 1101 & $<1.0$ & $<.05$ & $<.20$ & 145 & $<.06$ & $<7.0$ & e. 03 & $<.80$ & .02 & .70 & e9.4 \\
\hline $38^{\mathbf{F b}}$ & & $06 / 19 / 01$ & 1111 & $<1.0$ & $<.05$ & $<.20$ & $<1.0$ & $<.06$ & $<7.0$ & $<.04$ & $<.80$ & e. 01 & $<.20$ & $<10$ \\
\hline 39 & 465323114054301 & $06 / 20 / 01$ & 1300 & $<1.0$ & .06 & .23 & 498 & $<.06$ & 27 & $<.04$ & e. 46 & .09 & .43 & $<10$ \\
\hline 40 & 465127114055401 & $06 / 20 / 01$ & 1000 & $<1.0$ & .14 & .86 & 210 & $<.06$ & 20 & $<.04$ & e. 55 & .08 & .60 & $<10$ \\
\hline 41 & 464951114023701 & $06 / 19 / 01$ & 1600 & $<1.0$ & .10 & .93 & 288 & $<.06$ & 21 & $<.04$ & e. 55 & .10 & .54 & $<10$ \\
\hline 42 & 463932114035901 & $05 / 29 / 01$ & 1300 & $<1.0$ & $<.05$ & $<.20$ & 21 & $<.06$ & 8.6 & $<.04$ & $<.80$ & .07 & .53 & 486 \\
\hline 43 & 463827114001201 & $05 / 29 / 01$ & 1600 & $<1.0$ & $<.05$ & 2.2 & 112 & $<.06$ & 7.7 & $<.04$ & $<.80$ & .05 & e. 18 & 87 \\
\hline 44 & 463335114011701 & $05 / 30 / 01$ & 1600 & $<1.0$ & e. 04 & .51 & 153 & $<.06$ & 12 & $<.04$ & $<.80$ & .06 & .66 & 60 \\
\hline $44^{\mathbf{F b}}$ & 463335114011701 & $05 / 30 / 01$ & 1611 & $<1.0$ & $<.05$ & $<.20$ & $<1.0$ & $<.06$ & $<7.0$ & $<.04$ & $<.80$ & e. 01 & $<.20$ & $<10$ \\
\hline 45 & 463122114074701 & $05 / 30 / 01$ & 0900 & $<1.0$ & $<.05$ & $<.20$ & 26 & $<.06$ & $<7.0$ & $<.04$ & $<.80$ & .02 & .52 & 18 \\
\hline $45^{\mathbf{R}}$ & & $05 / 30 / 01$ & 0901 & $<1.0$ & $<.05$ & $<.20$ & 27 & $<.06$ & $<7.0$ & $<.04$ & $<.80$ & .02 & .51 & 19 \\
\hline 46 & 462948114060101 & $05 / 30 / 01$ & 1200 & $<1.0$ & .05 & .26 & 56 & $<.06$ & 14 & $<.04$ & $<.80$ & .05 & .80 & $<10$ \\
\hline 47 & 462859113574401 & $06 / 11 / 01$ & 1200 & $<1.0$ & .05 & .53 & 30 & $<.06$ & $<7.0$ & $<.04$ & $<.80$ & .03 & .77 & 11 \\
\hline 48 & 462818114074101 & $06 / 04 / 01$ & 1200 & $<1.0$ & e. 03 & $<.20$ & 20 & $<.06$ & e6.9 & $<.04$ & $<.80$ & .03 & .56 & $<10$ \\
\hline $48^{\mathbf{F b}}$ & & $06 / 04 / 01$ & 1211 & 12 & e. 02 & $<.20$ & $<1.0$ & $<.06$ & $<7.0$ & $<.04$ & $<.80$ & .02 & $<.20$ & $<10$ \\
\hline 49 & 462754113592701 & $06 / 05 / 01$ & 1500 & 1.1 & e. 04 & .87 & 51 & $<.06$ & 95 & .04 & e. 46 & .04 & 1.1 & $<10$ \\
\hline 50 & 462616114094301 & $06 / 05 / 01$ & 1300 & $<1.0$ & e. 05 & 1.3 & 39 & e. 04 & $\mathrm{e} 4.3$ & $<.04$ & $<.80$ & .04 & .96 & $<10$ \\
\hline 51 & 462545114034301 & $06 / 05 / 01$ & 1800 & $<1.0$ & .08 & 3.3 & 40 & $<.06$ & 20 & $<.04$ & e. 45 & .05 & .48 & $<10$ \\
\hline 52 & 462256114114501 & $06 / 04 / 01$ & 1700 & 5.8 & $<.05$ & $<.20$ & 1.3 & e. 03 & $\mathrm{e} 4.9$ & $<.04$ & $<.80$ & .02 & 2.8 & $\mathrm{e} 5.5$ \\
\hline 53 & 462228114030301 & $06 / 06 / 01$ & 1100 & $<1.0$ & .18 & 3.9 & 51 & $<.06$ & 9.8 & $<.04$ & $<.80$ & .05 & .46 & $<10$ \\
\hline 54 & 461823114050901 & $06 / 06 / 01$ & 1300 & $<1.0$ & .45 & 3.9 & 55 & $<.06$ & 20 & $<.04$ & $<.80$ & .10 & 2.6 & $<10$ \\
\hline 55 & 461807114123001 & $06 / 11 / 01$ & 1600 & $<1.0$ & e. 03 & e. 11 & 9.4 & $<.06$ & $<7.0$ & $<.04$ & $<.80$ & e. 01 & 1.5 & $<10$ \\
\hline 56 & 461638114023401 & $06 / 06 / 01$ & 0800 & $<1.0$ & .23 & 5.1 & 51 & $<.06$ & 19 & $<.04$ & $<.80$ & .10 & .55 & e6.3 \\
\hline 57 & 461451114090801 & $06 / 06 / 01$ & 1600 & $<1.0$ & .07 & .52 & 42 & $<.06$ & 13 & $<.04$ & $<.80$ & .07 & 1.7 & $<10$ \\
\hline 58 & 461320114121501 & $06 / 05 / 01$ & 0900 & $<1.0$ & $<.05$ & $<.20$ & 12 & $<.06$ & $<7.0$ & $<.04$ & $<.80$ & .02 & .42 & 35 \\
\hline 59 & 461055114112601 & $06 / 21 / 01$ & 0900 & 14 & e. 04 & $<.20$ & 5.0 & e.04 & $<7.0$ & e. 03 & $<.80$ & .02 & 4.2 & 14 \\
\hline 60 & 460110114110901 & $06 / 20 / 01$ & 1800 & $<1.0$ & e. 03 & 1.4 & 7.6 & $<.06$ & $<7.0$ & e. 02 & $<.80$ & e. 01 & .34 & $<10$ \\
\hline 61 & 455521114074801 & $06 / 20 / 01$ & 2000 & $<1.0$ & e. 04 & e. 11 & 20 & $<.06$ & e6.7 & .04 & $<.80$ & .04 & 2.5 & 36 \\
\hline
\end{tabular}


Table 17. Trace-element concentration data for ground-water samples collected from basin-fill aquifers, Northern Rockies Intermontane Basins study unit, 1999 and 2001 (Continued)

\begin{tabular}{|c|c|c|c|c|c|c|c|c|c|c|c|c|}
\hline $\begin{array}{c}\text { Lead, } \\
\text { dis- } \\
\text { solved } \\
(\mu \mathrm{g} / \mathrm{L})\end{array}$ & $\begin{array}{l}\text { Lith- } \\
\text { ium, } \\
\text { dis- } \\
\text { solved } \\
(\mu \mathrm{g} / \mathrm{L})\end{array}$ & $\begin{array}{c}\text { Man- } \\
\text { ganese, } \\
\text { dis- } \\
\text { solved } \\
(\mu \mathrm{g} / \mathrm{L})\end{array}$ & $\begin{array}{c}\text { Molyb- } \\
\text { denum, } \\
\text { dis- } \\
\text { solved } \\
(\mu \mathrm{g} / \mathrm{L})\end{array}$ & $\begin{array}{c}\text { Nickel, } \\
\text { dis- } \\
\text { solved } \\
(\mu \mathrm{g} / \mathrm{L})\end{array}$ & $\begin{array}{c}\text { Sele- } \\
\text { nium, } \\
\text { dis- } \\
\text { solved } \\
(\mu \mathrm{g} / \mathrm{L})\end{array}$ & $\begin{array}{c}\text { Silver, } \\
\text { dis- } \\
\text { solved } \\
(\mu \mathrm{g} / \mathrm{L})\end{array}$ & $\begin{array}{c}\text { Stron- } \\
\text { tium, } \\
\text { dis- } \\
\text { solved } \\
(\mu \mathrm{g} / \mathrm{L})\end{array}$ & $\begin{array}{c}\text { Thal- } \\
\text { lium, } \\
\text { dis- } \\
\text { solved } \\
(\mu \mathrm{g} / \mathrm{L})\end{array}$ & $\begin{array}{c}\text { Uran- } \\
\text { ium, } \\
\text { dis- } \\
\text { solved } \\
(\mu \mathrm{g} / \mathrm{L})\end{array}$ & $\begin{array}{c}\text { Vana- } \\
\text { dium, } \\
\text { dis- } \\
\text { solved } \\
(\mu \mathrm{g} / \mathrm{L})\end{array}$ & $\begin{array}{c}\text { Zinc, } \\
\text { dis- } \\
\text { solved } \\
(\mu \mathrm{g} / \mathrm{L})\end{array}$ & $\begin{array}{c}\text { Well } \\
\text { number } \\
\text { (figs. } 3 \\
\text { and 4) }\end{array}$ \\
\hline \multicolumn{13}{|c|}{ Subunit Survev 2, Montana } \\
\hline e. 06 & .52 & 1.1 & $<.20$ & e. 05 & .36 & $<1.0$ & 22 & $<.04$ & .02 & $<.20$ & 1.6 & 32 \\
\hline .31 & 1.2 & .85 & $<.20$ & $<.06$ & $<.30$ & $<1.0$ & 26 & $<.04$ & $<.02$ & e. 10 & 34 & 33 \\
\hline$<.08$ & 4.0 & $<1.0$ & .72 & $<.06$ & $<.30$ & $<1.0$ & 70 & $<.04$ & 2.1 & .40 & $<1.0$ & 34 \\
\hline$<.08$ & 3.8 & .48 & .47 & $<.06$ & .33 & $<1.0$ & 123 & e.03 & 1.7 & 1.5 & 3.2 & 35 \\
\hline$<.08$ & 4.9 & .19 & .67 & $<.06$ & $<.30$ & $<1.0$ & 79 & .11 & .74 & .29 & 6.7 & 36 \\
\hline$<.08$ & 5.4 & .20 & .64 & $<.06$ & $<.30$ & $<1.0$ & 80 & $<.04$ & .73 & .37 & 7.2 & $36^{\mathbf{R}}$ \\
\hline$<.08$ & $<.30$ & $<.10$ & $<.20$ & $<.06$ & $<.30$ & $<1.0$ & $<.08$ & $<.04$ & $<.02$ & $<.20$ & $<1.0$ & $36^{\mathrm{Fb}}$ \\
\hline e. 07 & 6.2 & e. 08 & $<.20$ & $<.06$ & $<.30$ & $<1.0$ & 107 & $<.04$ & .17 & .66 & 9.5 & 37 \\
\hline .12 & 1.5 & .48 & $<.20$ & .27 & $<.30$ & $<1.0$ & 49 & $<.04$ & .03 & e.14 & 48 & 38 \\
\hline .14 & 1.5 & .57 & $<.20$ & .26 & $<.30$ & $<1.0$ & 49 & $<.04$ & .04 & $<.20$ & 48 & $38^{\mathbf{R}}$ \\
\hline$<.08$ & $<.30$ & $<.10$ & $<.20$ & $<.06$ & $<.30$ & $<1.0$ & $<.08$ & $<.04$ & $<.02$ & $<.20$ & $<1.0$ & $38^{\mathbf{F b}}$ \\
\hline .19 & 3.7 & $<1.0$ & $<.20$ & $<.06$ & $<.30$ & $<1.0$ & 142 & $<.04$ & 1.1 & .40 & 21 & 39 \\
\hline e. 05 & 6.4 & $<1.0$ & .46 & $<.06$ & $<.30$ & $<1.0$ & 163 & $<.04$ & 1.4 & .85 & 5.2 & 40 \\
\hline e. 08 & 6.0 & $<1.0$ & .35 & $<.06$ & $<.30$ & $<1.0$ & 179 & $<.04$ & 1.5 & .88 & 7.8 & 41 \\
\hline .09 & 1.8 & 42 & .40 & .21 & $<.30$ & $<1.0$ & 60 & $<.04$ & .11 & .23 & 37 & 42 \\
\hline$<.08$ & .57 & 19 & 1.3 & .15 & $<.30$ & $<1.0$ & 101 & $<.04$ & .10 & .58 & 27 & 43 \\
\hline e. 06 & 10 & 5.4 & .87 & .24 & $<.30$ & $<1.0$ & 126 & e.03 & 1.0 & 2.0 & 20 & 44 \\
\hline$<.08$ & $<.30$ & $<.10$ & $<.20$ & $<.06$ & $<.30$ & $<1.0$ & .26 & $<.04$ & $<.02$ & $<.20$ & $<1.0$ & $44^{\mathbf{F b}}$ \\
\hline .12 & 1.4 & .77 & e. 15 & e. 03 & $<.30$ & $<1.0$ & 130 & $<.04$ & .19 & 1.3 & 7.8 & 45 \\
\hline .12 & 1.4 & .82 & e. 16 & e. 04 & $<.30$ & $<1.0$ & 131 & $<.04$ & .19 & 1.3 & 7.6 & $45^{\mathbf{R}}$ \\
\hline e. 05 & 1.5 & .16 & .37 & $<.06$ & $<.30$ & $<1.0$ & 51 & $<.04$ & .76 & .69 & 22 & 46 \\
\hline e. 06 & .97 & .21 & .70 & $<.06$ & e. 18 & $<1.0$ & 35 & $<.04$ & .72 & .62 & 17 & 47 \\
\hline$<.08$ & 1.4 & .10 & .28 & $<.06$ & $<.30$ & $<1.0$ & 181 & $<.04$ & .36 & .44 & 1.3 & 48 \\
\hline$<.08$ & $<.30$ & $<.10$ & $<.20$ & $<.06$ & $<.30$ & $<1.0$ & $<.08$ & $<.04$ & $<.02$ & $<.20$ & $<1.0$ & $48^{\mathbf{F b}}$ \\
\hline .19 & .38 & e. 08 & 18 & $<.06$ & $<.30$ & $<1.0$ & 433 & $<.04$ & 1.4 & 1.4 & 6.6 & 49 \\
\hline e. 05 & 1.4 & e.06 & .59 & .07 & $<.30$ & $<1.0$ & 66 & $<.04$ & .17 & 1.0 & 17 & 50 \\
\hline .40 & 4.8 & .96 & 3.1 & $<.06$ & e. 29 & $<1.0$ & 95 & $<.04$ & 4.5 & 4.7 & 143 & 51 \\
\hline$<.08$ & 1.4 & .70 & $<.20$ & $<.06$ & $<.30$ & $<1.0$ & 33 & .05 & .02 & $<.20$ & 1.3 & 52 \\
\hline$<.08$ & 4.1 & $<1.0$ & 13 & $<.06$ & $<.30$ & $<1.0$ & 39 & $<.04$ & 2.3 & 3.6 & 19 & 53 \\
\hline$<.08$ & 3.5 & e.07 & .39 & $<.06$ & e. 29 & $<1.0$ & 93 & $<.04$ & 7.9 & 1.6 & 2.6 & 54 \\
\hline .16 & 5.7 & .49 & $<.20$ & $<.06$ & $<.30$ & $<1.0$ & 81 & $<.04$ & .05 & .50 & 25 & 55 \\
\hline$<.08$ & 7.7 & .84 & 2.0 & $<.06$ & e. 21 & $<1.0$ & 133 & $<.04$ & 6.3 & 11 & 6.4 & 56 \\
\hline .11 & 1.5 & .12 & .37 & $<.06$ & $<.30$ & $<1.0$ & 54 & $<.04$ & 1.5 & .70 & 23 & 57 \\
\hline .09 & 3.4 & 1.5 & $<.20$ & $<.06$ & e. 18 & $<1.0$ & 151 & e.02 & .34 & $<.20$ & 4.6 & 58 \\
\hline .14 & 5.2 & .90 & $<.20$ & .12 & $<.30$ & $<1.0$ & 33 & $<.04$ & e. 02 & .37 & 24 & 59 \\
\hline .10 & 4.7 & 1.2 & $<.20$ & e. 03 & $<.30$ & $<1.0$ & 53 & $<.04$ & .03 & .81 & 36 & 60 \\
\hline .12 & 9.3 & .88 & .24 & $<.06$ & $<.30$ & $<1.0$ & 214 & $<.04$ & .68 & .41 & 9.0 & 61 \\
\hline
\end{tabular}


Table 18. Pesticide-concentration data for filtered ground-water samples collected from basin-fill aquifers, Northern Rockies Intermontane Basins study unit, 1999 and 2001

[Site identification number described in text. Bold text denotes detected values. Abbreviations: e, estimated; Fb, field blank; $\mu \mathrm{g} / \mathrm{L}$, micrograms per liter; R, replicate. Symbols: <, less than reporting level; --, no data]

\begin{tabular}{|c|c|c|c|c|c|c|c|c|c|c|c|}
\hline $\begin{array}{c}\text { Well } \\
\text { number } \\
\text { (figs. } 3 \\
\text { and 4) }\end{array}$ & $\begin{array}{c}\text { Site } \\
\text { identification } \\
\text { number }\end{array}$ & Date & Time & $\begin{array}{c}2,6- \\
\text { Diethyl- } \\
\text { aniline } \\
(\mu \mathrm{g} / \mathrm{L})\end{array}$ & $\begin{array}{l}\text { Aceto- } \\
\text { chlor } \\
(\mu \mathrm{g} / \mathrm{L})\end{array}$ & $\begin{array}{c}\text { Alachlor } \\
(\mu \mathrm{g} / \mathrm{L})\end{array}$ & $\begin{array}{c}\text { alpha- } \\
\text { HCH } \\
(\mu \mathrm{g} / \mathrm{L})\end{array}$ & $\begin{array}{l}\text { alpha- } \\
\text { HCH-d }_{6} \text {, } \\
\text { surrogate } \\
\text { (percent) }\end{array}$ & $\begin{array}{c}\text { Atra- } \\
\text { zine } \\
(\mu \mathrm{g} / \mathrm{L})\end{array}$ & $\begin{array}{l}\text { Azin- } \\
\text { phos- } \\
\text { methyl } \\
(\mu \mathrm{g} / \mathrm{L})\end{array}$ & $\begin{array}{c}\text { Ben- } \\
\text { fluralin } \\
(\mu \mathrm{g} / \mathrm{L})\end{array}$ \\
\hline \multicolumn{12}{|c|}{ Subunit Survey 1, Idaho and Washington } \\
\hline 1 & 481320116261101 & $07 / 14 / 99$ & 1700 & $<0.003$ & $<0.002$ & $<0.002$ & $<0.002$ & 90 & $<0.001$ & $<0.001$ & $<0.002$ \\
\hline 2 & 480950116324401 & 07/14/99 & 1100 & $<.003$ & $<.002$ & $<.002$ & $<.002$ & 102 & $<.001$ & $<.001$ & $<.002$ \\
\hline 3 & 480928117053201 & 07/07/99 & 1000 & $<.003$ & $<.002$ & $<.002$ & $<.002$ & 89 & $<.001$ & $<.001$ & $<.002$ \\
\hline 4 & 480813116593701 & 07/07/99 & 1600 & $<.003$ & $<.002$ & $<.002$ & $<.002$ & 107 & $<.001$ & $<.001$ & $<.002$ \\
\hline 5 & 480718117012501 & 07/06/99 & 1800 & $<.003$ & $<.002$ & $<.002$ & $<.002$ & 87 & $<.001$ & $<.001$ & $<.002$ \\
\hline 6 & 480249116510001 & 07/08/99 & 1000 & $<.003$ & $<.002$ & $<.002$ & $<.002$ & 102 & $<.001$ & $<.001$ & $<.002$ \\
\hline 7 & 480235116483001 & 07/13/99 & 1700 & $<.003$ & $<.002$ & $<.002$ & $<.002$ & 104 & $<.001$ & $<.001$ & $<.002$ \\
\hline 8 & 480203117200601 & $06 / 08 / 99$ & 0800 & $<.003$ & $<.002$ & $<.002$ & $<.002$ & 74 & $<.001$ & $<.001$ & $<.002$ \\
\hline 9 & 480128116374601 & 08/04/99 & 0900 & $<.003$ & $<.002$ & $<.002$ & $<.002$ & 94 & $<.001$ & $<.001$ & $<.002$ \\
\hline 10 & 480119117182101 & 06/08/99 & 1600 & $<.003$ & $<.002$ & $<.002$ & $<.002$ & 72 & $<.001$ & $<.001$ & $<.002$ \\
\hline 11 & 475925117153001 & 07/06/99 & 1100 & $<.003$ & $<.002$ & $<.002$ & $<.002$ & 106 & $<.001$ & $<.001$ & $<.002$ \\
\hline 12 & 475825117364401 & 06/09/99 & 1000 & $<.003$ & $<.002$ & $<.002$ & $<.002$ & 77 & $<.001$ & $<.001$ & $<.002$ \\
\hline 13 & 475731116371301 & $06 / 23 / 99$ & 1200 & $<.003$ & $<.002$ & $<.002$ & $<.002$ & 110 & $<.001$ & $<.001$ & $<.002$ \\
\hline 14 & 475637117262501 & 06/09/99 & 1700 & $<.003$ & $<.002$ & $<.002$ & $<.002$ & 74 & $<.001$ & $<.001$ & $<.002$ \\
\hline 15 & 475510116391201 & 08/03/99 & 1400 & $<.003$ & $<.002$ & $<.002$ & $<.002$ & 93 & $<.001$ & $<.001$ & $<.002$ \\
\hline 16 & 475400116404201 & $06 / 22 / 99$ & 1700 & $<.003$ & $<.002$ & $<.002$ & $<.002$ & 110 & $<.001$ & $<.001$ & $<.002$ \\
\hline 17 & 475343117225201 & $06 / 10 / 99$ & 0900 & $<.003$ & $<.002$ & $<.002$ & $<.002$ & 70 & $<.001$ & $<.001$ & $<.002$ \\
\hline 18 & 475322116522201 & $07 / 12 / 99$ & 1200 & $<.003$ & $<.002$ & $<.002$ & $<.002$ & 103 & $<.001$ & $<.001$ & $<.002$ \\
\hline $18^{\mathbf{R}}$ & & $07 / 12 / 99$ & 1201 & $<.003$ & $<.002$ & $<.002$ & $<.002$ & 108 & $<.001$ & $<.001$ & $<.002$ \\
\hline $18^{\mathbf{F b}}$ & & $07 / 12 / 99$ & 1205 & $<.003$ & $<.002$ & $<.002$ & $<.002$ & 95 & $<.001$ & $<.001$ & $<.002$ \\
\hline 19 & 475130117262201 & 06/07/99 & 0900 & $<.003$ & $<.002$ & $<.002$ & $<.002$ & 104 & $<.001$ & $<.001$ & $<.002$ \\
\hline 20 & 475035116424801 & $06 / 22 / 99$ & 1100 & $<.003$ & $<.002$ & $<.002$ & $<.002$ & 96 & $<.001$ & $<.001$ & $<.002$ \\
\hline 21 & 474718116530201 & $06 / 24 / 99$ & 0900 & $<.003$ & $<.002$ & $<.002$ & $<.002$ & 99 & $<.001$ & $<.001$ & $<.002$ \\
\hline $21^{R}$ & & $06 / 24 / 99$ & 0901 & $<.003$ & $<.002$ & $<.002$ & $<.002$ & 104 & $<.001$ & $<.001$ & $<.002$ \\
\hline $21^{\mathbf{F b}}$ & & $06 / 24 / 99$ & 0905 & $<.003$ & $<.002$ & $<.002$ & $<.002$ & 91 & $<.001$ & $<.001$ & $<.002$ \\
\hline 22 & 474708117250501 & 06/07/99 & 1600 & $<.003$ & $<.002$ & $<.002$ & $<.002$ & 79 & $<.001$ & $<.001$ & $<.002$ \\
\hline 23 & 474629117305101 & $05 / 25 / 99$ & 1100 & $<.003$ & $<.002$ & $<.002$ & $<.002$ & 100 & $<.001$ & $<.001$ & $<.002$ \\
\hline 24 & 474456116522001 & $06 / 21 / 99$ & 1700 & $<.003$ & $<.002$ & $<.002$ & $<.002$ & 104 & $<.001$ & $<.001$ & $<.002$ \\
\hline 25 & 474427117312101 & $05 / 24 / 99$ & 1400 & $<.003$ & $<.002$ & $<.002$ & $<.002$ & 93 & $<.001$ & $<.001$ & $<.002$ \\
\hline 26 & 474317117225301 & $05 / 27 / 99$ & 0900 & $<.003$ & $<.002$ & $<.002$ & $<.002$ & 103 & $<.001$ & $<.001$ & $<.002$ \\
\hline 27 & 474218116445601 & $07 / 15 / 99$ & 1000 & $<.003$ & $<.002$ & $<.002$ & $<.002$ & 90 & $<.001$ & $<.001$ & $<.002$ \\
\hline 28 & 474147116544001 & $07 / 26 / 99$ & 1300 & $<.003$ & $<.002$ & $<.002$ & $<.002$ & 106 & $<.001$ & $<.001$ & $<.002$ \\
\hline 29 & 474130117015401 & 07/29/99 & 1000 & $<.003$ & $<.002$ & $<.002$ & $<.002$ & 94 & $<.001$ & $<.001$ & $<.002$ \\
\hline 30 & 474050117084101 & 07/28/99 & 1700 & $<.003$ & $<.002$ & $<.002$ & $<.002$ & 92 & $<.001$ & $<.001$ & $<.002$ \\
\hline 31 & 473612117243601 & $05 / 26 / 99$ & 1100 & $<.003$ & $<.002$ & $<.002$ & $<.002$ & 79 & $<.001$ & $<.001$ & $<.002$ \\
\hline
\end{tabular}


Table 18. Pesticide-concentration data for filtered ground-water samples collected from basin-fill aquifers, Northern Rockies Intermontane Basins study unit, 1999 and 2001 (Continued)

\begin{tabular}{|c|c|c|c|c|c|c|c|c|c|c|c|}
\hline $\begin{array}{c}\text { Well } \\
\text { number } \\
\text { (figs. } 3 \\
\text { and 4) }\end{array}$ & $\begin{array}{c}\text { Butyl- } \\
\text { ate } \\
(\mu \mathrm{g} / \mathrm{L})\end{array}$ & $\begin{array}{c}\text { Carb- } \\
\text { aryl } \\
(\mu \mathrm{g} / \mathrm{L})\end{array}$ & $\begin{array}{c}\text { Carbo- } \\
\text { furan } \\
(\mu \mathrm{g} / \mathrm{L})\end{array}$ & $\begin{array}{c}\text { Chlor- } \\
\text { pyrifos } \\
(\mu \mathrm{g} / \mathrm{L})\end{array}$ & $\begin{array}{c}\text { cis-Per- } \\
\text { methrin } \\
(\mu \mathrm{g} / \mathrm{L})\end{array}$ & $\begin{array}{c}\text { Cyan- } \\
\text { azine } \\
(\mu \mathrm{g} / \mathrm{L})\end{array}$ & $\begin{array}{c}\text { Dacthal } \\
(\mu \mathrm{g} / \mathrm{L})\end{array}$ & $\begin{array}{c}\text { Deethyl- } \\
\text { atra- } \\
\text { zine } \\
(\mu \mathrm{g} / \mathrm{L})\end{array}$ & $\begin{array}{c}\text { Diaz- } \\
\text { inon } \\
(\mu \mathrm{g} / \mathrm{L})\end{array}$ & $\begin{array}{l}\text { Diazinon- } \\
\quad d_{10}, \\
\text { surrogate } \\
\text { (percent) }\end{array}$ & $\begin{array}{c}\text { Dieldrin } \\
(\mu \mathrm{g} / \mathrm{L})\end{array}$ \\
\hline \multicolumn{12}{|c|}{ Subunit Survey 1, Idaho and Washington } \\
\hline 1 & $<0.002$ & e0.010 & $<0.003$ & $<0.004$ & $<0.005$ & $<0.004$ & $<0.002$ & $<0.002$ & $<0.002$ & 106 & $<0.001$ \\
\hline 2 & $<.002$ & e.005 & $<.003$ & $<.004$ & $<.005$ & $<.004$ & $<.002$ & $<.002$ & $<.002$ & 110 & $<.001$ \\
\hline 3 & $<.002$ & $<.003$ & $<.003$ & $<.004$ & $<.005$ & $<.004$ & $<.002$ & $<.002$ & $<.002$ & 104 & $<.001$ \\
\hline 4 & $<.002$ & $<.003$ & $<.003$ & $<.004$ & $<.005$ & $<.004$ & $<.002$ & $<.002$ & $<.002$ & 127 & $<.001$ \\
\hline 5 & $<.002$ & $<.003$ & $<.003$ & $<.004$ & $<.005$ & $<.004$ & $<.002$ & $<.002$ & $<.002$ & 107 & $<.001$ \\
\hline 6 & $<.002$ & $<.003$ & $<.003$ & $<.004$ & $<.005$ & $<.004$ & $<.002$ & $<.002$ & $<.002$ & 120 & $<.001$ \\
\hline 7 & $<.002$ & $<.003$ & $<.003$ & $<.004$ & $<.005$ & $<.004$ & $<.002$ & $<.002$ & $<.002$ & 105 & $<.001$ \\
\hline 8 & $<.002$ & $<.003$ & $<.003$ & $<.004$ & $<.005$ & $<.004$ & $<.002$ & $<.002$ & $<.002$ & 96 & $<.001$ \\
\hline 9 & $<.002$ & $<.003$ & $<.003$ & $<.004$ & $<.005$ & $<.004$ & $<.002$ & $<.002$ & $<.002$ & 101 & $<.001$ \\
\hline 10 & $<.002$ & $<.003$ & $<.003$ & $<.004$ & $<.005$ & $<.004$ & $<.002$ & $<.002$ & $<.002$ & 96 & $<.001$ \\
\hline 11 & $<.002$ & $<.003$ & $<.003$ & $<.004$ & $<.005$ & $<.004$ & $<.002$ & $<.002$ & $<.002$ & 114 & $<.001$ \\
\hline 12 & $<.002$ & $<.003$ & $<.003$ & $<.004$ & $<.005$ & $<.004$ & $<.002$ & $<.002$ & $<.002$ & 96 & $<.001$ \\
\hline 13 & $<.002$ & $<.003$ & $<.003$ & $<.004$ & $<.005$ & $<.004$ & $<.002$ & $<.002$ & $<.002$ & 102 & $<.001$ \\
\hline 14 & $<.002$ & $<.003$ & $<.003$ & $<.004$ & $<.005$ & $<.004$ & $<.002$ & $<.002$ & $<.002$ & 87 & $<.001$ \\
\hline 15 & $<.002$ & $<.003$ & $<.003$ & $<.004$ & $<.005$ & $<.004$ & $<.002$ & $<.002$ & $<.002$ & 103 & $<.001$ \\
\hline 16 & $<.002$ & $<.003$ & $<.003$ & $<.004$ & $<.005$ & $<.004$ & $<.002$ & $<.002$ & $<.002$ & 99 & $<.001$ \\
\hline 17 & $<.002$ & $<.003$ & $<.003$ & $<.004$ & $<.005$ & $<.004$ & $<.002$ & $<.002$ & $<.002$ & 90 & $<.001$ \\
\hline 18 & $<.002$ & $<.003$ & $<.003$ & $<.004$ & $<.005$ & $<.004$ & $<.002$ & $<.002$ & $<.002$ & 99 & $<.001$ \\
\hline $18^{\mathbf{R}}$ & $<.002$ & $<.003$ & $<.003$ & $<.004$ & $<.005$ & $<.004$ & $<.002$ & $<.002$ & $<.002$ & 108 & $<.001$ \\
\hline $18^{\mathbf{F b}}$ & $<.002$ & $<.003$ & $<.003$ & $<.004$ & $<.005$ & $<.004$ & $<.002$ & $<.002$ & $<.002$ & 111 & $<.001$ \\
\hline 19 & $<.002$ & $<.003$ & $<.003$ & $<.004$ & $<.005$ & $<.004$ & $<.002$ & $<.002$ & $<.002$ & 91 & $<.001$ \\
\hline 20 & $<.002$ & $<.003$ & $<.003$ & $<.004$ & $<.005$ & $<.004$ & $<.002$ & $<.002$ & $<.002$ & 92 & $<.001$ \\
\hline 21 & $<.002$ & $<.003$ & $<.003$ & $<.004$ & $<.005$ & $<.004$ & $<.002$ & $<.002$ & $<.002$ & 106 & $<.001$ \\
\hline $21^{R}$ & $<.002$ & $<.003$ & $<.003$ & $<.004$ & $<.005$ & $<.004$ & $<.002$ & $<.002$ & $<.002$ & 113 & $<.001$ \\
\hline $21^{\mathbf{F b}}$ & $<.002$ & $<.003$ & $<.003$ & $<.004$ & $<.005$ & $<.004$ & $<.002$ & $<.002$ & $<.002$ & 117 & $<.001$ \\
\hline 22 & $<.002$ & $<.003$ & $<.003$ & $<.004$ & $<.005$ & $<.004$ & $<.002$ & $<.002$ & $<.002$ & 93 & $<.001$ \\
\hline 23 & $<.002$ & $<.003$ & $<.003$ & $<.004$ & $<.005$ & $<.004$ & $<.002$ & $<.002$ & $<.002$ & 100 & $<.001$ \\
\hline 24 & $<.002$ & $<.003$ & $<.003$ & $<.004$ & $<.005$ & $<.004$ & $<.002$ & $<.002$ & $<.002$ & 105 & $<.001$ \\
\hline 25 & $<.002$ & $<.003$ & $<.003$ & $<.004$ & $<.005$ & $<.004$ & $<.002$ & $<.002$ & $<.002$ & 93 & $<.001$ \\
\hline 26 & $<.002$ & $<.003$ & $<.003$ & $<.004$ & $<.005$ & $<.004$ & $<.002$ & $<.002$ & $<.002$ & 111 & $<.001$ \\
\hline 27 & $<.002$ & $<.003$ & $<.003$ & $<.004$ & $<.005$ & $<.004$ & $<.002$ & $<.002$ & $<.002$ & 104 & $<.001$ \\
\hline 28 & $<.002$ & $<.003$ & $<.003$ & $<.004$ & $<.005$ & $<.004$ & $<.002$ & $<.002$ & $<.002$ & 102 & $<.001$ \\
\hline 29 & $<.002$ & $<.003$ & $<.003$ & $<.004$ & $<.005$ & $<.004$ & $<.002$ & $<.002$ & $<.002$ & 118 & $<.001$ \\
\hline 30 & $<.002$ & $<.003$ & $<.003$ & $<.004$ & $<.005$ & $<.004$ & $<.002$ & $<.002$ & $<.002$ & 116 & $<.001$ \\
\hline 31 & $<.002$ & $<.003$ & $<.003$ & $<.004$ & $<.005$ & $<.004$ & $<.002$ & $<.002$ & $<.002$ & 94 & $<.001$ \\
\hline
\end{tabular}


Table 18. Pesticide-concentration data for filtered ground-water samples collected from basin-fill aquifers, Northern Rockies Intermontane Basins study unit, 1999 and 2001 (Continued)

\begin{tabular}{|c|c|c|c|c|c|c|c|c|c|c|}
\hline $\begin{array}{c}\text { Well } \\
\text { number } \\
\text { (figs. } 3 \\
\text { and 4) }\end{array}$ & $\begin{array}{c}\text { Disul- } \\
\text { foton } \\
(\mu \mathrm{g} / \mathrm{L})\end{array}$ & $\begin{array}{l}\text { EPTC } \\
(\mu \mathrm{g} / \mathrm{L})\end{array}$ & $\begin{array}{c}\text { Ethal- } \\
\text { fluralin } \\
(\mu \mathrm{g} / \mathrm{L})\end{array}$ & $\begin{array}{c}\text { Etho- } \\
\text { pro- } \\
\text { phos } \\
(\mu \mathrm{g} / \mathrm{L})\end{array}$ & $\begin{array}{c}\text { Fonofos } \\
(\mu \mathrm{g} / \mathrm{L})\end{array}$ & $\begin{array}{c}\text { Lindane } \\
(\mu \mathrm{g} / \mathrm{L})\end{array}$ & $\begin{array}{c}\text { Linuron } \\
(\mu \mathrm{g} / \mathrm{L})\end{array}$ & $\begin{array}{c}\text { Mala- } \\
\text { thion } \\
(\mu \mathrm{g} / \mathrm{L})\end{array}$ & $\begin{array}{l}\text { Metol- } \\
\text { achlor } \\
(\mu \mathrm{g} / \mathrm{L})\end{array}$ & $\begin{array}{c}\text { Metri- } \\
\text { buzin } \\
(\mu \mathrm{g} / \mathrm{L})\end{array}$ \\
\hline \multicolumn{11}{|c|}{ Subunit Survey 1, Idaho and Washington } \\
\hline 1 & $<0.017$ & $<0.002$ & $<0.004$ & $<0.003$ & $<0.003$ & $<0.004$ & $<0.002$ & $<0.005$ & $<0.002$ & $<0.004$ \\
\hline 2 & $<.017$ & $<.002$ & $<.004$ & $<.003$ & $<.003$ & $<.004$ & $<.002$ & $<.005$ & $<.002$ & $<.004$ \\
\hline 3 & $<.017$ & $<.002$ & $<.004$ & $<.003$ & $<.003$ & $<.004$ & $<.002$ & $<.005$ & $<.002$ & $<.004$ \\
\hline 4 & $<.017$ & $<.002$ & $<.004$ & $<.003$ & $<.003$ & $<.004$ & $<.002$ & $<.005$ & $<.002$ & $<.004$ \\
\hline 5 & $<.017$ & $<.002$ & $<.004$ & $<.003$ & $<.003$ & $<.004$ & $<.002$ & $<.005$ & $<.002$ & $<.004$ \\
\hline 6 & $<.017$ & $<.002$ & $<.004$ & $<.003$ & $<.003$ & $<.004$ & $<.002$ & $<.005$ & $<.002$ & $<.004$ \\
\hline 7 & $<.017$ & $<.002$ & $<.004$ & $<.003$ & $<.003$ & $<.004$ & $<.002$ & $<.005$ & $<.002$ & $<.004$ \\
\hline 8 & $<.017$ & $<.002$ & $<.004$ & $<.003$ & $<.003$ & $<.004$ & $<.002$ & $<.005$ & $<.002$ & $<.004$ \\
\hline 9 & $<.017$ & $<.002$ & $<.004$ & $<.003$ & $<.003$ & $<.004$ & $<.002$ & $<.005$ & $<.002$ & $<.004$ \\
\hline 10 & $<.017$ & $<.002$ & $<.004$ & $<.003$ & $<.003$ & $<.004$ & $<.002$ & $<.005$ & $<.002$ & $<.004$ \\
\hline 11 & $<.017$ & $<.002$ & $<.004$ & $<.003$ & $<.003$ & $<.004$ & $<.002$ & $<.005$ & $<.002$ & $<.004$ \\
\hline 12 & $<.017$ & $<.002$ & $<.004$ & $<.003$ & $<.003$ & $<.004$ & $<.002$ & $<.005$ & $<.002$ & $<.004$ \\
\hline 13 & $<.017$ & $<.002$ & $<.004$ & $<.003$ & $<.003$ & $<.004$ & $<.002$ & $<.005$ & $<.002$ & $<.004$ \\
\hline 14 & $<.017$ & $<.002$ & $<.004$ & $<.003$ & $<.003$ & $<.004$ & $<.002$ & $<.005$ & $<.002$ & $<.004$ \\
\hline 15 & $<.017$ & $<.002$ & $<.004$ & $<.003$ & $<.003$ & $<.004$ & $<.002$ & $<.005$ & $<.002$ & $<.004$ \\
\hline 16 & $<.017$ & $<.002$ & $<.004$ & $<.003$ & $<.003$ & $<.004$ & $<.002$ & $<.005$ & $<.002$ & $<.004$ \\
\hline 17 & $<.017$ & $<.002$ & $<.004$ & $<.003$ & $<.003$ & $<.004$ & $<.002$ & $<.005$ & $<.002$ & $<.004$ \\
\hline 18 & $<.017$ & $<.002$ & $<.004$ & $<.003$ & $<.003$ & $<.004$ & $<.002$ & $<.005$ & $<.002$ & $<.004$ \\
\hline $18^{\mathbf{R}}$ & $<.017$ & $<.002$ & $<.004$ & $<.003$ & $<.003$ & $<.004$ & $<.002$ & $<.005$ & $<.002$ & $<.004$ \\
\hline $18^{\mathbf{F b}}$ & $<.017$ & $<.002$ & $<.004$ & $<.003$ & $<.003$ & $<.004$ & $<.002$ & $<.005$ & $<.002$ & $<.004$ \\
\hline 19 & $<.017$ & $<.002$ & $<.004$ & $<.003$ & $<.003$ & $<.004$ & $<.002$ & $<.005$ & $<.002$ & $<.004$ \\
\hline 20 & $<.017$ & $<.002$ & $<.004$ & $<.003$ & $<.003$ & $<.004$ & $<.002$ & $<.005$ & $<.002$ & $<.004$ \\
\hline 21 & $<.017$ & $<.002$ & $<.004$ & $<.003$ & $<.003$ & $<.004$ & $<.002$ & $<.005$ & $<.002$ & $<.004$ \\
\hline $21^{\mathbf{R}}$ & $<.017$ & $<.002$ & $<.004$ & $<.003$ & $<.003$ & $<.004$ & $<.002$ & $<.005$ & $<.002$ & $<.004$ \\
\hline $21^{\mathbf{F b}}$ & $<.017$ & $<.002$ & $<.004$ & $<.003$ & $<.003$ & $<.004$ & $<.002$ & $<.005$ & $<.002$ & $<.004$ \\
\hline 22 & $<.017$ & $<.002$ & $<.004$ & $<.003$ & $<.003$ & $<.004$ & $<.002$ & $<.005$ & $<.002$ & $<.004$ \\
\hline 23 & $<.017$ & $<.002$ & $<.004$ & $<.003$ & $<.003$ & $<.004$ & $<.002$ & $<.005$ & $<.002$ & $<.004$ \\
\hline 24 & $<.017$ & $<.002$ & $<.004$ & $<.003$ & $<.003$ & $<.004$ & $<.002$ & $<.005$ & $<.002$ & $<.004$ \\
\hline 25 & $<.017$ & $<.002$ & $<.004$ & $<.003$ & $<.003$ & $<.004$ & $<.002$ & $<.005$ & $<.002$ & $<.004$ \\
\hline 26 & $<.017$ & $<.002$ & $<.004$ & $<.003$ & $<.003$ & $<.004$ & $<.002$ & $<.005$ & $<.002$ & $<.004$ \\
\hline 27 & $<.017$ & $<.002$ & $<.004$ & $<.003$ & $<.003$ & $<.004$ & $<.002$ & $<.005$ & $<.002$ & $<.004$ \\
\hline 28 & $<.017$ & $<.002$ & $<.004$ & $<.003$ & $<.003$ & $<.004$ & $<.002$ & $<.005$ & $<.002$ & $<.004$ \\
\hline 29 & $<.017$ & $<.002$ & $<.004$ & $<.003$ & $<.003$ & $<.004$ & $<.002$ & $<.005$ & $<.002$ & $<.004$ \\
\hline 30 & $<.017$ & $<.002$ & $<.004$ & $<.003$ & $<.003$ & $<.004$ & $<.002$ & $<.005$ & $<.002$ & $<.004$ \\
\hline 31 & $<.017$ & $<.002$ & $<.004$ & $<.003$ & $<.003$ & $<.004$ & $<.002$ & $<.005$ & $<.002$ & $<.004$ \\
\hline
\end{tabular}


Table 18. Pesticide-concentration data for filtered ground-water samples collected from basin-fill aquifers, Northern Rockies Intermontane Basins study unit, 1999 and 2001 (Continued)

\begin{tabular}{|c|c|c|c|c|c|c|c|c|c|}
\hline $\begin{array}{c}\text { Well } \\
\text { number } \\
\text { (figs. } 3 \\
\text { and 4) }\end{array}$ & $\begin{array}{c}\text { Molin- } \\
\text { ate } \\
(\mu \mathrm{g} / \mathrm{L})\end{array}$ & $\begin{array}{c}\text { Naprop- } \\
\text { amide } \\
(\mu \mathrm{g} / \mathrm{L})\end{array}$ & $\begin{array}{c}\text { Para- } \\
\text { thion } \\
(\mu \mathrm{g} / \mathrm{L})\end{array}$ & $\begin{array}{l}\text { Para- } \\
\text { thion- } \\
\text { methyl } \\
(\mu \mathrm{g} / \mathrm{L})\end{array}$ & $\begin{array}{c}\text { Pebulate } \\
(\mu \mathrm{g} / \mathbf{L})\end{array}$ & $\begin{array}{c}\text { Pendi- } \\
\text { meth- } \\
\text { alin } \\
(\mu \mathrm{g} / \mathrm{L})\end{array}$ & $\begin{array}{c}\text { Phorate } \\
(\mu \mathrm{g} / \mathrm{L})\end{array}$ & $\begin{array}{c}\text { p,p'-DDE } \\
(\mu \mathrm{g} / \mathrm{L})\end{array}$ & $\begin{array}{c}\text { Pro- } \\
\text { meton } \\
(\mu \mathrm{g} / \mathrm{L})\end{array}$ \\
\hline \multicolumn{10}{|c|}{ Subunit Survey 1, Idaho and Washington } \\
\hline 1 & $<0.004$ & $<0.003$ & $<0.004$ & $<0.006$ & $<0.004$ & $<0.004$ & $<0.002$ & $<0.006$ & $<0.018$ \\
\hline 2 & $<.004$ & $<.003$ & $<.004$ & $<.006$ & $<.004$ & $<.004$ & $<.002$ & $<.006$ & $<.018$ \\
\hline 3 & $<.004$ & $<.003$ & $<.004$ & $<.006$ & $<.004$ & $<.004$ & $<.002$ & $<.006$ & $<.018$ \\
\hline 4 & $<.004$ & $<.003$ & $<.004$ & $<.006$ & $<.004$ & $<.004$ & $<.002$ & $<.006$ & $<.018$ \\
\hline 5 & $<.004$ & $<.003$ & $<.004$ & $<.006$ & $<.004$ & $<.004$ & $<.002$ & $<.006$ & $<.018$ \\
\hline 6 & $<.004$ & $<.003$ & $<.004$ & $<.006$ & $<.004$ & $<.004$ & $<.002$ & $<.006$ & $<.018$ \\
\hline 7 & $<.004$ & $<.003$ & $<.004$ & $<.006$ & $<.004$ & $<.004$ & $<.002$ & $<.006$ & $<.018$ \\
\hline 8 & $<.004$ & $<.003$ & $<.004$ & $<.006$ & $<.004$ & $<.004$ & $<.002$ & $<.006$ & $<.018$ \\
\hline 9 & $<.004$ & $<.003$ & $<.004$ & $<.006$ & $<.004$ & $<.004$ & $<.002$ & $<.006$ & $<.018$ \\
\hline 10 & $<.004$ & $<.003$ & $<.004$ & $<.006$ & $<.004$ & $<.004$ & $<.002$ & $<.006$ & $<.018$ \\
\hline 11 & $<.004$ & $<.003$ & $<.004$ & $<.006$ & $<.004$ & $<.004$ & $<.002$ & $<.006$ & $<.018$ \\
\hline 12 & $<.004$ & $<.003$ & $<.004$ & $<.006$ & $<.004$ & $<.004$ & $<.002$ & $<.006$ & $<.018$ \\
\hline 13 & $<.004$ & $<.003$ & $<.004$ & $<.006$ & $<.004$ & $<.004$ & $<.002$ & $<.006$ & $<.018$ \\
\hline 14 & $<.004$ & $<.003$ & $<.004$ & $<.006$ & $<.004$ & $<.004$ & $<.002$ & $<.006$ & $<.018$ \\
\hline 15 & $<.004$ & $<.003$ & $<.004$ & $<.006$ & $<.004$ & $<.004$ & $<.002$ & $<.006$ & $<.018$ \\
\hline 16 & $<.004$ & $<.003$ & $<.004$ & $<.006$ & $<.004$ & $<.004$ & $<.002$ & $<.006$ & $<.018$ \\
\hline 17 & $<.004$ & $<.003$ & $<.004$ & $<.006$ & $<.004$ & $<.004$ & $<.002$ & $<.006$ & $<.018$ \\
\hline 18 & $<.004$ & $<.003$ & $<.004$ & $<.006$ & $<.004$ & $<.004$ & $<.002$ & $<.006$ & $<.018$ \\
\hline $18^{\mathbf{R}}$ & $<.004$ & $<.003$ & $<.004$ & $<.006$ & $<.004$ & $<.004$ & $<.002$ & $<.006$ & $<.018$ \\
\hline $18^{\mathbf{F b}}$ & $<.004$ & $<.003$ & $<.004$ & $<.006$ & $<.004$ & $<.004$ & $<.002$ & $<.006$ & $<.018$ \\
\hline 19 & $<.004$ & $<.003$ & $<.004$ & $<.006$ & $<.004$ & $<.004$ & $<.002$ & $<.006$ & $<.018$ \\
\hline 20 & $<.004$ & $<.003$ & $<.004$ & $<.006$ & $<.004$ & $<.004$ & $<.002$ & $<.006$ & $<.018$ \\
\hline 21 & $<.004$ & $<.003$ & $<.004$ & $<.006$ & $<.004$ & $<.004$ & $<.002$ & $<.006$ & $<.018$ \\
\hline $21^{\mathbf{R}}$ & $<.004$ & $<.003$ & $<.004$ & $<.006$ & $<.004$ & $<.004$ & $<.002$ & $<.006$ & $<.018$ \\
\hline $21^{\mathbf{F b}}$ & $<.004$ & $<.003$ & $<.004$ & $<.006$ & $<.004$ & $<.004$ & $<.002$ & $<.006$ & $<.018$ \\
\hline 22 & $<.004$ & $<.003$ & $<.004$ & $<.006$ & $<.004$ & $<.004$ & $<.002$ & $<.006$ & $<.018$ \\
\hline 23 & $<.004$ & $<.003$ & $<.004$ & $<.006$ & $<.004$ & $<.004$ & $<.002$ & $<.006$ & $<.018$ \\
\hline 24 & $<.004$ & $<.003$ & $<.004$ & $<.006$ & $<.004$ & $<.004$ & $<.002$ & $<.006$ & $<.018$ \\
\hline 25 & $<.004$ & $<.003$ & $<.004$ & $<.006$ & $<.004$ & $<.004$ & $<.002$ & $<.006$ & $<.018$ \\
\hline 26 & $<.004$ & $<.003$ & $<.004$ & $<.006$ & $<.004$ & $<.004$ & $<.002$ & $<.006$ & $<.018$ \\
\hline 27 & $<.004$ & $<.003$ & $<.004$ & $<.006$ & $<.004$ & $<.004$ & $<.002$ & $<.006$ & $<.018$ \\
\hline 28 & $<.004$ & $<.003$ & $<.004$ & $<.006$ & $<.004$ & $<.004$ & $<.002$ & $<.006$ & $<.018$ \\
\hline 29 & $<.004$ & $<.003$ & $<.004$ & $<.006$ & $<.004$ & $<.004$ & $<.002$ & $<.006$ & $<.018$ \\
\hline 30 & $<.004$ & $<.003$ & $<.004$ & $<.006$ & $<.004$ & $<.004$ & $<.002$ & $<.006$ & $<.018$ \\
\hline 31 & $<.004$ & $<.003$ & $<.004$ & $<.006$ & $<.004$ & $<.004$ & $<.002$ & $<.006$ & $<.018$ \\
\hline
\end{tabular}


Table 18. Pesticide-concentration data for filtered ground-water samples collected from basin-fill aquifers, Northern Rockies Intermontane Basins study unit, 1999 and 2001 (Continued)

\begin{tabular}{|c|c|c|c|c|c|c|c|c|c|c|c|}
\hline $\begin{array}{c}\text { Well } \\
\text { number } \\
\text { (figs. } 3 \\
\text { and 4) }\end{array}$ & $\begin{array}{l}\text { Prop- } \\
\text { achlor } \\
(\mu \mathrm{g} / \mathrm{L})\end{array}$ & $\begin{array}{c}\text { Prop- } \\
\text { anil } \\
(\mu \mathrm{g} / \mathrm{L})\end{array}$ & $\begin{array}{l}\text { Prop- } \\
\text { argite } \\
(\mu \mathrm{g} / \mathbf{L})\end{array}$ & $\begin{array}{c}\text { Propyz- } \\
\text { amide } \\
(\mu \mathrm{g} / \mathrm{L})\end{array}$ & $\begin{array}{c}\text { Sim- } \\
\text { azine } \\
(\mu \mathrm{g} / \mathrm{L})\end{array}$ & $\begin{array}{c}\text { Tebu- } \\
\text { thiuron } \\
(\mu \mathrm{g} / \mathrm{L})\end{array}$ & $\begin{array}{c}\text { Terbacil } \\
(\mu \mathrm{g} / \mathrm{L})\end{array}$ & $\begin{array}{c}\text { Terbufos } \\
(\mu \mathrm{g} / \mathrm{L})\end{array}$ & $\begin{array}{c}\text { Thio- } \\
\text { bencarb } \\
(\mu \mathrm{g} / \mathrm{L})\end{array}$ & $\begin{array}{c}\text { Tri- } \\
\text { allate } \\
(\mu \mathrm{g} / \mathrm{L})\end{array}$ & $\begin{array}{c}\text { Tri- } \\
\text { fluralin } \\
(\mu \mathrm{g} / \mathrm{L})\end{array}$ \\
\hline \multicolumn{12}{|c|}{ Subunt Survey 1, Idaho and Washington } \\
\hline 1 & $<0.007$ & $<0.004$ & $<0.013$ & $<0.003$ & $<0.005$ & $<0.010$ & $<0.007$ & $<0.013$ & $<0.002$ & $<0.001$ & $<0.002$ \\
\hline 2 & $<.007$ & $<.004$ & $<.013$ & $<.003$ & $<.005$ & $<.010$ & $<.007$ & $<.013$ & $<.002$ & $<.001$ & $<.002$ \\
\hline 3 & $<.007$ & $<.004$ & $<.013$ & $<.003$ & $<.005$ & $<.010$ & $<.007$ & $<.013$ & $<.002$ & $<.001$ & $<.002$ \\
\hline 4 & $<.007$ & $<.004$ & $<.013$ & $<.003$ & $<.005$ & $<.010$ & $<.007$ & $<.013$ & $<.002$ & $<.001$ & $<.002$ \\
\hline 5 & $<.007$ & $<.004$ & $<.013$ & $<.003$ & $<.005$ & $<.010$ & $<.007$ & $<.013$ & $<.002$ & $<.001$ & $<.002$ \\
\hline 6 & $<.007$ & $<.004$ & $<.013$ & $<.003$ & $<.005$ & $<.010$ & $<.007$ & $<.013$ & $<.002$ & $<.001$ & $<.002$ \\
\hline 7 & $<.007$ & $<.004$ & $<.013$ & $<.003$ & $<.005$ & $<.010$ & $<.007$ & $<.013$ & $<.002$ & $<.001$ & $<.002$ \\
\hline 8 & $<.007$ & $<.004$ & $<.013$ & $<.003$ & $<.005$ & $<.010$ & $<.007$ & $<.013$ & $<.002$ & $<.001$ & $<.002$ \\
\hline 9 & $<.007$ & $<.004$ & $<.013$ & $<.003$ & $<.005$ & $<.010$ & $<.007$ & $<.013$ & $<.002$ & $<.001$ & $<.002$ \\
\hline 10 & $<.007$ & $<.004$ & $<.013$ & $<.003$ & $<.005$ & $<.010$ & $<.007$ & $<.013$ & $<.002$ & $<.001$ & $<.002$ \\
\hline 11 & $<.007$ & $<.004$ & $<.013$ & $<.003$ & $<.005$ & $<.010$ & $<.007$ & $<.013$ & $<.002$ & $<.001$ & $<.002$ \\
\hline 12 & $<.007$ & $<.004$ & $<.013$ & $<.003$ & $<.005$ & $<.010$ & $<.007$ & $<.013$ & $<.002$ & $<.001$ & $<.002$ \\
\hline 13 & $<.007$ & $<.004$ & $<.013$ & $<.003$ & $<.005$ & $<.010$ & $<.007$ & $<.013$ & $<.002$ & $<.001$ & $<.002$ \\
\hline 14 & $<.007$ & $<.004$ & $<.013$ & $<.003$ & $<.005$ & $<.010$ & $<.007$ & $<.013$ & $<.002$ & $<.001$ & $<.002$ \\
\hline 15 & $<.007$ & $<.004$ & $<.013$ & $<.003$ & $<.005$ & $<.010$ & $<.007$ & $<.013$ & $<.002$ & $<.001$ & $<.002$ \\
\hline 16 & $<.007$ & $<.004$ & $<.013$ & $<.003$ & $<.005$ & $<.010$ & $<.007$ & $<.013$ & $<.002$ & $<.001$ & $<.002$ \\
\hline 17 & $<.007$ & $<.004$ & $<.013$ & $<.003$ & $<.005$ & $<.010$ & $<.007$ & $<.013$ & $<.002$ & $<.001$ & $<.002$ \\
\hline 18 & $<.007$ & $<.004$ & -- & $<.003$ & $<.005$ & $<.010$ & $<.007$ & $<.013$ & $<.002$ & $<.001$ & $<.002$ \\
\hline $18^{\mathbf{R}}$ & $<.007$ & $<.004$ & -- & $<.003$ & $<.005$ & $<.010$ & $<.007$ & $<.013$ & $<.002$ & $<.001$ & $<.002$ \\
\hline $18^{\mathbf{F b}}$ & $<.007$ & $<.004$ & $<.013$ & $<.003$ & $<.005$ & $<.010$ & $<.007$ & $<.013$ & $<.002$ & $<.001$ & $<.002$ \\
\hline 19 & $<.007$ & $<.004$ & $<.013$ & $<.003$ & $<.005$ & $<.010$ & $<.007$ & $<.013$ & $<.002$ & $<.001$ & $<.002$ \\
\hline 20 & $<.007$ & $<.004$ & $<.013$ & $<.003$ & $<.005$ & $<.010$ & $<.007$ & $<.013$ & $<.002$ & $<.001$ & $<.002$ \\
\hline 21 & $<.007$ & $<.004$ & $<.013$ & $<.003$ & $<.005$ & $<.010$ & $<.007$ & $<.013$ & $<.002$ & $<.001$ & $<.002$ \\
\hline $21^{\mathbf{R}}$ & $<.007$ & $<.004$ & $<.013$ & $<.003$ & $<.005$ & $<.010$ & $<.007$ & $<.013$ & $<.002$ & $<.001$ & $<.002$ \\
\hline $21^{\mathbf{F b}}$ & $<.007$ & $<.004$ & $<.013$ & $<.003$ & $<.005$ & $<.010$ & $<.007$ & $<.013$ & $<.002$ & $<.001$ & $<.002$ \\
\hline 22 & $<.007$ & $<.004$ & $<.013$ & $<.003$ & $<.005$ & $<.010$ & $<.007$ & $<.013$ & $<.002$ & $<.001$ & $<.002$ \\
\hline 23 & $<.007$ & $<.004$ & $<.013$ & $<.003$ & $<.005$ & $<.010$ & $<.007$ & $<.013$ & $<.002$ & $<.001$ & $<.002$ \\
\hline 24 & $<.007$ & $<.004$ & $<.013$ & $<.003$ & $<.005$ & $<.010$ & $<.007$ & $<.013$ & $<.002$ & $<.001$ & $<.002$ \\
\hline 25 & $<.007$ & $<.004$ & $<.013$ & $<.003$ & $<.005$ & $<.010$ & $<.007$ & $<.013$ & $<.002$ & $<.001$ & $<.002$ \\
\hline 26 & $<.007$ & $<.004$ & $<.013$ & $<.003$ & $<.005$ & $<.010$ & $<.007$ & $<.013$ & $<.002$ & $<.001$ & $<.002$ \\
\hline 27 & $<.007$ & $<.004$ & $<.013$ & $<.003$ & $<.005$ & $<.010$ & $<.007$ & $<.013$ & $<.002$ & $<.001$ & $<.002$ \\
\hline 28 & $<.007$ & $<.004$ & $<.013$ & $<.003$ & $<.005$ & $<.010$ & $<.007$ & $<.013$ & $<.002$ & $<.001$ & $<.002$ \\
\hline 29 & $<.007$ & $<.004$ & $<.013$ & $<.003$ & $<.005$ & $<.010$ & $<.007$ & $<.013$ & $<.002$ & $<.001$ & $<.002$ \\
\hline 30 & $<.007$ & $<.004$ & $<.013$ & $<.003$ & $<.005$ & $<.010$ & $<.007$ & $<.013$ & $<.002$ & $<.001$ & $<.002$ \\
\hline 31 & $<.007$ & $<.004$ & $<.013$ & $<.003$ & $<.005$ & $<.010$ & $<.007$ & $<.013$ & $<.002$ & $<.001$ & $<.002$ \\
\hline
\end{tabular}


Table 18. Pesticide-concentration data for filtered ground-water samples collected from basin-fill aquifers, Northern Rockies Intermontane Basins study unit, 1999 and 2001 (Continued)

\begin{tabular}{|c|c|c|c|c|c|c|c|c|c|c|c|}
\hline $\begin{array}{c}\text { Well } \\
\text { number } \\
\text { (figs. } 3 \\
\text { and 4) }\end{array}$ & $\begin{array}{c}\text { Site } \\
\text { identification } \\
\text { number }\end{array}$ & Date & Time & $\begin{array}{c}2,6- \\
\text { Diethyl- } \\
\text { aniline } \\
(\mu \mathrm{g} / \mathrm{L})\end{array}$ & $\begin{array}{l}\text { Aceto- } \\
\text { chlor } \\
(\mu \mathrm{g} / \mathrm{L})\end{array}$ & $\begin{array}{c}\text { Alachlor } \\
(\mu \mathrm{g} / \mathrm{L})\end{array}$ & $\begin{array}{l}\text { alpha- } \\
\text { HCH } \\
(\mu \mathrm{g} / \mathrm{L})\end{array}$ & $\begin{array}{l}\text { alpha- } \\
\text { HCH-d }_{6} \text {, } \\
\text { surrogate } \\
\text { (percent) }\end{array}$ & $\begin{array}{c}\text { Atra- } \\
\text { zine } \\
(\mu \mathrm{g} / \mathrm{L})\end{array}$ & $\begin{array}{c}\text { Azin- } \\
\text { phos- } \\
\text { methyl } \\
(\mu \mathrm{g} / \mathrm{L})\end{array}$ & $\begin{array}{c}\text { Ben- } \\
\text { fluralin } \\
(\mu \mathrm{g} / \mathrm{L})\end{array}$ \\
\hline \multicolumn{12}{|c|}{ Subunit Survey 2, Montana } \\
\hline 32 & 470719114301401 & $06 / 12 / 01$ & 1300 & $<.002$ & $<.004$ & $<.002$ & $<.005$ & 80 & $<.007$ & $<.050$ & $<.010$ \\
\hline 33 & 470502114265301 & $06 / 12 / 01$ & 0900 & $<.002$ & $<.004$ & $<.002$ & $<.005$ & 89 & $<.007$ & $<.050$ & $<.010$ \\
\hline 34 & 470328114164301 & $06 / 28 / 01$ & 0900 & $<.002$ & $<.004$ & $<.002$ & $<.005$ & 89 & $<.007$ & $<.050$ & $<.010$ \\
\hline 35 & 470112114144001 & $06 / 07 / 01$ & 0900 & $<.002$ & $<.004$ & $<.002$ & $<.005$ & 103 & $<.007$ & $<.050$ & $<.010$ \\
\hline 36 & 465838114074501 & $06 / 27 / 01$ & 1200 & $<.002$ & $<.004$ & $<.002$ & $<.005$ & 86 & $<.007$ & $<.050$ & $<.010$ \\
\hline $36^{\mathbf{R}}$ & & $06 / 27 / 01$ & 1201 & $<.002$ & $<.004$ & $<.002$ & $<.005$ & 94 & $<.007$ & $<.050$ & $<.010$ \\
\hline $36^{\mathbf{F b}}$ & & $06 / 27 / 01$ & 1205 & $<.002$ & $<.004$ & $<.002$ & $<.005$ & 88 & $<.007$ & $<.050$ & $<.010$ \\
\hline 37 & 465741114110601 & $06 / 27 / 01$ & 1700 & $<.002$ & $<.004$ & $<.002$ & $<.005$ & 95 & $<.007$ & $<.050$ & $<.010$ \\
\hline 38 & 465440114022101 & $06 / 19 / 01$ & 1100 & $<.002$ & $<.004$ & $<.002$ & $<.005$ & 78 & $<.007$ & $<.050$ & $<.010$ \\
\hline $38^{\mathbf{R}}$ & & 06/19/01 & 1101 & $<.002$ & $<.004$ & $<.002$ & $<.005$ & 106 & $<.009$ & $<.050$ & $<.010$ \\
\hline $38^{\mathbf{F b}}$ & & $06 / 19 / 01$ & 1105 & $<.002$ & $<.004$ & $<.002$ & $<.005$ & 87 & $<.007$ & $<.050$ & $<.010$ \\
\hline 39 & 465323114054301 & $06 / 20 / 01$ & 1300 & $<.002$ & $<.004$ & $<.002$ & $<.005$ & 88 & .031 & $<.050$ & $<.010$ \\
\hline 40 & 465127114055401 & $06 / 20 / 01$ & 1000 & $<.002$ & $<.004$ & $<.002$ & $<.005$ & 92 & $<.007$ & $<.050$ & $<.010$ \\
\hline 41 & 464951114023701 & $06 / 19 / 01$ & 1600 & $<.002$ & $<.004$ & $<.002$ & $<.005$ & 75 & .015 & $<.050$ & $<.010$ \\
\hline 42 & 463932114035901 & $05 / 29 / 01$ & 1300 & $<.002$ & $<.004$ & $<.002$ & $<.005$ & 95 & $<.007$ & $<.050$ & $<.010$ \\
\hline 43 & 463827114001201 & $05 / 29 / 01$ & 1600 & $<.002$ & $<.004$ & $<.002$ & $<.005$ & 85 & $<.007$ & $<.050$ & $<.010$ \\
\hline 44 & 463335114011701 & $05 / 30 / 01$ & 1600 & $<.002$ & $<.004$ & $<.002$ & $<.005$ & 89 & $<.007$ & $<.050$ & $<.010$ \\
\hline 45 & 463122114074701 & $05 / 30 / 01$ & 0900 & $<.002$ & $<.004$ & $<.002$ & $<.005$ & 59 & $<.007$ & $<.050$ & $<.010$ \\
\hline $45^{\mathbf{R}}$ & & $05 / 30 / 01$ & 0901 & $<.002$ & $<.004$ & $<.002$ & $<.005$ & 75 & $<.007$ & $<.050$ & $<.010$ \\
\hline 46 & 462948114060101 & $05 / 30 / 01$ & 1200 & $<.002$ & $<.004$ & $<.002$ & $<.005$ & 94 & $<.007$ & $<.050$ & $<.010$ \\
\hline 47 & 462859113574401 & $06 / 11 / 01$ & 1200 & $<.002$ & $<.004$ & $<.002$ & $<.005$ & 91 & $<.007$ & $<.050$ & $<.010$ \\
\hline 48 & 462818114074101 & $06 / 04 / 01$ & 1200 & $<.002$ & $<.004$ & $<.002$ & $<.005$ & 96 & $<.007$ & $<.050$ & $<.010$ \\
\hline $48^{\mathbf{F b}}$ & & $06 / 04 / 01$ & 1205 & $<.002$ & $<.004$ & $<.002$ & $<.005$ & 93 & $<.007$ & $<.050$ & $<.010$ \\
\hline 49 & 462754113592701 & $06 / 05 / 01$ & 1500 & $<.002$ & $<.004$ & $<.002$ & $<.005$ & 77 & $<.007$ & $<.050$ & $<.010$ \\
\hline 50 & 462616114094301 & $06 / 05 / 01$ & 1300 & $<.002$ & $<.004$ & $<.002$ & $<.005$ & 90 & $<.007$ & $<.050$ & $<.010$ \\
\hline 51 & 462545114034301 & $06 / 05 / 01$ & 1800 & $<.002$ & $<.004$ & $<.002$ & $<.005$ & 88 & $<.007$ & $<.050$ & $<.010$ \\
\hline 52 & 462256114114501 & $06 / 04 / 01$ & 1700 & $<.002$ & $<.004$ & $<.002$ & $<.005$ & 92 & $<.007$ & $<.050$ & $<.010$ \\
\hline 53 & 462228114030301 & $06 / 06 / 01$ & 1100 & $<.002$ & $<.004$ & $<.002$ & $<.005$ & 91 & $<.007$ & $<.050$ & $<.010$ \\
\hline 54 & 461823114050901 & $06 / 06 / 01$ & 1300 & $<.002$ & $<.004$ & $<.002$ & $<.005$ & 90 & $<.007$ & $<.050$ & $<.010$ \\
\hline 55 & 461807114123001 & $06 / 11 / 01$ & 1600 & $<.002$ & $<.004$ & $<.002$ & $<.005$ & 91 & $<.007$ & $<.050$ & $<.010$ \\
\hline 56 & 461638114023401 & $06 / 06 / 01$ & 0800 & $<.002$ & $<.004$ & $<.002$ & $<.005$ & 88 & $<.007$ & $<.050$ & $<.010$ \\
\hline 57 & 461451114090801 & $06 / 06 / 01$ & 1600 & $<.002$ & $<.004$ & $<.002$ & $<.005$ & 101 & $<.007$ & $<.050$ & $<.010$ \\
\hline 58 & 461320114121501 & $06 / 05 / 01$ & 0900 & $<.002$ & $<.004$ & $<.002$ & $<.005$ & 88 & $<.007$ & $<.050$ & $<.010$ \\
\hline 59 & 461055114112601 & $06 / 21 / 01$ & 0900 & $<.002$ & $<.004$ & $<.002$ & $<.005$ & 89 & $<.007$ & $<.050$ & $<.010$ \\
\hline 60 & 460110114110901 & $06 / 20 / 01$ & 1800 & $<.002$ & $<.004$ & $<.002$ & $<.005$ & 101 & $<.007$ & $<.050$ & $<.010$ \\
\hline 61 & 455521114074801 & $06 / 20 / 01$ & 2000 & $<.002$ & $<.004$ & $<.002$ & $<.005$ & 106 & $<.007$ & $<.050$ & $<.010$ \\
\hline
\end{tabular}


Table 18. Pesticide-concentration data for filtered ground-water samples collected from basin-fill aquifers, Northern Rockies Intermontane Basins study unit, 1999 and 2001 (Continued)

\begin{tabular}{|c|c|c|c|c|c|c|c|c|c|c|c|}
\hline $\begin{array}{c}\text { Well } \\
\text { number } \\
\text { (figs. } 3 \\
\text { and 4) }\end{array}$ & $\begin{array}{c}\text { Butyl- } \\
\text { ate } \\
(\mu \mathrm{g} / \mathrm{L})\end{array}$ & $\begin{array}{c}\text { Carb- } \\
\text { aryl } \\
(\mu \mathrm{g} / \mathrm{L})\end{array}$ & $\begin{array}{c}\text { Carbo- } \\
\text { furan } \\
(\mu \mathrm{g} / \mathrm{L})\end{array}$ & $\begin{array}{c}\text { Chlor- } \\
\text { pyrifos } \\
(\mu \mathrm{g} / \mathrm{L})\end{array}$ & $\begin{array}{c}\text { cis-Per- } \\
\text { methrin } \\
(\mu \mathrm{g} / \mathrm{L})\end{array}$ & $\begin{array}{l}\text { Cyan- } \\
\text { azine } \\
(\mu \mathrm{g} / \mathrm{L})\end{array}$ & $\begin{array}{c}\text { Dacthal } \\
(\mu \mathrm{g} / \mathrm{L})\end{array}$ & $\begin{array}{c}\text { Deethyl- } \\
\text { atra- } \\
\text { zine } \\
(\mu \mathrm{g} / \mathrm{L})\end{array}$ & $\begin{array}{c}\text { Diaz- } \\
\text { inon } \\
(\mu \mathrm{g} / \mathbf{L})\end{array}$ & $\begin{array}{l}\text { Diazinon- } \\
\quad d_{10}, \\
\text { surrogate } \\
\text { (percent) }\end{array}$ & $\begin{array}{c}\text { Dieldrin } \\
(\mu \mathrm{g} / \mathrm{L})\end{array}$ \\
\hline \multicolumn{12}{|c|}{ Subunit Survey 2, Montana } \\
\hline 32 & $<.002$ & $<.041$ & $<.020$ & $<.005$ & $<.006$ & $<.018$ & $<.003$ & $<.006$ & $<.005$ & 89 & $<.005$ \\
\hline 33 & $<.002$ & $<.041$ & $<.020$ & $<.005$ & $<.006$ & $<.018$ & $<.003$ & $<.006$ & $<.005$ & 103 & $<.005$ \\
\hline 34 & $<.002$ & $<.041$ & $<.020$ & $<.005$ & $<.006$ & $<.018$ & $<.003$ & $<.006$ & $<.005$ & 83 & $<.005$ \\
\hline 35 & $<.002$ & $<.041$ & $<.020$ & $<.005$ & $<.006$ & $<.018$ & $<.003$ & $<.006$ & $<.005$ & 102 & $<.005$ \\
\hline 36 & $<.002$ & $<.041$ & $<.020$ & $<.005$ & $<.006$ & $<.018$ & $<.003$ & $<.006$ & $<.005$ & 90 & $<.005$ \\
\hline $36^{\mathbf{R}}$ & $<.002$ & $<.041$ & $<.020$ & $<.005$ & $<.006$ & $<.018$ & $<.003$ & $<.006$ & $<.005$ & 91 & $<.005$ \\
\hline $36^{\mathbf{F b}}$ & $<.002$ & $<.041$ & $<.020$ & $<.005$ & $<.006$ & $<.018$ & $<.003$ & $<.006$ & $<.005$ & 82 & $<.005$ \\
\hline 37 & $<.002$ & $<.041$ & $<.020$ & $<.005$ & $<.006$ & $<.018$ & $<.003$ & $<.006$ & $<.005$ & 85 & $<.005$ \\
\hline 38 & $<.002$ & $<.041$ & $<.020$ & $<.005$ & $<.006$ & $<.018$ & $<.003$ & $<.006$ & $<.005$ & 97 & $<.005$ \\
\hline $38^{\mathbf{R}}$ & $<.002$ & $<.041$ & $<.020$ & $<.005$ & $<.006$ & $<.018$ & $<.003$ & $<.006$ & $<.005$ & 119 & $<.005$ \\
\hline $38^{\mathbf{F b}}$ & $<.002$ & $<.041$ & $<.020$ & $<.005$ & $<.006$ & $<.018$ & $<.003$ & $<.006$ & $<.005$ & 106 & $<.005$ \\
\hline 39 & $<.002$ & $<.041$ & $<.020$ & $<.005$ & $<.006$ & $<.018$ & $<.003$ & e.005 & $<.005$ & 107 & $<.005$ \\
\hline 40 & $<.002$ & $<.041$ & $<.020$ & $<.005$ & $<.006$ & $<.018$ & $<.003$ & $<.006$ & $<.005$ & 116 & $<.005$ \\
\hline 41 & $<.002$ & $<.041$ & $<.020$ & $<.005$ & $<.006$ & $<.018$ & $<.003$ & e.003 & $<.005$ & 112 & $<.005$ \\
\hline 42 & $<.002$ & $<.041$ & $<.020$ & $<.005$ & $<.006$ & $<.018$ & $<.003$ & $<.006$ & $<.005$ & 109 & $<.005$ \\
\hline 43 & $<.002$ & $<.041$ & $<.020$ & $<.005$ & $<.006$ & $<.018$ & $<.003$ & $<.006$ & $<.005$ & 95 & $<.005$ \\
\hline 44 & $<.002$ & $<.041$ & $<.020$ & $<.005$ & $<.006$ & $<.018$ & $<.003$ & $<.006$ & $<.005$ & 95 & $<.005$ \\
\hline 45 & $<.002$ & $<.041$ & $<.020$ & $<.005$ & $<.006$ & $<.018$ & $<.003$ & $<.006$ & $<.005$ & 84 & $<.005$ \\
\hline $45^{\mathbf{R}}$ & $<.002$ & $<.041$ & $<.020$ & $<.005$ & $<.006$ & $<.018$ & $<.003$ & $<.006$ & $<.005$ & 83 & $<.005$ \\
\hline 46 & $<.002$ & $<.041$ & $<.020$ & $<.005$ & $<.006$ & $<.018$ & $<.003$ & $<.006$ & $<.005$ & 98 & $<.005$ \\
\hline 47 & $<.002$ & $<.041$ & $<.020$ & $<.005$ & $<.006$ & $<.018$ & $<.003$ & $<.006$ & $<.005$ & 99 & $<.005$ \\
\hline 48 & $<.002$ & $<.041$ & $<.020$ & $<.005$ & $<.006$ & $<.018$ & $<.003$ & $<.006$ & $<.005$ & 95 & $<.005$ \\
\hline $48^{\mathbf{F b}}$ & $<.002$ & $<.041$ & $<.020$ & $<.005$ & $<.006$ & $<.018$ & $<.003$ & $<.006$ & $<.005$ & 91 & $<.005$ \\
\hline 49 & $<.002$ & $<.041$ & $<.020$ & $<.005$ & $<.006$ & $<.018$ & $<.003$ & $<.006$ & $<.005$ & 93 & $<.005$ \\
\hline 50 & $<.002$ & $<.041$ & $<.020$ & $<.005$ & $<.006$ & $<.018$ & $<.003$ & $<.006$ & $<.005$ & 93 & $<.005$ \\
\hline 51 & $<.002$ & $<.041$ & $<.020$ & $<.005$ & $<.006$ & $<.018$ & $<.003$ & $<.006$ & $<.005$ & 100 & $<.005$ \\
\hline 52 & $<.002$ & $<.041$ & $<.020$ & $<.005$ & $<.006$ & $<.018$ & $<.003$ & $<.006$ & $<.005$ & 96 & $<.005$ \\
\hline 53 & $<.002$ & $<.041$ & $<.020$ & $<.005$ & $<.006$ & $<.018$ & $<.003$ & $<.006$ & $<.005$ & 110 & $<.005$ \\
\hline 54 & $<.002$ & $<.041$ & $<.020$ & $<.005$ & $<.006$ & $<.018$ & $<.003$ & $<.006$ & $<.005$ & 102 & $<.005$ \\
\hline 55 & $<.002$ & $<.041$ & $<.020$ & $<.005$ & $<.006$ & $<.018$ & $<.003$ & $<.006$ & $<.005$ & 103 & $<.005$ \\
\hline 56 & $<.002$ & $<.041$ & $<.020$ & $<.005$ & $<.006$ & $<.018$ & $<.003$ & $<.006$ & $<.005$ & 97 & $<.005$ \\
\hline 57 & $<.002$ & $<.041$ & $<.020$ & $<.005$ & $<.006$ & $<.018$ & $<.003$ & $<.006$ & $<.005$ & 102 & $<.005$ \\
\hline 58 & $<.002$ & $<.041$ & $<.020$ & $<.005$ & $<.006$ & $<.018$ & $<.003$ & $<.006$ & $<.005$ & 90 & $<.005$ \\
\hline 59 & $<.002$ & $<.041$ & $<.020$ & $<.005$ & $<.006$ & $<.018$ & $<.003$ & $<.006$ & $<.005$ & 85 & $<.005$ \\
\hline 60 & $<.002$ & $<.041$ & $<.020$ & $<.005$ & $<.006$ & $<.018$ & $<.003$ & $<.006$ & $<.005$ & 98 & $<.005$ \\
\hline 61 & $<.002$ & $<.041$ & $<.020$ & $<.005$ & $<.006$ & $<.018$ & $<.003$ & $<.006$ & $<.005$ & 0 & $<.005$ \\
\hline
\end{tabular}


Table 18. Pesticide-concentration data for filtered ground-water samples collected from basin-fill aquifers, Northern Rockies Intermontane Basins study unit, 1999 and 2001 (Continued)

\begin{tabular}{|c|c|c|c|c|c|c|c|c|c|c|}
\hline $\begin{array}{c}\text { Well } \\
\text { number } \\
\text { (figs. } 3 \\
\text { and 4) }\end{array}$ & $\begin{array}{l}\text { Disul- } \\
\text { foton } \\
(\mu \mathrm{g} / \mathrm{L})\end{array}$ & $\begin{array}{l}\text { EPTC } \\
(\mu \mathrm{g} / \mathrm{L})\end{array}$ & $\begin{array}{c}\text { Ethal- } \\
\text { fluralin } \\
(\mu \mathrm{g} / \mathrm{L})\end{array}$ & $\begin{array}{c}\text { Etho- } \\
\text { pro- } \\
\text { phos } \\
(\mu \mathrm{g} / \mathrm{L})\end{array}$ & $\begin{array}{c}\text { Fonofos } \\
(\mu \mathrm{g} / \mathrm{L})\end{array}$ & $\begin{array}{l}\text { Lindane } \\
(\mu \mathrm{g} / \mathrm{L})\end{array}$ & $\begin{array}{l}\text { Linuron } \\
(\mu \mathrm{g} / \mathrm{L})\end{array}$ & $\begin{array}{c}\text { Mala- } \\
\text { thion } \\
(\mu \mathrm{g} / \mathrm{L})\end{array}$ & $\begin{array}{l}\text { Metol- } \\
\text { achlor } \\
(\mu \mathrm{g} / \mathrm{L})\end{array}$ & $\begin{array}{c}\text { Metri- } \\
\text { buzin } \\
(\mu \mathrm{g} / \mathrm{L})\end{array}$ \\
\hline \multicolumn{11}{|c|}{ Subunit Survey 2, Montana } \\
\hline 32 & $<.021$ & $<.002$ & $<.009$ & $<.005$ & $<.003$ & $<.004$ & $<.035$ & $<.027$ & $<.013$ & $<.006$ \\
\hline 33 & $<.021$ & $<.002$ & $<.009$ & $<.005$ & $<.003$ & $<.004$ & $<.035$ & $<.027$ & $<.013$ & $<.006$ \\
\hline 34 & $<.021$ & $<.002$ & $<.009$ & $<.005$ & $<.003$ & $<.004$ & $<.035$ & $<.027$ & $<.013$ & $<.006$ \\
\hline 35 & $<.021$ & $<.002$ & $<.009$ & $<.005$ & $<.003$ & $<.004$ & $<.035$ & $<.027$ & $<.013$ & $<.006$ \\
\hline 36 & $<.021$ & $<.002$ & $<.009$ & $<.005$ & $<.003$ & $<.004$ & $<.035$ & $<.027$ & $<.013$ & $<.006$ \\
\hline $36^{\mathbf{R}}$ & $<.021$ & $<.002$ & $<.009$ & $<.005$ & $<.003$ & $<.004$ & $<.035$ & $<.027$ & $<.013$ & $<.006$ \\
\hline $36^{\mathbf{F b}}$ & $<.021$ & $<.002$ & $<.009$ & $<.005$ & $<.003$ & $<.004$ & $<.035$ & $<.027$ & $<.013$ & $<.006$ \\
\hline 37 & $<.021$ & $<.002$ & $<.009$ & $<.005$ & $<.003$ & $<.004$ & $<.035$ & $<.027$ & $<.013$ & $<.006$ \\
\hline 38 & $<.021$ & $<.002$ & $<.009$ & $<.005$ & $<.003$ & $<.004$ & $<.035$ & $<.027$ & $<.013$ & $<.006$ \\
\hline $38^{\mathbf{R}}$ & $<.021$ & $<.002$ & $<.009$ & $<.005$ & $<.003$ & $<.004$ & $<.035$ & $<.027$ & $<.013$ & $<.006$ \\
\hline $38^{\mathbf{F b}}$ & $<.021$ & $<.002$ & $<.009$ & $<.005$ & $<.003$ & $<.004$ & $<.035$ & $<.027$ & $<.013$ & $<.006$ \\
\hline 39 & $<.021$ & $<.002$ & $<.009$ & $<.005$ & $<.003$ & $<.004$ & $<.035$ & $<.027$ & $<.013$ & $<.006$ \\
\hline 40 & $<.021$ & $<.002$ & $<.009$ & $<.005$ & $<.003$ & $<.004$ & $<.035$ & $<.027$ & $<.013$ & $<.006$ \\
\hline 41 & $<.021$ & $<.002$ & $<.009$ & $<.005$ & $<.003$ & $<.004$ & $<.035$ & $<.027$ & $<.013$ & $<.006$ \\
\hline 42 & $<.021$ & $<.002$ & $<.009$ & $<.005$ & $<.003$ & $<.004$ & $<.035$ & $<.027$ & $<.013$ & $<.006$ \\
\hline 43 & $<.021$ & $<.002$ & $<.009$ & $<.005$ & $<.003$ & $<.004$ & $<.035$ & $<.027$ & $<.013$ & $<.006$ \\
\hline 44 & $<.021$ & $<.002$ & $<.009$ & $<.005$ & $<.003$ & $<.004$ & $<.035$ & $<.027$ & $<.013$ & $<.006$ \\
\hline 45 & $<.021$ & $<.002$ & $<.009$ & $<.005$ & $<.003$ & $<.004$ & $<.035$ & $<.027$ & $<.013$ & $<.006$ \\
\hline $45^{\mathbf{R}}$ & $<.021$ & $<.002$ & $<.009$ & $<.005$ & $<.003$ & $<.004$ & $<.035$ & $<.027$ & $<.013$ & $<.006$ \\
\hline 46 & $<.021$ & $<.002$ & $<.009$ & $<.005$ & $<.003$ & $<.004$ & $<.035$ & $<.027$ & $<.013$ & $<.006$ \\
\hline 47 & $<.021$ & $<.002$ & $<.009$ & $<.005$ & $<.003$ & $<.004$ & $<.035$ & $<.027$ & $<.013$ & $<.006$ \\
\hline 48 & $<.021$ & $<.002$ & $<.009$ & $<.005$ & $<.003$ & $<.004$ & $<.035$ & $<.027$ & $<.013$ & $<.006$ \\
\hline $48^{\mathbf{F b}}$ & $<.021$ & $<.002$ & $<.009$ & $<.005$ & $<.003$ & $<.004$ & $<.035$ & $<.027$ & $<.013$ & $<.006$ \\
\hline 49 & $<.021$ & $<.002$ & $<.009$ & $<.005$ & $<.003$ & $<.004$ & $<.035$ & $<.027$ & $<.013$ & $<.006$ \\
\hline 50 & $<.021$ & $<.002$ & $<.009$ & $<.005$ & $<.003$ & $<.004$ & $<.035$ & $<.027$ & $<.013$ & $<.006$ \\
\hline 51 & $<.021$ & $<.002$ & $<.009$ & $<.005$ & $<.003$ & $<.004$ & $<.035$ & $<.027$ & $<.013$ & $<.006$ \\
\hline 52 & $<.021$ & $<.002$ & $<.009$ & $<.005$ & $<.003$ & $<.004$ & $<.035$ & $<.027$ & $<.013$ & $<.006$ \\
\hline 53 & $<.021$ & $<.002$ & $<.009$ & $<.005$ & $<.003$ & $<.004$ & $<.035$ & $<.027$ & $<.013$ & $<.006$ \\
\hline 54 & $<.021$ & $<.002$ & $<.009$ & $<.005$ & $<.003$ & $<.004$ & $<.035$ & $<.027$ & $<.013$ & $<.006$ \\
\hline 55 & $<.021$ & $<.002$ & $<.009$ & $<.005$ & $<.003$ & $<.004$ & $<.035$ & $<.027$ & $<.013$ & $<.006$ \\
\hline 56 & $<.021$ & $<.002$ & $<.009$ & $<.005$ & $<.003$ & $<.004$ & $<.035$ & $<.027$ & $<.013$ & $<.006$ \\
\hline 57 & $<.021$ & $<.002$ & $<.009$ & $<.005$ & $<.003$ & $<.004$ & $<.035$ & $<.027$ & $<.013$ & $<.006$ \\
\hline 58 & $<.021$ & $<.002$ & $<.009$ & $<.005$ & $<.003$ & $<.004$ & $<.035$ & $<.027$ & $<.013$ & $<.006$ \\
\hline 59 & $<.021$ & $<.002$ & $<.009$ & $<.005$ & $<.003$ & $<.004$ & $<.035$ & $<.027$ & $<.013$ & $<.006$ \\
\hline 60 & $<.021$ & $<.002$ & $<.009$ & $<.005$ & $<.003$ & $<.004$ & $<.035$ & $<.027$ & $<.013$ & $<.006$ \\
\hline 61 & $<.021$ & $<.002$ & $<.009$ & $<.005$ & $<.003$ & $<.004$ & $<.035$ & $<.027$ & $<.013$ & $<.006$ \\
\hline
\end{tabular}


Table 18. Pesticide-concentration data for filtered ground-water samples collected from basin-fill aquifers, Northern Rockies Intermontane Basins study unit, 1999 and 2001 (Continued)

\begin{tabular}{|c|c|c|c|c|c|c|c|c|c|}
\hline $\begin{array}{c}\text { Well } \\
\text { number } \\
\text { (figs. } 3 \\
\text { and 4) }\end{array}$ & $\begin{array}{c}\text { Molin- } \\
\text { ate } \\
(\mu \mathrm{g} / \mathrm{L})\end{array}$ & $\begin{array}{c}\text { Naprop- } \\
\text { amide } \\
(\mu \mathrm{g} / \mathrm{L})\end{array}$ & $\begin{array}{c}\text { Para- } \\
\text { thion } \\
(\mu \mathrm{g} / \mathrm{L})\end{array}$ & $\begin{array}{l}\text { Para- } \\
\text { thion- } \\
\text { methyl } \\
(\mu \mathrm{g} / \mathrm{L})\end{array}$ & $\begin{array}{c}\text { Pebulate } \\
(\mu \mathrm{g} / \mathrm{L})\end{array}$ & $\begin{array}{c}\text { Pendi- } \\
\text { meth- } \\
\text { alin } \\
(\mu \mathrm{g} / \mathrm{L})\end{array}$ & $\begin{array}{c}\text { Phorate } \\
(\mu \mathrm{g} / \mathrm{L})\end{array}$ & $\begin{array}{c}\text { p,p'-DDE } \\
(\mu \mathrm{g} / \mathrm{L})\end{array}$ & $\begin{array}{c}\text { Pro- } \\
\text { meton } \\
(\mu \mathrm{g} / \mathrm{L})\end{array}$ \\
\hline \multicolumn{10}{|c|}{ Subunit Survey 2, Montana } \\
\hline 32 & $<.002$ & $<.007$ & $<.007$ & $<.006$ & $<.002$ & $<.010$ & $<.011$ & $<.003$ & $<.015$ \\
\hline 33 & $<.002$ & $<.007$ & $<.007$ & $<.006$ & $<.002$ & $<.010$ & $<.011$ & $<.003$ & $<.015$ \\
\hline 34 & $<.002$ & $<.007$ & $<.007$ & $<.006$ & $<.002$ & $<.010$ & $<.011$ & $<.003$ & $<.015$ \\
\hline 35 & $<.002$ & $<.007$ & $<.007$ & $<.006$ & $<.002$ & $<.010$ & $<.011$ & $<.003$ & $<.015$ \\
\hline 36 & $<.002$ & $<.007$ & $<.007$ & $<.006$ & $<.002$ & $<.010$ & $<.011$ & $<.003$ & $<.015$ \\
\hline $36^{\mathbf{R}}$ & $<.002$ & $<.007$ & $<.007$ & $<.006$ & $<.002$ & $<.010$ & $<.011$ & $<.003$ & $<.015$ \\
\hline $36^{\mathbf{F b}}$ & $<.002$ & $<.007$ & $<.007$ & $<.006$ & $<.002$ & $<.010$ & $<.011$ & $<.003$ & $<.015$ \\
\hline 37 & $<.002$ & $<.007$ & $<.007$ & $<.006$ & $<.002$ & $<.010$ & $<.011$ & $<.003$ & $<.015$ \\
\hline 38 & $<.002$ & $<.007$ & $<.007$ & $<.006$ & $<.002$ & $<.010$ & $<.011$ & $<.003$ & $<.015$ \\
\hline $38^{\mathbf{R}}$ & $<.002$ & $<.007$ & $<.007$ & $<.006$ & $<.002$ & $<.010$ & $<.011$ & $<.003$ & $<.015$ \\
\hline $38^{\mathbf{F b}}$ & $<.002$ & $<.007$ & $<.007$ & $<.006$ & $<.002$ & $<.010$ & $<.011$ & $<.003$ & $<.015$ \\
\hline 39 & $<.002$ & $<.007$ & $<.007$ & $<.006$ & $<.002$ & $<.010$ & $<.011$ & $<.003$ & e.007 \\
\hline 40 & $<.002$ & $<.007$ & $<.007$ & $<.006$ & $<.002$ & $<.010$ & $<.011$ & $<.003$ & $<.015$ \\
\hline 41 & $<.002$ & $<.007$ & $<.007$ & $<.006$ & $<.002$ & $<.010$ & $<.011$ & $<.003$ & e.005 \\
\hline 42 & $<.002$ & $<.007$ & $<.007$ & $<.006$ & $<.002$ & $<.010$ & $<.011$ & $<.003$ & $<.015$ \\
\hline 43 & $<.002$ & $<.007$ & $<.007$ & $<.006$ & $<.002$ & $<.010$ & $<.011$ & $<.003$ & $<.015$ \\
\hline 44 & $<.002$ & $<.007$ & $<.007$ & $<.006$ & $<.002$ & $<.010$ & $<.011$ & $<.003$ & $<.015$ \\
\hline 45 & $<.002$ & $<.007$ & $<.007$ & $<.006$ & $<.002$ & $<.010$ & $<.011$ & $<.003$ & $<.015$ \\
\hline $45^{\mathbf{R}}$ & $<.002$ & $<.007$ & $<.007$ & $<.006$ & $<.002$ & $<.010$ & $<.011$ & $<.003$ & $<.015$ \\
\hline 46 & $<.002$ & $<.007$ & $<.007$ & $<.006$ & $<.002$ & $<.010$ & $<.011$ & $<.003$ & e.002 \\
\hline 47 & $<.002$ & $<.007$ & $<.007$ & $<.006$ & $<.002$ & $<.010$ & $<.011$ & $<.003$ & $<.015$ \\
\hline 48 & $<.002$ & $<.007$ & $<.007$ & $<.006$ & $<.002$ & $<.010$ & $<.011$ & $<.003$ & $<.015$ \\
\hline $48^{\mathbf{F b}}$ & $<.002$ & $<.007$ & $<.007$ & $<.006$ & $<.002$ & $<.010$ & $<.011$ & $<.002$ & $<.015$ \\
\hline 49 & $<.002$ & $<.007$ & $<.007$ & $<.006$ & $<.002$ & $<.010$ & $<.011$ & $<.003$ & $<.015$ \\
\hline 50 & $<.002$ & $<.007$ & $<.007$ & $<.006$ & $<.002$ & $<.010$ & $<.011$ & $<.003$ & $<.015$ \\
\hline 51 & $<.002$ & $<.007$ & $<.007$ & $<.006$ & $<.002$ & $<.010$ & $<.011$ & $<.003$ & $<.015$ \\
\hline 52 & $<.002$ & $<.007$ & $<.007$ & $<.006$ & $<.002$ & $<.010$ & $<.011$ & $<.003$ & $<.015$ \\
\hline 53 & $<.002$ & $<.007$ & $<.007$ & $<.006$ & $<.002$ & $<.010$ & $<.011$ & $<.003$ & $<.015$ \\
\hline 54 & $<.002$ & $<.007$ & $<.007$ & $<.006$ & $<.002$ & $<.010$ & $<.011$ & $<.003$ & $<.015$ \\
\hline 55 & $<.002$ & $<.007$ & $<.007$ & $<.006$ & $<.002$ & $<.010$ & $<.011$ & $<.003$ & $<.015$ \\
\hline 56 & $<.002$ & $<.007$ & $<.007$ & $<.006$ & $<.002$ & $<.010$ & $<.011$ & $<.003$ & $<.015$ \\
\hline 57 & $<.002$ & $<.007$ & $<.007$ & $<.006$ & $<.002$ & $<.010$ & $<.011$ & $<.003$ & e. 003 \\
\hline 58 & $<.002$ & $<.007$ & $<.007$ & $<.006$ & $<.002$ & $<.010$ & $<.011$ & $<.003$ & $<.015$ \\
\hline 59 & $<.002$ & $<.007$ & $<.007$ & $<.006$ & $<.002$ & $<.010$ & $<.011$ & $<.003$ & $<.015$ \\
\hline 60 & $<.002$ & $<.007$ & $<.007$ & $<.006$ & $<.002$ & $<.010$ & $<.011$ & $<.003$ & $<.015$ \\
\hline 61 & $<.002$ & $<.007$ & $<.007$ & $<.006$ & $<.002$ & $<.010$ & $<.011$ & $<.003$ & $<.015$ \\
\hline
\end{tabular}


Table 18. Pesticide-concentration data for filtered ground-water samples collected from basin-fill aquifers, Northern Rockies Intermontane Basins study unit, 1999 and 2001 (Continued)

\begin{tabular}{|c|c|c|c|c|c|c|c|c|c|c|c|}
\hline $\begin{array}{c}\text { Well } \\
\text { number } \\
\text { (figs. } 3 \\
\text { and 4) }\end{array}$ & $\begin{array}{l}\text { Prop- } \\
\text { achlor } \\
(\mu \mathrm{g} / \mathrm{L})\end{array}$ & $\begin{array}{c}\text { Prop- } \\
\text { anil } \\
(\mu \mathrm{g} / \mathrm{L})\end{array}$ & $\begin{array}{l}\text { Prop- } \\
\text { argite } \\
(\mu \mathrm{g} / \mathrm{L})\end{array}$ & $\begin{array}{c}\text { Propyz- } \\
\text { amide } \\
(\mu \mathrm{g} / \mathrm{L})\end{array}$ & $\begin{array}{l}\text { Sim- } \\
\text { azine } \\
(\mu \mathrm{g} / \mathrm{L})\end{array}$ & $\begin{array}{c}\text { Tebu- } \\
\text { thiuron } \\
(\mu \mathrm{g} / \mathrm{L})\end{array}$ & $\begin{array}{c}\text { Terbacil } \\
(\mu \mathrm{g} / \mathrm{L})\end{array}$ & $\begin{array}{c}\text { Terbufos } \\
(\mu \mathrm{g} / \mathrm{L})\end{array}$ & $\begin{array}{c}\text { Thio- } \\
\text { bencarb } \\
(\mu \mathrm{g} / \mathrm{L})\end{array}$ & $\begin{array}{c}\text { Tri- } \\
\text { allate } \\
(\mu \mathrm{g} / \mathrm{L})\end{array}$ & $\begin{array}{c}\text { Tri- } \\
\text { fluralin } \\
(\mu \mathrm{g} / \mathrm{L})\end{array}$ \\
\hline \multicolumn{12}{|c|}{ Subunit Survey 2, Montana } \\
\hline 32 & $<.010$ & $<.011$ & $<.023$ & $<.004$ & $<.011$ & $<.016$ & $<.034$ & $<.017$ & $<.005$ & $<.002$ & $<.009$ \\
\hline 33 & $<.010$ & $<.011$ & $<.023$ & $<.004$ & $<.011$ & $<.016$ & $<.034$ & $<.017$ & $<.005$ & $<.002$ & $<.009$ \\
\hline 34 & $<.010$ & $<.011$ & $<.023$ & $<.004$ & $<.011$ & $<.016$ & $<.034$ & $<.017$ & $<.005$ & $<.002$ & $<.009$ \\
\hline 35 & $<.010$ & $<.011$ & $<.023$ & $<.004$ & $<.011$ & $<.016$ & $<.034$ & $<.017$ & $<.005$ & $<.002$ & $<.009$ \\
\hline 36 & $<.010$ & $<.011$ & $<.023$ & $<.004$ & $<.011$ & $<.016$ & $<.034$ & $<.017$ & $<.005$ & $<.002$ & $<.009$ \\
\hline $36^{\mathbf{R}}$ & $<.010$ & $<.011$ & $<.023$ & $<.004$ & $<.011$ & $<.016$ & $<.034$ & $<.017$ & $<.005$ & $<.002$ & $<.009$ \\
\hline $36^{\mathbf{F b}}$ & $<.010$ & $<.011$ & $<.023$ & $<.004$ & $<.011$ & $<.016$ & $<.034$ & $<.017$ & $<.005$ & $<.002$ & $<.009$ \\
\hline 37 & $<.010$ & $<.011$ & $<.023$ & $<.004$ & $<.011$ & $<.016$ & $<.034$ & $<.017$ & $<.005$ & $<.002$ & $<.009$ \\
\hline 38 & $<.010$ & $<.011$ & $<.023$ & $<.004$ & $<.011$ & $<.016$ & $<.034$ & $<.017$ & $<.005$ & $<.002$ & $<.009$ \\
\hline $38^{\mathbf{R}}$ & $<.010$ & $<.011$ & $<.023$ & $<.004$ & $<.011$ & $<.016$ & $<.034$ & $<.017$ & $<.005$ & $<.002$ & $<.009$ \\
\hline $38^{\mathbf{F b}}$ & $<.010$ & $<.011$ & $<.023$ & $<.004$ & $<.011$ & $<.016$ & $<.034$ & $<.017$ & $<.005$ & $<.002$ & $<.009$ \\
\hline 39 & $<.010$ & $<.011$ & $<.023$ & $<.004$ & $<.011$ & .044 & $<.034$ & $<.017$ & $<.005$ & $<.002$ & $<.009$ \\
\hline 40 & $<.010$ & $<.011$ & $<.023$ & $<.004$ & $<.011$ & $<.016$ & $<.034$ & $<.017$ & $<.005$ & $<.002$ & $<.009$ \\
\hline 41 & $<.010$ & $<.011$ & $<.023$ & $<.004$ & $<.011$ & $<.016$ & $<.034$ & $<.017$ & $<.005$ & $<.002$ & $<.009$ \\
\hline 42 & $<.010$ & $<.011$ & $<.023$ & $<.004$ & $<.011$ & $<.016$ & $<.034$ & $<.017$ & $<.005$ & $<.002$ & $<.009$ \\
\hline 43 & $<.010$ & $<.011$ & $<.023$ & $<.004$ & $<.011$ & $<.016$ & $<.034$ & $<.017$ & $<.005$ & $<.002$ & $<.009$ \\
\hline 44 & $<.010$ & $<.011$ & $<.023$ & $<.004$ & $<.011$ & $<.016$ & $<.034$ & $<.017$ & $<.005$ & $<.002$ & $<.009$ \\
\hline 45 & $<.010$ & $<.011$ & $<.023$ & $<.004$ & $<.011$ & $<.016$ & $<.034$ & $<.017$ & $<.005$ & $<.002$ & $<.009$ \\
\hline $45^{\mathbf{R}}$ & $<.010$ & $<.011$ & $<.023$ & $<.004$ & $<.011$ & $<.016$ & $<.034$ & $<.017$ & $<.005$ & $<.002$ & $<.009$ \\
\hline 46 & $<.010$ & $<.011$ & $<.023$ & $<.004$ & $<.011$ & $<.016$ & $<.034$ & $<.017$ & $<.005$ & $<.002$ & $<.009$ \\
\hline 47 & $<.010$ & $<.011$ & $<.023$ & $<.004$ & $<.011$ & $<.016$ & $<.034$ & $<.017$ & $<.005$ & $<.002$ & $<.009$ \\
\hline 48 & $<.010$ & $<.011$ & $<.023$ & $<.004$ & $<.011$ & $<.016$ & $<.034$ & $<.017$ & $<.005$ & $<.002$ & $<.009$ \\
\hline $48^{\mathbf{F b}}$ & $<.010$ & $<.011$ & $<.023$ & $<.004$ & $<.011$ & $<.016$ & $<.034$ & $<.017$ & $<.005$ & $<.002$ & $<.009$ \\
\hline 49 & $<.010$ & $<.011$ & $<.023$ & $<.004$ & $<.011$ & $<.016$ & $<.034$ & $<.017$ & $<.005$ & $<.002$ & $<.009$ \\
\hline 50 & $<.010$ & $<.011$ & $<.023$ & $<.004$ & $<.011$ & $<.016$ & $<.034$ & $<.017$ & $<.005$ & $<.002$ & $<.009$ \\
\hline 51 & $<.010$ & $<.011$ & $<.023$ & $<.004$ & $<.011$ & $<.016$ & $<.034$ & $<.017$ & $<.005$ & $<.002$ & $<.009$ \\
\hline 52 & $<.010$ & $<.011$ & $<.023$ & $<.004$ & $<.011$ & $<.016$ & $<.034$ & $<.017$ & $<.005$ & $<.002$ & $<.009$ \\
\hline 53 & $<.010$ & $<.011$ & $<.023$ & $<.004$ & $<.011$ & $<.016$ & $<.034$ & $<.017$ & $<.005$ & $<.002$ & $<.009$ \\
\hline 54 & $<.010$ & $<.011$ & $<.023$ & $<.004$ & $<.011$ & $<.016$ & $<.034$ & $<.017$ & $<.005$ & $<.002$ & $<.009$ \\
\hline 55 & $<.010$ & $<.011$ & $<.023$ & $<.004$ & $<.011$ & $<.016$ & $<.034$ & $<.017$ & $<.005$ & $<.002$ & $<.009$ \\
\hline 56 & $<.010$ & $<.011$ & $<.023$ & $<.004$ & $<.011$ & $<.016$ & $<.034$ & $<.017$ & $<.005$ & $<.002$ & $<.009$ \\
\hline 57 & $<.010$ & $<.011$ & $<.023$ & $<.004$ & $<.011$ & $<.016$ & $<.034$ & $<.017$ & $<.005$ & $<.002$ & $<.009$ \\
\hline 58 & $<.010$ & $<.011$ & $<.023$ & $<.004$ & $<.011$ & $<.016$ & $<.034$ & $<.017$ & $<.005$ & $<.002$ & $<.009$ \\
\hline 59 & $<.010$ & $<.011$ & $<.023$ & $<.004$ & $<.011$ & $<.016$ & $<.034$ & $<.017$ & $<.005$ & $<.002$ & $<.009$ \\
\hline 60 & $<.010$ & $<.011$ & $<.023$ & $<.004$ & $<.011$ & $<.016$ & $<.034$ & $<.017$ & $<.005$ & $<.002$ & $<.009$ \\
\hline 61 & $<.010$ & $<.011$ & $<.023$ & $<.004$ & $<.011$ & $<.016$ & $<.034$ & $<.017$ & $<.005$ & $<.002$ & $<.009$ \\
\hline
\end{tabular}


Table 19. Pesticides and pesticide-degradate concentration data for filtered ground-water samples collected from basin-fill aquifers in Montana, Northern Rockies Intermontane Basins study unit, 2001

[Site identification number described in text. Bold text denotes detected values. Abbreviations: e, estimated; Fb, field blank; $\mu \mathrm{g} / \mathrm{L}$, micrograms per liter; R, replicate. Symbols: <, less than reporting level; --, no data]

\begin{tabular}{|c|c|c|c|c|c|c|c|c|c|c|}
\hline $\begin{array}{c}\text { Well } \\
\text { number } \\
\text { (figs. } 3 \\
\text { and 4) }\end{array}$ & $\begin{array}{c}\text { Site } \\
\text { identification } \\
\text { number }\end{array}$ & Date & Time & $\begin{array}{l}2,4,5,-T, \\
\text { surrogate } \\
\text { (percent) }\end{array}$ & $\begin{array}{c}\text { 2,4-D } \\
(\mu \mathrm{g} / \mathrm{L})\end{array}$ & $\begin{array}{c}\text { 2,4-D } \\
\text { methyl } \\
\text { ester } \\
(\mu \mathrm{g} / \mathrm{L})\end{array}$ & $\begin{array}{c}2,4-D B \\
(\mu \mathrm{g} / \mathrm{L})\end{array}$ & $\begin{array}{c}\text { 2-Hydroxy- } \\
\text { atrazine } \\
(\mu \mathrm{g} / \mathrm{L})\end{array}$ & $\begin{array}{c}\text { 3(4-Chloro- } \\
\text { phenyl)-1- } \\
\text { methyl } \\
\text { urea } \\
(\mu \mathrm{g} / \mathrm{L})\end{array}$ & $\begin{array}{c}\text { 3-Hydroxy- } \\
\text { carbo- } \\
\text { furan } \\
(\mu \mathrm{g} / \mathrm{L})\end{array}$ \\
\hline \multicolumn{11}{|c|}{ Subunit Survey 2, Montana } \\
\hline 32 & 470719114301401 & $06 / 12 / 01$ & 1300 & 81 & $<0.022$ & $<0.009$ & $<0.016$ & $<0.008$ & $<0.024$ & $<0.006$ \\
\hline 33 & 470502114265301 & $06 / 12 / 01$ & 0900 & 74 & $<.022$ & $<.009$ & $<.016$ & $<.008$ & $<.024$ & $<.006$ \\
\hline 34 & 470328114164301 & $06 / 28 / 01$ & 0900 & 81 & $<.022$ & $<.009$ & $<.016$ & $<.008$ & $<.024$ & $<.006$ \\
\hline 35 & 470112114144001 & 06/07/01 & 0900 & 61 & $<.022$ & $<.009$ & $<.016$ & $<.008$ & $<.024$ & $<.006$ \\
\hline 36 & 465838114074501 & $06 / 27 / 01$ & 1200 & 60 & $<.022$ & $<.028$ & $<.016$ & $<.008$ & $<.024$ & $<.006$ \\
\hline $36^{\mathrm{R}}$ & & $06 / 27 / 01$ & 1201 & 66 & $<.022$ & $<.032$ & $<.016$ & e.005 & $<.024$ & $<.006$ \\
\hline $36^{\mathbf{F b}}$ & & $06 / 27 / 01$ & 1205 & 63 & $<.022$ & $<.023$ & $<.016$ & $<.008$ & $<.024$ & $<.006$ \\
\hline 37 & 465741114110601 & $06 / 27 / 01$ & 1700 & 80 & $<.022$ & $<.009$ & $<.016$ & $<.008$ & $<.024$ & $<.006$ \\
\hline 38 & 465440114022101 & $06 / 19 / 01$ & 1100 & 62 & $<.022$ & $<.009$ & $<.016$ & $<.008$ & $<.024$ & $<.006$ \\
\hline $38^{\mathrm{R}}$ & & 06/19/01 & 1101 & 60 & $<.022$ & $<.009$ & $<.016$ & $<.008$ & $<.024$ & $<.006$ \\
\hline $38^{\mathbf{F b}}$ & & 06/19/01 & 1105 & e85 & $<.022$ & $<.009$ & $<.016$ & $<.008$ & $<.024$ & $<.006$ \\
\hline 39 & 465323114054301 & $06 / 20 / 01$ & 1300 & 60 & $<.022$ & $<.009$ & $<.016$ & $<.002$ & $<.024$ & $<.006$ \\
\hline 40 & 465127114055401 & $06 / 20 / 01$ & 1000 & 59 & $<.022$ & $<.009$ & $<.016$ & $<.008$ & $<.024$ & $<.006$ \\
\hline 41 & 464951114023701 & 06/19/01 & 1600 & 58 & $<.022$ & $<.009$ & $<.016$ & $<.001$ & $<.024$ & $<.006$ \\
\hline 42 & 463932114035901 & 05/29/01 & 1300 & 82 & $<.022$ & $<.009$ & $<.016$ & $<.008$ & $<.024$ & $<.006$ \\
\hline 43 & 463827114001201 & 05/29/01 & 1600 & 79 & $<.022$ & $<.009$ & $<.016$ & $<.008$ & $<.024$ & $<.006$ \\
\hline 44 & 463335114011701 & 05/30/01 & 1600 & e62 & $<.022$ & $<.009$ & $<.016$ & $<.008$ & $<.024$ & $<.006$ \\
\hline 45 & 463122114074701 & 05/30/01 & 0900 & e82 & $<.022$ & $<.009$ & $<.016$ & $<.008$ & $<.024$ & $<.006$ \\
\hline $45^{\mathrm{R}}$ & & $05 / 30 / 01$ & 0901 & e97 & $<.022$ & $<.009$ & $<.016$ & $<.008$ & $<.024$ & $<.006$ \\
\hline 46 & 462948114060101 & $05 / 30 / 01$ & 1200 & 67 & $<.022$ & $<.009$ & $<.016$ & $<.008$ & $<.024$ & $<.006$ \\
\hline 47 & 462859113574401 & $06 / 11 / 01$ & 1200 & 84 & $<.022$ & $<.009$ & $<.016$ & $<.008$ & $<.024$ & $<.006$ \\
\hline 48 & 462818114074101 & $06 / 04 / 01$ & 1200 & e91 & $<.022$ & $<.009$ & $<.016$ & $<.008$ & $<.024$ & $<.006$ \\
\hline $48^{\mathbf{F b}}$ & & $06 / 04 / 01$ & 1205 & e90 & $<.022$ & $<.009$ & $<.016$ & $<.008$ & $<.024$ & $<.006$ \\
\hline 49 & 462754113592701 & $06 / 05 / 01$ & 1500 & 58 & $<.022$ & $<.009$ & $<.016$ & $<.008$ & $<.024$ & $<.006$ \\
\hline 50 & 462616114094301 & $06 / 05 / 01$ & 1300 & e91 & $<.022$ & $<.009$ & $<.016$ & $<.008$ & $<.024$ & $<.006$ \\
\hline 51 & 462545114034301 & $06 / 05 / 01$ & 1800 & 37 & $<.022$ & $<.009$ & $<.016$ & $<.008$ & $<.024$ & $<.006$ \\
\hline 52 & 462256114114501 & $06 / 04 / 01$ & 1700 & 61 & $<.022$ & $<.009$ & $<.016$ & $<.008$ & $<.024$ & $<.006$ \\
\hline 53 & 462228114030301 & $06 / 06 / 01$ & 1100 & 58 & $<.022$ & $<.009$ & $<.016$ & $<.008$ & $<.024$ & $<.006$ \\
\hline 54 & 461823114050901 & $06 / 06 / 01$ & 1300 & $\mathrm{e} 25$ & $<.022$ & $<.009$ & $<.016$ & $<.008$ & $<.024$ & $<.006$ \\
\hline 55 & 461807114123001 & $06 / 11 / 01$ & 1600 & 73 & $<.022$ & $<.009$ & $<.016$ & $<.008$ & $<.024$ & $<.006$ \\
\hline 56 & 461638114023401 & $06 / 06 / 01$ & 0800 & 59 & $<.022$ & $<.009$ & $<.016$ & $<.008$ & $<.024$ & $<.006$ \\
\hline 57 & 461451114090801 & $06 / 06 / 01$ & 1600 & 63 & $<.022$ & $<.009$ & $<.016$ & $<.008$ & $<.024$ & $<.006$ \\
\hline 58 & 461320114121501 & $06 / 05 / 01$ & 0900 & 64 & $<.022$ & $<.009$ & $<.016$ & $<.008$ & $<.024$ & $<.006$ \\
\hline 59 & 461055114112601 & $06 / 21 / 01$ & 0900 & 55 & $<.022$ & $<.009$ & $<.016$ & $<.008$ & $<.024$ & $<.006$ \\
\hline 60 & 460110114110901 & $06 / 20 / 01$ & 1800 & 66 & $<.022$ & .014 & $<.016$ & $<.008$ & $<.024$ & $<.006$ \\
\hline 61 & 455521114074801 & $06 / 20 / 01$ & 2000 & 52 & $<.022$ & $<.009$ & $<.016$ & $<.008$ & $<.024$ & $<.006$ \\
\hline
\end{tabular}


Table 19. Pesticides and pesticide-degradate concentration data for filtered ground-water samples collected from basin-fill aquifers in Montana, Northern Rockies Intermontane Basins study unit, 2001 (Continued)

\begin{tabular}{|c|c|c|c|c|c|c|c|c|c|c|}
\hline $\begin{array}{c}\text { Well } \\
\text { number } \\
\text { (figs. } 3 \\
\text { and 4) }\end{array}$ & $\begin{array}{c}\text { 3-Keto- } \\
\text { carbo- } \\
\text { furan } \\
(\mu \mathrm{g} / \mathrm{L})\end{array}$ & $\begin{array}{c}\text { Acifluor- } \\
\text { fen } \\
(\mu \mathrm{g} / \mathrm{L})\end{array}$ & $\begin{array}{c}\text { Aldi- } \\
\text { carb } \\
(\mu \mathrm{g} / \mathrm{L})\end{array}$ & $\begin{array}{c}\text { Aldi- } \\
\text { carb } \\
\text { sulfone } \\
(\mu \mathrm{g} / \mathrm{L})\end{array}$ & $\begin{array}{c}\text { Aldi- } \\
\text { carb } \\
\text { sulfoxide } \\
(\mu \mathrm{g} / \mathrm{L})\end{array}$ & $\begin{array}{l}\text { Barban, } \\
\text { surrogate } \\
\text { ( percent) }\end{array}$ & $\begin{array}{c}\text { Bendio- } \\
\text { carb } \\
(\mu \mathrm{g} / \mathrm{L})\end{array}$ & $\begin{array}{c}\text { Benomyl } \\
(\mu \mathrm{g} / \mathrm{L})\end{array}$ & $\begin{array}{c}\text { Ben- } \\
\text { sul- } \\
\text { furon- } \\
\text { methyl } \\
(\mu \mathrm{g} / \mathrm{L})\end{array}$ & $\begin{array}{c}\text { Ben- } \\
\text { tazon } \\
(\mu \mathrm{g} / \mathrm{L})\end{array}$ \\
\hline \multicolumn{11}{|c|}{ Subunit Survey 2, Montana } \\
\hline 32 & $<1.50$ & $<0.007$ & $<0.040$ & $<0.02$ & $<0.008$ & 107 & $<0.025$ & $<0.004$ & $<0.016$ & $<0.011$ \\
\hline 33 & $<1.50$ & $<.007$ & $<.040$ & $<.02$ & $<.008$ & 94 & $<.025$ & $<.004$ & $<.016$ & $<.011$ \\
\hline 34 & $<1.50$ & $<.007$ & $<.040$ & $<.02$ & $<.008$ & 125 & $<.025$ & $<.004$ & $<.016$ & $<.011$ \\
\hline 35 & $<1.50$ & $<.007$ & $<.040$ & $<.02$ & $<.008$ & 101 & $<.025$ & $<.004$ & $<.016$ & $<.011$ \\
\hline 36 & $<1.50$ & $<.007$ & $<.040$ & $<.02$ & $<.008$ & e 86 & $<.025$ & $<.004$ & $<.016$ & $<.011$ \\
\hline $36^{\mathbf{R}}$ & $<1.50$ & $<.007$ & $<.040$ & $<.02$ & $<.008$ & e96 & $<.025$ & $<.004$ & $<.016$ & $<.011$ \\
\hline $36^{\mathbf{F b}}$ & $<1.50$ & $<.007$ & $<.040$ & $<.02$ & $<.008$ & e75 & $<.025$ & $<.004$ & $<.016$ & $<.011$ \\
\hline 37 & $<1.50$ & $<.007$ & $<.040$ & $<.02$ & $<.008$ & 107 & $<.025$ & $<.004$ & $<.016$ & $<.011$ \\
\hline 38 & $<1.50$ & $<.007$ & $<.040$ & $<.02$ & $<.008$ & 85 & $<.025$ & $<.004$ & $<.016$ & $<.011$ \\
\hline $38^{\mathbf{R}}$ & $<1.50$ & $<.007$ & $<.040$ & $<.02$ & $<.008$ & 84 & $<.025$ & $<.004$ & $<.016$ & $<.011$ \\
\hline $38^{\mathbf{F b}}$ & $<1.50$ & $<.007$ & $<.040$ & $<.02$ & $<.008$ & 79 & $<.025$ & $<.004$ & $<.016$ & $<.011$ \\
\hline 39 & $<1.50$ & $<.007$ & $<.040$ & $<.02$ & $<.008$ & 92 & $<.025$ & $<.004$ & $<.016$ & $<.011$ \\
\hline 40 & $<1.50$ & $<.007$ & $<.040$ & $<.02$ & $<.008$ & 95 & $<.025$ & $<.004$ & $<.016$ & $<.011$ \\
\hline 41 & $<1.50$ & $<.007$ & $<.040$ & $<.02$ & $<.008$ & 90 & $<.025$ & $<.004$ & $<.016$ & $<.011$ \\
\hline 42 & $<1.50$ & $<.007$ & $<.040$ & $<.02$ & $<.008$ & 107 & $<.025$ & $<.004$ & $<.016$ & $<.011$ \\
\hline 43 & $<1.50$ & $<.007$ & $<.040$ & $<.02$ & $<.008$ & 83 & $<.025$ & $<.004$ & $<.016$ & $<.011$ \\
\hline 44 & $<1.50$ & $<.007$ & $<.040$ & $<.02$ & $<.008$ & 62 & $<.025$ & $<.004$ & $<.016$ & $<.011$ \\
\hline 45 & $<1.50$ & $<.007$ & $<.040$ & $<.02$ & $<.008$ & 64 & $<.025$ & $<.004$ & $<.016$ & $<.011$ \\
\hline $45^{\mathbf{R}}$ & $<1.50$ & $<.007$ & $<.040$ & $<.02$ & $<.008$ & 91 & $<.025$ & $<.004$ & $<.016$ & $<.011$ \\
\hline 46 & $<1.50$ & $<.007$ & $<.040$ & $<.02$ & $<.008$ & 70 & $<.025$ & $<.004$ & $<.016$ & $<.011$ \\
\hline 47 & $<1.50$ & $<.007$ & $<.040$ & $<.02$ & $<.008$ & 111 & $<.025$ & $<.004$ & $<.016$ & $<.011$ \\
\hline 48 & $<1.50$ & $<.007$ & $<.040$ & $<.02$ & $<.008$ & 105 & $<.025$ & $<.004$ & $<.016$ & $<.011$ \\
\hline $48^{\mathbf{F b}}$ & $<1.50$ & $<.007$ & $<.040$ & $<.02$ & $<.008$ & 85 & $<.025$ & $<.004$ & $<.016$ & $<.011$ \\
\hline 49 & $<1.50$ & $<.007$ & $<.040$ & $<.02$ & $<.008$ & 91 & $<.025$ & $<.004$ & $<.016$ & $<.011$ \\
\hline 50 & $<1.50$ & $<.007$ & $<.040$ & $<.02$ & $<.008$ & 104 & $<.025$ & $<.004$ & $<.016$ & $<.011$ \\
\hline 51 & $<1.50$ & $<.007$ & $<.040$ & $<.02$ & $<.008$ & e8.9 & $<.025$ & $<.004$ & $<.016$ & $<.011$ \\
\hline 52 & $<1.50$ & $<.007$ & $<.040$ & $<.02$ & $<.008$ & 99 & $<.025$ & $<.004$ & $<.016$ & $<.011$ \\
\hline 53 & $<1.50$ & $<.007$ & $<.040$ & $<.02$ & $<.008$ & e16 & $<.025$ & $<.004$ & $<.016$ & $<.011$ \\
\hline 54 & $<1.50$ & $<.007$ & $<.040$ & $<.02$ & $<.008$ & $\mathrm{e} 0$ & $<.025$ & $<.004$ & $<.016$ & $<.011$ \\
\hline 55 & $<1.50$ & $<.007$ & $<.040$ & $<.02$ & $<.008$ & e95 & $<.025$ & $<.004$ & $<.016$ & $<.011$ \\
\hline 56 & $<1.50$ & $<.007$ & $<.040$ & $<.02$ & $<.008$ & 113 & $<.025$ & $<.004$ & $<.016$ & $<.011$ \\
\hline 57 & $<1.50$ & $<.007$ & $<.040$ & $<.02$ & $<.008$ & 103 & $<.025$ & $<.004$ & $<.016$ & $<.011$ \\
\hline 58 & $<1.50$ & $<.007$ & $<.040$ & $<.02$ & $<.008$ & 103 & $<.025$ & $<.004$ & $<.016$ & $<.011$ \\
\hline 59 & $<1.50$ & $<.007$ & $<.040$ & $<.02$ & $<.008$ & 81 & $<.025$ & $<.004$ & $<.016$ & $<.011$ \\
\hline 60 & $<1.50$ & $<.007$ & $<.040$ & $<.02$ & $<.008$ & 97 & $<.025$ & $<.004$ & $<.016$ & $<.011$ \\
\hline 61 & $<1.50$ & $<.007$ & $<.040$ & $<.02$ & $<.008$ & 104 & $<.025$ & $<.004$ & $<.016$ & $<.011$ \\
\hline
\end{tabular}


Table 19. Pesticides and pesticide-degradate concentration data for filtered ground-water samples collected from basin-fill aquifers in Montana, Northern Rockies Intermontane Basins study unit, 2001 (Continued)

\begin{tabular}{|c|c|c|c|c|c|c|c|c|}
\hline $\begin{array}{c}\text { Well } \\
\text { number } \\
\text { (figs. } 3 \\
\text { and 4) }\end{array}$ & $\begin{array}{c}\text { Bro- } \\
\text { macil } \\
(\mu \mathrm{g} / \mathrm{L})\end{array}$ & $\begin{array}{l}\text { Brom- } \\
\text { oxynil } \\
(\mu \mathrm{g} / \mathrm{L})\end{array}$ & $\begin{array}{c}\text { Caf- } \\
\text { feine } \\
(\mu \mathrm{g} / \mathrm{L})\end{array}$ & $\begin{array}{l}\text { Caffeine } \\
\text { C-13, } \\
\text { surrogate } \\
\text { ( percent) }\end{array}$ & $\begin{array}{c}\text { Chlor- } \\
\text { amben } \\
\text { methyl } \\
\text { ester } \\
(\mu \mathrm{g} / \mathrm{L})\end{array}$ & $\begin{array}{c}\text { Chlorimuron- } \\
\text { ethyl } \\
(\mu \mathrm{g} / \mathrm{L})\end{array}$ & $\begin{array}{c}\text { Chloro- } \\
\text { thalonil } \\
(\mu \mathrm{g} / \mathrm{L})\end{array}$ & $\begin{array}{c}\text { Clopyralid } \\
(\mu \mathrm{g} / \mathrm{L})\end{array}$ \\
\hline \multicolumn{9}{|c|}{ Subunit Survey 2, Montana } \\
\hline 32 & $<0.033$ & $<0.017$ & $<0.010$ & 116 & $<0.018$ & $<0.010$ & $<0.035$ & $<0.014$ \\
\hline 33 & $<.033$ & $<.017$ & e.004 & 103 & $<.018$ & $<.010$ & $<.035$ & $<.014$ \\
\hline 34 & $<.033$ & $<.017$ & $<.010$ & 111 & $<.018$ & $<.010$ & $<.035$ & $<.014$ \\
\hline 35 & $<.033$ & $<.017$ & $<.010$ & 113 & $<.018$ & $<.010$ & $<.035$ & $<.014$ \\
\hline 36 & $<.033$ & $<.017$ & $<.010$ & 100 & $<.018$ & $<.010$ & $<.035$ & $<.014$ \\
\hline $36^{\mathbf{R}}$ & $<.033$ & $<.017$ & $<.010$ & 100 & $<.018$ & $<.010$ & $<.035$ & $<.014$ \\
\hline $36^{\mathrm{Fb}}$ & $<.033$ & $<.017$ & $<.010$ & 105 & $<.018$ & $<.010$ & $<.035$ & $<.014$ \\
\hline 37 & $<.033$ & $<.017$ & $<.010$ & 113 & $<.018$ & $<.010$ & $<.035$ & $<.014$ \\
\hline 38 & $<.033$ & $<.017$ & $<.010$ & e107 & $<.018$ & $<.010$ & $<.035$ & $<.014$ \\
\hline $38^{\mathbf{R}}$ & $<.033$ & $<.017$ & $<.010$ & e109 & $<.018$ & $<.010$ & $<.035$ & $<.014$ \\
\hline $38^{\mathbf{F b}}$ & $<.033$ & $<.017$ & $<.010$ & e133 & $<.018$ & $<.010$ & $<.035$ & $<.014$ \\
\hline 39 & $<.033$ & $<.017$ & $<.010$ & e110 & $<.018$ & $<.010$ & $<.035$ & $<.014$ \\
\hline 40 & $<.033$ & $<.017$ & $<.010$ & e112 & $<.018$ & $<.010$ & $<.035$ & $<.014$ \\
\hline 41 & $<.033$ & $<.017$ & $<.010$ & e101 & $<.018$ & $<.010$ & $<.035$ & $<.014$ \\
\hline 42 & $<.033$ & $<.017$ & $<.002$ & 109 & $<.018$ & $<.010$ & $<.035$ & $<.014$ \\
\hline 43 & $<.033$ & $<.017$ & $<.010$ & 162 & $<.018$ & $<.010$ & $<.035$ & $<.014$ \\
\hline 44 & $<.033$ & $<.017$ & $<.010$ & 89 & $<.018$ & $<.010$ & $<.035$ & $<.014$ \\
\hline 45 & $<.033$ & $<.017$ & $<.010$ & 105 & $<.018$ & $<.010$ & $<.035$ & $<.014$ \\
\hline $45^{\mathrm{R}}$ & $<.033$ & $<.017$ & e.003 & 102 & $<.018$ & $<.010$ & $<.035$ & $<.014$ \\
\hline 46 & $<.033$ & $<.017$ & $<.010$ & 87 & $<.018$ & $<.010$ & $<.035$ & $<.014$ \\
\hline 47 & $<.033$ & $<.017$ & $<.010$ & 94 & $<.018$ & $<.010$ & $<.035$ & $<.014$ \\
\hline 48 & $<.033$ & $<.017$ & $<.010$ & 91 & $<.018$ & $<.010$ & $<.035$ & $<.014$ \\
\hline $48^{\mathbf{F b}}$ & $<.033$ & $<.017$ & $<.010$ & e149 & $<.018$ & $<.010$ & $<.035$ & $<.014$ \\
\hline 49 & $<.033$ & $<.017$ & $<.010$ & 98 & $<.018$ & $<.010$ & $<.035$ & $<.014$ \\
\hline 50 & $<.033$ & $<.017$ & $<.010$ & 93 & $<.018$ & $<.010$ & $<.035$ & $<.014$ \\
\hline 51 & $<.033$ & $<.017$ & $<.010$ & 78 & $<.018$ & $<.010$ & $<.035$ & $<.014$ \\
\hline 52 & $<.033$ & $<.017$ & $<.010$ & e19 & $<.018$ & $<.010$ & $<.035$ & $<.014$ \\
\hline 53 & $<.033$ & $<.017$ & $<.010$ & 105 & $<.018$ & $<.010$ & $<.035$ & $<.014$ \\
\hline 54 & $<.033$ & $<.017$ & $<.010$ & e265 & $<.018$ & $<.010$ & $<.035$ & $<.014$ \\
\hline 55 & $<.033$ & $<.017$ & e.004 & 131 & $<.018$ & $<.010$ & $<.035$ & $<.014$ \\
\hline 56 & $<.033$ & $<.017$ & $<.010$ & 89 & $<.018$ & $<.010$ & $<.035$ & $<.014$ \\
\hline 57 & $<.033$ & $<.017$ & $<.010$ & 97 & $<.018$ & $<.010$ & $<.035$ & $<.014$ \\
\hline 58 & $<.033$ & $<.017$ & $<.010$ & 94 & $<.018$ & $<.010$ & $<.035$ & $<.014$ \\
\hline 59 & $<.033$ & $<.017$ & $<.010$ & 52 & $<.018$ & $<.010$ & $<.035$ & $<.014$ \\
\hline 60 & $<.033$ & $<.017$ & $<.010$ & 86 & $<.018$ & $<.010$ & $<.035$ & $<.014$ \\
\hline 61 & $<.033$ & $<.017$ & $<.010$ & 71 & $<.018$ & $<.010$ & $<.035$ & $<.014$ \\
\hline
\end{tabular}

114 Water-Quality, Streambed-Sediment, and Biological Data from the Clark Fork-Pend Oreille and Spokane River Basins, Montana, Idaho, and Washington, 1998-2001 
Table 19. Pesticides and pesticide-degradate concentration data for filtered ground-water samples collected from basin-fill aquifers in Montana, Northern Rockies Intermontane Basins study unit, 2001 (Continued)

\begin{tabular}{|c|c|c|c|c|c|c|c|c|c|}
\hline $\begin{array}{c}\text { Well } \\
\text { number } \\
\text { (figs. } 3 \\
\text { and 4) }\end{array}$ & $\begin{array}{c}\text { Cycloate } \\
(\mu \mathrm{g} / \mathrm{L})\end{array}$ & $\begin{array}{c}\text { Dacthal } \\
\text { mono- } \\
\text { acid } \\
(\mu \mathrm{g} / \mathrm{L})\end{array}$ & $\begin{array}{c}\text { Deethyl- } \\
\text { deisopropyl- } \\
\text { atrazine } \\
(\mu \mathrm{g} / \mathrm{L})\end{array}$ & $\begin{array}{c}\text { Deiso- } \\
\text { propyl- } \\
\text { atrazine } \\
(\mu \mathrm{g} / \mathrm{L})\end{array}$ & $\begin{array}{c}\text { Di- } \\
\text { camba } \\
(\mu \mathrm{g} / \mathrm{L})\end{array}$ & $\begin{array}{c}\text { Dichlor- } \\
\text { prop } \\
(\mu \mathrm{g} / \mathrm{L})\end{array}$ & $\begin{array}{c}\text { Dinoseb } \\
(\mu \mathrm{g} / \mathrm{L})\end{array}$ & $\begin{array}{l}\text { Diphen- } \\
\text { amid } \\
(\mu \mathrm{g} / \mathrm{L})\end{array}$ & $\begin{array}{c}\text { Diuron } \\
(\mu \mathrm{g} / \mathrm{L})\end{array}$ \\
\hline \multicolumn{10}{|c|}{ Subunit Survey 2, Montana } \\
\hline 32 & $<0.013$ & $<0.012$ & $<0.010$ & $<0.044$ & $<0.013$ & $<0.014$ & $<0.012$ & $<0.026$ & $<0.015$ \\
\hline 33 & $<.013$ & $<.012$ & $<.010$ & $<.044$ & $<.013$ & $<.014$ & $<.012$ & $<.026$ & $<.015$ \\
\hline 34 & $<.013$ & $<.012$ & $<.010$ & $<.044$ & $<.013$ & $<.014$ & $<.012$ & $<.026$ & $<.015$ \\
\hline 35 & $<.013$ & $<.012$ & $<.010$ & $<.044$ & $<.013$ & $<.014$ & $<.012$ & $<.026$ & $<.015$ \\
\hline 36 & $<.013$ & $<.012$ & $<.010$ & $<.044$ & $<.013$ & $<.014$ & $<.012$ & $<.026$ & $<.015$ \\
\hline $36^{\mathbf{R}}$ & $<.013$ & $<.012$ & $<.010$ & $<.044$ & $<.013$ & $<.014$ & $<.012$ & $<.026$ & $<.007$ \\
\hline $36^{\mathrm{Fb}}$ & $<.013$ & $<.012$ & $<.010$ & $<.044$ & $<.013$ & $<.014$ & $<.012$ & $<.026$ & $<.015$ \\
\hline 37 & $<.013$ & $<.012$ & $<.010$ & $<.044$ & $<.013$ & $<.014$ & $<.012$ & $<.026$ & $<.015$ \\
\hline 38 & $<.013$ & $<.012$ & $<.010$ & $<.044$ & $<.013$ & $<.014$ & $<.012$ & $<.026$ & $<.015$ \\
\hline $38^{\mathbf{R}}$ & $<.013$ & $<.012$ & $<.010$ & $<.044$ & $<.013$ & $<.014$ & $<.012$ & $<.026$ & $<.015$ \\
\hline $38^{\mathbf{F b}}$ & $<.013$ & $<.012$ & $<.001$ & $<.044$ & $<.013$ & $<.014$ & $<.012$ & $<.026$ & $<.001$ \\
\hline 39 & $<.013$ & $<.012$ & $<.002$ & $<.044$ & $<.013$ & $<.014$ & $<.012$ & $<.026$ & $<.015$ \\
\hline 40 & $<.013$ & $<.012$ & $<.001$ & $<.044$ & $<.013$ & $<.014$ & $<.012$ & $<.026$ & $<.001$ \\
\hline 41 & $<.013$ & $<.012$ & $<.001$ & $<.044$ & $<.013$ & $<.014$ & $<.012$ & $<.026$ & $<.015$ \\
\hline 42 & $<.013$ & $<.012$ & $<.010$ & $<.044$ & $<.013$ & $<.014$ & $<.012$ & $<.026$ & $<.015$ \\
\hline 43 & $<.013$ & $<.012$ & $<.010$ & $<.044$ & $<.013$ & $<.014$ & $<.012$ & $<.026$ & $<.001$ \\
\hline 44 & $<.013$ & $<.012$ & $<.010$ & $<.044$ & $<.013$ & $<.014$ & $<.012$ & $<.026$ & $<.001$ \\
\hline 45 & $<.013$ & $<.012$ & $<.010$ & $<.044$ & $<.013$ & $<.014$ & $<.012$ & $<.026$ & $<.001$ \\
\hline $45^{\mathbf{R}}$ & $<.013$ & $<.012$ & $<.010$ & $<.044$ & $<.013$ & $<.014$ & $<.012$ & $<.026$ & $<.015$ \\
\hline 46 & $<.013$ & $<.012$ & $<.010$ & $<.044$ & $<.013$ & $<.014$ & $<.012$ & $<.026$ & $<.001$ \\
\hline 47 & $<.013$ & $<.012$ & $<.010$ & $<.044$ & $<.013$ & $<.014$ & $<.012$ & $<.026$ & $<.001$ \\
\hline 48 & $<.013$ & $<.012$ & $<.010$ & $<.044$ & $<.013$ & $<.014$ & $<.012$ & $<.026$ & $<.001$ \\
\hline $48^{\mathbf{F b}}$ & $<.013$ & $<.012$ & $<.010$ & $<.044$ & $<.013$ & $<.014$ & $<.012$ & $<.026$ & $<.001$ \\
\hline 49 & $<.013$ & $<.012$ & $<.010$ & $<.044$ & $<.013$ & $<.014$ & $<.012$ & $<.026$ & $<.015$ \\
\hline 50 & $<.013$ & $<.012$ & $<.010$ & $<.044$ & $<.013$ & $<.014$ & $<.012$ & $<.026$ & $<.001$ \\
\hline 51 & $<.013$ & $<.012$ & $<.010$ & $<.044$ & $<.013$ & $<.014$ & $<.012$ & $<.026$ & $<.015$ \\
\hline 52 & $<.013$ & $<.012$ & $<.010$ & $<.044$ & $<.013$ & $<.014$ & $<.012$ & $<.026$ & $<.015$ \\
\hline 53 & $<.013$ & $<.012$ & $<.010$ & $<.044$ & $<.013$ & $<.014$ & $<.012$ & $<.026$ & $<.015$ \\
\hline 54 & $<.013$ & $<.012$ & $<.010$ & $<.044$ & $<.013$ & $<.014$ & $<.012$ & $<.026$ & $<.015$ \\
\hline 55 & $<.013$ & $<.012$ & $<.010$ & $<.044$ & $<.013$ & $<.014$ & $<.012$ & $<.026$ & $<.015$ \\
\hline 56 & $<.013$ & $<.012$ & $<.010$ & $<.044$ & $<.013$ & $<.014$ & $<.012$ & $<.026$ & $<.015$ \\
\hline 57 & $<.013$ & $<.012$ & $<.010$ & $<.044$ & $<.013$ & $<.014$ & $<.012$ & $<.026$ & $<.015$ \\
\hline 58 & $<.013$ & $<.012$ & $<.010$ & $<.044$ & $<.013$ & $<.014$ & $<.012$ & $<.026$ & $<.015$ \\
\hline 59 & $<.013$ & $<.012$ & $<.001$ & $<.044$ & $<.013$ & $<.014$ & $<.012$ & $<.026$ & $<.015$ \\
\hline 60 & $<.013$ & $<.012$ & $<.001$ & $<.044$ & .034 & $<.014$ & $<.012$ & $<.026$ & $<.015$ \\
\hline 61 & $<.013$ & $<.012$ & $<.002$ & $<.044$ & $<.013$ & $<.014$ & $<.012$ & $<.026$ & $<.015$ \\
\hline
\end{tabular}


Table 19. Pesticides and pesticide-degradate concentration data for filtered ground-water samples collected from basin-fill aquifers in Montana, Northern Rockies Intermontane Basins study unit, 2001 (Continued)

\begin{tabular}{|c|c|c|c|c|c|c|c|c|c|}
\hline $\begin{array}{c}\text { Well } \\
\text { number } \\
\text { (figs. } 3 \\
\text { and 4) }\end{array}$ & $\begin{array}{c}\text { Fenuron } \\
(\mu \mathrm{g} / \mathrm{L})\end{array}$ & $\begin{array}{c}\text { Flumetsulam } \\
(\mu \mathrm{g} / \mathrm{L})\end{array}$ & $\begin{array}{c}\text { Fluo- } \\
\text { meturon } \\
(\mu \mathrm{g} / \mathrm{L})\end{array}$ & $\begin{array}{c}\text { Imazaquin } \\
(\mu \mathrm{g} / \mathrm{L})\end{array}$ & $\begin{array}{c}\text { Imazethapyr } \\
(\mu \mathrm{g} / \mathrm{L})\end{array}$ & $\begin{array}{c}\text { Imidacloprid } \\
(\mu \mathrm{g} / \mathrm{L})\end{array}$ & $\begin{array}{c}\text { MCPA } \\
(\mu \mathrm{g} / \mathrm{L})\end{array}$ & $\begin{array}{c}\text { MCPB } \\
(\mu \mathrm{g} / \mathrm{L})\end{array}$ & $\begin{array}{c}\text { Metalaxyl } \\
(\mu \mathrm{g} / \mathrm{L})\end{array}$ \\
\hline \multicolumn{10}{|c|}{ Subunit Survey 2, Montana } \\
\hline 32 & $<0.032$ & $<0.011$ & $<0.031$ & $<0.016$ & $<0.017$ & $<0.007$ & $<0.016$ & $<0.015$ & $<0.020$ \\
\hline 33 & $<.032$ & $<.011$ & $<.031$ & $<.016$ & $<.017$ & $<.007$ & $<.016$ & $<.015$ & $<.020$ \\
\hline 34 & $<.032$ & $<.011$ & $<.031$ & $<.016$ & $<.017$ & $<.007$ & $<.016$ & $<.015$ & $<.020$ \\
\hline 35 & $<.032$ & $<.011$ & $<.031$ & $<.016$ & $<.017$ & $<.007$ & $<.016$ & $<.015$ & $<.020$ \\
\hline 36 & $<.032$ & $<.011$ & $<.031$ & $<.016$ & e.014 & $<.007$ & $<.016$ & $<.015$ & $<.020$ \\
\hline $36^{\mathbf{R}}$ & $<.001$ & $<.011$ & e.004 & $<.016$ & $<.017$ & $<.007$ & $<.016$ & $<.015$ & $<.020$ \\
\hline $36^{\mathbf{F b}}$ & $<.001$ & $<.011$ & $<.031$ & $<.016$ & $<.017$ & $<.007$ & $<.016$ & $<.015$ & $<.020$ \\
\hline 37 & $<.032$ & $<.011$ & $<.031$ & $<.016$ & $<.017$ & $<.007$ & $<.016$ & $<.015$ & $<.020$ \\
\hline 38 & $<.032$ & $<.011$ & $<.031$ & $<.016$ & $<.017$ & $<.007$ & $<.016$ & $<.015$ & $<.020$ \\
\hline $38^{\mathbf{R}}$ & $<.032$ & $<.011$ & $<.031$ & $<.016$ & $<.017$ & $<.007$ & $<.016$ & $<.015$ & $<.020$ \\
\hline $38^{\mathbf{F b}}$ & $<.001$ & $<.011$ & $<.031$ & $<.016$ & $<.017$ & $<.007$ & $<.016$ & $<.015$ & $<.020$ \\
\hline 39 & $<.032$ & $<.011$ & $<.031$ & $<.016$ & $<.017$ & $<.007$ & $<.016$ & $<.015$ & $<.020$ \\
\hline 40 & $<.032$ & $<.011$ & $<.031$ & $<.016$ & $<.017$ & $<.007$ & $<.016$ & $<.015$ & $<.020$ \\
\hline 41 & $<.032$ & $<.011$ & $<.031$ & $<.016$ & $<.017$ & $<.007$ & $<.016$ & $<.015$ & $<.020$ \\
\hline 42 & $<.001$ & $<.011$ & $<.031$ & $<.016$ & $<.017$ & $<.007$ & $<.016$ & $<.015$ & $<.020$ \\
\hline 43 & $<.001$ & $<.011$ & $<.031$ & $<.016$ & $<.017$ & $<.007$ & $<.016$ & $<.015$ & $<.020$ \\
\hline 44 & $<.032$ & $<.011$ & $<.031$ & $<.016$ & $<.017$ & $<.007$ & $<.016$ & $<.015$ & $<.020$ \\
\hline 45 & $<.002$ & $<.011$ & $<.031$ & $<.016$ & $<.017$ & $<.007$ & $<.016$ & $<.015$ & $<.020$ \\
\hline $45^{\mathbf{R}}$ & $<.002$ & $<.011$ & $<.031$ & $<.016$ & $<.017$ & $<.007$ & $<.016$ & $<.015$ & $<.020$ \\
\hline 46 & $<.001$ & $<.011$ & $<.031$ & $<.016$ & $<.017$ & $<.007$ & $<.016$ & $<.015$ & $<.020$ \\
\hline 47 & $<.032$ & $<.011$ & $<.031$ & $<.016$ & $<.017$ & $<.007$ & $<.016$ & $<.015$ & $<.020$ \\
\hline 48 & $<.001$ & $<.011$ & $<.031$ & $<.016$ & $<.017$ & $<.007$ & $<.016$ & $<.015$ & $<.020$ \\
\hline $48^{\mathbf{F b}}$ & $<.001$ & $<.011$ & $<.031$ & $<.016$ & $<.017$ & $<.007$ & $<.016$ & $<.015$ & $<.020$ \\
\hline 49 & $<.032$ & $<.011$ & $<.031$ & $<.016$ & $<.017$ & $<.007$ & $<.016$ & $<.015$ & $<.020$ \\
\hline 50 & $<.032$ & $<.011$ & $<.031$ & $<.016$ & $<.017$ & $<.007$ & $<.016$ & $<.015$ & $<.020$ \\
\hline 51 & $<.032$ & $<.011$ & $<.031$ & $<.016$ & $<.017$ & $<.007$ & $<.016$ & $<.015$ & $<.020$ \\
\hline 52 & $<.032$ & $<.011$ & $<.031$ & $<.016$ & $<.017$ & $<.007$ & $<.016$ & $<.015$ & $<.020$ \\
\hline 53 & $<.032$ & $<.011$ & $<.031$ & $<.016$ & $<.017$ & $<.007$ & $<.016$ & $<.015$ & $<.020$ \\
\hline 54 & $<.032$ & $<.011$ & $<.031$ & $<.016$ & $<.017$ & $<.007$ & $<.016$ & $<.015$ & $<.020$ \\
\hline 55 & $<.032$ & $<.011$ & $<.031$ & $<.016$ & $<.017$ & $<.007$ & $<.016$ & $<.015$ & $<.020$ \\
\hline 56 & $<.032$ & $<.011$ & $<.031$ & $<.016$ & $<.017$ & $<.007$ & $<.016$ & $<.015$ & $<.020$ \\
\hline 57 & $<.032$ & $<.011$ & $<.031$ & $<.016$ & $<.017$ & $<.007$ & $<.016$ & $<.015$ & $<.020$ \\
\hline 58 & $<.032$ & $<.011$ & $<.031$ & $<.016$ & $<.017$ & $<.007$ & $<.016$ & $<.015$ & $<.020$ \\
\hline 59 & $<.032$ & $<.011$ & $<.031$ & $<.016$ & $<.017$ & $<.007$ & $<.016$ & $<.015$ & $<.020$ \\
\hline 60 & $<.032$ & $<.011$ & $<.031$ & $<.016$ & $<.017$ & $<.007$ & $<.016$ & $<.015$ & $<.020$ \\
\hline 61 & $<.032$ & $<.011$ & $<.031$ & $<.016$ & $<.017$ & $<.007$ & $<.016$ & $<.015$ & $<.020$ \\
\hline
\end{tabular}


Table 19. Pesticides and pesticide-degradate concentration data for filtered ground-water samples collected from basin-fill aquifers in Montana, Northern Rockies Intermontane Basins study unit, 2001 (Continued)

\begin{tabular}{|c|c|c|c|c|c|c|c|c|}
\hline $\begin{array}{c}\text { Well } \\
\text { number } \\
\text { (figs. } 3 \\
\text { and 4) }\end{array}$ & $\begin{array}{c}\text { Methio- } \\
\text { carb } \\
(\mu \mathrm{g} / \mathrm{L})\end{array}$ & $\begin{array}{c}\text { Methomyl } \\
(\mu \mathrm{g} / \mathrm{L})\end{array}$ & $\begin{array}{c}\text { Metsul- } \\
\text { furon } \\
\text { methyl } \\
(\mu \mathrm{g} / \mathrm{L})\end{array}$ & $\begin{array}{c}\text { Neburon } \\
(\mu \mathrm{g} / \mathrm{L})\end{array}$ & $\begin{array}{c}\text { Nico- } \\
\text { sulfuron } \\
(\mu \mathrm{g} / \mathrm{L})\end{array}$ & $\begin{array}{c}\text { Nor- } \\
\text { flurazon } \\
(\mu \mathrm{g} / \mathrm{L})\end{array}$ & $\begin{array}{c}\text { Oryzalin } \\
(\mu \mathrm{g} / \mathrm{L})\end{array}$ & $\begin{array}{c}\text { Oxamyl } \\
(\mu g / L)\end{array}$ \\
\hline \multicolumn{9}{|c|}{ Subunit Survey 2, Montana } \\
\hline 32 & $<0.008$ & $<0.004$ & $<0.025$ & $<0.012$ & $<0.013$ & $<0.016$ & $<0.018$ & $<0.012$ \\
\hline 33 & $<.008$ & $<.004$ & $<.025$ & $<.012$ & $<.013$ & $<.016$ & $<.018$ & $<.012$ \\
\hline 34 & $<.008$ & $<.004$ & $<.025$ & $<.012$ & $<.013$ & $<.016$ & $<.018$ & $<.012$ \\
\hline 35 & $<.008$ & $<.004$ & $<.025$ & $<.012$ & $<.013$ & $<.016$ & $<.018$ & $<.012$ \\
\hline 36 & $<.008$ & $<.004$ & $<.025$ & $<.012$ & $<.013$ & $<.016$ & $<.018$ & $<.012$ \\
\hline $36^{\mathbf{R}}$ & $<.008$ & $<.004$ & $<.025$ & $<.012$ & $<.013$ & e.006 & $<.018$ & $<.012$ \\
\hline $36^{\mathbf{F b}}$ & $<.008$ & $<.004$ & $<.025$ & $<.012$ & $<.013$ & $<.016$ & e.007 & $<.012$ \\
\hline 37 & $<.008$ & $<.004$ & $<.025$ & $<.012$ & $<.013$ & $<.016$ & $<.018$ & $<.012$ \\
\hline 38 & $<.008$ & $<.004$ & $<.025$ & $<.012$ & $<.013$ & $<.016$ & $<.018$ & $<.012$ \\
\hline $38^{\mathbf{R}}$ & $<.008$ & $<.004$ & $<.025$ & $<.012$ & $<.013$ & $<.016$ & $<.018$ & $<.012$ \\
\hline $38^{\mathbf{F b}}$ & $<.008$ & $<.004$ & $<.025$ & $<.012$ & $<.013$ & $<.016$ & $<.018$ & $<.012$ \\
\hline 39 & $<.008$ & $<.004$ & $<.025$ & $<.012$ & $<.013$ & $<.016$ & $<.018$ & $<.012$ \\
\hline 40 & $<.008$ & $<.004$ & $<.025$ & $<.012$ & $<.013$ & $<.016$ & $<.018$ & $<.012$ \\
\hline 41 & $<.008$ & $<.004$ & $<.017$ & $<.012$ & $<.013$ & $<.016$ & $<.018$ & $<.012$ \\
\hline 42 & $<.008$ & $<.004$ & $<.025$ & $<.012$ & $<.013$ & $<.016$ & $<.018$ & $<.012$ \\
\hline 43 & $<.008$ & $<.004$ & $<.025$ & $<.012$ & $<.013$ & $<.016$ & e.012 & $<.012$ \\
\hline 44 & $<.008$ & $<.004$ & $<.025$ & $<.012$ & $<.013$ & $<.016$ & $<.018$ & $<.012$ \\
\hline 45 & $<.008$ & $<.004$ & $<.025$ & $<.012$ & $<.013$ & $<.016$ & $<.018$ & $<.012$ \\
\hline $45^{\mathbf{R}}$ & $<.008$ & $<.004$ & $<.025$ & $<.012$ & $<.013$ & $<.016$ & $<.018$ & $<.012$ \\
\hline 46 & $<.008$ & $<.004$ & $<.025$ & $<.012$ & $<.013$ & $<.016$ & $<.018$ & $<.012$ \\
\hline 47 & $<.008$ & $<.004$ & $<.025$ & $<.012$ & $<.013$ & $<.016$ & $<.018$ & $<.012$ \\
\hline 48 & $<.008$ & $<.004$ & $<.025$ & $<.012$ & $<.013$ & $<.016$ & $<.018$ & $<.012$ \\
\hline $48^{\mathbf{F b}}$ & $<.008$ & $<.004$ & $<.025$ & $<.012$ & $<.013$ & $<.016$ & $<.018$ & $<.012$ \\
\hline 49 & $<.008$ & $<.004$ & $<.025$ & $<.012$ & $<.013$ & $<.016$ & $<.018$ & $<.012$ \\
\hline 50 & $<.008$ & $<.004$ & $<.025$ & $<.012$ & $<.013$ & $<.016$ & $<.018$ & $<.012$ \\
\hline 51 & $<.008$ & $<.004$ & $<.025$ & $<.012$ & $<.013$ & $<.016$ & $<.018$ & $<.012$ \\
\hline 52 & $<.008$ & $<.004$ & $<.025$ & $<.012$ & $<.013$ & $<.016$ & $<.018$ & $<.012$ \\
\hline 53 & $<.008$ & $<.004$ & $<.025$ & $<.012$ & $<.013$ & $<.016$ & $<.018$ & $<.012$ \\
\hline 54 & $<.008$ & $<.004$ & $<.025$ & $<.012$ & $<.013$ & $<.016$ & $<.018$ & $<.012$ \\
\hline 55 & $<.008$ & $<.004$ & $<.025$ & $<.012$ & $<.013$ & $<.016$ & $<.018$ & $<.012$ \\
\hline 56 & $<.008$ & $<.004$ & $<.025$ & $<.012$ & $<.013$ & $<.016$ & $<.018$ & $<.012$ \\
\hline 57 & $<.008$ & $<.004$ & $<.025$ & $<.012$ & $<.013$ & $<.016$ & $<.018$ & $<.012$ \\
\hline 58 & $<.008$ & $<.004$ & $<.025$ & $<.012$ & $<.013$ & $<.016$ & $<.018$ & $<.012$ \\
\hline 59 & $<.008$ & $<.004$ & $<.025$ & $<.012$ & $<.013$ & $<.016$ & $<.018$ & $<.012$ \\
\hline 60 & $<.008$ & $<.004$ & $<.025$ & $<.012$ & $<.013$ & $<.016$ & $<.018$ & $<.012$ \\
\hline 61 & $<.008$ & $<.004$ & $<.025$ & $<.012$ & $<.013$ & $<.016$ & $<.018$ & $<.012$ \\
\hline
\end{tabular}


Table 19. Pesticides and pesticide-degradate concentration data for filtered ground-water samples collected from basin-fill aquifers in Montana, Northern Rockies Intermontane Basins study unit, 2001 (Continued)

\begin{tabular}{|c|c|c|c|c|c|c|c|c|}
\hline $\begin{array}{c}\text { Well } \\
\text { number } \\
\text { (figs. } 3 \\
\text { and 4) }\end{array}$ & $\begin{array}{c}\text { Picloram } \\
(\mu \mathrm{g} / \mathrm{L})\end{array}$ & $\begin{array}{c}\text { Prop- } \\
\text { ham } \\
(\mu \mathrm{g} / \mathrm{L})\end{array}$ & $\begin{array}{c}\text { Propicon- } \\
\text { azole } \\
(\mu \mathrm{g} / \mathrm{L})\end{array}$ & $\begin{array}{c}\text { Propoxur } \\
(\mu \mathbf{g} / \mathbf{L})\end{array}$ & $\begin{array}{c}\text { Siduron } \\
(\mu \mathrm{g} / \mathrm{L})\end{array}$ & $\begin{array}{c}\text { Sulfo- } \\
\text { meturon- } \\
\text { methyl } \\
(\mu \mathrm{g} / \mathrm{L})\end{array}$ & $\begin{array}{c}\text { Triben- } \\
\text { uron- } \\
\text { methyl } \\
(\mu \mathrm{g} / \mathrm{L})\end{array}$ & $\begin{array}{c}\text { Triclopyr } \\
(\mu \mathrm{g} / \mathrm{L})\end{array}$ \\
\hline \multicolumn{9}{|c|}{ Subunit Survey 2, Montana } \\
\hline 32 & $<0.020$ & $<0.010$ & $<0.021$ & $<0.008$ & $<0.017$ & $<0.009$ & $<0.009$ & $<0.022$ \\
\hline 33 & $<.020$ & $<.010$ & $<.021$ & $<.008$ & $<.017$ & $<.009$ & $<.009$ & $<.022$ \\
\hline 34 & $<.020$ & $<.010$ & $<.008$ & $<.008$ & $<.017$ & $<.009$ & $<.009$ & $<.022$ \\
\hline 35 & $<.020$ & $<.010$ & $<.021$ & $<.008$ & $<.017$ & $<.009$ & $<.009$ & $<.022$ \\
\hline 36 & $<.020$ & $<.010$ & $<.019$ & $<.008$ & $<.017$ & $<.009$ & -- & $<.022$ \\
\hline $36^{\mathbf{R}}$ & $<.020$ & $<.010$ & $<.020$ & $<.008$ & $<.017$ & $<.009$ & -- & $<.022$ \\
\hline $36^{\mathbf{F b}}$ & $<.020$ & $<.010$ & $<.017$ & $<.008$ & $<.017$ & $<.009$ & -- & $<.022$ \\
\hline 37 & $<.020$ & $<.010$ & $<.007$ & $<.008$ & $<.017$ & $<.009$ & $<.009$ & $<.022$ \\
\hline 38 & $<.020$ & $<.010$ & $<.021$ & $<.008$ & $<.017$ & $<.009$ & $<.009$ & $<.022$ \\
\hline $38^{\mathbf{R}}$ & $<.020$ & $<.010$ & $<.021$ & $<.008$ & $<.017$ & $<.009$ & $<.009$ & $<.022$ \\
\hline $38^{\mathbf{F b}}$ & $<.020$ & $<.010$ & $<.021$ & $<.008$ & $<.017$ & $<.002$ & $<.009$ & $<.022$ \\
\hline 39 & $<.020$ & $<.010$ & $<.021$ & $<.008$ & $<.017$ & $<.009$ & $<.009$ & $<.022$ \\
\hline 40 & $<.020$ & $<.010$ & $<.021$ & $<.008$ & $<.017$ & $<.009$ & $<.009$ & $<.022$ \\
\hline 41 & $<.020$ & $<.010$ & $<.021$ & $<.008$ & $<.017$ & $<.009$ & $<.009$ & $<.022$ \\
\hline 42 & $<.020$ & $<.010$ & $<.021$ & $<.008$ & $<.001$ & $<.009$ & $<.009$ & $<.022$ \\
\hline 43 & $<.020$ & $<.010$ & $<.021$ & $<.008$ & $<.017$ & $<.009$ & $<.009$ & $<.022$ \\
\hline 44 & $<.020$ & $<.010$ & $<.021$ & $<.008$ & $<.017$ & $<.009$ & $<.009$ & $<.022$ \\
\hline 45 & $<.020$ & $<.010$ & $<.021$ & $<.008$ & $<.017$ & $<.009$ & $<.009$ & $<.022$ \\
\hline $45^{\mathbf{R}}$ & $<.020$ & $<.010$ & $<.021$ & $<.008$ & $<.017$ & $<.009$ & $<.009$ & $<.022$ \\
\hline 46 & $<.020$ & $<.010$ & $<.021$ & $<.008$ & $<.017$ & $<.009$ & $<.009$ & $<.022$ \\
\hline 47 & $<.020$ & $<.010$ & $<.021$ & $<.008$ & $<.017$ & $<.009$ & $<.009$ & $<.022$ \\
\hline 48 & $<.020$ & $<.010$ & $<.021$ & $<.008$ & $<.017$ & $<.009$ & -- & $<.022$ \\
\hline $48^{\mathbf{F b}}$ & $<.020$ & $<.010$ & $<.021$ & $<.008$ & $<.017$ & $<.001$ & $<.009$ & $<.022$ \\
\hline 49 & $<.020$ & $<.010$ & $<.021$ & $<.008$ & $<.017$ & $<.009$ & $<.009$ & $<.022$ \\
\hline 50 & $<.020$ & $<.010$ & $<.021$ & $<.008$ & $<.017$ & $<.009$ & -- & $<.022$ \\
\hline 51 & $<.020$ & $<.010$ & $<.021$ & $<.008$ & $<.017$ & $<.009$ & $<.009$ & $<.022$ \\
\hline 52 & $<.020$ & $<.010$ & $<.021$ & $<.008$ & $<.017$ & $<.009$ & $<.009$ & $<.022$ \\
\hline 53 & $<.020$ & $<.010$ & $<.021$ & $<.008$ & $<.017$ & $<.009$ & $<.009$ & $<.022$ \\
\hline 54 & $<.020$ & $<.010$ & $<.021$ & $<.008$ & $<.017$ & $<.009$ & $<.009$ & $<.022$ \\
\hline 55 & $<.020$ & $<.010$ & $<.021$ & $<.008$ & $<.017$ & $<.009$ & $<.009$ & $<.022$ \\
\hline 56 & $<.020$ & $<.010$ & $<.021$ & $<.008$ & $<.017$ & $<.009$ & $<.009$ & $<.022$ \\
\hline 57 & $<.020$ & $<.010$ & $<.021$ & $<.008$ & $<.017$ & $<.009$ & $<.009$ & $<.022$ \\
\hline 58 & $<.020$ & $<.010$ & $<.021$ & $<.008$ & $<.017$ & $<.009$ & $<.009$ & $<.022$ \\
\hline 59 & $<.020$ & $<.010$ & $<.021$ & $<.008$ & $<.017$ & $<.009$ & $<.009$ & $<.022$ \\
\hline 60 & $<.020$ & $<.010$ & $<.021$ & $<.008$ & $<.017$ & $<.009$ & $<.009$ & $<.022$ \\
\hline 61 & $<.020$ & $<.010$ & $<.021$ & $<.008$ & $<.017$ & $<.009$ & $<.009$ & $<.022$ \\
\hline
\end{tabular}


Table 20. Volatile organic-compound concentration data for ground-water samples collected from basin-fill aquifers, Northern Rockies Intermontane Basins study unit, 1999 and 2001

[Site identification number described in text. Bold text denotes detected concentrations. Abbreviations: e, estimated; Fb, field blank; $\mu \mathrm{g} / \mathrm{L}$, micrograms per liter; R, replicate; Sb, source-solution blank; Tb, trip blank. Symbols: <, less than reporting level; --, no data]

\begin{tabular}{|c|c|c|c|c|c|c|c|c|c|c|}
\hline $\begin{array}{c}\text { Well } \\
\text { number } \\
\text { (figs. } 3 \\
\text { and 4) }\end{array}$ & $\begin{array}{c}\text { Site } \\
\text { identification } \\
\text { number }\end{array}$ & Date & Time & $\begin{array}{l}\text { 1,1,1,2- } \\
\text { Tetra- } \\
\text { chloro- } \\
\text { ethane } \\
(\mu \mathrm{g} / \mathrm{L})\end{array}$ & $\begin{array}{c}\text { 1,1,1- } \\
\text { Trichloro- } \\
\text { ethane } \\
(\mu \mathrm{g} / \mathrm{L})\end{array}$ & $\begin{array}{c}\text { 1,1,2,2- } \\
\text { Tetra- } \\
\text { chloro- } \\
\text { ethane } \\
(\mu \mathrm{g} / \mathrm{L})\end{array}$ & $\begin{array}{c}\text { 1,1,2- } \\
\text { Trichloro- } \\
\text { ethane } \\
(\mu \mathrm{g} / \mathrm{L})\end{array}$ & $\begin{array}{c}\text { 1,1,2- } \\
\text { Trichloro- } \\
\text { trifluoro- } \\
\text { ethane } \\
(\mu \mathrm{g} / \mathrm{L})\end{array}$ & $\begin{array}{c}\text { 1,1- } \\
\text { Dichloro- } \\
\text { ethane } \\
(\mu \mathrm{g} / \mathrm{L})\end{array}$ & $\begin{array}{c}\text { 1,1- } \\
\text { Dichloro- } \\
\text { ethylene } \\
(\mu \mathrm{g} / \mathrm{L})\end{array}$ \\
\hline \multicolumn{11}{|c|}{ Subunit Survey 1, Idaho and Washington } \\
\hline 1 & 481320116261101 & 07/14/99 & 1700 & $<0.044$ & $<0.032$ & $<0.130$ & $<0.064$ & $<0.032$ & $<0.066$ & $<0.044$ \\
\hline 2 & 480950116324401 & 07/14/99 & 1100 & $<.044$ & $<.032$ & $<.130$ & $<.064$ & $<.032$ & $<.066$ & $<.044$ \\
\hline 3 & 480928117053201 & 07/07/99 & 1000 & $<.044$ & e.008 & $<.130$ & $<.064$ & $<.032$ & $<.066$ & $<.044$ \\
\hline 4 & 480813116593701 & 07/07/99 & 1600 & $<.044$ & $<.032$ & $<.130$ & $<.064$ & $<.032$ & $<.066$ & $<.044$ \\
\hline 5 & 480718117012501 & 07/06/99 & 1800 & $<.044$ & $<.032$ & $<.130$ & $<.064$ & $<.032$ & $<.066$ & $<.044$ \\
\hline 6 & 480249116510001 & 07/08/99 & 1000 & $<.044$ & e.014 & $<.130$ & $<.064$ & $<.032$ & $<.066$ & $<.044$ \\
\hline 7 & 480235116483001 & 07/13/99 & 1700 & $<.044$ & $<.032$ & $<.130$ & $<.064$ & $<.032$ & $<.066$ & $<.044$ \\
\hline 8 & 480203117200601 & 06/08/99 & 0800 & $<.044$ & $<.032$ & $<.130$ & $<.064$ & $<.032$ & $<.066$ & $<.044$ \\
\hline 9 & 480128116374601 & 08/04/99 & 0900 & $<.044$ & $<.032$ & $<.130$ & $<.064$ & $<.032$ & $<.066$ & $<.044$ \\
\hline 10 & 480119117182101 & 06/08/99 & 1600 & $<.044$ & $<.032$ & $<.130$ & $<.064$ & $<.032$ & $<.066$ & $<.044$ \\
\hline 11 & 475925117153001 & 07/06/99 & 1100 & $<.044$ & $<.032$ & $<.130$ & $<.064$ & $<.032$ & $<.066$ & $<.044$ \\
\hline 12 & 475825117364401 & 06/09/99 & 1000 & $<.044$ & $<.032$ & $<.130$ & $<.064$ & $<.032$ & $<.066$ & $<.044$ \\
\hline 13 & 475731116371301 & $06 / 23 / 99$ & 1200 & $<.044$ & $<.032$ & $<.130$ & $<.064$ & $<.032$ & $<.066$ & $<.044$ \\
\hline 13 & & 07/27/99 & 2000 & $<.044$ & $<.032$ & $<.130$ & $<.064$ & $<.032$ & $<.066$ & $<.044$ \\
\hline 14 & 475637117262501 & 06/09/99 & 1700 & $<.044$ & $<.032$ & $<.130$ & $<.064$ & $<.032$ & $<.066$ & $<.044$ \\
\hline 15 & 475510116391201 & 08/03/99 & 1400 & $<.044$ & e.005 & $<.130$ & $<.064$ & $<.032$ & $<.066$ & $<.044$ \\
\hline 16 & 475400116404201 & $06 / 22 / 99$ & 1700 & $<.044$ & $<.032$ & $<.130$ & $<.064$ & $<.032$ & $<.066$ & $<.044$ \\
\hline 17 & 475343117225201 & 06/10/99 & 0900 & $<.044$ & $<.032$ & $<.130$ & $<.064$ & $<.032$ & $<.066$ & $<.044$ \\
\hline 18 & 475322116522201 & $07 / 12 / 99$ & 1200 & $<.044$ & $<.032$ & $<.130$ & $<.064$ & $<.032$ & $<.066$ & $<.044$ \\
\hline $18^{\mathbf{R}}$ & & $07 / 12 / 99$ & 1201 & $<.044$ & $<.032$ & $<.130$ & $<.064$ & $<.032$ & $<.066$ & $<.044$ \\
\hline $18^{\mathbf{F b}}$ & & 07/12/99 & 1205 & $<.044$ & $<.032$ & $<.130$ & $<.064$ & $<.032$ & $<.066$ & $<.044$ \\
\hline $18^{\mathrm{Sb}}$ & & $07 / 12 / 99$ & 1207 & $<.044$ & $<.032$ & $<.130$ & $<.064$ & $<.032$ & $<.066$ & $<.044$ \\
\hline 19 & 475130117262201 & 06/07/99 & 0900 & $<.044$ & $<.032$ & $<.130$ & $<.064$ & $<.032$ & $<.066$ & $<.044$ \\
\hline 20 & 475035116424801 & $06 / 22 / 99$ & 1100 & $<.044$ & $<.032$ & $<.130$ & $<.064$ & $<.032$ & $<.066$ & $<.044$ \\
\hline 21 & 474718116530201 & $06 / 24 / 99$ & 0900 & $<.044$ & $<.032$ & $<.130$ & $<.064$ & $<.032$ & $<.066$ & $<.044$ \\
\hline $21^{\mathbf{R}}$ & & $06 / 24 / 99$ & 0901 & $<.044$ & $<.032$ & $<.130$ & $<.064$ & $<.032$ & $<.066$ & $<.044$ \\
\hline $21^{\mathbf{F b}}$ & & $06 / 24 / 99$ & 0905 & $<.044$ & $<.032$ & $<.130$ & $<.064$ & $<.032$ & $<.066$ & $<.044$ \\
\hline 22 & 474708117250501 & 06/07/99 & 1600 & $<.044$ & $<.032$ & $<.130$ & $<.064$ & $<.032$ & $<.066$ & $<.044$ \\
\hline 23 & 474629117305101 & $05 / 25 / 99$ & 1100 & $<.044$ & $<.032$ & $<.130$ & $<.064$ & $<.032$ & $<.066$ & $<.044$ \\
\hline $23^{\mathrm{Fb}}$ & & $05 / 26 / 99$ & 0905 & $<.044$ & $<.032$ & $<.130$ & $<.064$ & $<.032$ & $<.066$ & $<.044$ \\
\hline $23^{\mathrm{Sb}}$ & & $05 / 26 / 99$ & 0907 & $<.044$ & $<.032$ & $<.130$ & $<.064$ & $<.032$ & $<.066$ & $<.044$ \\
\hline 24 & 474456116522001 & $06 / 21 / 99$ & 1700 & $<.044$ & $<.032$ & $<.130$ & $<.064$ & $<.032$ & $<.066$ & $<.044$ \\
\hline 25 & 474427117312101 & $05 / 24 / 99$ & 1400 & $<.044$ & $<.032$ & $<.130$ & $<.064$ & $<.032$ & $<.066$ & $<.044$ \\
\hline 26 & 474317117225301 & 05/27/99 & 0900 & $<.044$ & e.082 & $<.130$ & $<.064$ & $<.032$ & $<.066$ & $<.044$ \\
\hline $26^{\mathbf{T b}}$ & & $05 / 27 / 99$ & 0908 & $<.044$ & $<.032$ & $<.130$ & $<.064$ & $<.032$ & $<.066$ & $<.044$ \\
\hline 27 & 474218116445601 & 07/15/99 & 1000 & $<.044$ & $<.032$ & $<.130$ & $<.064$ & $<.032$ & $<.066$ & $<.044$ \\
\hline 28 & 474147116544001 & 07/26/99 & 1300 & $<.044$ & $<.032$ & $<.130$ & $<.064$ & $<.032$ & $<.066$ & $<.044$ \\
\hline 29 & 474130117015401 & 07/29/99 & 1000 & $<.044$ & $<.032$ & $<.130$ & $<.064$ & $<.032$ & $<.066$ & $<.044$ \\
\hline 30 & 474050117084101 & 07/28/99 & 1700 & $<.044$ & $<.032$ & $<.130$ & $<.064$ & $<.032$ & $<.066$ & $<.044$ \\
\hline 31 & 473612117243601 & $05 / 26 / 99$ & 1100 & $<.044$ & $<.032$ & $<.130$ & $<.064$ & $<.032$ & .184 & $<.044$ \\
\hline
\end{tabular}


Table 20. Volatile organic-compound concentration data for ground-water samples collected from basin-fill aquifers, Northern Rockies Intermontane Basins study unit, 1999 and 2001 (Continued)

\begin{tabular}{|c|c|c|c|c|c|c|c|c|c|c|c|}
\hline $\begin{array}{c}\text { Well } \\
\text { number } \\
\text { (figs. } 3 \\
\text { and 4) }\end{array}$ & $\begin{array}{c}\text { 1,1- } \\
\text { Dichloro- } \\
\text { propene } \\
(\mu \mathrm{g} / \mathrm{L})\end{array}$ & $\begin{array}{c}1,2,3,4- \\
\text { Tetra- } \\
\text { methyl- } \\
\text { benzene } \\
(\mu \mathrm{g} / \mathrm{L})\end{array}$ & $\begin{array}{c}1,2,3,5- \\
\text { Tetra- } \\
\text { methyl- } \\
\text { benzene } \\
(\mu \mathrm{g} / \mathrm{L})\end{array}$ & $\begin{array}{c}\text { 1,2,3- } \\
\text { Trichloro- } \\
\text { benzene } \\
(\mu \mathrm{g} / \mathrm{L})\end{array}$ & $\begin{array}{c}\text { 1,2,3- } \\
\text { Trichloro- } \\
\text { propane } \\
(\mu \mathrm{g} / \mathrm{L})\end{array}$ & $\begin{array}{c}1,2,3- \\
\text { Trimethyl- } \\
\text { benzene } \\
(\mu \mathrm{g} / \mathrm{L})\end{array}$ & $\begin{array}{c}\text { 1,2,4- } \\
\text { Trichloro- } \\
\text { benzene } \\
(\mu \mathrm{g} / \mathrm{L})\end{array}$ & $\begin{array}{c}\text { 1,2,4- } \\
\text { Tri- } \\
\text { methyl- } \\
\text { benzene } \\
(\mu \mathrm{g} / \mathrm{L})\end{array}$ & $\begin{array}{c}\text { 1,2,- } \\
\text { Dibromo- } \\
\text { 3-chloro- } \\
\text { propane } \\
(\mu \mathrm{g} / \mathrm{L})\end{array}$ & $\begin{array}{c}\text { 1,2- } \\
\text { Dibromo- } \\
\text { ethane } \\
(\mu \mathrm{g} / \mathrm{L})\end{array}$ & $\begin{array}{c}\text { 1,2- } \\
\text { Dichloro- } \\
\text { benzene } \\
(\mu \mathrm{g} / \mathrm{L})\end{array}$ \\
\hline \multicolumn{12}{|c|}{ Subunit Survey 1, Idaho and Washington } \\
\hline 1 & $<0.026$ & $<0.230$ & $<0.200$ & $<0.270$ & $<0.160$ & $<0.120$ & $<0.190$ & $<0.056$ & $<0.210$ & $<0.036$ & $<0.048$ \\
\hline 2 & $<.026$ & $<.230$ & $<.200$ & $<.270$ & $<.160$ & $<.120$ & $<.190$ & $<.056$ & $<.210$ & $<.036$ & $<.048$ \\
\hline 3 & $<.026$ & $<.230$ & $<.200$ & $<.270$ & $<.160$ & $<.120$ & $<.190$ & $<.056$ & $<.210$ & $<.036$ & $<.048$ \\
\hline 4 & $<.026$ & $<.230$ & $<.200$ & $<.270$ & $<.160$ & $<.120$ & $<.190$ & $<.056$ & $<.210$ & $<.036$ & $<.048$ \\
\hline 5 & $<.026$ & $<.230$ & $<.200$ & $<.270$ & $<.160$ & $<.120$ & $<.190$ & $<.056$ & $<.210$ & $<.036$ & $<.048$ \\
\hline 6 & $<.026$ & $<.230$ & $<.200$ & $<.270$ & $<.160$ & $<.120$ & $<.190$ & $<.056$ & $<.210$ & $<.036$ & $<.048$ \\
\hline 7 & $<.026$ & $<.230$ & $<.200$ & $<.270$ & $<.160$ & $<.120$ & $<.190$ & $<.056$ & $<.210$ & $<.036$ & $<.048$ \\
\hline 8 & $<.026$ & $<.230$ & $<.200$ & $<.270$ & $<.160$ & $<.120$ & $<.190$ & $<.056$ & $<.210$ & $<.036$ & $<.048$ \\
\hline 9 & $<.026$ & $<.230$ & $<.200$ & $<.270$ & $<.160$ & $<.120$ & $<.190$ & $<.056$ & $<.210$ & $<.036$ & $<.048$ \\
\hline 10 & $<.026$ & $<.230$ & $<.200$ & $<.270$ & $<.160$ & $<.120$ & $<.190$ & $<.056$ & $<.210$ & $<.036$ & $<.048$ \\
\hline 11 & $<.026$ & $<.230$ & $<.200$ & $<.270$ & $<.160$ & $<.120$ & $<.190$ & $<.056$ & $<.210$ & $<.036$ & $<.048$ \\
\hline 12 & $<.026$ & $<.230$ & $<.200$ & $<.270$ & $<.160$ & $<.120$ & $<.190$ & $<.056$ & $<.210$ & $<.036$ & $<.048$ \\
\hline 13 & $<.026$ & $<.230$ & $<.200$ & $<.270$ & $<.160$ & $<.120$ & $<.190$ & $<.056$ & $<.210$ & $<.036$ & $<.048$ \\
\hline 13 & $<.026$ & $<.230$ & $<.200$ & $<.270$ & $<.160$ & $<.120$ & $<.190$ & $<.056$ & $<.210$ & $<.036$ & $<.048$ \\
\hline 14 & $<.026$ & $<.230$ & $<.200$ & $<.270$ & $<.160$ & $<.120$ & $<.190$ & $<.056$ & $<.210$ & $<.036$ & $<.048$ \\
\hline 15 & $<.026$ & $<.230$ & $<.200$ & $<.270$ & $<.160$ & $<.120$ & $<.190$ & $<.056$ & $<.210$ & $<.036$ & $<.048$ \\
\hline 16 & $<.026$ & $<.230$ & $<.200$ & $<.270$ & $<.160$ & $<.120$ & $<.190$ & $<.056$ & $<.210$ & $<.036$ & $<.048$ \\
\hline 17 & $<.026$ & $<.230$ & $<.200$ & $<.270$ & $<.160$ & $<.120$ & $<.190$ & $<.056$ & $<.210$ & $<.036$ & $<.048$ \\
\hline 18 & $<.026$ & $<.230$ & $<.200$ & $<.270$ & $<.160$ & $<.120$ & $<.190$ & $<.056$ & $<.210$ & $<.036$ & $<.048$ \\
\hline $18^{\mathbf{R}}$ & $<.026$ & $<.230$ & $<.200$ & $<.270$ & $<.160$ & $<.120$ & $<.190$ & $<.056$ & $<.210$ & $<.036$ & $<.048$ \\
\hline $18^{\mathbf{F b}}$ & $<.026$ & $<.230$ & $<.200$ & $<.270$ & $<.160$ & $<.120$ & $<.190$ & $<.056$ & $<.210$ & $<.036$ & $<.048$ \\
\hline $18^{\mathbf{S b}}$ & $<.026$ & $<.230$ & $<.200$ & $<.270$ & $<.160$ & $<.120$ & $<.190$ & $<.056$ & $<.210$ & $<.036$ & $<.048$ \\
\hline 19 & $<.026$ & $<.230$ & $<.200$ & $<.270$ & $<.160$ & $<.120$ & $<.190$ & $<.056$ & $<.210$ & $<.036$ & $<.048$ \\
\hline 20 & $<.026$ & $<.230$ & $<.200$ & $<.270$ & $<.160$ & $<.120$ & $<.190$ & $<.056$ & $<.210$ & $<.036$ & $<.048$ \\
\hline 21 & $<.026$ & $<.230$ & $<.200$ & $<.270$ & $<.160$ & $<.120$ & $<.190$ & $<.056$ & $<.210$ & $<.036$ & $<.048$ \\
\hline $21^{\mathbf{R}}$ & $<.026$ & $<.230$ & $<.200$ & $<.270$ & $<.160$ & $<.120$ & $<.190$ & $<.056$ & $<.210$ & $<.036$ & $<.048$ \\
\hline $21^{\mathbf{F b}}$ & $<.026$ & $<.230$ & $<.200$ & $<.270$ & $<.160$ & $<.120$ & $<.190$ & $<.056$ & $<.210$ & $<.036$ & $<.048$ \\
\hline 22 & $<.026$ & $<.230$ & $<.200$ & $<.270$ & $<.160$ & $<.120$ & $<.190$ & $<.056$ & $<.210$ & $<.036$ & $<.048$ \\
\hline 23 & $<.026$ & $<.230$ & $<.200$ & $<.270$ & $<.160$ & $<.120$ & $<.190$ & $<.056$ & $<.210$ & $<.036$ & $<.048$ \\
\hline $23^{\mathrm{Fb}}$ & $<.026$ & $<.230$ & $<.200$ & $<.270$ & $<.160$ & $<.120$ & $<.190$ & $<.056$ & $<.210$ & $<.036$ & $<.048$ \\
\hline $23^{\mathrm{Sb}}$ & $<.026$ & $<.230$ & $<.200$ & $<.270$ & $<.160$ & $<.120$ & $<.190$ & $<.056$ & $<.210$ & $<.036$ & $<.048$ \\
\hline 24 & $<.026$ & $<.230$ & $<.200$ & $<.270$ & $<.160$ & $<.120$ & $<.190$ & $<.056$ & $<.210$ & $<.036$ & $<.048$ \\
\hline 25 & $<.026$ & $<.230$ & $<.200$ & $<.270$ & $<.160$ & $<.120$ & $<.190$ & $<.056$ & $<.210$ & $<.036$ & $<.048$ \\
\hline 26 & $<.026$ & $<.230$ & $<.200$ & $<.270$ & $<.160$ & $<.120$ & $<.190$ & $<.056$ & $<.210$ & $<.036$ & $<.048$ \\
\hline $26^{\mathbf{T b}}$ & $<.026$ & $<.230$ & $<.200$ & $<.270$ & $<.160$ & $<.120$ & $<.190$ & $<.056$ & $<.210$ & $<.036$ & $<.048$ \\
\hline 27 & $<.026$ & $<.230$ & $<.200$ & $<.270$ & $<.160$ & $<.120$ & $<.190$ & $<.056$ & $<.210$ & $<.036$ & $<.048$ \\
\hline 28 & $<.026$ & $<.230$ & $<.200$ & $<.270$ & $<.160$ & $<.120$ & $<.190$ & $<.056$ & $<.210$ & $<.036$ & $<.048$ \\
\hline 29 & $<.026$ & $<.230$ & $<.200$ & $<.270$ & $<.160$ & $<.120$ & $<.190$ & $<.056$ & $<.210$ & $<.036$ & $<.048$ \\
\hline 30 & $<.026$ & $<.230$ & $<.200$ & $<.270$ & $<.160$ & $<.120$ & $<.190$ & $<.056$ & $<.210$ & $<.036$ & $<.048$ \\
\hline 31 & $<.026$ & $<.230$ & $<.200$ & $<.270$ & $<.160$ & $<.120$ & $<.190$ & $<.056$ & $<.210$ & $<.036$ & $<.048$ \\
\hline
\end{tabular}


Table 20. Volatile organic-compound concentration data for ground-water samples collected from basin-fill aquifers, Northern Rockies Intermontane Basins study unit, 1999 and 2001 (Continued)

\begin{tabular}{|c|c|c|c|c|c|c|c|c|c|c|}
\hline $\begin{array}{c}\text { Well } \\
\text { number } \\
\text { (figs. } 3 \\
\text { and 4) }\end{array}$ & $\begin{array}{c}\text { 1,2- } \\
\text { Dichloro- } \\
\text { ethane } \\
(\mu \mathrm{g} / \mathrm{L})\end{array}$ & $\begin{array}{c}1,2,- \\
\text { Dichloro- } \\
\text { ethane-d }{ }_{4}, \\
\text { surrogate } \\
\text { (percent) }\end{array}$ & $\begin{array}{c}\text { 1,2- } \\
\text { Dichloro- } \\
\text { propane } \\
(\mu \mathbf{g} / \mathbf{L})\end{array}$ & $\begin{array}{c}1,3,5- \\
\text { Trimethyl- } \\
\text { benzene } \\
(\mu \mathrm{g} / \mathrm{L})\end{array}$ & $\begin{array}{c}\text { 1,3- } \\
\text { Dichloro- } \\
\text { benzene } \\
(\mu \mathrm{g} / \mathrm{L})\end{array}$ & $\begin{array}{c}\text { 1,3- } \\
\text { Dichloro- } \\
\text { propane } \\
(\mu \mathrm{g} / \mathrm{L})\end{array}$ & $\begin{array}{c}\text { 1,4-Bromo- } \\
\text { fluoro- } \\
\text { benzene, } \\
\text { surrogate } \\
\text { (percent) }\end{array}$ & $\begin{array}{c}1,4- \\
\text { Dichloro- } \\
\text { benzene } \\
(\mu \mathrm{g} / \mathrm{L})\end{array}$ & $\begin{array}{c}2,2- \\
\text { Dichloro- } \\
\text { propane } \\
(\mu \mathrm{g} / \mathrm{L})\end{array}$ & $\begin{array}{c}\text { 2-Butanone } \\
(\mu \mathrm{g} / \mathrm{L})\end{array}$ \\
\hline \multicolumn{11}{|c|}{ Subunit Survey 1, Idaho and Washington } \\
\hline 1 & $<0.130$ & 105 & $<0.068$ & $<0.044$ & $<0.054$ & $<0.120$ & 101 & $<0.050$ & $<0.078$ & $<1.60$ \\
\hline 2 & $<.130$ & 103 & $<.068$ & $<.044$ & $<.054$ & $<.120$ & 102 & $<.050$ & $<.078$ & $<1.60$ \\
\hline 3 & $<.130$ & 110 & $<.068$ & $<.044$ & $<.054$ & $<.120$ & 94 & $<.050$ & $<.078$ & $<1.60$ \\
\hline 4 & $<.130$ & 101 & $<.068$ & $<.044$ & $<.054$ & $<.120$ & 81 & $<.050$ & $<.078$ & $<1.60$ \\
\hline 5 & $<.130$ & 109 & $<.068$ & $<.044$ & $<.054$ & $<.120$ & 94 & $<.050$ & $<.078$ & $<1.60$ \\
\hline 6 & $<.130$ & 100 & $<.068$ & $<.044$ & $<.054$ & $<.120$ & 81 & $<.050$ & $<.078$ & $<1.60$ \\
\hline 7 & $<.130$ & 101 & $<.068$ & $<.044$ & $<.054$ & $<.120$ & 101 & $<.050$ & $<.078$ & $<1.60$ \\
\hline 8 & $<.130$ & 108 & $<.068$ & $<.044$ & $<.054$ & $<.120$ & 103 & $<.050$ & $<.078$ & $<1.60$ \\
\hline 9 & $<.130$ & 99 & $<.068$ & $<.044$ & $<.054$ & $<.120$ & 93 & $<.050$ & $<.078$ & $<1.60$ \\
\hline 10 & $<.130$ & 110 & $<.068$ & $<.044$ & $<.054$ & $<.120$ & 110 & $<.050$ & $<.078$ & $<1.60$ \\
\hline 11 & $<.130$ & 107 & $<.068$ & $<.044$ & $<.054$ & $<.120$ & 94 & $<.050$ & $<.078$ & $<1.60$ \\
\hline 12 & $<.130$ & 109 & $<.068$ & $<.044$ & $<.054$ & $<.120$ & 108 & $<.050$ & $<.078$ & $<1.60$ \\
\hline 13 & $<.130$ & 112 & $<.068$ & $<.044$ & $<.054$ & $<.120$ & 103 & $<.050$ & $<.078$ & $<1.60$ \\
\hline 13 & $<.130$ & 100 & $<.068$ & $<.044$ & $<.054$ & $<.120$ & 99 & $<.050$ & $<.078$ & $<1.60$ \\
\hline 14 & $<.130$ & 105 & $<.068$ & $<.044$ & $<.054$ & $<.120$ & 108 & $<.050$ & $<.078$ & $<1.60$ \\
\hline 15 & $<.130$ & 100 & $<.068$ & $<.044$ & $<.054$ & $<.120$ & 93 & $<.050$ & $<.078$ & $<1.60$ \\
\hline 16 & $<.130$ & 109 & $<.068$ & $<.044$ & $<.054$ & $<.120$ & 104 & $<.050$ & $<.078$ & $<1.60$ \\
\hline 17 & $<.130$ & 105 & $<.068$ & $<.044$ & $<.054$ & $<.120$ & 112 & $<.050$ & $<.078$ & $<1.60$ \\
\hline 18 & $<.130$ & 103 & $<.068$ & $<.044$ & $<.054$ & $<.120$ & 101 & $<.050$ & $<.078$ & $<1.60$ \\
\hline $18^{\mathbf{R}}$ & $<.130$ & 100 & $<.068$ & $<.044$ & $<.054$ & $<.120$ & 101 & $<.050$ & $<.078$ & $<1.60$ \\
\hline $18^{\mathbf{F b}}$ & $<.130$ & 106 & $<.068$ & $<.044$ & $<.054$ & $<.120$ & 95 & $<.050$ & $<.078$ & $<1.60$ \\
\hline $18^{\mathbf{S b}}$ & $<.130$ & 106 & $<.068$ & $<.044$ & $<.054$ & $<.120$ & 95 & $<.050$ & $<.078$ & $<1.60$ \\
\hline 19 & $<.130$ & 105 & $<.068$ & $<.044$ & $<.054$ & $<.120$ & 103 & $<.050$ & $<.078$ & $<1.60$ \\
\hline 20 & $<.130$ & 106 & $<.068$ & $<.044$ & $<.054$ & $<.120$ & 102 & $<.050$ & $<.078$ & $<1.60$ \\
\hline 21 & $<.130$ & 106 & $<.068$ & $<.044$ & $<.054$ & $<.120$ & 96 & $<.050$ & $<.078$ & $<1.60$ \\
\hline $21^{\mathbf{R}}$ & $<.130$ & 112 & $<.068$ & $<.044$ & $<.054$ & $<.120$ & 99 & $<.050$ & $<.078$ & $<1.60$ \\
\hline $21^{\mathbf{F b}}$ & $<.130$ & 103 & $<.068$ & $<.044$ & $<.054$ & $<.120$ & 73 & $<.050$ & $<.078$ & $<1.60$ \\
\hline 22 & $<.130$ & 108 & $<.068$ & $<.044$ & $<.054$ & $<.120$ & 105 & $<.050$ & $<.078$ & $<1.60$ \\
\hline 23 & $<.130$ & 101 & $<.068$ & $<.044$ & $<.054$ & $<.120$ & 94 & $<.050$ & $<.078$ & $<1.60$ \\
\hline $23^{\mathbf{F b}}$ & $<.130$ & 102 & $<.068$ & $<.044$ & $<.054$ & $<.120$ & 92 & $<.050$ & $<.078$ & $<1.60$ \\
\hline $23^{\mathrm{Sb}}$ & $<.130$ & 103 & $<.068$ & $<.044$ & $<.054$ & $<.120$ & 92 & $<.050$ & $<.078$ & $<1.60$ \\
\hline 24 & $<.130$ & 110 & $<.068$ & $<.044$ & $<.054$ & $<.120$ & 103 & $<.050$ & $<.078$ & $<1.60$ \\
\hline 25 & $<.130$ & 97 & $<.068$ & $<.044$ & $<.054$ & $<.120$ & 92 & $<.050$ & $<.078$ & $<1.60$ \\
\hline 26 & $<.130$ & 105 & $<.068$ & $<.044$ & $<.054$ & $<.120$ & 94 & $<.050$ & $<.078$ & $<1.60$ \\
\hline $26^{\mathbf{T b}}$ & $<.130$ & 104 & $<.068$ & $<.044$ & $<.054$ & $<.120$ & 91 & $<.050$ & $<.078$ & $<1.60$ \\
\hline 27 & $<.130$ & 106 & $<.068$ & $<.044$ & $<.054$ & $<.120$ & 100 & $<.050$ & $<.078$ & $<1.60$ \\
\hline 28 & $<.130$ & 108 & $<.068$ & $<.044$ & $<.054$ & $<.120$ & 100 & $<.050$ & $<.078$ & $<1.60$ \\
\hline 29 & $<.130$ & 107 & $<.068$ & $<.044$ & $<.054$ & $<.120$ & 89 & $<.050$ & $<.078$ & $<1.60$ \\
\hline 30 & $<.130$ & 105 & $<.068$ & $<.044$ & $<.054$ & $<.120$ & 86 & $<.050$ & $<.078$ & $<1.60$ \\
\hline 31 & $<.130$ & 99 & $<.068$ & $<.044$ & $<.054$ & $<.120$ & 90 & $<.050$ & $<.078$ & $<1.60$ \\
\hline
\end{tabular}


Table 20. Volatile organic-compound concentration data for ground-water samples collected from basin-fill aquifers, Northern Rockies Intermontane Basins study unit, 1999 and 2001 (Continued)

\begin{tabular}{|c|c|c|c|c|c|c|c|c|c|c|}
\hline $\begin{array}{c}\text { Well } \\
\text { number } \\
\text { (figs. } 3 \\
\text { and 4) }\end{array}$ & $\begin{array}{c}\text { 2-Chloro- } \\
\text { toluene } \\
(\mu \mathrm{g} / \mathrm{L})\end{array}$ & $\begin{array}{c}2- \\
\text { Hexanone } \\
(\mu \mathrm{g} / \mathrm{L})\end{array}$ & $\begin{array}{c}\text { 3-Chloro- } \\
\text { propene } \\
(\mu \mathrm{g} / \mathrm{L})\end{array}$ & $\begin{array}{l}\text { 4-Chloro- } \\
\text { toluene } \\
(\mu \mathrm{g} / \mathrm{L})\end{array}$ & $\begin{array}{c}\text { 4-Isopro- } \\
\text { pyl-1- } \\
\text { methyl- } \\
\text { benzene } \\
(\mu \mathrm{g} / \mathrm{L})\end{array}$ & $\begin{array}{c}\text { 4-Methyl-2- } \\
\text { pentanone } \\
(\mu \mathrm{g} / \mathrm{L})\end{array}$ & $\begin{array}{c}\text { Ace- } \\
\text { tone } \\
(\mu \mathrm{g} / \mathrm{L})\end{array}$ & $\begin{array}{c}\text { Acrylo- } \\
\text { nitrile } \\
(\mu \mathrm{g} / \mathrm{L})\end{array}$ & $\begin{array}{c}\text { Benzene } \\
(\mu \mathrm{g} / \mathrm{L})\end{array}$ & $\begin{array}{c}\text { Bromo- } \\
\text { benzene } \\
(\mu \mathrm{g} / \mathrm{L})\end{array}$ \\
\hline \multicolumn{11}{|c|}{ Subunit Survey 1, Idaho and Washington } \\
\hline 1 & $<0.042$ & $<0.700$ & $<0.200$ & $<0.056$ & $<0.110$ & $<0.370$ & $<5.00$ & $<1.20$ & $<0.100$ & $<0.036$ \\
\hline 2 & $<.042$ & $<.700$ & $<.200$ & $<.056$ & $<.110$ & $<.370$ & $<5.00$ & $<1.20$ & $<.100$ & $<.036$ \\
\hline 3 & $<.042$ & $<.700$ & $<.200$ & $<.056$ & $<.110$ & $<.370$ & $<5.00$ & $<1.20$ & $<.100$ & $<.036$ \\
\hline 4 & $<.042$ & $<.700$ & $<.200$ & $<.056$ & $<.110$ & $<.370$ & $<5.00$ & $<1.20$ & $<.100$ & $<.036$ \\
\hline 5 & $<.042$ & $<.700$ & $<.200$ & $<.056$ & $<.110$ & $<.370$ & $<5.00$ & $<1.20$ & $<.100$ & $<.036$ \\
\hline 6 & $<.042$ & $<.700$ & $<.200$ & $<.056$ & $<.110$ & $<.370$ & $<5.00$ & $<1.20$ & $<.100$ & $<.036$ \\
\hline 7 & $<.042$ & $<.700$ & $<.200$ & $<.056$ & $<.110$ & $<.370$ & $<5.00$ & $<1.20$ & $<.100$ & $<.036$ \\
\hline 8 & $<.042$ & $<.700$ & $<.200$ & $<.056$ & $<.110$ & $<.370$ & $<5.00$ & $<1.20$ & $<.100$ & $<.036$ \\
\hline 9 & $<.042$ & $<.700$ & $<.200$ & $<.056$ & $<.110$ & $<.370$ & $<5.00$ & $<1.20$ & $<.100$ & $<.036$ \\
\hline 10 & $<.042$ & $<.700$ & $<.200$ & $<.056$ & $<.110$ & $<.370$ & $<5.00$ & $<1.20$ & $<.100$ & $<.036$ \\
\hline 11 & $<.042$ & $<.700$ & $<.200$ & $<.056$ & $<.110$ & $<.370$ & $<5.00$ & $<1.20$ & $<.100$ & $<.036$ \\
\hline 12 & $<.042$ & $<.700$ & $<.200$ & $<.056$ & $<.110$ & $<.370$ & $<5.00$ & $<1.20$ & $<.100$ & $<.036$ \\
\hline 13 & $<.042$ & $<.700$ & $<.200$ & $<.056$ & $<.110$ & $<.370$ & $<5.00$ & $<1.20$ & $<.100$ & $<.036$ \\
\hline 13 & $<.042$ & $<.700$ & $<.200$ & $<.056$ & $<.110$ & $<.370$ & $<5.00$ & $<1.20$ & $<.100$ & $<.036$ \\
\hline 14 & $<.042$ & $<.700$ & $<.200$ & $<.056$ & $<.110$ & $<.370$ & $<5.00$ & $<1.20$ & $<.100$ & $<.036$ \\
\hline 15 & $<.042$ & $<.700$ & $<.200$ & $<.056$ & $<.110$ & $<.370$ & $<5.00$ & $<1.20$ & $<.100$ & $<.036$ \\
\hline 16 & $<.042$ & $<.700$ & $<.200$ & $<.056$ & $<.110$ & $<.370$ & $<5.00$ & $<1.20$ & $<.100$ & $<.036$ \\
\hline 17 & $<.042$ & $<.700$ & $<.200$ & $<.056$ & $<.110$ & $<.370$ & $<5.00$ & $<1.20$ & $<.100$ & $<.036$ \\
\hline 18 & $<.042$ & $<.700$ & $<.200$ & $<.056$ & $<.110$ & $<.370$ & $<5.00$ & $<1.20$ & $<.100$ & $<.036$ \\
\hline $18^{\mathbf{R}}$ & $<.042$ & $<.700$ & $<.200$ & $<.056$ & $<.110$ & $<.370$ & $<5.00$ & $<1.20$ & $<.100$ & $<.036$ \\
\hline $18^{\mathbf{F b}}$ & $<.042$ & $<.700$ & $<.200$ & $<.056$ & $<.110$ & $<.370$ & $<5.00$ & $<1.20$ & $<.100$ & $<.036$ \\
\hline $18^{\mathbf{S b}}$ & $<.042$ & $<.700$ & $<.200$ & $<.056$ & $<.110$ & $<.370$ & $<5.00$ & $<1.20$ & $<.100$ & $<.036$ \\
\hline 19 & $<.042$ & $<.700$ & $<.200$ & $<.056$ & $<.110$ & $<.370$ & $<5.00$ & $<1.20$ & $<.100$ & $<.036$ \\
\hline 20 & $<.042$ & $<.700$ & $<.200$ & $<.056$ & $<.110$ & $<.370$ & $<5.00$ & $<1.20$ & $<.100$ & $<.036$ \\
\hline 21 & $<.042$ & $<.700$ & $<.200$ & $<.056$ & $<.110$ & $<.370$ & $<5.00$ & $<1.20$ & $<.100$ & $<.036$ \\
\hline $21^{R}$ & $<.042$ & $<.700$ & $<.200$ & $<.056$ & $<.110$ & $<.370$ & $<5.00$ & $<1.20$ & $<.100$ & $<.036$ \\
\hline $21^{\mathbf{F b}}$ & $<.042$ & $<.700$ & $<.200$ & $<.056$ & $<.110$ & $<.370$ & $<5.00$ & $<1.20$ & $<.100$ & $<.036$ \\
\hline 22 & $<.042$ & $<.700$ & $<.200$ & $<.056$ & $<.110$ & $<.370$ & $<5.00$ & $<1.20$ & $<.100$ & $<.036$ \\
\hline 23 & $<.042$ & $<.700$ & $<.200$ & $<.056$ & $<.110$ & $<.370$ & $<5.00$ & $<1.20$ & $<.100$ & $<.036$ \\
\hline $23^{\mathrm{Fb}}$ & $<.042$ & $<.700$ & $<.200$ & $<.056$ & $<.110$ & $<.370$ & $<5.00$ & $<1.20$ & $<.100$ & $<.036$ \\
\hline $23^{\mathbf{S b}}$ & $<.042$ & $<.700$ & $<.200$ & $<.056$ & $<.110$ & $<.370$ & $<5.00$ & $<1.20$ & $<.100$ & $<.036$ \\
\hline 24 & $<.042$ & $<.700$ & $<.200$ & $<.056$ & $<.110$ & $<.370$ & $<5.00$ & $<1.20$ & $<.100$ & $<.036$ \\
\hline 25 & $<.042$ & $<.700$ & $<.200$ & $<.056$ & $<.110$ & $<.370$ & $<5.00$ & $<1.20$ & $<.100$ & $<.036$ \\
\hline 26 & $<.042$ & $<.700$ & $<.200$ & $<.056$ & $<.110$ & $<.370$ & $<5.00$ & $<1.20$ & $<.100$ & $<.036$ \\
\hline $26^{\mathbf{T b}}$ & $<.042$ & $<.700$ & $<.200$ & $<.056$ & $<.110$ & $<.370$ & $<5.00$ & $<1.20$ & $<.100$ & $<.036$ \\
\hline 27 & $<.042$ & $<.700$ & $<.200$ & $<.056$ & $<.110$ & $<.370$ & $<5.00$ & $<1.20$ & $<.100$ & $<.036$ \\
\hline 28 & $<.042$ & $<.700$ & $<.200$ & $<.056$ & $<.110$ & $<.370$ & $<5.00$ & $<1.20$ & $<.100$ & $<.036$ \\
\hline 29 & $<.042$ & $<.700$ & $<.200$ & $<.056$ & $<.110$ & $<.370$ & $<5.00$ & $<1.20$ & $<.100$ & $<.036$ \\
\hline 30 & $<.042$ & $<.700$ & $<.200$ & $<.056$ & $<.110$ & $<.370$ & $<5.00$ & $<1.20$ & $<.100$ & $<.036$ \\
\hline 31 & $<.042$ & $<.700$ & $<.200$ & $<.056$ & $<.110$ & $<.370$ & $<5.00$ & $<1.20$ & $<.100$ & $<.036$ \\
\hline
\end{tabular}


Table 20. Volatile organic-compound concentration data for ground-water samples collected from basin-fill aquifers, Northern Rockies Intermontane Basins study unit, 1999 and 2001 (Continued)

\begin{tabular}{|c|c|c|c|c|c|c|c|c|c|c|}
\hline $\begin{array}{c}\text { Well } \\
\text { number } \\
\text { (figs. } 3 \\
\text { and 4) }\end{array}$ & $\begin{array}{c}\text { Bromo- } \\
\text { chloro- } \\
\text { meth- } \\
\text { thane } \\
(\mu \mathrm{g} / \mathrm{L})\end{array}$ & $\begin{array}{c}\text { Bromo- } \\
\text { dichloro- } \\
\text { methane } \\
(\mu \mathrm{g} / \mathrm{L})\end{array}$ & $\begin{array}{c}\text { Bromo- } \\
\text { ethane } \\
(\mu \mathrm{g} / \mathrm{L})\end{array}$ & $\begin{array}{c}\text { Bromo- } \\
\text { form } \\
(\mu \mathrm{g} / \mathrm{L})\end{array}$ & $\begin{array}{c}\text { Bromo- } \\
\text { methane } \\
(\mu \mathrm{g} / \mathrm{L})\end{array}$ & $\begin{array}{c}\text { Butyl- } \\
\text { benzene } \\
(\mu \mathrm{g} / \mathrm{L})\end{array}$ & $\begin{array}{c}\text { Carbon } \\
\text { disulfide } \\
(\mu \mathrm{g} / \mathrm{L})\end{array}$ & $\begin{array}{c}\text { Chloro- } \\
\text { benzene } \\
(\mu \mathrm{g} / \mathrm{L})\end{array}$ & $\begin{array}{c}\text { Chloro- } \\
\text { ethane } \\
(\mu \mathrm{g} / \mathrm{L})\end{array}$ & $\begin{array}{c}\text { Chloro- } \\
\text { form } \\
(\mu \mathrm{g} / \mathrm{L})\end{array}$ \\
\hline \multicolumn{11}{|c|}{ Subunit Survey 1, Idaho and Washington } \\
\hline 1 & $<0.044$ & $<0.048$ & $<0.100$ & $<0.100$ & $<0.150$ & $<0.190$ & $<0.370$ & $<0.028$ & $<0.120$ & $<0.052$ \\
\hline 2 & $<.044$ & $<.048$ & $<.100$ & $<.100$ & $<.150$ & $<.190$ & $<.370$ & $<.028$ & $<.120$ & $<.052$ \\
\hline 3 & $<.044$ & $<.048$ & $<.100$ & $<.100$ & $<.150$ & $<.190$ & $<.370$ & $<.028$ & $<.120$ & $<.052$ \\
\hline 4 & $<.044$ & $<.048$ & $<.100$ & $<.100$ & $<.150$ & $<.190$ & $<.370$ & $<.028$ & $<.120$ & $<.052$ \\
\hline 5 & $<.044$ & $<.048$ & $<.100$ & $<.100$ & $<.150$ & $<.190$ & $<.370$ & $<.028$ & $<.120$ & $<.052$ \\
\hline 6 & $<.044$ & $<.048$ & $<.100$ & $<.100$ & $<.150$ & $<.190$ & $<.370$ & $<.028$ & $<.120$ & $<.052$ \\
\hline 7 & $<.044$ & $<.048$ & $<.100$ & $<.100$ & $<.150$ & $<.190$ & $<.370$ & $<.028$ & $<.120$ & e.024 \\
\hline 8 & $<.044$ & $<.048$ & $<.100$ & $<.100$ & $<.150$ & $<.190$ & $<.370$ & $<.028$ & $<.120$ & $<.052$ \\
\hline 9 & $<.044$ & $<.048$ & $<.100$ & $<.100$ & $<.150$ & $<.190$ & $<.370$ & $<.028$ & $<.120$ & $<.052$ \\
\hline 10 & $<.044$ & $<.048$ & $<.100$ & $<.100$ & $<.150$ & $<.190$ & $<.370$ & $<.028$ & $<.120$ & $<.052$ \\
\hline 11 & $<.044$ & $<.048$ & $<.100$ & $<.100$ & $<.150$ & $<.190$ & $<.370$ & $<.028$ & $<.120$ & $<.052$ \\
\hline 12 & $<.044$ & $<.048$ & $<.100$ & $<.100$ & $<.150$ & $<.190$ & $<.370$ & $<.028$ & $<.120$ & $<.052$ \\
\hline 13 & $<.044$ & $<.048$ & $<.100$ & $<.100$ & $<.150$ & $<.190$ & $<.370$ & $<.028$ & $<.120$ & $<.052$ \\
\hline 13 & $<.044$ & $<.048$ & $<.100$ & $<.100$ & $<.150$ & $<.190$ & $<.370$ & $<.028$ & $<.120$ & $<.052$ \\
\hline 14 & $<.044$ & $<.048$ & $<.100$ & $<.100$ & $<.150$ & $<.190$ & $<.370$ & $<.028$ & $<.120$ & $<.052$ \\
\hline 15 & $<.044$ & $<.048$ & $<.100$ & $<.100$ & $<.150$ & $<.190$ & $<.370$ & $<.028$ & $<.120$ & $<.052$ \\
\hline 16 & $<.044$ & $<.048$ & $<.100$ & $<.100$ & $<.150$ & $<.190$ & $<.370$ & $<.028$ & $<.120$ & $<.052$ \\
\hline 17 & $<.044$ & $<.048$ & $<.100$ & $<.100$ & $<.150$ & $<.190$ & $<.370$ & $<.028$ & $<.120$ & $<.052$ \\
\hline 18 & $<.044$ & $<.048$ & $<.100$ & $<.100$ & $<.150$ & $<.190$ & $<.370$ & $<.028$ & $<.120$ & $<.052$ \\
\hline $18^{\mathbf{R}}$ & $<.044$ & $<.048$ & $<.100$ & $<.100$ & $<.150$ & $<.190$ & $<.370$ & $<.028$ & $<.120$ & $<.052$ \\
\hline $18^{\mathbf{F b}}$ & $<.044$ & $<.048$ & $<.100$ & $<.100$ & $<.150$ & $<.190$ & $<.370$ & $<.028$ & $<.120$ & $<.052$ \\
\hline $18^{\mathbf{S b}}$ & $<.044$ & $<.048$ & $<.100$ & $<.100$ & $<.150$ & $<.190$ & $<.370$ & $<.028$ & $<.120$ & $<.052$ \\
\hline 19 & $<.044$ & $<.048$ & $<.100$ & $<.100$ & $<.150$ & $<.190$ & $<.370$ & $<.028$ & $<.120$ & $<.052$ \\
\hline 20 & $<.044$ & $<.048$ & $<.100$ & $<.100$ & $<.150$ & $<.190$ & $<.370$ & $<.028$ & $<.120$ & $<.052$ \\
\hline 21 & $<.044$ & $<.048$ & $<.100$ & $<.100$ & $<.150$ & $<.190$ & $<.370$ & $<.028$ & $<.120$ & $<.052$ \\
\hline $21^{\mathbf{R}}$ & $<.044$ & $<.048$ & $<.100$ & $<.100$ & $<.150$ & $<.190$ & $<.370$ & $<.028$ & $<.120$ & $<.052$ \\
\hline $21^{\mathbf{F b}}$ & $<.044$ & $<.048$ & $<.100$ & $<.100$ & $<.150$ & $<.190$ & $<.370$ & $<.028$ & $<.120$ & $<.052$ \\
\hline 22 & $<.044$ & $<.048$ & $<.100$ & $<.100$ & $<.150$ & $<.190$ & $<.370$ & $<.028$ & $<.120$ & $<.052$ \\
\hline 23 & $<.044$ & $<.048$ & $<.100$ & $<.100$ & $<.150$ & $<.190$ & $<.370$ & $<.028$ & $<.120$ & $<.052$ \\
\hline $23^{\mathrm{Fb}}$ & $<.044$ & $<.048$ & $<.100$ & $<.100$ & $<.150$ & $<.190$ & $<.370$ & $<.028$ & $<.120$ & $<.052$ \\
\hline $23^{\mathrm{Sb}}$ & $<.044$ & $<.048$ & $<.100$ & $<.100$ & $<.150$ & $<.190$ & $<.370$ & $<.028$ & $<.120$ & $<.052$ \\
\hline 24 & $<.044$ & $<.048$ & $<.100$ & $<.100$ & $<.150$ & $<.190$ & $<.370$ & $<.028$ & $<.120$ & $<.052$ \\
\hline 25 & $<.044$ & $<.048$ & $<.100$ & $<.100$ & $<.150$ & $<.190$ & $<.370$ & $<.028$ & $<.120$ & $<.052$ \\
\hline 26 & $<.044$ & $<.048$ & $<.100$ & $<.100$ & $<.150$ & $<.190$ & $<.370$ & $<.028$ & $<.120$ & $<.052$ \\
\hline $26^{\mathbf{T b}}$ & $<.044$ & $<.048$ & $<.100$ & $<.100$ & $<.150$ & $<.190$ & $<.370$ & $<.028$ & $<.120$ & $<.052$ \\
\hline 27 & $<.044$ & $<.048$ & $<.100$ & $<.100$ & $<.150$ & $<.190$ & $<.370$ & $<.028$ & $<.120$ & $<.052$ \\
\hline 28 & $<.044$ & $<.048$ & $<.100$ & $<.100$ & $<.150$ & $<.190$ & $<.370$ & $<.028$ & $<.120$ & $<.052$ \\
\hline 29 & $<.044$ & $<.048$ & $<.100$ & $<.100$ & $<.150$ & $<.190$ & $<.370$ & $<.028$ & $<.120$ & $<.052$ \\
\hline 30 & $<.044$ & $<.048$ & $<.100$ & $<.100$ & $<.150$ & $<.190$ & $<.370$ & $<.028$ & $<.120$ & e.021 \\
\hline 31 & $<.044$ & $<.048$ & $<.100$ & $<.100$ & $<.150$ & $<.190$ & $<.370$ & $<.028$ & $<.120$ & $<.052$ \\
\hline
\end{tabular}


Table 20. Volatile organic-compound concentration data for ground-water samples collected from basin-fill aquifers, Northern Rockies Intermontane Basins study unit, 1999 and 2001 (Continued)

\begin{tabular}{|c|c|c|c|c|c|c|c|c|c|c|}
\hline $\begin{array}{c}\text { Well } \\
\text { number } \\
\text { (figs. } 3 \\
\text { and 4) }\end{array}$ & $\begin{array}{c}\text { Chloro- } \\
\text { methane } \\
(\mu \mathrm{g} / \mathrm{L})\end{array}$ & $\begin{array}{c}\text { cis- } \\
\text { 1,2- } \\
\text { Dichloro- } \\
\text { ethylene } \\
(\mu \mathrm{g} / \mathrm{L})\end{array}$ & $\begin{array}{c}\text { cis- } \\
\text { 1,3- } \\
\text { Dichloro- } \\
\text { propene } \\
(\mu \mathrm{g} / \mathrm{L})\end{array}$ & $\begin{array}{c}\text { Dibromo- } \\
\text { chloro- } \\
\text { methane } \\
(\mu \mathrm{g} / \mathrm{L})\end{array}$ & $\begin{array}{c}\text { Dibromo- } \\
\text { methane } \\
(\mu \mathrm{g} / \mathrm{L})\end{array}$ & $\begin{array}{c}\text { Dichloro- } \\
\text { difluoro- } \\
\text { methane } \\
(\mu \mathrm{g} / \mathrm{L})\end{array}$ & $\begin{array}{c}\text { Dichloro- } \\
\text { methane } \\
(\mu \mathrm{g} / \mathrm{L})\end{array}$ & $\begin{array}{l}\text { Diethyl } \\
\text { ether } \\
(\mu \mathrm{g} / \mathrm{L})\end{array}$ & $\begin{array}{l}\text { Diiso- } \\
\text { propyl } \\
\text { ether } \\
(\mu \mathrm{g} / \mathrm{L})\end{array}$ & $\begin{array}{c}\text { Ethyl } \\
\text { meth- } \\
\text { acrylate } \\
(\mu \mathrm{g} / \mathrm{L})\end{array}$ \\
\hline \multicolumn{11}{|c|}{ Subunit Survey 1, Idaho and Washington } \\
\hline 1 & $<0.250$ & $<0.038$ & $<0.090$ & $<0.180$ & $<0.050$ & $<0.140$ & $<0.380$ & $<0.170$ & $<0.098$ & $<0.280$ \\
\hline 2 & $<.250$ & $<.038$ & $<.090$ & $<.180$ & $<.050$ & $\mathrm{e} .254$ & $<.380$ & $<.170$ & $<.098$ & $<.280$ \\
\hline 3 & $<.250$ & $<.038$ & $<.090$ & $<.180$ & $<.050$ & $<.140$ & $<.380$ & $<.170$ & $<.098$ & $<.280$ \\
\hline 4 & $<.250$ & $<.038$ & $<.090$ & $<.180$ & $<.050$ & $<.140$ & $<.380$ & $<.170$ & $<.098$ & $<.280$ \\
\hline 5 & $<.250$ & $<.038$ & $<.090$ & $<.180$ & $<.050$ & $<.140$ & $<.380$ & $<.170$ & $<.098$ & $<.280$ \\
\hline 6 & $<.250$ & $<.038$ & $<.090$ & $<.180$ & $<.050$ & $<.140$ & $<.380$ & $<.170$ & $<.098$ & $<.280$ \\
\hline 7 & $<.250$ & $<.038$ & $<.090$ & $<.180$ & $<.050$ & $<.140$ & $<.380$ & $<.170$ & $<.098$ & $<.280$ \\
\hline 8 & $<.250$ & $<.038$ & $<.090$ & $<.180$ & $<.050$ & $<.140$ & $<.380$ & $<.170$ & $<.098$ & $<.280$ \\
\hline 9 & $<.250$ & $<.038$ & $<.090$ & $<.180$ & $<.050$ & $<.140$ & $<.380$ & $<.170$ & $<.098$ & $<.280$ \\
\hline 10 & $<.250$ & $<.038$ & $<.090$ & $<.180$ & $<.050$ & $<.140$ & $<.380$ & $<.170$ & $<.098$ & $<.280$ \\
\hline 11 & $<.250$ & $<.038$ & $<.090$ & $<.180$ & $<.050$ & $<.140$ & $<.380$ & $<.170$ & $<.098$ & $<.280$ \\
\hline 12 & $<.250$ & $<.038$ & $<.090$ & $<.180$ & $<.050$ & $<.140$ & $<.380$ & $<.170$ & $<.098$ & $<.280$ \\
\hline 13 & $<.250$ & $<.038$ & $<.090$ & $<.180$ & $<.050$ & $<.140$ & $<.380$ & $<.170$ & $<.098$ & $<.280$ \\
\hline 13 & $<.250$ & $<.038$ & $<.090$ & $<.180$ & $<.050$ & $<.140$ & $<.380$ & $<.170$ & $<.098$ & $<.280$ \\
\hline 14 & $<.250$ & $<.038$ & $<.090$ & $<.180$ & $<.050$ & $<.140$ & $<.380$ & $<.170$ & $<.098$ & $<.280$ \\
\hline 15 & $<.250$ & $<.038$ & $<.090$ & $<.180$ & $<.050$ & $<.140$ & $<.380$ & $<.170$ & $<.098$ & $<.280$ \\
\hline 16 & $<.250$ & $<.038$ & $<.090$ & $<.180$ & $<.050$ & $<.140$ & $<.380$ & $<.170$ & $<.098$ & $<.280$ \\
\hline 17 & $<.250$ & $<.038$ & $<.090$ & $<.180$ & $<.050$ & $<.140$ & $<.380$ & $<.170$ & $<.098$ & $<.280$ \\
\hline 18 & $<.250$ & $<.038$ & $<.090$ & $<.180$ & $<.050$ & $<.140$ & $<.380$ & $<.170$ & $<.098$ & $<.280$ \\
\hline $18^{\mathbf{R}}$ & $<.250$ & $<.038$ & $<.090$ & $<.180$ & $<.050$ & $<.140$ & $<.380$ & $<.170$ & $<.098$ & $<.280$ \\
\hline $18^{\mathbf{F b}}$ & $<.250$ & $<.038$ & $<.090$ & $<.180$ & $<.050$ & $<.140$ & $<.380$ & $<.170$ & $<.098$ & $<.280$ \\
\hline $18^{\mathbf{S b}}$ & $<.250$ & $<.038$ & $<.090$ & $<.180$ & $<.050$ & $<.140$ & $<.380$ & $<.170$ & $<.098$ & $<.280$ \\
\hline 19 & $<.250$ & $<.038$ & $<.090$ & $<.180$ & $<.050$ & $<.140$ & $<.380$ & $<.170$ & $<.098$ & $<.280$ \\
\hline 20 & $<.250$ & $<.038$ & $<.090$ & $<.180$ & $<.050$ & $<.140$ & $<.380$ & $<.170$ & $<.098$ & $<.280$ \\
\hline 21 & $<.250$ & $<.038$ & $<.090$ & $<.180$ & $<.050$ & $<.140$ & $<.380$ & $<.170$ & $<.098$ & $<.280$ \\
\hline $21^{\mathbf{R}}$ & $<.250$ & $<.038$ & $<.090$ & $<.180$ & $<.050$ & $<.140$ & $<.380$ & $<.170$ & $<.098$ & $<.280$ \\
\hline $21^{\mathbf{F b}}$ & $<.250$ & $<.038$ & $<.090$ & $<.180$ & $<.050$ & $<.140$ & $<.380$ & $<.170$ & $<.098$ & $<.280$ \\
\hline 22 & $<.250$ & $<.038$ & $<.090$ & $<.180$ & $<.050$ & $<.140$ & $<.380$ & $<.170$ & $<.098$ & $<.280$ \\
\hline 23 & $<.250$ & $<.038$ & $<.090$ & $<.180$ & $<.050$ & $<.140$ & $<.380$ & $<.170$ & $<.098$ & $<.280$ \\
\hline $23^{\mathbf{F b}}$ & $<.250$ & $<.038$ & $<.090$ & $<.180$ & $<.050$ & $<.140$ & $<.380$ & $<.170$ & $<.098$ & $<.280$ \\
\hline $23^{\mathrm{Sb}}$ & $<.250$ & $<.038$ & $<.090$ & $<.180$ & $<.050$ & $<.140$ & $<.380$ & $<.170$ & $<.098$ & $<.280$ \\
\hline 24 & $<.250$ & $<.038$ & $<.090$ & $<.180$ & $<.050$ & $<.140$ & $<.380$ & $<.170$ & $<.098$ & $<.280$ \\
\hline 25 & $<.250$ & $<.038$ & $<.090$ & $<.180$ & $<.050$ & $<.140$ & $<.380$ & $<.170$ & $<.098$ & $<.280$ \\
\hline 26 & $<.250$ & $<.038$ & $<.090$ & $<.180$ & $<.050$ & $<.140$ & $<.380$ & $<.170$ & $<.098$ & $<.280$ \\
\hline $26^{\mathrm{Tb}}$ & $<.250$ & $<.038$ & $<.090$ & $<.180$ & $<.050$ & $<.140$ & $<.380$ & $<.170$ & $<.098$ & $<.280$ \\
\hline 27 & $<.250$ & $<.038$ & $<.090$ & $<.180$ & $<.050$ & $<.140$ & $<.380$ & $<.170$ & $<.098$ & $<.280$ \\
\hline 28 & $<.250$ & $<.038$ & $<.090$ & $<.180$ & $<.050$ & $<.140$ & $<.380$ & $<.170$ & $<.098$ & $<.280$ \\
\hline 29 & $<.250$ & $<.038$ & $<.090$ & $<.180$ & $<.050$ & $<.140$ & $<.380$ & $<.170$ & $<.098$ & $<.280$ \\
\hline 30 & $<.250$ & $<.038$ & $<.090$ & $<.180$ & $<.050$ & $<.140$ & $<.380$ & $<.170$ & $<.098$ & $<.280$ \\
\hline 31 & $<.250$ & e.029 & $<.090$ & $<.180$ & $<.050$ & e1.60 & e.129 & $<.170$ & $<.098$ & $<.280$ \\
\hline
\end{tabular}


Table 20. Volatile organic-compound concentration data for ground-water samples collected from basin-fill aquifers, Northern Rockies Intermontane Basins study unit, 1999 and 2001 (Continued)

\begin{tabular}{|c|c|c|c|c|c|c|c|c|c|c|}
\hline $\begin{array}{c}\text { Well } \\
\text { number } \\
\text { (figs. } 3 \\
\text { and 4) }\end{array}$ & $\begin{array}{l}\text { Ethyl } \\
\text { tert- } \\
\text { butyl } \\
\text { ether } \\
(\mu \mathrm{g} / \mathrm{L})\end{array}$ & $\begin{array}{c}\text { Ethyl- } \\
\text { benzene } \\
(\mu \mathrm{g} / \mathrm{L})\end{array}$ & $\begin{array}{c}\text { Hexa- } \\
\text { chloro- } \\
\text { butadiene } \\
(\mu \mathrm{g} / \mathrm{L})\end{array}$ & $\begin{array}{l}\text { Hexa- } \\
\text { chloro- } \\
\text { ethane } \\
(\mu \mathrm{g} / \mathrm{L})\end{array}$ & $\begin{array}{l}\text { Isopropyl- } \\
\text { benzene } \\
(\mu \mathrm{g} / \mathrm{L})\end{array}$ & $\begin{array}{c}\text { m- and p- } \\
\text { Xylene } \\
(\mu \mathrm{g} / \mathrm{L})\end{array}$ & $\begin{array}{l}\text { Methyl } \\
\text { acrylate } \\
(\mu \mathrm{g} / \mathrm{L})\end{array}$ & $\begin{array}{l}\text { Methyl } \\
\text { acrylo- } \\
\text { nitrile } \\
(\mu \mathrm{g} / \mathrm{L})\end{array}$ & $\begin{array}{l}\text { Methyl } \\
\text { iodide } \\
(\mu \mathrm{g} / \mathrm{L})\end{array}$ & $\begin{array}{c}\text { Methyl } \\
\text { methacrylate } \\
(\mu \mathrm{g} / \mathrm{L})\end{array}$ \\
\hline
\end{tabular}

\begin{tabular}{|c|c|c|c|c|c|c|c|c|c|c|}
\hline \multicolumn{11}{|c|}{ Subunit Survey 1, Idaho and Washington } \\
\hline 1 & $<0.054$ & $<0.030$ & $<0.140$ & $<0.360$ & $<0.032$ & $<0.060$ & $<1.4$ & $<0.570$ & $<0.210$ & $<0.350$ \\
\hline 2 & $<.054$ & $<.030$ & $<.140$ & $<.360$ & $<.032$ & $<.060$ & $<1.4$ & $<.570$ & $<.210$ & $<.350$ \\
\hline 3 & $<.054$ & $<.030$ & $<.140$ & $<.360$ & $<.032$ & $<.060$ & $<1.4$ & $<.570$ & $<.210$ & $<.350$ \\
\hline 4 & $<.054$ & $<.030$ & $<.140$ & $<.360$ & $<.032$ & $<.060$ & $<1.4$ & $<.570$ & $<.210$ & $<.350$ \\
\hline 5 & $<.054$ & $<.030$ & $<.140$ & $<.360$ & $<.032$ & $<.060$ & $<1.4$ & $<.570$ & $<.210$ & $<.350$ \\
\hline 6 & $<.054$ & $<.030$ & $<.140$ & $<.360$ & $<.032$ & $<.060$ & $<1.4$ & $<.570$ & $<.210$ & $<.350$ \\
\hline 7 & $<.054$ & $<.030$ & $<.140$ & $<.360$ & $<.032$ & $<.060$ & $<1.4$ & $<.570$ & $<.210$ & $<.350$ \\
\hline 8 & $<.054$ & $<.030$ & $<.140$ & $<.360$ & $<.032$ & $<.060$ & $<1.4$ & $<.570$ & $<.210$ & $<.350$ \\
\hline 9 & $<.054$ & $<.030$ & $<.140$ & $<.360$ & $<.032$ & $<.060$ & $<1.4$ & $<.570$ & $<.210$ & $<.350$ \\
\hline 10 & $<.054$ & $<.030$ & $<.140$ & $<.360$ & $<.032$ & $<.060$ & $<1.4$ & $<.570$ & $<.210$ & $<.350$ \\
\hline 11 & $<.054$ & $<.030$ & $<.140$ & $<.360$ & $<.032$ & $<.060$ & $<1.4$ & $<.570$ & $<.210$ & $<.350$ \\
\hline 12 & $<.054$ & $<.030$ & $<.140$ & $<.360$ & $<.032$ & $<.060$ & $<1.4$ & $<.570$ & $<.210$ & $<.350$ \\
\hline 13 & $<.054$ & $<.030$ & $<.140$ & $<.360$ & $<.032$ & $<.060$ & $<1.4$ & $<.570$ & $<.210$ & $<.350$ \\
\hline 13 & $<.054$ & $<.030$ & $<.140$ & $<.360$ & $<.032$ & $<.060$ & $<1.4$ & $<.570$ & $<.210$ & $<.350$ \\
\hline 14 & $<.054$ & $<.030$ & $<.140$ & $<.360$ & $<.032$ & $<.060$ & $<1.4$ & $<.570$ & $<.210$ & $<.350$ \\
\hline 15 & $<.054$ & $<.030$ & $<.140$ & $<.360$ & $<.032$ & $<.060$ & $<1.4$ & $<.570$ & $<.210$ & $<.350$ \\
\hline 16 & $<.054$ & $<.030$ & $<.140$ & $<.360$ & $<.032$ & $<.060$ & $<1.4$ & $<.570$ & $<.210$ & $<.350$ \\
\hline 17 & $<.054$ & $<.030$ & $<.140$ & $<.360$ & $<.032$ & $<.060$ & $<1.4$ & $<.570$ & $<.210$ & $<.350$ \\
\hline 18 & $<.054$ & $<.030$ & $<.140$ & $<.360$ & $<.032$ & $<.060$ & $<1.4$ & $<.570$ & $<.210$ & $<.350$ \\
\hline $18^{\mathbf{R}}$ & $<.054$ & $<.030$ & $<.140$ & $<.360$ & $<.032$ & $<.060$ & $<1.4$ & $<.570$ & $<.210$ & $<.350$ \\
\hline $18^{\mathbf{F b}}$ & $<.054$ & $<.030$ & $<.140$ & $<.360$ & $<.032$ & $<.060$ & $<1.4$ & $<.570$ & $<.210$ & $<.350$ \\
\hline $18^{\mathbf{S b}}$ & $<.054$ & $<.030$ & $<.140$ & $<.360$ & $<.032$ & $<.060$ & $<1.4$ & $<.570$ & $<.210$ & $<.350$ \\
\hline 19 & $<.054$ & $<.030$ & $<.140$ & $<.360$ & $<.032$ & $<.060$ & $<1.4$ & $<.570$ & $<.210$ & $<.350$ \\
\hline 20 & $<.054$ & $<.030$ & $<.140$ & $<.360$ & $<.032$ & $<.060$ & $<1.4$ & $<.570$ & $<.210$ & $<.350$ \\
\hline 21 & $<.054$ & $<.030$ & $<.140$ & $<.360$ & $<.032$ & $<.060$ & $<1.4$ & $<.570$ & $<.210$ & $<.350$ \\
\hline $21^{\mathbf{R}}$ & $<.054$ & $<.030$ & $<.140$ & $<.360$ & $<.032$ & $<.060$ & $<1.4$ & $<.570$ & $<.210$ & $<.350$ \\
\hline $21^{\mathrm{Fb}}$ & $<.054$ & $<.030$ & $<.140$ & $<.360$ & $<.032$ & $<.060$ & $<1.4$ & $<.570$ & $<.210$ & $<.350$ \\
\hline 22 & $<.054$ & $<.030$ & $<.140$ & $<.360$ & $<.032$ & $<.060$ & $<1.4$ & $<.570$ & $<.210$ & $<.350$ \\
\hline 23 & $<.054$ & $<.030$ & $<.140$ & $<.360$ & $<.032$ & $<.060$ & $<1.4$ & $<.570$ & $<.210$ & $<.350$ \\
\hline $23^{\mathbf{F b}}$ & $<.054$ & $<.030$ & $<.140$ & $<.360$ & $<.032$ & $<.060$ & $<1.4$ & $<.570$ & $<.210$ & $<.350$ \\
\hline $23^{\mathbf{S b}}$ & $<.054$ & $<.030$ & $<.140$ & $<.360$ & $<.032$ & $<.060$ & $<1.4$ & $<.570$ & $<.210$ & $<.350$ \\
\hline 24 & $<.054$ & $<.030$ & $<.140$ & $<.360$ & $<.032$ & $<.060$ & $<1.4$ & $<.570$ & $<.210$ & $<.350$ \\
\hline 25 & $<.054$ & $<.030$ & $<.140$ & $<.360$ & $<.032$ & $<.060$ & $<1.4$ & $<.570$ & $<.210$ & $<.350$ \\
\hline 26 & $<.054$ & $<.030$ & $<.140$ & $<.360$ & $<.032$ & $<.060$ & $<1.4$ & $<.570$ & $<.210$ & $<.350$ \\
\hline $26^{\mathbf{T b}}$ & $<.054$ & $<.030$ & $<.140$ & $<.360$ & $<.032$ & $<.060$ & $<1.4$ & $<.570$ & $<.210$ & $<.350$ \\
\hline 27 & $<.054$ & $<.030$ & $<.140$ & $<.360$ & $<.032$ & $<.060$ & $<1.4$ & $<.570$ & $<.210$ & $<.350$ \\
\hline 28 & $<.054$ & $<.030$ & $<.140$ & $<.360$ & $<.032$ & $<.060$ & $<1.4$ & $<.570$ & $<.210$ & $<.350$ \\
\hline 29 & $<.054$ & $<.030$ & $<.140$ & $<.360$ & $<.032$ & $<.060$ & $<1.4$ & $<.570$ & $<.210$ & $<.350$ \\
\hline 30 & $<.054$ & $<.030$ & $<.140$ & $<.360$ & $<.032$ & $<.060$ & $<1.4$ & $<.570$ & $<.210$ & $<.350$ \\
\hline 31 & $<.054$ & $<.030$ & $<.140$ & $<.360$ & $<.032$ & $<.060$ & $<1.4$ & $<.570$ & $<.210$ & $<.350$ \\
\hline
\end{tabular}


Table 20. Volatile organic-compound concentration data for ground-water samples collected from basin-fill aquifers, Northern Rockies Intermontane Basins study unit, 1999 and 2001 (Continued)

\begin{tabular}{|c|c|c|c|c|c|c|c|c|c|c|}
\hline $\begin{array}{c}\text { Well } \\
\text { number } \\
\text { (figs. } 3 \\
\text { and 4) }\end{array}$ & $\begin{array}{l}\text { Naphthalene } \\
(\mu \mathrm{g} / \mathrm{L})\end{array}$ & $\begin{array}{c}\text { n-Propyl- } \\
\text { benzene } \\
(\mu \mathrm{g} / \mathrm{L})\end{array}$ & $\begin{array}{c}\text { o-Ethyl } \\
\text { toluene } \\
(\mu \mathrm{g} / \mathrm{L})\end{array}$ & $\begin{array}{c}\text { o-Xylene } \\
(\mu \mathrm{g} / \mathrm{L})\end{array}$ & $\begin{array}{c}\text { sec-Butyl- } \\
\text { benzene } \\
(\mu \mathrm{g} / \mathrm{L})\end{array}$ & $\begin{array}{c}\text { Styrene } \\
(\mu \mathrm{g} / \mathrm{L})\end{array}$ & $\begin{array}{c}\text { tert- } \\
\text { Butyl } \\
\text { methyl } \\
\text { ether } \\
(\mu \mathrm{g} / \mathrm{L})\end{array}$ & $\begin{array}{c}\text { tert- } \\
\text { Butyl- } \\
\text { benzene } \\
(\mu \mathrm{g} / \mathrm{L})\end{array}$ & $\begin{array}{c}\text { tert- } \\
\text { Pentyl } \\
\text { methyl } \\
\text { ether } \\
(\mu \mathrm{g} / \mathrm{L})\end{array}$ & $\begin{array}{r}\text { Tetra- } \\
\text { chloro- } \\
\text { ethylene } \\
(\mu \mathrm{g} / \mathrm{L})\end{array}$ \\
\hline \multicolumn{11}{|c|}{ Subunit Survey 1, Idaho and Washington } \\
\hline 1 & $<0.250$ & $<0.042$ & $<0.100$ & $<0.060$ & $<0.048$ & $<0.042$ & $<0.170$ & $<0.100$ & $<0.110$ & 1.64 \\
\hline 2 & $<.250$ & $<.042$ & $<.100$ & $<.060$ & $<.048$ & $<.042$ & $<.170$ & $<.100$ & $<.110$ & $<.100$ \\
\hline 3 & $<.250$ & $<.042$ & $<.100$ & $<.060$ & $<.048$ & $<.042$ & $<.170$ & $<.100$ & $<.110$ & $<.100$ \\
\hline 4 & $<.250$ & $<.042$ & $<.100$ & $<.060$ & $<.048$ & $<.042$ & $<.170$ & $<.100$ & $<.110$ & $<.100$ \\
\hline 5 & $<.250$ & $<.042$ & $<.100$ & $<.060$ & $<.048$ & $<.042$ & $<.170$ & $<.100$ & $<.110$ & $<.100$ \\
\hline 6 & $<.250$ & $<.042$ & $<.100$ & $<.060$ & $<.048$ & $<.042$ & $<.170$ & $<.100$ & $<.110$ & $<.100$ \\
\hline 7 & $<.250$ & $<.042$ & $<.100$ & $<.060$ & $<.048$ & $<.042$ & $<.170$ & $<.100$ & $<.110$ & $<.100$ \\
\hline 8 & $<.250$ & $<.042$ & $<.100$ & $<.060$ & $<.048$ & $<.042$ & $<.170$ & $<.100$ & $<.110$ & $<.100$ \\
\hline 9 & $<.250$ & $<.042$ & $<.100$ & $<.060$ & $<.048$ & $<.042$ & $<.170$ & $<.100$ & $<.110$ & $<.100$ \\
\hline 10 & $<.250$ & $<.042$ & $<.100$ & $<.060$ & $<.048$ & $<.042$ & $<.170$ & $<.100$ & $<.110$ & $<.100$ \\
\hline 11 & $<.250$ & $<.042$ & $<.100$ & $<.060$ & $<.048$ & $<.042$ & $<.170$ & $<.100$ & $<.110$ & $<.100$ \\
\hline 12 & $<.250$ & $<.042$ & $<.100$ & $<.060$ & $<.048$ & $<.042$ & $<.170$ & $<.100$ & $<.110$ & $<.100$ \\
\hline 13 & $<.250$ & $<.042$ & $<.100$ & $<.060$ & $<.048$ & $<.042$ & $<.170$ & $<.100$ & $<.110$ & $<.100$ \\
\hline 13 & $<.250$ & $<.042$ & $<.100$ & $<.060$ & $<.048$ & $<.042$ & $<.170$ & $<.100$ & $<.110$ & $<.100$ \\
\hline 14 & $<.250$ & $<.042$ & $<.100$ & $<.060$ & $<.048$ & $<.042$ & $<.170$ & $<.100$ & $<.110$ & $<.100$ \\
\hline 15 & $<.250$ & $<.042$ & $<.100$ & $<.060$ & $<.048$ & $<.042$ & $<.170$ & $<.100$ & $<.110$ & $<.100$ \\
\hline 16 & $<.250$ & $<.042$ & $<.100$ & $<.060$ & $<.048$ & $<.042$ & $<.170$ & $<.100$ & $<.110$ & $<.100$ \\
\hline 17 & $<.250$ & $<.042$ & $<.100$ & $<.060$ & $<.048$ & $<.042$ & $<.170$ & $<.100$ & $<.110$ & $<.100$ \\
\hline 18 & $<.250$ & $<.042$ & $<.100$ & $<.060$ & $<.048$ & $<.042$ & $<.170$ & $<.100$ & $<.110$ & $<.100$ \\
\hline $18^{\mathbf{R}}$ & $<.250$ & $<.042$ & $<.100$ & $<.060$ & $<.048$ & $<.042$ & $<.170$ & $<.100$ & $<.110$ & $<.100$ \\
\hline $18^{\mathbf{F b}}$ & $<.250$ & $<.042$ & $<.100$ & $<.060$ & $<.048$ & $<.042$ & $<.170$ & $<.100$ & $<.110$ & $<.100$ \\
\hline $18^{\mathbf{S b}}$ & $<.250$ & $<.042$ & $<.100$ & $<.060$ & $<.048$ & $<.042$ & $<.170$ & $<.100$ & $<.110$ & $<.100$ \\
\hline 19 & $<.250$ & $<.042$ & $<.100$ & $<.060$ & $<.048$ & $<.042$ & $<.170$ & $<.100$ & $<.110$ & $<.100$ \\
\hline 20 & $<.250$ & $<.042$ & $<.100$ & $<.060$ & $<.048$ & $<.042$ & $<.170$ & $<.100$ & $<.110$ & $<.100$ \\
\hline 21 & $<.250$ & $<.042$ & $<.100$ & $<.060$ & $<.048$ & $<.042$ & $<.170$ & $<.100$ & $<.110$ & $<.100$ \\
\hline $21^{\mathbf{R}}$ & $<.250$ & $<.042$ & $<.100$ & $<.060$ & $<.048$ & $<.042$ & $<.170$ & $<.100$ & $<.110$ & $<.100$ \\
\hline $21^{\mathbf{F b}}$ & $<.250$ & $<.042$ & $<.100$ & $<.060$ & $<.048$ & $<.042$ & $<.170$ & $<.100$ & $<.110$ & $<.100$ \\
\hline 22 & $<.250$ & $<.042$ & $<.100$ & $<.060$ & $<.048$ & $<.042$ & $<.170$ & $<.100$ & $<.110$ & $<.100$ \\
\hline 23 & $<.250$ & $<.042$ & $<.100$ & $<.060$ & $<.048$ & $<.042$ & $<.170$ & $<.100$ & $<.110$ & $<.100$ \\
\hline $23^{\mathrm{Fb}}$ & $<.250$ & $<.042$ & $<.100$ & $<.060$ & $<.048$ & $<.042$ & $<.170$ & $<.100$ & $<.110$ & $<.100$ \\
\hline $23^{\mathrm{Sb}}$ & $<.250$ & $<.042$ & $<.100$ & $<.060$ & $<.048$ & $<.042$ & $<.170$ & $<.100$ & $<.110$ & $<.100$ \\
\hline 24 & $<.250$ & $<.042$ & $<.100$ & $<.060$ & $<.048$ & $<.042$ & $<.170$ & $<.100$ & $<.110$ & $<.100$ \\
\hline 25 & $<.250$ & $<.042$ & $<.100$ & $<.060$ & $<.048$ & $<.042$ & $<.170$ & $<.100$ & $<.110$ & $<.100$ \\
\hline 26 & $<.250$ & $<.042$ & $<.100$ & $<.060$ & $<.048$ & $<.042$ & $<.170$ & $<.100$ & $<.110$ & $<.100$ \\
\hline $26^{\mathbf{T b}}$ & $<.250$ & $<.042$ & $<.100$ & $<.060$ & $<.048$ & $<.042$ & $<.170$ & $<.100$ & $<.110$ & $<.100$ \\
\hline 27 & $<.250$ & $<.042$ & $<.100$ & $<.060$ & $<.048$ & $<.042$ & $<.170$ & $<.100$ & $<.110$ & $<.100$ \\
\hline 28 & $<.250$ & $<.042$ & $<.100$ & $<.060$ & $<.048$ & $<.042$ & $<.170$ & $<.100$ & $<.110$ & $<.100$ \\
\hline 29 & $<.250$ & $<.042$ & $<.100$ & $<.060$ & $<.048$ & $<.042$ & $<.170$ & $<.100$ & $<.110$ & $<.100$ \\
\hline 30 & $<.250$ & $<.042$ & $<.100$ & $<.060$ & $<.048$ & $<.042$ & $<.170$ & $<.100$ & $<.110$ & $<.100$ \\
\hline 31 & $<.250$ & $<.042$ & $<.100$ & $<.060$ & $<.048$ & $<.042$ & $<.170$ & $<.100$ & $<.110$ & $<.100$ \\
\hline
\end{tabular}


Table 20. Volatile organic-compound concentration data for ground-water samples collected from basin-fill aquifers, Northern Rockies Intermontane Basins study unit, 1999 and 2001 (Continued)

\begin{tabular}{|c|c|c|c|c|c|c|c|c|c|c|}
\hline $\begin{array}{c}\text { Well } \\
\text { number } \\
\text { (figs. } 3 \\
\text { and 4) }\end{array}$ & $\begin{array}{c}\text { Tetra- } \\
\text { chloro- } \\
\text { methane } \\
(\mu \mathrm{g} / \mathrm{L})\end{array}$ & $\begin{array}{c}\text { Tetra- } \\
\text { hydrofuran } \\
(\mu \mathrm{g} / \mathrm{L})\end{array}$ & $\begin{array}{c}\text { Toluene } \\
(\mu \mathrm{g} / \mathrm{L})\end{array}$ & $\begin{array}{c}\text { Toluene-d } \\
\text { surrogate } \\
\text { (percent) }\end{array}$ & $\begin{array}{c}\text { trans- } \\
1,2- \\
\text { Dichloro- } \\
\text { ethylene } \\
(\mu \mathrm{g} / \mathrm{L})\end{array}$ & $\begin{array}{c}\text { trans- } \\
1,3- \\
\text { Dichloro- } \\
\text { propene } \\
(\mu \mathrm{g} / \mathrm{L})\end{array}$ & $\begin{array}{c}\text { trans- } \\
1,4,- \\
\text { Dichloro-2- } \\
\text { butene } \\
(\mu \mathrm{g} / \mathrm{L})\end{array}$ & $\begin{array}{c}\text { Trichloro- } \\
\text { ethylene } \\
(\mu \mathrm{g} / \mathrm{L})\end{array}$ & $\begin{array}{c}\text { Trichloro- } \\
\text { fluoro- } \\
\text { methane } \\
(\mu \mathrm{g} / \mathrm{L})\end{array}$ & $\begin{array}{c}\text { Vinyl } \\
\text { chloride } \\
(\mu \mathrm{g} / \mathrm{L})\end{array}$ \\
\hline
\end{tabular}

\begin{tabular}{|c|c|c|c|c|c|c|c|c|c|c|}
\hline \multicolumn{11}{|c|}{ Subunit Survey 1, Idaho and Washington } \\
\hline 1 & $<0.088$ & $<9.0$ & $<0.050$ & 100 & $<0.032$ & $<0.130$ & $<0.700$ & $<0.038$ & $<0.090$ & $<0.110$ \\
\hline 2 & $<.088$ & $<9.0$ & $<.050$ & 101 & $<.032$ & $<.130$ & $<.700$ & $<.038$ & $<.090$ & $<.110$ \\
\hline 3 & $<.088$ & $<9.0$ & $<.050$ & 100 & $<.032$ & $<.130$ & $<.700$ & $<.038$ & $<.090$ & $<.110$ \\
\hline 4 & $<.088$ & $<9.0$ & e.008 & 93 & $<.032$ & $<.130$ & $<.700$ & $<.038$ & $<.090$ & $<.110$ \\
\hline 5 & $<.088$ & $<9.0$ & $<.050$ & 101 & $<.032$ & $<.130$ & $<.700$ & $<.038$ & $<.090$ & $<.110$ \\
\hline 6 & $<.088$ & $<9.0$ & $<.050$ & 95 & $<.032$ & $<.130$ & $<.700$ & $<.038$ & $<.090$ & $<.110$ \\
\hline 7 & $<.088$ & $<9.0$ & $<.050$ & 102 & $<.032$ & $<.130$ & $<.700$ & $<.038$ & $<.090$ & $<.110$ \\
\hline 8 & $<.088$ & $<9.0$ & $<.050$ & 103 & $<.032$ & $<.130$ & $<.700$ & $<.038$ & $<.090$ & $<.110$ \\
\hline 9 & $<.088$ & $<9.0$ & $<.050$ & 98 & $<.032$ & $<.130$ & $<.700$ & $<.038$ & $<.090$ & $<.110$ \\
\hline 10 & $<.088$ & $<9.0$ & e.007 & 106 & $<.032$ & $<.130$ & $<.700$ & $<.038$ & $<.090$ & $<.110$ \\
\hline 11 & $<.088$ & $<9.0$ & $<.050$ & 101 & $<.032$ & $<.130$ & $<.700$ & $<.038$ & $<.090$ & $<.110$ \\
\hline 12 & $<.088$ & $<9.0$ & e.008 & 105 & $<.032$ & $<.130$ & $<.700$ & $<.038$ & $<.090$ & $<.110$ \\
\hline 13 & $<.088$ & $<9.0$ & 1.45 & 103 & $<.032$ & $<.130$ & $<.700$ & $<.038$ & $<.090$ & $<.110$ \\
\hline 13 & $<.088$ & $<9.0$ & 4.31 & 102 & $<.032$ & $<.130$ & $<.700$ & $<.038$ & $<.090$ & $<.110$ \\
\hline 14 & $<.088$ & $<9.0$ & $<.050$ & 106 & $<.032$ & $<.130$ & $<.700$ & $<.038$ & $<.090$ & $<.110$ \\
\hline 15 & $<.088$ & $<9.0$ & $<.050$ & 99 & $<.032$ & $<.130$ & $<.700$ & $<.038$ & $<.090$ & $<.110$ \\
\hline 16 & $<.088$ & $<9.0$ & e.016 & 103 & $<.032$ & $<.130$ & $<.700$ & $<.038$ & $<.090$ & $<.110$ \\
\hline 17 & $<.088$ & $<9.0$ & $<.050$ & 107 & $<.032$ & $<.130$ & $<.700$ & $<.038$ & $<.090$ & $<.110$ \\
\hline 18 & $<.088$ & $<9.0$ & $<.050$ & 102 & $<.032$ & $<.130$ & $<.700$ & $<.038$ & $<.090$ & $<.110$ \\
\hline $18^{\mathbf{R}}$ & $<.088$ & $<9.0$ & $<.050$ & 103 & $<.032$ & $<.130$ & $<.700$ & $<.038$ & $<.090$ & $<.110$ \\
\hline $18^{\mathbf{F b}}$ & $<.088$ & $<9.0$ & $<.050$ & 98 & $<.032$ & $<.130$ & $<.700$ & $<.038$ & $<.090$ & $<.110$ \\
\hline $18^{\mathbf{S b}}$ & $<.088$ & $<9.0$ & $<.050$ & 100 & $<.032$ & $<.130$ & $<.700$ & $<.038$ & $<.090$ & $<.110$ \\
\hline 19 & $<.088$ & $<9.0$ & $<.050$ & 101 & $<.032$ & $<.130$ & $<.700$ & $<.038$ & $<.090$ & $<.110$ \\
\hline 20 & $<.088$ & $<9.0$ & $<.050$ & 102 & $<.032$ & $<.130$ & $<.700$ & $<.038$ & $<.090$ & $<.110$ \\
\hline 21 & $<.088$ & $<9.0$ & e.017 & 102 & $<.032$ & $<.130$ & $<.700$ & $<.038$ & $<.090$ & $<.110$ \\
\hline $21^{\mathbf{R}}$ & $<.088$ & $<9.0$ & e.009 & 99 & $<.032$ & $<.130$ & $<.700$ & $<.038$ & $<.090$ & $<.110$ \\
\hline $21^{\mathbf{F b}}$ & $<.088$ & $<9.0$ & $<.050$ & 88 & $<.032$ & $<.130$ & $<.700$ & $<.038$ & $<.090$ & $<.110$ \\
\hline 22 & $<.088$ & $<9.0$ & $<.050$ & 104 & $<.032$ & $<.130$ & $<.700$ & $<.038$ & $<.090$ & $<.110$ \\
\hline 23 & $<.088$ & $<9.0$ & $<.050$ & 100 & $<.032$ & $<.130$ & $<.700$ & $<.038$ & $<.090$ & $<.110$ \\
\hline $23^{\mathrm{Fb}}$ & $<.088$ & $<9.0$ & e.013 & 96 & $<.032$ & $<.130$ & $<.700$ & $<.038$ & $<.090$ & $<.110$ \\
\hline $23^{\mathrm{Sb}}$ & $<.088$ & $<9.0$ & e.034 & 99 & $<.032$ & $<.130$ & $<.700$ & $<.038$ & $<.090$ & $<.110$ \\
\hline 24 & $<.088$ & $<9.0$ & $<.050$ & 102 & $<.032$ & $<.130$ & $<.700$ & $<.038$ & $<.090$ & $<.110$ \\
\hline 25 & $<.088$ & $<9.0$ & $<.050$ & 100 & $<.032$ & $<.130$ & $<.700$ & $<.038$ & $<.090$ & $<.110$ \\
\hline 26 & $<.088$ & $<9.0$ & $<.050$ & 101 & $<.032$ & $<.130$ & $<.700$ & $<.038$ & .111 & $<.110$ \\
\hline $26^{\mathbf{T b}}$ & $<.088$ & $<9.0$ & e.025 & 98 & $<.032$ & $<.130$ & $<.700$ & $<.038$ & $<.090$ & $<.110$ \\
\hline 27 & e.012 & $<9.0$ & $<.050$ & 100 & $<.032$ & $<.130$ & $<.700$ & $<.038$ & $<.090$ & $<.110$ \\
\hline 28 & $<.088$ & $<9.0$ & $<.050$ & 101 & $<.032$ & $<.130$ & $<.700$ & $<.038$ & $<.090$ & $<.110$ \\
\hline 29 & $<.088$ & $<9.0$ & $<.050$ & 98 & $<.032$ & $<.130$ & $<.700$ & $<.038$ & $<.090$ & $<.110$ \\
\hline 30 & $<.088$ & $<9.0$ & $<.050$ & 98 & $<.032$ & $<.130$ & $<.700$ & $<.038$ & $<.090$ & $<.110$ \\
\hline 31 & $<.088$ & $<9.0$ & $<.050$ & 100 & $<.032$ & $<.130$ & $<.700$ & $<.038$ & $<.090$ & $<.110$ \\
\hline
\end{tabular}


Table 20. Volatile organic-compound concentration data for ground-water samples collected from basin-fill aquifers, Northern Rockies Intermontane Basins study unit, 1999 and 2001 (Continued)

\begin{tabular}{|c|c|c|c|c|c|c|c|c|c|c|}
\hline $\begin{array}{c}\text { Well } \\
\text { number } \\
\text { (figs. } 3 \\
\text { and 4) }\end{array}$ & $\begin{array}{c}\text { Site } \\
\text { identification } \\
\text { number }\end{array}$ & Date & Time & $\begin{array}{l}\text { 1,1,1,2- } \\
\text { Tetra- } \\
\text { chloro- } \\
\text { ethane } \\
(\mu \mathrm{g} / \mathrm{L})\end{array}$ & $\begin{array}{c}\text { 1,1,1- } \\
\text { Trichloro- } \\
\text { ethane } \\
(\mu \mathrm{g} / \mathrm{L})\end{array}$ & $\begin{array}{l}\text { 1,1,2,2- } \\
\text { Tetra- } \\
\text { chloro- } \\
\text { ethane } \\
(\mu \mathrm{g} / \mathrm{L})\end{array}$ & $\begin{array}{c}\text { 1,1,2- } \\
\text { Trichloro- } \\
\text { ethane } \\
(\mu \mathrm{g} / \mathrm{L})\end{array}$ & $\begin{array}{c}\text { 1,1,2- } \\
\text { Trichloro- } \\
\text { trifluoro- } \\
\text { ethane } \\
(\mu \mathrm{g} / \mathrm{L})\end{array}$ & $\begin{array}{c}\text { 1,1- } \\
\text { Dichloro- } \\
\text { ethane } \\
(\mu \mathrm{g} / \mathrm{L})\end{array}$ & $\begin{array}{c}\text { 1,1- } \\
\text { Dichloro- } \\
\text { ethylene } \\
(\mu \mathrm{g} / \mathrm{L})\end{array}$ \\
\hline \multicolumn{11}{|c|}{ Subunit Survey 2, Montana } \\
\hline 32 & 470719114301401 & $06 / 12 / 01$ & 1300 & $<.030$ & $<.032$ & $<.090$ & $<.060$ & $<.060$ & $<.035$ & $<.040$ \\
\hline 33 & 470502114265301 & $06 / 12 / 01$ & 0900 & $<.030$ & $<.032$ & $<.090$ & $<.060$ & $<.060$ & $<.035$ & $<.040$ \\
\hline 34 & 470328114164301 & $06 / 28 / 01$ & 0900 & $<.030$ & $<.032$ & $<.090$ & $<.060$ & $<.060$ & $<.035$ & $<.040$ \\
\hline 35 & 470112114144001 & $06 / 07 / 01$ & 0900 & $<.030$ & $<.032$ & $<.090$ & $<.060$ & $<.060$ & $<.035$ & $<.040$ \\
\hline 36 & 465838114074501 & $06 / 27 / 01$ & 1200 & $<.030$ & $<.032$ & $<.090$ & $<.060$ & $<.060$ & $<.035$ & $<.040$ \\
\hline $36^{\mathbf{R}}$ & & $06 / 27 / 01$ & 1201 & $<.030$ & $<.032$ & $<.090$ & $<.060$ & $<.060$ & $<.035$ & $<.040$ \\
\hline $36^{\mathbf{F b}}$ & & $06 / 27 / 01$ & 1205 & $<.030$ & $<.032$ & $<.090$ & $<.060$ & $<.060$ & $<.035$ & $<.040$ \\
\hline 37 & 465741114110601 & $06 / 27 / 01$ & 1700 & $<.030$ & $<.032$ & $<.090$ & $<.060$ & $<.060$ & $<.035$ & $<.040$ \\
\hline 38 & 465440114022101 & $06 / 19 / 01$ & 1100 & $<.030$ & $<.032$ & $<.090$ & $<.060$ & $<.060$ & $<.035$ & $<.040$ \\
\hline $38^{\mathbf{R}}$ & & $06 / 19 / 01$ & 1101 & $<.030$ & $<.032$ & $<.090$ & $<.060$ & $<.060$ & $<.035$ & $<.040$ \\
\hline $38^{\mathbf{F b}}$ & & $06 / 19 / 01$ & 1105 & $<.030$ & $<.032$ & $<.090$ & $<.060$ & $<.060$ & $<.035$ & $<.040$ \\
\hline 39 & 465323114054301 & $06 / 20 / 01$ & 1300 & $<.030$ & .130 & $<.090$ & $<.060$ & $<.060$ & $<.035$ & $<.040$ \\
\hline 40 & 465127114055401 & $06 / 20 / 01$ & 1000 & $<.030$ & e.060 & $<.090$ & $<.060$ & $<.060$ & $<.035$ & $<.040$ \\
\hline 41 & 464951114023701 & $06 / 19 / 01$ & 1600 & $<.030$ & $<.032$ & $<.090$ & $<.060$ & $<.060$ & $<.035$ & $<.040$ \\
\hline 42 & 463932114035901 & $05 / 29 / 01$ & 1300 & $<.030$ & $<.032$ & $<.090$ & $<.060$ & $<.060$ & $<.035$ & $<.040$ \\
\hline 43 & 463827114001201 & $05 / 29 / 01$ & 1600 & $<.030$ & $<.032$ & $<.090$ & $<.060$ & $<.060$ & $<.035$ & $<.040$ \\
\hline 44 & 463335114011701 & $05 / 30 / 01$ & 1600 & $<.030$ & $<.032$ & $<.090$ & $<.060$ & $<.060$ & $<.035$ & $<.040$ \\
\hline 45 & 463122114074701 & $05 / 30 / 01$ & 0900 & $<.030$ & $<.032$ & $<.090$ & $<.060$ & $<.060$ & $<.035$ & $<.040$ \\
\hline 46 & 462948114060101 & $05 / 30 / 01$ & 1200 & $<.030$ & $<.032$ & $<.090$ & $<.060$ & $<.060$ & $<.035$ & $<.040$ \\
\hline 47 & 462859113574401 & $06 / 11 / 01$ & 1200 & $<.030$ & $<.032$ & $<.090$ & $<.060$ & $<.060$ & $<.035$ & $<.040$ \\
\hline 48 & 462818114074101 & $06 / 04 / 01$ & 1200 & $<.030$ & $<.032$ & $<.090$ & $<.060$ & $<.060$ & $<.035$ & $<.040$ \\
\hline $48^{\mathbf{F b}}$ & & $06 / 04 / 01$ & 1205 & $<.030$ & $<.032$ & $<.090$ & $<.060$ & $<.060$ & $<.035$ & $<.040$ \\
\hline 49 & 462754113592701 & $06 / 05 / 01$ & 1500 & $<.030$ & $<.032$ & $<.090$ & $<.060$ & $<.060$ & $<.035$ & $<.040$ \\
\hline 50 & 462616114094301 & $06 / 05 / 01$ & 1300 & $<.030$ & $<.032$ & $<.090$ & $<.060$ & $<.060$ & $<.035$ & $<.040$ \\
\hline 51 & 462545114034301 & $06 / 05 / 01$ & 1800 & $<.030$ & $<.032$ & $<.090$ & $<.060$ & $<.060$ & $<.035$ & $<.040$ \\
\hline 52 & 462256114114501 & $06 / 04 / 01$ & 1700 & $<.030$ & $<.032$ & $<.090$ & $<.060$ & $<.060$ & $<.035$ & $<.040$ \\
\hline 53 & 462228114030301 & $06 / 06 / 01$ & 1100 & $<.030$ & $<.032$ & $<.090$ & $<.060$ & $<.060$ & $<.035$ & $<.040$ \\
\hline 54 & 461823114050901 & $06 / 06 / 01$ & 1300 & $<.030$ & $<.032$ & $<.090$ & $<.060$ & $<.060$ & $<.035$ & $<.040$ \\
\hline 55 & 461807114123001 & $06 / 11 / 01$ & 1600 & $<.030$ & $<.032$ & $<.090$ & $<.060$ & $<.060$ & $<.035$ & $<.040$ \\
\hline 56 & 461638114023401 & $06 / 06 / 01$ & 0800 & $<.030$ & $<.032$ & $<.090$ & $<.060$ & $<.060$ & $<.035$ & $<.040$ \\
\hline 57 & 461451114090801 & $06 / 06 / 01$ & 1600 & $<.030$ & $<.032$ & $<.090$ & $<.060$ & $<.060$ & $<.035$ & $<.040$ \\
\hline 58 & 461320114121501 & $06 / 05 / 01$ & 0900 & $<.030$ & e.007 & $<.090$ & $<.060$ & $<.060$ & $<.035$ & $<.040$ \\
\hline $58^{\mathbf{R}}$ & & $06 / 05 / 01$ & 0901 & $<.030$ & e.008 & $<.090$ & $<.060$ & $<.060$ & $<.035$ & $<.040$ \\
\hline 59 & 461055114112601 & $06 / 21 / 01$ & 0900 & $<.030$ & $<.032$ & $<.090$ & $<.060$ & $<.060$ & $<.035$ & $<.040$ \\
\hline 60 & 460110114110901 & $06 / 20 / 01$ & 1800 & $<.030$ & $<.032$ & $<.090$ & $<.060$ & $<.060$ & $<.035$ & $<.040$ \\
\hline 61 & 455521114074801 & $06 / 20 / 01$ & 2000 & $<.030$ & $<.032$ & $<.090$ & $<.060$ & $<.060$ & $<.035$ & $<.040$ \\
\hline
\end{tabular}


Table 20. Volatile organic-compound concentration data for ground-water samples collected from basin-fill aquifers, Northern Rockies Intermontane Basins study unit, 1999 and 2001 (Continued)

\begin{tabular}{|c|c|c|c|c|c|c|c|c|c|c|c|}
\hline $\begin{array}{c}\text { Well } \\
\text { number } \\
\text { (figs. } 3 \\
\text { and 4) }\end{array}$ & $\begin{array}{c}\text { 1,1- } \\
\text { Dichloro- } \\
\text { propene } \\
(\mu \mathrm{g} / \mathrm{L})\end{array}$ & $\begin{array}{c}\text { 1,2,3,4- } \\
\text { Tetra- } \\
\text { methyl- } \\
\text { benzene } \\
(\mu \mathrm{g} / \mathrm{L})\end{array}$ & $\begin{array}{c}\text { 1,2,3,5- } \\
\text { Tetra- } \\
\text { methyl- } \\
\text { benzene } \\
(\mu \mathrm{g} / \mathrm{L})\end{array}$ & $\begin{array}{c}1,2,3- \\
\text { Trichloro- } \\
\text { benzene } \\
(\mu \mathrm{g} / \mathrm{L})\end{array}$ & $\begin{array}{c}\text { 1,2,3- } \\
\text { Trichloro- } \\
\text { propane } \\
(\mu \mathrm{g} / \mathrm{L})\end{array}$ & $\begin{array}{c}1,2,3- \\
\text { Trimethyl- } \\
\text { benzene } \\
(\mu \mathrm{g} / \mathrm{L})\end{array}$ & $\begin{array}{c}\text { 1,2,4- } \\
\text { Trichloro- } \\
\text { benzene } \\
(\mu \mathrm{g} / \mathrm{L})\end{array}$ & $\begin{array}{c}\text { 1,2,4- } \\
\text { Tri- } \\
\text { methyl- } \\
\text { benzene } \\
(\mu \mathrm{g} / \mathrm{L})\end{array}$ & $\begin{array}{c}\text { 1,2,- } \\
\text { Dibromo- } \\
\text { 3-chloro- } \\
\text { propane } \\
(\mu \mathrm{g} / \mathrm{L})\end{array}$ & $\begin{array}{c}\text { 1,2- } \\
\text { Dibromo- } \\
\text { ethane } \\
(\mu \mathrm{g} / \mathrm{L})\end{array}$ & $\begin{array}{c}\text { 1,2- } \\
\text { Dichloro- } \\
\text { benzene } \\
(\mu \mathrm{g} / \mathrm{L})\end{array}$ \\
\hline \multicolumn{12}{|c|}{ Subunit Survey 2, Montana } \\
\hline 32 & $<.026$ & $<.230$ & $<.200$ & $<.270$ & $<.160$ & $<.120$ & $<.190$ & $<.056$ & $<.210$ & $<.036$ & $<.031$ \\
\hline 33 & $<.026$ & $<.230$ & $<.200$ & $<.270$ & $<.160$ & $<.120$ & $<.190$ & $<.056$ & $<.210$ & $<.036$ & $<.031$ \\
\hline 34 & $<.026$ & $<.230$ & $<.200$ & $<.270$ & $<.160$ & $<.120$ & $<.190$ & $<.056$ & $<.210$ & $<.036$ & $<.031$ \\
\hline 35 & $<.026$ & $<.230$ & $<.200$ & $<.270$ & $<.160$ & $<.120$ & $<.190$ & $<.056$ & $<.210$ & $<.036$ & $<.031$ \\
\hline 36 & $<.026$ & $<.230$ & $<.200$ & $<.270$ & $<.160$ & $<.120$ & $<.190$ & $<.056$ & $<.210$ & $<.036$ & $<.031$ \\
\hline $36^{\mathbf{R}}$ & $<.026$ & $<.230$ & $<.200$ & $<.270$ & $<.160$ & $<.120$ & $<.190$ & $<.056$ & $<.210$ & $<.036$ & $<.031$ \\
\hline $36^{\mathbf{F b}}$ & $<.026$ & $<.230$ & $<.200$ & $<.270$ & $<.160$ & $<.120$ & $<.190$ & $<.056$ & $<.210$ & $<.036$ & $<.031$ \\
\hline 37 & $<.026$ & $<.230$ & $<.200$ & $<.270$ & $<.160$ & $<.120$ & $<.190$ & $<.056$ & $<.210$ & $<.036$ & $<.031$ \\
\hline 38 & $<.026$ & $<.230$ & $<.200$ & $<.270$ & $<.160$ & $<.120$ & $<.190$ & $<.056$ & $<.210$ & $<.036$ & $<.031$ \\
\hline $38^{\mathbf{R}}$ & $<.026$ & $<.230$ & $<.200$ & $<.270$ & $<.160$ & $<.120$ & $<.190$ & $<.056$ & $<.210$ & $<.036$ & $<.031$ \\
\hline $38^{\mathbf{F b}}$ & $<.026$ & $<.230$ & $<.200$ & $<.270$ & $<.160$ & $<.120$ & $<.190$ & $<.056$ & $<.210$ & $<.036$ & $<.031$ \\
\hline 39 & $<.026$ & $<.230$ & $<.200$ & $<.270$ & $<.160$ & $<.120$ & $<.190$ & $<.056$ & $<.210$ & $<.036$ & $<.031$ \\
\hline 40 & $<.026$ & $<.230$ & $<.200$ & $<.270$ & $<.160$ & $<.120$ & $<.190$ & $<.056$ & $<.210$ & $<.036$ & $<.031$ \\
\hline 41 & $<.026$ & $<.230$ & $<.200$ & $<.270$ & $<.160$ & $<.120$ & $<.190$ & $<.056$ & $<.210$ & $<.036$ & $<.031$ \\
\hline 42 & $<.026$ & $<.230$ & $<.200$ & $<.270$ & $<.160$ & $<.120$ & $<.190$ & $<.056$ & $<.210$ & $<.036$ & $<.031$ \\
\hline 43 & $<.026$ & $<.230$ & $<.200$ & $<.270$ & $<.160$ & $<.120$ & $<.190$ & $<.056$ & $<.210$ & $<.036$ & $<.031$ \\
\hline 44 & $<.026$ & $<.230$ & $<.200$ & $<.270$ & $<.160$ & $<.120$ & $<.190$ & $<.056$ & $<.210$ & $<.036$ & $<.031$ \\
\hline 45 & $<.026$ & $<.230$ & $<.200$ & $<.270$ & $<.160$ & $<.120$ & $<.190$ & $<.056$ & $<.210$ & $<.036$ & $<.031$ \\
\hline 46 & $<.026$ & $<.230$ & $<.200$ & $<.270$ & $<.160$ & $<.120$ & $<.190$ & $<.056$ & $<.210$ & $<.036$ & $<.031$ \\
\hline 47 & $<.026$ & $<.230$ & $<.200$ & $<.270$ & $<.160$ & $<.120$ & $<.190$ & $<.056$ & $<.210$ & $<.036$ & $<.031$ \\
\hline 48 & $<.026$ & $<.230$ & $<.200$ & $<.270$ & $<.160$ & $<.120$ & $<.190$ & $<.056$ & $<.210$ & $<.036$ & $<.031$ \\
\hline $48^{\mathbf{F b}}$ & $<.026$ & $<.230$ & $<.200$ & $<.270$ & $<.160$ & $<.120$ & $<.190$ & $<.056$ & $<.210$ & $<.036$ & $<.031$ \\
\hline 49 & $<.026$ & $<.230$ & $<.200$ & $<.270$ & $<.160$ & $<.120$ & $<.190$ & $<.056$ & $<.210$ & $<.036$ & $<.031$ \\
\hline 50 & $<.026$ & $<.230$ & $<.200$ & $<.270$ & $<.160$ & $<.120$ & $<.190$ & $<.056$ & $<.210$ & $<.036$ & $<.031$ \\
\hline 51 & $<.026$ & $<.230$ & $<.200$ & $<.270$ & $<.160$ & $<.120$ & $<.190$ & $<.056$ & $<.210$ & $<.036$ & $<.031$ \\
\hline 52 & $<.026$ & $<.230$ & $<.200$ & $<.270$ & $<.160$ & $<.120$ & $<.190$ & $<.056$ & $<.210$ & $<.036$ & $<.031$ \\
\hline 53 & $<.026$ & $<.230$ & $<.200$ & $<.270$ & $<.160$ & $<.120$ & $<.190$ & $<.056$ & $<.210$ & $<.036$ & $<.031$ \\
\hline 54 & $<.026$ & $<.230$ & $<.200$ & $<.270$ & $<.160$ & $<.120$ & $<.190$ & $<.056$ & $<.210$ & $<.036$ & $<.031$ \\
\hline 55 & $<.026$ & $<.230$ & $<.200$ & $<.270$ & $<.160$ & $<.120$ & $<.190$ & $<.056$ & $<.210$ & $<.036$ & $<.031$ \\
\hline 56 & $<.026$ & $<.230$ & $<.200$ & $<.270$ & $<.160$ & $<.120$ & $<.190$ & $<.056$ & $<.210$ & $<.036$ & $<.031$ \\
\hline 57 & $<.026$ & $<.230$ & $<.200$ & $<.270$ & $<.160$ & $<.120$ & $<.190$ & $<.056$ & $<.210$ & $<.036$ & $<.031$ \\
\hline 58 & $<.026$ & $<.230$ & $<.200$ & $<.270$ & $<.160$ & $<.120$ & $<.190$ & $<.056$ & $<.210$ & $<.036$ & $<.031$ \\
\hline $58^{\mathbf{R}}$ & $<.026$ & $<.230$ & $<.200$ & $<.270$ & $<.160$ & $<.120$ & $<.190$ & $<.056$ & $<.210$ & $<.036$ & $<.031$ \\
\hline 59 & $<.026$ & $<.230$ & $<.200$ & $<.270$ & $<.160$ & $<.120$ & $<.190$ & $<.056$ & $<.210$ & $<.036$ & $<.031$ \\
\hline 60 & $<.026$ & $<.230$ & $<.200$ & $<.270$ & $<.160$ & $<.120$ & $<.190$ & $<.056$ & $<.210$ & $<.036$ & $<.031$ \\
\hline 61 & $<.026$ & $<.230$ & $<.200$ & $<.270$ & $<.160$ & $<.120$ & $<.190$ & $<.056$ & $<.210$ & $<.036$ & $<.031$ \\
\hline
\end{tabular}


Table 20. Volatile organic-compound concentration data for ground-water samples collected from basin-fill aquifers, Northern Rockies Intermontane Basins study unit, 1999 and 2001 (Continued)

\begin{tabular}{|c|c|c|c|c|c|c|c|c|c|c|}
\hline $\begin{array}{c}\text { Well } \\
\text { number } \\
\text { (figs. } 3 \\
\text { and 4) }\end{array}$ & $\begin{array}{c}\text { 1,2- } \\
\text { Dichloro- } \\
\text { ethane } \\
(\mu \mathrm{g} / \mathrm{L})\end{array}$ & $\begin{array}{c}1,2,- \\
\text { Dichloro- } \\
\text { ethane-d } \\
\text { surrogate } \\
\text { (percent) }\end{array}$ & $\begin{array}{c}\text { 1,2- } \\
\text { Dichloro- } \\
\text { propane } \\
(\mu \mathrm{g} / \mathrm{L})\end{array}$ & $\begin{array}{c}\text { 1,3,5- } \\
\text { Trimethyl- } \\
\text { benzene } \\
(\mu \mathrm{g} / \mathrm{L})\end{array}$ & $\begin{array}{c}\text { 1,3- } \\
\text { Dichloro- } \\
\text { benzene } \\
(\mu \mathrm{g} / \mathrm{L})\end{array}$ & $\begin{array}{c}\text { 1,3- } \\
\text { Dichloro- } \\
\text { propane } \\
(\mu \mathrm{g} / \mathrm{L})\end{array}$ & $\begin{array}{l}\text { 1,4-Bromo- } \\
\text { fluoro- } \\
\text { benzene, } \\
\text { surrogate } \\
\text { (percent) }\end{array}$ & $\begin{array}{c}\text { 1,4- } \\
\text { Dichloro- } \\
\text { benzene } \\
(\mu \mathrm{g} / \mathrm{L})\end{array}$ & $\begin{array}{c}2,2- \\
\text { Dichloro- } \\
\text { propane } \\
(\mu \mathrm{g} / \mathrm{L})\end{array}$ & $\begin{array}{c}\text { 2-Butanone } \\
(\mu \mathrm{g} / \mathrm{L})\end{array}$ \\
\hline \multicolumn{11}{|c|}{ Subunit Survey 2, Montana } \\
\hline 32 & $<.130$ & 128 & $<.029$ & $<.044$ & $<.030$ & $<.120$ & 70 & $<.050$ & $<.050$ & $<1.60$ \\
\hline 33 & $<.130$ & 123 & $<.029$ & $<.044$ & $<.030$ & $<.120$ & 69 & $<.050$ & $<.050$ & $<1.60$ \\
\hline 34 & $<.130$ & 91 & $<.029$ & $<.044$ & $<.030$ & $<.120$ & 94 & $<.050$ & $<.050$ & $<1.60$ \\
\hline 35 & $<.130$ & 110 & $<.029$ & $<.044$ & $<.030$ & $<.120$ & 79 & $<.050$ & $<.050$ & $<1.60$ \\
\hline 36 & $<.130$ & 106 & $<.029$ & $<.044$ & $<.030$ & $<.120$ & 79 & $<.050$ & $<.050$ & $<1.60$ \\
\hline $36^{\mathbf{R}}$ & $<.130$ & 106 & $<.029$ & $<.044$ & $<.030$ & $<.120$ & 77 & $<.050$ & $<.050$ & $<1.60$ \\
\hline $36^{\mathbf{F b}}$ & $<.130$ & 104 & $<.029$ & $<.044$ & $<.030$ & $<.120$ & 81 & $<.050$ & $<.050$ & $<1.60$ \\
\hline 37 & $<.130$ & 89 & $<.029$ & $<.044$ & $<.030$ & $<.120$ & 95 & $<.050$ & $<.050$ & $<1.60$ \\
\hline 38 & $<.130$ & 106 & $<.029$ & $<.044$ & $<.030$ & $<.120$ & 67 & $<.050$ & $<.050$ & $<1.60$ \\
\hline $38^{\mathbf{R}}$ & $<.130$ & 108 & $<.029$ & $<.044$ & $<.030$ & $<.120$ & 66 & $<.050$ & $<.050$ & $<1.60$ \\
\hline $38^{\mathbf{F b}}$ & $<.130$ & 102 & $<.029$ & $<.044$ & $<.030$ & $<.120$ & 69 & $<.050$ & $<.050$ & $<1.60$ \\
\hline 39 & $<.130$ & 149 & $<.029$ & $<.044$ & $<.030$ & $<.120$ & 64 & $<.050$ & $<.050$ & $<1.60$ \\
\hline 40 & $<.130$ & 149 & $<.029$ & $<.044$ & $<.030$ & $<.120$ & 61 & $<.050$ & $<.050$ & $<1.60$ \\
\hline 41 & $<.130$ & 130 & $<.029$ & $<.044$ & $<.030$ & $<.120$ & 68 & $<.050$ & $<.050$ & $<1.60$ \\
\hline 42 & $<.130$ & 117 & $<.029$ & $<.044$ & $<.030$ & $<.120$ & 72 & $<.050$ & $<.050$ & $<1.60$ \\
\hline 43 & $<.130$ & 111 & $<.029$ & $<.044$ & $<.030$ & $<.120$ & 79 & $<.050$ & $<.050$ & $<1.60$ \\
\hline 44 & $<.130$ & 113 & $<.029$ & $<.044$ & $<.030$ & $<.120$ & 81 & $<.050$ & $<.050$ & $<1.60$ \\
\hline 45 & $<.130$ & 106 & $<.029$ & $<.044$ & $<.030$ & $<.120$ & 97 & $<.050$ & $<.050$ & $<1.60$ \\
\hline 46 & $<.130$ & 116 & $<.029$ & $<.044$ & $<.030$ & $<.120$ & 77 & $<.050$ & $<.050$ & $<1.60$ \\
\hline 47 & $<.130$ & 126 & $<.029$ & $<.044$ & $<.030$ & $<.120$ & 70 & $<.050$ & $<.050$ & $<1.60$ \\
\hline 48 & $<.130$ & 122 & $<.029$ & $<.044$ & $<.030$ & $<.120$ & 77 & $<.050$ & $<.050$ & $<1.60$ \\
\hline $48^{\mathbf{F b}}$ & $<.130$ & 112 & $<.029$ & $<.044$ & $<.030$ & $<.120$ & 81 & $<.050$ & $<.050$ & $<1.60$ \\
\hline 49 & $<.130$ & 112 & $<.029$ & $<.044$ & $<.030$ & $<.120$ & 78 & $<.050$ & $<.050$ & $<1.60$ \\
\hline 50 & $<.130$ & 120 & $<.029$ & $<.044$ & $<.030$ & $<.120$ & 74 & $<.050$ & $<.050$ & $<1.60$ \\
\hline 51 & $<.130$ & 109 & $<.029$ & $<.044$ & $<.030$ & $<.120$ & 79 & $<.050$ & $<.050$ & $<1.60$ \\
\hline 52 & $<.130$ & 114 & $<.029$ & $<.044$ & $<.030$ & $<.120$ & 83 & $<.050$ & $<.050$ & $<1.60$ \\
\hline 53 & $<.130$ & 109 & $<.029$ & $<.044$ & $<.030$ & $<.120$ & 82 & $<.050$ & $<.050$ & $<1.60$ \\
\hline 54 & $<.130$ & 111 & $<.029$ & $<.044$ & $<.030$ & $<.120$ & 80 & $<.050$ & $<.050$ & $<1.60$ \\
\hline 55 & $<.130$ & 116 & $<.029$ & $<.044$ & $<.030$ & $<.120$ & 70 & $<.050$ & $<.050$ & $<1.60$ \\
\hline 56 & $<.130$ & 106 & $<.029$ & $<.044$ & $<.030$ & $<.120$ & 77 & $<.050$ & $<.050$ & $<1.60$ \\
\hline 57 & $<.130$ & 107 & $<.029$ & $<.044$ & $<.030$ & $<.120$ & 75 & $<.050$ & $<.050$ & $<1.60$ \\
\hline 58 & $<.130$ & 111 & $<.029$ & $<.044$ & $<.030$ & $<.120$ & 83 & $<.050$ & $<.050$ & $<1.60$ \\
\hline $58^{\mathbf{R}}$ & $<.130$ & 111 & $<.029$ & $<.044$ & $<.030$ & $<.120$ & 84 & $<.050$ & $<.050$ & $<1.60$ \\
\hline 59 & $<.130$ & 108 & $<.029$ & $<.044$ & $<.030$ & $<.120$ & 76 & $<.050$ & $<.050$ & $<1.60$ \\
\hline 60 & $<.130$ & 104 & $<.029$ & $<.044$ & $<.030$ & $<.120$ & 80 & $<.050$ & $<.050$ & $<1.60$ \\
\hline 61 & $<.130$ & 101 & $<.029$ & $<.044$ & $<.030$ & $<.120$ & 83 & $<.050$ & $<.050$ & $<1.60$ \\
\hline
\end{tabular}


Table 20. Volatile organic-compound concentration data for ground-water samples collected from basin-fill aquifers, Northern Rockies Intermontane Basins study unit, 1999 and 2001 (Continued)

\begin{tabular}{|c|c|c|c|c|c|c|c|c|c|c|}
\hline $\begin{array}{c}\text { Well } \\
\text { number } \\
\text { (figs. } 3 \\
\text { and 4) }\end{array}$ & $\begin{array}{c}\text { 2-Chloro- } \\
\text { toluene } \\
(\mu \mathrm{g} / \mathrm{L})\end{array}$ & $\begin{array}{c}2- \\
\text { Hexanone } \\
(\mu \mathrm{g} / \mathrm{L})\end{array}$ & $\begin{array}{c}\text { 3-Chloro- } \\
\text { propene } \\
(\mu \mathrm{g} / \mathrm{L})\end{array}$ & $\begin{array}{l}\text { 4-Chloro- } \\
\text { toluene } \\
(\mu \mathrm{g} / \mathrm{L})\end{array}$ & $\begin{array}{l}\text { 4-Isopro- } \\
\text { pyl-1- } \\
\text { methyl- } \\
\text { benzene } \\
(\mu \mathrm{g} / \mathrm{L})\end{array}$ & $\begin{array}{l}\text { 4-Methyl-2- } \\
\text { pentanone } \\
(\mu \mathrm{g} / \mathrm{L})\end{array}$ & $\begin{array}{c}\begin{array}{c}\text { Ace- } \\
\text { tone } \\
(\mu \mathrm{g} / \mathrm{L})\end{array}\end{array}$ & $\begin{array}{c}\text { Acrylo- } \\
\text { nitrile } \\
(\mu \mathrm{g} / \mathrm{L})\end{array}$ & $\begin{array}{c}\text { Benzene } \\
(\mu \mathrm{g} / \mathrm{L})\end{array}$ & $\begin{array}{c}\text { Bromo- } \\
\text { benzene } \\
(\mu \mathrm{g} / \mathrm{L})\end{array}$ \\
\hline \multicolumn{11}{|c|}{ Subunit Survey 2, Montana } \\
\hline 32 & $<.026$ & $<.700$ & $<.070$ & $<.060$ & $<.070$ & $<.370$ & $<7.00$ & $<1.20$ & $<.035$ & $<.036$ \\
\hline 33 & $<.026$ & $<.700$ & $<.070$ & $<.060$ & $<.070$ & $<.370$ & $<7.00$ & $<1.20$ & $<.035$ & $<.036$ \\
\hline 34 & $<.026$ & $<.700$ & $<.070$ & $<.060$ & $<.070$ & $<.370$ & $<7.00$ & $<1.20$ & $<.035$ & $<.036$ \\
\hline 35 & $<.026$ & $<.700$ & $<.070$ & $<.060$ & $<.070$ & $<.370$ & $<7.00$ & $<1.20$ & $<.035$ & $<.036$ \\
\hline 36 & $<.026$ & $<.700$ & $<.070$ & $<.060$ & $<.070$ & $<.370$ & $<7.00$ & $<1.20$ & $<.035$ & $<.036$ \\
\hline $36^{\mathbf{R}}$ & $<.026$ & $<.700$ & $<.070$ & $<.060$ & $<.070$ & $<.370$ & $<7.00$ & $<1.20$ & $<.035$ & $<.036$ \\
\hline $36^{\mathbf{F b}}$ & $<.026$ & $<.700$ & $<.070$ & $<.060$ & $<.070$ & $<.370$ & $<7.00$ & $<1.20$ & $<.035$ & $<.036$ \\
\hline 37 & $<.026$ & $<.700$ & $<.070$ & $<.060$ & $<.070$ & $<.370$ & $<7.00$ & $<1.20$ & $<.035$ & $<.036$ \\
\hline 38 & $<.026$ & $<.700$ & $<.070$ & $<.060$ & $<.070$ & $<.370$ & $<7.00$ & $<1.20$ & $<.035$ & $<.036$ \\
\hline $38^{\mathbf{R}}$ & $<.026$ & $<.700$ & $<.070$ & $<.060$ & $<.070$ & $<.370$ & $<7.00$ & $<1.20$ & $<.035$ & $<.036$ \\
\hline $38^{\mathbf{F b}}$ & $<.026$ & $<.700$ & $<.070$ & $<.060$ & $<.070$ & $<.370$ & $<7.00$ & $<1.20$ & $<.035$ & $<.036$ \\
\hline 39 & $<.026$ & $<.700$ & $<.070$ & $<.060$ & $<.070$ & $<.370$ & $<7.00$ & $<1.20$ & $<.035$ & $<.036$ \\
\hline 40 & $<.026$ & $<.700$ & $<.070$ & $<.060$ & $<.070$ & $<.370$ & $<7.00$ & $<1.20$ & $<.035$ & $<.036$ \\
\hline 41 & $<.026$ & $<.700$ & $<.070$ & $<.060$ & $<.070$ & $<.370$ & $<7.00$ & $<1.20$ & $<.035$ & $<.036$ \\
\hline 42 & $<.026$ & $<.700$ & $<.070$ & $<.060$ & $<.070$ & $<.370$ & $<7.00$ & $<1.20$ & $<.035$ & $<.036$ \\
\hline 43 & $<.026$ & $<.700$ & $<.070$ & $<.060$ & $<.070$ & $<.370$ & $<7.00$ & $<1.20$ & $<.035$ & $<.036$ \\
\hline 44 & $<.026$ & $<.700$ & $<.070$ & $<.060$ & $<.070$ & $<.370$ & $<7.00$ & $<1.20$ & $<.035$ & $<.036$ \\
\hline 45 & $<.026$ & $<.700$ & $<.070$ & $<.060$ & $<.070$ & $<.370$ & $<7.00$ & $<1.20$ & $<.035$ & $<.036$ \\
\hline 46 & $<.026$ & $<.700$ & $<.070$ & $<.060$ & $<.070$ & $<.370$ & $<7.00$ & $<1.20$ & $<.035$ & $<.036$ \\
\hline 47 & $<.026$ & $<.700$ & $<.070$ & $<.060$ & $<.070$ & $<.370$ & $<7.00$ & $<1.20$ & $<.035$ & $<.036$ \\
\hline 48 & $<.026$ & $<.700$ & $<.070$ & $<.060$ & $<.070$ & $<.370$ & $<7.00$ & $<1.20$ & $<.035$ & $<.036$ \\
\hline $48^{\mathbf{F b}}$ & $<.026$ & $<.700$ & $<.070$ & $<.060$ & $<.070$ & $<.370$ & $<7.00$ & $<1.20$ & $<.035$ & $<.036$ \\
\hline 49 & $<.026$ & $<.700$ & $<.070$ & $<.060$ & $<.070$ & $<.370$ & $<7.00$ & $<1.20$ & $<.035$ & $<.036$ \\
\hline 50 & $<.026$ & $<.700$ & $<.070$ & $<.060$ & $<.070$ & $<.370$ & $<7.00$ & $<1.20$ & $<.035$ & $<.036$ \\
\hline 51 & $<.026$ & $<.700$ & $<.070$ & $<.060$ & $<.070$ & $<.370$ & $<7.00$ & $<1.20$ & $<.035$ & $<.036$ \\
\hline 52 & $<.026$ & $<.700$ & $<.070$ & $<.060$ & $<.070$ & $<.370$ & $<7.00$ & $<1.20$ & $<.035$ & $<.036$ \\
\hline 53 & $<.026$ & $<.700$ & $<.070$ & $<.060$ & $<.070$ & $<.370$ & $<7.00$ & $<1.20$ & $<.035$ & $<.036$ \\
\hline 54 & $<.026$ & $<.700$ & $<.070$ & $<.060$ & $<.070$ & $<.370$ & $<7.00$ & $<1.20$ & $<.035$ & $<.036$ \\
\hline 55 & $<.026$ & $<.700$ & $<.070$ & $<.060$ & $<.070$ & $<.370$ & $<7.00$ & $<1.20$ & $<.035$ & $<.036$ \\
\hline 56 & $<.026$ & $<.700$ & $<.070$ & $<.060$ & $<.070$ & $<.370$ & $<7.00$ & $<1.20$ & $<.035$ & $<.036$ \\
\hline 57 & $<.026$ & $<.700$ & $<.070$ & $<.060$ & $<.070$ & $<.370$ & $<7.00$ & $<1.20$ & $<.035$ & $<.036$ \\
\hline 58 & $<.026$ & $<.700$ & $<.070$ & $<.060$ & $<.070$ & $<.370$ & $<7.00$ & $<1.20$ & $<.035$ & $<.036$ \\
\hline $58^{\mathbf{R}}$ & $<.026$ & $<.700$ & $<.070$ & $<.060$ & $<.070$ & $<.370$ & $<7.00$ & $<1.20$ & $<.035$ & $<.036$ \\
\hline 59 & $<.026$ & $<.700$ & $<.070$ & $<.060$ & $<.070$ & $<.370$ & $<7.00$ & $<1.20$ & $<.035$ & $<.036$ \\
\hline 60 & $<.026$ & $<.700$ & $<.070$ & $<.060$ & $<.070$ & $<.370$ & $<7.00$ & $<1.20$ & $<.035$ & $<.036$ \\
\hline 61 & $<.026$ & $<.700$ & $<.070$ & $<.060$ & $<.070$ & $<.370$ & $<7.00$ & $<1.20$ & $<.035$ & $<.036$ \\
\hline
\end{tabular}


Table 20. Volatile organic-compound concentration data for ground-water samples collected from basin-fill aquifers, Northern Rockies Intermontane Basins study unit, 1999 and 2001 (Continued)

\begin{tabular}{|c|c|c|c|c|c|c|c|c|c|c|}
\hline $\begin{array}{c}\text { Well } \\
\text { number } \\
\text { (figs. } 3 \\
\text { and 4) }\end{array}$ & $\begin{array}{c}\text { Bromo- } \\
\text { chloro- } \\
\text { meth- } \\
\text { thane } \\
(\mu \mathrm{g} / \mathrm{L})\end{array}$ & $\begin{array}{l}\text { Bromo- } \\
\text { dichloro- } \\
\text { methane } \\
(\mu \mathrm{g} / \mathrm{L})\end{array}$ & $\begin{array}{c}\text { Bromo- } \\
\text { ethane } \\
(\mu \mathrm{g} / \mathrm{L})\end{array}$ & $\begin{array}{c}\text { Bromo- } \\
\text { form } \\
(\mu \mathrm{g} / \mathrm{L})\end{array}$ & $\begin{array}{c}\text { Bromo- } \\
\text { methane } \\
(\mu \mathrm{g} / \mathrm{L})\end{array}$ & $\begin{array}{c}\text { Butyl- } \\
\text { benzene } \\
(\mu \mathrm{g} / \mathrm{L})\end{array}$ & $\begin{array}{c}\text { Carbon } \\
\text { disulfide } \\
(\mu \mathrm{g} / \mathrm{L})\end{array}$ & $\begin{array}{c}\text { Chloro- } \\
\text { benzene } \\
(\mu \mathrm{g} / \mathrm{L})\end{array}$ & $\begin{array}{c}\text { Chloro- } \\
\text { ethane } \\
(\mu \mathrm{g} / \mathrm{L})\end{array}$ & $\begin{array}{c}\text { Chloro- } \\
\text { form } \\
(\mu \mathrm{g} / \mathrm{L})\end{array}$ \\
\hline \multicolumn{11}{|c|}{ Subunit Survey 2, Montana } \\
\hline 32 & $<.044$ & $<.048$ & $<.100$ & $<.060$ & $<.260$ & $<.190$ & $<.070$ & $<.028$ & $<.120$ & $<.024$ \\
\hline 33 & $<.044$ & $<.048$ & $<.100$ & $<.060$ & $<.260$ & $<.190$ & $<.070$ & $<.028$ & $<.120$ & $<.024$ \\
\hline 34 & $<.044$ & $<.048$ & $<.100$ & $<.060$ & $<.260$ & $<.190$ & $<.070$ & $<.028$ & $<.120$ & $<.024$ \\
\hline 35 & $<.044$ & $<.048$ & $<.100$ & $<.060$ & $<.260$ & $<.190$ & $<.070$ & $<.028$ & $<.120$ & $<.024$ \\
\hline 36 & $<.044$ & $<.048$ & $<.100$ & $<.060$ & $<.260$ & $<.190$ & $<.070$ & $<.028$ & $<.120$ & $<.024$ \\
\hline $36^{\mathbf{R}}$ & $<.044$ & $<.048$ & $<.100$ & $<.060$ & $<.260$ & $<.190$ & $<.070$ & $<.028$ & $<.120$ & $<.024$ \\
\hline $36^{\mathbf{F b}}$ & $<.044$ & $<.048$ & $<.100$ & $<.060$ & $<.260$ & $<.190$ & $<.070$ & $<.028$ & $<.120$ & $<.024$ \\
\hline 37 & $<.044$ & $<.048$ & $<.100$ & $<.060$ & $<.260$ & $<.190$ & $<.070$ & $<.028$ & $<.120$ & $<.024$ \\
\hline 38 & $<.044$ & $<.048$ & $<.100$ & $<.060$ & $<.260$ & $<.190$ & $<.070$ & $<.028$ & $<.120$ & $<.024$ \\
\hline $38^{\mathbf{R}}$ & $<.044$ & $<.048$ & $<.100$ & $<.060$ & $<.260$ & $<.190$ & $<.070$ & $<.028$ & $<.120$ & $<.024$ \\
\hline $38^{\mathbf{F b}}$ & $<.044$ & $<.048$ & $<.100$ & $<.060$ & $<.260$ & $<.190$ & $<.070$ & $<.028$ & $<.120$ & $<.024$ \\
\hline 39 & $<.044$ & e.062 & $<.100$ & $<.060$ & $<.260$ & $<.190$ & $<.070$ & $<.028$ & $<.120$ & .111 \\
\hline 40 & $<.044$ & $<.048$ & $<.100$ & $<.060$ & $<.260$ & $<.190$ & $<.070$ & $<.028$ & $<.120$ & e.023 \\
\hline 41 & $<.044$ & .165 & $<.100$ & $<.060$ & $<.260$ & $<.190$ & $<.070$ & $<.028$ & $<.120$ & .214 \\
\hline 42 & $<.044$ & $<.048$ & $<.100$ & $<.060$ & $<.260$ & $<.190$ & $<.070$ & $<.028$ & $<.120$ & $<.024$ \\
\hline 43 & $<.044$ & $<.048$ & $<.100$ & $<.060$ & $<.260$ & $<.190$ & $<.070$ & $<.028$ & $<.120$ & $<.024$ \\
\hline 44 & $<.044$ & $<.048$ & $<.100$ & $<.060$ & $<.260$ & $<.190$ & $<.070$ & $<.028$ & $<.120$ & $<.024$ \\
\hline 45 & $<.044$ & $<.048$ & $<.100$ & $<.060$ & $<.260$ & $<.190$ & $<.070$ & $<.028$ & $<.120$ & $<.024$ \\
\hline 46 & $<.044$ & $<.048$ & $<.100$ & $<.060$ & $<.260$ & $<.190$ & $<.070$ & $<.028$ & $<.120$ & $<.024$ \\
\hline 47 & $<.044$ & $<.048$ & $<.100$ & $<.060$ & $<.260$ & $<.190$ & $<.070$ & $<.028$ & $<.120$ & $<.024$ \\
\hline 48 & $<.044$ & $<.048$ & $<.100$ & $<.060$ & $<.260$ & $<.190$ & $<.070$ & $<.028$ & $<.120$ & $<.024$ \\
\hline $48^{\mathbf{F b}}$ & $<.044$ & $<.048$ & $<.100$ & $<.060$ & $<.260$ & $<.190$ & $<.070$ & $<.028$ & $<.120$ & $<.024$ \\
\hline 49 & $<.044$ & $<.048$ & $<.100$ & $<.060$ & $<.260$ & $<.190$ & $<.070$ & $<.028$ & $<.120$ & $<.024$ \\
\hline 50 & $<.044$ & $<.048$ & $<.100$ & $<.060$ & $<.260$ & $<.190$ & $<.070$ & $<.028$ & $<.120$ & $<.024$ \\
\hline 51 & $<.044$ & $<.048$ & $<.100$ & $<.060$ & $<.260$ & $<.190$ & $<.070$ & $<.028$ & $<.120$ & $<.024$ \\
\hline 52 & $<.044$ & $<.048$ & $<.100$ & $<.060$ & $<.260$ & $<.190$ & $<.070$ & $<.028$ & $<.120$ & $<.024$ \\
\hline 53 & $<.044$ & $<.048$ & $<.100$ & $<.060$ & $<.260$ & $<.190$ & $<.070$ & $<.028$ & $<.120$ & $<.024$ \\
\hline 54 & $<.044$ & $<.048$ & $<.100$ & $<.060$ & $<.260$ & $<.190$ & $<.070$ & $<.028$ & $<.120$ & $<.024$ \\
\hline 55 & $<.044$ & $<.048$ & $<.100$ & $<.060$ & $<.260$ & $<.190$ & $<.070$ & $<.028$ & $<.120$ & $<.024$ \\
\hline 56 & $<.044$ & $<.048$ & $<.100$ & $<.060$ & $<.260$ & $<.190$ & $<.070$ & $<.028$ & $<.120$ & $<.024$ \\
\hline 57 & $<.044$ & $<.048$ & $<.100$ & $<.060$ & $<.260$ & $<.190$ & $<.070$ & $<.028$ & $<.120$ & e.007 \\
\hline 58 & $<.044$ & $<.048$ & $<.100$ & $<.060$ & $<.260$ & $<.190$ & $<.070$ & $<.028$ & $<.120$ & $<.024$ \\
\hline $58^{\mathbf{R}}$ & $<.044$ & $<.048$ & $<.100$ & $<.060$ & $<.260$ & $<.190$ & $<.070$ & $<.028$ & $<.120$ & $<.024$ \\
\hline 59 & $<.044$ & $<.048$ & $<.100$ & $<.060$ & $<.260$ & $<.190$ & $<.070$ & $<.028$ & $<.120$ & $<.024$ \\
\hline 60 & $<.044$ & $<.048$ & $<.100$ & $<.060$ & $<.260$ & $<.190$ & $<.070$ & $<.028$ & $<.120$ & $<.024$ \\
\hline 61 & $<.044$ & .163 & $<.100$ & $<.060$ & $<.260$ & $<.190$ & $<.070$ & $<.028$ & $<.120$ & .540 \\
\hline
\end{tabular}


Table 20. Volatile organic-compound concentration data for ground-water samples collected from basin-fill aquifers, Northern Rockies Intermontane Basins study unit, 1999 and 2001 (Continued)

\begin{tabular}{|c|c|c|c|c|c|c|c|c|c|c|}
\hline $\begin{array}{c}\text { Well } \\
\text { number } \\
\text { (figs. } 3 \\
\text { and 4) }\end{array}$ & $\begin{array}{c}\text { Chloro- } \\
\text { methane } \\
(\mu \mathrm{g} / \mathrm{L})\end{array}$ & $\begin{array}{c}\text { cis- } \\
1,2- \\
\text { Dichloro- } \\
\text { ethylene } \\
(\mu \mathrm{g} / \mathrm{L})\end{array}$ & $\begin{array}{c}\text { cis- } \\
\text { 1,3- } \\
\text { Dichloro- } \\
\text { propene } \\
(\mu \mathrm{g} / \mathrm{L})\end{array}$ & $\begin{array}{c}\text { Dibromo- } \\
\text { chloro- } \\
\text { methane } \\
(\mu \mathrm{g} / \mathrm{L})\end{array}$ & $\begin{array}{c}\text { Dibromo- } \\
\text { methane } \\
(\mu \mathrm{g} / \mathrm{L})\end{array}$ & $\begin{array}{c}\text { Dichloro- } \\
\text { difluoro- } \\
\text { methane } \\
(\mu \mathrm{g} / \mathrm{L})\end{array}$ & $\begin{array}{c}\text { Dichloro- } \\
\text { methane } \\
(\mu \mathrm{g} / \mathrm{L})\end{array}$ & $\begin{array}{c}\text { Diethyl } \\
\text { ether } \\
(\mu \mathrm{g} / \mathrm{L})\end{array}$ & $\begin{array}{l}\text { Diiso- } \\
\text { propyl } \\
\text { ether } \\
(\mu \mathrm{g} / \mathrm{L})\end{array}$ & $\begin{array}{c}\text { Ethyl } \\
\text { meth- } \\
\text { acrylate } \\
(\mu \mathrm{g} / \mathrm{L})\end{array}$ \\
\hline \multicolumn{11}{|c|}{ Subunit Survey 2, Montana } \\
\hline 32 & $<.250$ & $<.038$ & $<.090$ & $<.180$ & $<.050$ & $<.270$ & $<.160$ & $<.170$ & $<.100$ & $<.180$ \\
\hline 33 & $<.250$ & $<.038$ & $<.090$ & $<.180$ & $<.050$ & $<.270$ & $<.160$ & $<.170$ & $<.100$ & $<.180$ \\
\hline 34 & $<.250$ & $<.038$ & $<.090$ & $<.180$ & $<.050$ & $<.270$ & $<.160$ & $<.170$ & $<.100$ & $<.180$ \\
\hline 35 & $<.250$ & $<.038$ & $<.090$ & $<.180$ & $<.050$ & $<.270$ & $<.160$ & $<.170$ & $<.100$ & $<.180$ \\
\hline 36 & $<.250$ & $<.038$ & $<.090$ & $<.180$ & $<.050$ & $<.270$ & $<.160$ & $<.170$ & $<.100$ & $<.180$ \\
\hline $36^{\mathbf{R}}$ & $<.250$ & $<.038$ & $<.090$ & $<.180$ & $<.050$ & $<.270$ & $<.160$ & $<.170$ & $<.100$ & $<.180$ \\
\hline $36^{\mathbf{F b}}$ & $<.250$ & $<.038$ & $<.090$ & $<.180$ & $<.050$ & $<.270$ & $<.160$ & $<.170$ & $<.100$ & $<.180$ \\
\hline 37 & $<.250$ & $<.038$ & $<.090$ & $<.180$ & $<.050$ & $<.270$ & $<.160$ & $<.170$ & $<.100$ & $<.180$ \\
\hline 38 & $<.250$ & $<.038$ & $<.090$ & $<.180$ & $<.050$ & $<.270$ & $<.160$ & $<.170$ & $<.100$ & $<.180$ \\
\hline $38^{\mathbf{R}}$ & $<.250$ & $<.038$ & $<.090$ & $<.180$ & $<.050$ & $<.270$ & $<.160$ & $<.170$ & $<.100$ & $<.180$ \\
\hline $38^{\mathbf{F b}}$ & $<.250$ & $<.038$ & $<.090$ & $<.180$ & $<.050$ & $<.270$ & $<.160$ & $<.170$ & $<.100$ & $<.180$ \\
\hline 39 & $<.250$ & $<.038$ & $<.090$ & $<.180$ & $<.050$ & $<.270$ & $<.160$ & $<.170$ & $<.100$ & $<.180$ \\
\hline 40 & $<.250$ & $<.038$ & $<.090$ & $<.180$ & $<.050$ & $<.270$ & $<.160$ & $<.170$ & $<.100$ & $<.180$ \\
\hline 41 & $<.250$ & $<.038$ & $<.090$ & e.062 & $<.050$ & e.131 & $<.160$ & $<.170$ & $<.100$ & $<.180$ \\
\hline 42 & $<.250$ & $<.038$ & $<.090$ & $<.180$ & $<.050$ & $<.270$ & $<.160$ & $<.170$ & $<.100$ & $<.180$ \\
\hline 43 & $<.250$ & $<.038$ & $<.090$ & $<.180$ & $<.050$ & $<.270$ & $<.160$ & $<.170$ & $<.100$ & $<.180$ \\
\hline 44 & $<.250$ & $<.038$ & $<.090$ & $<.180$ & $<.050$ & $<.270$ & $<.160$ & $<.170$ & $<.100$ & $<.180$ \\
\hline 45 & $<.250$ & $<.038$ & $<.090$ & $<.180$ & $<.050$ & e.475 & $<.160$ & $<.170$ & $<.100$ & $<.180$ \\
\hline 46 & $<.250$ & $<.038$ & $<.090$ & $<.180$ & $<.050$ & $<.270$ & $<.160$ & $<.170$ & $<.100$ & $<.180$ \\
\hline 47 & $<.250$ & $<.038$ & $<.090$ & $<.180$ & $<.050$ & $<.270$ & $<.160$ & $<.170$ & $<.100$ & $<.180$ \\
\hline 48 & $<.250$ & $<.038$ & $<.090$ & $<.180$ & $<.050$ & $<.270$ & $<.160$ & $<.170$ & $<.100$ & $<.180$ \\
\hline $48^{\mathbf{F b}}$ & $<.250$ & $<.038$ & $<.090$ & $<.180$ & $<.050$ & $<.270$ & $<.160$ & $<.170$ & $<.100$ & $<.180$ \\
\hline 49 & $<.250$ & $<.038$ & $<.090$ & $<.180$ & $<.050$ & $<.270$ & $<.160$ & $<.170$ & $<.100$ & $<.180$ \\
\hline 50 & $<.250$ & $<.038$ & $<.090$ & $<.180$ & $<.050$ & $<.270$ & $<.160$ & $<.170$ & $<.100$ & $<.180$ \\
\hline 51 & $<.250$ & $<.038$ & $<.090$ & $<.180$ & $<.050$ & $<.270$ & $<.160$ & $<.170$ & $<.100$ & $<.180$ \\
\hline 52 & $<.250$ & $<.038$ & $<.090$ & $<.180$ & $<.050$ & $<.270$ & $<.160$ & $<.170$ & $<.100$ & $<.180$ \\
\hline 53 & $<.250$ & $<.038$ & $<.090$ & $<.180$ & $<.050$ & $<.270$ & $<.160$ & $<.170$ & $<.100$ & $<.180$ \\
\hline 54 & $<.250$ & $<.038$ & $<.090$ & $<.180$ & $<.050$ & $<.270$ & $<.160$ & $<.170$ & $<.100$ & $<.180$ \\
\hline 55 & $<.250$ & $<.038$ & $<.090$ & $<.180$ & $<.050$ & $<.270$ & $<.160$ & $<.170$ & $<.100$ & $<.180$ \\
\hline 56 & $<.250$ & $<.038$ & $<.090$ & $<.180$ & $<.050$ & $<.270$ & $<.160$ & $<.170$ & $<.100$ & $<.180$ \\
\hline 57 & $<.250$ & $<.038$ & $<.090$ & $<.180$ & $<.050$ & $<.270$ & $<.160$ & $<.170$ & $<.100$ & $<.180$ \\
\hline 58 & $<.250$ & $<.038$ & $<.090$ & $<.180$ & $<.050$ & $<.270$ & $<.160$ & $<.170$ & $<.100$ & $<.180$ \\
\hline $58^{\mathbf{R}}$ & $<.250$ & $<.038$ & $<.090$ & $<.180$ & $<.050$ & $<.270$ & $<.160$ & $<.170$ & $<.100$ & $<.180$ \\
\hline 59 & $<.250$ & $<.038$ & $<.090$ & $<.180$ & $<.050$ & $<.270$ & $<.160$ & $<.170$ & $<.100$ & $<.180$ \\
\hline 60 & $<.250$ & $<.038$ & $<.090$ & $<.180$ & $<.050$ & $<.270$ & $<.160$ & $<.170$ & $<.100$ & $<.180$ \\
\hline 61 & $<.250$ & $<.038$ & $<.090$ & e.054 & $<.050$ & $<.270$ & $<.160$ & $<.170$ & $<.100$ & $<.180$ \\
\hline
\end{tabular}


Table 20. Volatile organic-compound concentration data for ground-water samples collected from basin-fill aquifers, Northern Rockies Intermontane Basins study unit, 1999 and 2001 (Continued)

\begin{tabular}{|c|c|c|c|c|c|c|c|c|c|c|}
\hline $\begin{array}{c}\text { Well } \\
\text { number } \\
\text { (figs. } 3 \\
\text { and 4) }\end{array}$ & $\begin{array}{l}\text { Ethyl } \\
\text { tert- } \\
\text { butyl } \\
\text { ether } \\
(\mu \mathrm{g} / \mathrm{L})\end{array}$ & $\begin{array}{c}\text { Ethyl- } \\
\text { benzene } \\
(\mu \mathrm{g} / \mathrm{L})\end{array}$ & $\begin{array}{c}\text { Hexa- } \\
\text { chloro- } \\
\text { butadiene } \\
(\mu \mathrm{g} / \mathrm{L})\end{array}$ & $\begin{array}{l}\text { Hexa- } \\
\text { chloro- } \\
\text { ethane } \\
(\mu \mathrm{g} / \mathrm{L})\end{array}$ & $\begin{array}{c}\text { Isopropyl- } \\
\text { benzene } \\
(\mu \mathrm{g} / \mathrm{L})\end{array}$ & $\begin{array}{l}\text { m- and p- } \\
\text { Xylene } \\
(\mu \mathrm{g} / \mathrm{L})\end{array}$ & $\begin{array}{c}\text { Methyl } \\
\text { acrylate } \\
(\mu \mathrm{g} / \mathrm{L})\end{array}$ & $\begin{array}{l}\text { Methyl } \\
\text { acrylo- } \\
\text { nitrile } \\
(\mu \mathrm{g} / \mathrm{L})\end{array}$ & $\begin{array}{l}\text { Methyl } \\
\text { iodide } \\
(\mu \mathrm{g} / \mathrm{L})\end{array}$ & $\begin{array}{c}\text { Methyl } \\
\text { methacrylate } \\
(\mu \mathrm{g} / \mathrm{L})\end{array}$ \\
\hline \multicolumn{11}{|c|}{ Subunit Survey 2, Montana } \\
\hline 32 & $<.054$ & $<.030$ & $<.140$ & $<.190$ & $<.032$ & $<.060$ & $<1.4$ & $<.600$ & $<.120$ & $<.350$ \\
\hline 33 & $<.054$ & $<.030$ & $<.140$ & $<.190$ & $<.032$ & $<.060$ & $<1.4$ & $<.600$ & $<.120$ & $<.350$ \\
\hline 34 & $<.054$ & $<.030$ & $<.140$ & $<.190$ & $<.032$ & $<.060$ & $<1.4$ & $<.600$ & $<.120$ & $<.350$ \\
\hline 35 & $<.054$ & $<.030$ & $<.140$ & $<.190$ & $<.032$ & $<.060$ & $<1.4$ & $<.600$ & $<.120$ & $<.350$ \\
\hline 36 & $<.054$ & $<.030$ & $<.140$ & $<.190$ & $<.032$ & $<.060$ & $<1.4$ & $<.600$ & $<.120$ & $<.350$ \\
\hline $36^{\mathbf{R}}$ & $<.054$ & $<.030$ & $<.140$ & $<.190$ & $<.032$ & $<.060$ & $<1.4$ & $<.600$ & $<.120$ & $<.350$ \\
\hline $36^{\mathbf{F b}}$ & $<.054$ & $<.030$ & $<.140$ & $<.190$ & $<.032$ & $<.060$ & $<1.4$ & $<.600$ & $<.120$ & $<.350$ \\
\hline 37 & $<.054$ & $<.030$ & $<.140$ & $<.190$ & $<.032$ & $<.060$ & $<1.4$ & $<.600$ & $<.120$ & $<.350$ \\
\hline 38 & $<.054$ & $<.030$ & $<.140$ & $<.190$ & $<.032$ & $<.060$ & $<1.4$ & $<.600$ & $<.120$ & $<.350$ \\
\hline $38^{\mathbf{R}}$ & $<.054$ & $<.030$ & $<.140$ & $<.190$ & $<.032$ & $<.060$ & $<1.4$ & $<.600$ & $<.120$ & $<.350$ \\
\hline $38^{\mathbf{F b}}$ & $<.054$ & $<.030$ & $<.140$ & $<.190$ & $<.032$ & $<.060$ & $<1.4$ & $<.600$ & $<.120$ & $<.350$ \\
\hline 39 & $<.054$ & $<.030$ & $<.140$ & $<.190$ & $<.032$ & $<.060$ & $<1.4$ & $<.600$ & $<.120$ & $<.350$ \\
\hline 40 & $<.054$ & $<.030$ & $<.140$ & $<.190$ & $<.032$ & $<.060$ & $<1.4$ & $<.600$ & $<.120$ & $<.350$ \\
\hline 41 & $<.054$ & $<.030$ & $<.140$ & $<.190$ & $<.032$ & $<.060$ & $<1.4$ & $<.600$ & $<.120$ & $<.350$ \\
\hline 42 & $<.054$ & $<.030$ & $<.140$ & $<.190$ & $<.032$ & $<.060$ & $<1.4$ & $<.600$ & $<.120$ & $<.350$ \\
\hline 43 & $<.054$ & $<.030$ & $<.140$ & $<.190$ & $<.032$ & $<.060$ & $<1.4$ & $<.600$ & $<.120$ & $<.350$ \\
\hline 44 & $<.054$ & $<.030$ & $<.140$ & $<.190$ & $<.032$ & $<.060$ & $<1.4$ & $<.600$ & $<.120$ & $<.350$ \\
\hline 45 & $<.054$ & $<.030$ & $<.140$ & $<.190$ & $<.032$ & $<.060$ & $<1.4$ & $<.600$ & $<.120$ & $<.350$ \\
\hline 46 & $<.054$ & $<.030$ & $<.140$ & $<.190$ & $<.032$ & $<.060$ & $<1.4$ & $<.600$ & $<.120$ & $<.350$ \\
\hline 47 & $<.054$ & $<.030$ & $<.140$ & $<.190$ & $<.032$ & $<.060$ & $<1.4$ & $<.600$ & $<.120$ & $<.350$ \\
\hline 48 & $<.054$ & $<.030$ & $<.140$ & $<.190$ & $<.032$ & $<.060$ & $<1.4$ & $<.600$ & $<.120$ & $<.350$ \\
\hline $48^{\mathbf{F b}}$ & $<.054$ & $<.030$ & $<.140$ & $<.190$ & $<.032$ & $<.060$ & $<1.4$ & $<.600$ & $<.120$ & $<.350$ \\
\hline 49 & $<.054$ & $<.030$ & $<.140$ & $<.190$ & $<.032$ & $<.060$ & $<1.4$ & $<.600$ & $<.120$ & $<.350$ \\
\hline 50 & $<.054$ & $<.030$ & $<.140$ & $<.190$ & $<.032$ & $<.060$ & $<1.4$ & $<.600$ & $<.120$ & $<.350$ \\
\hline 51 & $<.054$ & $<.030$ & $<.140$ & $<.190$ & $<.032$ & $<.060$ & $<1.4$ & $<.600$ & $<.120$ & $<.350$ \\
\hline 52 & $<.054$ & $<.030$ & $<.140$ & $<.190$ & $<.032$ & $<.060$ & $<1.4$ & $<.600$ & $<.120$ & $<.350$ \\
\hline 53 & $<.054$ & $<.030$ & $<.140$ & $<.190$ & $<.032$ & $<.060$ & $<1.4$ & $<.600$ & $<.120$ & $<.350$ \\
\hline 54 & $<.054$ & $<.030$ & $<.140$ & $<.190$ & $<.032$ & $<.060$ & $<1.4$ & $<.600$ & $<.120$ & $<.350$ \\
\hline 55 & $<.054$ & $<.030$ & $<.140$ & $<.190$ & $<.032$ & $<.060$ & $<1.4$ & $<.600$ & $<.120$ & $<.350$ \\
\hline 56 & $<.054$ & $<.030$ & $<.140$ & $<.190$ & $<.032$ & $<.060$ & $<1.4$ & $<.600$ & $<.120$ & $<.350$ \\
\hline 57 & $<.054$ & $<.030$ & $<.140$ & $<.190$ & $<.032$ & $<.060$ & $<1.4$ & $<.600$ & $<.120$ & $<.350$ \\
\hline 58 & $<.054$ & $<.030$ & $<.140$ & $<.190$ & $<.032$ & $<.060$ & $<1.4$ & $<.600$ & $<.120$ & $<.350$ \\
\hline $58^{\mathbf{R}}$ & $<.054$ & $<.030$ & $<.140$ & $<.190$ & $<.032$ & $<.060$ & $<1.4$ & $<.600$ & $<.120$ & $<.350$ \\
\hline 59 & $<.054$ & $<.030$ & $<.140$ & $<.190$ & $<.032$ & $<.060$ & $<1.4$ & $<.600$ & $<.120$ & $<.350$ \\
\hline 60 & $<.054$ & $<.030$ & $<.140$ & $<.190$ & $<.032$ & $<.060$ & $<1.4$ & $<.600$ & $<.120$ & $<.350$ \\
\hline 61 & $<.054$ & $<.030$ & $<.140$ & $<.190$ & $<.032$ & $<.060$ & $<1.4$ & $<.600$ & $<.120$ & $<.350$ \\
\hline
\end{tabular}


Table 20. Volatile organic-compound concentration data for ground-water samples collected from basin-fill aquifers, Northern Rockies Intermontane Basins study unit, 1999 and 2001 (Continued)

\begin{tabular}{|c|c|c|c|c|c|c|c|c|c|c|}
\hline $\begin{array}{c}\text { Well } \\
\text { number } \\
\text { (figs. } 3 \\
\text { and 4) }\end{array}$ & $\begin{array}{l}\text { Naphthalene } \\
(\mu \mathrm{g} / \mathrm{L})\end{array}$ & $\begin{array}{c}\text { n-Propyl- } \\
\text { benzene } \\
(\mu \mathrm{g} / \mathrm{L})\end{array}$ & $\begin{array}{c}\text { o-Ethyl } \\
\text { toluene } \\
(\mu \mathrm{g} / \mathrm{L})\end{array}$ & $\begin{array}{c}\text { o-Xylene } \\
(\mu \mathrm{g} / \mathrm{L})\end{array}$ & $\begin{array}{c}\text { sec-Butyl- } \\
\text { benzene } \\
(\mu \mathrm{g} / \mathrm{L})\end{array}$ & $\begin{array}{c}\text { Styrene } \\
(\mu \mathrm{g} / \mathrm{L})\end{array}$ & $\begin{array}{l}\text { tert- } \\
\text { Butyl } \\
\text { methyl } \\
\text { ether } \\
(\mu \mathrm{g} / \mathrm{L})\end{array}$ & $\begin{array}{c}\text { tert- } \\
\text { Butyl- } \\
\text { benzene } \\
(\mu \mathrm{g} / \mathrm{L})\end{array}$ & $\begin{array}{c}\text { tert- } \\
\text { Pentyl } \\
\text { methyl } \\
\text { ether } \\
(\mu \mathrm{g} / \mathrm{L})\end{array}$ & $\begin{array}{c}\text { Tetra- } \\
\text { chloro- } \\
\text { ethylene } \\
(\mu \mathrm{g} / \mathrm{L})\end{array}$ \\
\hline \multicolumn{11}{|c|}{ Subunit Survey 2, Montana } \\
\hline 32 & $<.250$ & $<.042$ & $<.060$ & $<.038$ & $<.032$ & $<.042$ & $<.170$ & $<.060$ & $<.110$ & $<.100$ \\
\hline 33 & $<.250$ & $<.042$ & $<.060$ & $<.038$ & $<.032$ & $<.042$ & $<.170$ & $<.060$ & $<.110$ & $<.100$ \\
\hline 34 & $<.250$ & $<.042$ & $<.060$ & $<.038$ & $<.032$ & $<.042$ & $<.170$ & $<.060$ & $<.110$ & $<.100$ \\
\hline 35 & $<.250$ & $<.042$ & $<.060$ & $<.038$ & $<.032$ & $<.042$ & $<.170$ & $<.060$ & $<.110$ & $<.100$ \\
\hline 36 & $<.250$ & $<.042$ & $<.060$ & $<.038$ & $<.032$ & $<.042$ & $<.170$ & $<.060$ & $<.110$ & $<.100$ \\
\hline $36^{\mathrm{R}}$ & $<.250$ & $<.042$ & $<.060$ & $<.038$ & $<.032$ & $<.042$ & $<.170$ & $<.060$ & $<.110$ & $<.100$ \\
\hline $36^{\mathbf{F b}}$ & $<.250$ & $<.042$ & $<.060$ & $<.038$ & $<.032$ & $<.042$ & $<.170$ & $<.060$ & $<.110$ & $<.100$ \\
\hline 37 & $<.250$ & $<.042$ & $<.060$ & $<.038$ & $<.032$ & $<.042$ & $<.170$ & $<.060$ & $<.110$ & $<.100$ \\
\hline 38 & $<.250$ & $<.042$ & $<.060$ & $<.038$ & $<.032$ & $<.042$ & $<.170$ & $<.060$ & $<.110$ & $<.100$ \\
\hline $38^{R}$ & $<.250$ & $<.042$ & $<.060$ & $<.038$ & $<.032$ & $<.042$ & $<.170$ & $<.060$ & $<.110$ & $<.100$ \\
\hline $38^{\mathbf{F b}}$ & $<.250$ & $<.042$ & $<.060$ & $<.038$ & $<.032$ & $<.042$ & $<.170$ & $<.060$ & $<.110$ & $<.100$ \\
\hline 39 & $<.250$ & $<.042$ & $<.060$ & $<.038$ & $<.032$ & $<.042$ & $<.170$ & $<.060$ & $<.110$ & .171 \\
\hline 40 & $<.250$ & $<.042$ & $<.060$ & $<.038$ & $<.032$ & $<.042$ & $<.170$ & $<.060$ & $<.110$ & e.071 \\
\hline 41 & $<.250$ & $<.042$ & $<.060$ & $<.038$ & $<.032$ & $<.042$ & $<.170$ & $<.060$ & $<.110$ & .303 \\
\hline 42 & $<.250$ & $<.042$ & $<.060$ & $<.038$ & $<.032$ & $<.042$ & $<.170$ & $<.060$ & $<.110$ & $<.100$ \\
\hline 43 & $<.250$ & $<.042$ & $<.060$ & $<.038$ & $<.032$ & $<.042$ & $<.170$ & $<.060$ & $<.110$ & $<.100$ \\
\hline 44 & $<.250$ & $<.042$ & $<.060$ & $<.038$ & $<.032$ & $<.042$ & $<.170$ & $<.060$ & $<.110$ & $<.100$ \\
\hline 45 & $<.250$ & $<.042$ & $<.060$ & $<.038$ & $<.032$ & $<.042$ & $<.170$ & $<.060$ & $<.110$ & e.020 \\
\hline 46 & $<.250$ & $<.042$ & $<.060$ & $<.038$ & $<.032$ & $<.042$ & $<.170$ & $<.060$ & $<.110$ & $<.100$ \\
\hline 47 & $<.250$ & $<.042$ & $<.060$ & $<.038$ & $<.032$ & $<.042$ & $<.170$ & $<.060$ & $<.110$ & $<.100$ \\
\hline 48 & $<.250$ & $<.042$ & $<.060$ & $<.038$ & $<.032$ & $<.042$ & $<.170$ & $<.060$ & $<.110$ & $<.100$ \\
\hline $48^{\mathbf{F b}}$ & $<.250$ & $<.042$ & $<.060$ & $<.038$ & $<.032$ & $<.042$ & $<.170$ & $<.060$ & $<.110$ & $<.100$ \\
\hline 49 & $<.250$ & $<.042$ & $<.060$ & $<.038$ & $<.032$ & $<.042$ & $<.170$ & $<.060$ & $<.110$ & $<.100$ \\
\hline 50 & $<.250$ & $<.042$ & $<.060$ & $<.038$ & $<.032$ & $<.042$ & $<.170$ & $<.060$ & $<.110$ & $<.100$ \\
\hline 51 & $<.250$ & $<.042$ & $<.060$ & $<.038$ & $<.032$ & $<.042$ & $<.170$ & $<.060$ & $<.110$ & $<.100$ \\
\hline 52 & $<.250$ & $<.042$ & $<.060$ & $<.038$ & $<.032$ & $<.042$ & $<.170$ & $<.060$ & $<.110$ & $<.100$ \\
\hline 53 & $<.250$ & $<.042$ & $<.060$ & $<.038$ & $<.032$ & $<.042$ & $<.170$ & $<.060$ & $<.110$ & $<.100$ \\
\hline 54 & $<.250$ & $<.042$ & $<.060$ & $<.038$ & $<.032$ & $<.042$ & $<.170$ & $<.060$ & $<.110$ & $<.100$ \\
\hline 55 & $<.250$ & $<.042$ & $<.060$ & $<.038$ & $<.032$ & $<.042$ & $<.170$ & $<.060$ & $<.110$ & $<.100$ \\
\hline 56 & $<.250$ & $<.042$ & $<.060$ & $<.038$ & $<.032$ & $<.042$ & $<.170$ & $<.060$ & $<.110$ & e.021 \\
\hline 57 & $<.250$ & $<.042$ & $<.060$ & $<.038$ & $<.032$ & $<.042$ & $<.170$ & $<.060$ & $<.110$ & $<.100$ \\
\hline 58 & $<.250$ & $<.042$ & $<.060$ & $<.038$ & $<.032$ & $<.042$ & $<.170$ & $<.060$ & $<.110$ & $<.100$ \\
\hline $58^{\mathrm{R}}$ & $<.250$ & $<.042$ & $<.060$ & $<.038$ & $<.032$ & $<.042$ & $<.170$ & $<.060$ & $<.110$ & $<.100$ \\
\hline 59 & $<.250$ & $<.042$ & $<.060$ & $<.038$ & $<.032$ & $<.042$ & $<.170$ & $<.060$ & $<.110$ & $<.100$ \\
\hline 60 & $<.250$ & $<.042$ & $<.060$ & $<.038$ & $<.032$ & $<.042$ & $<.170$ & $<.060$ & $<.110$ & $<.100$ \\
\hline 61 & $<.250$ & $<.042$ & $<.060$ & $<.038$ & $<.032$ & $<.042$ & $<.170$ & $<.060$ & $<.110$ & $<.100$ \\
\hline
\end{tabular}


Table 20. Volatile organic-compound concentration data for ground-water samples collected from basin-fill aquifers, Northern Rockies Intermontane Basins study unit, 1999 and 2001 (Continued)

\begin{tabular}{|c|c|c|c|c|c|c|c|c|c|c|}
\hline $\begin{array}{c}\text { Well } \\
\text { number } \\
\text { (figs. } 3 \\
\text { and 4) }\end{array}$ & $\begin{array}{c}\text { Tetra- } \\
\text { chloro- } \\
\text { methane } \\
(\mu \mathrm{g} / \mathrm{L})\end{array}$ & $\begin{array}{c}\text { Tetra- } \\
\text { hydrofuran } \\
(\mu \mathrm{g} / \mathrm{L})\end{array}$ & $\begin{array}{c}\text { Toluene } \\
(\mu \mathrm{g} / \mathrm{L})\end{array}$ & $\begin{array}{c}\text { Toluene-d } \\
\text { surrogate } \\
\text { (percent) }\end{array}$ & $\begin{array}{c}\text { trans- } \\
\text { 1,2- } \\
\text { Dichloro- } \\
\text { ethylene } \\
(\mu \mathrm{g} / \mathrm{L})\end{array}$ & $\begin{array}{c}\text { trans- } \\
1,3- \\
\text { Dichloro- } \\
\text { propene } \\
(\mu \mathrm{g} / \mathrm{L})\end{array}$ & $\begin{array}{c}\text { trans- } \\
1,4,- \\
\text { Dichloro-2- } \\
\text { butene } \\
(\mu \mathrm{g} / \mathrm{L})\end{array}$ & $\begin{array}{c}\text { Trichloro- } \\
\text { ethylene } \\
(\mu \mathrm{g} / \mathrm{L})\end{array}$ & $\begin{array}{l}\text { Trichloro- } \\
\text { fluoro- } \\
\text { methane } \\
(\mu \mathrm{g} / \mathrm{L})\end{array}$ & $\begin{array}{c}\text { Vinyl } \\
\text { chloride } \\
(\mu \mathrm{g} / \mathrm{L})\end{array}$ \\
\hline \multicolumn{11}{|c|}{ Subunit Survey 2, Montana } \\
\hline 32 & $<.060$ & $<2.2$ & $<.050$ & 99 & $<.032$ & $<.090$ & $<.700$ & $<.038$ & $<.090$ & $<.110$ \\
\hline 33 & $<.060$ & $<2.2$ & $<.050$ & 96 & $<.032$ & $<.090$ & $<.700$ & $<.038$ & $<.090$ & $<.110$ \\
\hline 34 & $<.060$ & $<2.2$ & $<.050$ & 98 & $<.032$ & $<.090$ & $<.700$ & $<.038$ & $<.090$ & $<.110$ \\
\hline 35 & $<.060$ & $<2.2$ & $<.050$ & 100 & $<.032$ & $<.090$ & $<.700$ & $<.038$ & $<.090$ & $<.110$ \\
\hline 36 & $<.060$ & $<2.2$ & $<.050$ & 96 & $<.032$ & $<.090$ & $<.700$ & $<.038$ & $<.090$ & $<.110$ \\
\hline $36^{\mathbf{R}}$ & $<.060$ & $<2.2$ & $<.050$ & 96 & $<.032$ & $<.090$ & $<.700$ & $<.038$ & $<.090$ & $<.110$ \\
\hline $36^{\mathbf{F b}}$ & $<.060$ & $<2.2$ & $<.050$ & 96 & $<.032$ & $<.090$ & $<.700$ & $<.038$ & $<.090$ & $<.110$ \\
\hline 37 & $<.060$ & $<2.2$ & $<.050$ & 100 & $<.032$ & $<.090$ & $<.700$ & $<.038$ & $<.090$ & $<.110$ \\
\hline 38 & $<.060$ & $<2.2$ & $<.050$ & 96 & $<.032$ & $<.090$ & $<.700$ & $<.038$ & $<.090$ & $<.110$ \\
\hline $38^{\mathbf{R}}$ & $<.060$ & $<2.2$ & $<.050$ & 94 & $<.032$ & $<.090$ & $<.700$ & $<.038$ & $<.090$ & $<.110$ \\
\hline $38^{\mathbf{F b}}$ & $<.060$ & $<2.2$ & $<.050$ & 97 & $<.032$ & $<.090$ & $<.700$ & $<.038$ & $<.090$ & $<.110$ \\
\hline 39 & $<.060$ & $<2.2$ & e.010 & 98 & $<.032$ & $<.090$ & $<.700$ & $<.038$ & $<.090$ & $<.110$ \\
\hline 40 & $<.060$ & $<2.2$ & e.010 & 98 & $<.032$ & $<.090$ & $<.700$ & $<.038$ & $<.090$ & $<.110$ \\
\hline 41 & $<.060$ & $<2.2$ & $<.050$ & 97 & $<.032$ & $<.090$ & $<.700$ & $<.038$ & $<.090$ & $<.110$ \\
\hline 42 & $<.060$ & $<2.2$ & $<.050$ & 95 & $<.032$ & $<.090$ & $<.700$ & $<.038$ & $<.090$ & $<.110$ \\
\hline 43 & $<.060$ & $<2.2$ & $<.050$ & 94 & $<.032$ & $<.090$ & $<.700$ & $<.038$ & $<.090$ & $<.110$ \\
\hline 44 & $<.060$ & $<2.2$ & $<.050$ & 94 & $<.032$ & $<.090$ & $<.700$ & $<.038$ & $<.090$ & $<.110$ \\
\hline 45 & $<.060$ & $<2.2$ & $<.050$ & 102 & $<.032$ & $<.090$ & $<.700$ & $<.038$ & $<.090$ & $<.110$ \\
\hline 46 & $<.060$ & $<2.2$ & $<.050$ & 96 & $<.032$ & $<.090$ & $<.700$ & $<.038$ & $<.090$ & $<.110$ \\
\hline 47 & $<.060$ & $<2.2$ & $<.050$ & 98 & $<.032$ & $<.090$ & $<.700$ & $<.038$ & $<.090$ & $<.110$ \\
\hline 48 & $<.060$ & $<2.2$ & $<.050$ & 96 & $<.032$ & $<.090$ & $<.700$ & $<.038$ & $<.090$ & $<.110$ \\
\hline $48^{\mathbf{F b}}$ & $<.060$ & $<2.2$ & $<.050$ & 95 & $<.032$ & $<.090$ & $<.700$ & $<.038$ & $<.090$ & $<.110$ \\
\hline 49 & $<.060$ & $<2.2$ & $<.050$ & 99 & $<.032$ & $<.090$ & $<.700$ & $<.038$ & $<.090$ & $<.110$ \\
\hline 50 & $<.060$ & $<2.2$ & $<.050$ & 98 & $<.032$ & $<.090$ & $<.700$ & $<.038$ & $<.090$ & $<.110$ \\
\hline 51 & $<.060$ & $<2.2$ & e.009 & 100 & $<.032$ & $<.090$ & $<.700$ & $<.038$ & $<.090$ & $<.110$ \\
\hline 52 & $<.060$ & $<2.2$ & $<.050$ & 99 & $<.032$ & $<.090$ & $<.700$ & $<.038$ & $<.090$ & $<.110$ \\
\hline 53 & $<.060$ & $<2.2$ & $<.050$ & 99 & $<.032$ & $<.090$ & $<.700$ & $<.038$ & $<.090$ & $<.110$ \\
\hline 54 & $<.060$ & $<2.2$ & $<.050$ & 99 & $<.032$ & $<.090$ & $<.700$ & $<.038$ & $<.090$ & $<.110$ \\
\hline 55 & $<.060$ & $<2.2$ & $<.050$ & 98 & $<.032$ & $<.090$ & $<.700$ & $<.038$ & $<.090$ & $<.110$ \\
\hline 56 & $<.060$ & $<2.2$ & $<.050$ & 99 & $<.032$ & $<.090$ & $<.700$ & $<.038$ & $<.090$ & $<.110$ \\
\hline 57 & $<.060$ & $<2.2$ & $<.050$ & 100 & $<.032$ & $<.090$ & $<.700$ & $<.038$ & $<.090$ & $<.110$ \\
\hline 58 & $<.060$ & $<2.2$ & $<.050$ & 100 & $<.032$ & $<.090$ & $<.700$ & $<.038$ & $<.090$ & $<.110$ \\
\hline $58^{\mathbf{R}}$ & $<.060$ & $<2.2$ & $<.050$ & 99 & $<.032$ & $<.090$ & $<.700$ & $<.038$ & $<.090$ & $<.110$ \\
\hline 59 & $<.060$ & $<2.2$ & e.006 & 100 & $<.032$ & $<.090$ & $<.700$ & $<.038$ & $<.090$ & $<.110$ \\
\hline 60 & $<.060$ & $<2.2$ & $<.050$ & 92 & $<.032$ & $<.090$ & $<.700$ & $<.038$ & $<.090$ & $<.110$ \\
\hline 61 & $<.060$ & $<2.2$ & $<.050$ & 91 & $<.032$ & $<.090$ & $<.700$ & $<.038$ & $<.090$ & $<.110$ \\
\hline
\end{tabular}


Table 21. Tritium and radon concentration and stable-isotope data for ground-water samples collected from basin-fill aquifers, Northern Rockies Intermontane Basins study unit, 1999 and 2001

[Site identification number described in text. The 2-sigma (2 standard deviations) precision represents the amount of uncertainty $( \pm$ in $\mathrm{pCi} / \mathrm{L})$ associated with the reported concentration. Stable-isotope ratios are reported relative to the Vienna Standard Mean Ocean Water (VSMOW).

Abbreviations: permil, parts per thousand; $\mathrm{pCi} / \mathrm{L}$, picocuries per liter; R, replicate. Symbol: --, no data]

\begin{tabular}{|c|c|c|c|c|c|c|c|c|c|}
\hline \multirow{2}{*}{$\begin{array}{c}\text { Well } \\
\text { number } \\
\text { (figs. } 3 \\
\text { and 4) }\end{array}$} & \multirow{2}{*}{$\begin{array}{c}\text { Site } \\
\text { identification } \\
\text { number }\end{array}$} & \multirow{2}{*}{ Date } & \multirow{2}{*}{ Time } & \multirow{2}{*}{$\begin{array}{c}\text { Tritium, } \\
\text { total } \\
(\mathbf{p C i} / \mathbf{L})\end{array}$} & \multirow{2}{*}{$\begin{array}{c}\text { Tritium } \\
\text { 2-sigma } \\
\text { precision } \\
(\mathrm{pCi} / \mathrm{L})\end{array}$} & \multirow{2}{*}{$\begin{array}{c}\text { Radon-222, } \\
\text { total } \\
(\mathrm{pCi} / \mathrm{L})\end{array}$} & \multirow{2}{*}{$\begin{array}{c}\text { Radon-222 } \\
\text { 2-sigma } \\
\text { precision } \\
\text { (pCi/L) }\end{array}$} & \multicolumn{2}{|c|}{$\begin{array}{c}\text { Stable-isotope ratio } \\
\text { (permil) }\end{array}$} \\
\hline & & & & & & & & $\begin{array}{l}\text { Hydrogen-2/ } \\
\text { Hydrogen-1 }\end{array}$ & $\begin{array}{r}\text { Oxygen-18/ } \\
\text { Oxygen-16 }\end{array}$ \\
\hline \multicolumn{10}{|c|}{ Subunit Survey 1, Idaho and Washington } \\
\hline 1 & 481320116261101 & 07/14/99 & 1700 & 1.0 & 1.0 & 1,140 & 35 & -120.5 & -16.16 \\
\hline 2 & 480950116324401 & $07 / 14 / 99$ & 1100 & -- & -- & 2,450 & 44 & -118.6 & -15.64 \\
\hline 3 & 480928117053201 & 07/07/99 & 1000 & -- & -- & 830 & 29 & -117.1 & -15.55 \\
\hline 4 & 480813116593701 & 07/07/99 & 1600 & -- & -- & 839 & 30 & -116.8 & -15.46 \\
\hline 5 & 480718117012501 & $07 / 06 / 99$ & 1800 & 1.9 & 1.0 & 423 & 25 & -119.7 & -15.91 \\
\hline 6 & 480249116510001 & $07 / 08 / 99$ & 1000 & -- & -- & 376 & 21 & -115.9 & -15.57 \\
\hline 7 & 480235116483001 & 07/13/99 & 1700 & -- & -- & 533 & 26 & -117.6 & -15.57 \\
\hline 8 & 480203117200601 & $06 / 08 / 99$ & 0800 & -- & -- & 769 & 28 & -115.8 & -15.34 \\
\hline 9 & 480128116374601 & $08 / 04 / 99$ & 0900 & -- & -- & 632 & 28 & -118.3 & -15.86 \\
\hline 10 & 480119117182101 & $06 / 08 / 99$ & 1600 & -- & -- & 986 & 33 & -116.8 & -15.48 \\
\hline 11 & 475925117153001 & $07 / 06 / 99$ & 1100 & -- & -- & 2,077 & 41 & -114.1 & -15.10 \\
\hline 12 & 475825117364401 & 06/09/99 & 1000 & -- & -- & 740 & 28 & -116.1 & -15.45 \\
\hline 13 & 475731116371301 & $06 / 23 / 99$ & 1200 & -- & -- & 814 & 29 & -121.2 & -15.86 \\
\hline 14 & 475637117262501 & 06/09/99 & 1700 & -- & -- & 642 & 28 & -120.1 & -15.81 \\
\hline 15 & 475510116391201 & 08/03/99 & 1400 & -- & -- & 646 & 30 & -110.7 & -15.15 \\
\hline 16 & 475400116404201 & $06 / 22 / 99$ & 1700 & -- & -- & 733 & 30 & -111.1 & -14.96 \\
\hline 17 & 475343117225201 & $06 / 10 / 99$ & 0900 & -- & -- & 1,091 & 32 & -116.2 & -15.48 \\
\hline 18 & 475322116522201 & 07/12/99 & 1200 & -- & -- & 530 & 23 & -102.2 & -13.76 \\
\hline $18^{\mathbf{R}}$ & & $07 / 12 / 99$ & 1201 & -- & -- & 525 & 23 & -105.6 & -13.69 \\
\hline 19 & 475130117262201 & $06 / 07 / 99$ & 0900 & -- & -- & 788 & 27 & -115.3 & -15.52 \\
\hline 20 & 475035116424801 & $06 / 22 / 99$ & 1100 & -- & -- & 2,943 & 52 & -115.2 & -15.47 \\
\hline 21 & 474718116530201 & $06 / 24 / 99$ & 0900 & -- & -- & 767 & 28 & -105.6 & -14.06 \\
\hline $21^{\mathbf{R}}$ & & $06 / 24 / 99$ & 0901 & -- & -- & 804 & 28 & -104.7 & -13.98 \\
\hline 22 & 474708117250501 & 06/07/99 & 1600 & -- & -- & 870 & 32 & -116.1 & -15.35 \\
\hline 23 & 474629117305101 & $05 / 25 / 99$ & 1100 & 54.7 & 3.2 & 460 & 23 & -113.3 & -14.94 \\
\hline $23^{R}$ & & $05 / 25 / 99$ & 1101 & -- & -- & 477 & 23 & -- & -- \\
\hline 24 & 474456116522001 & $06 / 21 / 99$ & 1700 & -- & -- & 742 & 32 & -115.0 & -15.39 \\
\hline 25 & 474427117312101 & $05 / 24 / 99$ & 1400 & 48.6 & 3.2 & 387 & 22 & -117.3 & -15.45 \\
\hline 26 & 474317117225301 & $05 / 27 / 99$ & 0900 & -- & -- & 731 & 43 & -110.1 & -14.61 \\
\hline 27 & 474218116445601 & $07 / 15 / 99$ & 1000 & -- & -- & 1,592 & 38 & -114.6 & -15.37 \\
\hline 28 & 474147116544001 & 07/26/99 & 1300 & -- & -- & 893 & 29 & -110.5 & -14.76 \\
\hline 29 & 474130117015401 & 07/29/99 & 1000 & -- & -- & 781 & 28 & -113.3 & -15.01 \\
\hline 30 & 474050117084101 & $07 / 28 / 99$ & 1700 & -- & -- & 635 & 28 & -115.1 & -15.41 \\
\hline 31 & 473612117243601 & 05/26/99 & 1100 & -- & -- & 984 & 32 & -109.5 & -14.21 \\
\hline \multicolumn{10}{|c|}{ Subunit Survey 2, Montana } \\
\hline 32 & 470719114301401 & $06 / 12 / 01$ & 1300 & - & - & 1,116 & 34 & -- & -- \\
\hline 33 & 470502114265301 & $06 / 12 / 01$ & 0900 & -- & -- & 1,336 & 36 & -- & -- \\
\hline 34 & 470328114164301 & $06 / 28 / 01$ & 0900 & -- & -- & 853 & 28 & -- & -- \\
\hline 35 & 470112114144001 & $06 / 07 / 01$ & 0900 & 33.3 & 2.6 & 600 & 25 & -- & -- \\
\hline 36 & 465838114074501 & $06 / 27 / 01$ & 1200 & 26.9 & 1.9 & 949 & 29 & -- & -- \\
\hline $36^{\mathbf{R}}$ & & $06 / 27 / 01$ & 1201 & - & -- & 942 & 29 & -- & -- \\
\hline 37 & 465741114110601 & $06 / 27 / 01$ & 1700 & 37.1 & 2.6 & 931 & 31 & -- & -- \\
\hline 38 & 465440114022101 & $06 / 19 / 01$ & 1100 & 38.4 & 2.6 & 2,289 & 47 & -- & -- \\
\hline $38^{\mathbf{R}}$ & & $06 / 19 / 01$ & 1101 & -- & -- & 2,448 & 49 & -- & -- \\
\hline 39 & 465323114054301 & $06 / 20 / 01$ & 1300 & 36.2 & 2.6 & 358 & 23 & -- & -- \\
\hline
\end{tabular}


Table 21. Tritium and radon concentration and stable-isotope data for ground-water samples collected from basin-fill aquifers, Northern Rockies Intermontane Basins study unit, 1999 and 2001 (Continued)

\begin{tabular}{|c|c|c|c|c|c|c|c|c|c|}
\hline \multirow{2}{*}{$\begin{array}{c}\text { Well } \\
\text { number } \\
\text { (figs. } 3 \\
\text { and 4) }\end{array}$} & \multirow{2}{*}{$\begin{array}{c}\text { Site } \\
\text { identification } \\
\text { number }\end{array}$} & \multirow{2}{*}{ Date } & \multirow{2}{*}{ Time } & \multirow{2}{*}{$\begin{array}{c}\text { Tritium, } \\
\text { total } \\
(\mathbf{p C i} / \mathbf{L})\end{array}$} & \multirow{2}{*}{$\begin{array}{c}\text { Tritium } \\
\text { 2-sigma } \\
\text { precision } \\
(\mathbf{p C i} / \mathrm{L})\end{array}$} & \multirow{2}{*}{$\begin{array}{c}\text { Radon-222, } \\
\text { total } \\
(\mathrm{pCi} / \mathrm{L})\end{array}$} & \multirow{2}{*}{$\begin{array}{c}\text { Radon-222 } \\
\text { 2-sigma } \\
\text { precision } \\
\text { (pCi/L) }\end{array}$} & \multicolumn{2}{|c|}{$\begin{array}{l}\text { Stable-isotope ratio } \\
\text { (permil) }\end{array}$} \\
\hline & & & & & & & & $\begin{array}{l}\text { Hydrogen-2/ } \\
\text { Hydrogen-1 }\end{array}$ & $\begin{array}{r}\text { Oxygen-18/ } \\
\text { Oxygen-16 }\end{array}$ \\
\hline \multicolumn{10}{|c|}{ Subunit Survey 2, Montana--Continued } \\
\hline 40 & 465127114055401 & $06 / 20 / 01$ & 1000 & 39.4 & 2.6 & 401 & 23 & -- & -- \\
\hline 41 & 464951114023701 & $06 / 19 / 01$ & 1600 & 38.7 & 2.6 & 683 & 30 & -- & -- \\
\hline 42 & 463932114035901 & $05 / 29 / 01$ & 1300 & 36.5 & 2.6 & 958 & 29 & -- & -- \\
\hline 43 & 463827114001201 & $05 / 29 / 01$ & 1600 & -- & -- & 1,314 & 36 & -- & -- \\
\hline 44 & 463335114011701 & $05 / 30 / 01$ & 1600 & 32.3 & 2.6 & 1,424 & 35 & -- & -- \\
\hline 45 & 463122114074701 & 05/30/01 & 0900 & -- & -- & 2,559 & 47 & -- & -- \\
\hline $45^{\mathrm{R}}$ & & $05 / 30 / 01$ & 0901 & -- & -- & 2,555 & 47 & -- & -- \\
\hline 46 & 462948114060101 & $05 / 30 / 01$ & 1200 & -- & -- & 2,169 & 43 & -- & -- \\
\hline 47 & 462859113574401 & $06 / 11 / 01$ & 1200 & -- & -- & 1,399 & 34 & -- & -- \\
\hline 48 & 462818114074101 & $06 / 04 / 01$ & 1200 & -- & -- & 1,678 & 36 & -- & -- \\
\hline 49 & 462754113592701 & $06 / 05 / 01$ & 1500 & -- & -- & 1,359 & 38 & -- & -- \\
\hline 50 & 462616114094301 & $06 / 05 / 01$ & 1300 & -- & -- & 2,129 & 42 & -- & -- \\
\hline 51 & 462545114034301 & $06 / 05 / 01$ & 1800 & 6.4 & 1.0 & 253 & 22 & -- & -- \\
\hline 52 & 462256114114501 & $06 / 04 / 01$ & 1700 & -- & -- & 1,472 & 38 & -- & -- \\
\hline 53 & 462228114030301 & $06 / 06 / 01$ & 1100 & -- & -- & 367 & 22 & -- & -- \\
\hline 54 & 461823114050901 & $06 / 06 / 01$ & 1300 & -- & -- & 478 & 24 & -- & -- \\
\hline 55 & 461807114123001 & $06 / 11 / 01$ & 1600 & -- & -- & 1,227 & 37 & -- & -- \\
\hline 56 & 461638114023401 & $06 / 06 / 01$ & 0800 & -- & -- & 332 & 22 & -- & -- \\
\hline 57 & 461451114090801 & $06 / 06 / 01$ & 1600 & -- & -- & 1,626 & 40 & -- & -- \\
\hline 58 & 461320114121501 & $06 / 05 / 01$ & 0900 & -- & -- & 3,047 & 50 & -- & -- \\
\hline 59 & 461055114112601 & $06 / 21 / 01$ & 0900 & -- & -- & 490 & 24 & -- & -- \\
\hline 60 & 460110114110901 & $06 / 20 / 01$ & 1800 & -- & -- & 1,814 & 42 & -- & -- \\
\hline 61 & 455521114074801 & $06 / 20 / 01$ & 2000 & -- & -- & 1,690 & 41 & -- & -- \\
\hline
\end{tabular}


Table 22. Radionuclide data from filtered ground-water samples collected from basin-fill aquifers in Idaho and Washington, Northern Rockies Intermontane Basins study unit, 1999

[Site identification number described in text. Radiation concentrations are listed as reported by the respective laboratories. The 2 -sigma ( 2 standard deviations) precision represents the amount of uncertainty $( \pm$ in $\mathrm{pCi} / \mathrm{L})$ associated with the reported concentration. Sample-specific minimum detectable concentration at the 95 -percent confidence level was computed for each radium analysis based on instrument operating conditions. Where the combined value of the radium concentration plus the 2-sigma quantity equals or exceeds the MDC, there is less uncertainty compared to those combined values that are less than the MDC. Abbreviations: MDC, minimum detectable concentration; pCi/L, picocuries per liter; Cs, Cesium; Th, Thorium. Symbols: <, less than reporting level; --, no data]

\begin{tabular}{|c|c|c|c|c|c|c|c|}
\hline $\begin{array}{c}\text { Well } \\
\text { number } \\
\text { (figs. } 3 \\
\text { and 4) }\end{array}$ & $\begin{array}{c}\text { Site } \\
\text { identification } \\
\text { number }\end{array}$ & Date & Time & $\begin{array}{c}\text { Gross alpha } \\
\text { radioactivity } \\
\text { (pCi/L as } \mathrm{Th}-230)\end{array}$ & $\begin{array}{c}\text { Gross alpha } \\
\text { radioactivity } \\
\text { 2-sigma precision } \\
(\mathrm{pCi} / \mathrm{L} \text { as } \mathrm{Th}-230)\end{array}$ & $\begin{array}{c}\text { Gross beta } \\
\text { radioactivity } \\
(\mathbf{p C i} / \mathrm{L} \text { as Cs-137) }\end{array}$ & $\begin{array}{c}\text { Gross beta } \\
\text { radioactivity } \\
\text { 2-sigma precision } \\
\text { (pCi/L as Cs-137) }\end{array}$ \\
\hline \multicolumn{8}{|c|}{ Subunit Survev 1, Idaho and Washington } \\
\hline 1 & 481320116261101 & $07 / 14 / 99$ & 1700 & $<3.0$ & 2.9 & 4.9 & 4.3 \\
\hline 2 & 480950116324401 & $07 / 14 / 99$ & 1100 & 4.0 & 2.9 & $<4.0$ & 4.0 \\
\hline 3 & 480928117053201 & 07/07/99 & 1000 & $<3.0$ & 3.2 & 4.1 & 4.3 \\
\hline 4 & 480813116593701 & 07/07/99 & 1600 & $<3.0$ & 2.9 & $<4.0$ & 4.0 \\
\hline 5 & 480718117012501 & $07 / 06 / 99$ & 1800 & 4.0 & 3.4 & 6.3 & 4.7 \\
\hline 6 & 480249116510001 & $07 / 08 / 99$ & 1000 & 3.7 & 3.3 & $<4.0$ & 4.3 \\
\hline 7 & 480235116483001 & $07 / 13 / 99$ & 1700 & 3.9 & 3.3 & 7.6 & 4.7 \\
\hline 8 & 480203117200601 & 06/08/99 & 0800 & 3.1 & 2.8 & 5.5 & 4.1 \\
\hline 9 & 480128116374601 & $08 / 04 / 99$ & 0900 & $<3.0$ & 2.2 & $<4.0$ & 4.0 \\
\hline 10 & 480119117182101 & $06 / 08 / 99$ & 1600 & $<3.0$ & 2.8 & 4.4 & 4.3 \\
\hline 11 & 475925117153001 & 07/06/99 & 1100 & $<3.0$ & 2.7 & $<4.0$ & 4.2 \\
\hline 12 & 475825117364401 & $06 / 09 / 99$ & 1000 & $<3.0$ & 2.1 & $<4.0$ & 3.8 \\
\hline 13 & 475731116371301 & $06 / 23 / 99$ & 1200 & $<3.0$ & 2.8 & 6.1 & 4.3 \\
\hline 14 & 475637117262501 & 06/09/99 & 1700 & $<3.0$ & 2.7 & $<4.0$ & 4.0 \\
\hline 15 & 475510116391201 & 08/03/99 & 1400 & $<3.0$ & 2.6 & $<4.0$ & 4.0 \\
\hline 16 & 475400116404201 & $06 / 22 / 99$ & 1700 & 4.0 & 3.1 & 4.3 & 4.1 \\
\hline 17 & 475343117225201 & $06 / 10 / 99$ & 0900 & 5.5 & 3.4 & 5.4 & 4.4 \\
\hline 18 & 475322116522201 & $07 / 12 / 99$ & 1200 & $<3.0$ & 2.7 & $<4.0$ & 4.1 \\
\hline 19 & 475130117262201 & 06/07/99 & 0900 & $<3.0$ & 2.7 & $<4.0$ & 4.3 \\
\hline 20 & 475035116424801 & $06 / 22 / 99$ & 1100 & $<3.0$ & 2.1 & $<4.0$ & 4.0 \\
\hline 21 & 474718116530201 & $06 / 24 / 99$ & 0900 & $<3.0$ & 2.9 & $<4.0$ & 4.1 \\
\hline 22 & 474708117250501 & $06 / 07 / 99$ & 1600 & 8.6 & 3.9 & 6.8 & 4.6 \\
\hline 23 & 474629117305101 & $05 / 25 / 99$ & 1100 & 3.3 & 2.9 & 2.6 & 4.1 \\
\hline 24 & 474456116522001 & $06 / 21 / 99$ & 1700 & 3.1 & 3.3 & $<4.0$ & 4.4 \\
\hline 25 & 474427117312101 & $05 / 24 / 99$ & 1400 & 6.2 & 3.5 & 7.2 & 4.4 \\
\hline 26 & 474317117225301 & $05 / 27 / 99$ & 0900 & 9.6 & 4.0 & 7.4 & 4.3 \\
\hline 27 & 474218116445601 & $07 / 15 / 99$ & 1000 & $<3.0$ & 2.7 & $<4.0$ & 4.1 \\
\hline 28 & 474147116544001 & $07 / 26 / 99$ & 1300 & $<3.0$ & 2.1 & $<4.0$ & 3.9 \\
\hline 29 & 474130117015401 & $07 / 29 / 99$ & 1000 & $<3.0$ & 1.7 & $<4.0$ & 3.8 \\
\hline 30 & 474050117084101 & $07 / 28 / 99$ & 1700 & $<3.0$ & 2.2 & $<4.0$ & 3.7 \\
\hline 31 & 473612117243601 & $05 / 26 / 99$ & 1100 & $<3.0$ & 2.7 & 4.7 & 4.3 \\
\hline
\end{tabular}

${ }^{1}$ Radiation measurement is less than long-term average background radiation and it is unlikely that the radionuclide is present in a quantifiable amount. 
Table 22. Radionuclide data from filtered ground-water samples collected from basin-fill aquifers in Idaho and Washington, Northern Rockies Intermontane Basins study unit, 1999 (Continued)

\begin{tabular}{|c|c|c|c|c|c|c|c|c|c|}
\hline $\begin{array}{l}\text { Radium- } \\
224 \\
(\mathrm{pCi} / \mathrm{L})\end{array}$ & $\begin{array}{c}\text { Radium-224 } \\
\text { 2-sigma } \\
\text { precision } \\
\text { (pCi/L) }\end{array}$ & $\begin{array}{c}\text { Radium- } \\
\text { 224, } \\
\text { MDC } \\
\text { (pCi/L) }\end{array}$ & $\begin{array}{l}\text { Radium- } \\
226 \\
(\mathrm{pCi} / \mathrm{L})\end{array}$ & $\begin{array}{c}\text { Radium-226 } \\
\text { 2-sigma } \\
\text { precision } \\
(\mathbf{p C i} / \mathrm{L})\end{array}$ & $\begin{array}{c}\text { Radium- } \\
\text { 226, } \\
\text { MDC } \\
\text { (pCi/L) }\end{array}$ & $\begin{array}{c}\text { Radium- } \\
228 \\
(\mathbf{p C i} / \mathbf{L})\end{array}$ & $\begin{array}{c}\text { Radium-228 } \\
\text { 2-sigma } \\
\text { precision } \\
\text { (pCi/L) }\end{array}$ & $\begin{array}{c}\text { Radium-228, } \\
\text { MDC } \\
(p C i / L)\end{array}$ & $\begin{array}{c}\text { Well } \\
\text { number } \\
\text { (figs. } 3 \\
\text { and 4) }\end{array}$ \\
\hline \multicolumn{10}{|c|}{ Subunit Survey 1, Idaho and Washington } \\
\hline 0.01 & 0.19 & 0.42 & 0.08 & 0.07 & 0.07 & 0.32 & 0.25 & 0.48 & 1 \\
\hline${ }^{1}-.04$ & .10 & .18 & .01 & .03 & .05 & .35 & .24 & .46 & 2 \\
\hline .26 & .12 & .17 & .13 & .04 & .05 & .68 & .38 & .59 & 3 \\
\hline $1_{-.005}$ & .13 & .31 & ${ }^{1}-.005$ & .04 & .09 & .81 & .32 & .39 & 4 \\
\hline .52 & .18 & .22 & .23 & .06 & .05 & 1.40 & .64 & .92 & 5 \\
\hline${ }^{1}-.05$ & .16 & .32 & .04 & .04 & .07 & .22 & .22 & .41 & 6 \\
\hline .10 & .17 & .28 & .14 & .05 & .06 & .95 & .35 & .51 & 7 \\
\hline .01 & .16 & .31 & .06 & .04 & .06 & .62 & .31 & .52 & 8 \\
\hline .05 & .21 & .38 & -.004 & .07 & .15 & .56 & .26 & .36 & 9 \\
\hline .22 & .13 & .19 & .11 & .04 & .05 & .70 & .33 & .56 & 10 \\
\hline .05 & .25 & .46 & .19 & .08 & .06 & .61 & .58 & 1.1 & 11 \\
\hline${ }^{1}-.001$ & .10 & .17 & .03 & .03 & .05 & .45 & .27 & .50 & 12 \\
\hline .12 & .83 & 1.4 & .02 & .12 & .22 & .67 & .29 & .40 & 13 \\
\hline .03 & .14 & .26 & .03 & .02 & .03 & .23 & .21 & .38 & 14 \\
\hline .08 & .10 & .16 & .03 & .03 & .04 & .28 & .20 & .33 & 15 \\
\hline .17 & .51 & .92 & .02 & .08 & .16 & .52 & .24 & .32 & 16 \\
\hline .02 & .14 & .24 & .08 & .04 & .04 & .41 & .24 & .38 & 17 \\
\hline${ }^{1}-.02$ & .48 & .96 & $-{ }^{1} .04$ & .13 & .32 & .45 & .30 & .56 & 18 \\
\hline .23 & .12 & .15 & .49 & .12 & .06 & .54 & .28 & .49 & 19 \\
\hline .06 & .09 & .15 & .01 & .02 & .04 & .16 & .20 & .38 & 20 \\
\hline .05 & .19 & .37 & .01 & .04 & .09 & .73 & .30 & .39 & 21 \\
\hline .18 & .30 & .49 & .12 & .10 & .13 & .26 & .21 & .36 & 22 \\
\hline-- & -- & -- & -- & -- & -- & -- & .39 & -- & 23 \\
\hline .33 & .15 & .21 & .09 & .06 & .08 & .55 & .27 & .39 & 24 \\
\hline .67 & .14 & .12 & .37 & .06 & .02 & .86 & .42 & .69 & 25 \\
\hline .04 & .24 & .42 & .06 & .03 & .05 & 1.2 & .54 & .82 & 26 \\
\hline .00 & .18 & .35 & .02 & .05 & .09 & .72 & .31 & .50 & 27 \\
\hline${ }^{1}-.01$ & .08 & .16 & .06 & .04 & .05 & .38 & .29 & .50 & 28 \\
\hline${ }^{1}-.001$ & .10 & .18 & .02 & .02 & .04 & .96 & .34 & .36 & 29 \\
\hline${ }^{1}-.02$ & .13 & .27 & .02 & .04 & .07 & .11 & .29 & .62 & 30 \\
\hline .03 & .06 & .10 & .06 & .02 & .02 & -- & -- & -- & 31 \\
\hline
\end{tabular}


Table 23. Water-level, specific-conductance, and ancillary data for selected ground-water sites inventoried for the surface-water/ground-water interaction study of the Spokane River, Idaho and Washington

[See figure 5 for well locations. All wells are completed in Quaternary alluvium. Location number described in text. Altitude of land surface referenced to National Geodetic Vertical Datum of 1929 (NGVD 29). Depth of well: in feet below land surface. Depth to water: in feet below land surface. Method of measurement: A, air line; R, reported by driller; S, steel tape; T, electric tape. Abbreviations: $\mathrm{ft}$, feet; $\mu \mathrm{S} / \mathrm{cm}, \mathrm{microsiemens}$ per centimeter at 25 degrees Celsius. Symbol: --, no data or not applicable]

\begin{tabular}{|c|c|c|c|c|c|c|c|c|}
\hline \multirow[b]{2}{*}{$\begin{array}{c}\text { Well } \\
\text { number }\end{array}$} & \multirow[b]{2}{*}{ Location number } & \multirow[b]{2}{*}{$\begin{array}{l}\text { Altitude of } \\
\text { land surface } \\
\text { (ft) }\end{array}$} & \multirow[b]{2}{*}{$\begin{array}{c}\text { Depth of } \\
\text { well } \\
\text { (ft) }\end{array}$} & \multicolumn{3}{|c|}{ Water level } & \multirow{2}{*}{$\begin{array}{c}\text { Specific } \\
\text { conduct- } \\
\text { ance } \\
(\mu \mathrm{S} / \mathrm{cm})\end{array}$} & \multirow[b]{2}{*}{$\begin{array}{c}\text { Date of } \\
\text { meas- } \\
\text { urement }\end{array}$} \\
\hline & & & & $\begin{array}{l}\text { Depth to } \\
\text { water } \\
\text { (ft) }\end{array}$ & $\begin{array}{l}\text { Method } \\
\text { of meas- } \\
\text { urement }\end{array}$ & $\begin{array}{c}\text { Date of } \\
\text { meas- } \\
\text { urement }\end{array}$ & & \\
\hline \multicolumn{9}{|c|}{ Sites located in Idaho } \\
\hline-- & 50N05W01ACBB01 & 2,238 & 243 & 187.54 & $\mathrm{~T}$ & $03 / 16 / 00$ & 292 & 03/16/00 \\
\hline-- & 50N05W01CBBB01 & $2,194.1$ & 279 & 198.93 & $\mathrm{~S}$ & $09 / 26 / 78$ & 230 & $12 / 09 / 99$ \\
\hline-- & 50N05W04CABD01 & 2,100 & 160 & 130 & $\mathrm{R}$ & $03 / 01 / 78$ & 60 & $12 / 10 / 99$ \\
\hline-- & 50N05W04CACB01 & 2,107 & 165 & 92.98 & S & $03 / 16 / 00$ & 53 & $03 / 16 / 00$ \\
\hline-- & 50N05W04CACC02 & $2,083.4$ & 140 & 89.80 & $\mathrm{~T}$ & $08 / 16 / 00$ & 59 & $08 / 16 / 00$ \\
\hline-- & 50N05W06DCDC01 & $2,110.7$ & 190 & 121.67 & $\mathrm{~T}$ & $03 / 15 / 00$ & 62 & $03 / 15 / 00$ \\
\hline-- & 50N05W07ADDD01 & 2,065 & 110 & 74.03 & S & 03/16/00 & 53 & 03/16/00 \\
\hline-- & 50N05W07BCCC01 & $2,063.8$ & 140 & 80.05 & $\mathrm{~S}$ & $08 / 03 / 00$ & 55 & 08/03/00 \\
\hline M1 & 50N05W07DABC01 & $2,069.5$ & 79.3 & 63.37 & $\mathrm{~T}$ & $08 / 08 / 00$ & 148 & $08 / 08 / 00$ \\
\hline M2 & 50N05W07DABC02 & $2,070.3$ & 45.8 & 32.74 & $\mathrm{~T}$ & $09 / 27 / 00$ & 45 & 09/27/00 \\
\hline-- & 50N05W07DBBA01 & $2,082.8$ & 200 & 39.10 & $\mathrm{~T}$ & $08 / 18 / 00$ & 110 & $08 / 18 / 00$ \\
\hline-- & 50N05W07DBBA02 & 2,040 & 135 & 95 & $\mathrm{R}$ & $08 / 03 / 93$ & 52 & $12 / 15 / 99$ \\
\hline-- & 50N05W08BBBB01 & 2,090 & 196 & 110 & $\mathrm{R}$ & $09 / 05 / 92$ & 55 & 09/30/99 \\
\hline-- & 50N05W10BAAA01 & 2,150 & 59 & 23.06 & $\mathrm{~S}$ & $03 / 14 / 00$ & 279 & $03 / 14 / 00$ \\
\hline-- & 50N05W12BBAB01 & 2,165 & 206 & 180 & $\mathrm{R}$ & $06 / 12 / 77$ & 94 & $02 / 08 / 00$ \\
\hline-- & 50N05W12BBBD01 & 2,165 & 217.5 & 163.12 & S & $03 / 14 / 00$ & 153 & $03 / 14 / 00$ \\
\hline-- & $50 \mathrm{~N} 05 \mathrm{~W} 12 \mathrm{BBBD} 02$ & 2,160 & 200 & 160.13 & S & $03 / 14 / 00$ & 95 & $03 / 14 / 00$ \\
\hline-- & 50N05W12BBDA01 & 2,160 & 180 & 120.86 & S & $12 / 16 / 99$ & 93 & 03/15/00 \\
\hline-- & 50N05W12BBDA02 & 2,160 & 185 & 138 & $\mathrm{R}$ & $10 / 02 / 79$ & 105 & $12 / 16 / 99$ \\
\hline-- & 50N05W12BCAD01 & 2,130 & 200 & 115.40 & S & $03 / 14 / 00$ & 128 & $03 / 14 / 00$ \\
\hline-- & 50N05W12BCDA01 & 2,183 & 250 & 115.79 & S & $03 / 21 / 00$ & 169 & $03 / 21 / 00$ \\
\hline-- & 50N06W12BDAC01 & 2,065 & 137 & 95 & $\mathrm{R}$ & $06 / 06 / 95$ & 112 & 08/03/00 \\
\hline-- & 50N06W12CABA01 & 2,030 & 133 & 56.20 & S & 11/17/99 & 76 & $11 / 17 / 99$ \\
\hline-- & 50N06W12CACA01 & $2,044.9$ & 137 & 62.39 & $\mathrm{~S}$ & $08 / 18 / 00$ & 57 & $08 / 18 / 00$ \\
\hline-- & 50N06W12CBDB01 & $2,081.2$ & 150 & 98.44 & S & $08 / 18 / 00$ & 81 & $08 / 18 / 00$ \\
\hline-- & 50N06W12CCAD01 & 2,105 & 157 & 122.19 & S & $03 / 14 / 00$ & 89 & $03 / 14 / 00$ \\
\hline-- & 50N06W12CCAD02 & $2,102.6$ & 157 & 119.68 & S & $03 / 14 / 00$ & 139 & $08 / 03 / 00$ \\
\hline-- & 50N06W12DBAD01 & $2,058.9$ & 126 & 75.94 & S & $08 / 18 / 00$ & 55 & $08 / 18 / 00$ \\
\hline-- & 50N06W12DBCD01 & $2,069.2$ & 137 & 86.40 & S & $08 / 18 / 00$ & 56 & $08 / 18 / 00$ \\
\hline-- & 50N06W12DCDD01 & 2,110 & 165 & 137 & $\mathrm{R}$ & $12 / 16 / 92$ & 252 & $11 / 19 / 99$ \\
\hline-- & 50N06W12DDAB01 & $2,100.3$ & 157 & 116.68 & S & $08 / 03 / 00$ & 106 & $08 / 03 / 00$ \\
\hline-- & 50N06W12DDCD01 & $2,100.6$ & 168 & 122.32 & S & $03 / 14 / 00$ & 456 & $08 / 17 / 00$ \\
\hline-- & 50N06W12DDDB02 & $2,103.2$ & 170 & 120.34 & S & $08 / 18 / 00$ & 444 & $08 / 18 / 00$ \\
\hline-- & 50N06W13CABA01 & $2,123.8$ & 209 & 138.10 & S & $08 / 18 / 00$ & 207 & $08 / 18 / 00$ \\
\hline-- & 51N04W20CBCD01 & 2,245 & 290 & 239.68 & $\mathrm{~T}$ & $03 / 15 / 00$ & 390 & $03 / 15 / 00$ \\
\hline-- & 51N05W19DBCC01 & $2,128.6$ & 212 & 135.54 & S & 08/30/99 & 354 & 08/30/99 \\
\hline-- & 51N05W26AAAA01 & 2,229 & 268 & 238.46 & $\mathrm{~S}$ & $04 / 29 / 77$ & 286 & $09 / 23 / 88$ \\
\hline-- & 51N05W26BBDD01 & $2,241.1$ & 274 & 253.10 & S & $05 / 19 / 77$ & 293 & $08 / 14 / 90$ \\
\hline-- & 51N05W27DCCC01 & 2,228 & 328 & 245 & $\mathrm{R}$ & $10 / 07 / 80$ & 247 & $12 / 09 / 99$ \\
\hline-- & 51N05W28DADA01 & 2,153 & 276 & 160.57 & $\mathrm{R}$ & $03 / 31 / 75$ & 251 & $09 / 23 / 88$ \\
\hline-- & $51 \mathrm{~N} 05 \mathrm{~W} 31 \mathrm{BCCB} 01$ & 2,106 & 156 & 117.90 & $\mathrm{~T}$ & $08 / 16 / 00$ & 328 & $08 / 16 / 00$ \\
\hline-- & 51N06W36DAAA01 & $2,102.9$ & 150 & 115.66 & S & $08 / 18 / 00$ & 340 & $08 / 18 / 00$ \\
\hline-- & 51N06W36DDAA01 & $2,099.3$ & 162 & 112.01 & $\mathrm{~T}$ & $03 / 14 / 00$ & 312 & $03 / 14 / 00$ \\
\hline \multicolumn{9}{|c|}{ Sites located in Washington } \\
\hline-- & 25N44E01BBCC01 & $2,051.6$ & 150 & 107.10 & S & $03 / 15 / 00$ & 362 & $10 / 20 / 99$ \\
\hline-- & 25N44E01CBAA01 & 2,020 & 160 & 76.2 & $\mathrm{R}$ & $09 / 10 / 70$ & 293 & $10 / 21 / 99$ \\
\hline-- & 25N44E01DBDD01 & 2,020 & 120 & -- & -- & -- & 304 & $10 / 20 / 99$ \\
\hline
\end{tabular}


Table 23. Water-level, specific-conductance, and ancillary data for selected ground-water sites inventoried for the surface-water/ground-water interaction study of the Spokane River, Idaho and Washington (Continued)

\begin{tabular}{|c|c|c|c|c|c|c|c|c|}
\hline \multirow[b]{2}{*}{$\begin{array}{c}\text { Well } \\
\text { number }^{1}\end{array}$} & \multirow[b]{2}{*}{ Location number } & \multirow[b]{2}{*}{$\begin{array}{l}\text { Altitude of } \\
\text { land surface } \\
\text { (ft) }\end{array}$} & \multirow[b]{2}{*}{$\begin{array}{c}\text { Depth of } \\
\text { well } \\
\text { (ft) }\end{array}$} & \multicolumn{3}{|c|}{ Water level } & \multirow{2}{*}{$\begin{array}{c}\text { Specific } \\
\text { conduct- } \\
\text { ance } \\
(\mu \mathrm{S} / \mathrm{cm})\end{array}$} & \multirow[b]{2}{*}{$\begin{array}{c}\text { Date of } \\
\text { meas- } \\
\text { urement }\end{array}$} \\
\hline & & & & $\begin{array}{l}\text { Depth to } \\
\text { water } \\
\text { (ft) }\end{array}$ & $\begin{array}{l}\text { Method } \\
\text { of meas- } \\
\text { urement }\end{array}$ & $\begin{array}{c}\text { Date of } \\
\text { meas- } \\
\text { urement }\end{array}$ & & \\
\hline \multicolumn{9}{|c|}{ Sites located in Washington (Continued) } \\
\hline-- & 25N44E01DCDD01 & $2,018.2$ & 150 & 72.66 & $\mathrm{~T}$ & $08 / 11 / 00$ & 325 & $08 / 11 / 00$ \\
\hline M25 & 25N44E11DDAC01 & $1,965.9$ & 67 & 33.79 & $\mathrm{~T}$ & $08 / 10 / 00$ & 264 & $08 / 10 / 00$ \\
\hline-- & 25N44E11DDAD01 & $1,967.2$ & 69 & 34.58 & $\mathrm{~T}$ & $08 / 10 / 00$ & 262 & $08 / 10 / 00$ \\
\hline-- & 25N44E11DDDD01 & $1,987.4$ & 85 & 55.65 & $\mathrm{~T}$ & $08 / 10 / 00$ & 268 & $08 / 10 / 00$ \\
\hline-- & 25N44E12BBBD01 & 2,004 & 117 & 67.8 & $\mathrm{R}$ & $09 / 10 / 70$ & 332 & $10 / 21 / 99$ \\
\hline-- & 25N44E12CBCD01 & $1,998.1$ & 100 & 54.42 & $\mathrm{~T}$ & $08 / 10 / 00$ & -- & -- \\
\hline-- & 25N44E12CCAB01 & $1,987.2$ & -- & 50.35 & $\mathrm{~T}$ & $08 / 10 / 00$ & 275 & $03 / 14 / 00$ \\
\hline-- & $25 \mathrm{~N} 44 \mathrm{E} 12 \mathrm{CCDC} 01^{2}$ & 1,960 & -- & -- & -- & -- & 108 & $01 / 23 / 01$ \\
\hline-- & 25N44E12DABB01 & $2,005.7$ & -- & 61.19 & $\mathrm{~T}$ & $08 / 10 / 00$ & 217 & $08 / 10 / 00$ \\
\hline-- & 25N44E24BDAA01 & 2,040 & 127 & 96.39 & S & $03 / 17 / 00$ & 359 & $11 / 04 / 99$ \\
\hline M4 & 25N45E01ABDD01 & $2,035.4$ & 74.3 & 62.64 & $\mathrm{~T}$ & $08 / 07 / 00$ & 54 & $08 / 07 / 00$ \\
\hline M5 & 25N45E01ABDD02 & $2,035.3$ & 60.7 & 57.17 & $\mathrm{~T}$ & $08 / 07 / 00$ & 54 & $08 / 07 / 00$ \\
\hline M6 & 25N45E01ABDD03 & $2,034.9$ & 77.8 & 61.93 & $\mathrm{~T}$ & $08 / 07 / 00$ & 55 & $08 / 07 / 00$ \\
\hline M7 & 25N45E01ACAD01 & $2,045.8$ & 86.9 & 73.46 & $\mathrm{~T}$ & $08 / 08 / 00$ & 87 & $08 / 08 / 00$ \\
\hline-- & 25N45E01ADBB01 & 2,051 & 185 & 77.17 & $\mathrm{~S}$ & $08 / 09 / 00$ & 82 & $08 / 09 / 00$ \\
\hline-- & 25N45E01BBAA01 & $2,078.2$ & 150 & 107.25 & S & $08 / 07 / 00$ & 228 & $08 / 07 / 00$ \\
\hline M10 & $25 \mathrm{~N} 45 \mathrm{E} 01 \mathrm{CBBC} 01$ & $2,031.2$ & 71.0 & 46.36 & $\mathrm{~T}$ & $08 / 09 / 00$ & 53 & $08 / 09 / 00$ \\
\hline M9 & 25N45E01CBBD01 & $2,024.9$ & 76.1 & 28.68 & $\mathrm{~T}$ & $08 / 09 / 00$ & 52 & $08 / 09 / 00$ \\
\hline M8 & 25N45E01CBBD02 & 2,025 & 32.9 & 11.95 & $\mathrm{~T}$ & $08 / 09 / 00$ & 51 & $08 / 09 / 00$ \\
\hline-- & 25N45E02ACCD01 & 2,070 & 235 & 104 & $\mathrm{R}$ & $12 / 29 / 64$ & 266 & $08 / 09 / 00$ \\
\hline-- & 25N45E02DDDD01 & 2,060 & 128 & 104 & $\mathrm{R}$ & -- & 58 & 09/30/99 \\
\hline M16 & 25N45E03BDDA01 & $2,052.6$ & 117.3 & 89.53 & $\mathrm{~T}$ & $08 / 10 / 00$ & 324 & $08 / 10 / 00$ \\
\hline-- & 25N45E03BDDA02 & 2,050 & 200 & 92.8 & $\mathrm{R}$ & $09 / 08 / 64$ & 327 & $08 / 16 / 00$ \\
\hline-- & 25N45E03CBDD01 & $2,048.2$ & 138 & 83.50 & S & $03 / 13 / 00$ & 303 & $08 / 07 / 00$ \\
\hline M15 & 25N45E03CDDA01 & $2,040.2$ & 97.5 & 77.05 & $\mathrm{~T}$ & $08 / 10 / 00$ & 302 & $08 / 10 / 00$ \\
\hline M14 & 25N45E03CDDD01 & $2,033.1$ & 88.8 & 70.52 & $\mathrm{~T}$ & $09 / 20 / 00$ & 55 & $09 / 26 / 00$ \\
\hline-- & 25N45E04BAAC02 & 2,060 & 195 & 105.1 & $\mathrm{R}$ & $10 / 30 / 64$ & 293 & $08 / 16 / 00$ \\
\hline-- & $25 \mathrm{~N} 45 \mathrm{E} 05 \mathrm{BBBC} 01$ & $2,045.2$ & 128 & 91.50 & $\mathrm{~T}$ & $08 / 11 / 00$ & ${ }^{3} 373$ & $05 / 21 / 99$ \\
\hline-- & 25N45E05DDBA01 & $2,024.3$ & 96 & 68.42 & $\mathrm{~T}$ & $08 / 09 / 00$ & 332 & $08 / 09 / 00$ \\
\hline-- & 25N45E06BBCA01 & $2,063.3$ & 147 & 115.55 & S & $08 / 08 / 00$ & 405 & $08 / 08 / 00$ \\
\hline-- & 25N45E07AAAA02 & 2,020 & 168 & 71.7 & $\mathrm{R}$ & $08 / 31 / 64$ & 340 & $08 / 09 / 00$ \\
\hline M24 & 25N45E07AAAA04 & $2,021.7$ & 100 & 69.78 & $\mathrm{~T}$ & $08 / 10 / 00$ & 349 & $08 / 10 / 00$ \\
\hline M23 & 25N45E07ADDD01 & $2,001.5$ & 80 & 48.86 & $\mathrm{~T}$ & $08 / 10 / 00$ & 55 & $08 / 10 / 00$ \\
\hline-- & 25N45E08BDAA01 & $2,018.3$ & 128 & 64.63 & S & $08 / 09 / 00$ & 58 & $08 / 09 / 00$ \\
\hline M22 & $25 \mathrm{~N} 45 \mathrm{E} 08 \mathrm{CBBC} 01$ & $2,017.5$ & 97 & 66.29 & $\mathrm{~T}$ & $08 / 10 / 00$ & 55 & $08 / 10 / 00$ \\
\hline M21 & 25N45E08CBBC02 & 2,018 & 98 & 67.67 & $\mathrm{~T}$ & $08 / 10 / 00$ & 55 & $08 / 10 / 00$ \\
\hline-- & 25N45E09ABCD01 & 2,029 & 107 & 70.00 & S & $08 / 08 / 00$ & -- & -- \\
\hline M19 & 25N45E09ADAB01 & $2,021.3$ & 70.9 & 55.05 & $\mathrm{~T}$ & $08 / 08 / 00$ & 55 & $08 / 08 / 00$ \\
\hline M18 & 25N45E09ADAD01 & $2,023.1$ & 77.4 & 59.44 & $\mathrm{~T}$ & 08/08/00 & 55 & $08 / 08 / 00$ \\
\hline M11 & 25N45E10BAAA01 & $2,030.6$ & 71.6 & 45.25 & $\mathrm{~T}$ & $08 / 09 / 00$ & 52 & $08 / 09 / 00$ \\
\hline M13 & 25N45E10BAAA02 & $2,036.5$ & 75.5 & 59.14 & $\mathrm{~T}$ & $08 / 09 / 00$ & 53 & $08 / 09 / 00$ \\
\hline M12 & 25N45E10BAAA03 & $2,031.5$ & 136.7 & 69.50 & $\mathrm{~T}$ & $09 / 21 / 00$ & 48 & $10 / 04 / 00$ \\
\hline-- & 25N45E10BDAD01 & $2,030.6$ & 80 & 67.82 & $\mathrm{~T}$ & $08 / 08 / 00$ & 86 & $08 / 08 / 00$ \\
\hline M17 & 25N45E10CBDA01 & 2,038 & 97.1 & 76.95 & $\mathrm{~T}$ & $08 / 08 / 00$ & 95 & $08 / 08 / 00$ \\
\hline-- & 25N45E10DBCB01 & $2,058.3$ & 140 & 95.64 & S & $08 / 07 / 00$ & -- & -- \\
\hline-- & 25N45E11CCAA01 & $2,107.3$ & 210 & 144.00 & S & $09 / 26 / 00$ & -- & -- \\
\hline-- & 25N45E14BACD01 & $2,143.3$ & 238 & 172 & A & $09 / 26 / 00$ & 185 & $09 / 26 / 00$ \\
\hline
\end{tabular}


Table 23. Water-level, specific-conductance, and ancillary data for selected ground-water sites inventoried for the surface-water/ground-water interaction study of the Spokane River, Idaho and Washington (Continued)

\begin{tabular}{|c|c|c|c|c|c|c|c|c|}
\hline \multirow[b]{2}{*}{$\begin{array}{c}\text { Well } \\
\text { number }^{1}\end{array}$} & \multirow[b]{2}{*}{ Location number } & \multirow[b]{2}{*}{$\begin{array}{l}\text { Altitude of } \\
\text { land surface } \\
\text { (ft) }\end{array}$} & \multirow[b]{2}{*}{$\begin{array}{c}\text { Depth of } \\
\text { well } \\
\text { (ft) }\end{array}$} & \multicolumn{3}{|c|}{ Water level } & \multirow{2}{*}{$\begin{array}{c}\text { Specific } \\
\text { conduct- } \\
\text { ance } \\
(\mu \mathrm{S} / \mathrm{cm})\end{array}$} & \multirow[b]{2}{*}{$\begin{array}{c}\text { Date of } \\
\text { meas- } \\
\text { urement }\end{array}$} \\
\hline & & & & $\begin{array}{l}\text { Depth to } \\
\text { water } \\
\text { (ft) }\end{array}$ & $\begin{array}{l}\text { Method } \\
\text { of meas- } \\
\text { urement }\end{array}$ & $\begin{array}{c}\text { Date of } \\
\text { meas- } \\
\text { urement }\end{array}$ & & \\
\hline \multicolumn{9}{|c|}{ Sites located in Washington (Continued) } \\
\hline-- & 25N45E14CABB01 & $2,139.2$ & 250 & 169.07 & $\mathrm{~S}$ & $03 / 15 / 00$ & 261 & $09 / 26 / 00$ \\
\hline-- & 25N45E14CCDD01 & 2,110 & 186 & 163.5 & $\mathrm{R}$ & $11 / 25 / 57$ & 114 & $09 / 26 / 00$ \\
\hline-- & 25N45E15BADA01 & $2,081.6$ & 157 & 116.46 & $\mathrm{~T}$ & $03 / 16 / 00$ & 253 & $09 / 26 / 00$ \\
\hline-- & 25N45E15BADC01 & 2,080 & 173 & 117.94 & S & $03 / 14 / 00$ & -- & -- \\
\hline-- & 25N45E15DDCC01 & $2,072.5$ & 155 & 106.16 & S & $03 / 16 / 00$ & 272 & $09 / 26 / 00$ \\
\hline-- & 25N45E16ACAB01 & 2,050 & 124 & ${ }^{3} 92.78$ & $\mathrm{~T}$ & $05 / 19 / 99$ & ${ }^{3} 305$ & 05/19/99 \\
\hline-- & 25N45E17BBAA01 & 2,040 & 230 & 87.9 & $\mathrm{R}$ & $11 / 02 / 64$ & 150 & 08/16/00 \\
\hline M20 & 25N45E17BBAA05 & $2,035.6$ & 113 & 83.80 & $\mathrm{~T}$ & $08 / 10 / 00$ & 130 & $08 / 10 / 00$ \\
\hline-- & 25N45E17CDDD01 & 2,044 & 207 & 94.2 & $\mathrm{R}$ & $08 / 18 / 64$ & 217 & $08 / 16 / 00$ \\
\hline-- & 25N45E17DCCB01 & $2,044.8$ & 287 & 92.47 & S & $08 / 11 / 00$ & -- & -- \\
\hline-- & 25N45E18DDCB01 & 2,040 & 190 & 88.6 & $\mathrm{R}$ & $04 / 24 / 63$ & 174 & $08 / 16 / 00$ \\
\hline-- & 25N45E23BBAA01 & $2,121.5$ & 191 & 157.67 & $\mathrm{~T}$ & $09 / 26 / 00$ & 129 & $09 / 26 / 00$ \\
\hline-- & 25N46E06BBCB01 & $2,046.8$ & 120 & 72.78 & S & $08 / 03 / 00$ & 57 & $08 / 03 / 00$ \\
\hline-- & 25N46E06BCDD01 & $2,081.2$ & 180 & 100.02 & S & $08 / 18 / 00$ & -- & -- \\
\hline-- & 25N46E06CCDD01 & $2,074.4$ & 175 & 90.50 & $\mathrm{~S}$ & $08 / 03 / 00$ & -- & -- \\
\hline-- & 25N46E07BCAC01 & 2,150 & 241 & 163.44 & S & $09 / 14 / 99$ & 350 & 09/14/99 \\
\hline-- & 25N46E07BCAD01 & $2,174.3$ & 248 & 189.25 & S & $08 / 03 / 00$ & -- & -- \\
\hline-- & 25N46E07BCDA01 & 2,170 & 240 & 195.45 & S & $09 / 15 / 99$ & 296 & 09/15/99 \\
\hline-- & 26N45E24DDDA01 & $2,080.3$ & 178 & 122.80 & $\mathrm{~T}$ & $08 / 11 / 00$ & -- & -- \\
\hline-- & 26N45E25ABBC01 & 2,095 & 213 & 130 & $\mathrm{R}$ & $11 / 18 / 94$ & 288 & 09/17/99 \\
\hline-- & 26N45E25BAAA01 & 2,095 & 190 & 113.13 & S & $03 / 13 / 00$ & 287 & 09/16/99 \\
\hline-- & 26N45E25BAAA02 & 2,095 & 195 & 124.05 & S & $09 / 16 / 99$ & 304 & 09/16/99 \\
\hline-- & 26N45E25BCCC01 & $2,077.2$ & 169 & 103.35 & $\mathrm{~T}$ & $08 / 11 / 00$ & 291 & 09/17/99 \\
\hline-- & 26N45E25CCAC01 & $2,087.2$ & 150 & 112.18 & $\mathrm{~T}$ & $08 / 11 / 00$ & 282 & $08 / 11 / 00$ \\
\hline-- & 26N45E25CCBB01 & 2,080 & 150 & 110.39 & S & $03 / 13 / 00$ & 289 & 03/13/00 \\
\hline-- & 26N45E25DAAB01 & 2,090 & 159 & 110.39 & S & $03 / 13 / 00$ & 293 & $03 / 13 / 00$ \\
\hline-- & 26N45E25DAAC01 & 2,081 & 140 & 101.40 & S & $08 / 11 / 00$ & 296 & $08 / 11 / 00$ \\
\hline-- & 26N45E25DAAC02 & 2,080 & 155 & 100.70 & S & $03 / 14 / 00$ & 287 & 09/15/99 \\
\hline-- & 26N45E25DDAA01 & $2,080.3$ & 146 & 100.91 & $\mathrm{~T}$ & $08 / 11 / 00$ & 304 & $12 / 14 / 99$ \\
\hline-- & 26N45E32ADAA01 & 2,106 & 190 & 144.30 & S & $03 / 13 / 00$ & 421 & 03/13/00 \\
\hline-- & 26N45E32DBDA01 & 2,075 & 157 & 114.50 & S & $03 / 13 / 00$ & -- & -- \\
\hline-- & 26N45E32DCBC01 & 2,061 & 157 & 105.73 & S & $08 / 11 / 00$ & 336 & $08 / 11 / 00$ \\
\hline-- & 26N45E32DCCD01 & 2,060 & 141 & 100.12 & S & $03 / 13 / 00$ & 383 & 03/13/00 \\
\hline-- & 26N45E34CADB01 & 2,067 & 199.7 & 105.10 & S & $03 / 08 / 00$ & -- & -- \\
\hline-- & 26N45E34CADB02 & 2,067 & 238 & 105.3 & $\mathrm{R}$ & $01 / 16 / 65$ & 273 & $08 / 16 / 00$ \\
\hline-- & 26N45E35BDBD03 & 2,080 & 249 & 117.3 & $\mathrm{R}$ & $01 / 27 / 65$ & 294 & $08 / 16 / 00$ \\
\hline M3 & 26N46E31CBBC01 & $2,090.2$ & 151 & 112.60 & $\mathrm{~T}$ & $08 / 11 / 00$ & 279 & 03/07/00 \\
\hline-- & 26N46E31DBAD03 & 2,090 & 222 & 120 & $\mathrm{R}$ & $12 / 10 / 64$ & 261 & $08 / 16 / 00$ \\
\hline
\end{tabular}

${ }^{1}$ Well numbers were assigned only for monitoring wells (table 4, figure 2).

${ }^{2}$ Site is a spring.

${ }^{3}$ From Marti and Garrigues (2001). 
Table 24. Physical and major-ion concentration data for ground-water samples collected for the surface-water/ground-water interaction study of the Spokane River and the surrounding basin-fill aquifers, Idaho and Washington, 2000-01

[Site identification number described in text. Constituents are reported as dissolved $\left(0.45 \mu \mathrm{m}\right.$ filtration), unless otherwise indicated. Abbreviations: ${ }^{\circ} \mathrm{C}$, degrees

Celsius; lab, laboratory; $\mu \mathrm{m}$, micrometer; $\mu \mathrm{S} / \mathrm{cm}$, microsiemens per centimeter at 25 degrees Celsius; $\mathrm{mg} / \mathrm{L}$, milligrams per liter; NTU, nephelometric turbidity units;

R, replicate. Symbols: <, less than reporting level; --, no data]

\begin{tabular}{|c|c|c|c|c|c|c|c|c|c|c|c|c|}
\hline $\begin{array}{c}\text { Well } \\
\text { number } \\
\text { (fig. 5) }\end{array}$ & $\begin{array}{c}\text { Site } \\
\text { identification } \\
\text { number }\end{array}$ & Date & Time & $\begin{array}{c}\text { Spe- } \\
\text { cific } \\
\text { conduc- } \\
\text { tance, } \\
\text { field } \\
(\mu \mathrm{S} / \mathrm{cm})\end{array}$ & $\begin{array}{c}\text { pH, } \\
\text { field } \\
\text { (stan- } \\
\text { dard } \\
\text { units) }\end{array}$ & $\begin{array}{c}\text { Temper- } \\
\text { ature, } \\
\text { water } \\
\left({ }^{0} \mathrm{C}\right)\end{array}$ & $\begin{array}{c}\text { Tur- } \\
\text { bidity, } \\
\text { field } \\
\text { (NTU) }\end{array}$ & $\begin{array}{c}\text { Oxy- } \\
\text { gen } \\
(\mathrm{mg} / \mathrm{L})\end{array}$ & $\begin{array}{c}\text { Hard- } \\
\text { ness } \\
(\mathrm{mg} / \mathrm{L} \\
\text { as } \\
\mathrm{CaCO}_{3}\end{array}$ & $\begin{array}{c}\text { Cal- } \\
\text { cium } \\
(\mathrm{mg} / \mathrm{L})\end{array}$ & $\begin{array}{l}\text { Magne- } \\
\text { sium } \\
(\mathrm{mg} / \mathrm{L})\end{array}$ & $\begin{array}{c}\text { Sodium } \\
\text { adsorp- } \\
\text { tion ratio }\end{array}$ \\
\hline \multirow[t]{7}{*}{ M1 } & 474134117002201 & $08 / 08 / 00$ & 0800 & 148 & 6.5 & 11.0 & 1.6 & 7.9 & 66 & 18 & 5.3 & 0.2 \\
\hline & & $09 / 27 / 00$ & 1300 & 56 & 6.7 & 15.5 & 1.0 & 6.4 & 22 & 6.1 & 1.7 & .2 \\
\hline & & $12 / 15 / 00$ & 0800 & 90 & 6.9 & 10.5 & .52 & 7.0 & 39 & 11 & 3.1 & .2 \\
\hline & & $03 / 28 / 01$ & 1400 & 54 & 6.9 & 9.0 & .19 & 9.5 & 20 & 5.6 & 1.5 & .2 \\
\hline & & $05 / 03 / 01$ & 0900 & 55 & 6.8 & 7.5 & .22 & 8.6 & 22 & 6.0 & 1.7 & .2 \\
\hline & & $05 / 23 / 01$ & 0800 & 74 & 6.7 & 10.0 & .33 & 7.7 & 33 & 9.2 & 2.5 & .2 \\
\hline & & $08 / 14 / 01$ & 1200 & 107 & 6.7 & 11.0 & 1.1 & 6.9 & 45 & 13 & 3.6 & .2 \\
\hline \multirow[t]{9}{*}{ M2 } & 474134117002202 & $09 / 27 / 00$ & 1400 & 45 & 6.9 & 16.0 & .37 & 7.4 & 19 & 5.2 & 1.5 & .2 \\
\hline & & $12 / 15 / 00$ & 0900 & 49 & 7.2 & 3.5 & .73 & 10.9 & 20 & 5.4 & 1.7 & .2 \\
\hline & & $12 / 15 / 00^{\mathbf{R}}$ & 0901 & -- & -- & -- & -- & -- & 20 & 5.4 & 1.7 & .2 \\
\hline & & $03 / 28 / 01$ & 1300 & 53 & 7.2 & 5.5 & .67 & 11.3 & 20 & 5.5 & 1.6 & .2 \\
\hline & & $04 / 30 / 01$ & 1700 & 49 & 7.2 & 8.0 & 1.3 & 11.0 & 22 & 5.8 & 1.8 & .2 \\
\hline & & 05/03/01 & 0800 & 54 & 7.2 & 7.5 & .67 & 10.6 & 22 & 5.9 & 1.8 & .2 \\
\hline & & 05/03/01 & 1600 & 55 & 7.0 & 8.0 & .51 & 10.7 & 22 & 5.9 & 1.8 & .2 \\
\hline & & $05 / 23 / 01$ & 0700 & 56 & 6.9 & 12.0 & 1.2 & 7.4 & 23 & 6.3 & 1.8 & .2 \\
\hline & & $08 / 14 / 01$ & 1100 & 68 & 6.7 & 22.5 & 2.6 & 1.7 & 25 & 6.7 & 2.0 & .2 \\
\hline \multirow[t]{5}{*}{ M3 } & 474226117024801 & $10 / 04 / 00$ & 1500 & 269 & 7.9 & 13.0 & .62 & 6.0 & 140 & 32 & 14 & .1 \\
\hline & & $12 / 20 / 00$ & 0800 & 284 & 8.0 & 11.5 & .67 & 6.8 & 136 & 31 & 14 & .1 \\
\hline & & $05 / 01 / 01$ & 1700 & 270 & 8.0 & 12.5 & .57 & 3.6 & 141 & 32 & 15 & .1 \\
\hline & & $05 / 23 / 01$ & 1900 & 308 & 8.0 & -- & -- & -- & 148 & 34 & 15 & .1 \\
\hline & & $08 / 14 / 01$ & 1400 & 299 & 7.7 & 14.0 & .22 & 6.7 & 145 & 33 & 15 & .1 \\
\hline \multirow[t]{8}{*}{ M4 } & 474151117031101 & $06 / 19 / 00$ & 1800 & 47 & -- & 15.0 & 3.8 & -- & 22 & 6.1 & 1.5 & .2 \\
\hline & & $08 / 07 / 00$ & 1300 & 54 & 6.6 & 23.5 & .22 & 3.3 & 20 & 5.4 & 1.5 & .2 \\
\hline & & $09 / 25 / 00$ & 1600 & 47 & 6.7 & 18.0 & .27 & 6.3 & 19 & 5.3 & 1.5 & .2 \\
\hline & & $12 / 14 / 00$ & 1300 & 50 & 7.4 & 5.5 & .52 & 9.3 & 21 & 5.6 & 1.7 & .2 \\
\hline & & $03 / 26 / 01$ & 1300 & 54 & 7.1 & 6.0 & 1.0 & 9.6 & 22 & 5.8 & 1.7 & .2 \\
\hline & & $04 / 30 / 01$ & 1300 & 51 & 7.1 & 7.5 & 1.3 & 10.6 & 21 & 5.7 & 1.7 & .2 \\
\hline & & $05 / 22 / 01$ & 0900 & 56 & 7.0 & 10.5 & .36 & 8.0 & 22 & 6.1 & 1.7 & .2 \\
\hline & & $08 / 13 / 01$ & 1900 & 63 & 6.7 & 22.5 & .48 & 3.2 & 23 & 6.3 & 1.8 & .2 \\
\hline \multirow[t]{7}{*}{ M5 } & 474151117031102 & $06 / 19 / 00$ & 1545 & 49 & 6.6 & 14.0 & 5.9 & -- & 20 & 5.6 & 1.5 & .2 \\
\hline & & $08 / 07 / 00$ & 1500 & 54 & 6.6 & 21.0 & 3.8 & 3.7 & 20 & 5.6 & 1.6 & .2 \\
\hline & & $09 / 26 / 00$ & 1300 & 48 & 6.7 & 18.0 & .52 & 6.1 & 20 & 5.4 & 1.5 & .2 \\
\hline & & $03 / 26 / 01$ & 1400 & 54 & 7.0 & 6.0 & 1.4 & 9.9 & 21 & 5.8 & 1.7 & .2 \\
\hline & & $04 / 30 / 01$ & 1200 & 54 & 7.3 & 7.0 & 6.4 & 11 & 21 & 5.8 & 1.7 & .2 \\
\hline & & $05 / 22 / 01$ & 0800 & 57 & 7.0 & 10.0 & 1.2 & 8.0 & 23 & 6.1 & 1.7 & .2 \\
\hline & & $08 / 13 / 01$ & 1800 & 63 & 6.8 & 20.5 & 5.3 & 4.3 & 24 & 6.5 & 1.9 & .2 \\
\hline \multirow[t]{7}{*}{ M6 } & 474149117031101 & $08 / 07 / 00$ & 1600 & 55 & 6.6 & 20.0 & .32 & 3.8 & 21 & 5.6 & 1.6 & .2 \\
\hline & & $09 / 26 / 00$ & 1500 & 48 & 6.6 & 18.5 & .59 & 6.0 & 19 & 5.3 & 1.5 & .2 \\
\hline & & $12 / 14 / 00$ & 1400 & 49 & 7.3 & 9.5 & .49 & 8.5 & 21 & 5.6 & 1.6 & .2 \\
\hline & & $03 / 26 / 01$ & 1500 & 54 & 6.9 & 7.5 & 1.7 & 9.8 & 22 & 5.8 & 1.7 & .2 \\
\hline & & $04 / 30 / 01$ & 1500 & 51 & 7.0 & 7.0 & 1.0 & 11 & 22 & 5.9 & 1.7 & .2 \\
\hline & & $05 / 22 / 01$ & 1000 & 55 & 6.9 & 10.0 & .47 & 8.4 & 23 & 6.2 & 1.8 & .2 \\
\hline & & $08 / 13 / 01$ & 2000 & 63 & 6.7 & 18.5 & .33 & 3.9 & 24 & 6.5 & 1.9 & .2 \\
\hline \multirow[t]{7}{*}{ M7 } & 474144117031401 & $08 / 08 / 00$ & 1000 & 87 & 7.2 & 12.0 & .71 & 7.7 & 37 & 10 & 2.8 & .1 \\
\hline & & $09 / 27 / 00$ & 1600 & 81 & 7.2 & 13.0 & 1.5 & 7.8 & 38 & 11 & 2.7 & .1 \\
\hline & & $12 / 14 / 00$ & 1500 & 79 & 7.6 & 10.5 & .47 & 7.6 & 37 & 10 & 2.8 & .1 \\
\hline & & $03 / 26 / 01$ & 1600 & 87 & 7.2 & 11.5 & 4.0 & 7.5 & 39 & 11 & 2.8 & .1 \\
\hline & & $04 / 30 / 01$ & 1600 & 81 & 7.4 & 11.0 & 2.5 & 8.5 & 38 & 11 & 2.8 & .1 \\
\hline & & $05 / 22 / 01$ & 1100 & 90 & 7.3 & 13.0 & 2.4 & 7.4 & 41 & 12 & 2.9 & .1 \\
\hline & & 08/14/01 & 0800 & 95 & 7.1 & 12.0 & 2.1 & 7.4 & 40 & 11 & 3.0 & .2 \\
\hline
\end{tabular}

144 Water-Quality, Streambed-Sediment, and Biological Data from the Clark Fork-Pend Oreille and Spokane River Basins, Montana, Idaho, and Washington, 1998-2001 
Table 24. Physical and major-ion concentration data for ground-water samples collected for the surface-water/ground-water interaction study of the Spokane River and surrounding basin-fill aquifers, Idaho and Washington, 2000-01 (Continued)

\begin{tabular}{|c|c|c|c|c|c|c|c|c|c|c|c|c|c|}
\hline $\begin{array}{c}\text { Sodium } \\
(\mathrm{mg} / \mathrm{L})\end{array}$ & $\begin{array}{c}\text { Potas- } \\
\text { sium } \\
(\mathrm{mg} / \mathrm{L})\end{array}$ & $\begin{array}{c}\text { Bicar- } \\
\text { bonate } \\
(\mathrm{mg} / \mathrm{L} \\
\text { as } \\
\left.\mathrm{HCO}_{3}\right)\end{array}$ & $\begin{array}{c}\text { Carbo- } \\
\text { nate } \\
(\mathrm{mg} / \mathrm{L} \\
\left.\text { as } \mathrm{CO}_{3}\right)\end{array}$ & $\begin{array}{c}\text { Alka- } \\
\text { linity, } \\
\text { field } \\
(\mathrm{mg} / \mathrm{L} \\
\text { as } \\
\left.\mathrm{CaCO}_{3}\right)\end{array}$ & $\begin{array}{c}\text { Alka- } \\
\text { linity, } \\
\text { lab } \\
(\mathrm{mg} / \mathrm{L} \\
\text { as } \\
\left.\mathrm{CaCO}_{3}\right)\end{array}$ & $\begin{array}{c}\text { Sulfate } \\
(\mathrm{mg} / \mathrm{L} \\
\left.\text { as } \mathrm{SO}_{4}\right)\end{array}$ & $\begin{array}{c}\text { Chlo- } \\
\text { ride } \\
(\mathrm{mg} / \mathrm{L})\end{array}$ & $\begin{array}{c}\text { Fluo- } \\
\text { ride } \\
(\mathrm{mg} / \mathrm{L})\end{array}$ & $\begin{array}{c}\text { Bro- } \\
\text { mide } \\
(\mathrm{mg} / \mathrm{L})\end{array}$ & $\begin{array}{c}\text { Silica } \\
(\mathrm{mg} / \mathrm{L} \\
\left.\text { as } \mathrm{SiO}_{2}\right)\end{array}$ & $\begin{array}{c}\text { Dis- } \\
\text { solved } \\
\text { solids, } \\
\text { sum } \\
(\mathrm{mg} / \mathrm{L})\end{array}$ & $\begin{array}{c}\text { Dis- } \\
\text { solved } \\
\text { solids, } \\
\text { residue } \\
\text { at } 180^{\circ} \mathrm{C} \\
(\mathrm{mg} / \mathrm{L})\end{array}$ & $\begin{array}{c}\text { Well } \\
\text { number } \\
\text { (fig. 5) }\end{array}$ \\
\hline 4.3 & 1.0 & 73 & 0 & 60 & -- & 6.8 & 3.0 & $<.1$ & 0.01 & 16 & 90 & 101 & M1 \\
\hline 2.3 & .82 & 26 & 0 & 22 & 24 & 4.2 & 1.2 & $<.1$ & .01 & 13 & 43 & 41 & \\
\hline 3.5 & .92 & 49 & 0 & -- & 40 & 5.1 & 1.9 & $<.16$ & $<.01$ & 18 & 68 & 71 & \\
\hline 2.2 & .56 & 26 & 0 & -- & 21 & 4.5 & 1.2 & $<.16$ & $<.01$ & 11 & 40 & 46 & \\
\hline 1.8 & .58 & 26 & 0 & -- & 21 & 5.0 & .97 & $<.16$ & $<.01$ & 10 & 39 & 50 & \\
\hline 2.5 & .75 & 39 & 0 & -- & 32 & 5.0 & 1.4 & $<.2$ & .01 & 13 & 54 & 47 & \\
\hline 3.8 & .89 & 55 & 0 & -- & 45 & 5.4 & 1.6 & $<.2$ & $<.01$ & 17 & 72 & 75 & \\
\hline 1.6 & .63 & 22 & 0 & 18 & 19 & 3.9 & .84 & $<.1$ & $<.01$ & 8.2 & 33 & 30 & M2 \\
\hline 1.9 & .58 & 24 & 0 & -- & 20 & 4.4 & 1.1 & $<.16$ & $<.01$ & 9.2 & 37 & 38 & \\
\hline 1.9 & .65 & 24 & 0 & -- & 20 & 4.2 & 1.1 & $<.16$ & $<.01$ & 9.2 & 36 & 38 & \\
\hline 1.8 & .64 & 24 & 0 & -- & 20 & 4.8 & 1.0 & $<.16$ & $<.01$ & 9.0 & 36 & 47 & \\
\hline 1.7 & .75 & 13 & 0 & -- & 11 & 5.2 & .94 & $<.2$ & .01 & 9.4 & 32 & 40 & \\
\hline 1.8 & .65 & 24 & 0 & -- & 20 & 5.6 & .89 & $<.16$ & $<.01$ & 9.1 & 38 & 45 & \\
\hline 1.7 & .66 & 26 & 0 & -- & 21 & 5.6 & .89 & $<.16$ & $<.01$ & 8.9 & 38 & 50 & \\
\hline 1.9 & .81 & 27 & 0 & -- & 22 & 4.9 & .87 & $<.2$ & $<.01$ & 9.4 & 39 & 33 & \\
\hline 2.6 & .85 & 29 & 0 & -- & 24 & 5.1 & 1.6 & $<.2$ & $<.01$ & 9.9 & 43 & 46 & \\
\hline 2.9 & 2.3 & 155 & 0 & 127 & 128 & 12 & 2.8 & $<.16$ & .02 & 13 & 156 & 163 & M3 \\
\hline 2.7 & 2.1 & 156 & 0 & -- & 128 & 12 & 2.4 & $<.16$ & .02 & 13 & 154 & 163 & \\
\hline 2.9 & 2.3 & 142 & 0 & -- & 116 & 12 & 2.4 & $<.2$ & .01 & 14 & 151 & 120 & \\
\hline 3.0 & 2.1 & 170 & 0 & -- & 139 & 12 & 2.4 & $<.2$ & .03 & 13 & 166 & 162 & \\
\hline 2.9 & 2.2 & 165 & 0 & -- & 135 & 13 & 2.5 & $<.2$ & .01 & 13 & 163 & 177 & \\
\hline 2.6 & .99 & 27 & 0 & -- & 22 & 4.2 & 2.0 & $<.1$ & .07 & 10 & 41 & 46 & M4 \\
\hline 1.9 & .88 & 24 & 0 & 20 & -- & 3.5 & 99 & $<.1$ & .02 & 9.6 & 36 & 41 & \\
\hline 1.7 & .72 & 23 & 0 & 19 & 20 & 4.0 & .88 & $<.1$ & $<.01$ & 8.3 & 34 & 30 & \\
\hline 1.8 & .56 & 24 & 0 & -- & 20 & 4.3 & 1.1 & $<.16$ & $<.01$ & 8.7 & 36 & 36 & \\
\hline 1.9 & .66 & 26 & 0 & -- & 21 & 4.9 & 1.1 & $<.16$ & $<.01$ & 8.9 & 38 & 46 & \\
\hline 1.8 & .75 & 26 & 0 & -- & 21 & 5.4 & .86 & $<.16$ & $<.01$ & 9.0 & 38 & 38 & \\
\hline 1.9 & .80 & 27 & 0 & -- & 22 & 4.9 & .86 & $<.2$ & $<.01$ & 9.1 & 38 & 26 & \\
\hline 2.5 & .91 & 28 & 0 & - & 23 & 5.4 & 1.6 & $<.2$ & $<.01$ & 9.3 & 42 & 46 & \\
\hline 2.1 & 1.2 & 28 & 0 & -- & 23 & 4.1 & .73 & $<.1$ & .07 & 10 & 39 & 48 & M5 \\
\hline 1.9 & .91 & 24 & 0 & 20 & -- & 3.5 & .98 & $<.1$ & $<.01$ & 10 & 36 & 43 & \\
\hline 1.7 & .76 & 22 & 0 & 18 & 20 & 3.9 & .93 & $<.1$ & $<.01$ & 8.8 & 34 & 30 & \\
\hline 1.9 & .61 & 26 & 0 & -- & 21 & 4.8 & 1.1 & $<.16$ & $<.01$ & 8.7 & 37 & 45 & \\
\hline 2.0 & .68 & 26 & 0 & -- & 21 & 5.6 & 1.0 & $<.16$ & $<.01$ & 8.9 & 38 & 39 & \\
\hline 2.0 & .77 & 27 & 0 & -- & 22 & 4.9 & .87 & $<.2$ & $<.01$ & 9.6 & 39 & 37 & \\
\hline 2.4 & .91 & 24 & 0 & -- & 20 & 5.1 & 1.6 & $<.2$ & $<.01$ & 9.9 & 40 & 48 & \\
\hline 1.9 & .88 & 23 & 0 & 19 & -- & 3.5 & .93 & $<.1$ & $<.01$ & 11 & 37 & 44 & M6 \\
\hline 1.7 & .83 & 22 & 0 & 18 & 19 & 3.9 & .91 & $<.1$ & $<.01$ & 9.0 & 34 & 32 & \\
\hline 1.7 & .55 & 24 & 0 & -- & 20 & 4.4 & 1.1 & $<.16$ & $<.01$ & 8.4 & 35 & 33 & \\
\hline 1.9 & .54 & 26 & 0 & -- & 21 & 4.7 & 1.1 & $<.16$ & $<.01$ & 8.6 & 37 & 48 & \\
\hline 1.7 & .63 & 26 & 0 & -- & 21 & 5.2 & .84 & $<.16$ & $<.01$ & 9.0 & 38 & 40 & \\
\hline 1.9 & .76 & 26 & 0 & -- & 21 & 5.0 & .90 & $<.2$ & $<.01$ & 9.6 & 39 & 32 & \\
\hline 2.3 & .93 & 28 & 0 & -- & 23 & 5.0 & 1.5 & $<.2$ & $<.01$ & 10 & 42 & 45 & \\
\hline 1.9 & .93 & 46 & 0 & 37 & -- & 4.9 & .94 & $<.1$ & $<.01$ & 11 & 56 & 61 & M7 \\
\hline 2.1 & 1.0 & 43 & 0 & 35 & 37 & 5.0 & 1.2 & $<.1$ & $<.01$ & 12 & 56 & 55 & \\
\hline 1.9 & .95 & 44 & 0 & -- & 36 & 4.8 & 1.1 & $<.16$ & .01 & 12 & 55 & 54 & \\
\hline 2.1 & 1.0 & 46 & 0 & -- & 38 & 5.1 & 1.2 & $<.16$ & .01 & 11 & 57 & $<10$ & \\
\hline 1.9 & .94 & 46 & 0 & -- & 38 & 5.2 & 1.2 & $<.16$ & $<.01$ & 11 & 57 & 66 & \\
\hline 2.0 & 1.2 & 46 & 0 & -- & 38 & 4.8 & 1.2 & $<.2$ & .01 & 12 & 59 & 55 & \\
\hline 2.1 & .98 & 48 & 0 & -- & 39 & 5.0 & 1.1 & $<.2$ & $<.01$ & 12 & 59 & 62 & \\
\hline
\end{tabular}


Table 24. Physical and major-ion concentration data for ground-water samples collected for the surface-water/ground-water interaction study of the Spokane River and the surrounding basin-fill aquifers, Idaho and Washington, 2000-01 (Continued)

\begin{tabular}{|c|c|c|c|c|c|c|c|c|c|c|c|c|}
\hline $\begin{array}{c}\text { Well } \\
\text { number } \\
\text { (fig. 5) }\end{array}$ & $\begin{array}{c}\text { Site } \\
\text { identification } \\
\text { number }\end{array}$ & Date & Time & $\begin{array}{c}\text { Spe- } \\
\text { cific } \\
\text { conduc- } \\
\text { tance, } \\
\text { field } \\
(\mu \mathrm{S} / \mathrm{cm})\end{array}$ & $\begin{array}{c}\text { pH, } \\
\text { field } \\
\text { (stan- } \\
\text { dard } \\
\text { units) }\end{array}$ & $\begin{array}{l}\text { Temper- } \\
\text { ature, } \\
\text { water } \\
\left({ }^{\circ} \mathrm{C}\right)\end{array}$ & $\begin{array}{c}\text { Tur- } \\
\text { bidity, } \\
\text { field } \\
\text { (NTU) }\end{array}$ & $\begin{array}{c}\text { Oxy- } \\
\text { gen } \\
(\mathrm{mg} / \mathrm{L})\end{array}$ & $\begin{array}{c}\text { Hard- } \\
\text { ness } \\
(\mathrm{mg} / \mathrm{L} \\
\text { as } \\
\mathrm{CaCO}_{3}\end{array}$ & $\begin{array}{c}\text { Cal- } \\
\text { cium } \\
(\mathrm{mg} / \mathrm{L})\end{array}$ & $\begin{array}{l}\text { Magne- } \\
\text { sium } \\
(\mathrm{mg} / \mathrm{L})\end{array}$ & $\begin{array}{l}\text { Sodium } \\
\text { adsorp- } \\
\text { tion ratio }\end{array}$ \\
\hline \multirow[t]{12}{*}{ M8 } & 474130117035902 & $07 / 12 / 00$ & 1300 & 44 & 7.0 & 21.0 & 8.0 & 6.6 & 18 & 4.8 & 1.3 & .2 \\
\hline & & $08 / 09 / 00$ & 1000 & 51 & 6.8 & 25.0 & 1.8 & 4.8 & 19 & 5.1 & 1.6 & .2 \\
\hline & & $08 / 09 / 00^{1}$ & 1020 & 51 & 6.8 & 25.0 & 1.8 & 4.8 & 19 & 5.1 & 1.6 & .2 \\
\hline & & $09 / 26 / 00$ & 0800 & 47 & 7.0 & 15.0 & 4.4 & 8.1 & 20 & 5.4 & 1.6 & .2 \\
\hline & & $12 / 14 / 00$ & 1000 & 50 & 7.5 & 2.5 & 6.9 & 11 & 21 & 5.5 & 1.7 & .2 \\
\hline & & $03 / 28 / 01$ & 1100 & 54 & 7.3 & 5.0 & 5.9 & 11 & 21 & 5.7 & 1.7 & .2 \\
\hline & & $05 / 02 / 01$ & 1100 & 54 & 7.5 & 7.5 & 3.1 & 11 & 23 & 6.0 & 1.8 & .2 \\
\hline & & $05 / 02 / 01^{\mathbf{R}}$ & 1101 & -- & -1.0 & -1.0 & --1 & - & 23 & 6.0 & 1.8 & .2 \\
\hline & & $05 / 23 / 01$ & 1000 & 55 & 6.9 & 12.5 & 11.0 & 7.5 & 22 & 5.8 & 1.8 & .2 \\
\hline & & 08/13/01 & 1400 & 62 & 7.0 & 25.0 & 1.3 & 5.0 & 22 & 6.0 & 1.8 & .2 \\
\hline & & $08 / 14 / 01$ & 0600 & 65 & 6.9 & 24.5 & .85 & 4.1 & 23 & 6.1 & 1.8 & .2 \\
\hline & & $08 / 14 / 01^{\mathbf{R}}$ & 0601 & -- & -- & -- & -- & -- & 23 & 6.2 & 1.8 & .2 \\
\hline \multirow[t]{9}{*}{ M9 } & 474130117035901 & 08/09/00 & 0900 & 52 & 6.8 & 24.5 & .52 & 4.6 & 20 & 5.2 & 1.6 & .2 \\
\hline & & $08 / 09 / 00^{1}$ & 0920 & - & -0.0 & -- & $-{ }_{-}^{-52}$ & -3.0 & 19 & 5.2 & 1.6 & .2 \\
\hline & & $09 / 26 / 00$ & 0900 & 47 & 6.9 & 22.0 & 3.7 & 7.4 & 20 & 5.4 & 1.6 & .2 \\
\hline & & $12 / 14 / 00$ & 0900 & 50 & 7.4 & 3.5 & 2.5 & 11 & 21 & 5.6 & 1.7 & .2 \\
\hline & & $03 / 28 / 01$ & 1000 & 54 & 7.4 & 5.0 & 2.6 & 11 & 21 & 5.6 & 1.6 & .2 \\
\hline & & $05 / 02 / 01$ & 1200 & 54 & 7.2 & 7.5 & 2.9 & 10 & 22 & 5.9 & 1.8 & .2 \\
\hline & & $05 / 23 / 01$ & 0900 & 54 & 6.8 & 12.0 & 7.6 & 7.5 & 22 & 5.9 & 1.7 & .2 \\
\hline & & $08 / 13 / 01$ & 1600 & 62 & 7.0 & 23.0 & .93 & 4.5 & 22 & 6.0 & 1.8 & .2 \\
\hline & & $08 / 14 / 01$ & 0500 & 64 & 7.0 & 23.0 & .80 & 4.2 & 22 & 6.0 & 1.8 & .2 \\
\hline \multirow[t]{7}{*}{ M10 } & 474131117040401 & $08 / 09 / 00$ & 1100 & 53 & 6.7 & 25.0 & 1.8 & 2.7 & 20 & 5.4 & 1.6 & .2 \\
\hline & & $09 / 26 / 00$ & 1100 & 47 & 6.8 & 18.5 & 6.6 & 6.4 & 19 & 5.3 & 1.5 & .2 \\
\hline & & $12 / 14 / 00$ & 1100 & 49 & 7.4 & 5.5 & 12.0 & 10 & 21 & 5.5 & 1.7 & .2 \\
\hline & & $03 / 28 / 01$ & 1200 & 54 & 7.2 & 5.5 & 11.0 & 10 & 21 & 5.7 & 1.7 & .2 \\
\hline & & $05 / 02 / 01$ & 1300 & 55 & 7.1 & 8.5 & 6.3 & 9.3 & 22 & 5.9 & 1.7 & .2 \\
\hline & & $05 / 23 / 01$ & 1100 & 60 & 6.9 & 12.0 & 25.0 & 7.0 & 23 & 6.3 & 1.8 & .2 \\
\hline & & $08 / 13 / 01$ & 1200 & 62 & 7.0 & 24.0 & 3.2 & 3.1 & 23 & 6.2 & 1.8 & .2 \\
\hline \multirow[t]{10}{*}{ M11 } & 474106117060501 & 08/09/00 & 1300 & 52 & 6.8 & 25.0 & 2.0 & 3.7 & 19 & 5.1 & 1.5 & .2 \\
\hline & & $08 / 09 / 00^{\mathbf{R}}$ & 1301 & - & -- & -- & $-{ }_{--}^{2.0}$ & --1 & 19 & 5.1 & 1.6 & .2 \\
\hline & & $08 / 09 / 00^{\mathbf{R}, 1}$ & 1321 & -- & -- & -- & -- & -- & 19 & 5.2 & 1.6 & .2 \\
\hline & & $10 / 04 / 00$ & 1000 & 44 & 7.1 & 15.5 & 9.3 & 7.2 & 20 & 5.3 & 1.5 & .2 \\
\hline & & $12 / 19 / 00$ & 1000 & 50 & 7.1 & 4.0 & 11.0 & 11 & 21 & 5.6 & 1.7 & .2 \\
\hline & & $03 / 27 / 01$ & 1400 & 53 & 7.0 & 5.5 & 13.0 & 9.7 & 22 & 5.9 & 1.7 & .2 \\
\hline & & $05 / 01 / 01$ & 1000 & 48 & 7.1 & 7.5 & 14.0 & 8.4 & 21 & 5.7 & 1.7 & .2 \\
\hline & & $05 / 23 / 01$ & 1400 & 55 & 6.9 & 12.0 & 12.0 & 7.2 & 22 & 5.8 & 1.7 & .2 \\
\hline & & $05 / 23 / 01^{\mathbf{R}}$ & 1401 & -- & -- & -- & -- & -1.2 & 22 & 6.0 & 1.7 & .2 \\
\hline & & $08 / 16 / 01$ & 0800 & 65 & 6.8 & 23.5 & 4.9 & 3.7 & 23 & 6.2 & 1.8 & .2 \\
\hline \multirow[t]{6}{*}{ M12 } & 474107117060502 & $10 / 04 / 00$ & 1200 & 48 & 7.0 & 16.0 & 1.6 & 6.9 & 21 & 5.8 & 1.5 & .2 \\
\hline & & $12 / 19 / 00$ & 0900 & 49 & 7.1 & 6.0 & .85 & 10 & 21 & 5.7 & 1.6 & .2 \\
\hline & & $03 / 27 / 01$ & 1200 & 54 & 7.0 & 5.5 & 4.0 & 9.7 & 22 & 5.9 & 1.7 & .2 \\
\hline & & $05 / 01 / 01$ & 0900 & 49 & 7.1 & 7.0 & .49 & 7.8 & 23 & 6.3 & 1.8 & .2 \\
\hline & & $05 / 23 / 01$ & 1500 & 56 & 6.8 & 10.5 & 1.7 & 7.3 & 23 & 6.1 & 1.8 & .2 \\
\hline & & $08 / 16 / 01$ & 0600 & 64 & 6.8 & 20.5 & 1.6 & 4.1 & 23 & 6.4 & 1.8 & .2 \\
\hline \multirow[t]{7}{*}{ M13 } & 474107117060501 & $08 / 09 / 00$ & 1500 & 53 & 6.8 & 23.5 & 2.1 & 4.2 & 20 & 5.2 & 1.6 & .2 \\
\hline & & $10 / 04 / 00$ & 0800 & 45 & 7.0 & 16.5 & 4.1 & 7.4 & 20 & 5.3 & 1.5 & .2 \\
\hline & & $12 / 19 / 00$ & 1100 & 49 & 7.0 & 8.0 & 7.6 & 9.7 & 21 & 5.6 & 1.6 & .2 \\
\hline & & $03 / 27 / 01$ & 1500 & 56 & 7.0 & 7.5 & 3.9 & 9.9 & 23 & 6.2 & 1.8 & .2 \\
\hline & & $05 / 01 / 01$ & 0800 & 49 & 7.4 & 7.0 & .56 & 9.2 & 23 & 6.2 & 1.8 & .2 \\
\hline & & $05 / 23 / 01$ & 1300 & 55 & 6.9 & 12.5 & .50 & 7.6 & 23 & 6.1 & 1.8 & .2 \\
\hline & & $08 / 16 / 01$ & 0900 & 64 & 6.8 & 23.0 & .64 & 4.2 & 23 & 6.2 & 1.8 & .2 \\
\hline
\end{tabular}


Table 24. Physical and major-ion concentration data for ground-water samples collected for the surface-water/ground-water interaction study of the Spokane River and surrounding basin-fill aquifers, Idaho and Washington, 2000-01 (Continued)

\begin{tabular}{|c|c|c|c|c|c|c|c|c|c|c|c|c|c|}
\hline $\begin{array}{c}\text { Sodium } \\
(\mathrm{mg} / \mathrm{L})\end{array}$ & $\begin{array}{c}\text { Potas- } \\
\text { sium } \\
(\mathrm{mg} / \mathrm{L})\end{array}$ & $\begin{array}{c}\text { Bicar- } \\
\text { bonate } \\
(\mathrm{mg} / \mathrm{L} \\
\text { as } \\
\left.\mathrm{HCO}_{3}\right)\end{array}$ & $\begin{array}{c}\text { Carbo- } \\
\text { nate } \\
(\mathrm{mg} / \mathrm{L} \\
\left.\text { as } \mathrm{CO}_{3}\right)\end{array}$ & $\begin{array}{c}\text { Alka- } \\
\text { linity, } \\
\text { field } \\
(\mathrm{mg} / \mathrm{L} \\
\text { as } \\
\left.\mathrm{CaCO}_{3}\right)\end{array}$ & $\begin{array}{c}\text { Alka- } \\
\text { linity, } \\
\text { lab } \\
(\mathrm{mg} / \mathrm{L} \\
\text { as } \\
\left.\mathrm{CaCO}_{3}\right)\end{array}$ & $\begin{array}{c}\text { Sulfate } \\
(\mathrm{mg} / \mathrm{L} \\
\left.\text { as } \mathrm{SO}_{4}\right)\end{array}$ & $\begin{array}{c}\text { Chlo- } \\
\text { ride } \\
(\mathrm{mg} / \mathrm{L})\end{array}$ & $\begin{array}{c}\text { Fluo- } \\
\text { ride } \\
(\mathrm{mg} / \mathrm{L})\end{array}$ & $\begin{array}{c}\text { Bro- } \\
\text { mide } \\
(\mathrm{mg} / \mathrm{L})\end{array}$ & $\begin{array}{c}\text { Silica } \\
(\mathrm{mg} / \mathrm{L} \\
\left.\text { as } \mathrm{SiO}_{2}\right)\end{array}$ & $\begin{array}{c}\text { Dis- } \\
\text { solved } \\
\text { solids, } \\
\text { sum } \\
(\mathrm{mg} / \mathrm{L})\end{array}$ & $\begin{array}{c}\text { Dis- } \\
\text { solved } \\
\text { solids, } \\
\text { residue } \\
\text { at } 180^{\circ} \mathrm{C} \\
(\mathrm{mg} / \mathrm{L})\end{array}$ & $\begin{array}{c}\text { Well } \\
\text { number } \\
\text { (fig. 5) }\end{array}$ \\
\hline 1.6 & .65 & 16 & 0 & 13 & -- & 3.0 & .65 & $<.1$ & $<.01$ & 8.6 & 29 & 34 & M8 \\
\hline 1.9 & .75 & 23 & 0 & 19 & -- & 3.6 & 1.1 & $<.1$ & $<.01$ & 8.7 & 34 & 42 & \\
\hline 1.8 & .71 & 23 & 0 & 19 & -- & 3.6 & 1.1 & $<.1$ & $<.01$ & 8.6 & 34 & 39 & \\
\hline 1.7 & .67 & 22 & 0 & 18 & 20 & 3.9 & .84 & $<.1$ & $<.01$ & 8.3 & 33 & 31 & \\
\hline 1.9 & .66 & 26 & 0 & -- & 21 & 4.3 & 1.1 & $<.16$ & $<.01$ & 9.4 & 37 & 38 & \\
\hline 1.8 & .66 & 26 & 0 & -- & 21 & 4.8 & .98 & $<.16$ & $<.01$ & 9.1 & 37 & 49 & \\
\hline 1.7 & .73 & 26 & 0 & -- & 21 & 5.4 & .90 & $<.16$ & $<.01$ & 9.1 & 39 & 46 & \\
\hline 1.7 & .74 & 26 & 0 & -- & 21 & 5.4 & .86 & $<.16$ & $<.01$ & 9.1 & 38 & 42 & \\
\hline 1.8 & .80 & 26 & 0 & -- & 21 & 5.0 & .92 & $<.2$ & $<.01$ & 9.3 & 38 & 36 & \\
\hline 2.5 & .79 & 27 & 0 & -- & 22 & 5.1 & 1.6 & $<.2$ & $<.01$ & 8.3 & 40 & 44 & \\
\hline 2.5 & .82 & 28 & 0 & -- & 23 & 5.1 & 1.6 & $<.2$ & $<.01$ & 8.4 & 40 & 44 & \\
\hline 2.5 & .80 & 28 & 0 & -- & 23 & 5.0 & 1.5 & $<.2$ & $<.01$ & 8.4 & 40 & 45 & \\
\hline 1.9 & .73 & 23 & 0 & 19 & -- & 3.6 & 1.1 & $<.1$ & $<.01$ & 8.7 & 34 & 42 & M9 \\
\hline 1.8 & .71 & -- & 0 & -- & -- & 3.6 & 1.1 & $<.1$ & $<.01$ & 8.7 & -- & 38 & \\
\hline 1.6 & .70 & 22 & 0 & 18 & 20 & 4.0 & .86 & $<.1$ & $<.01$ & 8.4 & 33 & 42 & \\
\hline 1.9 & .63 & 26 & 0 & -- & 21 & 4.4 & 1.1 & $<.16$ & $<.01$ & 9.4 & 37 & 40 & \\
\hline 1.8 & .65 & 26 & 0 & -- & 21 & 4.7 & 1.0 & $<.16$ & $<.01$ & 8.8 & 37 & 52 & \\
\hline 1.7 & .70 & 26 & 0 & -- & 21 & 5.3 & .91 & $<.16$ & $<.01$ & 9.0 & 38 & 37 & \\
\hline 1.8 & .79 & 26 & 0 & -- & 21 & 4.9 & .94 & $<.2$ & $<.01$ & 9.0 & 38 & 29 & \\
\hline 2.5 & .82 & 24 & 0 & -- & 20 & 5.2 & 1.6 & $<.2$ & $<.01$ & 8.8 & 39 & 45 & \\
\hline 2.4 & .82 & 28 & 0 & -- & 23 & 5.1 & 1.6 & $<.2$ & $<.01$ & 8.7 & 40 & 47 & \\
\hline 1.8 & .83 & 23 & 0 & 19 & -- & 3.6 & 1.0 & $<.1$ & $<.01$ & 10 & 36 & 42 & M10 \\
\hline 1.7 & .74 & 22 & 0 & 18 & 20 & 4.0 & .88 & $<.1$ & $<.01$ & 8.5 & 33 & 50 & \\
\hline 1.8 & .57 & 24 & 0 & -- & 20 & 4.3 & 1.1 & $<.16$ & $<.01$ & 8.7 & 36 & 37 & \\
\hline 1.9 & .59 & 26 & 0 & -- & 21 & 4.8 & 1.1 & $<.16$ & $<.01$ & 8.8 & 37 & 47 & \\
\hline 1.7 & .58 & 26 & 0 & -- & 21 & 5.0 & .96 & $<.16$ & $<.01$ & 8.8 & 37 & 49 & \\
\hline 1.9 & .78 & 27 & 0 & -- & 22 & 5.0 & .88 & $<.2$ & $<.01$ & 9.7 & 40 & 37 & \\
\hline 2.4 & .93 & 28 & 0 & -- & 23 & 5.1 & 1.6 & $<.2$ & $<.01$ & 9.9 & 42 & 44 & \\
\hline 1.9 & .75 & 23 & 0 & 18 & -- & 3.7 & 1.1 & $<.1$ & $<.01$ & 9.6 & 35 & 40 & M11 \\
\hline 1.8 & .83 & 23 & 0 & 19 & -- & 3.7 & 1.1 & $<.1$ & $<.01$ & 9.6 & 35 & 40 & \\
\hline 1.8 & .94 & -- & -- & -- & -- & -- & -- & -- & -- & 9.5 & -- & -- & \\
\hline 1.7 & .69 & 22 & 0 & 18 & 20 & 3.9 & .76 & $<.16$ & $<.01$ & 8.4 & 33 & 36 & \\
\hline 1.8 & .58 & 24 & 0 & -- & 20 & 4.2 & 1.1 & $<.16$ & $<.01$ & 8.7 & 36 & 41 & \\
\hline 2.0 & .63 & 26 & 0 & -- & 21 & 4.8 & 1.0 & $<.16$ & $<.01$ & 9.0 & 38 & 45 & \\
\hline 1.8 & .79 & 26 & 0 & -- & 21 & 5.6 & .97 & $<.16$ & $<.01$ & 8.8 & 38 & 63 & \\
\hline 1.8 & .67 & 24 & 0 & -- & 20 & 5.0 & .91 & $<.2$ & $<.01$ & 9.4 & 38 & 39 & \\
\hline 1.9 & .66 & 26 & 0 & -- & 21 & 5.0 & .94 & $<.2$ & $<.01$ & 9.3 & 38 & 32 & \\
\hline 2.6 & .92 & 28 & 0 & -- & 23 & 5.2 & 1.6 & $<.2$ & $<.01$ & 8.7 & 41 & 37 & \\
\hline 1.8 & .74 & 24 & 0 & 20 & 21 & 4.0 & .79 & $<.16$ & $<.01$ & 9.0 & 35 & 38 & M12 \\
\hline 1.7 & .60 & 24 & 0 & -- & 20 & 4.1 & 1.1 & $<.16$ & $<.01$ & 8.4 & 35 & 38 & \\
\hline 1.9 & .66 & 26 & 0 & -- & 21 & 4.8 & 1.1 & $<.16$ & $<.01$ & 8.6 & 37 & 42 & \\
\hline 1.9 & .67 & 26 & 0 & -- & 21 & 5.0 & .90 & $<.16$ & $<.01$ & 9.1 & 38 & 45 & \\
\hline 1.9 & .73 & 27 & 0 & -- & 22 & 4.9 & .90 & $<.2$ & $<.01$ & 9.4 & 39 & 37 & \\
\hline 2.4 & .91 & 28 & 0 & -- & 23 & 5.0 & 1.5 & $<.2$ & $<.01$ & 9.6 & 41 & 41 & \\
\hline 1.8 & .97 & 22 & 0 & 18 & -- & 3.6 & 1.1 & $<.1$ & $<.01$ & 10 & 35 & 42 & M13 \\
\hline 1.7 & .74 & 23 & 0 & 19 & 20 & 4.0 & .74 & $<.16$ & $<.01$ & 8.7 & 34 & 36 & \\
\hline 1.7 & .63 & 24 & 0 & -- & 20 & 4.1 & 1.1 & $<.16$ & $<.01$ & 8.0 & 35 & 40 & \\
\hline 1.9 & .56 & 26 & 0 & -- & 21 & 4.9 & 1.3 & $<.16$ & $<.01$ & 8.2 & 38 & 47 & \\
\hline 1.9 & .65 & 26 & 0 & -- & 21 & 5.4 & .99 & $<.16$ & $<.01$ & 9.0 & 38 & 47 & \\
\hline 1.9 & .61 & 27 & 0 & -- & 22 & 5.0 & .92 & $<.2$ & $<.01$ & 9.5 & 39 & 25 & \\
\hline 2.6 & .91 & 27 & 0 & -- & 22 & 5.2 & 1.6 & $<.2$ & $<.01$ & 8.9 & 41 & 40 & \\
\hline
\end{tabular}


Table 24. Physical and major-ion concentration data for ground-water samples collected for the surface-water/ground-water interaction study of the Spokane River and the surrounding basin-fill aquifers, Idaho and Washington, 2000-01 (Continued)

\begin{tabular}{|c|c|c|c|c|c|c|c|c|c|c|c|c|}
\hline $\begin{array}{c}\text { Well } \\
\text { number } \\
\text { (fig. 5) }\end{array}$ & $\begin{array}{c}\text { Site } \\
\text { identification } \\
\text { number }\end{array}$ & Date & Time & $\begin{array}{c}\text { Spe- } \\
\text { cific } \\
\text { conduc- } \\
\text { tance, } \\
\text { field } \\
(\mu S / c m)\end{array}$ & $\begin{array}{c}\text { pH, } \\
\text { field } \\
\text { (stan- } \\
\text { dard } \\
\text { units) }\end{array}$ & $\begin{array}{l}\text { Temper- } \\
\text { ature, } \\
\text { water } \\
\left({ }^{0} \mathrm{C}\right)\end{array}$ & $\begin{array}{c}\text { Tur- } \\
\text { bidity, } \\
\text { field } \\
\text { (NTU) }\end{array}$ & $\begin{array}{c}\text { Oxy- } \\
\text { gen } \\
(\mathrm{mg} / \mathrm{L})\end{array}$ & $\begin{array}{c}\text { Hard- } \\
\text { ness } \\
(\mathrm{mg} / \mathrm{L} \\
\text { as } \\
\mathrm{CaCO}_{3}\end{array}$ & $\begin{array}{c}\text { Cal- } \\
\text { cium } \\
(\mathrm{mg} / \mathrm{L})\end{array}$ & $\begin{array}{c}\text { Magne- } \\
\text { sium } \\
(\mathrm{mg} / \mathrm{L})\end{array}$ & $\begin{array}{c}\text { Sodium } \\
\text { adsorp- } \\
\text { tion ratio }\end{array}$ \\
\hline \multirow[t]{6}{*}{ M14 } & 474110117060601 & $10 / 03 / 00$ & 1800 & 50 & 7.1 & 15.5 & 2.9 & 7.3 & 21 & 5.9 & 1.5 & .2 \\
\hline & & $12 / 19 / 00$ & 1400 & 53 & 7.2 & 5.5 & 2.3 & 11 & 21 & 5.9 & 1.6 & .2 \\
\hline & & $03 / 27 / 01$ & 1100 & 58 & 7.1 & 6.0 & 3.6 & 9.9 & 23 & 6.5 & 1.7 & .2 \\
\hline & & $05 / 02 / 01$ & 0800 & 51 & 7.3 & 6.5 & .51 & 9.8 & 24 & 6.6 & 1.9 & .2 \\
\hline & & $05 / 24 / 01$ & 1200 & 61 & 7.2 & 9.5 & 2.0 & 8.5 & 26 & 7.1 & 1.9 & .2 \\
\hline & & $08 / 15 / 01$ & 1600 & 65 & 6.9 & 23.0 & .21 & 4.1 & 24 & 6.7 & 1.8 & .2 \\
\hline \multirow[t]{7}{*}{ M15 } & 474115117060301 & $08 / 10 / 00$ & 1000 & 302 & 7.7 & 10.5 & .16 & 6.8 & 155 & 35 & 16 & .1 \\
\hline & & $10 / 03 / 00$ & 1700 & 307 & 7.8 & 10.0 & .29 & 6.9 & 157 & 35 & 17 & .1 \\
\hline & & $12 / 19 / 00$ & 1300 & 304 & 7.9 & 9.0 & 4.0 & 7.2 & 155 & 35 & 17 & .1 \\
\hline & & $03 / 28 / 01$ & 0900 & 309 & 7.9 & 9.5 & 3.1 & 7.8 & 156 & 35 & 17 & .1 \\
\hline & & $05 / 02 / 01$ & 1000 & 308 & 7.9 & 9.5 & 1.5 & 7.9 & 163 & 37 & 17 & .1 \\
\hline & & $05 / 24 / 01$ & 1100 & 314 & 7.8 & 10.0 & 2.0 & 8.3 & 159 & 35 & 17 & .1 \\
\hline & & $08 / 13 / 01$ & 1100 & 304 & 7.8 & 11.0 & 1.3 & 7.6 & 156 & 35 & 17 & .1 \\
\hline \multirow[t]{6}{*}{ M16 } & 474140117060401 & $08 / 10 / 00$ & 0800 & 324 & 7.6 & 7.5 & .19 & 8.7 & 163 & 36 & 18 & .1 \\
\hline & & $10 / 04 / 00$ & 1400 & 324 & 7.7 & 9.0 & .28 & 9.0 & 168 & 37 & 18 & .1 \\
\hline & & $12 / 19 / 00$ & 1200 & 321 & 7.8 & 8.5 & 1.5 & 8.7 & 164 & 37 & 18 & .1 \\
\hline & & $05 / 01 / 01$ & 1600 & 302 & 7.8 & 9.5 & .69 & 5.1 & 164 & 37 & 17 & .1 \\
\hline & & $05 / 23 / 01$ & 1700 & 331 & 7.7 & 11.0 & .30 & 8.7 & 165 & 37 & 18 & .1 \\
\hline & & $08 / 15 / 01$ & 1400 & 322 & 7.6 & 10.0 & .65 & 8.8 & 165 & 37 & 18 & .1 \\
\hline \multirow[t]{7}{*}{ M17 } & 474033117062501 & $08 / 08 / 00$ & 1300 & 95 & 7.7 & 13.0 & 4.7 & 7.8 & 43 & 13 & 2.8 & .1 \\
\hline & & $10 / 03 / 00$ & 1500 & 91 & 7.6 & 12.0 & 1.7 & 7.8 & 42 & 12 & 2.8 & .1 \\
\hline & & $12 / 14 / 00$ & 1600 & 85 & 8.2 & 11.0 & 1.9 & 6.8 & 41 & 12 & 2.7 & .1 \\
\hline & & $03 / 27 / 01$ & 0800 & 89 & 8.0 & 12.0 & 1.7 & 6.6 & 41 & 12 & 2.6 & .1 \\
\hline & & $05 / 01 / 01$ & 1500 & 81 & 7.8 & 12.0 & 1.9 & 5.6 & 41 & 12 & 2.6 & .1 \\
\hline & & $05 / 22 / 01$ & 1200 & 92 & 7.9 & 14.5 & 1.6 & 6.8 & 42 & 12 & 2.7 & .1 \\
\hline & & $08 / 15 / 01$ & 1100 & 91 & 7.7 & 13.0 & .40 & 7.4 & 40 & 12 & 2.7 & .1 \\
\hline \multirow[t]{7}{*}{ M18 } & 474050117064201 & $08 / 08 / 00$ & 1500 & 55 & 6.9 & 13.5 & 5.5 & 5.4 & 23 & 6.4 & 1.7 & .2 \\
\hline & & $10 / 03 / 00$ & 1200 & 58 & 6.8 & 16.5 & 155 & 6.2 & 25 & 7.1 & 1.7 & .2 \\
\hline & & $12 / 19 / 00$ & 1600 & 57 & 6.9 & 14.0 & 3.5 & 7.1 & 23 & 6.4 & 1.6 & .2 \\
\hline & & $03 / 27 / 01$ & 0900 & 63 & 7.0 & 13.0 & 1.3 & 7.0 & 27 & 7.7 & 1.9 & .2 \\
\hline & & $05 / 01 / 01$ & 1200 & 57 & 7.0 & 13.0 & 2.5 & 7.4 & 26 & 7.2 & 1.9 & .1 \\
\hline & & $05 / 22 / 01$ & 1500 & 63 & 7.0 & 14.0 & 2.1 & 8.4 & 26 & 7.5 & 1.8 & .2 \\
\hline & & $08 / 15 / 01$ & 1200 & 70 & 7.0 & 13.5 & .27 & 7.2 & 29 & 8.1 & 2.2 & .2 \\
\hline \multirow[t]{7}{*}{ M19 } & 474053117064701 & $08 / 08 / 00$ & 1600 & 55 & 6.7 & 15.0 & 1.8 & 4.9 & 22 & 5.9 & 1.7 & .2 \\
\hline & & $10 / 03 / 00$ & 1400 & 52 & 6.8 & 17.0 & 1.9 & 6.9 & 21 & 5.9 & 1.6 & .2 \\
\hline & & $12 / 19 / 00$ & 1500 & 53 & 6.9 & 13.5 & 3.5 & 7.5 & 21 & 5.7 & 1.5 & .2 \\
\hline & & $03 / 27 / 01$ & 1000 & 53 & 7.0 & 7.5 & 1.2 & 9.8 & 21 & 5.8 & 1.6 & .2 \\
\hline & & $05 / 01 / 01$ & 1300 & 51 & 6.9 & 8.5 & 1.5 & 7.8 & 22 & 6.0 & 1.6 & .2 \\
\hline & & $05 / 22 / 01$ & 1300 & 56 & 7.0 & 11.0 & .37 & 8.4 & 23 & 6.2 & 1.7 & .2 \\
\hline & & $08 / 15 / 01$ & 1300 & 64 & 6.7 & 12.5 & .61 & 3.9 & 25 & 6.7 & 2.0 & .2 \\
\hline \multirow[t]{6}{*}{ M20 } & 474016117085601 & $10 / 05 / 00$ & 0800 & 117 & 7.4 & 11.0 & .15 & 7.8 & 56 & 14 & 4.9 & .1 \\
\hline & & $12 / 20 / 00$ & 1000 & 121 & 7.4 & 10.5 & 1.2 & 7.0 & 54 & 14 & 4.7 & .1 \\
\hline & & 03/29/01 & 0800 & 136 & 7.6 & 12.0 & .88 & 7.4 & 64 & 17 & 5.4 & .1 \\
\hline & & $05 / 02 / 01$ & 1600 & 141 & 7.4 & 12.5 & .31 & 7.1 & 68 & 18 & 5.9 & .1 \\
\hline & & $05 / 21 / 01$ & 1300 & 136 & 7.4 & 13.5 & .07 & 7.5 & 64 & 16 & 5.7 & .1 \\
\hline & & $08 / 15 / 01$ & 0600 & 132 & 7.4 & 12.5 & .96 & 7.6 & 61 & 15 & 5.4 & .1 \\
\hline \multirow[t]{6}{*}{ M21 } & 474037117091301 & $10 / 05 / 00$ & 1200 & 52 & 6.6 & 17.0 & .69 & 4.2 & 22 & 6.0 & 1.6 & .2 \\
\hline & & $12 / 20 / 00$ & 1400 & 49 & 6.7 & 13.0 & .91 & 7.6 & 20 & 5.7 & 1.5 & .2 \\
\hline & & $03 / 29 / 01$ & 0900 & 54 & 7.0 & 8.0 & .39 & 9.0 & 22 & 5.9 & 1.6 & .2 \\
\hline & & $05 / 03 / 01$ & 1200 & 51 & 6.8 & 8.0 & .35 & 9.5 & 23 & 6.1 & 1.8 & .2 \\
\hline & & $05 / 21 / 01$ & 1600 & 52 & 6.9 & 9.5 & .19 & 8.2 & 22 & 6.0 & 1.7 & .2 \\
\hline & & $08 / 15 / 01$ & 0700 & 64 & 6.8 & 14.0 & .12 & 1.7 & 25 & 6.9 & 1.9 & .2 \\
\hline
\end{tabular}

148 Water-Quality, Streambed-Sediment, and Biological Data from the Clark Fork-Pend Oreille and Spokane River Basins, Montana, Idaho, and Washington, 1998-2001 
Table 24. Physical and major-ion concentration data for ground-water samples collected for the surface-water/ground-water interaction study of the Spokane River and surrounding basin-fill aquifers, Idaho and Washington, 2000-01 (Continued)

\begin{tabular}{|c|c|c|c|c|c|c|c|c|c|c|c|c|c|}
\hline $\begin{array}{c}\text { Sodium } \\
(\mathrm{mg} / \mathrm{L})\end{array}$ & $\begin{array}{c}\text { Potas- } \\
\text { sium } \\
(\mathrm{mg} / \mathrm{L})\end{array}$ & $\begin{array}{c}\text { Bicar- } \\
\text { bonate } \\
(\mathrm{mg} / \mathrm{L} \\
\text { as } \\
\left.\mathrm{HCO}_{3}\right)\end{array}$ & $\begin{array}{c}\text { Carbo- } \\
\text { nate } \\
(\mathrm{mg} / \mathrm{L} \\
\left.\text { as } \mathrm{CO}_{3}\right) \\
\end{array}$ & $\begin{array}{c}\text { Alka- } \\
\text { linity, } \\
\text { field } \\
(\mathrm{mg} / \mathrm{L} \\
\text { as } \\
\left.\mathrm{CaCO}_{3}\right)\end{array}$ & $\begin{array}{c}\text { Alka- } \\
\text { linity, } \\
\text { lab } \\
(\mathrm{mg} / \mathrm{L} \\
\mathrm{as} \\
\left.\mathrm{CaCO}_{3}\right) \\
\end{array}$ & $\begin{array}{c}\text { Sulfate } \\
(\mathrm{mg} / \mathrm{L} \\
\left.\text { as } \mathrm{SO}_{4}\right)\end{array}$ & $\begin{array}{c}\text { Chlo- } \\
\text { ride } \\
(\mathrm{mg} / \mathrm{L})\end{array}$ & $\begin{array}{c}\text { Fluo- } \\
\text { ride } \\
(\mathrm{mg} / \mathrm{L})\end{array}$ & $\begin{array}{c}\text { Bro- } \\
\text { mide } \\
(\mathrm{mg} / \mathrm{L})\end{array}$ & $\begin{array}{c}\text { Silica } \\
(\mathrm{mg} / \mathrm{L} \\
\left.\text { as } \mathrm{SiO}_{2}\right)\end{array}$ & $\begin{array}{c}\text { Dis- } \\
\text { solved } \\
\text { solids, } \\
\text { sum } \\
(\mathrm{mg} / \mathrm{L})\end{array}$ & $\begin{array}{c}\text { Dis- } \\
\text { solved } \\
\text { solids, } \\
\text { residue } \\
\text { at } 180{ }^{\circ} \mathrm{C} \\
(\mathrm{mg} / \mathrm{L})\end{array}$ & $\begin{array}{c}\text { Well } \\
\text { number } \\
\text { (fig. 5) }\end{array}$ \\
\hline 1.9 & .77 & 24 & 0 & 19 & 21 & 4.0 & .74 & $<.16$ & $<.01$ & 8.3 & 35 & 38 & M14 \\
\hline 1.7 & .54 & 24 & 0 & -- & 20 & 4.5 & 1.2 & $<.16$ & $<.01$ & 7.6 & 35 & 36 & \\
\hline 2.1 & .64 & 28 & 0 & -- & 23 & 4.8 & 1.2 & $<.16$ & $<.01$ & 8.3 & 39 & 48 & \\
\hline 1.9 & .65 & 28 & 0 & -- & 23 & 5.2 & .90 & $<.16$ & $<.01$ & 9.1 & 40 & 47 & \\
\hline 2.1 & .70 & 29 & 0 & -- & 24 & 5.0 & .89 & $<.2$ & $<.01$ & 9.7 & 42 & 39 & \\
\hline 2.4 & 1.0 & 28 & 0 & -- & 23 & 5.3 & 1.6 & $<.2$ & $<.01$ & 10 & 43 & 41 & \\
\hline 2.7 & 2.2 & 171 & 0 & 140 & -- & 14 & 1.7 & $<.10$ & .02 & 12 & 168 & 179 & M15 \\
\hline 3.0 & 2.2 & 176 & 0 & 144 & 147 & 14 & 1.8 & $<.16$ & .02 & 12 & 172 & 179 & \\
\hline 2.8 & 2.1 & 181 & 0 & -- & 148 & 15 & 2.1 & $<.16$ & .03 & 11 & 174 & 176 & \\
\hline 2.9 & 2.1 & 183 & 0 & -- & 150 & 15 & 1.9 & $<.16$ & .02 & 12 & 175 & 185 & \\
\hline 2.8 & 2.3 & 183 & 0 & -- & 150 & 15 & 1.7 & $<.16$ & .02 & 12 & 178 & 178 & \\
\hline 3.1 & 2.2 & 185 & -- & -- & 152 & 15 & 1.7 & $<.2$ & .02 & 12 & 178 & 176 & \\
\hline 2.8 & 2.0 & 181 & -- & -- & 148 & 14 & 1.8 & $<.2$ & $<.01$ & 12 & 173 & 176 & \\
\hline 2.9 & 2.1 & 184 & 0 & 154 & -- & 15 & 1.6 & $<.10$ & .04 & 11 & 177 & 190 & M16 \\
\hline 3.2 & 2.2 & 191 & 0 & 157 & 156 & 16 & 2.0 & $<.16$ & .01 & 11 & 184 & 191 & \\
\hline 3.0 & 2.0 & 192 & -- & -- & 157 & 16 & 2.0 & $<.16$ & .02 & 11 & 183 & 190 & \\
\hline 2.9 & 2.0 & 192 & -- & -- & 157 & 16 & 1.4 & $<.16$ & $<.01$ & 11 & 181 & 184 & \\
\hline 3.1 & 1.9 & 192 & -- & -- & 157 & 15 & 1.4 & $<.2$ & .02 & 11 & 182 & 180 & \\
\hline 3.1 & 2.1 & 188 & -- & -- & 154 & 15 & 1.6 & $<.2$ & $<.01$ & 11 & 181 & 179 & \\
\hline 1.9 & .94 & 49 & 0 & 40 & -- & 4.4 & 1.1 & $<.10$ & $<.01$ & 9.9 & 58 & 64 & M17 \\
\hline 1.8 & 1.0 & 46 & 0 & 38 & 41 & 4.9 & .92 & $<.16$ & $<.01$ & 10 & 56 & 59 & \\
\hline 1.8 & .92 & 49 & -- & -- & 40 & 4.4 & 1.2 & $<.16$ & $<.01$ & 10 & 57 & 56 & \\
\hline 1.8 & 1.1 & 50 & -- & -- & 41 & 4.6 & 1.0 & $<.16$ & $<.01$ & 10 & 58 & 68 & \\
\hline 1.7 & .90 & 50 & -- & -- & 41 & 4.5 & .95 & $<.16$ & $<.01$ & 10 & 57 & 63 & \\
\hline 1.8 & .97 & 50 & -- & -- & 41 & 4.2 & 1.0 & $<.2$ & $<.01$ & 11 & 58 & 50 & \\
\hline 1.8 & .94 & 48 & -- & -- & 39 & 4.5 & 1.1 & $<.2$ & $<.01$ & 11 & 57 & 53 & \\
\hline 1.7 & .68 & 27 & 0 & 22 & -- & 3.4 & .83 & $<.10$ & $<.01$ & 11 & 39 & 45 & M18 \\
\hline 2.0 & .93 & 28 & 0 & 23 & 25 & 4.0 & .86 & $<.16$ & $<.01$ & 12 & 42 & 45 & \\
\hline 1.7 & .76 & 28 & 0 & -- & 23 & 3.9 & .98 & $<.16$ & $<.01$ & 10 & 39 & 41 & \\
\hline 1.8 & .81 & 32 & 0 & -- & 26 & 4.2 & 1.2 & $<.16$ & $<.01$ & 10 & 44 & 50 & \\
\hline 1.7 & .73 & 31 & 0 & -- & 25 & 4.5 & 1.6 & $<.16$ & $<.01$ & 9.5 & 42 & 44 & \\
\hline 1.8 & .81 & 31 & 0 & -- & 25 & 5.3 & 1.3 & $<.2$ & .01 & 10 & 44 & 48 & \\
\hline 1.9 & .82 & 34 & 0 & -- & 28 & 4.8 & 1.1 & $<.2$ & $<.01$ & 11 & 46 & 44 & \\
\hline 2.0 & .79 & 26 & 0 & 21 & -- & 3.6 & .84 & $<.10$ & $<.01$ & 11 & 39 & 46 & M19 \\
\hline 2.3 & .93 & 25 & 0 & 21 & 23 & 4.0 & .77 & $<.16$ & $<.01$ & 11 & 39 & 43 & \\
\hline 1.7 & .79 & 26 & 0 & -- & 21 & 4.0 & 1.1 & $<.16$ & .01 & 9.3 & 37 & 40 & \\
\hline 1.8 & .65 & 26 & 0 & -- & 21 & 4.6 & 1.1 & $<.16$ & $<.01$ & 8.5 & 36 & 45 & \\
\hline 1.9 & .69 & 27 & 0 & -- & 22 & 5.3 & .82 & $<.16$ & $<.01$ & 9.3 & 39 & 41 & \\
\hline 1.8 & .69 & 27 & 0 & -- & 22 & 5.0 & .88 & $<.2$ & $<.01$ & 9.5 & 39 & 38 & \\
\hline 2.2 & .85 & 29 & 0 & -- & 24 & 5.0 & 1.3 & $<.2$ & $<.01$ & 10 & 43 & 43 & \\
\hline 2.1 & 1.2 & 61 & 0 & 50 & 53 & 5.8 & 1.1 & $<.16$ & $<.01$ & 11 & 70 & 76 & M20 \\
\hline 2.0 & 1.1 & 65 & 0 & -- & 53 & 6.0 & 1.4 & $<.16$ & .02 & 11 & 71 & 75 & \\
\hline 2.2 & 1.3 & 74 & 0 & -- & 61 & 6.9 & 1.6 & $<.16$ & .02 & 11 & 81 & 84 & \\
\hline 2.5 & 1.2 & 74 & 0 & -- & 61 & 7.1 & 1.8 & $<.16$ & .01 & 11 & 84 & 92 & \\
\hline 2.6 & 1.3 & 73 & 0 & -- & 60 & 6.6 & 1.6 & $<.2$ & .01 & 11 & 82 & 74 & \\
\hline 2.2 & 1.2 & 70 & 0 & -- & 57 & 6.4 & 1.6 & $<.2$ & $<.01$ & 11 & 78 & 84 & \\
\hline 2.2 & .92 & 26 & 0 & 22 & 23 & 4.1 & .84 & $<.16$ & $<.01$ & 11 & 39 & 41 & M21 \\
\hline 1.7 & .77 & 24 & 0 & -- & 20 & 4.0 & 1.1 & $<.16$ & $<.01$ & 9.3 & 36 & 41 & \\
\hline 1.6 & .61 & 26 & 0 & -- & 21 & 4.6 & 1.3 & $<.16$ & $<.01$ & 7.7 & 35 & 39 & \\
\hline 1.8 & .59 & 26 & 0 & -- & 21 & 5.2 & .91 & $<.16$ & $<.01$ & 8.9 & 38 & 42 & \\
\hline 1.9 & .56 & 26 & 0 & -- & 21 & 5.1 & .93 & $<.2$ & $<.01$ & 8.8 & 38 & 30 & \\
\hline 2.3 & .92 & 29 & 0 & -- & 24 & 4.8 & 1.3 & $<.2$ & $<.01$ & 11 & 43 & 44 & \\
\hline
\end{tabular}


Table 24. Physical and major-ion concentration data for ground-water samples collected for the surface-water/ground-water interaction study of the Spokane River and the surrounding basin-fill aquifers, Idaho and Washington, 2000-01 (Continued)

\begin{tabular}{|c|c|c|c|c|c|c|c|c|c|c|c|c|}
\hline $\begin{array}{c}\text { Well } \\
\text { number } \\
\text { (fig. 5) }\end{array}$ & $\begin{array}{c}\text { Site } \\
\text { identification } \\
\text { number }\end{array}$ & Date & Time & $\begin{array}{c}\text { Spe- } \\
\text { cific } \\
\text { conduc- } \\
\text { tance, } \\
\text { field } \\
(\mu \mathrm{S} / \mathrm{cm})\end{array}$ & $\begin{array}{c}\text { pH, } \\
\text { field } \\
\text { (stan- } \\
\text { dard } \\
\text { units) }\end{array}$ & $\begin{array}{l}\text { Temper- } \\
\text { ature, } \\
\text { water } \\
\left({ }^{0} \mathrm{C}\right)\end{array}$ & $\begin{array}{c}\text { Tur- } \\
\text { bidity, } \\
\text { field } \\
\text { (NTU) }\end{array}$ & $\begin{array}{c}\text { Oxy- } \\
\text { gen } \\
(\mathrm{mg} / \mathrm{L})\end{array}$ & $\begin{array}{c}\text { Hard- } \\
\text { ness } \\
(\mathrm{mg} / \mathrm{L} \\
\text { as } \\
\mathrm{CaCO}_{3}\end{array}$ & $\begin{array}{c}\text { Cal- } \\
\text { cium } \\
(\mathrm{mg} / \mathrm{L})\end{array}$ & $\begin{array}{l}\text { Magne- } \\
\text { sium } \\
(\mathrm{mg} / \mathrm{L})\end{array}$ & $\begin{array}{c}\text { Sodium } \\
\text { adsorp- } \\
\text { tion ratio }\end{array}$ \\
\hline \multirow[t]{8}{*}{ M22 } & 474038117091201 & $10 / 05 / 00$ & 1300 & 52 & 6.9 & 17.5 & .73 & 3.1 & 21 & 5.9 & 1.6 & .2 \\
\hline & & $10 / 05 / 00^{\mathbf{R}}$ & 1301 & -- & -- & -- & -- & -- & 22 & 5.9 & 1.6 & .2 \\
\hline & & $12 / 20 / 00$ & 1500 & 50 & 6.6 & 13.5 & .99 & 5.2 & 21 & 5.8 & 1.6 & .2 \\
\hline & & $12 / 20 / 00^{\mathbf{R}}$ & 1501 & - & -- & -- & -- & -- & 21 & 5.8 & 1.6 & .2 \\
\hline & & $03 / 29 / 01$ & 1000 & 54 & 6.9 & 6.5 & .29 & 7.9 & 21 & 5.8 & 1.6 & .2 \\
\hline & & $05 / 03 / 01$ & 1300 & 52 & 6.7 & 7.0 & .39 & 7.1 & 22 & 5.8 & 1.8 & .2 \\
\hline & & $05 / 21 / 01$ & 1700 & 55 & 6.9 & 8.5 & .17 & 6.5 & 22 & 5.9 & 1.8 & .2 \\
\hline & & $08 / 15 / 01$ & 0800 & 63 & 6.7 & 12.5 & .75 & 1.4 & 25 & 6.8 & 1.9 & .2 \\
\hline \multirow[t]{6}{*}{ M23 } & 474046117091501 & $10 / 05 / 00$ & 1100 & 48 & 6.4 & 16.5 & .77 & 5.9 & 20 & 5.6 & 1.5 & .2 \\
\hline & & $12 / 20 / 00$ & 1300 & 50 & 6.8 & 7.5 & 4.2 & 11 & 21 & 5.8 & 1.7 & .2 \\
\hline & & $03 / 29 / 01$ & 1100 & 62 & 6.8 & 6.0 & .47 & 11 & 24 & 6.6 & 1.9 & .2 \\
\hline & & 05/03/01 & 1100 & 61 & 6.8 & 7.5 & .76 & 10 & 25 & 6.7 & 2.0 & .2 \\
\hline & & $05 / 21 / 01$ & 1500 & 55 & 6.9 & 10.0 & .27 & 7.9 & 23 & 6.2 & 1.8 & .2 \\
\hline & & $08 / 15 / 01$ & 1000 & 66 & 6.6 & 20.5 & .27 & 1.8 & 24 & 6.5 & 1.9 & .2 \\
\hline \multirow[t]{7}{*}{ M24 } & 474109117091701 & $10 / 05 / 00$ & 1000 & 353 & 7.7 & 9.0 & .06 & 8.7 & 187 & 44 & 19 & .1 \\
\hline & & $12 / 20 / 00$ & 1200 & 361 & 7.7 & 8.5 & .46 & 9.1 & 180 & 42 & 18 & .1 \\
\hline & & $03 / 29 / 01$ & 1200 & 340 & 7.7 & 9.0 & .04 & 9.0 & 170 & 40 & 17 & .1 \\
\hline & & $03 / 29 / 01^{\mathbf{R}}$ & 1201 & -- & -- & -- & -- & -- & 169 & 40 & 17 & .1 \\
\hline & & $05 / 03 / 01$ & 1000 & 332 & 7.6 & 9.5 & .09 & 8.9 & 177 & 42 & 17 & .1 \\
\hline & & $05 / 21 / 01$ & 1400 & 338 & 7.7 & 10.0 & .07 & 8.8 & 179 & 42 & 18 & .1 \\
\hline & & $08 / 15 / 01$ & 0900 & 352 & 7.4 & 9.5 & .07 & 8.6 & 180 & 42 & 18 & .1 \\
\hline \multirow[t]{6}{*}{ M25 } & 474026117115301 & $10 / 04 / 00$ & 1700 & 271 & 7.8 & 10.5 & .07 & 9.0 & 142 & 32 & 15 & .1 \\
\hline & & $12 / 20 / 00$ & 0900 & 287 & 7.9 & 9.0 & .09 & 9.4 & 140 & 32 & 14 & .1 \\
\hline & & $03 / 29 / 01$ & 1400 & 285 & 8.0 & 8.5 & .12 & 9.6 & 142 & 33 & 15 & .1 \\
\hline & & $05 / 02 / 01$ & 1700 & 74 & 8.2 & 9.0 & .27 & 11 & 36 & 8.3 & 3.7 & .1 \\
\hline & & $05 / 21 / 01$ & 1900 & 229 & 7.9 & 11.0 & .16 & 9.4 & 115 & 26 & 12 & .1 \\
\hline & & $08 / 14 / 01$ & 1500 & 247 & 7.8 & 12.5 & .06 & 8.4 & 119 & 27 & 12 & .1 \\
\hline
\end{tabular}

${ }^{1}$ Sample filtered through $0.10-\mu \mathrm{m}$ capsule filter. 
Table 24. Physical and major-ion concentration data for ground-water samples collected for the surface-water/ground-water interaction study of the Spokane River and surrounding basin-fill aquifers, Idaho and Washington, 2000-01 (Continued)

\begin{tabular}{|c|c|c|c|c|c|c|c|c|c|c|c|c|c|}
\hline $\begin{array}{c}\text { Sodium } \\
(\mathrm{mg} / \mathrm{L})\end{array}$ & $\begin{array}{c}\text { Potas- } \\
\text { sium } \\
(\mathrm{mg} / \mathrm{L})\end{array}$ & $\begin{array}{c}\text { Bicar- } \\
\text { bonate } \\
(\mathrm{mg} / \mathrm{L} \\
\text { as } \\
\left.\mathrm{HCO}_{3}\right)\end{array}$ & $\begin{array}{c}\text { Carbo- } \\
\text { nate } \\
(\mathrm{mg} / \mathrm{L} \\
\left.\text { as } \mathrm{CO}_{3}\right)\end{array}$ & $\begin{array}{c}\text { Alka- } \\
\text { linity, } \\
\text { field } \\
(\mathrm{mg} / \mathrm{L} \\
\text { as } \\
\left.\mathrm{CaCO}_{3}\right)\end{array}$ & $\begin{array}{c}\text { Alka- } \\
\text { linity, } \\
\text { lab } \\
(\mathrm{mg} / \mathrm{L} \\
\text { as } \\
\left.\mathrm{CaCO}_{3}\right)\end{array}$ & $\begin{array}{c}\text { Sulfate } \\
(\mathrm{mg} / \mathrm{L} \\
\left.\text { as } \mathrm{SO}_{4}\right)\end{array}$ & $\begin{array}{c}\text { Chlo- } \\
\text { ride } \\
(\mathrm{mg} / \mathrm{L})\end{array}$ & $\begin{array}{c}\text { Fluo- } \\
\text { ride } \\
(\mathrm{mg} / \mathrm{L})\end{array}$ & $\begin{array}{c}\text { Bro- } \\
\text { mide } \\
(\mathrm{mg} / \mathrm{L})\end{array}$ & $\begin{array}{c}\text { Silica } \\
(\mathrm{mg} / \mathrm{L} \\
\left.\text { as } \mathrm{SiO}_{2}\right)\end{array}$ & $\begin{array}{c}\text { Dis- } \\
\text { solved } \\
\text { solids, } \\
\text { sum } \\
(\mathrm{mg} / \mathrm{L})\end{array}$ & $\begin{array}{c}\text { Dis- } \\
\text { solved } \\
\text { solids, } \\
\text { residue } \\
\text { at } 180^{\circ} \mathrm{C} \\
(\mathrm{mg} / \mathrm{L})\end{array}$ & $\begin{array}{c}\text { Well } \\
\text { number } \\
\text { (fig. 5) }\end{array}$ \\
\hline 2.1 & .92 & 24 & 0 & 20 & 22 & 4.0 & .87 & $<.16$ & $<.01$ & 10 & 38 & 40 & M22 \\
\hline 2.0 & .87 & 27 & 0 & -- & 22 & 4.0 & .96 & $<.16$ & $<.01$ & 10 & 39 & 37 & \\
\hline 1.8 & .78 & 26 & 0 & -- & 21 & 4.0 & 1.1 & $<.16$ & $<.01$ & 9.5 & 37 & 39 & \\
\hline 1.8 & .76 & 26 & 0 & -- & 21 & 4.0 & 1.1 & $<.16$ & $<.01$ & 9.4 & 37 & 39 & \\
\hline 1.7 & .58 & 24 & 0 & -- & 20 & 4.6 & 1.2 & $<.16$ & $<.01$ & 8.1 & 36 & 38 & \\
\hline 1.8 & .66 & 26 & 0 & -- & 21 & 5.4 & .90 & $<.16$ & $<.01$ & 9.0 & 38 & 47 & \\
\hline 1.8 & .70 & 26 & 0 & -- & 21 & 5.0 & .95 & $<.2$ & $<.01$ & 9.0 & 38 & 30 & \\
\hline 2.2 & .80 & 29 & 0 & -- & 24 & 4.8 & 1.4 & $<.2$ & $<.01$ & 10 & 43 & 44 & \\
\hline 1.9 & .84 & 23 & 0 & 18 & 21 & 4.0 & .78 & $<.16$ & $<.01$ & 9.0 & 35 & 38 & M23 \\
\hline 1.7 & .54 & 24 & 0 & -- & 20 & 4.4 & 1.4 & $<.16$ & $<.01$ & 7.9 & 36 & 41 & \\
\hline 1.8 & .56 & 27 & 0 & -- & 22 & 4.8 & 2.4 & $<.16$ & .01 & 8.3 & 40 & 45 & \\
\hline 1.9 & .73 & 28 & 0 & -- & 23 & 5.6 & 1.2 & $<.16$ & $<.01$ & 9.5 & 42 & 38 & \\
\hline 2.1 & .75 & 26 & 0 & -- & 21 & 5.1 & 1.0 & $<.2$ & $<.01$ & 9.5 & 39 & 35 & \\
\hline 2.4 & .99 & 28 & 0 & -- & 23 & 5.0 & 1.6 & $<.2$ & $<.01$ & 10 & 43 & 42 & \\
\hline 3.3 & 2.2 & 210 & 0 & 172 & 177 & 16 & 1.5 & $<.16$ & $<.01$ & 12 & 200 & 210 & M24 \\
\hline 3.1 & 2.1 & 212 & 0 & -- & 174 & 16 & 2.0 & $<.16$ & .02 & 11 & 199 & 207 & \\
\hline 2.9 & 2.0 & 206 & 0 & -- & 169 & 16 & 1.4 & $<.16$ & .02 & 11 & 191 & 201 & \\
\hline 2.9 & 2.0 & 205 & 0 & -- & 168 & 16 & 1.4 & $<.16$ & .02 & 11 & 191 & 201 & \\
\hline 3.1 & 2.0 & 206 & 0 & -- & 169 & 15 & 1.3 & $<.16$ & .02 & 11 & 194 & 205 & \\
\hline 3.3 & 2.1 & 206 & 0 & -- & 169 & 15 & 1.3 & $<.2$ & $<.02$ & 12 & 195 & 186 & \\
\hline 3.1 & 2.1 & 210 & 0 & -- & 172 & 15 & 1.5 & $<.2$ & .01 & 12 & 197 & 208 & \\
\hline 3.1 & 1.9 & 160 & 0 & 131 & 132 & 14 & 1.6 & $<.16$ & .01 & 12 & 157 & 162 & M25 \\
\hline 2.8 & 1.6 & 162 & 0 & -- & 133 & 14 & 1.9 & $<.16$ & .02 & 11 & 158 & 163 & \\
\hline 2.7 & 1.7 & 168 & 0 & -- & 138 & 14 & 1.8 & $<.16$ & .02 & 10 & 161 & 171 & \\
\hline 1.4 & .80 & 39 & 0 & -- & 32 & 5.7 & 1.3 & $<.16$ & $<.01$ & 9.5 & 50 & 55 & \\
\hline 2.8 & 1.7 & 134 & 0 & -- & 110 & 11 & 2.0 & $<.2$ & .01 & 12 & 134 & 135 & \\
\hline 2.7 & 1.8 & 139 & 0 & -- & 114 & 11 & 1.4 & $<.2$ & .01 & 12 & 137 & 143 & \\
\hline
\end{tabular}


Table 25. Trace-element concentration data for ground-water samples collected for the surface-water/ground-water interaction study of the Spokane River and surrounding basin-fill aquifers, Idaho and Washington, 2000-01

[Site identification number described in text. Constituents are reported as dissolved ( $0.45 \mu \mathrm{m}$ filtration), unless otherwise indicated. Abbreviations: e, estimated; Fb, field blank; $\mu \mathrm{g} /$ L, microgram per liter; $\mu \mathrm{m}$, micrometer; R, replicate. Symbols: <, less than reporting level; --, no data]

\begin{tabular}{|c|c|c|c|c|c|c|c|c|c|}
\hline $\begin{array}{c}\text { Well } \\
\text { number } \\
\text { (fig. 5) }\end{array}$ & $\begin{array}{c}\text { Site } \\
\text { identification } \\
\text { number }\end{array}$ & Date & Time & $\begin{array}{l}\text { Alumi- } \\
\text { num, } \\
\text { dis- } \\
\text { solved } \\
(\mu \mathrm{g} / \mathrm{L})\end{array}$ & $\begin{array}{c}\text { Anti- } \\
\text { mony, } \\
\text { dis- } \\
\text { solved } \\
(\mu \mathrm{g} / \mathrm{L})\end{array}$ & $\begin{array}{c}\text { Arsenic, } \\
\text { total } \\
\text { recov- } \\
\text { erable } \\
(\mu \mathrm{g} / \mathrm{L})\end{array}$ & $\begin{array}{c}\text { Arsenic, } \\
\text { dis- } \\
\text { solved } \\
(\mu \mathrm{g} / \mathrm{L})\end{array}$ & $\begin{array}{c}\text { Barium, } \\
\text { dis- } \\
\text { solved } \\
(\mu \mathrm{g} / \mathrm{L})\end{array}$ & $\begin{array}{l}\text { Beryl- } \\
\text { lium, } \\
\text { dis- } \\
\text { solved } \\
(\mu \mathrm{g} / \mathrm{L})\end{array}$ \\
\hline \multirow[t]{8}{*}{ M1 } & 474134117002201 & $08 / 08 / 00$ & 0800 & 5.7 & $<1.0$ & -- & $<0.90$ & 26 & $<1.0$ \\
\hline & & $09 / 27 / 00$ & 1300 & 3.7 & $<1.0$ & -- & $<.90$ & 10 & $<1.0$ \\
\hline & & $12 / 15 / 00$ & 0800 & 3.5 & .05 & -- & e. 17 & 17 & $<.06$ \\
\hline & & $03 / 28 / 01$ & 1400 & $<1.0$ & .17 & -- & e. 12 & 8.2 & $<.06$ \\
\hline & & $05 / 03 / 01$ & 0900 & $<1.0$ & .34 & -- & e.09 & 8.6 & $<.06$ \\
\hline & & $05 / 23 / 01$ & 0800 & $<1.0$ & .21 & -- & e. 10 & 14 & $<.06$ \\
\hline & & $08 / 14 / 01$ & 1200 & 6.4 & .20 & $<2.0$ & e. 13 & 17 & $<.06$ \\
\hline & & 08/14/01 ${ }^{\mathbf{F b}}$ & 1211 & $<1.0$ & $<.05$ & $<2.0$ & $<.20$ & $<1.0$ & $<.06$ \\
\hline \multirow[t]{10}{*}{ M2 } & 474134117002202 & $09 / 27 / 00$ & 1400 & $<1.0$ & $<1.0$ & -- & $<.90$ & 12 & $<1.0$ \\
\hline & & $12 / 15 / 00$ & 0900 & 5.0 & .40 & -- & .36 & 12 & $<.06$ \\
\hline & & $12 / 15 / 00^{\mathbf{R}}$ & 0901 & $<1.0$ & .43 & -- & .41 & 12 & $<.06$ \\
\hline & & $12 / 15 / 00^{\mathbf{F b}}$ & 0911 & $<.30$ & $<.20$ & -- & -- & $<.2$ & $<.20$ \\
\hline & & $03 / 28 / 01$ & 1300 & $<1.0$ & .45 & -- & .31 & 12 & $<.06$ \\
\hline & & $04 / 30 / 01$ & 1700 & 6.0 & .56 & -- & .32 & 14 & $<.06$ \\
\hline & & 05/03/01 & 0800 & 4.3 & .51 & -- & .27 & 13 & $<.06$ \\
\hline & & 05/03/01 & 1600 & $<1.0$ & .52 & -- & .30 & 13 & $<.06$ \\
\hline & & $05 / 23 / 01$ & 0700 & 5.0 & .51 & -- & .38 & 16 & $<.06$ \\
\hline & & 08/14/01 & 1100 & 4.6 & .70 & $<2.0$ & .48 & 18 & $<.06$ \\
\hline \multirow[t]{5}{*}{ M3 } & 474226117024801 & $10 / 04 / 00$ & 1500 & 5.7 & .17 & -- & 4.4 & 20 & $<.06$ \\
\hline & & $12 / 20 / 00$ & 0800 & 5.2 & .24 & -- & 4.3 & 21 & $<.06$ \\
\hline & & $05 / 01 / 01$ & 1700 & 5.7 & .20 & -- & 4.1 & 19 & $<.06$ \\
\hline & & $05 / 23 / 01$ & 1900 & 1.3 & .11 & -- & 4.4 & 23 & $<.06$ \\
\hline & & $08 / 14 / 01$ & 1400 & $<1.0$ & .19 & -- & 4.0 & 17 & $<.06$ \\
\hline \multirow[t]{8}{*}{ M4 } & 474151117031101 & $06 / 19 / 00$ & 1800 & 1.4 & $<1.0$ & -- & 1.3 & 9.6 & $<1.0$ \\
\hline & & $08 / 07 / 00$ & 1300 & $<1.0$ & $<1.0$ & $<2.6$ & e. 52 & 16 & $<1.0$ \\
\hline & & $09 / 25 / 00$ & 1600 & $<1.0$ & $<1.0$ & -- & $<.90$ & 13 & $<1.0$ \\
\hline & & $12 / 14 / 00$ & 1300 & $<1.0$ & .40 & -- & .28 & 13 & $<.06$ \\
\hline & & $03 / 26 / 01$ & 1300 & $<1.0$ & .43 & -- & .32 & 14 & $<.06$ \\
\hline & & $04 / 30 / 01$ & 1300 & 5.4 & .58 & -- & .42 & 14 & $<.06$ \\
\hline & & $05 / 22 / 01$ & 0900 & $<1.0$ & .53 & -- & .38 & 16 & $<.06$ \\
\hline & & 08/13/01 & 1900 & 5.7 & .66 & $<2.0$ & .42 & 17 & $<.06$ \\
\hline \multirow[t]{7}{*}{ M5 } & 474151117031102 & $06 / 19 / 00$ & 1545 & 2.0 & $<1.0$ & -- & e. 83 & 1.6 & $<1.0$ \\
\hline & & $08 / 07 / 00$ & 1500 & 3.9 & $<1.0$ & $<2.6$ & $<.90$ & 17 & $<1.0$ \\
\hline & & $09 / 26 / 00$ & 1300 & $<1.0$ & $<1.0$ & -- & $<.90$ & 14 & $<1.0$ \\
\hline & & $03 / 26 / 01$ & 1400 & $<1.0$ & .41 & -- & .30 & 14 & $<.06$ \\
\hline & & $04 / 30 / 01$ & 1200 & 5.1 & .58 & -- & .37 & 14 & $<.06$ \\
\hline & & $05 / 22 / 01$ & 0800 & 3.6 & .49 & -- & .36 & 17 & $<.06$ \\
\hline & & 08/13/01 & 1800 & 5.4 & .64 & $<2.0$ & .37 & 18 & $<.06$ \\
\hline
\end{tabular}

152 Water-Quality, Streambed-Sediment, and Biological Data from the Clark Fork-Pend Oreille and Spokane River Basins, Montana, Idaho, and Washington, 1998-2001 
Table 25. Trace-element concentration data for ground-water samples collected for the surface-water/ground-water interaction study of the Spokane River and surrounding basin-fill aquifers, Idaho and Washington, 2000-01 (Continued)

\begin{tabular}{|c|c|c|c|c|c|c|c|c|c|}
\hline $\begin{array}{c}\text { Boron, } \\
\text { dis- } \\
\text { solved } \\
(\mu \mathrm{g} / \mathrm{L})\end{array}$ & $\begin{array}{c}\text { Cadmium, } \\
\text { total } \\
\text { recov- } \\
\text { erable } \\
(\mu \mathrm{g} / \mathrm{L})\end{array}$ & $\begin{array}{c}\text { Cad- } \\
\text { mium, } \\
\text { dis- } \\
\text { solved } \\
(\mu \mathrm{g} / \mathrm{L})\end{array}$ & $\begin{array}{c}\text { Chro- } \\
\text { mium, } \\
\text { dis- } \\
\text { solved } \\
(\mu \mathrm{g} / \mathrm{L})\end{array}$ & $\begin{array}{c}\text { Cobalt, } \\
\text { dis- } \\
\text { solved } \\
(\mu \mathrm{g} / \mathrm{L})\end{array}$ & $\begin{array}{c}\text { Copper, } \\
\text { dis- } \\
\text { solved } \\
(\mu \mathrm{g} / \mathrm{L})\end{array}$ & $\begin{array}{l}\text { Iron, } \\
\text { total } \\
\text { recov- } \\
\text { erable } \\
(\mu \mathrm{g} / \mathrm{L})\end{array}$ & $\begin{array}{c}\text { Iron, } \\
\text { dis- } \\
\text { solved } \\
(\mu \mathrm{g} / \mathrm{L})\end{array}$ & $\begin{array}{c}\text { Lead, } \\
\text { total } \\
\text { recov- } \\
\text { erable } \\
(\mu \mathrm{g} / \mathrm{L})\end{array}$ & $\begin{array}{c}\text { Well } \\
\text { number } \\
\text { (fig. 5) }\end{array}$ \\
\hline 16 & -- & $<1.0$ & $<0.80$ & $<1.0$ & $<1.0$ & -- & $<10$ & -- & M1 \\
\hline 13 & -- & $<1.0$ & e. 55 & $<1.0$ & $<1.0$ & -- & $<10$ & -- & \\
\hline 9.1 & -- & $<.04$ & $<.80$ & .03 & 1.4 & -- & $<10$ & -- & \\
\hline$<7.0$ & -- & $<.04$ & $<.80$ & .02 & .30 & -- & $<10$ & -- & \\
\hline$<7.0$ & -- & $<.04$ & $<.80$ & e. 01 & .30 & -- & $<10$ & -- & \\
\hline$<7.0$ & -- & $<.04$ & e. 50 & e.01 & .30 & -- & $<10$ & -- & \\
\hline 14 & $<.04$ & $<.04$ & .86 & .04 & $<.20$ & 163 & $<10$ & $<1.0$ & \\
\hline$<7.0$ & $<.04$ & e. 02 & $<.80$ & .03 & $<.20$ & $\mathrm{e} 10$ & $<10$ & $<1.0$ & \\
\hline$<12$ & -- & $<1.0$ & $<.80$ & $<1.0$ & $<1.0$ & -- & $<10$ & -- & M2 \\
\hline 7.2 & -- & .35 & $<.80$ & .02 & 1.2 & -- & $<10$ & -- & \\
\hline$<7.0$ & -- & .34 & $<.80$ & .02 & .69 & -- & $<10$ & -- & \\
\hline$<2.0$ & -- & $<.30$ & $<.20$ & $<.20$ & $<.20$ & & $<3.0$ & & \\
\hline$<7.0$ & -- & .32 & $<.80$ & .02 & .74 & -- & $<10$ & -- & \\
\hline 17 & -- & .36 & $<.80$ & .02 & .97 & -- & $<10$ & -- & \\
\hline 10 & -- & .36 & 1.1 & .02 & .59 & -- & e8.1 & -- & \\
\hline$<7.0$ & -- & .37 & e. 43 & e. 01 & .62 & -- & $<10$ & -- & \\
\hline 12 & -- & .40 & .85 & .02 & .70 & -- & $<10$ & -- & \\
\hline 14 & .70 & .68 & e. 67 & .02 & .48 & 69 & $<10$ & 2.6 & \\
\hline 11 & -- & e. 03 & 2.4 & .13 & .37 & -- & 10 & -- & M3 \\
\hline 15 & -- & $<.04$ & e. 47 & .06 & .29 & -- & $<10$ & -- & \\
\hline 17 & -- & $<.04$ & 1.5 & .05 & .29 & -- & $<10$ & -- & \\
\hline e5.6 & -- & $<.04$ & 2.1 & .06 & .31 & -- & e5.6 & -- & \\
\hline$<7.0$ & -- & $<.04$ & e. 59 & .04 & e. 23 & -- & e6.0 & -- & \\
\hline 25 & -- & $<1.0$ & $<.80$ & $<1.0$ & 4.7 & -- & $<10$ & -- & M4 \\
\hline$<12$ & $<1.0$ & $<1.0$ & $<.80$ & $<1.0$ & $<1.0$ & $<21$ & $<10$ & $<1.0$ & \\
\hline$<12$ & -- & $<1.0$ & 1.0 & $<1.0$ & $<1.0$ & -- & e5.2 & -- & \\
\hline$<7.0$ & -- & .30 & $<.80$ & .02 & 1.1 & -- & $<10$ & -- & \\
\hline$<7.0$ & -- & .33 & $<.80$ & .02 & .78 & -- & $<10$ & -- & \\
\hline 14 & -- & .33 & e. 54 & .02 & .78 & -- & $<10$ & -- & \\
\hline$<7.0$ & -- & .35 & e. 70 & .02 & .66 & -- & $<10$ & -- & \\
\hline 12 & .45 & .42 & .83 & .03 & .65 & 31 & $<10$ & $<1.0$ & \\
\hline e9.9 & -- & $<1.0$ & $<.80$ & $<1.0$ & 7.3 & -- & $<10$ & -- & M5 \\
\hline 12 & $<1.0$ & $<1.0$ & $<.80$ & $<1.0$ & $<1.0$ & 135 & $<10$ & $<1.0$ & \\
\hline$<12$ & -- & $<1.0$ & e. 72 & $<1.0$ & $<1.0$ & -- & $<10$ & -- & \\
\hline$<7.0$ & -- & .26 & $<.80$ & .02 & .73 & -- & $<10$ & -- & \\
\hline 11 & -- & .27 & $<.80$ & .02 & .72 & -- & $<10$ & -- & \\
\hline 10 & -- & .31 & e. 71 & e. 01 & .77 & -- & $<10$ & -- & \\
\hline 11 & .33 & .28 & $<.80$ & .03 & .53 & 393 & $<10$ & 1.5 & \\
\hline
\end{tabular}


Table 25. Trace-element concentration data for ground-water samples collected for the surface-water/ground-water interaction study of the Spokane River and surrounding basin-fill aquifers, Idaho and Washington, 2000-01 (Continued)

\begin{tabular}{|c|c|c|c|c|c|c|c|c|}
\hline $\begin{array}{c}\text { Well } \\
\text { number } \\
\text { (fig. 5) }\end{array}$ & Date & Time & $\begin{array}{c}\text { Lead, } \\
\text { dis- } \\
\text { solved } \\
(\mu \mathrm{g} / \mathrm{L})\end{array}$ & $\begin{array}{c}\text { Lithium, } \\
\text { dis- } \\
\text { solved } \\
(\mu \mathrm{g} / \mathrm{L})\end{array}$ & $\begin{array}{c}\text { Manganese, } \\
\text { total } \\
\text { recov- } \\
\text { erable } \\
(\mu \mathrm{g} / \mathrm{L})\end{array}$ & $\begin{array}{c}\text { Manganese, } \\
\text { dis- } \\
\text { solved } \\
(\mu \mathrm{g} / \mathrm{L})\end{array}$ & $\begin{array}{c}\text { Molyb- } \\
\text { denum, } \\
\text { dis- } \\
\text { solved } \\
(\mu \mathrm{g} / \mathrm{L})\end{array}$ & $\begin{array}{c}\text { Nickel, } \\
\text { dis- } \\
\text { solved } \\
(\mu \mathrm{g} / \mathrm{L})\end{array}$ \\
\hline \multirow[t]{8}{*}{ M1 } & $08 / 08 / 00$ & 0800 & $<1.0$ & 2.4 & -- & $<1.0$ & $<1.0$ & $<1.0$ \\
\hline & $09 / 27 / 00$ & 1300 & $<1.0$ & 1.1 & -- & $<1.0$ & $<1.0$ & $<1.0$ \\
\hline & $12 / 15 / 00$ & 0800 & $<.08$ & 1.3 & -- & .10 & e.11 & .34 \\
\hline & 03/28/01 & 1400 & $<.08$ & .75 & -- & e. 07 & e. 12 & .37 \\
\hline & 05/03/01 & 0900 & $<.08$ & .88 & -- & $<.10$ & e. 12 & .14 \\
\hline & 05/23/01 & 0800 & $<.08$ & .79 & -- & .11 & $<.20$ & .23 \\
\hline & 08/14/01 & 1200 & $<.08$ & 1.1 & 6.6 & $<.10$ & .22 & .33 \\
\hline & $08 / 14 / 01^{\mathbf{F b}}$ & 1211 & $<.08$ & $<.3$ & $<1.0$ & e. 08 & $<.20$ & .21 \\
\hline \multirow[t]{10}{*}{ M2 } & 09/27/00 & 1400 & $<1.0$ & .38 & -- & $<1.0$ & $<1.0$ & $<1.0$ \\
\hline & $12 / 15 / 00$ & 0900 & .12 & .51 & -- & .11 & $<.20$ & .49 \\
\hline & $12 / 15 / 00^{\mathbf{R}}$ & 0901 & .10 & e.19 & -- & $<.10$ & $<.20$ & .30 \\
\hline & $12 / 15 / 00^{\mathbf{F b}}$ & 0911 & $<.30$ & -- & -- & $<.10$ & $<.20$ & $<.50$ \\
\hline & $03 / 28 / 01$ & 1300 & .09 & .44 & -- & $<.10$ & $<.20$ & .36 \\
\hline & $04 / 30 / 01$ & 1700 & .09 & .49 & -- & .15 & e. 17 & .55 \\
\hline & 05/03/01 & 0800 & .11 & .58 & -- & .13 & e. 18 & .81 \\
\hline & 05/03/01 & 1600 & .12 & .59 & -- & $<.10$ & $<.20$ & .25 \\
\hline & $05 / 23 / 01$ & 0700 & .12 & .84 & -- & e. 07 & e. 14 & .51 \\
\hline & $08 / 14 / 01$ & 1100 & $<.08$ & .60 & 5.0 & $<.10$ & e. 17 & .60 \\
\hline \multirow[t]{5}{*}{ M3 } & $10 / 04 / 00$ & 1500 & $<.08$ & 3.9 & -- & .27 & 1.0 & 2.0 \\
\hline & $12 / 20 / 00$ & 0800 & $<.08$ & 3.6 & -- & .12 & .98 & .34 \\
\hline & 05/01/01 & 1700 & $<.08$ & 3.1 & -- & .20 & 1.0 & .33 \\
\hline & 05/23/01 & 1900 & $<.08$ & 3.6 & -- & .26 & .86 & 1.0 \\
\hline & 08/14/01 & 1400 & $<.08$ & 3.2 & -- & $<.10$ & .79 & $<.06$ \\
\hline \multirow[t]{8}{*}{ M4 } & 06/19/00 & 1800 & $<1.0$ & .96 & -- & 5.9 & $<1.0$ & $<1.0$ \\
\hline & 08/07/00 & 1300 & $<1.0$ & .53 & $<1.0$ & $<1.0$ & $<1.0$ & $<1.0$ \\
\hline & $09 / 25 / 00$ & 1600 & $<1.0$ & .44 & -- & $<1.0$ & $<1.0$ & $<1.0$ \\
\hline & $12 / 14 / 00$ & 1300 & e.05 & .47 & -- & e.09 & $<.20$ & .37 \\
\hline & $03 / 26 / 01$ & 1300 & $<.08$ & .56 & -- & $<.10$ & e. 10 & .40 \\
\hline & $04 / 30 / 01$ & 1300 & $<.08$ & .44 & -- & .16 & e. 10 & .40 \\
\hline & $05 / 22 / 01$ & 0900 & $<.08$ & .65 & -- & e. 05 & e. 11 & .26 \\
\hline & 08/13/01 & 1900 & $<.08$ & .53 & 1.3 & $<.10$ & e. 19 & .50 \\
\hline \multirow[t]{7}{*}{ M5 } & $06 / 19 / 00$ & 1545 & $<1.0$ & .67 & -- & 4.7 & $<1.0$ & $<1.0$ \\
\hline & $08 / 07 / 00$ & 1500 & $<1.0$ & .46 & 4.7 & $<1.0$ & $<1.0$ & $<1.0$ \\
\hline & $09 / 26 / 00$ & 1300 & $<1.0$ & .45 & -- & $<1.0$ & $<1.0$ & $<1.0$ \\
\hline & 03/26/01 & 1400 & $<.08$ & .39 & -- & $<.10$ & $<.20$ & .23 \\
\hline & 04/30/01 & 1200 & $<.08$ & .39 & -- & .91 & e. 11 & .37 \\
\hline & $05 / 22 / 01$ & 0800 & $<.08$ & .44 & -- & e. 10 & $<.20$ & .39 \\
\hline & 08/13/01 & 1800 & $<.08$ & .43 & 14 & $<.10$ & e. 16 & .54 \\
\hline
\end{tabular}


Table 25. Trace-element concentration data for ground-water samples collected for the surface-water/ground-water interaction study of the Spokane River and surrounding basin-fill aquifers, Idaho and Washington, 2000-01 (Continued)

\begin{tabular}{|c|c|c|c|c|c|c|c|c|}
\hline $\begin{array}{c}\text { Sele- } \\
\text { nium, } \\
\text { dis- } \\
\text { solved } \\
(\mu \mathrm{g} / \mathrm{L})\end{array}$ & $\begin{array}{c}\text { Silver, } \\
\text { dis- } \\
\text { solved } \\
(\mu \mathrm{g} / \mathrm{L})\end{array}$ & $\begin{array}{c}\text { Stron- } \\
\text { tium, } \\
\text { dis- } \\
\text { solved } \\
(\mu \mathrm{g} / \mathrm{L})\end{array}$ & $\begin{array}{l}\text { Thal- } \\
\text { lium, } \\
\text { dis- } \\
\text { solved } \\
(\mu \mathrm{g} / \mathrm{L}\end{array}$ & $\begin{array}{c}\text { Uranium, } \\
\text { dis- } \\
\text { solved } \\
(\mu \mathrm{g} / \mathrm{L})\end{array}$ & $\begin{array}{c}\text { Vana- } \\
\text { dium, } \\
\text { dis- } \\
\text { solved } \\
(\mu \mathrm{g} / \mathrm{L})\end{array}$ & $\begin{array}{l}\text { Zinc, } \\
\text { total } \\
\text { recov- } \\
\text { erable } \\
(\mu \mathrm{g} / \mathrm{L})\end{array}$ & $\begin{array}{c}\text { Zinc, } \\
\text { dis- } \\
\text { solved } \\
(\mu \mathrm{g} / \mathrm{L})\end{array}$ & $\begin{array}{c}\text { Well } \\
\text { number } \\
\text { (fig. 5) }\end{array}$ \\
\hline$<0.70$ & $<1.0$ & 81 & $<0.90$ & $<1.0$ & $<1.0$ & -- & $<1.0$ & M1 \\
\hline$<.70$ & $<1.0$ & 25 & $<.90$ & $<1.0$ & $<1.0$ & -- & $<1.0$ & \\
\hline$<.33$ & $<1.0$ & 44 & $<.04$ & e. 02 & .29 & -- & 1.3 & \\
\hline$<.33$ & $<1.0$ & 20 & $<.04$ & $<.02$ & e. 16 & -- & $<1.0$ & \\
\hline$<.33$ & $<1.0$ & 24 & $<.04$ & $<.02$ & $<.21$ & -- & $<1.0$ & \\
\hline$<.30$ & $<1.0$ & 35 & $<.04$ & $<.02$ & .21 & -- & $<1.0$ & \\
\hline$<.30$ & $<1.0$ & 49 & $<.04$ & $<.02$ & e. 15 & 1.7 & $<1.0$ & \\
\hline$<.30$ & $<1.0$ & $<.08$ & $<.04$ & $<.02$ & $<.20$ & 1.1 & $<1.0$ & \\
\hline$<.70$ & $<1.0$ & 16 & $<.90$ & $<1.0$ & $<1.0$ & -- & 54 & M2 \\
\hline$<.33$ & $<1.0$ & 18 & $<.04$ & .04 & $<.21$ & -- & 61 & \\
\hline$<.33$ & $<1.0$ & 17 & $<.04$ & e. 01 & $<.21$ & -- & 58 & \\
\hline -- & $<.20$ & $<.10$ & $<.10$ & $<.20$ & -- & -- & $<.50$ & \\
\hline$<.33$ & $<1.0$ & 16 & $<.04$ & $<.02$ & e. 12 & -- & 56 & \\
\hline$<.30$ & $<1.0$ & 20 & $<.04$ & e. 02 & e. 11 & -- & 63 & \\
\hline$<.33$ & $<1.0$ & 20 & e. 03 & $<.02$ & $<.21$ & -- & 60 & \\
\hline$<.33$ & $<1.0$ & 20 & $<.04$ & $<.02$ & $<.21$ & -- & 61 & \\
\hline$<.30$ & $<1.0$ & 21 & e. 02 & e. 02 & e. 16 & -- & 71 & \\
\hline$<.30$ & $<1.0$ & 22 & $<.04$ & $<.02$ & e. 18 & 74 & 66 & \\
\hline$<.33$ & $<1.0$ & 101 & $<.04$ & 2.0 & .26 & -- & $<1.0$ & M3 \\
\hline$<.33$ & $<1.0$ & 98 & $<.04$ & 2.3 & .39 & -- & $<1.0$ & \\
\hline$<.30$ & $<1.0$ & 103 & $<.04$ & 2.4 & e. 20 & -- & $<1.0$ & \\
\hline$<.30$ & $<1.0$ & 105 & .08 & 2.4 & 1.0 & -- & $<1.0$ & \\
\hline$<.30$ & $<1.0$ & 98 & $<.04$ & 2.1 & e. 20 & -- & $<1.0$ & \\
\hline$<.70$ & $<1.0$ & 19 & $<.90$ & $<1.0$ & $<1.0$ & -- & 25 & M4 \\
\hline$<.70$ & $<1.0$ & 18 & $<.90$ & $<1.0$ & $<1.0$ & 35 & 34 & \\
\hline$<.70$ & $<1.0$ & 17 & $<.90$ & $<1.0$ & $<1.0$ & -- & 38 & \\
\hline$<.33$ & $<1.0$ & 18 & $<.04$ & e. 01 & e.11 & -- & 43 & \\
\hline$<.33$ & $<1.0$ & 16 & .18 & $<.02$ & e. 12 & -- & 46 & \\
\hline$<.33$ & $<1.0$ & 22 & $<.04$ & $<.02$ & .25 & -- & 49 & \\
\hline$<.30$ & $<1.0$ & 21 & $<.04$ & e. 02 & $<.20$ & -- & 49 & \\
\hline$<.30$ & $<1.0$ & 21 & $<.04$ & $<.02$ & e. 16 & 46 & 42 & \\
\hline e.43 & $<1.0$ & 18 & $<.90$ & $<1.0$ & $<1.0$ & -- & 25 & M5 \\
\hline$<.70$ & $<1.0$ & 18 & $<.90$ & $<1.0$ & $<1.0$ & 22 & 21 & \\
\hline$<.70$ & $<1.0$ & 17 & $<.90$ & $<1.0$ & $<1.0$ & -- & 24 & \\
\hline$<.33$ & $<1.0$ & 16 & $<.04$ & .02 & e.13 & -- & 34 & \\
\hline$<.33$ & $<1.0$ & 23 & $<.04$ & e. 01 & .24 & -- & 36 & \\
\hline$<.30$ & $<1.0$ & 21 & $<.04$ & $<.02$ & e.11 & -- & 41 & \\
\hline$<.30$ & $<1.0$ & 21 & $<.04$ & $<.02$ & e. 21 & 36 & 29 & \\
\hline
\end{tabular}


Table 25. Trace-element concentration data for ground-water samples collected for the surface-water/ground-water interaction study of the Spokane River and surrounding basin-fill aquifers, Idaho and Washington, 2000-01 (Continued)

\begin{tabular}{|c|c|c|c|c|c|c|c|c|c|}
\hline $\begin{array}{c}\text { Well } \\
\text { number } \\
\text { (fig. 5) }\end{array}$ & $\begin{array}{c}\text { Site } \\
\text { identification } \\
\text { number }\end{array}$ & Date & Time & $\begin{array}{l}\text { Alumi- } \\
\text { num, } \\
\text { dis- } \\
\text { solved } \\
(\mu \mathrm{g} / \mathrm{L})\end{array}$ & $\begin{array}{l}\text { Anti- } \\
\text { mony, } \\
\text { dis- } \\
\text { solved } \\
(\mu \mathrm{g} / \mathrm{L})\end{array}$ & $\begin{array}{c}\text { Arsenic, } \\
\text { total } \\
\text { recov- } \\
\text { erable } \\
(\mu \mathrm{g} / \mathrm{L})\end{array}$ & $\begin{array}{c}\text { Arsenic, } \\
\text { dis- } \\
\text { solved } \\
(\mu \mathrm{g} / \mathrm{L})\end{array}$ & $\begin{array}{c}\text { Barium, } \\
\text { dis- } \\
\text { solved } \\
(\mu \mathrm{g} / \mathrm{L})\end{array}$ & $\begin{array}{c}\text { Beryl- } \\
\text { lium, } \\
\text { dis- } \\
\text { solved } \\
(\mu \mathrm{g} / \mathrm{L})\end{array}$ \\
\hline \multirow[t]{7}{*}{ M6 } & 474149117031101 & $08 / 07 / 00$ & 1600 & 3.1 & $<1.0$ & -- & e.48 & 19 & $<1.0$ \\
\hline & & $09 / 26 / 00$ & 1500 & $<1.0$ & $<1.0$ & -- & $<.90$ & 16 & $<1.0$ \\
\hline & & $12 / 14 / 00$ & 1400 & 3.6 & .48 & -- & .27 & 16 & $<.06$ \\
\hline & & $03 / 26 / 01$ & 1500 & 4.5 & .44 & -- & .21 & 15 & $<.06$ \\
\hline & & $04 / 30 / 01$ & 1500 & 5.6 & .65 & -- & .27 & 17 & $<.06$ \\
\hline & & $05 / 22 / 01$ & 1000 & 5.6 & .53 & -- & .33 & 18 & $<.06$ \\
\hline & & $08 / 13 / 01$ & 2000 & $<1.0$ & .75 & $<2.0$ & .37 & 20 & $<.06$ \\
\hline \multirow[t]{7}{*}{ M7 } & 474144117031401 & $08 / 08 / 00$ & 1000 & $<1.0$ & $<1.0$ & -- & e.52 & 12 & $<1.0$ \\
\hline & & $09 / 27 / 00$ & 1600 & 1.0 & $<1.0$ & -- & e. 47 & 12 & $<1.0$ \\
\hline & & $12 / 14 / 00$ & 1500 & 3.9 & $<.05$ & -- & .45 & 12 & $<.06$ \\
\hline & & $03 / 26 / 01$ & 1600 & 4.8 & e. 05 & -- & .37 & 12 & $<.06$ \\
\hline & & $04 / 30 / 01$ & 1600 & 6.1 & .05 & -- & .50 & 13 & $<.06$ \\
\hline & & $05 / 22 / 01$ & 1100 & 5.9 & $<.05$ & -- & .45 & 13 & $<.06$ \\
\hline & & $08 / 14 / 01$ & 0800 & 3.0 & .05 & -- & .42 & 12 & $<.06$ \\
\hline \multirow[t]{16}{*}{ M8 } & 474130117035902 & $07 / 12 / 00$ & 1300 & 2.3 & $<1.0$ & -- & e. 45 & 12 & $<1.0$ \\
\hline & & $07 / 12 / 00^{\mathbf{F b}}$ & 1308 & $<.30$ & $<.20$ & -- & -- & $<.20$ & $<.20$ \\
\hline & & 08/09/00 & 1000 & 5.6 & $<1.0$ & $<2.6$ & $<.90$ & 13 & $<1.0$ \\
\hline & & $08 / 09 / 00^{2}$ & 1020 & $<1.0$ & $<1.0$ & -- & e.49 & 13 & $<1.0$ \\
\hline & & 09/26/00 & 0800 & $<1.0$ & $<1.0$ & -- & $<.90$ & 11 & $<1.0$ \\
\hline & & $12 / 14 / 00$ & 1000 & 3.5 & .45 & -- & .46 & 12 & $<.06$ \\
\hline & & $03 / 28 / 01$ & 1100 & $<1.0$ & .40 & -- & .35 & 12 & $<.06$ \\
\hline & & $05 / 02 / 01$ & 1100 & 3.9 & .48 & $<1.9$ & .30 & 13 & $<.06$ \\
\hline & & $05 / 02 / 01^{\mathbf{R}}$ & 1101 & $<1.0$ & .46 & e.94 & .21 & 12 & $<.06$ \\
\hline & & $05 / 02 / 01^{1}$ & 1130 & 1.7 & .54 & -- & .29 & 12 & $<.06$ \\
\hline & & $05 / 02 / 01^{\mathbf{R}, 1}$ & 1131 & $<1.0$ & .50 & -- & .33 & 13 & $<.06$ \\
\hline & & $05 / 02 / 01^{\mathbf{F b}, 1}$ & 1132 & $<.30$ & $<.20$ & & $<.20$ & $<.20$ & $<.20$ \\
\hline & & $05 / 23 / 01$ & 1000 & 6.3 & .42 & -- & .44 & 15 & $<.06$ \\
\hline & & 08/13/01 & 1400 & $<1.0$ & .49 & $<2.0$ & .54 & 14 & $<.06$ \\
\hline & & $08 / 14 / 01$ & 0600 & $<1.0$ & .48 & $<2.0$ & .52 & 14 & $<.06$ \\
\hline & & $08 / 14 / 01^{\mathbf{R}}$ & 0601 & $<1.0$ & .49 & $<2.0$ & .55 & 14 & $<.06$ \\
\hline \multirow[t]{9}{*}{ M9 } & 474130117035901 & $08 / 09 / 00$ & 0900 & 4.7 & $<1.0$ & $<2.6$ & e. 47 & 14 & $<1.0$ \\
\hline & & $08 / 09 / 00^{2}$ & 0920 & $<1.0$ & $<1.0$ & -- & e. 48 & 14 & $<1.0$ \\
\hline & & $09 / 26 / 00$ & 0900 & $<1.0$ & $<1.0$ & -- & $<.90$ & 11 & $<1.0$ \\
\hline & & $12 / 14 / 00$ & 0900 & $<1.0$ & .41 & -- & .39 & 13 & $<.06$ \\
\hline & & $03 / 28 / 01$ & 1000 & $<1.0$ & .46 & -- & .30 & 12 & $<.06$ \\
\hline & & $05 / 02 / 01$ & 1200 & 5.6 & .51 & -- & .28 & 13 & $<.06$ \\
\hline & & $05 / 23 / 01$ & 0900 & 6.4 & .45 & -- & .38 & 15 & $<.06$ \\
\hline & & $08 / 13 / 01$ & 1600 & $<1.0$ & .51 & $<2.0$ & .51 & 14 & $<.06$ \\
\hline & & $08 / 14 / 01$ & 0500 & $<1.0$ & .64 & $<2.0$ & .45 & 14 & $<.06$ \\
\hline
\end{tabular}


Table 25. Trace-element concentration data for ground-water samples collected for the surface-water/ground-water interaction study of the Spokane River and surrounding basin-fill aquifers, Idaho and Washington, 2000-01 (Continued)

\begin{tabular}{|c|c|c|c|c|c|c|c|c|c|}
\hline $\begin{array}{c}\text { Boron, } \\
\text { dis- } \\
\text { solved } \\
(\mu \mathrm{g} / \mathrm{L})\end{array}$ & $\begin{array}{c}\text { Cadmium, } \\
\text { total } \\
\text { recov- } \\
\text { erable } \\
(\mu \mathrm{g} / \mathrm{L})\end{array}$ & $\begin{array}{c}\text { Cad- } \\
\text { mium, } \\
\text { dis- } \\
\text { solved } \\
(\mu \mathrm{g} / \mathrm{L})\end{array}$ & $\begin{array}{l}\text { Chro- } \\
\text { mium, } \\
\text { dis- } \\
\text { solved } \\
(\mu \mathrm{g} / \mathrm{L})\end{array}$ & $\begin{array}{l}\text { Cobalt, } \\
\text { dis- } \\
\text { solved } \\
(\mu \mathrm{g} / \mathrm{L})\end{array}$ & $\begin{array}{c}\text { Copper, } \\
\text { dis- } \\
\text { solved } \\
(\mu \mathrm{g} / \mathrm{L})\end{array}$ & $\begin{array}{l}\text { Iron, } \\
\text { total } \\
\text { recov- } \\
\text { erable } \\
(\mu \mathrm{g} / \mathrm{L})\end{array}$ & $\begin{array}{c}\text { Iron, } \\
\text { dis- } \\
\text { solved } \\
(\mu \mathrm{g} / \mathrm{L})\end{array}$ & $\begin{array}{l}\text { Lead, } \\
\text { total } \\
\text { recov- } \\
\text { erable } \\
(\mu \mathrm{g} / \mathrm{L})\end{array}$ & $\begin{array}{c}\text { Well } \\
\text { number } \\
\text { (fig. 5) }\end{array}$ \\
\hline $\mathrm{e} 10$ & -- & $<1.0$ & $<.80$ & $<1.0$ & $<1.0$ & -- & $<10$ & -- & M6 \\
\hline$<12$ & -- & $<1.0$ & e.61 & $<1.0$ & $<1.0$ & -- & $<10$ & -- & \\
\hline e6.3 & -- & .33 & 1.6 & .03 & .82 & -- & $<10$ & -- & \\
\hline 14 & -- & .32 & e. 74 & .02 & .76 & -- & $<10$ & -- & \\
\hline 12 & -- & .29 & e. 74 & .02 & .69 & -- & $<10$ & -- & \\
\hline 13 & -- & .32 & .87 & e. 01 & .72 & -- & $<10$ & -- & \\
\hline$<7.0$ & .38 & .34 & $<.80$ & .03 & .53 & 17 & $<10$ & $<1.0$ & \\
\hline$<12$ & -- & $<1.0$ & $<.80$ & $<1.0$ & $<1.0$ & -- & $<10$ & -- & M7 \\
\hline$<12$ & -- & $<1.0$ & e. 68 & $<1.0$ & $<1.0$ & -- & $<10$ & -- & \\
\hline e6.2 & -- & $<.04$ & e. 48 & .03 & 1.3 & -- & $<10$ & -- & \\
\hline 12 & -- & $<.04$ & $<.80$ & .03 & .23 & -- & $<10$ & -- & \\
\hline 12 & -- & $<.04$ & 1.2 & .05 & .40 & -- & $<10$ & -- & \\
\hline 15 & -- & $<.04$ & 1.1 & .02 & .27 & -- & $<10$ & -- & \\
\hline e6.7 & -- & $<.04$ & 2.4 & .05 & $<.20$ & -- & 14 & -- & \\
\hline$<12$ & $<1.0$ & $<1.0$ & .81 & $<1.0$ & $<1.0$ & 234 & $<10$ & $<1.0$ & M8 \\
\hline 2.2 & -- & $<.30$ & $<.20$ & $<.20$ & .25 & -- & $<3.0$ & -- & \\
\hline 13 & $<1.0$ & $<1.0$ & e. 54 & $<1.0$ & $<1.0$ & 46 & $<10$ & $<1.0$ & \\
\hline$<12$ & -- & $<1.0$ & e. 50 & $<1.0$ & $<1.0$ & -- & $<10$ & -- & \\
\hline$<12$ & -- & $<1.0$ & e.77 & $<1.0$ & $<1.0$ & -- & $<10$ & -- & \\
\hline e6.5 & -- & .18 & $<.80$ & .02 & .67 & -- & $<10$ & -- & \\
\hline$<7.0$ & -- & .21 & $<.80$ & .02 & .69 & -- & $<10$ & -- & \\
\hline 11 & .24 & .21 & e.71 & .02 & .50 & 133 & e8.7 & 1.3 & \\
\hline$<7.0$ & .22 & .20 & $<.80$ & e. 01 & .51 & 78 & e9.3 & $<1.0$ & \\
\hline$<7.0$ & -- & .20 & $<.80$ & .02 & .52 & -- & -- & -- & \\
\hline$<7.0$ & -- & .21 & $<.80$ & .02 & .57 & -- & -- & -- & \\
\hline$<2.0$ & -- & $<.30$ & $<.20$ & $<.20$ & .35 & -- & $<3.0$ & -- & \\
\hline 13 & -- & .24 & 1.1 & .02 & .62 & -- & e6.7 & -- & \\
\hline$<7.0$ & .22 & .20 & $<.80$ & .02 & .46 & 63 & $<10$ & $<1.0$ & \\
\hline$<7.0$ & .22 & .19 & $<.80$ & e. 01 & .45 & 40 & $<10$ & $<1.0$ & \\
\hline$<7.0$ & .21 & .19 & $<.80$ & .02 & .48 & 46 & $<10$ & $<1.0$ & \\
\hline 13 & $<1.0$ & $<1.0$ & $<.80$ & $<1.0$ & $<1.0$ & e17 & $<10$ & $<1.0$ & M9 \\
\hline$<12$ & -- & $<1.0$ & $<.80$ & $<1.0$ & $<1.0$ & -- & $<10$ & -- & \\
\hline$<12$ & -- & $<1.0$ & e. 57 & $<1.0$ & $<1.0$ & -- & $<10$ & -- & \\
\hline$<7.0$ & -- & .16 & $<.80$ & .02 & .76 & -- & $<10$ & -- & \\
\hline $\mathrm{e} 4.7$ & -- & .15 & $<.80$ & .02 & .71 & -- & e7.1 & -- & \\
\hline 11 & -- & .17 & .89 & .02 & .73 & -- & e8.1 & -- & \\
\hline 15 & -- & .18 & .97 & .02 & .59 & -- & $\mathrm{e} 7.2$ & -- & \\
\hline$<7.0$ & .16 & .15 & $<.80$ & .02 & .49 & 38 & $<10$ & $<1.0$ & \\
\hline$<7.0$ & .16 & .13 & $<.80$ & e. 01 & .51 & 31 & $<10$ & $<1.0$ & \\
\hline
\end{tabular}


Table 25. Trace-element concentration data for ground-water samples collected for the surface-water/ground-water interaction study of the Spokane River and surrounding basin-fill aquifers, Idaho and Washington, 2000-01 (Continued)

\begin{tabular}{|c|c|c|c|c|c|c|c|c|}
\hline $\begin{array}{c}\text { Well } \\
\text { number } \\
\text { (fig. 5) }\end{array}$ & Date & Time & $\begin{array}{c}\text { Lead, } \\
\text { dis- } \\
\text { solved } \\
(\mu \mathrm{g} / \mathrm{L})\end{array}$ & $\begin{array}{l}\text { Lithium, } \\
\text { dis- } \\
\text { solved } \\
(\mu \mathrm{g} / \mathrm{L})\end{array}$ & $\begin{array}{c}\text { Manganese, } \\
\text { total } \\
\text { recov- } \\
\text { erable } \\
(\mu \mathrm{g} / \mathrm{L})\end{array}$ & $\begin{array}{c}\text { Manganese, } \\
\text { dis- } \\
\text { solved } \\
(\mu \mathrm{g} / \mathrm{L})\end{array}$ & $\begin{array}{l}\text { Molyb- } \\
\text { denum, } \\
\text { dis- } \\
\text { solved } \\
(\mu \mathrm{g} / \mathrm{L})\end{array}$ & $\begin{array}{c}\text { Nickel, } \\
\text { dis- } \\
\text { solved } \\
(\mu \mathrm{g} / \mathrm{L})\end{array}$ \\
\hline \multirow[t]{7}{*}{ M6 } & $08 / 07 / 00$ & 1600 & $<1.0$ & .46 & -- & $<1.0$ & $<1.0$ & $<1.0$ \\
\hline & $09 / 26 / 00$ & 1500 & $<1.0$ & .47 & -- & $<1.0$ & $<1.0$ & $<1.0$ \\
\hline & $12 / 14 / 00$ & 1400 & $<.08$ & .49 & -- & .24 & e. 12 & 1.1 \\
\hline & $03 / 26 / 01$ & 1500 & $<.08$ & .36 & -- & e. 07 & $<.20$ & .54 \\
\hline & $04 / 30 / 01$ & 1500 & $<.08$ & .33 & -- & .12 & e. 10 & .46 \\
\hline & $05 / 22 / 01$ & 1000 & $<.08$ & .42 & -- & e. 07 & $<.20$ & .36 \\
\hline & 08/13/01 & 2000 & $<.08$ & .45 & $<1.0$ & $<.10$ & $<.20$ & .06 \\
\hline \multirow[t]{7}{*}{ M7 } & $08 / 08 / 00$ & 1000 & $<1.0$ & .42 & -- & $<1.0$ & $<1.0$ & $<1.0$ \\
\hline & $09 / 27 / 00$ & 1600 & $<1.0$ & .50 & -- & $<1.0$ & $<1.0$ & $<1.0$ \\
\hline & $12 / 14 / 00$ & 1500 & e.06 & .52 & -- & .33 & e.17 & .77 \\
\hline & $03 / 26 / 01$ & 1600 & $<.08$ & .61 & -- & .16 & e. 17 & .43 \\
\hline & $04 / 30 / 01$ & 1600 & $<.08$ & .60 & -- & .40 & .26 & .90 \\
\hline & $05 / 22 / 01$ & 1100 & $<.08$ & .56 & -- & .23 & e. 18 & .78 \\
\hline & $08 / 14 / 01$ & 0800 & $<.08$ & .36 & -- & $<.10$ & .36 & 1.3 \\
\hline \multirow[t]{16}{*}{ M8 } & $07 / 12 / 00$ & 1300 & $<1.0$ & .42 & 7.8 & $<1.0$ & $<1.0$ & $<1.0$ \\
\hline & $07 / 12 / 00^{\mathbf{F b}}$ & 1308 & $<.30$ & -- & -- & $<.10$ & $<.20$ & $<.50$ \\
\hline & 08/09/00 & 1000 & $<1.0$ & .41 & 2.9 & $<1.0$ & $<1.0$ & $<1.0$ \\
\hline & $08 / 09 / 00^{2}$ & 1020 & $<1.0$ & .41 & -- & $<1.0$ & $<1.0$ & $<1.0$ \\
\hline & $09 / 26 / 00$ & 0800 & $<1.0$ & .43 & -- & $<1.0$ & $<1.0$ & $<1.0$ \\
\hline & $12 / 14 / 00$ & 1000 & e.07 & e. 18 & -- & e. 05 & $<.20$ & .49 \\
\hline & $03 / 28 / 01$ & 1100 & .13 & .54 & -- & e. 07 & e. 10 & .46 \\
\hline & $05 / 02 / 01$ & 1100 & e. 06 & .48 & 9.3 & .14 & e. 13 & .56 \\
\hline & $05 / 02 / 01^{\mathbf{R}}$ & 1101 & e.06 & .50 & 5.6 & e.09 & $<.20$ & .37 \\
\hline & $05 / 02 / 01^{1}$ & 1130 & $<.08$ & .94 & -- & .12 & e.11 & .51 \\
\hline & $05 / 02 / 01^{\mathbf{R}, 1}$ & 1131 & $<.08$ & .65 & -- & .10 & $<.20$ & .43 \\
\hline & $05 / 02 / 01^{\mathbf{F b}, 1}$ & 1132 & $<.30$ & -- & -- & .30 & $<.20$ & $<.50$ \\
\hline & $05 / 23 / 01$ & 1000 & e.06 & .58 & -- & .23 & e. 10 & .61 \\
\hline & 08/13/01 & 1400 & $<.08$ & .44 & 4.0 & $<.10$ & e. 15 & .15 \\
\hline & $08 / 14 / 01$ & 0600 & $<.08$ & .42 & 2.5 & $<.10$ & e.16 & .19 \\
\hline & $08 / 14 / 01^{\mathbf{R}}$ & 0601 & $<.08$ & .40 & 2.6 & $<.10$ & .20 & .38 \\
\hline \multirow[t]{9}{*}{ M9 } & 08/09/00 & 0900 & $<1.0$ & .41 & $<1.0$ & $<1.0$ & $<1.0$ & $<1.0$ \\
\hline & $08 / 09 / 00^{2}$ & 0920 & $<1.0$ & .42 & -- & $<1.0$ & $<1.0$ & $<1.0$ \\
\hline & $09 / 26 / 00$ & 0900 & $<1.0$ & .39 & -- & $<1.0$ & $<1.0$ & $<1.0$ \\
\hline & $12 / 14 / 00$ & 0900 & e.07 & .57 & -- & .12 & $<.20$ & .44 \\
\hline & $03 / 28 / 01$ & 1000 & $<.08$ & .63 & -- & e. 07 & e. 10 & .33 \\
\hline & $05 / 02 / 01$ & 1200 & e.07 & .79 & -- & .20 & e. 15 & .72 \\
\hline & $05 / 23 / 01$ & 0900 & e. 05 & .60 & -- & .23 & e. 15 & .73 \\
\hline & $08 / 13 / 01$ & 1600 & $<.08$ & .39 & 2.4 & $<.10$ & e. 14 & .09 \\
\hline & $08 / 14 / 01$ & 0500 & $<.08$ & .35 & 2.4 & $<.10$ & e.13 & .12 \\
\hline
\end{tabular}


Table 25. Trace-element concentration data for ground-water samples collected for the surface-water/ground-water interaction study of the Spokane River and surrounding basin-fill aquifers, Idaho and Washington, 2000-01 (Continued)

\begin{tabular}{|c|c|c|c|c|c|c|c|c|}
\hline $\begin{array}{c}\text { Sele- } \\
\text { nium, } \\
\text { dis- } \\
\text { solved } \\
(\mu \mathrm{g} / \mathrm{L})\end{array}$ & $\begin{array}{c}\text { Silver, } \\
\text { dis- } \\
\text { solved } \\
(\mu \mathrm{g} / \mathrm{L})\end{array}$ & $\begin{array}{c}\text { Stron- } \\
\text { tium, } \\
\text { dis- } \\
\text { solved } \\
(\mu \mathrm{g} / \mathrm{L})\end{array}$ & $\begin{array}{l}\text { Thal- } \\
\text { lium, } \\
\text { dis- } \\
\text { solved } \\
(\mu \mathrm{g} / \mathrm{L}\end{array}$ & $\begin{array}{c}\text { Uranium, } \\
\text { dis- } \\
\text { solved } \\
(\mu \mathrm{g} / \mathrm{L})\end{array}$ & $\begin{array}{c}\text { Vana- } \\
\text { dium, } \\
\text { dis- } \\
\text { solved } \\
(\mu \mathrm{g} / \mathrm{L})\end{array}$ & $\begin{array}{l}\text { Zinc, } \\
\text { total } \\
\text { recov- } \\
\text { erable } \\
(\mu \mathrm{g} / \mathrm{L})\end{array}$ & $\begin{array}{c}\text { Zinc, } \\
\text { dis- } \\
\text { solved } \\
(\mu \mathrm{g} / \mathrm{L})\end{array}$ & $\begin{array}{c}\text { Well } \\
\text { number } \\
\text { (fig. 5) }\end{array}$ \\
\hline$<.70$ & $<1.0$ & 19 & $<.90$ & $<1.0$ & $<1.0$ & -- & 29 & M6 \\
\hline$<.70$ & $<1.0$ & 17 & $<.90$ & $<1.0$ & $<1.0$ & -- & 29 & \\
\hline$<.33$ & $<1.0$ & 18 & $<.04$ & e.01 & e.14 & -- & 34 & \\
\hline$<.33$ & $<1.0$ & 16 & $<.04$ & e. 01 & $<.21$ & -- & 35 & \\
\hline$<.33$ & $<1.0$ & 22 & $<.04$ & e. 01 & .23 & -- & 42 & \\
\hline$<.30$ & $<1.0$ & 20 & $<.04$ & $<.02$ & $<.20$ & -- & 38 & \\
\hline$<.30$ & $<1.0$ & 21 & $<.04$ & $<.02$ & e. 17 & 41 & 32 & \\
\hline$<.70$ & $<1.0$ & 27 & $<.90$ & $<1.0$ & $<1.0$ & -- & $<1.0$ & M7 \\
\hline$<.70$ & $<1.0$ & 28 & $<.90$ & $<1.0$ & $<1.0$ & -- & $<1.0$ & \\
\hline$<.33$ & $<1.0$ & 27 & $<.04$ & .10 & e.19 & -- & $<1.0$ & \\
\hline$<.33$ & $<1.0$ & 25 & $<.04$ & .10 & .21 & -- & $<1.0$ & \\
\hline$<.33$ & $<1.0$ & 33 & .08 & .10 & .45 & -- & $<1.0$ & \\
\hline$<.30$ & $<1.0$ & 30 & $<.04$ & .10 & .34 & -- & 6.8 & \\
\hline$<.30$ & $<1.0$ & 29 & $<.04$ & .04 & e. 14 & -- & $<1.0$ & \\
\hline$<.70$ & $<1.0$ & 22 & $<.90$ & $<1.0$ & $<1.0$ & 31 & 26 & M8 \\
\hline -- & $<.20$ & $<.10$ & $<.10$ & $<.20$ & -- & -- & 2.0 & \\
\hline$<.70$ & $<1.0$ & 17 & $<.90$ & $<1.0$ & $<1.0$ & 34 & 32 & \\
\hline$<.70$ & $<1.0$ & 17 & $<.90$ & $<1.0$ & $<1.0$ & -- & 32 & \\
\hline$<.70$ & $<1.0$ & 16 & $<.90$ & $<1.0$ & $<1.0$ & -- & 33 & \\
\hline$<.33$ & $<1.0$ & 17 & $<.04$ & e. 01 & e. 20 & -- & 53 & \\
\hline$<.33$ & $<1.0$ & 16 & $<.04$ & e. 01 & e. 14 & -- & 54 & \\
\hline$<.33$ & $<1.0$ & 19 & $<.04$ & $<.02$ & $<.21$ & 62 & 56 & \\
\hline$<.33$ & $<1.0$ & 19 & $<.04$ & $<.02$ & $<.21$ & 60 & 54 & \\
\hline$<.33$ & $<1.0$ & 19 & .06 & $<.02$ & e. 10 & -- & 54 & \\
\hline$<.33$ & $<1.0$ & 19 & $<.04$ & $<.02$ & .31 & -- & 57 & \\
\hline$<.30$ & $<.20$ & $<.10$ & $<.10$ & $<.20$ & -- & -- & $<.50$ & \\
\hline$<.30$ & $<1.0$ & 20 & $<.04$ & $<.02$ & e. 20 & -- & 62 & \\
\hline$<.30$ & $<1.0$ & 19 & $<.04$ & $<.02$ & .25 & 35 & 32 & \\
\hline$<.30$ & $<1.0$ & 20 & $<.04$ & $<.02$ & .25 & 43 & 31 & \\
\hline$<.30$ & $<1.0$ & 20 & $<.04$ & $<.02$ & .24 & 32 & 31 & \\
\hline$<.70$ & $<1.0$ & 18 & $<.90$ & $<1.0$ & $<1.0$ & 22 & 22 & M9 \\
\hline$<.70$ & $<1.0$ & 18 & $<.90$ & $<1.0$ & $<1.0$ & -- & 22 & \\
\hline$<.70$ & $<1.0$ & 17 & $<.90$ & $<1.0$ & $<1.0$ & -- & 23 & \\
\hline$<.33$ & $<1.0$ & 19 & $<.04$ & e. 02 & e. 14 & -- & 45 & \\
\hline$<.33$ & $<1.0$ & 16 & .12 & e. 01 & e. 12 & -- & 41 & \\
\hline$<.33$ & $<1.0$ & 20 & $<.04$ & $<.02$ & $<.21$ & -- & 45 & \\
\hline$<.30$ & $<1.0$ & 21 & $<.04$ & e. 02 & e. 16 & -- & 48 & \\
\hline$<.30$ & $<1.0$ & 20 & $<.04$ & $<.02$ & .23 & 26 & 24 & \\
\hline$<.30$ & $<1.0$ & 20 & $<.04$ & $<.02$ & .24 & 24 & 23 & \\
\hline
\end{tabular}


Table 25. Trace-element concentration data for ground-water samples collected for the surface-water/ground-water interaction study of the Spokane River and surrounding basin-fill aquifers, Idaho and Washington, 2000-01 (Continued)

\begin{tabular}{|c|c|c|c|c|c|c|c|c|c|}
\hline $\begin{array}{c}\text { Well } \\
\text { number } \\
\text { (fig. 5) }\end{array}$ & $\begin{array}{c}\text { Site } \\
\text { identification } \\
\text { number }\end{array}$ & Date & Time & $\begin{array}{c}\text { Alumi- } \\
\text { num, } \\
\text { dis- } \\
\text { solved } \\
(\mu \mathrm{g} / \mathrm{L})\end{array}$ & $\begin{array}{c}\text { Anti- } \\
\text { mony, } \\
\text { dis- } \\
\text { solved } \\
(\mu \mathrm{g} / \mathrm{L})\end{array}$ & $\begin{array}{c}\text { Arsenic, } \\
\text { total } \\
\text { recov- } \\
\text { erable } \\
(\mu \mathrm{g} / \mathrm{L})\end{array}$ & $\begin{array}{c}\text { Arsenic, } \\
\text { dis- } \\
\text { solved } \\
(\mu \mathrm{g} / \mathrm{L})\end{array}$ & $\begin{array}{c}\text { Barium, } \\
\text { dis- } \\
\text { solved } \\
(\mu \mathrm{g} / \mathrm{L})\end{array}$ & $\begin{array}{c}\text { Beryl- } \\
\text { lium, } \\
\text { dis- } \\
\text { solved } \\
(\mu \mathrm{g} / \mathrm{L})\end{array}$ \\
\hline \multirow[t]{7}{*}{ M10 } & 474131117040401 & $08 / 09 / 00$ & 1100 & $<1.0$ & $<1.0$ & -- & e.46 & 15 & $<1.0$ \\
\hline & & $09 / 26 / 00$ & 1100 & 6.0 & $<1.0$ & -- & $<.90$ & 12 & $<1.0$ \\
\hline & & $12 / 14 / 00$ & 1100 & 3.6 & .37 & -- & .30 & 12 & $<.06$ \\
\hline & & $03 / 28 / 01$ & 1200 & $<1.0$ & .41 & -- & .29 & 12 & $<.06$ \\
\hline & & $05 / 02 / 01$ & 1300 & $<1.0$ & .51 & - & e. 18 & 16 & $<.06$ \\
\hline & & $05 / 23 / 01$ & 1100 & $<1.0$ & .38 & -- & .33 & 15 & $<.06$ \\
\hline & & 08/13/01 & 1200 & 3.5 & .50 & $<2.0$ & .39 & 16 & $<.06$ \\
\hline \multirow[t]{14}{*}{ M11 } & 474106117060501 & 08/09/00 & 1300 & $<1.0$ & $<1.0$ & $<2.6$ & e.49 & 15 & $<1.0$ \\
\hline & & $08 / 09 / 00^{\mathbf{R}}$ & 1301 & $<1.0$ & $<1.0$ & 2.8 & e. 48 & 15 & $<1.0$ \\
\hline & & $08 / 09 / 00^{\mathbf{F b}}$ & 1311 & $<.30$ & $<.20$ & -- & $<2.0$ & $<.20$ & $<.20$ \\
\hline & & $08 / 09 / 00^{2}$ & 1320 & $<1.0$ & $<1.0$ & -- & e. 50 & 14 & $<1.0$ \\
\hline & & $08 / 09 / 00^{\mathbf{R}, 2}$ & 1321 & $<1.0$ & $<1.0$ & -- & e. 51 & 14 & $<1.0$ \\
\hline & & $10 / 04 / 00$ & 1000 & $<1.0$ & .46 & -- & .35 & 13 & $<.06$ \\
\hline & & $12 / 19 / 00$ & 1000 & 3.9 & .41 & -- & .34 & 13 & $<.06$ \\
\hline & & $03 / 27 / 01$ & 1400 & $<1.0$ & .43 & -- & .32 & 14 & $<.06$ \\
\hline & & $05 / 01 / 01$ & 1000 & 5.7 & .53 & $<1.9$ & .39 & 15 & $<.06$ \\
\hline & & $05 / 01 / 01^{1}$ & 1030 & $<1.0$ & .50 & -- & .37 & 14 & $<.06$ \\
\hline & & $05 / 23 / 01$ & 1400 & $<1.0$ & .38 & -- & .40 & 16 & $<.06$ \\
\hline & & $05 / 23 / 01^{\mathbf{R}}$ & 1401 & $<1.0$ & .38 & -- & .39 & 16 & $<.06$ \\
\hline & & $05 / 23 / 01^{\mathbf{F b}}$ & 1411 & $<.30$ & $<.20$ & -- & $<.20$ & $<.20$ & $<.20$ \\
\hline & & $08 / 16 / 01$ & 0800 & 1.2 & .63 & $<2.0$ & .51 & 17 & $<.06$ \\
\hline \multirow[t]{7}{*}{ M12 } & 474107117060502 & $10 / 04 / 00$ & 1200 & 4.7 & .52 & -- & .51 & 10 & $<.06$ \\
\hline & & $12 / 19 / 00$ & 0900 & 3.8 & .47 & -- & .31 & 11 & $<.06$ \\
\hline & & $03 / 27 / 01$ & 1200 & 5.5 & .46 & -- & .27 & 12 & $<.06$ \\
\hline & & $05 / 01 / 01$ & 0900 & $<1.0$ & .51 & $<1.9$ & .28 & 12 & $<.06$ \\
\hline & & $05 / 01 / 01^{1}$ & 0930 & $<1.0$ & .51 & -- & .34 & 12 & $<.06$ \\
\hline & & $05 / 23 / 01$ & 1500 & 5.1 & .44 & -- & .34 & 14 & $<.06$ \\
\hline & & $08 / 16 / 01$ & 0600 & $<1.0$ & .64 & $<2.0$ & .43 & 14 & $<.06$ \\
\hline \multirow[t]{9}{*}{ M13 } & 474107117060501 & $08 / 09 / 00$ & 1500 & $<1.0$ & $<1.0$ & $<2.6$ & e. 46 & 14 & $<1.0$ \\
\hline & & $08 / 09 / 00^{2}$ & 1520 & $<1.0$ & $<1.0$ & -- & e.49 & 13 & $<1.0$ \\
\hline & & $10 / 04 / 00$ & 0800 & $<1.0$ & .43 & -- & .39 & 13 & $<.06$ \\
\hline & & $12 / 19 / 00$ & 1100 & $<1.0$ & .34 & -- & .20 & 12 & $<.06$ \\
\hline & & $03 / 27 / 01$ & 1500 & $<1.0$ & .35 & -- & e. 17 & 13 & $<.06$ \\
\hline & & $05 / 01 / 01$ & 0800 & $<1.0$ & .53 & $<1.9$ & .23 & 14 & $<.06$ \\
\hline & & $05 / 01 / 01^{1}$ & 0830 & $<1.0$ & .48 & -- & .29 & 14 & $<.06$ \\
\hline & & 05/23/01 & 1300 & 4.7 & .35 & -- & .30 & 16 & $<.06$ \\
\hline & & $08 / 16 / 01$ & 0900 & 3.4 & .59 & $<2.0$ & .47 & 16 & $<.06$ \\
\hline \multirow[t]{7}{*}{ M14 } & 474110117060601 & $10 / 03 / 00$ & 1800 & 6.4 & .39 & -- & .37 & 9.4 & $<.06$ \\
\hline & & $12 / 19 / 00$ & 1400 & $<1.0$ & .32 & -- & .20 & 9.1 & $<.06$ \\
\hline & & $03 / 27 / 01$ & 1100 & $<1.0$ & .32 & -- & .21 & 9.8 & $<.06$ \\
\hline & & $05 / 02 / 01$ & 0800 & 3.8 & .40 & $<1.9$ & e. 17 & 10 & $<.06$ \\
\hline & & $05 / 02 / 01^{1}$ & 0830 & $<1.0$ & .40 & -- & .18 & 9.6 & $<.06$ \\
\hline & & $05 / 24 / 01$ & 1200 & 1.6 & .34 & -- & .27 & 12 & $<.06$ \\
\hline & & $08 / 15 / 01$ & 1600 & 3.1 & .57 & $<2.0$ & .47 & 13 & $<.06$ \\
\hline
\end{tabular}

160 Water-Quality, Streambed-Sediment, and Biological Data from the Clark Fork-Pend Oreille and Spokane River Basins, Montana, Idaho, and Washington, 1998-2001 
Table 25. Trace-element concentration data for ground-water samples collected for the surface-water/ground-water interaction study of the Spokane River and surrounding basin-fill aquifers, Idaho and Washington, 2000-01 (Continued)

\begin{tabular}{|c|c|c|c|c|c|c|c|c|c|}
\hline $\begin{array}{c}\text { Boron, } \\
\text { dis- } \\
\text { solved } \\
(\mu \mathrm{g} / \mathrm{L})\end{array}$ & $\begin{array}{c}\text { Cadmium, } \\
\text { total } \\
\text { recov- } \\
\text { erable } \\
(\mu \mathrm{g} / \mathrm{L})\end{array}$ & $\begin{array}{c}\text { Cad- } \\
\text { mium, } \\
\text { dis- } \\
\text { solved } \\
(\mu \mathrm{g} / \mathrm{L})\end{array}$ & $\begin{array}{l}\text { Chro- } \\
\text { mium, } \\
\text { dis- } \\
\text { solved } \\
(\mu \mathrm{g} / \mathrm{L})\end{array}$ & $\begin{array}{l}\text { Cobalt, } \\
\text { dis- } \\
\text { solved } \\
(\mu \mathrm{g} / \mathrm{L})\end{array}$ & $\begin{array}{c}\text { Copper, } \\
\text { dis- } \\
\text { solved } \\
(\mu \mathrm{g} / \mathrm{L})\end{array}$ & $\begin{array}{l}\text { Iron, } \\
\text { total } \\
\text { recov- } \\
\text { erable } \\
(\mu \mathrm{g} / \mathrm{L})\end{array}$ & $\begin{array}{c}\text { Iron, } \\
\text { dis- } \\
\text { solved } \\
(\mu \mathrm{g} / \mathrm{L})\end{array}$ & $\begin{array}{c}\text { Lead, } \\
\text { total } \\
\text { recov- } \\
\text { erable } \\
(\mu \mathrm{g} / \mathrm{L})\end{array}$ & $\begin{array}{c}\text { Well } \\
\text { number } \\
\text { (fig. 5) }\end{array}$ \\
\hline$<12$ & -- & $<1.0$ & e. 57 & $<1.0$ & $<1.0$ & -- & $<10$ & -- & M10 \\
\hline $\mathrm{e} 12$ & -- & $<1.0$ & e. 69 & $<1.0$ & $<1.0$ & -- & $<10$ & -- & \\
\hline e4.4 & -- & .09 & e. 66 & .02 & 1.2 & -- & $<10$ & -- & \\
\hline e4.5 & -- & .09 & $<.80$ & .02 & .69 & -- & $<10$ & -- & \\
\hline$<7.0$ & -- & .24 & $<.80$ & e. 01 & .56 & -- & $<10$ & -- & \\
\hline$<7.0$ & -- & .11 & e.79 & .02 & .63 & -- & $<10$ & -- & \\
\hline 7.8 & .16 & .10 & 11 & .18 & .88 & 151 & 45 & $<1.0$ & \\
\hline$<12$ & $<1.0$ & $<1.0$ & e. 53 & $<1.0$ & $<1.0$ & 97 & $<10$ & $<1.0$ & M11 \\
\hline$<12$ & $<1.0$ & $<1.0$ & e. 49 & $<1.0$ & $<1.0$ & 100 & $<10$ & 1.2 & \\
\hline$<2.0$ & -- & $<.30$ & $<.20$ & $<.20$ & .35 & -- & $<3.0$ & -- & \\
\hline$<12$ & -- & $<1.0$ & $<.80$ & $<1.0$ & $<1.0$ & -- & -- & -- & \\
\hline e6.8 & -- & $<1.0$ & $<.80$ & $<1.0$ & $<1.0$ & -- & $<10$ & -- & \\
\hline$<7.0$ & -- & .25 & e. 42 & .02 & .56 & -- & $<10$ & -- & \\
\hline 7.8 & -- & .22 & e. 63 & .02 & .70 & -- & $<10$ & -- & \\
\hline$<7.0$ & -- & .29 & e. 48 & .02 & .64 & -- & $<10$ & -- & \\
\hline 13 & .31 & .27 & 1.2 & .03 & .79 & 405 & e8.7 & 2.1 & \\
\hline$<7.0$ & -- & .27 & $<.80$ & .02 & .90 & -- & -- & -- & \\
\hline$<7.0$ & -- & $<.30$ & .97 & .02 & .65 & -- & $<10$ & -- & \\
\hline e5.6 & -- & .30 & e. 51 & .02 & .71 & -- & $<10$ & -- & \\
\hline 2.5 & -- & $<.30$ & $<.20$ & $<.20$ & $<.20$ & -- & $<3.0$ & -- & \\
\hline e 5.5 & .34 & .27 & $<.80$ & .02 & .76 & 400 & $<10$ & 4.2 & \\
\hline 8.2 & -- & .05 & e. 78 & .03 & .71 & -- & $<10$ & -- & M12 \\
\hline 7.7 & -- & .07 & $<.80$ & .02 & .81 & -- & $<10$ & -- & \\
\hline 14 & -- & .12 & e. 49 & .02 & 1.0 & -- & e5.8 & -- & \\
\hline$<7.0$ & .10 & .10 & $<.80$ & .02 & 1.1 & 22 & $<10$ & $<1.0$ & \\
\hline$<7.0$ & -- & .10 & $<.80$ & e. 01 & 1.2 & -- & -- & -- & \\
\hline 13 & -- & .10 & e. 73 & .02 & 1.3 & -- & $<10$ & -- & \\
\hline $\mathrm{e} 5.2$ & .11 & .08 & $<.80$ & .02 & 2.5 & 81 & $<10$ & $<1.0$ & \\
\hline$<12$ & $<1.0$ & $<1.0$ & .95 & $<1.0$ & $<1.0$ & 92 & $<10$ & $<1.0$ & M13 \\
\hline$<12$ & -- & $<1.0$ & e.41 & $<1.0$ & $<1.0$ & - & -- & -- & \\
\hline$<7.0$ & -- & .12 & e.55 & .02 & .61 & -- & $<10$ & -- & \\
\hline$<7.0$ & -- & .08 & e. 62 & .03 & 7.4 & -- & $<10$ & -- & \\
\hline$<7.0$ & -- & .08 & e. 57 & .02 & .60 & -- & e6.4 & -- & \\
\hline$<7.0$ & .15 & .15 & $<.80$ & e.01 & .78 & 22 & e7.5 & $<1.0$ & \\
\hline$<7.0$ & -- & .15 & $<.80$ & e. 01 & .77 & -- & -- & -- & \\
\hline 13 & -- & .18 & $<.80$ & .02 & .70 & -- & $<10$ & -- & \\
\hline e6.4 & .16 & .15 & $<.80$ & e. 01 & .85 & 27 & $<10$ & $<1.0$ & \\
\hline 14 & -- & $<.04$ & 1.0 & .04 & .65 & -- & $<10$ & -- & M14 \\
\hline$<7.0$ & -- & $<.04$ & e.74 & .03 & 5.3 & -- & $<10$ & -- & \\
\hline$<7.0$ & -- & $<.04$ & 1.1 & .03 & 1.0 & -- & 13 & -- & \\
\hline 9.3 & $<.04$ & $<.04$ & .89 & .02 & .71 & 31 & e6.2 & $<1.0$ & \\
\hline$<7.0$ & -- & $<.04$ & $<.80$ & .02 & 1.2 & -- & & -- & \\
\hline$<7.0$ & -- & $<.04$ & e. 69 & .04 & .74 & -- & $<10$ & -- & \\
\hline e5.3 & $<.04$ & $<.04$ & $<.80$ & .02 & 1.7 & e8.1 & $<10$ & $<1.0$ & \\
\hline$<12$ & -- & $<1.0$ & .94 & $<1.0$ & $<1.0$ & -- & $<10$ & -- & M15 \\
\hline
\end{tabular}


Table 25. Trace-element concentration data for ground-water samples collected for the surface-water/ground-water interaction study of the Spokane River and surrounding basin-fill aquifers, Idaho and Washington, 2000-01 (Continued)

\begin{tabular}{|c|c|c|c|c|c|c|c|c|}
\hline $\begin{array}{c}\text { Well } \\
\text { number } \\
\text { (fig. 5) }\end{array}$ & Date & Time & $\begin{array}{c}\text { Lead, } \\
\text { dis- } \\
\text { solved } \\
(\mu \mathrm{g} / \mathrm{L})\end{array}$ & $\begin{array}{c}\text { Lithium, } \\
\text { dis- } \\
\text { solved } \\
(\mu \mathrm{g} / \mathrm{L})\end{array}$ & $\begin{array}{c}\text { Manganese, } \\
\text { total } \\
\text { recov- } \\
\text { erable } \\
(\mu \mathrm{g} / \mathrm{L})\end{array}$ & $\begin{array}{c}\text { Manganese, } \\
\text { dis- } \\
\text { solved } \\
(\mu \mathrm{g} / \mathrm{L})\end{array}$ & $\begin{array}{l}\text { Molyb- } \\
\text { denum, } \\
\text { dis- } \\
\text { solved } \\
(\mu \mathrm{g} / \mathrm{L})\end{array}$ & $\begin{array}{c}\text { Nickel, } \\
\text { dis- } \\
\text { solved } \\
(\mu \mathrm{g} / \mathrm{L})\end{array}$ \\
\hline \multirow[t]{7}{*}{ M10 } & $08 / 09 / 00$ & 1100 & $<1.0$ & .47 & -- & $<1.0$ & $<1.0$ & $<1.0$ \\
\hline & $09 / 26 / 00$ & 1100 & $<1.0$ & .41 & -- & $<1.0$ & $<1.0$ & $<1.0$ \\
\hline & $12 / 14 / 00$ & 1100 & $<.08$ & $<.30$ & -- & .13 & $<.20$ & .66 \\
\hline & $03 / 28 / 01$ & 1200 & $<.08$ & .46 & -- & $<.10$ & $<.20$ & .38 \\
\hline & $05 / 02 / 01$ & 1300 & $<.08$ & .46 & -- & $<.10$ & $<.20$ & .22 \\
\hline & $05 / 23 / 01$ & 1100 & $<.08$ & .49 & -- & .34 & $<.20$ & .57 \\
\hline & 08/13/01 & 1200 & $<.08$ & .44 & 11 & 2.2 & .97 & 6.4 \\
\hline \multirow[t]{14}{*}{ M11 } & $08 / 09 / 00$ & 1300 & $<1.0$ & .48 & 4.6 & $<1.0$ & $<1.0$ & $<1.0$ \\
\hline & $08 / 09 / 00^{\mathbf{R}}$ & 1301 & $<1.0$ & .48 & 5.4 & $<1.0$ & $<1.0$ & $<1.0$ \\
\hline & $08 / 09 / 00^{\mathbf{F b}}$ & 1311 & $<.30$ & -- & -- & $<.10$ & $<.20$ & $<.50$ \\
\hline & $08 / 09 / 00^{2}$ & 1320 & $<1.0$ & .51 & -- & $<1.0$ & $<1.0$ & $<1.0$ \\
\hline & $08 / 09 / 00^{\mathbf{R}, 2}$ & 1321 & $<1.0$ & .55 & -- & $<1.0$ & $<1.0$ & $<1.0$ \\
\hline & $10 / 04 / 00$ & 1000 & $<.08$ & .45 & -- & .68 & e.12 & .42 \\
\hline & $12 / 19 / 00$ & 1000 & e.06 & .40 & -- & .12 & e. 10 & .48 \\
\hline & $03 / 27 / 01$ & 1400 & $<.08$ & .45 & -- & e.09 & $<.20$ & .58 \\
\hline & $05 / 01 / 01$ & 1000 & $<.08$ & .54 & 18 & .50 & e. 17 & .99 \\
\hline & $05 / 01 / 01^{1}$ & 1030 & $<.08$ & -- & -- & .58 & e.13 & .80 \\
\hline & 05/23/01 & 1400 & $<.08$ & .61 & -- & e. 28 & $<.20$ & .64 \\
\hline & $05 / 23 / 01^{\mathbf{R}}$ & 1401 & $<.08$ & -- & -- & .28 & $<.20$ & .65 \\
\hline & $05 / 23 / 01^{\mathbf{F b}}$ & 1411 & $<.30$ & -- & -- & $<.10$ & $<.20$ & $<.50$ \\
\hline & 08/16/01 & 0800 & $<.08$ & .63 & 21 & $<.10$ & e. 15 & .36 \\
\hline \multirow[t]{7}{*}{ M12 } & $10 / 04 / 00$ & 1200 & $<.08$ & .47 & -- & 3.0 & e. 12 & .53 \\
\hline & $12 / 19 / 00$ & 0900 & e.06 & .32 & -- & .20 & $<.20$ & .33 \\
\hline & $03 / 27 / 01$ & 1200 & $<.08$ & .42 & -- & .17 & $<.20$ & .58 \\
\hline & $05 / 01 / 01$ & 0900 & $<.08$ & .48 & $<1.0$ & .11 & $<.20$ & .23 \\
\hline & $05 / 01 / 01^{1}$ & 0930 & $<.08$ & -- & -- & .15 & e. 10 & .34 \\
\hline & $05 / 23 / 01$ & 1500 & $<.08$ & .50 & -- & .23 & e. 11 & .64 \\
\hline & $08 / 16 / 01$ & 0600 & $<.08$ & .60 & 3.6 & $<.10$ & e. 14 & .24 \\
\hline \multirow[t]{9}{*}{ M13 } & 08/09/00 & 1500 & $<1.0$ & .47 & 4.8 & $<1.0$ & $<1.0$ & $<1.0$ \\
\hline & $08 / 09 / 00^{2}$ & 1520 & $<1.0$ & .50 & -- & $<1.0$ & $<1.0$ & $<1.0$ \\
\hline & $10 / 04 / 00$ & 0800 & $<.08$ & .42 & -- & e. 07 & $<.20$ & .38 \\
\hline & $12 / 19 / 00$ & 1100 & 1.6 & .35 & -- & .18 & $<.20$ & .56 \\
\hline & $03 / 27 / 01$ & 1500 & $<.08$ & .34 & -- & e. 10 & $<.20$ & .66 \\
\hline & $05 / 01 / 01$ & 0800 & $<.08$ & .90 & 1.3 & .27 & e. 12 & .19 \\
\hline & $05 / 01 / 01^{1}$ & 0830 & $<.08$ & -- & -- & .31 & $<.20$ & .32 \\
\hline & $05 / 23 / 01$ & 1300 & e. 04 & .58 & -- & .14 & $<.20$ & .65 \\
\hline & 08/16/01 & 0900 & $<.08$ & .60 & 1.0 & $<.10$ & e. 17 & .19 \\
\hline \multirow[t]{7}{*}{ M14 } & $10 / 03 / 00$ & 1800 & $<.08$ & .40 & -- & .59 & e. 13 & .51 \\
\hline & $12 / 19 / 00$ & 1400 & 1.1 & e. 21 & -- & .18 & $<.20$ & .46 \\
\hline & $03 / 27 / 01$ & 1100 & $<.08$ & .31 & -- & .48 & $<.20$ & .88 \\
\hline & 05/02/01 & 0800 & $<.08$ & e. 29 & $<1.0$ & .16 & e.13 & .48 \\
\hline & $05 / 02 / 01^{1}$ & 0830 & $<.08$ & e. 28 & -- & .12 & $<.20$ & .25 \\
\hline & 05/24/01 & 1200 & $<.08$ & .37 & -- & .38 & e. 10 & .22 \\
\hline & $08 / 15 / 01$ & 1600 & $<.08$ & .57 & $<1.0$ & $<.10$ & e. 12 & $<.06$ \\
\hline
\end{tabular}

162 Water-Quality, Streambed-Sediment, and Biological Data from the Clark Fork-Pend Oreille and Spokane River Basins, Montana, Idaho, and Washington, 1998-2001 
Table 25. Trace-element concentration data for ground-water samples collected for the surface-water/ground-water interaction study of the Spokane River and surrounding basin-fill aquifers, Idaho and Washington, 2000-01 (Continued)

\begin{tabular}{|c|c|c|c|c|c|c|c|c|}
\hline $\begin{array}{c}\text { Sele- } \\
\text { nium, } \\
\text { dis- } \\
\text { solved } \\
(\mu \mathrm{g} / \mathrm{L})\end{array}$ & $\begin{array}{c}\text { Silver, } \\
\text { dis- } \\
\text { solved } \\
(\mu \mathrm{g} / \mathrm{L})\end{array}$ & $\begin{array}{c}\text { Stron- } \\
\text { tium, } \\
\text { dis- } \\
\text { solved } \\
(\mu \mathrm{g} / \mathrm{L})\end{array}$ & $\begin{array}{l}\text { Thal- } \\
\text { lium, } \\
\text { dis- } \\
\text { solved } \\
(\mu \mathrm{g} / \mathrm{L}\end{array}$ & $\begin{array}{c}\text { Uranium, } \\
\text { dis- } \\
\text { solved } \\
(\mu \mathrm{g} / \mathrm{L})\end{array}$ & $\begin{array}{c}\text { Vana- } \\
\text { dium, } \\
\text { dis- } \\
\text { solved } \\
(\mu \mathrm{g} / \mathrm{L})\end{array}$ & $\begin{array}{l}\text { Zinc, } \\
\text { total } \\
\text { recov- } \\
\text { erable } \\
(\mu \mathrm{g} / \mathrm{L})\end{array}$ & $\begin{array}{c}\text { Zinc, } \\
\text { dis- } \\
\text { solved } \\
(\mu \mathrm{g} / \mathrm{L})\end{array}$ & $\begin{array}{c}\text { Well } \\
\text { number } \\
\text { (fig. 5) }\end{array}$ \\
\hline$<.70$ & $<1.0$ & 17 & $<.90$ & $<1.0$ & $<1.0$ & -- & 14 & M10 \\
\hline$<.70$ & $<1.0$ & 17 & $<.90$ & $<1.0$ & $<1.0$ & -- & 15 & \\
\hline$<.33$ & $<1.0$ & 17 & $<.04$ & $<.02$ & e.13 & -- & 19 & \\
\hline$<.33$ & $<1.0$ & 16 & $<.04$ & e.01 & e.11 & -- & 20 & \\
\hline$<.33$ & $<1.0$ & 19 & $<.04$ & $<.02$ & $<.21$ & -- & 40 & \\
\hline$<.30$ & $<1.0$ & 20 & $<.04$ & $<.02$ & e. 12 & -- & 25 & \\
\hline$<.30$ & $<1.0$ & 20 & $<.04$ & $<.02$ & e. 10 & 23 & 16 & \\
\hline$<.70$ & $<1.0$ & 17 & $<.90$ & $<1.0$ & $<1.0$ & 27 & 23 & M11 \\
\hline$<.70$ & $<1.0$ & 17 & $<.90$ & $<1.0$ & $<1.0$ & 27 & 23 & \\
\hline$<2.4$ & $<.20$ & $<.10$ & $<.10$ & $<.20$ & -- & -- & $<.50$ & \\
\hline$<.70$ & $<1.0$ & 17 & $<.90$ & $<1.0$ & $<1.0$ & -- & 23 & \\
\hline$<.70$ & $<1.0$ & 17 & $<.90$ & $<1.0$ & $<1.0$ & -- & 23 & \\
\hline$<.33$ & $<1.0$ & 16 & .04 & e. 01 & e. 12 & -- & 28 & \\
\hline$<.33$ & $<1.0$ & 18 & $<.04$ & e. 01 & $<.21$ & -- & 40 & \\
\hline$<.33$ & $<1.0$ & 16 & $<.04$ & $<.02$ & e. 11 & -- & 45 & \\
\hline$<.33$ & $<1.0$ & 21 & $<.04$ & $<.02$ & .22 & 51 & 43 & \\
\hline$<.33$ & $<1.0$ & 20 & $<.04$ & $<.02$ & .28 & -- & 43 & \\
\hline$<.30$ & $<1.0$ & 20 & $<.04$ & $<.02$ & e.16 & -- & 48 & \\
\hline$<.30$ & $<1.0$ & 20 & $<.04$ & $<.02$ & e. 16 & -- & 48 & \\
\hline$<.30$ & $<.20$ & $<.10$ & $<.10$ & $<.20$ & -- & -- & $<.50$ & \\
\hline$<.30$ & $<1.0$ & 21 & $<.04$ & $<.02$ & .24 & 42 & 27 & \\
\hline$<.33$ & $<1.0$ & 18 & $<.04$ & e. 01 & e. 21 & -- & 4.0 & M12 \\
\hline$<.33$ & $<1.0$ & 18 & $<.04$ & e. 01 & e.11 & -- & 11 & \\
\hline$<.33$ & $<1.0$ & 16 & $<.04$ & $<.02$ & e. 12 & -- & 18 & \\
\hline$<.33$ & $<1.0$ & 19 & $<.04$ & $<.02$ & e. 18 & 18 & 18 & \\
\hline$<.33$ & $<1.0$ & 19 & $<.04$ & $<.02$ & .41 & -- & 19 & \\
\hline$<.30$ & $<1.0$ & 20 & $<.04$ & $<.02$ & e. 16 & -- & 18 & \\
\hline$<.30$ & $<1.0$ & 20 & $<.04$ & $<.02$ & e. 17 & 12 & 8.4 & \\
\hline$<.70$ & $<1.0$ & 17 & $<.90$ & $<1.0$ & $<1.0$ & 14 & 12 & M13 \\
\hline$<.70$ & $<1.0$ & 17 & $<.90$ & $<1.0$ & $<1.0$ & -- & 11 & \\
\hline$<.33$ & $<1.0$ & 16 & $<.04$ & e. 01 & e. 14 & -- & 13 & \\
\hline$<.33$ & $<1.0$ & 18 & $<.04$ & e. 02 & e. 10 & -- & 12 & \\
\hline$<.33$ & $<1.0$ & 17 & $<.04$ & e. 01 & $<.21$ & -- & 10 & \\
\hline$<.33$ & $<1.0$ & 20 & .11 & $<.02$ & $<.21$ & 23 & 23 & \\
\hline$<.33$ & $<1.0$ & 19 & $<.04$ & $<.02$ & .27 & -- & 23 & \\
\hline$<.30$ & $<1.0$ & 21 & $<.04$ & e. 01 & e.11 & -- & 25 & \\
\hline$<.30$ & $<1.0$ & 21 & $<.04$ & $<.02$ & e. 19 & 15 & 14 & \\
\hline$<.33$ & $<1.0$ & 15 & $<.04$ & .03 & e. 18 & -- & $<1.0$ & M14 \\
\hline$<.33$ & $<1.0$ & 17 & $<.04$ & .03 & $<.21$ & -- & $<1.0$ & \\
\hline$<.33$ & $<1.0$ & 16 & $<.04$ & .03 & e. 10 & -- & $<1.0$ & \\
\hline$<.33$ & $<1.0$ & 19 & $<.04$ & e. 01 & $<.21$ & 1.1 & $<1.0$ & \\
\hline$<.33$ & $<1.0$ & 18 & $<.04$ & e. 01 & $<.21$ & -- & $<1.0$ & \\
\hline$<.30$ & $<1.0$ & 20 & e. 03 & .03 & e. 15 & -- & $<1.0$ & \\
\hline$<.30$ & $<1.0$ & 20 & $<.04$ & $<.02$ & .26 & 1.5 & $<1.0$ & \\
\hline
\end{tabular}


Table 25. Trace-element concentration data for ground-water samples collected for the surface-water/ground-water interaction study of the Spokane River and surrounding basin-fill aquifers, Idaho and Washington, 2000-01 (Continued)

\begin{tabular}{|c|c|c|c|c|c|c|c|c|c|}
\hline $\begin{array}{c}\text { Well } \\
\text { number } \\
\text { (fig. 5) }\end{array}$ & $\begin{array}{c}\text { Site } \\
\text { identification } \\
\text { number }\end{array}$ & Date & Time & $\begin{array}{l}\text { Alumi- } \\
\text { num, } \\
\text { dis- } \\
\text { solved } \\
(\mu \mathrm{g} / \mathrm{L})\end{array}$ & $\begin{array}{l}\text { Anti- } \\
\text { mony, } \\
\text { dis- } \\
\text { solved } \\
(\mu \mathrm{g} / \mathrm{L})\end{array}$ & $\begin{array}{c}\text { Arsenic, } \\
\text { total } \\
\text { recov- } \\
\text { erable } \\
(\mu \mathrm{g} / \mathrm{L})\end{array}$ & $\begin{array}{c}\text { Arsenic, } \\
\text { dis- } \\
\text { solved } \\
(\mu \mathrm{g} / \mathrm{L})\end{array}$ & $\begin{array}{c}\text { Barium, } \\
\text { dis- } \\
\text { solved } \\
(\mu \mathrm{g} / \mathrm{L})\end{array}$ & $\begin{array}{c}\text { Beryl- } \\
\text { lium, } \\
\text { dis- } \\
\text { solved } \\
(\mu \mathrm{g} / \mathrm{L})\end{array}$ \\
\hline \multirow[t]{8}{*}{ M15 } & 474115117060301 & $08 / 10 / 00$ & 1000 & $<1.0$ & $<1.0$ & -- & 4.6 & 23 & $<1.0$ \\
\hline & & $10 / 03 / 00$ & 1700 & 3.9 & .19 & -- & 4.5 & 24 & $<.06$ \\
\hline & & $12 / 19 / 00$ & 1300 & 5.3 & .19 & -- & 4.3 & 26 & $<.06$ \\
\hline & & $03 / 28 / 01$ & 0900 & $<1.0$ & .18 & -- & 3.6 & 24 & $<.06$ \\
\hline & & $05 / 02 / 01$ & 1000 & $<1.0$ & .19 & 4.8 & 4.0 & 24 & $<.06$ \\
\hline & & $05 / 02 / 01^{1}$ & 1030 & $<1.0$ & .18 & -- & 4.0 & 23 & $<.06$ \\
\hline & & $05 / 24 / 01$ & 1100 & $<1.0$ & .20 & -- & 4.2 & 26 & $<.06$ \\
\hline & & $08 / 13 / 01$ & 1100 & $<1.0$ & .22 & 4.6 & 4.2 & 20 & $<.06$ \\
\hline \multirow[t]{6}{*}{ M16 } & 474140117060401 & $08 / 10 / 00$ & 0800 & 8.8 & $<1.0$ & 3.0 & 3.1 & 27 & $<1.0$ \\
\hline & & $10 / 04 / 00$ & 1400 & 4.3 & .22 & -- & 2.9 & 27 & $<.06$ \\
\hline & & $12 / 19 / 00$ & 1200 & 5.4 & .24 & -- & 3.1 & 28 & $<.06$ \\
\hline & & $05 / 01 / 01$ & 1600 & 6.5 & .22 & -- & 3.4 & 27 & $<.06$ \\
\hline & & $05 / 23 / 01$ & 1700 & 5.1 & .07 & -- & 3.0 & 28 & $<.06$ \\
\hline & & $08 / 15 / 01$ & 1400 & 14 & .30 & -- & 2.9 & 24 & $<.06$ \\
\hline \multirow[t]{7}{*}{ M17 } & 474033117062501 & $08 / 08 / 00$ & 1300 & 3.1 & $<1.0$ & -- & $<.90$ & 12 & $<1.0$ \\
\hline & & $10 / 03 / 00$ & 1500 & 2.7 & .06 & -- & .32 & 12 & $<.06$ \\
\hline & & $12 / 14 / 00$ & 1600 & 2.6 & $<.05$ & -- & .28 & 12 & $<.06$ \\
\hline & & $03 / 27 / 01$ & 0800 & 6.4 & .06 & -- & .29 & 12 & $<.06$ \\
\hline & & $05 / 01 / 01$ & 1500 & 2.8 & .05 & -- & .32 & 12 & $<.06$ \\
\hline & & $05 / 22 / 01$ & 1200 & 2.7 & $<.05$ & -- & .30 & 13 & $<.06$ \\
\hline & & $08 / 15 / 01$ & 1100 & 2.1 & .07 & -- & .28 & 11 & $<.06$ \\
\hline \multirow[t]{7}{*}{ M18 } & 474050117064201 & $08 / 08 / 00$ & 1500 & $<1.0$ & $<1.0$ & -- & $<.90$ & 8.8 & $<1.0$ \\
\hline & & $10 / 03 / 00$ & 1200 & 5.8 & .61 & -- & .31 & 10 & $<.06$ \\
\hline & & $12 / 19 / 00$ & 1600 & $<1.0$ & .52 & -- & .20 & 9.5 & $<.06$ \\
\hline & & $03 / 27 / 01$ & 0900 & $<1.0$ & .41 & -- & e. 17 & 9.9 & $<.06$ \\
\hline & & $05 / 01 / 01$ & 1200 & $<1.0$ & .50 & -- & .24 & 9.8 & $<.06$ \\
\hline & & $05 / 22 / 01$ & 1500 & 4.5 & .40 & -- & e. 17 & 10 & $<.06$ \\
\hline & & $08 / 15 / 01$ & 1200 & 1.0 & .43 & -- & .22 & 11 & $<.06$ \\
\hline \multirow[t]{7}{*}{ M19 } & 474053117064701 & $08 / 08 / 00$ & 1600 & 4.4 & $<1.0$ & -- & $<.90$ & 15 & $<1.0$ \\
\hline & & $10 / 03 / 00$ & 1400 & 4.8 & .71 & -- & .52 & 15 & $<.06$ \\
\hline & & $12 / 19 / 00$ & 1500 & $<1.0$ & .59 & -- & .25 & 15 & $<.06$ \\
\hline & & $03 / 27 / 01$ & 1000 & 4.6 & .42 & -- & .23 & 14 & $<.06$ \\
\hline & & $05 / 01 / 01$ & 1300 & 6.7 & .55 & -- & .38 & 15 & $<.06$ \\
\hline & & $05 / 22 / 01$ & 1300 & 5.3 & .47 & -- & .26 & 15 & $<.06$ \\
\hline & & 08/15/01 & 1300 & 4.8 & .70 & $<2.0$ & .35 & 18 & $<.06$ \\
\hline \multirow[t]{6}{*}{ M20 } & 474016117085601 & $10 / 05 / 00$ & 0800 & 3.7 & .17 & -- & .94 & 13 & $<.06$ \\
\hline & & $12 / 20 / 00$ & 1000 & 5.1 & .24 & -- & 1.0 & 13 & $<.06$ \\
\hline & & $03 / 29 / 01$ & 0800 & $<1.0$ & .23 & -- & 1.0 & 15 & $<.06$ \\
\hline & & $05 / 02 / 01$ & 1600 & 5.4 & .18 & -- & .98 & 15 & $<.06$ \\
\hline & & $05 / 21 / 01$ & 1300 & 1.0 & .12 & -- & 1.0 & 16 & $<.06$ \\
\hline & & $08 / 15 / 01$ & 0600 & 4.9 & .21 & -- & 1.1 & 13 & $<.06$ \\
\hline
\end{tabular}

164 Water-Quality, Streambed-Sediment, and Biological Data from the Clark Fork-Pend Oreille and Spokane River Basins, Montana, Idaho, and Washington, 1998-2001 
Table 25. Trace-element concentration data for ground-water samples collected for the surface-water/ground-water interaction study of the Spokane River and surrounding basin-fill aquifers, Idaho and Washington, 2000-01 (Continued)

\begin{tabular}{|c|c|c|c|c|c|c|c|c|c|}
\hline $\begin{array}{c}\text { Boron, } \\
\text { dis- } \\
\text { solved } \\
(\mu \mathrm{g} / \mathrm{L})\end{array}$ & $\begin{array}{c}\text { Cadmium, } \\
\text { total } \\
\text { recov- } \\
\text { erable } \\
(\mu \mathrm{g} / \mathrm{L})\end{array}$ & $\begin{array}{c}\text { Cad- } \\
\text { mium, } \\
\text { dis- } \\
\text { solved } \\
(\mu \mathrm{g} / \mathrm{L})\end{array}$ & $\begin{array}{c}\text { Chro- } \\
\text { mium, } \\
\text { dis- } \\
\text { solved } \\
(\mu \mathrm{g} / \mathrm{L})\end{array}$ & $\begin{array}{c}\text { Cobalt, } \\
\text { dis- } \\
\text { solved } \\
(\mu \mathrm{g} / \mathrm{L})\end{array}$ & $\begin{array}{c}\text { Copper, } \\
\text { dis- } \\
\text { solved } \\
(\mu \mathrm{g} / \mathrm{L})\end{array}$ & $\begin{array}{l}\text { Iron, } \\
\text { total } \\
\text { recov- } \\
\text { erable } \\
(\mu \mathrm{g} / \mathrm{L})\end{array}$ & $\begin{array}{c}\text { Iron, } \\
\text { dis- } \\
\text { solved } \\
(\mu \mathrm{g} / \mathrm{L})\end{array}$ & $\begin{array}{l}\text { Lead, } \\
\text { total } \\
\text { recov- } \\
\text { erable } \\
(\mu \mathrm{g} / \mathrm{L})\end{array}$ & $\begin{array}{c}\text { Well } \\
\text { number } \\
\text { (fig. 5) }\end{array}$ \\
\hline 9.2 & -- & .15 & 1.2 & .13 & .63 & -- & $<10$ & -- & \\
\hline 12 & -- & $<.04$ & e. 50 & .07 & .28 & -- & $<10$ & -- & \\
\hline$<7.0$ & -- & $<.04$ & $<.80$ & .06 & .25 & -- & $<10$ & -- & \\
\hline$<7.0$ & $<.04$ & $<.04$ & e.54 & .04 & e. 20 & 81 & e6.8 & $<1.0$ & \\
\hline$<7.0$ & -- & $<.04$ & e. 52 & .04 & .29 & -- & -- & -- & \\
\hline e5.5 & -- & $<.04$ & e.74 & .08 & .28 & -- & $<10$ & -- & \\
\hline$<7.0$ & .04 & e. 02 & $<.80$ & .09 & e. 17 & 40 & $<10$ & $<1.0$ & \\
\hline e11 & $<1.0$ & $<1.0$ & e. 65 & $<1.0$ & 1.3 & $<21$ & $<10$ & $<1.0$ & M16 \\
\hline 10 & -- & $<.04$ & e. 60 & .12 & .34 & -- & $<10$ & -- & \\
\hline 14 & -- & $<.04$ & e. 44 & .07 & .31 & -- & $<10$ & -- & \\
\hline 14 & -- & $<.04$ & 1.5 & .08 & .32 & -- & $<10$ & -- & \\
\hline 16 & -- & $<.04$ & e. 72 & .06 & .36 & -- & $<10$ & -- & \\
\hline e6.1 & -- & $<.04$ & $<.80$ & .04 & .47 & -- & $<10$ & -- & \\
\hline$<12$ & -- & $<1.0$ & $<.80$ & $<1.0$ & $<1.0$ & -- & $<10$ & -- & M17 \\
\hline$<7.0$ & -- & $<.04$ & .85 & .04 & .30 & -- & $<10$ & -- & \\
\hline$<7.0$ & -- & $<.04$ & $<.80$ & .03 & 1.0 & -- & $<10$ & -- & \\
\hline 12 & -- & $<.04$ & e. 63 & .03 & .24 & -- & $<10$ & -- & \\
\hline$<7.0$ & -- & $<.04$ & e.77 & .04 & e. 16 & -- & $<10$ & -- & \\
\hline$<7.0$ & -- & $<.04$ & 1.3 & .02 & e. 15 & -- & $<10$ & -- & \\
\hline$<7.0$ & -- & $<.04$ & $<.80$ & e. 01 & e. 18 & -- & $<10$ & -- & \\
\hline$<12$ & -- & $<1.0$ & $<.80$ & $<1.0$ & $<1.0$ & -- & $<10$ & -- & M18 \\
\hline 16 & -- & $<.04$ & 1.2 & .08 & .41 & -- & e5.4 & -- & \\
\hline$<7.0$ & -- & $<.04$ & $<.80$ & .02 & .39 & -- & $<10$ & -- & \\
\hline$<7.0$ & -- & $<.04$ & e. 53 & .02 & .31 & -- & $<10$ & -- & \\
\hline$<7.0$ & -- & $<.04$ & $<.80$ & .03 & .36 & -- & $<10$ & -- & \\
\hline 12 & -- & $<.04$ & .90 & .02 & .40 & -- & $<10$ & -- & \\
\hline e4.3 & -- & $<.04$ & 2.3 & e. 01 & .23 & -- & e7.7 & -- & \\
\hline 14 & -- & $<1.0$ & $<.80$ & $<1.0$ & $<1.0$ & -- & $<10$ & -- & M19 \\
\hline 14 & -- & .09 & e. 63 & .04 & .64 & -- & $<10$ & -- & \\
\hline$<7.0$ & -- & .07 & $<.80$ & .02 & 4.1 & -- & $<10$ & -- & \\
\hline 14 & -- & .16 & .88 & .02 & .82 & -- & $<10$ & -- & \\
\hline 11 & -- & .06 & e. 55 & .02 & .91 & -- & $<10$ & -- & \\
\hline 12 & -- & .26 & 1.2 & .02 & .76 & -- & $<10$ & -- & \\
\hline 9.9 & .17 & .16 & 9.1 & .12 & .89 & 60 & 34 & $<1.0$ & \\
\hline 8.1 & -- & $<.04$ & e. 45 & .04 & .61 & -- & $<10$ & -- & M20 \\
\hline 14 & -- & $<.04$ & $<.80$ & .04 & 1.1 & -- & $<10$ & -- & \\
\hline$<7.0$ & -- & $<.04$ & e. 44 & .03 & .62 & -- & $<10$ & -- & \\
\hline 15 & -- & $<.04$ & .99 & .05 & .39 & -- & e8.1 & -- & \\
\hline e6.2 & -- & $<.04$ & $<.80$ & .03 & .30 & -- & $<10$ & -- & \\
\hline 11 & -- & $<.04$ & 1.0 & .03 & .29 & -- & e6.4 & -- & \\
\hline
\end{tabular}


Table 25. Trace-element concentration data for ground-water samples collected for the surface-water/ground-water interaction study of the Spokane River and surrounding basin-fill aquifers, Idaho and Washington, 2000-01 (Continued)

\begin{tabular}{|c|c|c|c|c|c|c|c|c|}
\hline $\begin{array}{c}\text { Well } \\
\text { number } \\
\text { (fig. 5) }\end{array}$ & Date & Time & $\begin{array}{c}\text { Lead, } \\
\text { dis- } \\
\text { solved } \\
(\mu \mathrm{g} / \mathrm{L})\end{array}$ & $\begin{array}{c}\text { Lithium, } \\
\text { dis- } \\
\text { solved } \\
(\mu \mathrm{g} / \mathrm{L})\end{array}$ & $\begin{array}{c}\text { Manganese, } \\
\text { total } \\
\text { recov- } \\
\text { erable } \\
(\mu \mathrm{g} / \mathrm{L})\end{array}$ & $\begin{array}{c}\text { Manganese, } \\
\text { dis- } \\
\text { solved } \\
(\mu \mathrm{g} / \mathrm{L})\end{array}$ & $\begin{array}{c}\text { Molyb- } \\
\text { denum, } \\
\text { dis- } \\
\text { solved } \\
(\mu \mathrm{g} / \mathrm{L})\end{array}$ & $\begin{array}{c}\text { Nickel, } \\
\text { dis- } \\
\text { solved } \\
(\mu \mathrm{g} / \mathrm{L})\end{array}$ \\
\hline \multirow[t]{8}{*}{ M15 } & $08 / 10 / 00$ & 1000 & $<1.0$ & 3.5 & -- & $<1.0$ & 1.6 & $<1.0$ \\
\hline & $10 / 03 / 00$ & 1700 & e. 05 & 4.2 & -- & .11 & 1.5 & .94 \\
\hline & $12 / 19 / 00$ & 1300 & $<.08$ & 3.9 & -- & .11 & 1.4 & .22 \\
\hline & 03/28/01 & 0900 & $<.08$ & 3.8 & -- & e. 08 & 1.4 & .27 \\
\hline & 05/02/01 & 1000 & $<.08$ & 3.7 & 2.1 & e. 08 & 1.4 & e. 03 \\
\hline & $05 / 02 / 01^{1}$ & 1030 & $<.08$ & -- & -- & e. 10 & 1.4 & e. 03 \\
\hline & $05 / 24 / 01$ & 1100 & $<.08$ & 3.6 & -- & .22 & 1.5 & .24 \\
\hline & 08/13/01 & 1100 & $<.08$ & 3.2 & 1.2 & $<.10$ & 1.4 & $<.06$ \\
\hline \multirow[t]{6}{*}{ M16 } & $08 / 10 / 00$ & 0800 & $<1.0$ & 3.8 & $<1.0$ & $<1.0$ & 1.9 & $<1.0$ \\
\hline & $10 / 04 / 00$ & 1400 & $<.08$ & 4.6 & -- & e.06 & 1.8 & .89 \\
\hline & $12 / 19 / 00$ & 1200 & $<.08$ & 4.2 & -- & .13 & 1.8 & .30 \\
\hline & 05/01/01 & 1600 & .10 & 4.1 & -- & .19 & 1.9 & .19 \\
\hline & 05/23/01 & 1700 & $<.08$ & 4.3 & -- & .13 & 1.9 & .50 \\
\hline & 08/15/01 & 1400 & e. 08 & 3.7 & -- & $<.10$ & 1.8 & $<.06$ \\
\hline \multirow[t]{7}{*}{ M17 } & 08/08/00 & 1300 & $<1.0$ & .51 & -- & 1.2 & $<1.0$ & $<1.0$ \\
\hline & $10 / 03 / 00$ & 1500 & $<.08$ & .54 & -- & .44 & e. 17 & .49 \\
\hline & $12 / 14 / 00$ & 1600 & $<.08$ & .70 & -- & .32 & e. 17 & .57 \\
\hline & 03/27/01 & 0800 & $<.08$ & .55 & -- & .17 & e. 16 & .58 \\
\hline & 05/01/01 & 1500 & $<.08$ & .52 & -- & .21 & e. 14 & .26 \\
\hline & $05 / 22 / 01$ & 1200 & $<.08$ & .54 & -- & .16 & e. 14 & .46 \\
\hline & $08 / 15 / 01$ & 1100 & 1.0 & .53 & -- & $<.10$ & e. 15 & $<.06$ \\
\hline \multirow[t]{7}{*}{ M18 } & $08 / 08 / 00$ & 1500 & $<1.0$ & e. 20 & -- & $<1.0$ & $<1.0$ & $<1.0$ \\
\hline & $10 / 03 / 00$ & 1200 & $<.08$ & .46 & -- & .71 & .21 & 1.4 \\
\hline & $12 / 19 / 00$ & 1600 & $<.08$ & e. 28 & -- & .13 & $<.20$ & .45 \\
\hline & 03/27/01 & 0900 & $<.08$ & .36 & -- & e. 08 & $<.20$ & .23 \\
\hline & 05/01/01 & 1200 & $<.08$ & e. 30 & -- & .12 & e.11 & .27 \\
\hline & $05 / 22 / 01$ & 1500 & $<.08$ & e. 30 & -- & .19 & e. 11 & .58 \\
\hline & 08/15/01 & 1200 & $<.08$ & .31 & -- & $<.10$ & e. 12 & $<.06$ \\
\hline \multirow[t]{7}{*}{ M19 } & 08/08/00 & 1600 & $<1.0$ & .44 & -- & 1.7 & $<1.0$ & $<1.0$ \\
\hline & $10 / 03 / 00$ & 1400 & $<.08$ & .51 & -- & 6.0 & e.14 & .51 \\
\hline & $12 / 19 / 00$ & 1500 & .34 & .36 & -- & .15 & $<.20$ & .39 \\
\hline & 03/27/01 & 1000 & $<.08$ & .36 & -- & e.09 & $<.20$ & .65 \\
\hline & 05/01/01 & 1300 & e. 04 & .36 & -- & 3.4 & e. 11 & .47 \\
\hline & $05 / 22 / 01$ & 1300 & $<.08$ & .38 & -- & .16 & $<.20$ & .60 \\
\hline & 08/15/01 & 1300 & $<.08$ & .48 & 3.8 & .81 & .60 & 9.8 \\
\hline \multirow[t]{6}{*}{ M20 } & $10 / 05 / 00$ & 0800 & $<.08$ & .97 & -- & e. 06 & .30 & .40 \\
\hline & $12 / 20 / 00$ & 1000 & .23 & 1.0 & -- & e. 08 & .33 & .34 \\
\hline & 03/29/01 & 0800 & $<.08$ & .98 & -- & e. 07 & .23 & .29 \\
\hline & 05/02/01 & 1600 & $<.08$ & 1.1 & -- & .13 & .36 & .61 \\
\hline & $05 / 21 / 01$ & 1300 & $<.08$ & 1.2 & -- & .11 & .31 & .30 \\
\hline & 08/15/01 & 0600 & $<.08$ & .76 & -- & $<.10$ & .36 & .23 \\
\hline
\end{tabular}


Table 25. Trace-element concentration data for ground-water samples collected for the surface-water/ground-water interaction study of the Spokane River and surrounding basin-fill aquifers, Idaho and Washington, 2000-01 (Continued)

\begin{tabular}{|c|c|c|c|c|c|c|c|c|}
\hline $\begin{array}{c}\text { Sele- } \\
\text { nium, } \\
\text { dis- } \\
\text { solved } \\
(\mu \mathrm{g} / \mathrm{L})\end{array}$ & $\begin{array}{c}\text { Silver, } \\
\text { dis- } \\
\text { solved } \\
(\mu \mathrm{g} / \mathrm{L})\end{array}$ & $\begin{array}{c}\text { Stron- } \\
\text { tium, } \\
\text { dis- } \\
\text { solved } \\
(\mu \mathrm{g} / \mathrm{L})\end{array}$ & $\begin{array}{l}\text { Thal- } \\
\text { lium, } \\
\text { dis- } \\
\text { solved } \\
(\mu \mathrm{g} / \mathrm{L}\end{array}$ & $\begin{array}{c}\text { Uranium, } \\
\text { dis- } \\
\text { solved } \\
(\mu \mathrm{g} / \mathrm{L})\end{array}$ & $\begin{array}{c}\text { Vana- } \\
\text { dium, } \\
\text { dis- } \\
\text { solved } \\
(\mu \mathrm{g} / \mathrm{L})\end{array}$ & $\begin{array}{c}\text { Zinc, } \\
\text { total } \\
\text { recov- } \\
\text { erable } \\
(\mu \mathrm{g} / \mathrm{L})\end{array}$ & $\begin{array}{c}\text { Zinc, } \\
\text { dis- } \\
\text { solved } \\
(\mu \mathrm{g} / \mathrm{L})\end{array}$ & $\begin{array}{c}\text { Well } \\
\text { number } \\
\text { (fig. 5) }\end{array}$ \\
\hline e.36 & $<1.0$ & 106 & $<.90$ & 3.1 & $<1.0$ & -- & $<1.0$ & M15 \\
\hline e. 33 & $<1.0$ & 111 & $<.04$ & 3.0 & .43 & -- & $<1.0$ & \\
\hline e. 20 & $<1.0$ & 108 & $<.04$ & 3.5 & .24 & -- & $<1.0$ & \\
\hline$<.33$ & $<1.0$ & 104 & $<.04$ & 3.1 & .44 & -- & $<1.0$ & \\
\hline$<.33$ & $<1.0$ & 104 & $<.04$ & 3.3 & e. 18 & $<1.0$ & $<1.0$ & \\
\hline$<.33$ & $<1.0$ & 102 & $<.04$ & 3.3 & .27 & -- & $<1.0$ & \\
\hline$<.30$ & $<1.0$ & 111 & e.04 & 3.6 & .59 & -- & $<1.0$ & \\
\hline$<.30$ & $<1.0$ & 104 & $<.04$ & 3.0 & e. 20 & 1.1 & $<1.0$ & \\
\hline e. 40 & $<1.0$ & 118 & $<.90$ & 3.6 & $<1.0$ & $<1.0$ & $<1.0$ & M16 \\
\hline e. 29 & $<1.0$ & 122 & $<.04$ & 3.9 & e.12 & -- & $<1.0$ & \\
\hline e. 23 & $<1.0$ & 119 & $<.04$ & 3.8 & .37 & -- & $<1.0$ & \\
\hline e. 21 & $<1.0$ & 117 & $<.04$ & 3.6 & .93 & -- & $<1.0$ & \\
\hline e. 19 & $<1.0$ & 117 & $<.04$ & 3.8 & .65 & -- & $<1.0$ & \\
\hline e. 25 & $<1.0$ & 112 & $<.04$ & 3.3 & e. 14 & -- & 2.3 & \\
\hline$<.70$ & $<1.0$ & 26 & $<.90$ & $<1.0$ & $<1.0$ & -- & $<1.0$ & M17 \\
\hline$<.33$ & $<1.0$ & 25 & $<.04$ & .10 & $<.21$ & -- & $<1.0$ & \\
\hline$<.33$ & $<1.0$ & 26 & e. 04 & .12 & e. 16 & -- & 1.2 & \\
\hline$<.33$ & $<1.0$ & 24 & $<.04$ & .11 & e. 16 & -- & $<1.0$ & \\
\hline$<.33$ & $<1.0$ & 30 & $<.04$ & .11 & .35 & -- & $<1.0$ & \\
\hline$<.30$ & $<1.0$ & 27 & $<.04$ & .10 & e. 12 & -- & $<1.0$ & \\
\hline$<.30$ & $<1.0$ & 26 & $<.04$ & .03 & e. 11 & -- & $<1.0$ & \\
\hline$<.70$ & $<1.0$ & 17 & $<.90$ & $<1.0$ & $<1.0$ & -- & $<1.0$ & M18 \\
\hline$<.33$ & $<1.0$ & 18 & $<.04$ & .03 & e. 15 & -- & $<1.0$ & \\
\hline$<.33$ & $<1.0$ & 18 & $<.04$ & .02 & e. 16 & -- & $<1.0$ & \\
\hline$<.33$ & $<1.0$ & 18 & $<.04$ & .02 & e. 18 & -- & $<1.0$ & \\
\hline$<.33$ & $<1.0$ & 23 & $<.04$ & .03 & .28 & -- & $<1.0$ & \\
\hline$<.30$ & $<1.0$ & 21 & $<.04$ & .02 & e. 14 & -- & $<1.0$ & \\
\hline$<.30$ & $<1.0$ & 22 & $<.04$ & $<.02$ & e. 14 & -- & $<1.0$ & \\
\hline$<.70$ & $<1.0$ & 20 & $<.90$ & $<1.0$ & $<1.0$ & -- & 8.9 & M19 \\
\hline$<.33$ & $<1.0$ & 19 & $<.04$ & e. 02 & e. 18 & -- & 9.9 & \\
\hline$<.33$ & $<1.0$ & 19 & $<.04$ & e. 01 & e. 17 & -- & 9.3 & \\
\hline$<.33$ & $<1.0$ & 17 & $<.04$ & e. 01 & e. 12 & -- & 19 & \\
\hline$<.33$ & $<1.0$ & 29 & $<.04$ & e. 02 & .31 & -- & 12 & \\
\hline$<.30$ & $<1.0$ & 20 & $<.04$ & e. 01 & e. 13 & -- & 26 & \\
\hline$<.30$ & $<1.0$ & 23 & $<.04$ & $<.02$ & $<.20$ & 20 & 20 & \\
\hline$<.33$ & $<1.0$ & 36 & $<.04$ & .38 & $<.21$ & -- & $<1.0$ & M20 \\
\hline$<.33$ & $<1.0$ & 38 & $<.04$ & .45 & .33 & -- & $<1.0$ & \\
\hline$<.33$ & $<1.0$ & 45 & $<.04$ & .62 & .42 & -- & $<1.0$ & \\
\hline$<.33$ & $<1.0$ & 46 & $<.04$ & .59 & $<.21$ & -- & $<1.0$ & \\
\hline$<.30$ & $<1.0$ & 44 & $<.04$ & .63 & .38 & -- & 1.1 & \\
\hline$<.30$ & $<1.0$ & 42 & $<.04$ & .40 & $<.20$ & -- & $<1.0$ & \\
\hline
\end{tabular}


Table 25. Trace-element concentration data for ground-water samples collected for the surface-water/ground-water interaction study of the Spokane River and surrounding basin-fill aquifers, Idaho and Washington, 2000-01 (Continued)

\begin{tabular}{|c|c|c|c|c|c|c|c|c|c|}
\hline $\begin{array}{c}\text { Well } \\
\text { number } \\
\text { (fig. 5) }\end{array}$ & $\begin{array}{c}\text { Site } \\
\text { identification } \\
\text { number }\end{array}$ & Date & Time & $\begin{array}{l}\text { Alumi- } \\
\text { num, } \\
\text { dis- } \\
\text { solved } \\
(\mu \mathrm{g} / \mathrm{L})\end{array}$ & $\begin{array}{l}\text { Anti- } \\
\text { mony, } \\
\text { dis- } \\
\text { solved } \\
(\mu \mathrm{g} / \mathrm{L})\end{array}$ & $\begin{array}{c}\text { Arsenic, } \\
\text { total } \\
\text { recov- } \\
\text { erable } \\
(\mu \mathrm{g} / \mathrm{L})\end{array}$ & $\begin{array}{c}\text { Arsenic, } \\
\text { dis- } \\
\text { solved } \\
(\mu \mathrm{g} / \mathrm{L})\end{array}$ & $\begin{array}{c}\text { Barium, } \\
\text { dis- } \\
\text { solved } \\
(\mu \mathrm{g} / \mathrm{L})\end{array}$ & $\begin{array}{c}\text { Beryl- } \\
\text { lium, } \\
\text { dis- } \\
\text { solved } \\
(\mu \mathrm{g} / \mathrm{L})\end{array}$ \\
\hline \multirow[t]{6}{*}{ M21 } & 474037117091301 & $10 / 05 / 00$ & 1200 & 3.7 & .94 & -- & .27 & 18 & $<.06$ \\
\hline & & $12 / 20 / 00$ & 1400 & $<1.0$ & .84 & -- & .29 & 13 & $<.06$ \\
\hline & & $03 / 29 / 01$ & 0900 & $<1.0$ & .34 & -- & .22 & 13 & $<.06$ \\
\hline & & 05/03/01 & 1200 & $<1.0$ & .62 & -- & .12 & 15 & $<.06$ \\
\hline & & $05 / 21 / 01$ & 1600 & 4.5 & .49 & -- & e. 18 & 17 & $<.06$ \\
\hline & & $08 / 15 / 01$ & 0700 & 3.2 & .83 & $<2.0$ & .22 & 18 & $<.06$ \\
\hline \multirow[t]{10}{*}{ M22 } & 474038117091201 & $10 / 05 / 00$ & 1300 & $<1.0$ & .74 & -- & .33 & 19 & $<.06$ \\
\hline & & $10 / 05 / 00^{\mathbf{R}}$ & 1301 & 4.6 & .75 & -- & .33 & 18 & $<.06$ \\
\hline & & $10 / 05 / 00^{\mathbf{F b}}$ & 1305 & $<.30$ & $<.20$ & -- & -- & $<.20$ & $<.20$ \\
\hline & & $12 / 20 / 00$ & 1500 & 5.1 & .71 & -- & .25 & 17 & $<.06$ \\
\hline & & $12 / 20 / 00^{\mathbf{R}}$ & 1501 & 5.2 & .68 & -- & .26 & 17 & $<.06$ \\
\hline & & $12 / 20 / 00^{\mathbf{F b}}$ & 1511 & $<.30$ & $<.20$ & -- & -- & $<.20$ & $<.20$ \\
\hline & & $03 / 29 / 01$ & 1000 & $<1.0$ & .24 & -- & .22 & 16 & $<.06$ \\
\hline & & $05 / 03 / 01$ & 1300 & $<1.0$ & .45 & -- & .28 & 13 & $<.06$ \\
\hline & & $05 / 21 / 01$ & 1700 & 4.9 & .48 & -- & .21 & 18 & $<.06$ \\
\hline & & $08 / 15 / 01$ & 0800 & 4.8 & .70 & $<2.0$ & .20 & 18 & $<.06$ \\
\hline \multirow[t]{6}{*}{ M23 } & 474046117091501 & $10 / 05 / 00$ & 1100 & $<1.0$ & .65 & -- & .35 & 17 & $<.06$ \\
\hline & & $12 / 20 / 00$ & 1300 & 4.9 & .50 & -- & .22 & 16 & $<.06$ \\
\hline & & $03 / 29 / 01$ & 1100 & $<1.0$ & .26 & -- & .24 & 19 & $<.06$ \\
\hline & & $05 / 03 / 01$ & 1100 & $<1.0$ & .59 & -- & .21 & 18 & $<.06$ \\
\hline & & $05 / 21 / 01$ & 1500 & 5.1 & .58 & -- & .33 & 19 & $<.06$ \\
\hline & & $08 / 15 / 01$ & 1000 & $<1.0$ & .85 & $<2.0$ & .49 & 22 & $<.06$ \\
\hline \multirow[t]{8}{*}{ M24 } & 474109117091701 & $10 / 05 / 00$ & 1000 & 3.8 & .19 & -- & 1.7 & 32 & $<.06$ \\
\hline & & $12 / 20 / 00$ & 1200 & $<1.0$ & .18 & -- & 1.8 & 32 & $<.06$ \\
\hline & & 03/29/01 & 1200 & $<1.0$ & $<.05$ & -- & 2.0 & 30 & $<.06$ \\
\hline & & $03 / 29 / 01^{\mathbf{R}}$ & 1201 & $<1.0$ & $<.05$ & -- & 1.9 & 30 & $<.06$ \\
\hline & & $03 / 29 / 01^{\mathbf{F b}}$ & 1211 & $<.30$ & $<.20$ & -- & $<.20$ & $<.20$ & $<.20$ \\
\hline & & $05 / 03 / 01$ & 1000 & 4.4 & .21 & -- & 1.8 & 29 & $<.06$ \\
\hline & & $05 / 21 / 01$ & 1400 & $<5.1$ & .13 & -- & 2.0 & 32 & $<.06$ \\
\hline & & $08 / 15 / 01$ & 0900 & 2.7 & .20 & -- & 1.8 & 26 & $<.06$ \\
\hline \multirow[t]{6}{*}{ M25 } & 474026117115301 & $10 / 04 / 00$ & 1700 & 4.3 & .30 & -- & 2.4 & 25 & e.04 \\
\hline & & $12 / 20 / 00$ & 0900 & 5.5 & .28 & -- & 2.0 & 25 & $<.06$ \\
\hline & & $03 / 29 / 01$ & 1400 & 1.0 & $<.05$ & -- & 2.6 & 24 & $<.06$ \\
\hline & & $05 / 02 / 01$ & 1700 & 5.5 & .44 & -- & 3.2 & 5.7 & $<.06$ \\
\hline & & $05 / 21 / 01$ & 1900 & 1.3 & .27 & -- & 2.6 & 21 & $<.06$ \\
\hline & & 08/14/01 & 1500 & 1.2 & .31 & 3.0 & 2.7 & 18 & $<.06$ \\
\hline
\end{tabular}

${ }^{1}$ Sample filtered through 0.001-tangential-flow filtration unit.

${ }^{2}$ Sample filtered through $0.1-\mu \mathrm{m}$ capsule filter. 
Table 25. Trace-element concentration data for ground-water samples collected for the surface-water/ground-water interaction study of the Spokane River and surrounding basin-fill aquifers, Idaho and Washington, 2000-01 (Continued)

\begin{tabular}{|c|c|c|c|c|c|c|c|c|c|}
\hline $\begin{array}{c}\text { Boron, } \\
\text { dis- } \\
\text { solved } \\
(\mu \mathrm{g} / \mathrm{L})\end{array}$ & $\begin{array}{c}\text { Cadmium, } \\
\text { total } \\
\text { recov- } \\
\text { erable } \\
(\mu \mathrm{g} / \mathrm{L})\end{array}$ & $\begin{array}{c}\text { Cad- } \\
\text { mium, } \\
\text { dis- } \\
\text { solved } \\
(\mu \mathrm{g} / \mathrm{L})\end{array}$ & $\begin{array}{l}\text { Chro- } \\
\text { mium, } \\
\text { dis- } \\
\text { solved } \\
(\mu \mathrm{g} / \mathrm{L})\end{array}$ & $\begin{array}{c}\text { Cobalt, } \\
\text { dis- } \\
\text { solved } \\
(\mu \mathrm{g} / \mathrm{L})\end{array}$ & $\begin{array}{c}\text { Copper, } \\
\text { dis- } \\
\text { solved } \\
(\mu \mathrm{g} / \mathrm{L})\end{array}$ & $\begin{array}{l}\text { Iron, } \\
\text { total } \\
\text { recov- } \\
\text { erable } \\
(\mu \mathrm{g} / \mathrm{L})\end{array}$ & $\begin{array}{c}\text { Iron, } \\
\text { dis- } \\
\text { solved } \\
(\mu \mathrm{g} / \mathrm{L})\end{array}$ & $\begin{array}{l}\text { Lead, } \\
\text { total } \\
\text { recov- } \\
\text { erable } \\
(\mu \mathrm{g} / \mathrm{L})\end{array}$ & $\begin{array}{c}\text { Well } \\
\text { number } \\
\text { (fig. 5) }\end{array}$ \\
\hline 8.9 & -- & .06 & $<.80$ & .03 & .90 & -- & $<10$ & -- & M21 \\
\hline$<7.0$ & -- & $<.04$ & $<.80$ & .02 & 2.3 & -- & $<10$ & -- & \\
\hline$<7.0$ & -- & $<.04$ & $<.80$ & e. 01 & .82 & -- & $<10$ & -- & \\
\hline$<7.0$ & -- & .05 & e. 47 & .02 & .58 & -- & e6.8 & -- & \\
\hline 15 & -- & .04 & e. 63 & .02 & .55 & -- & $<10$ & -- & \\
\hline 9.9 & .06 & .05 & 1.8 & .04 & .54 & e9.1 & 10 & $<1.0$ & \\
\hline$<7.0$ & -- & .21 & e. 48 & .02 & .50 & -- & $<10$ & -- & M22 \\
\hline 9.8 & -- & .22 & e.78 & .04 & .56 & -- & $<10$ & -- & \\
\hline$<2.0$ & -- & $<.30$ & $<.20$ & $<.20$ & $<.20$ & -- & $<3.0$ & -- & \\
\hline 14 & -- & .13 & $<.80$ & .03 & .56 & -- & $<10$ & -- & \\
\hline 15 & -- & .13 & $<.80$ & .02 & .44 & -- & $<10$ & -- & \\
\hline$<2.0$ & -- & $<.30$ & $<.20$ & $<.20$ & $<.20$ & -- & $<3.0$ & -- & \\
\hline$<7.0$ & -- & .27 & $<.80$ & e. 01 & .92 & -- & $<10$ & -- & \\
\hline$<7.0$ & -- & .08 & e. 62 & .02 & .64 & -- & e8.7 & -- & \\
\hline 12 & -- & .25 & 1.3 & .02 & .58 & -- & $<10$ & -- & \\
\hline 15 & .15 & .12 & $<.80$ & .03 & .41 & 27 & e5.2 & $<1.0$ & \\
\hline$<7.0$ & -- & .41 & $<.80$ & .02 & .64 & -- & $<10$ & -- & M23 \\
\hline 13 & -- & .41 & .88 & .04 & 1.3 & -- & $<10$ & -- & \\
\hline$<7.0$ & -- & .46 & $<.80$ & e. 01 & .79 & -- & $<10$ & -- & \\
\hline$<7.0$ & -- & .42 & $<.80$ & .02 & .62 & -- & e5.6 & -- & \\
\hline 15 & -- & .39 & $<.80$ & .02 & .76 & -- & $<10$ & -- & \\
\hline e 4.7 & .50 & .52 & $<.80$ & e. 01 & .72 & 16 & $<10$ & $<1.0$ & \\
\hline 9.2 & -- & $<.04$ & e. 58 & .11 & .30 & -- & $<10$ & -- & M24 \\
\hline e 4.3 & -- & $<.04$ & $<.80$ & .08 & .38 & -- & $<10$ & -- & \\
\hline$<7.0$ & -- & $<.04$ & $<.80$ & .05 & .45 & -- & $<10$ & -- & \\
\hline$<7.0$ & -- & $<.04$ & e. .55 & .05 & .72 & -- & $<10$ & -- & \\
\hline$<2.0$ & -- & $<.30$ & $<.20$ & $<.20$ & $<.20$ & -- & $<3.0$ & -- & \\
\hline 12 & -- & $<.04$ & 1.3 & .06 & .31 & -- & e7.5 & -- & \\
\hline$<13$ & -- & $<.04$ & 1.1 & .06 & .26 & -- & $<10$ & -- & \\
\hline 7.2 & -- & $<.04$ & 6.2 & .10 & e. 20 & -- & 28 & -- & \\
\hline 11 & -- & $<.04$ & $<.80$ & .11 & .32 & -- & $<10$ & -- & M25 \\
\hline 16 & -- & $<.04$ & $<.80$ & .06 & .28 & -- & $<10$ & -- & \\
\hline$<7.0$ & -- & $<.04$ & $<.80$ & .04 & 1.8 & -- & $<10$ & -- & \\
\hline 12 & -- & $<.04$ & $<.80$ & .02 & .67 & -- & e6.2 & -- & \\
\hline e5.3 & -- & $<.04$ & 1.1 & .04 & .24 & -- & $<10$ & -- & \\
\hline$<7.0$ & $<.04$ & $<.04$ & .94 & .02 & e. 14 & $<14$ & $<10$ & $<1.0$ & \\
\hline
\end{tabular}


Table 25. Trace-element concentration data for ground-water samples collected for the surface-water/ground-water interaction study of the Spokane River and surrounding basin-fill aquifers, Idaho and Washington, 2000-01 (Continued)

\begin{tabular}{|c|c|c|c|c|c|c|c|c|}
\hline $\begin{array}{c}\text { Well } \\
\text { number } \\
\text { (fig. 5) }\end{array}$ & Date & Time & $\begin{array}{c}\text { Lead, } \\
\text { dis- } \\
\text { solved } \\
(\mu \mathrm{g} / \mathrm{L})\end{array}$ & $\begin{array}{c}\text { Lithium, } \\
\text { dis- } \\
\text { solved } \\
(\mu \mathrm{g} / \mathrm{L})\end{array}$ & $\begin{array}{c}\text { Manganese, } \\
\text { total } \\
\text { recov- } \\
\text { erable } \\
(\mu \mathrm{g} / \mathrm{L})\end{array}$ & $\begin{array}{c}\text { Manganese, } \\
\text { dis- } \\
\text { solved } \\
(\mu \mathrm{g} / \mathrm{L})\end{array}$ & $\begin{array}{c}\text { Molyb- } \\
\text { denum, } \\
\text { dis- } \\
\text { solved } \\
(\mu \mathrm{g} / \mathrm{L})\end{array}$ & $\begin{array}{c}\text { Nickel } \\
\text { dis- } \\
\text { solved } \\
(\mu \mathrm{g} / \mathrm{L})\end{array}$ \\
\hline \multirow[t]{6}{*}{ M21 } & $10 / 05 / 00$ & 1200 & $<.08$ & .45 & -- & e. 08 & e. 13 & .38 \\
\hline & $12 / 20 / 00$ & 1400 & .14 & .34 & -- & .13 & e. 10 & .35 \\
\hline & $03 / 29 / 01$ & 0900 & $<.08$ & e. 26 & -- & $<.10$ & $<.20$ & .10 \\
\hline & 05/03/01 & 1200 & $<.08$ & .52 & -- & e. 10 & e. 13 & .42 \\
\hline & $05 / 21 / 01$ & 1600 & $<.08$ & .35 & -- & e. 08 & $<.20$ & .50 \\
\hline & $08 / 15 / 01$ & 0700 & $<.08$ & e. 29 & $<1.0$ & $<.10$ & .25 & 1.1 \\
\hline \multirow[t]{10}{*}{ M22 } & $10 / 05 / 00$ & 1300 & $<.08$ & .52 & -- & e. 05 & $<.20$ & .45 \\
\hline & $10 / 05 / 00^{\mathbf{R}}$ & 1301 & $<.08$ & .54 & -- & $<1.0$ & e. 18 & 1.1 \\
\hline & $10 / 05 / 00^{\mathbf{F b}}$ & 1305 & $<.30$ & -- & -- & $<.10$ & $<.20$ & $<.50$ \\
\hline & $12 / 20 / 00$ & 1500 & $<.08$ & .59 & -- & e. 06 & e. 13 & .43 \\
\hline & $12 / 20 / 00^{\mathbf{R}}$ & 1501 & $<.08$ & .38 & -- & e. 07 & $<.20$ & .38 \\
\hline & $12 / 20 / 00^{\mathbf{F b}}$ & 1511 & $<.30$ & -- & -- & $<.10$ & $<.20$ & $<.50$ \\
\hline & 03/29/01 & 1000 & $<.08$ & e. 25 & -- & $<.10$ & $<.20$ & .18 \\
\hline & $05 / 03 / 01$ & 1300 & $<.08$ & .59 & -- & .17 & e. 11 & .48 \\
\hline & $05 / 21 / 01$ & 1700 & $<.08$ & .40 & -- & .11 & $<.20$ & .64 \\
\hline & $08 / 15 / 01$ & 0800 & $<.08$ & .34 & 1.2 & .10 & e. 14 & .36 \\
\hline \multirow[t]{6}{*}{ M23 } & $10 / 05 / 00$ & 1100 & $<.08$ & .54 & -- & $<.10$ & $<.20$ & .29 \\
\hline & $12 / 20 / 00$ & 1300 & .09 & .32 & -- & .16 & $<.20$ & .82 \\
\hline & $03 / 29 / 01$ & 1100 & e. 04 & .32 & -- & $<.10$ & $<.20$ & .19 \\
\hline & $05 / 03 / 01$ & 1100 & e. 05 & .48 & -- & $<.10$ & $<.20$ & .22 \\
\hline & $05 / 21 / 01$ & 1500 & $<.08$ & .52 & -- & e. 07 & $<.20$ & .34 \\
\hline & $08 / 15 / 01$ & 1000 & $<.08$ & .70 & $<1.0$ & $<.10$ & e. 10 & .10 \\
\hline \multirow[t]{8}{*}{ M24 } & $10 / 05 / 00$ & 1000 & $<.08$ & 4.2 & -- & $<.10$ & 1.6 & .55 \\
\hline & $12 / 20 / 00$ & 1200 & e. 04 & 4.2 & -- & $<.10$ & 1.5 & $<.06$ \\
\hline & 03/29/01 & 1200 & e. 07 & 4.1 & -- & $<.10$ & 1.6 & $<.06$ \\
\hline & $03 / 29 / 01^{\mathbf{R}}$ & 1201 & e. 04 & 4.0 & -- & $<.10$ & 1.6 & $<.06$ \\
\hline & $03 / 29 / 01^{\mathbf{F b}}$ & 1211 & $<.30$ & -- & -- & $<.10$ & $<.20$ & $<.50$ \\
\hline & 05/03/01 & 1000 & $<.08$ & 4.1 & -- & .11 & 1.8 & .32 \\
\hline & $05 / 21 / 01$ & 1400 & $<.08$ & 3.9 & -- & e.08 & $<1.7$ & .14 \\
\hline & $08 / 15 / 01$ & 0900 & $<.08$ & 3.7 & -- & .31 & 2.0 & 2.7 \\
\hline \multirow[t]{6}{*}{ M25 } & $10 / 04 / 00$ & 1700 & $<.08$ & 3.7 & -- & $<.10$ & 1.5 & .80 \\
\hline & $12 / 20 / 00$ & 0900 & $<.08$ & 3.1 & -- & $<.10$ & 1.4 & .10 \\
\hline & $03 / 29 / 01$ & 1400 & e.07 & 3.4 & -- & $<.10$ & 1.3 & $<.06$ \\
\hline & $05 / 02 / 01$ & 1700 & e. 05 & 1.5 & -- & e. 06 & .98 & .23 \\
\hline & $05 / 21 / 01$ & 1900 & $<.08$ & 3.2 & -- & $<.10$ & 1.5 & .09 \\
\hline & $08 / 14 / 01$ & 1500 & $<.08$ & 2.7 & $<1.0$ & $<.10$ & 1.4 & $<.06$ \\
\hline
\end{tabular}


Table 25. Trace-element concentration data for ground-water samples collected for the surface-water/ground-water interaction study of the Spokane River and surrounding basin-fill aquifers, Idaho and Washington, 2000-01 (Continued)

\begin{tabular}{|c|c|c|c|c|c|c|c|c|}
\hline $\begin{array}{c}\text { Sele- } \\
\text { nium, } \\
\text { dis- } \\
\text { solved } \\
(\mu \mathrm{g} / \mathrm{L})\end{array}$ & $\begin{array}{l}\text { Silver, } \\
\text { dis- } \\
\text { solved } \\
(\mu \mathrm{g} / \mathrm{L})\end{array}$ & $\begin{array}{c}\text { Stron- } \\
\text { tium, } \\
\text { dis- } \\
\text { solved } \\
(\mu \mathrm{g} / \mathrm{L})\end{array}$ & $\begin{array}{l}\text { Thal- } \\
\text { lium, } \\
\text { dis- } \\
\text { solved } \\
(\mu \mathrm{g} / \mathrm{L}\end{array}$ & $\begin{array}{c}\text { Uranium, } \\
\text { dis- } \\
\text { solved } \\
(\mu \mathrm{g} / \mathrm{L})\end{array}$ & $\begin{array}{c}\text { Vana- } \\
\text { dium, } \\
\text { dis- } \\
\text { solved } \\
(\mu \mathrm{g} / \mathrm{L})\end{array}$ & $\begin{array}{c}\text { Zinc, } \\
\text { total } \\
\text { recov- } \\
\text { erable } \\
(\mu \mathrm{g} / \mathrm{L})\end{array}$ & $\begin{array}{c}\text { Zinc, } \\
\text { dis- } \\
\text { solved } \\
(\mu \mathrm{g} / \mathrm{L})\end{array}$ & $\begin{array}{c}\text { Well } \\
\text { number } \\
\text { (fig. 5) }\end{array}$ \\
\hline$<.33$ & $<1.0$ & 19 & $<.04$ & e.02 & .22 & -- & 10 & M21 \\
\hline$<.33$ & $<1.0$ & 19 & $<.04$ & e. 02 & e. 17 & -- & 1.6 & \\
\hline$<.33$ & $<1.0$ & 20 & $<.04$ & $<.02$ & .24 & -- & 3.7 & \\
\hline$<.33$ & $<1.0$ & 19 & .18 & $<.02$ & $<.21$ & -- & 11 & \\
\hline$<.30$ & $<1.0$ & 20 & $<.04$ & e. 01 & e. 13 & -- & 12 & \\
\hline$<.30$ & $<1.0$ & 22 & $<.04$ & $<.02$ & e. 12 & 12 & 9.8 & \\
\hline$<.33$ & $<1.0$ & 18 & $<.04$ & e. 01 & e. 14 & -- & 27 & M22 \\
\hline$<.33$ & $<1.0$ & 17 & $<.04$ & e. 01 & e. 18 & -- & 23 & \\
\hline -- & $<.20$ & $<.10$ & $<.10$ & $<.20$ & -- & -- & $<.50$ & \\
\hline$<.33$ & $<1.0$ & 21 & $<.04$ & e. 02 & e. 17 & -- & 19 & \\
\hline$<.33$ & $<1.0$ & 19 & $<.04$ & e. 01 & e. 16 & -- & 18 & \\
\hline-- & $<.20$ & $<.10$ & $<.10$ & $<.20$ & -- & -- & $<.50$ & \\
\hline$<.33$ & $<1.0$ & 19 & $<.04$ & $<.02$ & .22 & -- & 43 & \\
\hline$<.33$ & $<1.0$ & 19 & $<.04$ & $<.02$ & $<.21$ & -- & 21 & \\
\hline$<.30$ & $<1.0$ & 20 & $<.04$ & $<.02$ & e. 14 & -- & 42 & \\
\hline$<.30$ & $<1.0$ & 21 & $<.04$ & $<.02$ & $<.20$ & 18 & 17 & \\
\hline$<.33$ & $<1.0$ & 17 & $<.04$ & e. 01 & e. 18 & -- & 42 & M23 \\
\hline$<.33$ & $<1.0$ & 20 & $<.04$ & e. 01 & e. 11 & -- & 48 & \\
\hline$<.33$ & $<1.0$ & 22 & $<.04$ & $<.02$ & e. 18 & -- & 60 & \\
\hline$<.33$ & $<1.0$ & 22 & $<.04$ & $<.02$ & $<.21$ & -- & 53 & \\
\hline$<.30$ & $<1.0$ & 20 & $<.04$ & e. 01 & e. 19 & -- & 49 & \\
\hline$<.30$ & $<1.0$ & 22 & $<.04$ & $<.02$ & e. 16 & 44 & 44 & \\
\hline .40 & $<1.0$ & 137 & $<.04$ & 3.3 & e. 32 & -- & $<1.0$ & M24 \\
\hline e. 22 & $<1.0$ & 130 & $<.04$ & 3.8 & $<.21$ & -- & $<1.0$ & \\
\hline e. 31 & $<1.0$ & 129 & $<.04$ & 3.7 & .63 & -- & $<1.0$ & \\
\hline e. 25 & $<1.0$ & 127 & $<.04$ & 3.7 & .72 & -- & 1.1 & \\
\hline$<.30$ & $<.20$ & $<.10$ & $<.10$ & $<.20$ & -- & -- & $<.50$ & \\
\hline$<.33$ & $<1.0$ & 122 & $<.04$ & 3.6 & e. 18 & -- & $<1.0$ & \\
\hline e. 26 & $<1.0$ & 127 & $<.04$ & $<3.7$ & e. 87 & -- & $<1.0$ & \\
\hline$<.30$ & $<1.0$ & 123 & $<.04$ & 3.2 & $<.20$ & -- & $<1.0$ & \\
\hline e. 25 & $<1.0$ & 109 & .10 & 3.2 & e. 16 & -- & $<1.0$ & M25 \\
\hline$<.33$ & $<1.0$ & 102 & $<.04$ & 3.4 & .31 & -- & $<1.0$ & \\
\hline e. 26 & $<1.0$ & 109 & $<.04$ & 3.6 & .91 & -- & 1.2 & \\
\hline$<.33$ & $<1.0$ & 25 & $<.04$ & .28 & e. 15 & -- & 1.8 & \\
\hline$<.30$ & $<1.0$ & 86 & e.02 & 2.6 & .53 & -- & $<1.0$ & \\
\hline$<.30$ & $<1.0$ & 85 & $<.04$ & 2.4 & $\mathrm{e} 1.7$ & $<1.0$ & $<1.0$ & \\
\hline
\end{tabular}


Table 26. Stable-isotope data for ground-water samples collected during the surface-water/groundwater interaction study of the Spokane River and the surrounding basin-fill aquifers, Idaho and Washington, 1999-2001

[Location number described in text. Abbreviation: per mil, parts per thousand. Symbol: --, no well number assigned]

\begin{tabular}{|c|c|c|c|c|}
\hline \multirow{2}{*}{$\begin{array}{c}\text { Well } \\
\text { number } \\
\text { (fig. 5) }\end{array}$} & \multirow{2}{*}{$\begin{array}{c}\text { Location } \\
\text { number }\end{array}$} & \multirow{2}{*}{ Date } & \multicolumn{2}{|c|}{$\begin{array}{c}\text { Stable-isotope ratio } \\
\text { (per mil) }\end{array}$} \\
\hline & & & $\begin{array}{l}\text { Hydrogen-2/ } \\
\text { Hydrogen-1 }\end{array}$ & $\begin{array}{r}\text { Oxygen-18 } \\
\text { Oxygen-16 }\end{array}$ \\
\hline \multicolumn{5}{|c|}{ Sites located in Washington } \\
\hline 31 & 24N43E05CBBD01 & $05 / 26 / 99$ & -109.54 & -14.21 \\
\hline-- & 25N44E01DCDD01 & $08 / 11 / 00$ & -111.82 & -14.81 \\
\hline M25 & 25N44E11DDAC01 & $\begin{array}{l}08 / 10 / 00 \\
12 / 20 / 00 \\
05 / 02 / 01 \\
08 / 14 / 01\end{array}$ & $\begin{array}{l}-113.22 \\
-114.02 \\
-111.12 \\
-112.12\end{array}$ & $\begin{array}{l}-14.98 \\
-14.94 \\
-14.75 \\
-14.90\end{array}$ \\
\hline-- & 25N44E11DDAD01 & $08 / 10 / 00$ & -112.80 & -15.03 \\
\hline-- & 25N44E11DDDD01 & $08 / 10 / 00$ & -112.87 & -14.91 \\
\hline-- & 25N44E12DABB01 & $08 / 10 / 00$ & -113.94 & -15.06 \\
\hline M4 & 25N45E01ABDD01 & $\begin{array}{l}08 / 07 / 00 \\
12 / 14 / 00 \\
04 / 30 / 01 \\
08 / 13 / 01\end{array}$ & $\begin{array}{l}-111.59 \\
-111.06 \\
-110.37 \\
-108.25\end{array}$ & $\begin{array}{l}-14.78 \\
-14.81 \\
-14.65 \\
-14.31\end{array}$ \\
\hline M5 & 25N45E01ABDD02 & $08 / 07 / 00$ & -111.74 & -14.82 \\
\hline M6 & 25N45E01ABDD03 & 08/07/00 & -111.71 & -14.83 \\
\hline M7 & 25N45E01ACAD01 & $\begin{array}{l}08 / 08 / 00 \\
12 / 14 / 00 \\
04 / 30 / 01 \\
08 / 14 / 01\end{array}$ & $\begin{array}{l}-113.59 \\
-111.09 \\
-111.02 \\
-111.80\end{array}$ & $\begin{array}{l}-15.01 \\
-15.01 \\
-14.95 \\
-14.88\end{array}$ \\
\hline-- & 25N45E01ADBB01 & $08 / 09 / 00$ & -112.54 & -15.05 \\
\hline-- & 25N45E01BBAA01 & $08 / 07 / 00$ & -110.76 & -14.50 \\
\hline M10 & 25N45E01CBBC01 & $\begin{array}{l}08 / 09 / 00 \\
12 / 14 / 00 \\
05 / 02 / 01 \\
08 / 13 / 01\end{array}$ & $\begin{array}{l}-113.51 \\
-111.93 \\
-110.28 \\
-108.42\end{array}$ & $\begin{array}{l}-14.75 \\
-14.80 \\
-14.73 \\
-14.32\end{array}$ \\
\hline M9 & 25N45E01CBBD01 & $08 / 09 / 00$ & -110.39 & -14.75 \\
\hline M8 & 25N45E01CBBD02 & $\begin{array}{l}08 / 09 / 00 \\
12 / 14 / 00 \\
05 / 02 / 01 \\
08 / 13 / 01\end{array}$ & $\begin{array}{l}-111.84 \\
-110.79 \\
-111.40 \\
-109.24\end{array}$ & $\begin{array}{l}-14.71 \\
-14.80 \\
-14.80 \\
-14.26\end{array}$ \\
\hline-- & 25N45E02ACCD01 & $08 / 09 / 00$ & -110.36 & -14.68 \\
\hline M16 & 25N45E03BDDA01 & $\begin{array}{l}08 / 10 / 00 \\
12 / 19 / 00\end{array}$ & $\begin{array}{l}-114.15 \\
-114.56\end{array}$ & $\begin{array}{l}-14.99 \\
-14.99\end{array}$ \\
\hline-- & 25N45E03BDDA02 & $08 / 09 / 00$ & -113.86 & -15.06 \\
\hline-- & 25N45E03CBDD01 & $08 / 07 / 00$ & -112.65 & -14.76 \\
\hline M15 & 25N45E03CDDA01 & $\begin{array}{l}08 / 10 / 00 \\
12 / 19 / 00 \\
05 / 02 / 01 \\
08 / 13 / 01\end{array}$ & $\begin{array}{l}-110.92 \\
-110.66 \\
-112.24 \\
-111.94\end{array}$ & $\begin{array}{l}-14.72 \\
-14.76 \\
-14.71 \\
-14.68\end{array}$ \\
\hline
\end{tabular}


Table 26. Stable-isotope data for ground-water samples collected during the surface-water/groundwater interaction study of the Spokane River and the surrounding basin-fill aquifers, Idaho and Washington, 1999-2001 (Continued)

\begin{tabular}{|c|c|c|c|c|}
\hline \multirow{2}{*}{$\begin{array}{c}\text { Well } \\
\text { number } \\
\text { (fig. 5) }\end{array}$} & \multirow{2}{*}{$\begin{array}{c}\text { Location } \\
\text { number }\end{array}$} & \multirow{2}{*}{ Date } & \multicolumn{2}{|c|}{$\begin{array}{c}\text { Stable-isotope ratio } \\
\text { (per mil) }\end{array}$} \\
\hline & & & $\begin{array}{l}\text { Hydrogen-2/ } \\
\text { Hydrogen-1 }\end{array}$ & $\begin{array}{l}\text { Oxygen-18/ } \\
\text { Oxygen-16 }\end{array}$ \\
\hline \multicolumn{5}{|c|}{ Sites located in Washington (Continued) } \\
\hline M14 & 25N45E03CDDD01 & $\begin{array}{l}12 / 19 / 00 \\
05 / 02 / 01 \\
08 / 15 / 01\end{array}$ & $\begin{array}{l}-111.53 \\
-110.97 \\
-108.64\end{array}$ & $\begin{array}{l}-14.75 \\
-14.73 \\
-14.30\end{array}$ \\
\hline-- & 25N45E04BAAC02 & 08/09/00 & -110.39 & -14.42 \\
\hline-- & 25N45E05DDBA01 & 08/09/00 & -115.14 & -15.08 \\
\hline-- & 25N45E06BBCA01 & $08 / 08 / 00$ & -113.06 & -14.75 \\
\hline-- & 25N45E07AAAA02 & 08/09/00 & -112.34 & -14.94 \\
\hline M24 & 25N45E07AAAA04 & $\begin{array}{l}04 / 01 / 99 \\
08 / 01 / 99 \\
12 / 01 / 99 \\
01 / 31 / 00 \\
03 / 09 / 00 \\
08 / 10 / 00 \\
12 / 20 / 00 \\
05 / 03 / 01 \\
08 / 18 / 01\end{array}$ & $\begin{array}{l}-113.94 \\
-114.44 \\
-114.02 \\
-113.17 \\
-113.95 \\
-113.92 \\
-113.28 \\
-113.46 \\
-113.74\end{array}$ & $\begin{array}{l}-14.91 \\
-14.96 \\
-14.94 \\
-14.95 \\
-14.92 \\
-14.92 \\
-14.91 \\
-14.93 \\
-14.94\end{array}$ \\
\hline M23 & 25N45E07ADDD01 & $\begin{array}{l}04 / 01 / 99 \\
08 / 01 / 99 \\
12 / 01 / 99 \\
01 / 31 / 00 \\
03 / 09 / 00 \\
04 / 25 / 00 \\
08 / 10 / 00\end{array}$ & $\begin{array}{l}-112.84 \\
-113.03 \\
-113.59 \\
-114.34 \\
-112.29 \\
-113.71 \\
-111.66\end{array}$ & $\begin{array}{l}-14.97 \\
-15.16 \\
-15.06 \\
-15.11 \\
-15.02 \\
-15.12 \\
-14.72\end{array}$ \\
\hline 30 & 25N45E08BDAA01 & $08 / 09 / 00$ & -112.02 & -14.78 \\
\hline M22 & $25 \mathrm{~N} 45 \mathrm{E} 08 \mathrm{CBBC} 01$ & $\begin{array}{l}04 / 01 / 99 \\
08 / 01 / 99 \\
12 / 01 / 99 \\
01 / 31 / 00 \\
03 / 09 / 00 \\
04 / 25 / 00 \\
08 / 10 / 00 \\
12 / 20 / 00 \\
05 / 03 / 01 \\
08 / 15 / 01\end{array}$ & $\begin{array}{l}-111.76 \\
-112.50 \\
-113.43 \\
-113.27 \\
-112.04 \\
-112.93 \\
-113.02 \\
-111.55 \\
-111.36 \\
-109.98\end{array}$ & $\begin{array}{l}-14.93 \\
-15.21 \\
-15.07 \\
-14.99 \\
-14.96 \\
-14.71 \\
-14.88 \\
-14.77 \\
-14.74 \\
-14.58\end{array}$ \\
\hline M21 & 25N45E08CBBC02 & $\begin{array}{l}04 / 01 / 99 \\
08 / 01 / 99 \\
12 / 01 / 99 \\
01 / 31 / 00 \\
03 / 09 / 00 \\
04 / 25 / 00 \\
08 / 10 / 00\end{array}$ & $\begin{array}{l}-110.30 \\
-113.89 \\
-111.59 \\
-113.85 \\
-113.37 \\
-113.86 \\
-112.30\end{array}$ & $\begin{array}{l}-14.71 \\
-15.19 \\
-15.10 \\
-15.02 \\
-15.00 \\
-15.06 \\
-14.90\end{array}$ \\
\hline M19 & 25N45E09ADAB01 & $\begin{array}{l}08 / 08 / 00 \\
12 / 19 / 00 \\
05 / 01 / 01 \\
08 / 15 / 01\end{array}$ & $\begin{array}{l}-111.26 \\
-111.11 \\
-111.38 \\
-109.22\end{array}$ & $\begin{array}{l}-14.95 \\
-14.81 \\
-14.84 \\
-14.44\end{array}$ \\
\hline M18 & 25N45E09ADAD01 & $\begin{array}{l}08 / 08 / 00 \\
12 / 19 / 00 \\
05 / 01 / 01 \\
08 / 15 / 01\end{array}$ & $\begin{array}{l}-112.82 \\
-110.52 \\
-111.63 \\
-108.94\end{array}$ & $\begin{array}{l}-14.99 \\
-14.85 \\
-14.78 \\
-14.69\end{array}$ \\
\hline
\end{tabular}


Table 26. Stable-isotope data for ground-water samples collected during the surface-water/groundwater interaction study of the Spokane River and the surrounding basin-fill aquifers, Idaho and Washington, 1999-2001 (Continued)

\begin{tabular}{|c|c|c|c|c|}
\hline \multirow{2}{*}{$\begin{array}{c}\text { Well } \\
\text { number } \\
\text { (fig. 5) }\end{array}$} & \multirow{2}{*}{$\begin{array}{c}\text { Location } \\
\text { number }\end{array}$} & \multirow{2}{*}{ Date } & \multicolumn{2}{|c|}{$\begin{array}{c}\text { Stable-isotope ratio } \\
\text { (per mil) }\end{array}$} \\
\hline & & & $\begin{array}{r}\text { Hydrogen-2/ } \\
\text { Hydrogen-1 }\end{array}$ & $\begin{array}{r}\text { Oxygen-18 } \\
\text { Oxygen-16 }\end{array}$ \\
\hline \multicolumn{5}{|c|}{ Sites located in Washington (Continued) } \\
\hline M11 & 25N45E10BAAA01 & $\begin{array}{l}08 / 09 / 00 \\
12 / 19 / 00 \\
05 / 01 / 01 \\
08 / 16 / 01\end{array}$ & $\begin{array}{l}-112.99 \\
-110.27 \\
-110.34 \\
-109.52\end{array}$ & $\begin{array}{l}-14.74 \\
-14.78 \\
-14.70 \\
-14.27\end{array}$ \\
\hline M13 & 25N45E10BAAA02 & $08 / 09 / 00$ & -112.33 & -14.72 \\
\hline M12 & 25N45E10BAAA03 & $\begin{array}{l}12 / 19 / 00 \\
05 / 01 / 01 \\
08 / 16 / 01\end{array}$ & $\begin{array}{l}-112.30 \\
-110.42 \\
-109.22\end{array}$ & $\begin{array}{l}-14.78 \\
-14.74 \\
-14.34\end{array}$ \\
\hline-- & 25N45E10BDAD01 & $08 / 08 / 00$ & -112.83 & -15.10 \\
\hline M17 & 25N45E10CBDA01 & $\begin{array}{l}08 / 08 / 00 \\
12 / 14 / 00 \\
05 / 01 / 01 \\
08 / 15 / 01\end{array}$ & $\begin{array}{l}-112.64 \\
-112.66 \\
-112.62 \\
-111.28\end{array}$ & $\begin{array}{l}-15.02 \\
-14.95 \\
-14.88 \\
-14.83\end{array}$ \\
\hline-- & 25N45E17BBAA01 & $08 / 09 / 00$ & -110.03 & -14.86 \\
\hline M20 & 25N45E17BBAA05 & $\begin{array}{l}08 / 16 / 99 \\
12 / 14 / 99 \\
01 / 31 / 00 \\
03 / 08 / 00 \\
04 / 25 / 00 \\
08 / 10 / 00 \\
12 / 20 / 00 \\
05 / 02 / 01 \\
08 / 15 / 01\end{array}$ & $\begin{array}{l}-109.81 \\
-111.69 \\
-111.96 \\
-111.12 \\
-110.66 \\
-110.52 \\
-110.60 \\
-111.03 \\
-111.18\end{array}$ & $\begin{array}{l}-14.87 \\
-14.98 \\
-14.89 \\
-14.83 \\
-14.85 \\
-14.92 \\
-14.79 \\
-14.79 \\
-14.84\end{array}$ \\
\hline-- & 25N45E17CDDD01 & $08 / 09 / 00$ & -105.80 & -13.70 \\
\hline-- & 25N45E18DDCB01 & $08 / 09 / 00$ & -108.07 & -14.42 \\
\hline-- & 25N46E06BBCB01 & $08 / 03 / 00$ & -110.51 & -14.89 \\
\hline-- & 26N45E25CCAC01 & $08 / 11 / 00$ & -109.83 & -14.62 \\
\hline-- & 26N45E25DAAC01 & $08 / 11 / 00$ & -114.02 & -15.02 \\
\hline-- & 26N45E32DCBC01 & $08 / 11 / 00$ & -116.38 & -15.26 \\
\hline-- & 26N45E34CADB02 & $08 / 09 / 00$ & -108.32 & -14.24 \\
\hline-- & 26N45E35BDBD03 & 08/09/00 & -109.06 & -14.72 \\
\hline M3 & 26N46E31CBBC01 & $\begin{array}{l}04 / 26 / 99 \\
08 / 17 / 99 \\
12 / 13 / 99 \\
03 / 07 / 00 \\
12 / 20 / 00 \\
05 / 01 / 01 \\
08 / 14 / 01\end{array}$ & $\begin{array}{l}-110.54 \\
-106.07 \\
-108.67 \\
-109.58 \\
-109.23 \\
-111.29 \\
-110.05\end{array}$ & $\begin{array}{l}-14.56 \\
-14.53 \\
-14.54 \\
-14.56 \\
-14.60 \\
-14.57 \\
-14.50\end{array}$ \\
\hline-- & 26N46E31DBAD03 & 08/09/00 & -109.34 & -14.61 \\
\hline
\end{tabular}


Table 26. Stable-isotope data for ground-water samples collected during the surface-water/groundwater interaction study of the Spokane River and the surrounding basin-fill aquifers, Idaho and Washington, 1999-2001 (Continued)

\begin{tabular}{|c|c|c|c|c|}
\hline \multirow{2}{*}{$\begin{array}{c}\text { Well } \\
\text { number } \\
\text { (fig. 5) }\end{array}$} & \multirow{2}{*}{$\begin{array}{c}\text { Location } \\
\text { number }\end{array}$} & \multirow{2}{*}{ Date } & \multicolumn{2}{|c|}{$\begin{array}{c}\text { Stable-isotope ratio } \\
\text { (per mil) }\end{array}$} \\
\hline & & & $\begin{array}{l}\text { Hydrogen-2/ } \\
\text { Hydrogen-1 }\end{array}$ & $\begin{array}{r}\text { Oxygen-18/ } \\
\text { Oxygen-16 }\end{array}$ \\
\hline \multicolumn{5}{|c|}{ Sites located in Idaho } \\
\hline-- & 50N05W04CACC02 & $08 / 17 / 00$ & -112.42 & -14.94 \\
\hline-- & 50N05W06DCDC01 & $08 / 17 / 00$ & -113.00 & -15.04 \\
\hline-- & 50N05W07BCCC01 & $08 / 03 / 00$ & -112.48 & -15.16 \\
\hline M1 & 50N05W07DABC01 & $\begin{array}{l}08 / 08 / 00 \\
12 / 15 / 00 \\
05 / 03 / 01 \\
08 / 14 / 01\end{array}$ & $\begin{array}{l}-115.04 \\
-114.79 \\
-111.28 \\
-110.09\end{array}$ & $\begin{array}{l}-15.11 \\
-15.10 \\
-14.74 \\
-14.24\end{array}$ \\
\hline M2 & 50N05W07DABC02 & $\begin{array}{l}12 / 15 / 00 \\
04 / 30 / 01 \\
08 / 14 / 01\end{array}$ & $\begin{array}{l}-111.62 \\
-110.83 \\
-109.07\end{array}$ & $\begin{array}{l}-14.81 \\
-14.71 \\
-14.33\end{array}$ \\
\hline-- & 50N05W07DBBA01 & $08 / 18 / 00$ & -110.20 & -14.74 \\
\hline-- & 50N06W12BDAC01 & $10 / 01 / 99$ & -108.20 & -14.34 \\
\hline-- & 50N06W12BDAC01 & $12 / 01 / 99$ & -109.96 & -14.64 \\
\hline-- & 50N06W12BDAC01 & $08 / 03 / 00$ & -111.31 & -15.00 \\
\hline-- & 50N06W12CBDB01 & $08 / 18 / 00$ & -110.07 & -14.91 \\
\hline-- & 50N06W12CCAD02 & $08 / 03 / 00$ & -109.08 & -14.71 \\
\hline-- & 50N06W12DBAD01 & $08 / 18 / 00$ & -110.24 & -14.69 \\
\hline 29 & 50N06W12DBCD01 & $\begin{array}{l}12 / 01 / 99 \\
08 / 18 / 00\end{array}$ & $\begin{array}{l}-112.45 \\
-111.24\end{array}$ & $\begin{array}{l}-15.00 \\
-14.77\end{array}$ \\
\hline-- & 50N06W12DDAB01 & $08 / 03 / 00$ & -112.65 & -15.11 \\
\hline-- & 50N06W12DDCD01 & $08 / 18 / 00$ & -107.06 & -13.51 \\
\hline-- & 50N06W12DDDB02 & $08 / 18 / 00$ & -112.65 & -14.79 \\
\hline $\begin{array}{l}-- \\
--\end{array}$ & $\begin{array}{l}\text { 50N06W13CABA01 } \\
51 \mathrm{~N} 05 \mathrm{~W} 31 \mathrm{BCCB} 01\end{array}$ & $\begin{array}{l}08 / 18 / 00 \\
08 / 18 / 00\end{array}$ & $\begin{array}{l}-113.43 \\
-112.98\end{array}$ & $\begin{array}{l}-15.03 \\
-14.98\end{array}$ \\
\hline-- & 51N06W36DAAA01 & $08 / 18 / 00$ & -114.10 & -15.08 \\
\hline
\end{tabular}


Table 27. Trace-element and carbon concentration data for streambed-sediment samples, Northern Rockies Intermontane Basins study unit, 1998-99 1

[Samples were wet sieved in the field through a 63-micrometer nylon-mesh sieve. Abbreviation: $\mu \mathrm{g} / \mathrm{g}$, micrograms per gram, dry weight; R, replicate. Symbol: <, less than reporting level]

\begin{tabular}{|c|c|c|c|c|c|c|c|}
\hline $\begin{array}{c}\text { Site } \\
\text { number } \\
\text { (fig. 1) }\end{array}$ & Site name & Date & Time & $\begin{array}{c}\text { Alumi- } \\
\text { num } \\
\text { (percent) }\end{array}$ & $\begin{array}{l}\text { Anti- } \\
\text { mony } \\
(\mu \mathrm{g} / \mathrm{g})\end{array}$ & $\begin{array}{c}\text { Arsenic } \\
(\mu \mathrm{g} / \mathrm{g})\end{array}$ & $\begin{array}{c}\text { Barium } \\
(\mu \mathrm{g} / \mathrm{g})\end{array}$ \\
\hline 1 & Clark Fork near Galen, Mont. & $08 / 18 / 98$ & 1600 & 6.8 & 9.9 & 180 & 803 \\
\hline 2 & Rock Creek near Clinton, Mont. & $08 / 11 / 98$ & 1300 & 6.1 & 2.2 & 11 & 675 \\
\hline 3 & Clark Fork at Turah Bridge, near Bonner, Mont. & $08 / 11 / 98$ & 1030 & 6.7 & 6.1 & 66 & 856 \\
\hline $3^{\mathbf{R}}$ & Clark Fork at Turah Bridge, near Bonner, Mont. & $08 / 11 / 98$ & 1045 & 7.2 & 6.3 & 63 & 880 \\
\hline $3^{\mathbf{R}}$ & Clark Fork at Turah Bridge, near Bonner, Mont. & $08 / 11 / 98$ & 1130 & 7.3 & 6.3 & 75 & 849 \\
\hline 4 & Blackfoot River above Nevada Creek, near Helmville, Mont. & $08 / 17 / 98$ & 1510 & 6.5 & 1.7 & 12 & 794 \\
\hline 5 & Bitterroot River near Missoula, Mont. & 08/10/98 & 1715 & 6.9 & .57 & 2.6 & 819 \\
\hline 9 & Clark Fork at St. Regis, Mont. & 09/08/98 & 1330 & 6.1 & 2.2 & 22 & 760 \\
\hline 10 & North Fork Flathead River near Columbia Falls, Mont. & $07 / 22 / 98$ & 1100 & 6.5 & .76 & 6.1 & 656 \\
\hline 11 & Middle Fork Flathead River near West Glacier, Mont. & $07 / 21 / 98$ & 1430 & 5.6 & .78 & 5.6 & 706 \\
\hline 12 & Flathead River at Perma, Mont. & 09/09/98 & 1105 & 6.7 & .80 & 5.4 & 760 \\
\hline 13 & Rock Creek near Noxon, Mont. & $06 / 21 / 98$ & 1500 & 6.0 & 1.1 & 9.2 & 740 \\
\hline 14 & Lightning Creek at Clark Fork, Idaho & $07 / 29 / 98$ & 1005 & 8.0 & .80 & 8.3 & 667 \\
\hline 15 & Priest River near Priest River, Idaho & $07 / 28 / 98$ & 1300 & 8.3 & .51 & 7.0 & 883 \\
\hline 16 & Pend Oreille River above Priest River, Idaho & $07 / 28 / 98$ & 1400 & 8.0 & 1.3 & 10 & 851 \\
\hline 23 & North Fork Coeur d'Alene River at Enaville, Idaho & $06 / 16 / 98$ & 1130 & 6.4 & 1.9 & 14 & 620 \\
\hline 24 & South Fork Coeur d'Alene River at Shoshone Park, near Mullan, Idaho & $06 / 23 / 98$ & 1000 & 7.0 & 4.7 & 17 & 920 \\
\hline 31 & South Fork Coeur d'Alene River near Pinehurst, Idaho & $06 / 17 / 98$ & 1100 & 5.3 & 54 & 120 & 760 \\
\hline $31^{\mathbf{R}}$ & South Fork Coeur d'Alene River near Pinehurst, Idaho & $06 / 17 / 98$ & 1105 & 6.0 & 74 & 140 & 900 \\
\hline $31^{\mathbf{R}}$ & South Fork Coeur d'Alene River near Pinehurst, Idaho & $06 / 18 / 98$ & 1100 & 5.9 & 88 & 120 & 860 \\
\hline 31 & South Fork Coeur d'Alene River near Pinehurst, Idaho & 08/17/99 & 1200 & 6.6 & 66 & 170 & 750 \\
\hline 32 & Coeur d'Alene River near Harrison, Idaho & $06 / 18 / 98$ & 1430 & 5.0 & 58 & 190 & 820 \\
\hline 33 & St. Joe River at Red Ives Ranger Station, Idaho & 07/06/98 & 1540 & 7.7 & .26 & 8.5 & 511 \\
\hline 33 & St. Joe River at Red Ives Ranger Station, Idaho & 08/30/99 & 1500 & 7.0 & .19 & 4.9 & 450 \\
\hline 34 & St. Joe River at Calder, Idaho & 07/08/98 & 0945 & 6.8 & .75 & 7.0 & 576 \\
\hline 35 & Spokane River near Post Falls, Idaho & 08/03/98 & 1045 & 8.2 & 12 & 27 & 630 \\
\hline 40 & Hangman Creek at Spokane, Wash. & $06 / 19 / 98$ & 1130 & 6.6 & .90 & 7.8 & 650 \\
\hline 41 & Spokane River at Seven Mile Bridge, near Spokane, Wash. & 08/05/98 & 1215 & 7.6 & 1.1 & 7.8 & 670 \\
\hline
\end{tabular}

${ }^{1}$ Some data previously published in Beckwith (2002). 
Table 27. Trace-element and carbon concentration data for streambed-sediment samples, Northern Rockies Intermontane Basins study unit, 1998-99 (Continued)

\begin{tabular}{|c|c|c|c|c|c|c|c|c|c|c|c|}
\hline $\begin{array}{c}\text { Site } \\
\text { number } \\
\text { (fig. 1) }\end{array}$ & $\begin{array}{c}\text { Beryllium } \\
(\mu \mathrm{g} / \mathrm{g})\end{array}$ & $\begin{array}{c}\text { Bismuth } \\
(\mu \mathbf{g} / \mathbf{g})\end{array}$ & $\begin{array}{l}\text { Cadmium } \\
(\mu \mathrm{g} / \mathrm{g})\end{array}$ & $\begin{array}{l}\text { Calcium } \\
\text { (percent) }\end{array}$ & $\begin{array}{l}\text { Cerium } \\
(\mu \mathrm{g} / \mathrm{g})\end{array}$ & $\begin{array}{c}\text { Cobalt } \\
(\mu \mathrm{g} / \mathrm{g})\end{array}$ & $\begin{array}{l}\text { Chro- } \\
\text { mium } \\
(\mu \mathrm{g} / \mathrm{g})\end{array}$ & $\begin{array}{c}\text { Copper } \\
(\mu \mathbf{g} / \mathbf{g})\end{array}$ & $\begin{array}{c}\text { Europium } \\
\quad(\mu \mathrm{g} / \mathbf{g})\end{array}$ & $\begin{array}{c}\text { Gallium } \\
(\mu \mathbf{g} / \mathbf{g})\end{array}$ & $\begin{array}{r}\text { Gold } \\
(\mu \mathrm{g} / \mathrm{g})\end{array}$ \\
\hline 1 & 3.2 & 9.4 & 6.9 & 3.3 & 72 & 12 & 65 & 1,200 & 1.4 & 17 & $<<1.0$ \\
\hline 2 & 2.4 & $<1.0$ & .22 & .70 & 96 & 6.2 & 45 & 20 & 1.9 & 14 & $<1.0$ \\
\hline 3 & 2.5 & 2.8 & 4.2 & 1.7 & 81 & 11 & 57 & 490 & 1.6 & 16 & $<1.0$ \\
\hline $3^{R}$ & 2.5 & 2.9 & 4.2 & 1.8 & 84 & 11 & 66 & 500 & 1.6 & 17 & $<1.0$ \\
\hline $3^{R}$ & 2.7 & 3.3 & 4.8 & 1.9 & 81 & 11 & 60 & 570 & 1.5 & 17 & $<1.0$ \\
\hline 4 & 1.9 & $<1.0$ & .21 & 2.0 & 106 & 9.5 & 61 & 42 & 1.7 & 16 & $<1.0$ \\
\hline 5 & 2.1 & $<1.0$ & .22 & 1.0 & 96 & 5.4 & 45 & 26 & 1.4 & 17 & $<1.0$ \\
\hline 9 & 2.4 & 1.0 & 1.0 & .86 & 76 & 8.1 & 48 & 140 & 1.4 & 15 & $<1.0$ \\
\hline 10 & 2.1 & $<1.0$ & .32 & 3.7 & 96 & 7.6 & 44 & 19 & 1.7 & 14 & $<1.0$ \\
\hline 11 & 1.7 & $<1.0$ & $<.10$ & 2.5 & 89 & 7.0 & 35 & 18 & 1.4 & 12 & $<1.0$ \\
\hline 12 & 2.5 & $<1.0$ & .14 & .85 & 84 & 9.8 & 48 & 25 & 1.4 & 16 & $<1.0$ \\
\hline 13 & 2.0 & $<1.0$ & .50 & .40 & 92 & 9.1 & 36 & 48 & 1.7 & 13 & $<.05$ \\
\hline 14 & 2.5 & $<1.0$ & .17 & 1.2 & 79 & 12 & 33 & 26 & 1.6 & 17 & $<1.0$ \\
\hline 15 & 3.0 & $<1.0$ & .13 & 1.7 & 128 & 8.7 & 40 & 19 & 1.9 & 19 & $<1.0$ \\
\hline 16 & 2.5 & $<1.0$ & .75 & 1.0 & 73 & 8.6 & 56 & 93 & 1.4 & 18 & $<1.0$ \\
\hline 23 & 2.4 & $<1.0$ & 1.1 & .40 & 99 & 8.9 & 43 & 30 & 1.8 & 13 & $<.05$ \\
\hline 24 & 2.2 & $<1.0$ & 1.4 & .69 & 95 & 12 & 43 & 38 & 1.7 & 16 & $<.05$ \\
\hline 31 & 1.9 & 1.3 & 28 & .40 & 78 & 14 & 36 & 140 & 1.7 & 12 & $<.05$ \\
\hline $31^{\mathbf{R}}$ & 2.2 & 1.5 & 43 & .40 & 92 & 17 & 42 & 190 & 2.0 & 14 & $<.05$ \\
\hline $31^{\mathbf{R}}$ & 2.3 & 1.5 & 83 & .30 & 70 & 22 & 42 & 210 & 1.8 & 13 & $<.05$ \\
\hline 31 & 2.3 & 1.2 & 41 & .44 & 72 & 14 & 43 & 180 & 1.6 & 14 & $<1.0$ \\
\hline 32 & 1.8 & 1.5 & 26 & .40 & 72 & 14 & 38 & 140 & 2.1 & 11 & $<.05$ \\
\hline 33 & 2.6 & $<1.0$ & .10 & .72 & 115 & 10 & 44 & 24 & 2.0 & 19 & $<1.0$ \\
\hline 33 & 2.2 & $<1.0$ & $<1.0$ & .69 & 100 & 8.2 & 42 & 20 & 1.7 & 16 & $<1.0$ \\
\hline 34 & 2.2 & $<1.0$ & .16 & .82 & 106 & 9.4 & 40 & 26 & 1.8 & 16 & $<1.0$ \\
\hline 35 & 2.2 & 1.0 & 24 & 1.0 & 75 & 13 & 44 & 62 & 1.7 & 18 & $<1.0$ \\
\hline 40 & 2.0 & $<1.0$ & .40 & 1.5 & 92 & 12 & 48 & 20 & 1.9 & 14 & $<.05$ \\
\hline 41 & 1.8 & $<1.0$ & 3.3 & 1.5 & 85 & 9.8 & 38 & 26 & 1.6 & 16 & $<1.0$ \\
\hline
\end{tabular}


Table 27. Trace-element and carbon concentration data for streambed-sediment samples, Northern Rockies Intermontane Basins study unit, 1998-99 (Continued)

\begin{tabular}{|c|c|c|c|c|c|c|c|c|c|c|c|}
\hline $\begin{array}{c}\text { Site } \\
\text { number } \\
\text { (fig. 1) }\end{array}$ & $\begin{array}{l}\text { Holmium } \\
(\mu \mathrm{g} / \mathrm{g})\end{array}$ & $\begin{array}{c}\text { Iron } \\
\text { (percent) }\end{array}$ & $\begin{array}{l}\text { Lanthanum } \\
\quad(\mu \mathrm{g} / \mathrm{g})\end{array}$ & $\begin{array}{c}\text { Lead } \\
(\mu \mathrm{g} / \mathrm{g})\end{array}$ & $\begin{array}{c}\text { Lithium } \\
(\mu \mathrm{g} / \mathrm{g})\end{array}$ & $\begin{array}{c}\text { Magnesium } \\
\text { (percent) }\end{array}$ & $\begin{array}{c}\text { Manganese } \\
(\mu \mathbf{g} / \mathbf{g})\end{array}$ & $\begin{array}{c}\text { Mercury } \\
(\mu \mathrm{g} / \mathrm{g})\end{array}$ & $\begin{array}{c}\text { Molyb- } \\
\text { denum } \\
(\mu \mathrm{g} / \mathrm{g})\end{array}$ & $\begin{array}{c}\text { Neody- } \\
\text { mium } \\
(\mu \mathrm{g} / \mathrm{g})\end{array}$ & $\begin{array}{l}\text { Nickel } \\
(\mu \mathrm{g} / \mathrm{g})\end{array}$ \\
\hline 1 & 1.5 & 4.1 & 40 & 200 & 46 & 1.5 & 2,710 & 0.65 & 2.7 & 34 & 24 \\
\hline 2 & 1.6 & 2.5 & 52 & 17 & 31 & .82 & 267 & .07 & .63 & 49 & 15 \\
\hline 3 & 1.5 & 3.1 & 43 & 120 & 33 & 1.0 & 1,740 & 1.1 & .89 & 38 & 21 \\
\hline $3^{R}$ & 1.6 & 3.3 & 46 & 120 & 34 & 1.1 & 1,340 & .90 & .95 & 41 & 21 \\
\hline $3^{R}$ & 1.6 & 3.3 & 44 & 120 & 35 & 1.1 & 1,120 & .80 & 1.2 & 39 & 23 \\
\hline 4 & 1.8 & 3.0 & 57 & 24 & 56 & 1.4 & 574 & .03 & $<.50$ & 52 & 20 \\
\hline 5 & 1.2 & 2.3 & 56 & 49 & 37 & .67 & 194 & .09 & .54 & 48 & 12 \\
\hline 6 & 1.2 & 3.1 & 44 & 53 & 39 & .99 & 270 & .33 & .51 & 37 & 17 \\
\hline 10 & 1.6 & 2.5 & 54 & 17 & 51 & 2.3 & 395 & .04 & .50 & 47 & 18 \\
\hline 11 & 1.3 & 2.3 & 48 & 14 & 44 & 1.9 & 314 & .03 & $<.50$ & 42 & 14 \\
\hline 12 & 1.3 & 2.8 & 45 & 23 & 64 & 1.6 & 400 & .03 & $<.50$ & 39 & 19 \\
\hline 13 & 1.0 & 2.5 & 51 & 58 & 35 & .71 & 720 & .02 & .70 & 43 & 16 \\
\hline 14 & 1.5 & 3.8 & 43 & 25 & 24 & .88 & 581 & .02 & 1.4 & 39 & 14 \\
\hline 15 & 1.4 & 2.8 & 72 & 26 & 33 & 1.1 & 519 & .02 & $<.50$ & 58 & 15 \\
\hline 16 & 1.1 & 2.8 & 42 & 47 & 47 & 1.4 & 282 & .18 & .56 & 37 & 23 \\
\hline 23 & $<1.0$ & 2.6 & 51 & 120 & 37 & .74 & 410 & .09 & .70 & 46 & 19 \\
\hline 24 & $<1.0$ & 2.9 & 50 & 190 & 44 & .48 & 1,200 & .19 & .90 & 43 & 18 \\
\hline 31 & $<1.0$ & 5.6 & 42 & 4,400 & 27 & .37 & 3,600 & 4.5 & 1.4 & 37 & 19 \\
\hline 31 & $<1.0$ & 6.5 & 48 & 6,300 & 30 & .41 & 3,400 & 5.9 & 1.8 & 44 & 22 \\
\hline $31^{2}$ & $<1.0$ & 6.2 & 37 & 9,100 & 32 & .39 & 2,700 & 6.2 & 1.5 & 33 & 25 \\
\hline $31^{2}$ & $<1.0$ & 6.5 & 38 & 5,600 & 34 & .58 & 3,200 & 4.5 & 2.0 & 34 & 20 \\
\hline 32 & $<1.0$ & 10 & 36 & 6,600 & 26 & .48 & 7,600 & 3.9 & 1.1 & 34 & 22 \\
\hline 33 & 1.6 & 3.3 & 63 & 12 & 30 & 1.1 & 452 & .02 & .71 & 56 & 19 \\
\hline 33 & 1.0 & 2.6 & 54 & 4.4 & 26 & .91 & 370 & .02 & $<.50$ & 48 & 15 \\
\hline 34 & 1.4 & 2.9 & 57 & 17 & 38 & 1.1 & 390 & .04 & $<.50$ & 52 & 18 \\
\hline 35 & 1.6 & 3.7 & 39 & 1,600 & 40 & 1.1 & 2,000 & .71 & .80 & 36 & 29 \\
\hline 40 & 1.1 & 3.4 & 53 & 37 & 25 & .78 & 740 & .02 & .50 & 42 & 21 \\
\hline 41 & 1.2 & 3.2 & 48 & 47 & 27 & .87 & 488 & .05 & $<.50$ & 40 & 17 \\
\hline
\end{tabular}


Table 27. Trace-element and carbon concentration data for streambed-sediment samples, Northern Rockies Intermontane Basins study unit, $1998-99^{1}$ (Continued)

\begin{tabular}{|c|c|c|c|c|c|c|c|c|c|c|c|c|}
\hline $\begin{array}{c}\text { Site } \\
\text { number } \\
\text { (fig. 1) }\end{array}$ & $\begin{array}{c}\text { Niobium } \\
(\mu \mathrm{g} / \mathrm{g})\end{array}$ & $\begin{array}{c}\text { Phosphorus } \\
\text { (percent) }\end{array}$ & $\begin{array}{c}\text { Potassium } \\
\text { (percent) }\end{array}$ & $\begin{array}{l}\text { Scandium } \\
(\mu \mathbf{g} / \mathbf{g})\end{array}$ & $\begin{array}{l}\text { Selenium } \\
(\mu \mathrm{g} / \mathrm{g})\end{array}$ & $\begin{array}{l}\text { Silver } \\
(\mu \mathrm{g} / \mathrm{g})\end{array}$ & $\begin{array}{l}\text { Sodium } \\
\text { (percent) }\end{array}$ & $\begin{array}{l}\text { Strontium } \\
\quad(\mu \mathbf{g} / \mathbf{g})\end{array}$ & $\begin{array}{c}\text { Sulfur } \\
\text { (percent) }\end{array}$ & $\begin{array}{c}\text { Tantalum } \\
(\mu \mathrm{g} / \mathrm{g})\end{array}$ & $\begin{array}{c}\text { Thallium } \\
(\mu \mathrm{g} / \mathrm{g})\end{array}$ & $\begin{array}{c}\text { Thorium } \\
(\mu \mathrm{g} / \mathrm{g})\end{array}$ \\
\hline 1 & 10 & 0.15 & 1.9 & 10 & 0.92 & 3.8 & 0.87 & 256 & 0.51 & 1.3 & $<1.0$ & 16 \\
\hline 2 & 7.8 & .11 & 1.9 & 7.2 & .47 & .90 & .88 & 86 & .08 & 1.0 & $<1.0$ & 15 \\
\hline 3 & 11 & .13 & 1.9 & 8.9 & .54 & 2.0 & .90 & 212 & .16 & 1.4 & $<1.0$ & 14 \\
\hline $3^{R}$ & 12 & .13 & 2.1 & 9.2 & .44 & 2.0 & 1.0 & 235 & .13 & 1.7 & $<1.0$ & 14 \\
\hline $3^{R}$ & 12 & .12 & 2.1 & 9.0 & .78 & 2.3 & .93 & 224 & .23 & 1.6 & $<1.0$ & 14 \\
\hline 4 & 11 & .09 & 1.4 & 10 & .23 & 1.1 & .75 & 148 & $<.05$ & 1.3 & $<1.0$ & 15 \\
\hline 5 & 9.4 & .08 & 1.9 & 5.6 & .29 & .92 & 1.7 & 245 & .12 & 1.2 & $<1.0$ & 17 \\
\hline 9 & 13 & .12 & 2.1 & 7.8 & .36 & .70 & 1.1 & 130 & .10 & $<1.0$ & $<1.0$ & 13 \\
\hline 10 & 7.2 & .10 & 2.4 & 10 & .33 & .37 & .79 & 75 & .05 & $<1.0$ & $<1.0$ & 13 \\
\hline 11 & 5.7 & .08 & 2.5 & 8.5 & .15 & .26 & .84 & 64 & $<.05$ & $<1.0$ & $<1.0$ & 12 \\
\hline 12 & 10 & .06 & 2.7 & 9.4 & .19 & .41 & .81 & 49 & $<.05$ & $<1.0$ & $<1.0$ & 12 \\
\hline 13 & 9.8 & .08 & 2.0 & 9.1 & .34 & .45 & 1.0 & 62 & $<.05$ & $<1.0$ & $<1.0$ & 12 \\
\hline 14 & 11 & .07 & 2.5 & 16 & .15 & .61 & 1.7 & 156 & $<.05$ & 1.3 & $<1.0$ & 12 \\
\hline 15 & 15 & .15 & 2.6 & 12 & $<.10$ & .85 & 1.9 & 322 & .05 & 1.9 & $<1.0$ & 20 \\
\hline 16 & 9.1 & .07 & 2.6 & 13 & .38 & .54 & 1.0 & 137 & .12 & $<1.0$ & $<1.0$ & 12 \\
\hline 23 & 10 & .07 & 2.2 & 10 & .25 & .44 & .75 & 61 & $<.05$ & $<1.0$ & $<1.0$ & 13 \\
\hline 24 & 11 & .11 & 2.3 & 10 & .44 & .55 & .81 & 90 & .06 & $<1.0$ & $<1.0$ & 12 \\
\hline 31 & 7.4 & .10 & 1.8 & 8.8 & .32 & .19 & .70 & 72 & .23 & $<1.0$ & $<1.0$ & 11 \\
\hline $31^{R}$ & 10 & .14 & 2.0 & 9.9 & .43 & 28 & .69 & 72 & .29 & $<1.0$ & 1.0 & 13 \\
\hline $31^{\mathbf{R}}$ & 7.5 & .22 & 2.0 & 9.7 & .54 & 29 & .59 & 58 & .55 & $<1.0$ & 1.1 & 11 \\
\hline 31 & 9.8 & .18 & 2.2 & 10 & .45 & 25 & .76 & 75 & .20 & 1.0 & $<1.0$ & 11 \\
\hline 32 & 6.3 & .07 & 1.8 & 8.5 & .28 & 21 & .42 & 41 & .52 & $<1.0$ & $<1.0$ & 9.9 \\
\hline 33 & 11 & .13 & 1.9 & 12 & .24 & .56 & 1.9 & 71 & $<.05$ & 1.4 & $<1.0$ & 16 \\
\hline 33 & 17 & .11 & 1.7 & 10 & .18 & .16 & 2.0 & 66 & $<.05$ & 1.2 & $<1.0$ & 14 \\
\hline 34 & 7.3 & .10 & 1.8 & 11 & .24 & .35 & 1.5 & 75 & $<.05$ & $<1.0$ & $<1.0$ & 14 \\
\hline 35 & 7.7 & .16 & 1.7 & 15 & .54 & 3.0 & .91 & 121 & .09 & $<1.0$ & $<1.0$ & 12 \\
\hline 40 & 13 & .08 & 1.6 & 13 & .16 & .44 & 1.3 & 230 & $<.05$ & $<1.0$ & $<1.0$ & 11 \\
\hline 41 & 8.9 & .10 & 1.8 & 13 & .20 & .50 & 1.6 & 217 & $<.05$ & $<1.0$ & $<1.0$ & 11 \\
\hline
\end{tabular}


Table 27. Trace-element and carbon concentration data for streambed-sediment samples, Northern Rockies Intermontane Basins study unit, 1998-99 (Continued)

\begin{tabular}{|c|c|c|c|c|c|c|c|c|c|c|}
\hline $\begin{array}{c}\begin{array}{c}\text { Site } \\
\text { number } \\
\text { (fig. 1) }\end{array} \\
\text {. }\end{array}$ & $\begin{array}{c}\text { Tin } \\
(\mu \mathrm{g} / \mathrm{g})\end{array}$ & $\begin{array}{l}\text { Titanium } \\
\text { (percent) }\end{array}$ & $\begin{array}{c}\text { Uranium } \\
(\mu \mathrm{g} / \mathrm{g})\end{array}$ & $\begin{array}{c}\text { Vanadium } \\
(\mu \mathbf{g} / \mathbf{g})\end{array}$ & $\underset{(\mu \mathbf{g} / \mathbf{g})}{\text { Yttrium }}$ & $\begin{array}{l}\text { Ytterbium } \\
\quad(\mu \mathrm{g} / \mathrm{g})\end{array}$ & $\begin{array}{c}\text { Zinc } \\
(\mu \mathrm{g} / \mathrm{g})\end{array}$ & $\begin{array}{c}\text { Inorganic } \\
\text { carbon } \\
\text { (percent) }\end{array}$ & $\begin{array}{c}\text { Organic } \\
\text { carbon } \\
\text { (percent) }\end{array}$ & $\begin{array}{c}\text { Carbon, } \\
\text { total } \\
\text { (percent) }\end{array}$ \\
\hline 1 & 6.7 & 0.36 & 6.5 & 67 & 24 & 2.2 & 1,300 & 0.42 & 3.0 & 3.4 \\
\hline 2 & 2.1 & .27 & 6.4 & 44 & 25 & 2.9 & 54 & .02 & 3.9 & 4.0 \\
\hline 3 & 3.4 & .36 & 4.5 & 65 & 25 & 2.7 & 1,000 & .17 & 2.6 & 2.7 \\
\hline $3^{R}$ & 3.8 & .37 & 4.6 & 70 & 27 & 2.7 & 1,200 & .14 & 2.0 & 2.2 \\
\hline $3^{R}$ & 3.7 & .37 & 5.7 & 69 & 26 & 2.8 & 1,000 & .20 & 2.4 & 2.6 \\
\hline 4 & 2.0 & .41 & 3.5 & 62 & 28 & 3.1 & 96 & .56 & 1.4 & 1.9 \\
\hline 5 & 6.5 & .25 & 8.0 & 35 & 20 & 1.8 & 94 & .01 & 2.9 & 2.9 \\
\hline 9 & 2.6 & .27 & 2.9 & 58 & 25 & 2.9 & 320 & .06 & 2.5 & 2.6 \\
\hline 10 & 1.9 & .42 & 4.0 & 61 & 26 & 3.0 & 110 & 1.2 & 1.1 & 2.4 \\
\hline 11 & 1.6 & .36 & 3.6 & 55 & 22 & 2.6 & 50 & .86 & .67 & 1.5 \\
\hline 12 & 2.4 & .23 & 2.3 & 58 & 23 & 3.1 & 74 & .17 & 1.4 & 1.5 \\
\hline 13 & 2.0 & .30 & 5.4 & 47 & 27 & 2.6 & 92 & .02 & 3.0 & 3.1 \\
\hline 14 & 2.7 & .79 & 4.2 & 89 & 24 & 3.3 & 79 & .01 & 1.2 & 1.2 \\
\hline 15 & 2.7 & .49 & 6.7 & 64 & 23 & 2.5 & 75 & .02 & .88 & .90 \\
\hline 16 & 2.8 & .39 & 3.4 & 64 & 19 & 2.1 & 210 & .06 & 1.8 & 1.9 \\
\hline 23 & 2.0 & .30 & 4.3 & 54 & 24 & 2.7 & 210 & .01 & 2.1 & 2.1 \\
\hline 24 & 3.0 & .30 & 5.6 & 58 & 23 & 2.7 & 260 & .03 & 4.9 & 5.0 \\
\hline 31 & 5.0 & .20 & 3.4 & 47 & 18 & 2.1 & 3,700 & .47 & 1.4 & 1.9 \\
\hline $31^{\mathbf{R}}$ & 6.0 & .30 & 4.2 & 54 & 22 & 2.9 & 4,700 & .43 & 1.7 & 2.1 \\
\hline $31^{\mathbf{R}}$ & 5.8 & .30 & 4.4 & 52 & 20 & 2.6 & 10,000 & .30 & 2.3 & 2.6 \\
\hline 31 & 6.5 & .23 & 4.0 & 54 & 17 & 1.8 & 5,300 & .32 & 2.3 & 2.6 \\
\hline 32 & 5.3 & .20 & 3.4 & 43 & 18 & 2.4 & 4,600 & 1.3 & 1.3 & 2.6 \\
\hline 33 & 2.4 & .72 & 7.0 & 62 & 26 & 2.7 & 47 & .02 & 2.7 & 2.7 \\
\hline 33 & 2.3 & .54 & 4.9 & 56 & 22 & 2.3 & 38 & .01 & 2.3 & 2.3 \\
\hline 34 & 2.3 & .54 & 5.4 & 62 & 23 & 3.5 & 65 & .02 & 2.4 & 2.4 \\
\hline 35 & 5.9 & .49 & 7.0 & 70 & 25 & 3.1 & 3,200 & .02 & 3.7 & 3.7 \\
\hline 40 & 2.0 & .60 & 2.5 & 92 & 29 & 3.0 & 100 & .06 & 1.4 & 1.5 \\
\hline 41 & 3.0 & .72 & 3.3 & 81 & 21 & 2.4 & 320 & .02 & 1.4 & 1.4 \\
\hline
\end{tabular}


Table 28. Trace-element and carbon concentration data for streambed-sediment samples collected for a synoptic study of mining-affected areas, Northern Rockies Intermontane Basins study unit, August-September 2000

[Samples were wet-sieved in the field through a 63 -micrometer nylon-mesh sieve. Abbreviations: ${ }^{\circ} \mathrm{C}$, degrees Celsius; $\mu \mathrm{g} / \mathrm{g}$, micrograms per gram, dry weight; R, replicate. Symbols: <, less than reporting level; --, no data]

\begin{tabular}{|c|c|c|c|c|c|c|c|}
\hline $\begin{array}{c}\text { Site } \\
\text { num- } \\
\text { ber } \\
\text { (fig. 1) }\end{array}$ & Station name & Date & Time & $\begin{array}{c}\text { Water } \\
\text { temper- } \\
\text { ature } \\
\left({ }^{\circ} \mathrm{C}\right)\end{array}$ & $\begin{array}{l}\text { Alumi- } \\
\text { num } \\
\text { (per- } \\
\text { cent) }\end{array}$ & $\begin{array}{l}\text { Anti- } \\
\text { mony } \\
(\mu g / g)\end{array}$ & $\begin{array}{c}\text { Arsenic } \\
(\mu \mathrm{g} / \mathrm{g})\end{array}$ \\
\hline 6 & St. Regis River above Rainy Creek, near Saltese, Mont. & $08 / 02 / 00$ & 0930 & 9.5 & 5.3 & 2.2 & 13 \\
\hline 7 & St Regis River near Haugan, Mont. & $08 / 02 / 00$ & 1330 & 15.0 & 5.4 & 2.4 & 10 \\
\hline 8 & St. Regis River near St. Regis, Mont. & 08/03/00 & 0930 & 13.5 & 5.0 & 1.1 & 5.1 \\
\hline 17 & North Fork Coeur d'Alene River above Shoshone Creek, near Prichard, Idaho & 09/06/00 & 1000 & 12.0 & 5.9 & 1.4 & 16 \\
\hline 18 & Prichard Creek near Murray, Idaho & $08 / 22 / 00$ & 0900 & 14.0 & 7.7 & 1.9 & 87 \\
\hline 19 & East Fork Eagle Creek near mouth, near Prichard, Idaho & $08 / 21 / 00$ & 1530 & 16.5 & 7.1 & 4.1 & 21 \\
\hline 20 & West Fork Eagle Creek below Settlers Grove, Idaho & $08 / 21 / 00$ & 1330 & 11.5 & 5.9 & 1.7 & 11 \\
\hline 21 & Beaver Creek near mouth, near Murray, Idaho & $08 / 21 / 00$ & 1130 & 14.5 & 7.2 & 4.5 & 26 \\
\hline 22 & Prichard Creek at mouth, at Prichard, Idaho & $08 / 22 / 00$ & 1200 & 14.5 & 6.8 & 2.5 & 51 \\
\hline 23 & North Fork Coeur d'Alene River at Enaville, Idaho & 09/07/00 & 1530 & 15.0 & 6.1 & 2.2 & 14 \\
\hline 24 & South Fork Coeur d'Alene River at Shoshone Park, near Mullan, Idaho & $08 / 24 / 00$ & 1000 & 11.5 & 6.2 & 3.7 & 22 \\
\hline 25 & South Fork Coeur d'Alene River above Deadman Gulch, near Mullan, Idaho & $08 / 24 / 00$ & 1430 & 13.0 & 4.5 & 28 & 50 \\
\hline 26 & Canyon Creek near Burke, Idaho & $08 / 23 / 00$ & 1030 & 8.0 & 5.9 & 3.7 & 9.4 \\
\hline 27 & Canyon Creek at Woodland Park, Idaho & $08 / 23 / 00$ & 1500 & 19.0 & 4.7 & 110 & 79 \\
\hline 28 & South Fork Coeur d'Alene River at Silverton, Idaho & $08 / 25 / 00$ & 1000 & 12.5 & 5.0 & 45 & 78 \\
\hline 29 & East Fork Pine Creek above Nabob Creek, near Pinehurst, Idaho & $08 / 22 / 00$ & 1400 & 17.5 & 6.1 & 10 & 60 \\
\hline 30 & Pine Creek below Amy Gulch, near Pinehurst, Idaho & $08 / 22 / 00$ & 1600 & 14.0 & 6.1 & 6.4 & 33 \\
\hline 31 & South Fork Coeur d'Alene River near Pinehurst, Idaho & $09 / 06 / 00$ & 1400 & 13.0 & 4.7 & 62 & 140 \\
\hline 33 & St. Joe River at Red Ives Ranger Station, Idaho & $09 / 05 / 00$ & 1230 & 8.5 & 5.7 & .24 & 8.1 \\
\hline 34 & St. Joe River at Calder, Idaho & 09/07/00 & 1100 & 12.5 & 6.2 & .73 & 7.5 \\
\hline $34^{\mathbf{R}}$ & St. Joe River at Calder, Idaho & 09/07/00 & 1101 & -- & 6.4 & .80 & 6.5 \\
\hline
\end{tabular}


Table 28. Trace-element and carbon concentration data for streambed-sediment samples collected for a synoptic study of mining-affected areas, Northern Rockies Intermontane Basins study unit, August-September 2000 (Continued)

\begin{tabular}{|c|c|c|c|c|c|c|c|c|c|c|c|}
\hline $\begin{array}{c}\text { Site } \\
\text { num- } \\
\text { ber } \\
\text { (fig. 1) }\end{array}$ & $\begin{array}{c}\text { Barium } \\
(\mu \mathbf{g} / \mathbf{g})\end{array}$ & $\begin{array}{c}\text { Beryllium } \\
(\mu \mathrm{g} / \mathrm{g})\end{array}$ & $\begin{array}{c}\text { Bismuth } \\
(\mu \mathrm{g} / \mathrm{g})\end{array}$ & $\begin{array}{c}\text { Cadmiuim } \\
(\mu \mathrm{g} / \mathrm{g})\end{array}$ & $\begin{array}{c}\text { Calcium } \\
\text { (percent) }\end{array}$ & $\begin{array}{c}\text { Cerium } \\
(\mu \mathrm{g} / \mathrm{g})\end{array}$ & $\begin{array}{c}\text { Cobalt } \\
(\mu \mathrm{g} / \mathrm{g})\end{array}$ & $\begin{array}{c}\text { Chromium } \\
(\mu \mathrm{g} / \mathrm{g})\end{array}$ & $\begin{array}{c}\text { Copper } \\
(\mu \mathrm{g} / \mathrm{g})\end{array}$ & $\begin{array}{c}\text { Europium } \\
(\mu \mathrm{g} / \mathrm{g})\end{array}$ & $\begin{array}{c}\text { Gallium } \\
(\mu \mathrm{g} / \mathrm{g})\end{array}$ \\
\hline 6 & 600 & 1.8 & $<1.0$ & 1.1 & 0.67 & 76 & 7.7 & 35 & 26 & 1.1 & 14 \\
\hline 7 & 580 & 1.6 & $<1.0$ & .52 & .51 & 66 & 6.5 & 32 & 21 & 1.0 & 13 \\
\hline 8 & 530 & 1.8 & $<1.0$ & $<.1$ & .36 & 76 & 5.6 & 28 & 18 & 1.1 & 12 \\
\hline 17 & 540 & 1.8 & $<1.0$ & $<.1$ & .51 & 84 & 8.4 & 45 & 18 & 1.3 & 14 \\
\hline 18 & 630 & 2.6 & $<1.0$ & 3.1 & .48 & 85 & 16 & 49 & 52 & 1.2 & 19 \\
\hline 19 & 540 & 2.4 & $<1.0$ & 3.0 & .34 & 72 & 10 & 46 & 130 & 1.1 & 18 \\
\hline 20 & 770 & 1.9 & $<1.0$ & .62 & .63 & 83 & 7.6 & 37 & 38 & 1.2 & 15 \\
\hline 21 & 620 & 2.1 & $<1.0$ & 2.2 & .52 & 96 & 11 & 47 & 31 & 1.4 & 18 \\
\hline 22 & 600 & 2.0 & $<1.0$ & 2.1 & .35 & 87 & 9.4 & 45 & 50 & 1.2 & 18 \\
\hline 23 & 610 & 2.1 & $<1.0$ & 1.1 & .40 & 95 & 9.2 & 45 & 30 & 1.3 & 16 \\
\hline 24 & 820 & 2.3 & $<1.0$ & 1.4 & .76 & 63 & 10 & 40 & 32 & 1.1 & 16 \\
\hline 25 & 1,100 & 1.4 & $<1.0$ & 6.4 & .78 & 40 & 60 & 30 & 220 & $<1.0$ & 13 \\
\hline 26 & 690 & 1.8 & $<1.0$ & 1.7 & .74 & 72 & 10 & 36 & 78 & 1.2 & 15 \\
\hline 27 & 500 & 1.7 & $<1.0$ & 62 & .34 & 54 & 12 & 35 & 230 & 1.0 & 13 \\
\hline 28 & 790 & 1.6 & 1.0 & 43 & .55 & 47 & 13 & 36 & 170 & $<1.0$ & 13 \\
\hline 29 & 490 & 1.7 & $<1.0$ & 3.6 & .41 & 79 & 11 & 39 & 50 & 1.1 & 15 \\
\hline 30 & 540 & 2.1 & $<1.0$ & 3.2 & .37 & 98 & 9.5 & 44 & 39 & 1.3 & 15 \\
\hline 31 & 630 & 1.8 & 1.3 & 150 & .43 & 38 & 15 & 34 & 180 & $<1.0$ & 12 \\
\hline 33 & 420 & 1.7 & $<1.0$ & $<.1$ & .71 & 66 & 8.9 & 38 & 18 & 1.1 & 14 \\
\hline 34 & 530 & 1.9 & $<1.0$ & $<.1$ & .82 & 78 & 9.8 & 42 & 25 & 1.2 & 15 \\
\hline $34^{\mathbf{R}}$ & 550 & 2.4 & $<1.0$ & $<.1$ & .84 & 88 & 10 & 44 & 29 & 1.4 & 16 \\
\hline
\end{tabular}


Table 28. Trace-element and carbon concentration data for streambed-sediment samples collected for a synoptic study of mining-affected areas, Northern Rockies Intermontane Basins study unit, August-September 2000 (Continued)

\begin{tabular}{|c|c|c|c|c|c|c|c|c|c|c|c|}
\hline $\begin{array}{c}\text { Site } \\
\text { num- } \\
\text { ber } \\
\text { (fig. 1) }\end{array}$ & $\begin{array}{c}\text { Gold } \\
(\mu \mathrm{g} / \mathrm{g})\end{array}$ & $\begin{array}{c}\text { Holmium } \\
\quad(\mu \mathrm{g} / \mathrm{g})\end{array}$ & $\begin{array}{c}\text { Iron } \\
\text { (percent) }\end{array}$ & $\begin{array}{c}\text { Lantha- } \\
\text { num } \\
(\mu \mathrm{g} / \mathrm{g})\end{array}$ & $\begin{array}{c}\text { Lead } \\
(\mu \mathrm{g} / \mathrm{g})\end{array}$ & $\begin{array}{l}\text { Lithium } \\
\qquad(\mu \mathrm{g} / \mathrm{g})\end{array}$ & $\begin{array}{l}\text { Magne- } \\
\text { sium } \\
\text { (percent) }\end{array}$ & $\begin{array}{c}\text { Manga- } \\
\text { nese } \\
(\mu \mathrm{g} / \mathrm{g})\end{array}$ & $\begin{array}{c}\text { Mercury } \\
(\mu \mathbf{g} / \mathbf{g})\end{array}$ & $\begin{array}{c}\text { Molyb- } \\
\text { denum } \\
(\mu \mathrm{g} / \mathrm{g})\end{array}$ & $\begin{array}{c}\text { Neody- } \\
\text { mium } \\
(\mu \mathrm{g} / \mathrm{g})\end{array}$ \\
\hline 6 & $<1.0$ & $<1.0$ & 2.6 & 39 & 81 & 41 & 0.77 & 640 & 0.15 & 0.50 & 34 \\
\hline 7 & $<1.0$ & $<1.0$ & 2.3 & 34 & 41 & 38 & .75 & 410 & .05 & $<.50$ & 30 \\
\hline 8 & $<1.0$ & $<1.0$ & 2.1 & 39 & 20 & 34 & .72 & 210 & .03 & $<.50$ & 35 \\
\hline 17 & $<1.0$ & $<1.0$ & 3.1 & 43 & 20 & 46 & .72 & 370 & .05 & $<.50$ & 38 \\
\hline 18 & $<1.0$ & $<1.0$ & 4.0 & 42 & 710 & 31 & .71 & 860 & .71 & 2.1 & 37 \\
\hline 19 & $<1.0$ & $<1.0$ & 3.4 & 39 & 1,000 & 27 & .55 & 580 & .13 & 1.2 & 33 \\
\hline 20 & $<1.0$ & $<1.0$ & 2.4 & 41 & 69 & 29 & .50 & 680 & .06 & .80 & 36 \\
\hline 21 & $<1.0$ & $<1.0$ & 3.3 & 48 & 200 & 45 & .56 & 720 & .12 & .94 & 42 \\
\hline 22 & $<1.0$ & $<1.0$ & 3.3 & 44 & 460 & 28 & .55 & 500 & .18 & 1.3 & 38 \\
\hline 23 & $<1.0$ & $<1.0$ & 2.7 & 48 & 160 & 41 & .67 & 580 & .09 & .66 & 42 \\
\hline 24 & $<1.0$ & $<1.0$ & 2.9 & 34 & 150 & 39 & .54 & 1,900 & .13 & .83 & 31 \\
\hline 25 & $<1.0$ & $<1.0$ & 4.7 & 21 & 850 & 29 & .42 & 21,000 & .30 & 1.8 & 19 \\
\hline 26 & $<1.0$ & $<1.0$ & 2.8 & 37 & 200 & 27 & .44 & 1,200 & .19 & 1.4 & 34 \\
\hline 27 & $<1.0$ & $<1.0$ & 5.6 & 28 & 11,000 & 21 & .34 & 2,100 & 5.1 & 1.6 & 25 \\
\hline 28 & $<1.0$ & $<1.0$ & 4.4 & 24 & 4,400 & 31 & .51 & 2,800 & 2.3 & 1.4 & 22 \\
\hline 29 & $<1.0$ & $<1.0$ & 3.5 & 40 & 1,000 & 28 & .44 & 670 & .32 & .78 & 34 \\
\hline 30 & $<1.0$ & $<1.0$ & 3.2 & 49 & 550 & 29 & .43 & 560 & .16 & .71 & 43 \\
\hline 31 & $<1.0$ & $<1.0$ & 6.1 & 20 & 6,400 & 25 & .40 & 3,300 & 4.0 & 1.1 & 18 \\
\hline 33 & $<1.0$ & $<1.0$ & 2.8 & 34 & 8.8 & 24 & .73 & 480 & .02 & .65 & 30 \\
\hline 34 & $<1.0$ & $<1.0$ & 2.9 & 41 & 17 & 38 & .95 & 440 & .03 & .52 & 36 \\
\hline $34^{\mathbf{R}}$ & $<1.0$ & $<1.0$ & 3.0 & 46 & 22 & 39 & .96 & 470 & .03 & $<.50$ & 40 \\
\hline
\end{tabular}


Table 28. Trace-element and carbon concentration data for streambed-sediment samples collected for a synoptic study of mining-affected areas, Northern Rockies Intermontane Basins study unit, August-September 2000 (Continued)

\begin{tabular}{|c|c|c|c|c|c|c|c|c|c|c|c|}
\hline $\begin{array}{c}\text { Site } \\
\text { num- } \\
\text { ber } \\
\text { (fig. 1) }\end{array}$ & $\begin{array}{c}\text { Nickel } \\
(\mu \mathrm{g} / \mathrm{g})\end{array}$ & $\begin{array}{c}\text { Niobium } \\
(\mu \mathrm{g} / \mathrm{g})\end{array}$ & $\begin{array}{c}\text { Phosphorus } \\
\text { (percent) }\end{array}$ & $\begin{array}{c}\text { Potassium } \\
\text { (percent) }\end{array}$ & $\begin{array}{c}\text { Sandium } \\
(\mu \mathrm{g} / \mathrm{g})\end{array}$ & $\begin{array}{c}\text { Selenium } \\
(\mu \mathrm{g} / \mathrm{g})\end{array}$ & $\begin{array}{l}\text { Silver } \\
(\mu \mathrm{g} / \mathrm{g})\end{array}$ & $\begin{array}{c}\text { Sodium } \\
(\mu \mathrm{g} / \mathrm{g})\end{array}$ & $\begin{array}{l}\text { Strontium } \\
\quad(\mu \mathrm{g} / \mathrm{g})\end{array}$ & $\begin{array}{c}\text { Sulfur } \\
\text { (percent) }\end{array}$ & $\begin{array}{l}\text { Tantalum } \\
\quad(\mu \mathrm{g} / \mathrm{g})\end{array}$ \\
\hline 6 & 14 & 9.0 & 0.10 & 1.7 & 8.1 & 0.45 & 0.15 & 1.1 & 88 & 0.06 & $<1.0$ \\
\hline 7 & 13 & 7.7 & .09 & 1.8 & 8.3 & .29 & .15 & 1.0 & 67 & .06 & $<1.0$ \\
\hline 8 & 11 & 5.5 & .07 & 1.7 & 7.0 & .19 & $<.1$ & 1.2 & 51 & $<.05$ & $<1.0$ \\
\hline 17 & 17 & 8.9 & .10 & 2.1 & 10 & .32 & .10 & .65 & 67 & .06 & $<1.0$ \\
\hline 18 & 34 & 15 & .08 & 2.4 & 14 & .45 & .44 & 1.1 & 110 & $<.05$ & 1.2 \\
\hline 19 & 23 & 14 & .06 & 2.3 & 12 & .35 & .49 & 1.0 & 88 & $<.05$ & 1.0 \\
\hline 20 & 15 & 12 & .10 & 1.8 & 9.3 & .45 & .19 & .93 & 110 & .06 & $<1.0$ \\
\hline 21 & 25 & 15 & .08 & 2.1 & 12 & .42 & .32 & .72 & 100 & $<.05$ & 1.1 \\
\hline 22 & 21 & 13 & .06 & 2.2 & 11 & .26 & .30 & .89 & 90 & $<.05$ & 1.1 \\
\hline 23 & 18 & 11 & .07 & 2.1 & 10 & .25 & .19 & .70 & 71 & $<.05$ & $<1.0$ \\
\hline 24 & 15 & 10 & .12 & 2.0 & 9.8 & .69 & .37 & .78 & 110 & .08 & $<1.0$ \\
\hline 25 & 21 & 5.8 & .15 & 1.6 & 7.0 & .72 & 4.2 & .55 & 88 & .18 & $<1.0$ \\
\hline 26 & 15 & 12 & .12 & 1.7 & 8.7 & .72 & .54 & 1.0 & 120 & .07 & $<1.0$ \\
\hline 27 & 18 & 9.0 & .07 & 1.8 & 7.4 & .43 & 26 & .64 & 79 & .43 & $<1.0$ \\
\hline 28 & 17 & 8.1 & .09 & 1.8 & 7.9 & .46 & 13 & .73 & 86 & .27 & $<1.0$ \\
\hline 29 & 19 & 11 & .06 & 2.2 & 10 & .33 & 1.9 & .69 & 85 & .07 & $<1.0$ \\
\hline 30 & 19 & 11 & .07 & 2.1 & 10 & .38 & .54 & .68 & 75 & .05 & $<1.0$ \\
\hline 31 & 20 & 6.7 & .37 & 1.6 & 7.3 & .68 & 18 & .56 & 74 & .41 & $<1.0$ \\
\hline 33 & 16 & 14 & .12 & 1.4 & 9.2 & .26 & .14 & 1.5 & 69 & .08 & 1.1 \\
\hline 34 & 18 & 13 & .09 & 1.6 & 11 & .19 & .12 & 1.3 & 84 & $<.05$ & 1.0 \\
\hline $34^{\mathbf{R}}$ & 18 & 14 & .09 & 1.7 & 12 & .22 & .15 & 1.4 & 89 & $<.05$ & 1.0 \\
\hline
\end{tabular}


Table 28. Trace-element and carbon concentration data for streambed-sediment samples collected for a synoptic study of mining-affected areas, Northern Rockies Intermontane Basins study unit, August-September 2000 (Continued)

\begin{tabular}{|c|c|c|c|c|c|c|c|c|c|c|c|c|}
\hline $\begin{array}{c}\text { Site } \\
\text { num- } \\
\text { ber } \\
\text { (fig. 1) }\end{array}$ & $\begin{array}{c}\text { Thallium } \\
(\mu \mathbf{g} / \mathbf{g})\end{array}$ & $\begin{array}{c}\text { Thorium } \\
(\mu \mathrm{g} / \mathrm{g})\end{array}$ & $\begin{array}{c}\text { Tin } \\
(\mu \mathrm{g} / \mathrm{g})\end{array}$ & $\begin{array}{c}\text { Titanium } \\
(\mu \mathbf{g} / \mathbf{g})\end{array}$ & $\begin{array}{l}\text { Uranium } \\
(\mu \mathrm{g} / \mathrm{g})\end{array}$ & $\begin{array}{l}\text { Vanadium } \\
(\mu \mathrm{g} / \mathrm{g})\end{array}$ & $\begin{array}{c}\text { Yttrium } \\
(\mu \mathrm{g} / \mathrm{g})\end{array}$ & $\begin{array}{c}\text { Ytterbium } \\
(\mu \mathrm{g} / \mathrm{g})\end{array}$ & $\begin{array}{c}\text { Zinc } \\
(\mu \mathrm{g} / \mathrm{g})\end{array}$ & $\begin{array}{c}\text { Inorganic } \\
\text { carbon } \\
\text { (percent) }\end{array}$ & $\begin{array}{l}\text { Organic } \\
\text { carbon } \\
\text { (percent) }\end{array}$ & $\begin{array}{c}\text { Total } \\
\text { carbon } \\
\text { (percent) }\end{array}$ \\
\hline 6 & $<1.0$ & 11 & 2.8 & 0.23 & 4.6 & 49 & 20 & 2.1 & 430 & 0.03 & 4.9 & 4.9 \\
\hline 7 & $<1.0$ & 11 & 3.5 & .20 & 4.3 & 46 & 17 & 1.9 & 180 & .02 & 3.7 & 3.7 \\
\hline 8 & $<1.0$ & 11 & 2.4 & .16 & 3.0 & 38 & 13 & 1.4 & 74 & .02 & 1.8 & 1.8 \\
\hline 17 & $<1.0$ & 14 & 2.7 & .22 & 5.8 & 54 & 24 & 2.6 & 62 & .03 & 4.3 & 4.3 \\
\hline 18 & $<1.0$ & 14 & 3.8 & .36 & 4.8 & 79 & 18 & 2.4 & 840 & .02 & 2.2 & 2.2 \\
\hline 19 & $<1.0$ & 14 & 7.4 & .27 & 4.2 & 66 & 14 & 1.8 & 790 & .03 & 2.3 & 2.3 \\
\hline 20 & $<1.0$ & 13 & 2.9 & .28 & 7.6 & 55 & 16 & 1.7 & 100 & .03 & 5.2 & 5.2 \\
\hline 21 & $<1.0$ & 15 & 3.3 & .31 & 5.2 & 65 & 24 & 2.6 & 620 & .02 & 2.3 & 2.3 \\
\hline 22 & $<1.0$ & 14 & 3.5 & .28 & 4.1 & 64 & 15 & 1.9 & 480 & .03 & 1.9 & 2.0 \\
\hline 23 & $<1.0$ & 16 & 3.2 & .24 & 5.1 & 58 & 21 & 2.2 & 290 & .01 & 2.4 & 2.4 \\
\hline 24 & $<1.0$ & 11 & 3.2 & .25 & 7.6 & 53 & 21 & 2.2 & 340 & .04 & 6.1 & 6.1 \\
\hline 25 & $<1.0$ & 7.1 & 2.6 & .16 & 5.4 & 40 & 14 & 1.5 & 2,200 & .21 & 6.6 & 6.8 \\
\hline 26 & $<1.0$ & 12 & 2.8 & .29 & 9.3 & 52 & 17 & 1.9 & 260 & .03 & 8.0 & 8.0 \\
\hline 27 & $<1.0$ & 11 & 9.9 & .20 & 4.7 & 46 & 13 & 1.7 & 8,200 & .27 & 2.3 & 2.6 \\
\hline 28 & $<1.0$ & 10 & 6.4 & .20 & 4.4 & 49 & 16 & 1.9 & 5,400 & .26 & 3.9 & 4.2 \\
\hline 29 & $<1.0$ & 15 & 4.2 & .29 & 3.8 & 58 & 15 & 2.1 & 1,100 & .05 & 2.1 & 2.2 \\
\hline 30 & $<1.0$ & 16 & 5.1 & .25 & 3.7 & 58 & 16 & 1.9 & 740 & .02 & 2.8 & 2.9 \\
\hline 31 & $<1.0$ & 8.7 & 5.8 & .16 & 3.6 & 45 & 16 & 1.5 & 10,000 & .28 & 5.1 & 5.4 \\
\hline 33 & $<1.0$ & 10 & 2.4 & .37 & 5.4 & 50 & 23 & 2.1 & 39 & .02 & 4.8 & 4.8 \\
\hline 34 & $<1.0$ & 12 & 3.0 & .38 & 5.0 & 63 & 24 & 2.6 & 65 & .02 & 2.4 & 2.4 \\
\hline $34^{\mathbf{R}}$ & $<1.0$ & 13 & 3.3 & .40 & 5.0 & 67 & 25 & 3.6 & 72 & .02 & 2.4 & 2.4 \\
\hline
\end{tabular}


Table 29. Organochlorine-compound and carbon concentration data for streambed-sediment samples, Northern Rockies Intermontane Basins study unit, 1998-99

[Samples were wet-sieved in the field through a 2-millimeter mesh stainless-steel screen. Bold text denotes detected concentrations. Abbreviations: e, estimated; $\mathrm{g} / \mathrm{kg}$, grams per kilogram; $\mu \mathrm{g} / \mathrm{kg}$, micrograms per kilogram, dry weight; R, replicate. Symbols: <, less than reporting level; --, no data]

\begin{tabular}{|c|c|c|c|c|c|c|c|c|}
\hline $\begin{array}{c}\text { Site } \\
\text { num- } \\
\text { ber } \\
\text { (fig. 1) }\end{array}$ & Site name & Date & Time & 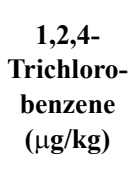 & $\begin{array}{c}\text { 1,2- } \\
\text { Dichloro- } \\
\text { benzene } \\
(\mu \mathrm{g} / \mathbf{k g})\end{array}$ & $\begin{array}{c}1,2- \\
\text { Dimethyl- } \\
\text { naphtha- } \\
\text { lene } \\
(\mu \mathrm{g} / \mathrm{kg})\end{array}$ & $\begin{array}{c}\text { 1,3- } \\
\text { Dichloro- } \\
\text { benzene } \\
(\mu \mathrm{g} / \mathrm{kg})\end{array}$ & $\begin{array}{c}1,4- \\
\text { Dichloro- } \\
\text { benzene } \\
(\mu \mathrm{g} / \mathrm{kg})\end{array}$ \\
\hline 3 & Clark Fork at Turah Bridge, near Bonner, Mont. & $08 / 11 / 98$ & 1035 & $<50$ & $<50$ & $<50$ & $<50$ & $<50$ \\
\hline $3^{\mathbf{R}}$ & Clark Fork at Turah Bridge, near Bonner, Mont. & $08 / 11 / 98$ & 1050 & $<50$ & $<50$ & $<50$ & $<50$ & $<50$ \\
\hline $3^{\mathbf{R}}$ & Clark Fork at Turah Bridge, near Bonner, Mont. & $08 / 11 / 98$ & 1135 & $<50$ & $<50$ & $<50$ & $<50$ & $<50$ \\
\hline 4 & Blackfoot River above Nevada Creek, near Helmville, Mont. & $08 / 17 / 98$ & 1500 & $<50$ & $<50$ & $<50$ & $<50$ & $<50$ \\
\hline 5 & Bitterroot River near Missoula, Mont. & $08 / 10 / 98$ & 1720 & $<50$ & $<50$ & $<50$ & $<50$ & $<50$ \\
\hline 9 & Clark Fork at St. Regis, Mont. & $09 / 08 / 98$ & 1340 & $<50$ & $<50$ & $<50$ & $<50$ & $<50$ \\
\hline 9 & Clark Fork at St. Regis, Mont. & $08 / 23 / 99$ & 1200 & $<50$ & $<50$ & $<50$ & $<50$ & $<50$ \\
\hline 10 & North Fork Flathead River near Columbia Falls, Mont. & $07 / 22 / 98$ & 1105 & $<50$ & $<50$ & $<50$ & $<50$ & $<50$ \\
\hline 11 & Middle Fork Flathead River near West Glacier, Mont. & $07 / 21 / 98$ & 1435 & $<50$ & $<50$ & $<50$ & $<50$ & $<50$ \\
\hline 12 & Flathead River at Perma, Mont. & $09 / 09 / 98$ & 1100 & $<50$ & $<50$ & $<50$ & $<50$ & $<50$ \\
\hline 14 & Lightning Creek at Clark Fork, Idaho & $07 / 29 / 98$ & 1000 & $<50$ & $<50$ & $<50$ & $<50$ & $<50$ \\
\hline 23 & North Fork Coeur d'Alene River at Enaville, Idaho & 07/20/98 & 1000 & $<50$ & $<50$ & $<50$ & $<50$ & $<50$ \\
\hline 31 & South Fork Coeur d'Alene River near Pinehurst, Idaho & $06 / 18 / 98$ & 1105 & $<50$ & $<50$ & $<50$ & $<50$ & $<50$ \\
\hline 33 & St. Joe River at Red Ives Ranger Station, Idaho & 07/06/98 & 1535 & $<50$ & $<50$ & $<50$ & $<50$ & $<50$ \\
\hline 35 & Spokane River near Post Falls, Idaho & $08 / 03 / 98$ & 1100 & $<50$ & $<50$ & $<50$ & $<50$ & $<50$ \\
\hline 40 & Hangman Creek at Spokane, Wash. & $06 / 19 / 98$ & 1135 & $<50$ & $<50$ & $<50$ & $<50$ & $<50$ \\
\hline 41 & Spokane River at Seven Mile Bridge, near Spokane, Wash. & $08 / 05 / 98$ & 1115 & $<50$ & $<50$ & $<50$ & $<50$ & $<50$ \\
\hline $41^{\mathbf{R}}$ & Spokane River at Seven Mile Bridge, near Spokane, Wash. & 08/05/98 & 1130 & $<50$ & $<50$ & $<50$ & $<50$ & $<50$ \\
\hline $41^{\mathbf{R}}$ & Spokane River at Seven Mile Bridge, near Spokane, Wash. & 08/05/98 & 1145 & $<50$ & $<50$ & $<50$ & $<50$ & $<50$ \\
\hline 41 & Spokane River at Seven Mile Bridge, near Spokane, Wash. & 07/30/99 & 1100 & $<50$ & $<50$ & $<50$ & $<50$ & $<50$ \\
\hline
\end{tabular}


Table 29. Organochlorine-compound and carbon concentration data for streambed-sediment samples, Northern Rockies Intermontane Basins study unit, 1998-99 (Continued)

\begin{tabular}{|c|c|c|c|c|c|c|c|c|c|c|}
\hline $\begin{array}{c}\text { Site } \\
\text { num- } \\
\text { ber } \\
\text { (fig. 1) }\end{array}$ & $\begin{array}{c}\text { 1,6- } \\
\text { Dimethyl- } \\
\text { naphtha- } \\
\text { lene } \\
(\mu \mathrm{g} / \mathrm{kg})\end{array}$ & $\begin{array}{c}\text { 1- } \\
\text { Methyl- } \\
\text { 9H- } \\
\text { fluorene } \\
(\mu \mathrm{g} / \mathrm{kg})\end{array}$ & $\begin{array}{c}\text { 1- } \\
\text { Methyl- } \\
\text { phenan- } \\
\text { threne } \\
(\mu \mathrm{g} / \mathrm{kg})\end{array}$ & $\begin{array}{c}\text { 1-Methyl- } \\
\text { pyrene } \\
(\mu \mathrm{g} / \mathrm{kg})\end{array}$ & $\begin{array}{c}2,2^{\prime}- \\
\text { Biquino- } \\
\text { line } \\
(\mu \mathrm{g} / \mathbf{k g})\end{array}$ & $\begin{array}{c}2,3,6- \\
\text { Trimethyl- } \\
\text { naphtha- } \\
\text { lene } \\
(\mu \mathbf{g} / \mathbf{k g})\end{array}$ & $\begin{array}{l}\text { 2,4-Dinitro- } \\
\text { toluene } \\
(\mu \mathrm{g} / \mathrm{kg})\end{array}$ & $\begin{array}{c}2,6- \\
\text { Dimethyl- } \\
\text { naphtha- } \\
\text { lene } \\
(\mu \mathrm{g} / \mathrm{kg})\end{array}$ & $\begin{array}{l}\text { 2,6-Dinitro- } \\
\text { toluene } \\
(\mu \mathrm{g} / \mathrm{kg})\end{array}$ & $\begin{array}{c}\text { 2-Chloro- } \\
\text { naphtha- } \\
\text { lene } \\
(\mu \mathrm{g} / \mathrm{kg})\end{array}$ \\
\hline 3 & $<50$ & $<50$ & $<50$ & $<50$ & $<50$ & $<50$ & $<50$ & $<50$ & $<50$ & $<50$ \\
\hline $3^{R}$ & $<50$ & $<50$ & $<50$ & $<50$ & $<50$ & $<50$ & $<50$ & $<50$ & $<50$ & $<50$ \\
\hline $3^{R}$ & $<50$ & $<50$ & $<50$ & $<50$ & $<50$ & $<50$ & $<50$ & $\mathrm{e} 32$ & $<50$ & $<50$ \\
\hline 4 & $<50$ & $<50$ & $<50$ & $<50$ & $<50$ & $<50$ & $<50$ & $<50$ & $<50$ & $<50$ \\
\hline 5 & $<50$ & $<50$ & $<50$ & $<50$ & $<50$ & $<50$ & $<50$ & $<50$ & $<50$ & $<50$ \\
\hline 9 & $<50$ & $<50$ & $<50$ & $<50$ & $<50$ & $<50$ & $<50$ & $<50$ & $<50$ & $<50$ \\
\hline 9 & $<50$ & $<50$ & $<50$ & $<50$ & $<50$ & $<50$ & $<50$ & $<50$ & $<50$ & $<50$ \\
\hline 10 & $<50$ & $<50$ & $<50$ & $<50$ & $<50$ & $<50$ & $<50$ & $<50$ & $<50$ & $<50$ \\
\hline 11 & $<50$ & $<50$ & $<50$ & $<50$ & $<50$ & $<50$ & $<50$ & $<50$ & $<50$ & $<50$ \\
\hline 12 & $<50$ & $<50$ & $<50$ & $<50$ & $<50$ & $<50$ & $<50$ & $<50$ & $<50$ & $<50$ \\
\hline 14 & $<50$ & $<50$ & $<50$ & $<50$ & $<50$ & $<50$ & $<50$ & $<50$ & $<50$ & $<50$ \\
\hline 23 & $<50$ & $<50$ & $<50$ & $<50$ & $<50$ & $<50$ & $<50$ & $<50$ & $<50$ & $<50$ \\
\hline 31 & $<50$ & $<50$ & $<50$ & $<50$ & $<50$ & $<50$ & $<50$ & 94 & $<50$ & $<50$ \\
\hline 33 & $<50$ & $<50$ & $<50$ & $<50$ & $<50$ & $<50$ & $<50$ & $<50$ & $<50$ & $<50$ \\
\hline 35 & $<50$ & $<50$ & $<50$ & $<50$ & $<50$ & $<50$ & $<50$ & $<50$ & $<50$ & $<50$ \\
\hline 40 & $<50$ & $<50$ & $<50$ & $<50$ & $<50$ & $<50$ & $<50$ & e8.8 & $<50$ & $<50$ \\
\hline 41 & $<50$ & $<50$ & $<50$ & e11 & $\mathrm{e} 33$ & $<50$ & $<50$ & $<50$ & $<50$ & $<50$ \\
\hline $41^{\mathbf{R}}$ & $<50$ & $<50$ & $<50$ & $<50$ & $<50$ & $<50$ & $<50$ & $<50$ & $<50$ & $<50$ \\
\hline $41^{\mathbf{R}}$ & $<50$ & $<50$ & $<50$ & $<50$ & $<50$ & $<50$ & $<50$ & $\mathrm{e} 31$ & $<50$ & $<50$ \\
\hline 41 & $<50$ & $<50$ & $<50$ & $<50$ & $<50$ & $<50$ & $<50$ & $<50$ & $<50$ & $<50$ \\
\hline
\end{tabular}


Table 29. Organochlorine-compound and carbon concentration data for streambed-sediment samples, Northern Rockies Intermontane Basins study unit, 1998-99 (Continued)

\begin{tabular}{|c|c|c|c|c|c|c|c|c|c|c|c|}
\hline $\begin{array}{c}\text { Site } \\
\text { num- } \\
\text { ber } \\
\text { (fig. 1) }\end{array}$ & $\begin{array}{c}\text { 2-Chloro- } \\
\text { phenol } \\
(\mu \mathrm{g} / \mathrm{kg})\end{array}$ & $\begin{array}{c}\text { 2-Ethyl- } \\
\text { naphtha- } \\
\text { lene } \\
(\mu \mathrm{g} / \mathrm{kg})\end{array}$ & $\begin{array}{c}\text { 2-Fluoro- } \\
\text { biphenyl, } \\
\text { surrogate } \\
\text { (percent) }\end{array}$ & $\begin{array}{c}\text { 2-Methyl- } \\
\text { anthra- } \\
\text { cene } \\
(\mu \mathrm{g} / \mathrm{kg})\end{array}$ & $\begin{array}{c}\text { 3,5- } \\
\text { Dimethyl- } \\
\text { phenol } \\
(\mu \mathrm{g} / \mathbf{k g})\end{array}$ & $\begin{array}{c}\text { 4-Bromo- } \\
\text { phenyl- } \\
\text { phenyl- } \\
\text { ether } \\
(\mu \mathrm{g} / \mathrm{kg})\end{array}$ & $\begin{array}{c}\text { 4-Chloro- } \\
\text { 3-methyl- } \\
\text { phenol } \\
(\mu \mathrm{g} / \mathrm{kg})\end{array}$ & $\begin{array}{c}\text { 4-Chloro- } \\
\text { phenyl- } \\
\text { phenyl- } \\
\text { ether } \\
(\mu \mathrm{g} / \mathrm{kg})\end{array}$ & $\begin{array}{c}\text { 4H-Cyclo- } \\
\text { penta[def]- } \\
\text { phenan- } \\
\text { threne } \\
(\mu \mathrm{g} / \mathrm{kg})\end{array}$ & $\begin{array}{c}\text { Acenaph- } \\
\text { thene } \\
(\mu \mathrm{g} / \mathbf{k g})\end{array}$ & $\begin{array}{c}\text { Acenaph- } \\
\text { thylene } \\
(\mu \mathrm{g} / \mathrm{kg})\end{array}$ \\
\hline 3 & $<50$ & $<50$ & 38 & $<50$ & $<50$ & $<50$ & $<50$ & $<50$ & $<50$ & $<50$ & $<50$ \\
\hline $3^{\mathbf{R}}$ & $<50$ & $<50$ & 58 & $<50$ & $<50$ & $<50$ & $<50$ & $<50$ & $<50$ & $<50$ & $<50$ \\
\hline $3^{R}$ & $<50$ & $<50$ & 57 & $<50$ & $<50$ & $<50$ & $<50$ & $<50$ & $<50$ & $<50$ & $<50$ \\
\hline 4 & $<50$ & $<50$ & 58 & $<50$ & $<50$ & $<50$ & $<50$ & $<50$ & $<50$ & $<50$ & $<50$ \\
\hline 5 & $<50$ & $<50$ & 58 & $<50$ & $<50$ & $<50$ & $<50$ & $<50$ & $<50$ & $<50$ & $<50$ \\
\hline 9 & $<50$ & $<50$ & 55 & $<50$ & $<50$ & $<50$ & $<50$ & $<50$ & $<50$ & $<50$ & $<50$ \\
\hline 9 & $<50$ & $<50$ & 53 & $<50$ & $<50$ & $<50$ & $<50$ & $<50$ & $<50$ & $<50$ & $<50$ \\
\hline 10 & $<50$ & e1.8 & 51 & $<50$ & $<50$ & $<50$ & $<50$ & $<50$ & $<50$ & $<50$ & $<50$ \\
\hline 11 & $<50$ & $<50$ & 64 & $<50$ & $<50$ & $<50$ & $<50$ & $<50$ & $<50$ & $<50$ & $<50$ \\
\hline 12 & $<50$ & $<50$ & 56 & $<50$ & $<50$ & $<50$ & $<50$ & $<50$ & $<50$ & $<50$ & $<50$ \\
\hline 14 & $<50$ & $<50$ & 54 & $<50$ & $<50$ & $<50$ & $<50$ & $<50$ & $<50$ & $<50$ & $<50$ \\
\hline 23 & $<50$ & $<50$ & 52 & $<50$ & $<50$ & $<50$ & $<50$ & $<50$ & $<50$ & $<50$ & $<50$ \\
\hline 31 & -- & $<50$ & 82 & $<50$ & $<50$ & $<50$ & $<50$ & $<50$ & $<50$ & $<50$ & $<50$ \\
\hline 33 & $<50$ & $<50$ & 59 & $<50$ & $<50$ & $<50$ & $<50$ & $<50$ & $<50$ & $<50$ & $<50$ \\
\hline 35 & $<50$ & $<50$ & 47 & $<50$ & $<50$ & $<50$ & $<50$ & $<50$ & $<50$ & $<50$ & $<50$ \\
\hline 40 & -- & $<50$ & 76 & $<50$ & $<50$ & $<50$ & $<50$ & $<50$ & $<50$ & $<50$ & $<50$ \\
\hline 41 & $<50$ & $<50$ & 48 & $<50$ & $<50$ & $<50$ & $<50$ & $<50$ & $<50$ & $<50$ & $<50$ \\
\hline $41^{\mathbf{R}}$ & $<50$ & $<50$ & 48 & $<50$ & $<50$ & $<50$ & $<50$ & $<50$ & $<50$ & $<50$ & $<50$ \\
\hline $41^{\mathbf{R}}$ & $<50$ & $<50$ & 51 & $<50$ & $<50$ & $<50$ & $<50$ & $<50$ & $<50$ & $<50$ & e17 \\
\hline 41 & $<50$ & $<50$ & 57 & $<50$ & $<50$ & $<50$ & $<50$ & $<50$ & $<50$ & $<50$ & e18 \\
\hline
\end{tabular}


Table 29. Organochlorine-compound and carbon concentration data for streambed-sediment samples, Northern Rockies Intermontane Basins study unit, 1998-99 (Continued)

\begin{tabular}{|c|c|c|c|c|c|c|c|c|c|c|c|}
\hline $\begin{array}{c}\text { Site } \\
\text { num- } \\
\text { ber } \\
\text { (fig. 1) }\end{array}$ & $\begin{array}{c}\text { Acridine } \\
(\mu \mathrm{g} / \mathrm{kg})\end{array}$ & $\begin{array}{l}\text { Aldrin } \\
(\mu \mathrm{g} / \mathrm{kg})\end{array}$ & $\begin{array}{l}\text { alpha- } \\
\text { Endo- } \\
\text { sulfan } \\
(\mu \mathrm{g} / \mathrm{kg})\end{array}$ & $\begin{array}{c}\text { alpha- } \\
\text { HCH } \\
(\mu \mathrm{g} / \mathrm{kg})\end{array}$ & $\begin{array}{c}\text { alpha- } \\
\text { HCH-d }_{6} \text {, } \\
\text { sur- } \\
\text { rogate } \\
\text { (percent) }\end{array}$ & $\begin{array}{c}\text { Anthra- } \\
\text { cene } \\
(\mu \mathrm{g} / \mathrm{kg})\end{array}$ & $\begin{array}{c}\text { Anthra- } \\
\text { quinone } \\
(\mu \mathrm{g} / \mathrm{kg})\end{array}$ & $\begin{array}{c}\text { Azo- } \\
\text { benzene } \\
(\mu \mathrm{g} / \mathrm{kg})\end{array}$ & $\begin{array}{c}\text { Benz[a]- } \\
\text { anthra- } \\
\text { cene } \\
(\mu \mathrm{g} / \mathbf{k g})\end{array}$ & $\begin{array}{c}\text { Benzo- } \\
{[\mathrm{a}]-} \\
\text { pyrene } \\
(\mu \mathrm{g} / \mathrm{kg})\end{array}$ & $\begin{array}{c}\text { Benzo- } \\
\text { [b]-fluor- } \\
\text { anthene } \\
(\mu \mathrm{g} / \mathrm{kg})\end{array}$ \\
\hline 3 & $<50$ & $<1.0$ & $<1.0$ & $<1.0$ & 71 & $<50$ & $<50$ & $<50$ & $<50$ & $<50$ & $<50$ \\
\hline $3^{R}$ & $<50$ & $<1.0$ & $<1.0$ & $<1.0$ & 78 & $<50$ & $<50$ & $<50$ & $<50$ & $<50$ & $<50$ \\
\hline $3^{\mathbf{R}}$ & $<50$ & $<1.0$ & $<1.0$ & $<1.0$ & 74 & $<50$ & $<50$ & $<50$ & $<50$ & $<50$ & $<50$ \\
\hline 4 & $<50$ & $<1.0$ & $<1.0$ & $<1.0$ & 82 & $<50$ & $<50$ & $<50$ & $<50$ & $<50$ & $<50$ \\
\hline 5 & $<50$ & $<1.0$ & $<1.0$ & $<1.0$ & 71 & $<50$ & $<50$ & $<50$ & e14 & $<50$ & e22 \\
\hline 9 & $<50$ & $<1.0$ & $<1.0$ & $<1.0$ & 73 & $<50$ & $<50$ & $<50$ & $<50$ & $<50$ & $<50$ \\
\hline 9 & $<50$ & $<1.0$ & $<1.0$ & $<1.0$ & 64 & $<50$ & $<50$ & $<50$ & $<50$ & $<50$ & $<50$ \\
\hline 10 & $<50$ & $<1.0$ & $<1.0$ & $<1.0$ & 98 & $<50$ & $<50$ & $<50$ & $<50$ & $<50$ & $<50$ \\
\hline 11 & $<50$ & $<1.0$ & $<1.0$ & $<1.0$ & 95 & $<50$ & $<50$ & $<50$ & $<50$ & $<50$ & $<50$ \\
\hline 12 & $<50$ & $<1.0$ & $<1.0$ & $<1.0$ & 90 & $<50$ & $<50$ & $<50$ & $<50$ & $<50$ & $<50$ \\
\hline 14 & $<50$ & $<1.0$ & $<1.0$ & $<1.0$ & 84 & $<50$ & $<50$ & $<50$ & $<50$ & $<50$ & $<50$ \\
\hline 23 & $<50$ & $<1.0$ & $<1.0$ & $<1.0$ & 91 & $<50$ & $<50$ & $<50$ & $<50$ & $<50$ & $<50$ \\
\hline 31 & $<50$ & $<1.0$ & $<1.0$ & $<1.0$ & 77 & $<50$ & $<50$ & $<50$ & e13 & $<50$ & $<50$ \\
\hline 33 & $<50$ & $<1.0$ & $<1.0$ & $<1.0$ & 88 & $<50$ & $<50$ & $<50$ & $<50$ & $<50$ & $<50$ \\
\hline 35 & $<50$ & $<1.0$ & $<1.0$ & $<1.0$ & 111 & $<50$ & $<50$ & $<50$ & e9.8 & e15 & e20 \\
\hline 40 & $<50$ & $<1.0$ & $<1.0$ & $<1.0$ & 75 & $<50$ & $<50$ & $<50$ & $<50$ & $<50$ & $<50$ \\
\hline 41 & $<50$ & $<1.0$ & $<1.0$ & $<1.0$ & 79 & e6.6 & $<50$ & $<50$ & e17 & e24 & $<50$ \\
\hline $41^{R}$ & $<50$ & $<1.0$ & $<1.0$ & $<1.0$ & 76 & e14 & $<50$ & $<50$ & e26 & e30 & $<50$ \\
\hline $41^{\mathbf{R}}$ & $<50$ & $<1.0$ & $<1.0$ & $<1.0$ & 78 & $\mathrm{e} 25$ & $<50$ & $<50$ & 96 & 99 & 68 \\
\hline 41 & $<50$ & $<1.0$ & $<1.0$ & $<1.0$ & 68 & $<50$ & $<50$ & $<50$ & $\mathrm{e} 24$ & e25 & e6.2 \\
\hline
\end{tabular}


Table 29. Organochlorine-compound and carbon concentration data for streambed-sediment samples, Northern Rockies Intermontane Basins study unit, 1998-99 (Continued)

\begin{tabular}{|c|c|c|c|c|c|c|c|c|c|c|}
\hline $\begin{array}{c}\text { Site } \\
\text { num- } \\
\text { ber } \\
\text { (fig. 1 } \\
\text { and } \\
\text { table 1) }\end{array}$ & $\begin{array}{c}\text { Benzo- } \\
{[c]-} \\
\text { cinnoline } \\
(\mu \mathrm{g} / \mathbf{k g})\end{array}$ & $\begin{array}{c}\text { Benzo- } \\
\text { [ghi]- } \\
\text { perylene } \\
(\mu \mathrm{g} / \mathrm{kg})\end{array}$ & $\begin{array}{c}\text { Benzo- } \\
{[\mathrm{k}]-} \\
\text { fluoran- } \\
\text { thene } \\
(\mu \mathrm{g} / \mathrm{kg})\end{array}$ & $\begin{array}{c}\text { beta- } \\
\text { HCH } \\
(\mu \mathrm{g} / \mathrm{kg})\end{array}$ & $\begin{array}{l}\text { bis(2- } \\
\text { Chloro- } \\
\text { ethoxy)- } \\
\text { methane } \\
(\mu \mathrm{g} / \mathrm{kg})\end{array}$ & $\begin{array}{c}\text { bis(2- } \\
\text { Chloro- } \\
\text { ethyl) } \\
\text { ether } \\
(\mu \mathrm{g} / \mathrm{kg})\end{array}$ & $\begin{array}{c}\text { bis(2- } \\
\text { Ethyl- } \\
\text { hexyl) } \\
\text { phthalate } \\
(\mu \mathrm{g} / \mathrm{kg})\end{array}$ & $\begin{array}{c}\text { Butyl- } \\
\text { benzyl } \\
\text { phthalate } \\
(\mu \mathrm{g} / \mathrm{kg})\end{array}$ & $\begin{array}{c}\text { C8-Alkyl- } \\
\text { phenol } \\
(\mu \mathrm{g} / \mathrm{kg})\end{array}$ & $\begin{array}{c}\text { Carbazole } \\
(\mu \mathrm{g} / \mathbf{k g})\end{array}$ \\
\hline 3 & $<50$ & $<50$ & $<50$ & $<1.0$ & $<50$ & -- & $<50$ & $<50$ & $<50$ & $<50$ \\
\hline $3^{\mathbf{R}}$ & $<50$ & e9.6 & $<50$ & $<1.0$ & $<50$ & -- & $<50$ & $<50$ & $<50$ & $<50$ \\
\hline $3^{\mathrm{R}}$ & $<50$ & $<50$ & $<50$ & $<1.0$ & $<50$ & -- & $<50$ & $<50$ & $<50$ & $<50$ \\
\hline 4 & $<50$ & $<50$ & $<50$ & $<1.0$ & $<50$ & -- & e29 & e20 & $<50$ & $<50$ \\
\hline 5 & $<50$ & e24 & e22 & $<1.0$ & $<50$ & -- & e32 & e20 & $<50$ & $<50$ \\
\hline 9 & $<50$ & $<50$ & $<50$ & $<1.0$ & $<50$ & -- & e33 & $<50$ & $<50$ & $<50$ \\
\hline 9 & $<50$ & $<50$ & $<50$ & $<1.0$ & $<50$ & $<50$ & 66 & 102 & $<50$ & $<50$ \\
\hline 10 & $<50$ & $<50$ & $<50$ & $<1.0$ & $<50$ & -- & e 48 & e23 & $<50$ & $<50$ \\
\hline 11 & $<50$ & $<50$ & $<50$ & $<1.0$ & $<50$ & -- & e25 & $<50$ & $<50$ & $<50$ \\
\hline 12 & $<50$ & $<50$ & $<50$ & $<1.0$ & $<50$ & -- & $<50$ & $<50$ & $<50$ & $<50$ \\
\hline 14 & $<50$ & $<50$ & $<50$ & $<1.0$ & $<50$ & -- & e23 & e20 & $<50$ & $<50$ \\
\hline 23 & $<50$ & $<50$ & $<50$ & $<1.0$ & $<50$ & -- & e40 & e16 & $<50$ & $<50$ \\
\hline 31 & -- & $<50$ & $<50$ & $<1.0$ & $<50$ & -- & 120 & e28 & $<50$ & $<50$ \\
\hline 33 & $<50$ & $<50$ & $<50$ & $<1.0$ & $<50$ & -- & e15 & $<50$ & $<50$ & $<50$ \\
\hline 35 & $<50$ & e13 & e19 & $<1.0$ & $<50$ & -- & e36 & e30 & $<50$ & $<50$ \\
\hline 40 & -- & $<50$ & $<50$ & $<1.0$ & $<50$ & -- & 60 & $\mathrm{e} 20$ & $<50$ & $<50$ \\
\hline 41 & $<50$ & $<50$ & $<50$ & $<1.0$ & $<50$ & -- & e45 & e26 & $<50$ & $<50$ \\
\hline $41^{\mathbf{R}}$ & $<50$ & $<50$ & $<50$ & $<1.0$ & $<50$ & -- & 72 & e18 & $<50$ & $<50$ \\
\hline $41^{\mathbf{R}}$ & $<50$ & 52 & 56 & $<1.0$ & $<50$ & -- & e49 & $<50$ & $<50$ & $<50$ \\
\hline 41 & $<50$ & e5.4 & $\mathrm{e} 47$ & $<1.0$ & $<50$ & $<50$ & 92 & $\mathrm{e} 31$ & $<50$ & $<50$ \\
\hline
\end{tabular}


Table 29. Organochlorine-compound and carbon concentration data for streambed-sediment samples, Northern Rockies Intermontane Basins study unit, 1998-99 (Continued)

\begin{tabular}{|c|c|c|c|c|c|c|c|c|c|c|c|}
\hline $\begin{array}{c}\text { Site } \\
\text { num- } \\
\text { ber } \\
\text { (fig. 1) }\end{array}$ & $\begin{array}{c}\text { Chlor- } \\
\text { oneb } \\
(\mu \mathrm{g} / \mathrm{kg})\end{array}$ & $\begin{array}{c}\text { Chry- } \\
\text { sene } \\
(\mu \mathrm{g} / \mathbf{k g})\end{array}$ & $\begin{array}{c}\text { cis-Chlor- } \\
\text { dane } \\
(\mu \mathrm{g} / \mathrm{kg})\end{array}$ & $\begin{array}{l}\text { cis-Nona- } \\
\text { chlor } \\
(\mu \mathrm{g} / \mathrm{kg})\end{array}$ & $\begin{array}{c}\text { cis- } \\
\text { Permethrin } \\
(\mu \mathrm{g} / \mathbf{k g})\end{array}$ & $\begin{array}{c}\text { Dacthal } \\
(\mu \mathrm{g} / \mathrm{kg})\end{array}$ & $\begin{array}{c}\text { Dibenz- } \\
{[\mathbf{a}, \mathbf{h}]-} \\
\text { anthra- } \\
\text { cene } \\
(\mu \mathrm{g} / \mathrm{kg})\end{array}$ & $\begin{array}{c}\text { Dibenzo- } \\
\text { thiophene } \\
(\mu \mathrm{g} / \mathrm{kg})\end{array}$ & $\begin{array}{l}\text { Deldrin } \\
(\mu \mathrm{g} / \mathbf{k g})\end{array}$ & $\begin{array}{c}\text { Diethyl } \\
\text { phthalate } \\
(\mu \mathrm{g} / \mathrm{kg})\end{array}$ & $\begin{array}{c}\text { Dimethyl } \\
\text { phthalate } \\
(\mu \mathrm{g} / \mathrm{kg})\end{array}$ \\
\hline 3 & $<5.0$ & e20 & $<1.0$ & $<1.0$ & $<5.0$ & $<5.0$ & $<50$ & $<50$ & $<<1.0$ & $<50$ & $<50$ \\
\hline $3^{R}$ & $<5.0$ & $<50$ & $<1.0$ & $<1.0$ & $<5.0$ & $<5.0$ & $<50$ & $<50$ & $<1.0$ & $<50$ & $<50$ \\
\hline $3^{R}$ & $<5.0$ & $<50$ & $<1.0$ & $<1.0$ & $<5.0$ & $<5.0$ & $<50$ & $<50$ & $<1.0$ & $<50$ & $<50$ \\
\hline 4 & $<5.0$ & $<50$ & $<1.0$ & $<1.0$ & $<5.0$ & $<5.0$ & $<50$ & $<50$ & $<1.0$ & $<50$ & $<50$ \\
\hline 5 & $<5.0$ & e 9.3 & $<1.0$ & $<1.0$ & $<5.0$ & $<5.0$ & $<50$ & $<50$ & $<1.0$ & $<50$ & $<50$ \\
\hline 9 & $<5.0$ & $<50$ & $<1.0$ & $<1.0$ & $<5.0$ & $<5.0$ & $<50$ & $<50$ & $<1.0$ & $<50$ & $<50$ \\
\hline 9 & $<5.0$ & $<50$ & $<1.0$ & $<1.0$ & $<5.0$ & $<5.0$ & $<50$ & $<50$ & $<1.0$ & $<50$ & $<50$ \\
\hline 10 & $<5.0$ & $<50$ & $<1.0$ & $<1.0$ & $<5.0$ & $<5.0$ & $<50$ & $<50$ & $<1.0$ & $<50$ & $<50$ \\
\hline 11 & $<5.0$ & $<50$ & $<1.0$ & $<1.0$ & $<5.0$ & $<5.0$ & $<50$ & $<50$ & $<1.0$ & $<50$ & $<50$ \\
\hline 12 & $<5.0$ & $<50$ & $<1.0$ & $<1.0$ & $<5.0$ & $<5.0$ & $<50$ & $<50$ & $<1.0$ & $<50$ & $<50$ \\
\hline 14 & $<5.0$ & $<50$ & $<1.0$ & $<1.0$ & $<5.0$ & $<5.0$ & $<50$ & $<50$ & $<1.0$ & $<50$ & $<50$ \\
\hline 23 & $<5.0$ & $<50$ & $<1.0$ & $<1.0$ & $<5.0$ & $<5.0$ & $<50$ & $<50$ & $<1.0$ & $<50$ & $<50$ \\
\hline 31 & $<5.0$ & e6.9 & $<1.0$ & $<1.0$ & $<5.0$ & $<5.0$ & $<50$ & $<50$ & $<1.0$ & e35 & $<50$ \\
\hline 33 & $<5.0$ & $<50$ & $<1.0$ & $<1.0$ & $<5.0$ & $<5.0$ & $<50$ & $<50$ & $<1.0$ & e1.1 & $<50$ \\
\hline 35 & $<5.0$ & e5.1 & $<1.0$ & $<1.0$ & $<5.0$ & $<5.0$ & $<50$ & $<50$ & $<1.0$ & $<50$ & $<50$ \\
\hline 40 & $<5.0$ & $<50$ & $<1.0$ & $<1.0$ & $<5.0$ & $<5.0$ & $<50$ & $<50$ & $<1.0$ & e12 & $<50$ \\
\hline 41 & $<5.0$ & e17 & $<1.0$ & $<1.0$ & $<5.0$ & $<5.0$ & $<50$ & $<50$ & $<1.0$ & $<50$ & $<50$ \\
\hline $41^{\mathbf{R}}$ & $<5.0$ & e25 & $<1.0$ & $<1.0$ & $<5.0$ & $<5.0$ & $<50$ & $<50$ & $<1.0$ & $<50$ & $<50$ \\
\hline $41^{\mathbf{R}}$ & $<5.0$ & 86 & $<1.0$ & $<1.0$ & $<5.0$ & $<5.0$ & $<50$ & $<50$ & $<1.0$ & $<50$ & $<50$ \\
\hline 41 & $<5.0$ & $\mathrm{e} 35$ & $<1.0$ & $<1.0$ & $<5.0$ & $<5.0$ & $<50$ & $<50$ & $<1.0$ & $<50$ & $<50$ \\
\hline
\end{tabular}


Table 29. Organochlorine-compound and carbon concentration data for streambed-sediment samples, Northern Rockies Intermontane Basins study unit, 1998-99 (Continued)

\begin{tabular}{|c|c|c|c|c|c|c|c|c|c|c|}
\hline $\begin{array}{l}\text { Site } \\
\text { num- } \\
\text { ber } \\
\text { (fig. 1) }\end{array}$ & $\begin{array}{c}\text { Di-n- } \\
\text { butyl } \\
\text { phtha- } \\
\text { late } \\
(\mu \mathrm{g} / \mathrm{kg})\end{array}$ & $\begin{array}{c}\text { Di-n- } \\
\text { octyl } \\
\text { phthalate } \\
(\mu \mathrm{g} / \mathrm{kg})\end{array}$ & $\begin{array}{l}\text { Endrin } \\
(\mu \mathrm{g} / \mathrm{kg})\end{array}$ & $\begin{array}{c}\text { Fluor- } \\
\text { anthene } \\
(\mu \mathrm{g} / \mathrm{kg})\end{array}$ & $\begin{array}{c}\text { Fluo- } \\
\text { rene } \\
(\mu \mathrm{g} / \mathbf{k g})\end{array}$ & $\begin{array}{l}\text { Hepta- } \\
\text { chlor } \\
(\mu \mathrm{g} / \mathrm{kg})\end{array}$ & $\begin{array}{l}\text { Hepta- } \\
\text { chlor } \\
\text { epoxide } \\
(\mu \mathrm{g} / \mathrm{kg})\end{array}$ & $\begin{array}{c}\text { Hexa- } \\
\text { chloro- } \\
\text { benzene } \\
(\mu \mathrm{g} / \mathrm{kg})\end{array}$ & $\begin{array}{c}\text { Indeno- } \\
{[1,2,3-c d]-} \\
\text { pyrene } \\
(\mu \mathrm{g} / \mathrm{kg})\end{array}$ & $\begin{array}{l}\text { Isodrin } \\
(\mu \mathrm{g} / \mathbf{k g})\end{array}$ \\
\hline 3 & e36 & $<50$ & $<2.0$ & e25 & $<50$ & $<1.0$ & $<1.0$ & $<1.0$ & $<50$ & $<1.0$ \\
\hline $3^{R}$ & e27 & $<50$ & $<2.0$ & e19 & $<50$ & $<1.0$ & $<1.0$ & $<1.0$ & $<50$ & $<1.0$ \\
\hline $3^{R}$ & e35 & $<50$ & $<2.0$ & e24 & $<50$ & $<1.0$ & $<1.0$ & $<1.0$ & $<50$ & $<1.0$ \\
\hline 4 & e41 & $<50$ & $<2.0$ & $<50$ & $<50$ & $<1.0$ & $<1.0$ & $<1.0$ & $<50$ & $<1.0$ \\
\hline 5 & $\mathrm{e} 42$ & $<50$ & $<2.0$ & e7.2 & $<50$ & $<1.0$ & $<1.0$ & $<1.0$ & e24 & $<1.0$ \\
\hline 9 & $<50$ & $<50$ & $<2.0$ & $\mathrm{e} 13$ & $<50$ & $<1.0$ & $<1.0$ & $<1.0$ & $<50$ & $<1.0$ \\
\hline 9 & 91 & $<50$ & $<2.0$ & $<50$ & $<50$ & $<1.0$ & $<1.0$ & $<1.0$ & $<50$ & $<1.0$ \\
\hline 10 & $\mathrm{e} 43$ & $<50$ & $<2.0$ & $<50$ & $<50$ & $<1.0$ & $<1.0$ & $<1.0$ & $<50$ & $<1.0$ \\
\hline 11 & $\mathrm{e} 42$ & $<50$ & $<2.0$ & e11 & $<50$ & $<1.0$ & $<1.0$ & $<1.0$ & $<50$ & $<1.0$ \\
\hline 12 & e30 & $<50$ & $<2.0$ & $<50$ & $<50$ & $<1.0$ & $<1.0$ & $<1.0$ & $<50$ & $<1.0$ \\
\hline 14 & e30 & $<50$ & $<2.0$ & $<50$ & $<50$ & $<1.0$ & $<1.0$ & $<1.0$ & e5.9 & $<1.0$ \\
\hline 23 & e39 & $<50$ & $<2.0$ & e15 & $<50$ & $<1.0$ & $<1.0$ & $<1.0$ & $<50$ & $<1.0$ \\
\hline 31 & e56 & $<50$ & $<2.0$ & -- & $<50$ & $<1.0$ & $<1.0$ & $<1.0$ & $<50$ & $<1.0$ \\
\hline 33 & e47 & $<50$ & $<2.0$ & $<50$ & $<50$ & $<1.0$ & $<1.0$ & $<1.0$ & $<50$ & $<1.0$ \\
\hline 35 & $\mathrm{e} 33$ & $<50$ & $<2.0$ & e33 & $<50$ & $<1.0$ & $<1.0$ & $<1.0$ & e11 & $<1.0$ \\
\hline 40 & $\mathrm{e} 42$ & $<50$ & $<2.0$ & -- & $<50$ & $<1.0$ & $<1.0$ & $<1.0$ & $<50$ & $<1.0$ \\
\hline 41 & e28 & $<50$ & $<2.0$ & e39 & $<50$ & $<1.0$ & $<1.0$ & $<1.0$ & $<50$ & $<1.0$ \\
\hline $41^{\mathbf{R}}$ & e41 & $<50$ & $<2.0$ & 53 & $<50$ & $<1.0$ & $<1.0$ & $<1.0$ & $<50$ & $<1.0$ \\
\hline $41^{\mathbf{R}}$ & e27 & $<50$ & $<2.0$ & 130 & $<50$ & $<1.0$ & $<1.0$ & $<1.0$ & 51 & $<1.0$ \\
\hline 41 & $<50$ & $<50$ & $<2.0$ & e25 & $<50$ & $<1.0$ & $<1.0$ & $<1.0$ & $<50$ & $<1.0$ \\
\hline
\end{tabular}


Table 29. Organochlorine-compound and carbon concentration data for streambed-sediment samples, Northern Rockies Intermontane Basins study unit, 1998-99 (Continued)

\begin{tabular}{|c|c|c|c|c|c|c|c|c|c|c|c|}
\hline $\begin{array}{c}\text { Site } \\
\text { num- } \\
\text { ber } \\
\text { (fig. 1) }\end{array}$ & $\begin{array}{c}\text { Iso- } \\
\text { phorone } \\
(\mu \mathrm{g} / \mathbf{k g})\end{array}$ & $\begin{array}{l}\text { Iso- } \\
\text { quin- } \\
\text { oline } \\
(\mu \mathrm{g} / \mathbf{k g})\end{array}$ & $\begin{array}{c}\text { Lindane } \\
(\mu \mathrm{g} / \mathrm{kg})\end{array}$ & $\begin{array}{c}\text { Mirex } \\
(\mu \mathrm{g} / \mathbf{k g})\end{array}$ & $\begin{array}{l}\text { Naptha- } \\
\text { lene } \\
(\mu \mathrm{g} / \mathrm{kg})\end{array}$ & $\begin{array}{c}\text { N-Nitrosodin- } \\
\text { n-propyl- } \\
\text { amine } \\
(\mu \mathrm{g} / \mathrm{kg})\end{array}$ & $\begin{array}{l}\text { N-Nitro- } \\
\text { sodo- } \\
\text { diphenyl- } \\
\text { amine } \\
(\mu \mathrm{g} / \mathrm{kg})\end{array}$ & $\begin{array}{c}\text { Nitro- } \\
\text { benzene } \\
(\mu \mathrm{g} / \mathrm{kg})\end{array}$ & $\begin{array}{c}\text { Nitro- } \\
\text { benzene-d } \\
\text { surrogate } \\
\text { (percent) }\end{array}$ & $\begin{array}{c}\text { o,p'-DDD } \\
(\mu \mathbf{g} / \mathbf{k g})\end{array}$ & $\begin{array}{c}\mathbf{0 , p} \text { '-DDE } \\
(\mu \mathrm{g} / \mathbf{k g})\end{array}$ \\
\hline 3 & $<50$ & $<50$ & $<1.0$ & $<1.0$ & $<50$ & $<50$ & $<50$ & $<50$ & 60 & $<1.0$ & $<1.0$ \\
\hline $3^{R}$ & $<50$ & $<50$ & $<1.0$ & $<1.0$ & $<50$ & $<50$ & $<50$ & $<50$ & 54 & $<1.0$ & $<1.0$ \\
\hline $3^{R}$ & $<50$ & $<50$ & $<1.0$ & $<1.0$ & $<50$ & $<50$ & $<50$ & $<50$ & 63 & $<1.0$ & $<1.0$ \\
\hline 4 & $<50$ & $<50$ & $<1.0$ & $<1.0$ & $<50$ & $<50$ & $<50$ & $<50$ & 55 & $<1.0$ & $<1.0$ \\
\hline 5 & $<50$ & $<50$ & $<1.0$ & $<1.0$ & $<50$ & $<50$ & $<50$ & $<50$ & 52 & $<1.0$ & $<1.0$ \\
\hline 9 & $<50$ & $<50$ & $<1.0$ & $<1.0$ & $<50$ & $<50$ & $<50$ & $<50$ & 61 & $<1.0$ & $<1.0$ \\
\hline 9 & $<50$ & $<50$ & $<1.0$ & $<1.0$ & $<50$ & $<50$ & $<50$ & $<50$ & 39 & $<1.0$ & $<1.0$ \\
\hline 10 & $<50$ & $<50$ & $<1.0$ & $<1.0$ & $<50$ & $<50$ & $<50$ & $<50$ & 52 & $<1.0$ & $<1.0$ \\
\hline 11 & $<50$ & $<50$ & $<1.0$ & $<1.0$ & $<50$ & $<50$ & $<50$ & $<50$ & 90 & $<1.0$ & $<1.0$ \\
\hline 12 & $<50$ & $<50$ & $<1.0$ & $<1.0$ & $<50$ & $<50$ & $<50$ & $<50$ & 62 & $<1.0$ & $<1.0$ \\
\hline 14 & $<50$ & $<50$ & $<1.0$ & $<1.0$ & $<50$ & $<50$ & $<50$ & $<50$ & 73 & $<1.0$ & $<1.0$ \\
\hline 23 & $<50$ & $<50$ & $<1.0$ & $<1.0$ & $<50$ & $<50$ & $<50$ & $<50$ & 86 & $<1.0$ & $<1.0$ \\
\hline 31 & $<50$ & $<50$ & $<1.0$ & $<1.0$ & $<50$ & $<50$ & $<50$ & $<50$ & 103 & $<1.0$ & $<1.0$ \\
\hline 33 & $<50$ & $<50$ & $<1.0$ & $<1.0$ & $<50$ & $<50$ & $<50$ & $<50$ & 70 & $<1.0$ & $<1.0$ \\
\hline 35 & $<50$ & $<50$ & $<1.0$ & $<1.0$ & e6.9 & $<50$ & $<50$ & $<50$ & 56 & $<1.0$ & $<1.0$ \\
\hline 40 & $<50$ & $<50$ & $<1.0$ & $<1.0$ & $<50$ & $<50$ & $<50$ & $<50$ & 72 & $<1.0$ & $<1.0$ \\
\hline 41 & $<50$ & $<50$ & $<1.0$ & $<1.0$ & e7.1 & $<50$ & $<50$ & $<50$ & 41 & $<1.0$ & $<1.0$ \\
\hline $41^{\mathbf{R}}$ & $<50$ & $<50$ & $<1.0$ & $<1.0$ & e15 & $<50$ & $<50$ & $<50$ & 44 & $<1.0$ & $<1.0$ \\
\hline $41^{\mathbf{R}}$ & $<50$ & $<50$ & $<1.0$ & $<1.0$ & $\mathrm{e} 20$ & $<50$ & $<50$ & $<50$ & 43 & $<1.0$ & $<1.0$ \\
\hline 41 & $<50$ & $<50$ & $<1.0$ & $<1.0$ & $<50$ & $<50$ & $<50$ & $<50$ & 49 & $<1.0$ & $<1.0$ \\
\hline
\end{tabular}


Table 29. Organochlorine-compound and carbon concentration data for streambed-sediment samples, Northern Rockies Intermontane Basins study unit, 1998-99 (Continued)

\begin{tabular}{|c|c|c|c|c|c|c|c|c|c|c|c|c|}
\hline $\begin{array}{c}\text { Site } \\
\text { num- } \\
\text { ber } \\
\text { (fig. 1 } \\
\text { and } \\
\text { table 1) }\end{array}$ & $\begin{array}{c}\text { o,p'- } \\
\text { DDT } \\
(\mu \mathrm{g} / \mathbf{k g})\end{array}$ & $\begin{array}{c}\text { o,p'-Meth- } \\
\text { oxychlor } \\
(\mu \mathrm{g} / \mathrm{kg})\end{array}$ & $\begin{array}{c}\text { Oxychlor- } \\
\text { dane } \\
(\mu \mathrm{g} / \mathrm{kg})\end{array}$ & $\begin{array}{c}\text { p,p'- } \\
\text { DDD } \\
(\mu \mathrm{g} / \mathbf{k g})\end{array}$ & $\begin{array}{c}\text { p,p'- } \\
\text { DDE } \\
(\mu \mathbf{g} / \mathbf{k g})\end{array}$ & $\begin{array}{c}\text { p,p'- } \\
\text { DDT } \\
(\mu \mathrm{g} / \mathbf{k g})\end{array}$ & $\begin{array}{c}\text { p,p'- } \\
\text { Methoxy- } \\
\text { chlor } \\
(\mu \mathrm{g} / \mathbf{k g})\end{array}$ & $\begin{array}{c}\text { p- } \\
\text { Cresol } \\
(\mu \mathrm{g} / \mathrm{kg})\end{array}$ & $\begin{array}{l}\text { Penta- } \\
\text { chloro- } \\
\text { anisole } \\
(\mu \mathrm{g} / \mathrm{kg})\end{array}$ & $\begin{array}{c}\text { Penta- } \\
\text { chloro- } \\
\text { nitro- } \\
\text { benzene } \\
(\mu \mathrm{g} / \mathrm{kg})\end{array}$ & $\begin{array}{c}\text { Pen- } \\
\text { anthrene } \\
(\mu \mathrm{g} / \mathrm{kg})\end{array}$ & $\begin{array}{c}\text { Pen- } \\
\text { anthri- } \\
\text { dine } \\
(\mu \mathrm{g} / \mathrm{kg})\end{array}$ \\
\hline 3 & $<2.0$ & $<5.0$ & $<1.0$ & $<1.0$ & $<1.0$ & $<2.0$ & $<5.0$ & 680 & $<1.0$ & $<50$ & $<50$ & $<50$ \\
\hline $3^{R}$ & $<2.0$ & $<5.0$ & $<1.0$ & $<1.0$ & $<1.0$ & $<2.0$ & $<5.0$ & 540 & $<1.0$ & $<50$ & $<50$ & $<50$ \\
\hline $3^{R}$ & $<2.0$ & $<5.0$ & $<1.0$ & $<1.0$ & $<1.0$ & $<2.0$ & $<5.0$ & 78 & $<1.0$ & $<50$ & e6.4 & $<50$ \\
\hline 4 & $<2.0$ & $<5.0$ & $<1.0$ & $<1.0$ & $<1.0$ & $<2.0$ & $<5.0$ & $\mathrm{e} 31$ & $<1.0$ & $<50$ & $<50$ & $<50$ \\
\hline 5 & $<2.0$ & $<5.0$ & $<1.0$ & $<1.0$ & $<1.0$ & $<2.0$ & $<5.0$ & e47 & $<1.0$ & $<50$ & $<50$ & $<50$ \\
\hline 9 & $<2.0$ & $<5.0$ & $<1.0$ & $<1.0$ & $<1.0$ & $<2.0$ & $<5.0$ & 1,100 & $<1.0$ & $<50$ & $<50$ & $<50$ \\
\hline 9 & $<2.0$ & $<5.0$ & $<1.0$ & $<1.0$ & $<1.0$ & $<2.0$ & $<5.0$ & 2,690 & $<1.0$ & $<50$ & $<50$ & $<50$ \\
\hline 10 & $<2.0$ & $<5.0$ & $<1.0$ & $<1.0$ & $<1.0$ & $<2.0$ & $<5.0$ & 190 & $<1.0$ & $<50$ & $<50$ & $<50$ \\
\hline 11 & $<2.0$ & $<5.0$ & $<1.0$ & $<1.0$ & $<1.0$ & $<2.0$ & $<5.0$ & 87 & $<1.0$ & $<50$ & $<50$ & $<50$ \\
\hline 12 & $<2.0$ & $<5.0$ & $<1.0$ & $<1.0$ & $<1.0$ & $<2.0$ & $<5.0$ & 120 & $<1.0$ & $<50$ & $<50$ & $<50$ \\
\hline 14 & $<2.0$ & $<5.0$ & $<1.0$ & $<1.0$ & $<1.0$ & $<2.0$ & $<5.0$ & $<50$ & $<1.0$ & $<50$ & $<50$ & $<50$ \\
\hline 23 & $<2.0$ & $<5.0$ & $<1.0$ & $<1.0$ & $<1.0$ & $<2.0$ & $<5.0$ & 100 & $<1.0$ & $<50$ & $<50$ & $<50$ \\
\hline 31 & $<2.0$ & $<5.0$ & $<1.0$ & $<1.0$ & $<1.0$ & $<2.0$ & $<5.0$ & 88 & $<1.0$ & $<50$ & e6.4 & -- \\
\hline 33 & $<2.0$ & $<5.0$ & $<1.0$ & $<1.0$ & $<1.0$ & $<2.0$ & $<5.0$ & 200 & $<1.0$ & $<50$ & $<50$ & $<50$ \\
\hline 35 & $<2.0$ & $<5.0$ & $<1.0$ & $<1.0$ & $<1.0$ & $<2.0$ & $<5.0$ & $<50$ & $<1.0$ & $<50$ & e9.0 & $<50$ \\
\hline 40 & $<2.0$ & $<5.0$ & $<1.0$ & $<1.0$ & 1.4 & $<2.0$ & $<5.0$ & 84 & $<1.0$ & $<50$ & $<50$ & -- \\
\hline 41 & $<2.0$ & $<5.0$ & $<1.0$ & 1.2 & 2.9 & $<2.0$ & $<5.0$ & 160 & $<1.0$ & $<50$ & e15 & $<50$ \\
\hline $41^{\mathbf{R}}$ & $<2.0$ & $<5.0$ & $<1.0$ & 1.8 & 4.5 & $<2.0$ & $<5.0$ & 120 & $<1.0$ & $<50$ & $\mathrm{e} 27$ & $<50$ \\
\hline $41^{\mathbf{R}}$ & $<2.0$ & $<5.0$ & $<1.0$ & 1.4 & 4.0 & $<2.0$ & $<5.0$ & 270 & $<1.0$ & $<50$ & $\mathrm{e} 27$ & $<50$ \\
\hline 41 & $<2.0$ & $<5.0$ & $<1.0$ & $<1.0$ & 1.2 & $<2.0$ & $<5.0$ & 1,350 & $<1.0$ & $<50$ & $<50$ & $<50$ \\
\hline
\end{tabular}


Table 29. Organochlorine-compound and carbon concentration data for streambed-sediment samples, Northern Rockies Intermontane Basins study unit, 1998-99 (Continued)

\begin{tabular}{|c|c|c|c|c|c|c|c|c|c|c|c|c|}
\hline $\begin{array}{c}\text { Site } \\
\text { num- } \\
\text { ber } \\
\text { (fig. 1) }\end{array}$ & $\begin{array}{l}\text { Phenol } \\
(\mu \mathrm{g} / \mathbf{k g})\end{array}$ & $\begin{array}{c}\text { Poly- } \\
\text { chlorinated } \\
\text { biphenyls } \\
(\mu \mathrm{g} / \mathbf{k g})\end{array}$ & $\begin{array}{l}\text { Pyrene } \\
(\mu \mathrm{g} / \mathrm{kg})\end{array}$ & $\begin{array}{l}\text { Quin- } \\
\text { oline } \\
(\mu \mathrm{g} / \mathrm{kg})\end{array}$ & $\begin{array}{c}\text { Ter- } \\
\text { phenyl- } \\
\text { d }_{14}, \\
\text { surrogate } \\
(\mu \mathrm{g} / \mathbf{k g})\end{array}$ & $\begin{array}{c}\text { Toxa- } \\
\text { phene } \\
(\mu \mathrm{g} / \mathrm{kg})\end{array}$ & $\begin{array}{c}\text { trans- } \\
\text { Chlora- } \\
\text { dane } \\
(\mu \mathrm{g} / \mathrm{kg})\end{array}$ & $\begin{array}{l}\text { trans- } \\
\text { Nona- } \\
\text { chlor } \\
(\mu \mathrm{g} / \mathbf{k g})\end{array}$ & $\begin{array}{c}\text { trans- } \\
\text { Permethrin } \\
(\mu \mathrm{g} / \mathbf{k g})\end{array}$ & $\begin{array}{c}\text { Inor- } \\
\text { ganic } \\
\text { carbon } \\
(\mathrm{g} / \mathrm{kg})\end{array}$ & $\begin{array}{c}\text { Organic } \\
\text { carbon } \\
(\mathrm{g} / \mathrm{kg})\end{array}$ & $\begin{array}{c}\text { Total } \\
\text { carbon } \\
(\mathrm{g} / \mathrm{kg})\end{array}$ \\
\hline 3 & 51 & $<50$ & e27 & $<50$ & 60 & $<200$ & $<1.0$ & $<1.0$ & $<5.0$ & 1.0 & 32 & 33 \\
\hline $3^{R}$ & e50 & $<50$ & e 7.2 & $<50$ & 72 & $<200$ & $<1.0$ & $<1.0$ & $<5.0$ & .40 & 24 & 24 \\
\hline $3^{R}$ & $<\mathbf{5 0}$ & $<50$ & e10 & $<50$ & 74 & $<200$ & $<1.0$ & $<1.0$ & $<5.0$ & 1.2 & 22 & 23 \\
\hline 4 & $<\mathbf{5 0}$ & $<50$ & $<50$ & $<50$ & 70 & $<200$ & $<1.0$ & $<1.0$ & $<5.0$ & 3.4 & 14 & 14 \\
\hline 5 & e9.0 & $<50$ & e9.2 & $<50$ & 70 & $<200$ & $<1.0$ & $<1.0$ & $<5.0$ & $<.10$ & 11 & 11 \\
\hline 9 & 72 & $<50$ & $<50$ & $<50$ & 66 & $<200$ & $<1.0$ & $<1.0$ & $<5.0$ & .60 & 20 & 20 \\
\hline 9 & 75 & $<50$ & $<50$ & $<50$ & 74 & $<200$ & $<1.0$ & $<1.0$ & $<5.0$ & 1.0 & 20 & 21 \\
\hline 10 & $<50$ & $<50$ & $<50$ & $<50$ & 64 & $<200$ & $<1.0$ & $<1.0$ & $<5.0$ & 6.7 & 11 & 18 \\
\hline 11 & $<50$ & $<50$ & $<50$ & $<50$ & 69 & $<200$ & $<1.0$ & $<1.0$ & $<5.0$ & 5.4 & 4.6 & 10 \\
\hline 12 & e11 & $<50$ & $<50$ & $<50$ & 72 & $<200$ & $<1.0$ & $<1.0$ & $<5.0$ & 2.3 & 4.7 & 7.0 \\
\hline 14 & $<50$ & $<50$ & $<50$ & $<50$ & 69 & $<200$ & $<1.0$ & $<1.0$ & $<5.0$ & $<.10$ & 3.1 & 3.1 \\
\hline 23 & e7.8 & $<50$ & e3.9 & $<50$ & 60 & $<200$ & $<1.0$ & $<1.0$ & $<5.0$ & $<.10$ & 15 & 15 \\
\hline 31 & e22 & e26 & e14 & $<50$ & 86 & $<200$ & $<1.0$ & $<1.0$ & $<5.0$ & .40 & 15 & 15 \\
\hline 33 & e9.1 & $<50$ & $<50$ & $<50$ & 70 & $<200$ & $<1.0$ & $<1.0$ & $<5.0$ & $<.10$ & 7.6 & 7.6 \\
\hline 35 & $<50$ & $<50$ & e20 & $<50$ & 65 & $<200$ & $<1.0$ & $<1.0$ & $<5.0$ & $<.10$ & 9.2 & 9.2 \\
\hline 40 & e16 & $<50$ & $<50$ & $<50$ & 84 & $<200$ & $<1.0$ & $<1.0$ & $<5.0$ & .40 & 15 & 11 \\
\hline 41 & $<50$ & $<50$ & e30 & $<50$ & 74 & $<200$ & $<1.0$ & $<1.0$ & $<5.0$ & .30 & 8.7 & 13 \\
\hline $41^{\mathbf{R}}$ & $<50$ & $<50$ & e46 & $<50$ & 76 & $<200$ & $<1.0$ & $<1.0$ & $<5.0$ & $<.10$ & 13 & 9.0 \\
\hline $41^{\mathbf{R}}$ & e38 & $<50$ & 160 & $<50$ & 60 & $<200$ & $<1.0$ & $<1.0$ & $<5.0$ & $<.10$ & 14 & 14 \\
\hline 41 & e8.9 & $<50$ & e18 & $<50$ & 73 & $<200$ & $<1.0$ & $<1.0$ & $<5.0$ & 1.0 & 13 & 14 \\
\hline
\end{tabular}


Table 30. Trace-element concentration data for fish-tissue samples, Northern Rockies Intermontane Basins study unit, 1998-99

[Body part: L, liver; F, fillet. Abbreviations: $\mu \mathrm{g} / \mathrm{g}$, micrograms per gram, dry weight; R, replicate. Symbol: <, less than reporting level]

\begin{tabular}{|c|c|c|c|c|c|c|}
\hline $\begin{array}{c}\text { Site num- } \\
\text { ber } \\
\text { (fig. 1) }\end{array}$ & Site name & Date & Time & $\begin{array}{l}\text { Body } \\
\text { part } \\
\text { ana- } \\
\text { lyzed }\end{array}$ & $\begin{array}{c}\text { Fish } \\
\text { species }\end{array}$ & $\begin{array}{l}\text { Num- } \\
\text { ber } \\
\text { of fish } \\
\text { com- } \\
\text { posited }\end{array}$ \\
\hline 1 & Clark Fork near Galen, Mont. & $08 / 19 / 98$ & 1130 & $\mathrm{~L}$ & Largescale sucker & 8 \\
\hline 1 & Clark Fork near Galen, Mont. & 08/19/98 & 1145 & $\mathrm{~F}$ & Mountain whitefish & 5 \\
\hline 1 & Clark Fork near Galen, Mont. & $08 / 19 / 98$ & 1200 & $\mathrm{~F}$ & Brown trout & 5 \\
\hline 2 & Rock Creek near Clinton, Mont. & $08 / 11 / 98$ & 1305 & $\mathrm{~L}$ & Brown trout & 7 \\
\hline 2 & Rock Creek near Clinton, Mont. & $08 / 11 / 98$ & 1310 & $\mathrm{~F}$ & Brown trout & 7 \\
\hline 3 & Clark Fork at Turah Bridge, near Bonner, Mont. & $08 / 12 / 98$ & 1315 & $\mathrm{~L}$ & Largescale sucker & 8 \\
\hline 3 & Clark Fork at Turah Bridge, near Bonner, Mont. & $08 / 12 / 98$ & 1330 & $\mathrm{~F}$ & Mountain whitefish & 6 \\
\hline 3 & Clark Fork at Turah Bridge, near Bonner, Mont. & $08 / 12 / 98$ & 1415 & $\mathrm{~L}$ & Largescale sucker & 8 \\
\hline $3^{\mathbf{R}}$ & Clark Fork at Turah Bridge, near Bonner, Mont. & $08 / 12 / 98$ & 1515 & $\mathrm{~L}$ & Largescale sucker & 8 \\
\hline 4 & Blackfoot River above Nevada Creek, near Helmville, Mont. & $08 / 18 / 98$ & 1205 & $\mathrm{~L}$ & Largescale sucker & 8 \\
\hline 4 & Blackfoot River above Nevada Creek, near Helmville, Mont. & $08 / 18 / 98$ & 1210 & $\mathrm{~F}$ & Mountain whitefish & 4 \\
\hline 5 & Bitterroot River near Missoula, Mont. & 08/10/98 & 1705 & $\mathrm{~F}$ & Mountain whitefish & 8 \\
\hline 5 & Bitterroot River near Missoula, Mont. & 08/10/98 & 1710 & $\mathrm{~L}$ & Mountain whitefish & 17 \\
\hline 9 & Clark Fork at St. Regis, Mont. & $09 / 08 / 98$ & 1505 & $\mathrm{~L}$ & Largescale sucker & 6 \\
\hline 9 & Clark Fork at St. Regis, Mont. & $09 / 08 / 98$ & 1510 & $\mathrm{~F}$ & Mountain whitefish & 6 \\
\hline 11 & Middle Fork Flathead River near West Glacier, Mont. & $07 / 21 / 98$ & 1305 & $\mathrm{~L}$ & Mountain whitefish & 8 \\
\hline 11 & Middle Fork Flathead River near West Glacier, Mont. & $07 / 21 / 98$ & 1310 & $\mathrm{~F}$ & Mountain whitefish & 8 \\
\hline $11^{\mathbf{R}}$ & Middle Fork Flathead River near West Glacier, Mont. & $07 / 21 / 98$ & 1315 & $\mathrm{~F}$ & Mountain whitefish & 8 \\
\hline 12 & Flathead River at Perma, Mont. & $10 / 16 / 98$ & 0115 & $\mathrm{~L}$ & Largescale sucker & 6 \\
\hline 12 & Flathead River at Perma, Mont. & $10 / 16 / 98$ & 0130 & $\mathrm{~F}$ & Mountain whitefish & 6 \\
\hline 15 & Priest River near Priest River, Idaho & 09/10/98 & 1405 & $\mathrm{~L}$ & Largescale sucker & 8 \\
\hline 23 & North Fork Coeur d'Alene River at Enaville, Idaho & $06 / 16 / 98$ & 1600 & $\mathrm{~L}$ & Mountain whitefish & 7 \\
\hline 23 & North Fork Coeur d'Alene River at Enaville, Idaho & $06 / 16 / 98$ & 1610 & $\mathrm{~F}$ & Mountain whitefish & 7 \\
\hline 25 & South Fork Coeur d'Alene River above Deadman Gulch, near Mullan, Idaho & $06 / 23 / 98$ & 1200 & $\mathrm{~L}$ & Rainbow trout & 7 \\
\hline 25 & South Fork Coeur d'Alene River above Deadman Gulch, near Mullan, Idaho & $06 / 23 / 98$ & 1205 & $\mathrm{~F}$ & Rainbow trout & 7 \\
\hline 31 & South Fork Coeur d'Alene River near Pinehurst, Idaho & $06 / 17 / 98$ & 1615 & $\mathrm{~L}$ & Brook trout & 6 \\
\hline 31 & South Fork Coeur d'Alene River near Pinehurst, Idaho & $06 / 17 / 98$ & 1620 & $\mathrm{~F}$ & Brook trout & 6 \\
\hline 31 & South Fork Coeur d'Alene River near Pinehurst, Idaho & $08 / 17 / 99$ & 1205 & $\mathrm{~F}$ & Brook trout & 5 \\
\hline 31 & South Fork Coeur d'Alene River near Pinehurst, Idaho & $08 / 17 / 99$ & 1210 & $\mathrm{~L}$ & Brook trout & 10 \\
\hline 33 & St. Joe River at Red Ives Ranger Station, Idaho & 07/07/98 & 1345 & $\mathrm{~L}$ & Cutthroat trout & 8 \\
\hline 33 & St. Joe River at Red Ives Ranger Station, Idaho & 07/07/98 & 1350 & $\mathrm{~F}$ & Cutthroat trout & 8 \\
\hline 33 & St. Joe River at Red Ives Ranger Station, Idaho & 09/01/99 & 1530 & $\mathrm{~L}$ & Cutthroat trout & 9 \\
\hline 34 & St. Joe River at Calder, Idaho & $07 / 08 / 98$ & 1405 & $\mathrm{~L}$ & Largescale sucker & 8 \\
\hline 34 & St. Joe River at Calder, Idaho & $07 / 08 / 98$ & 1410 & $\mathrm{~F}$ & Mountain whitefish & 8 \\
\hline 35 & Spokane River near Post Falls, Idaho & $07 / 12 / 99$ & 1305 & $\mathrm{~L}$ & Largescale sucker & 5 \\
\hline 36 & Spokane River above Liberty Bridge, near Otis Orchard, Wash. & $07 / 27 / 99$ & 1205 & $\mathrm{~L}$ & Largescale sucker & 5 \\
\hline 38 & Spokane River at Sullivan Bridge, near Trentwood, Wash. & $07 / 28 / 99$ & 1205 & $\mathrm{~L}$ & Largescale sucker & 10 \\
\hline 40 & Hangman Creek at Spokane, Wash. & $06 / 19 / 98$ & 1505 & $\mathrm{~L}$ & Largescale sucker & 8 \\
\hline 40 & Hangman Creek at Spokane, Wash. & $06 / 19 / 98$ & 1510 & $\mathrm{~F}$ & Rainbow trout & 5 \\
\hline 41 & Spokane River at Seven Mile Bridge, near Spokane, Wash. & $08 / 05 / 98$ & 1115 & $\mathrm{~L}$ & Largescale sucker & 8 \\
\hline 41 & Spokane River at Seven Mile Bridge, near Spokane, Wash. & $08 / 05 / 98$ & 1145 & $\mathrm{~F}$ & Rainbow trout & 5 \\
\hline
\end{tabular}


Table 30. Trace-element concentration data for fish-tissue samples, Northern Rockies Intermontane Basins study unit, $1998-99$ (Continued)

\begin{tabular}{|c|c|c|c|c|c|c|c|c|c|c|c|c|}
\hline $\begin{array}{c}\text { Site num- } \\
\text { ber } \\
\text { (fig. 1) }\end{array}$ & $\begin{array}{c}\text { Alumi- } \\
\text { num } \\
(\mu \mathrm{g} / \mathrm{g})\end{array}$ & $\begin{array}{l}\text { Anti- } \\
\text { mony } \\
(\mu \mathrm{g} / \mathrm{g})\end{array}$ & $\begin{array}{c}\text { Arsenic } \\
(\mu \mathrm{g} / \mathrm{g})\end{array}$ & $\begin{array}{c}\text { Barium } \\
(\mu \mathrm{g} / \mathrm{g})\end{array}$ & $\begin{array}{c}\text { Beryl- } \\
\text { lium } \\
(\mu \mathrm{g} / \mathrm{g})\end{array}$ & $\begin{array}{l}\text { Boron } \\
(\mu \mathrm{g} / \mathrm{g})\end{array}$ & $\begin{array}{l}\text { Cad- } \\
\text { mium } \\
(\mu \mathrm{g} / \mathrm{g})\end{array}$ & $\begin{array}{l}\text { Chro- } \\
\text { mium } \\
(\mu \mathrm{g} / \mathrm{g})\end{array}$ & $\begin{array}{c}\text { Cobalt } \\
(\mu \mathrm{g} / \mathrm{g})\end{array}$ & $\begin{array}{c}\text { Copper } \\
(\mu \mathrm{g} / \mathrm{g})\end{array}$ & $\begin{array}{c}\text { Iron } \\
(\mu \mathrm{g} / \mathrm{g})\end{array}$ & $\begin{array}{c}\text { Lead } \\
(\mu \mathrm{g} / \mathrm{g})\end{array}$ \\
\hline 1 & 2.1 & 0.44 & 0.89 & $<0.10$ & $<0.16$ & 0.30 & 9.0 & $<0.50$ & $<0.16$ & 52 & 281 & $<0.16$ \\
\hline 1 & $<1.0$ & $<.15$ & .32 & .13 & $<.15$ & .58 & $<.15$ & $<.50$ & $<.15$ & 1.2 & 15 & $<.15$ \\
\hline 1 & $<1.0$ & $<.18$ & .25 & $<.10$ & $<.18$ & .35 & $<.18$ & .54 & $<.18$ & 1.2 & 18 & $<.18$ \\
\hline 2 & 22 & $<.14$ & .28 & .11 & $<.14$ & .44 & .62 & .62 & .27 & 387 & 1,300 & $<.14$ \\
\hline 2 & $<1.0$ & $<.21$ & .99 & .12 & $<.21$ & .45 & $<.21$ & $<.50$ & $<.21$ & 1.0 & 25 & $<.21$ \\
\hline 3 & 7.0 & .27 & 1.3 & .20 & $<.16$ & .63 & 2.5 & $<.50$ & $<.16$ & 71 & 498 & .28 \\
\hline 3 & $<1.0$ & $<.18$ & $<.18$ & .11 & $<.18$ & .65 & $<.18$ & $<.50$ & $<.18$ & 1.4 & 16 & $<.18$ \\
\hline 3 & 6.0 & .29 & 1.4 & .35 & $<.20$ & .94 & 5.6 & $<.50$ & $<.20$ & 70 & 1,020 & .43 \\
\hline $3^{R}$ & 2.4 & $<.19$ & .99 & .29 & $<.19$ & .78 & 4.4 & $<.50$ & $<.19$ & 83 & 501 & .25 \\
\hline 4 & 3.1 & $<.14$ & .55 & .15 & $<.14$ & .32 & 1.0 & $<.50$ & .19 & 70 & 719 & $<.14$ \\
\hline 4 & $<1.0$ & $<.18$ & .28 & .73 & $<.18$ & .28 & $<.18$ & .59 & $<.18$ & 1.6 & 19 & $<.18$ \\
\hline 5 & 1.2 & $<.18$ & $<.18$ & .32 & $<.18$ & .50 & $<.18$ & .57 & $<.18$ & 1.2 & 23 & $<.18$ \\
\hline 5 & 2.0 & $<.17$ & $<.17$ & $<.10$ & $<.17$ & .48 & .36 & .57 & .30 & 11 & 529 & $<.17$ \\
\hline 9 & 2.1 & $<.18$ & .76 & $<.10$ & $<.18$ & .25 & .30 & $<.50$ & $<.18$ & 57 & 483 & $<.18$ \\
\hline 9 & $<1.0$ & $<.18$ & .24 & .19 & $<.18$ & .40 & $<.18$ & $<.50$ & $<.18$ & 1.4 & 17 & $<.18$ \\
\hline 11 & 1.7 & $<.19$ & .32 & .12 & $<.19$ & .70 & .32 & .51 & .64 & 8.8 & 602 & $<.19$ \\
\hline 11 & 1.1 & $<.18$ & .21 & .44 & $<.18$ & .48 & $<.18$ & $<.50$ & $<.18$ & 1.5 & 24 & $<.18$ \\
\hline $11^{\mathbf{R}}$ & $<1.0$ & $<.20$ & .31 & 1.4 & $<.20$ & .53 & $<.20$ & .66 & $<.20$ & 1.6 & 26 & $<.20$ \\
\hline 12 & 19 & $<.10$ & .71 & 1.2 & $<.10$ & .37 & .93 & .61 & .30 & 67 & 824 & .16 \\
\hline 12 & 5.1 & $<.09$ & .32 & .40 & $<.09$ & .45 & $<.09$ & .61 & $<.09$ & 1.1 & 13 & $<.09$ \\
\hline 15 & 154 & $<.21$ & .56 & 1.9 & $<.21$ & .29 & 1.0 & .62 & .31 & 42 & 1,970 & .29 \\
\hline 23 & $<1.0$ & $<.24$ & $<.24$ & $<.10$ & $<.24$ & .39 & 8.2 & .55 & .72 & 13 & 253 & 2.9 \\
\hline 23 & $<1.0$ & $<.12$ & .22 & .35 & $<.12$ & .36 & $<.12$ & $<.50$ & $<.12$ & 1.5 & 19 & .72 \\
\hline 25 & 5.2 & $<.17$ & 1.5 & $<.10$ & $<.17$ & .66 & .33 & .54 & $<.17$ & 346 & 671 & 1.3 \\
\hline 25 & $<1.0$ & $<.16$ & 1.8 & .51 & $<.16$ & .48 & $<.16$ & .54 & $<.16$ & 2.2 & 17 & $<.16$ \\
\hline 31 & $<1.0$ & $<.18$ & .24 & $<.10$ & $<.18$ & .38 & 10 & $<.50$ & .55 & 153 & 597 & 12 \\
\hline 31 & $<1.0$ & $<.17$ & $<.17$ & .14 & $<.17$ & .65 & .57 & $<.50$ & $<.17$ & 1.8 & 18 & 1.7 \\
\hline 31 & $<1.0$ & $<.20$ & .21 & .25 & $<.20$ & .79 & .22 & $<.50$ & $<.20$ & 1.8 & 17 & 2.1 \\
\hline 31 & 2.7 & $<.25$ & .34 & $<.10$ & $<.25$ & .46 & 17 & $<.50$ & .40 & 209 & 493 & 13 \\
\hline 33 & 1.0 & $<.02$ & $<.10$ & $<.10$ & $<.02$ & $<.20$ & $<.10$ & $<.50$ & $<.10$ & 1.4 & 91 & $<.02$ \\
\hline 33 & 14 & $<.21$ & .47 & .41 & $<.21$ & .61 & $<.21$ & .62 & $<.21$ & 1.8 & 39 & $<.21$ \\
\hline 33 & 20 & $<.16$ & .67 & $<.10$ & $<.16$ & .23 & .51 & .64 & .48 & 17 & 1,010 & $<.16$ \\
\hline 34 & 20 & $<.22$ & .65 & .14 & $<.22$ & .36 & 3.8 & .56 & .52 & 85 & 1,850 & 3.4 \\
\hline 34 & 1.5 & $<.18$ & $<.18$ & .26 & $<.18$ & .39 & $<.18$ & $<.50$ & $<.18$ & 1.5 & 21 & $<.18$ \\
\hline 35 & 35 & $<.21$ & .78 & .10 & $<.21$ & .80 & 3.4 & .56 & $<.21$ & 35 & 1,080 & 2.3 \\
\hline 36 & 5.1 & $<.17$ & 1.1 & $<.10$ & $<.17$ & $<.20$ & 1.6 & $<.50$ & $<.17$ & 8.9 & 309 & 1.7 \\
\hline 38 & 3.7 & $<.22$ & 1.2 & $<.10$ & $<.22$ & .49 & 1.7 & $<.50$ & $<.22$ & 23 & 594 & 1.4 \\
\hline 40 & 35 & $<.21$ & 1.2 & .52 & $<.21$ & .53 & 2.7 & $<.50$ & .30 & 47 & 997 & 1.4 \\
\hline 40 & $<1.0$ & $<.20$ & $<.20$ & .56 & $<.20$ & .65 & $<.20$ & .61 & $<.20$ & 1.4 & 17 & $<.20$ \\
\hline 41 & 8.8 & $<.24$ & .99 & .10 & $<.24$ & .79 & 3.2 & $<.50$ & $<.24$ & 50 & 829 & 1.4 \\
\hline 41 & $<1.0$ & $<.22$ & $<.22$ & .56 & $<.22$ & .56 & $<.22$ & .54 & $<.22$ & 1.2 & 16 & .83 \\
\hline
\end{tabular}


Table 30. Trace-element concentration data for fish-tissue samples, Northern Rockies Intermontane Basins study unit, $1998-99$ (Continued)

\begin{tabular}{|c|c|c|c|c|c|c|c|c|c|c|c|}
\hline $\begin{array}{l}\text { Site num- } \\
\text { ber } \\
\text { (fig. 1) }\end{array}$ & $\begin{array}{c}\text { Manga- } \\
\text { nese } \\
(\mu \mathrm{g} / \mathrm{g})\end{array}$ & $\begin{array}{l}\text { Mer- } \\
\text { cury } \\
(\mu \mathrm{g} / \mathrm{g})\end{array}$ & $\begin{array}{c}\text { Molyb- } \\
\text { denum } \\
(\mu \mathrm{g} / \mathrm{g})\end{array}$ & $\begin{array}{l}\text { Nickel } \\
(\mu \mathrm{g} / \mathrm{g})\end{array}$ & $\begin{array}{c}\text { Sele- } \\
\text { nium } \\
(\mu \mathrm{g} / \mathrm{g})\end{array}$ & $\begin{array}{l}\text { Silver } \\
(\mu \mathrm{g} / \mathrm{g})\end{array}$ & $\begin{array}{c}\text { Stron- } \\
\text { tium } \\
(\mu \mathrm{g} / \mathrm{g})\end{array}$ & $\begin{array}{c}\text { Ura- } \\
\text { nium } \\
(\mu \mathrm{g} / \mathrm{g})\end{array}$ & $\begin{array}{c}\text { Vana- } \\
\text { dium } \\
(\mu \mathrm{g} / \mathrm{g})\end{array}$ & $\begin{array}{c}\text { Zinc } \\
(\mu \mathrm{g} / \mathrm{g})\end{array}$ & $\begin{array}{c}\text { Water } \\
\text { present } \\
\text { in tissue } \\
\text { (percent) }\end{array}$ \\
\hline 1 & 8.5 & 0.15 & 1.1 & $<0.16$ & 3.1 & $<0.16$ & 0.16 & $<0.16$ & 0.21 & 94 & 70 \\
\hline 1 & 2.1 & .15 & $<.15$ & $<.15$ & .70 & $<.15$ & 1.9 & $<.15$ & .23 & 11 & 67 \\
\hline 1 & 1.6 & .37 & .22 & $<.18$ & 1.3 & $<.18$ & 1.8 & $<.18$ & $<.18$ & 15 & 77 \\
\hline 2 & 4.5 & .20 & 1.1 & $<.14$ & 24 & .46 & .12 & $<.14$ & 1.3 & 99 & 70 \\
\hline 2 & .35 & 1.9 & .34 & $<.21$ & .61 & $<.21$ & .31 & $<.21$ & $<.21$ & 15 & 77 \\
\hline 3 & 6.0 & .32 & .90 & $<.16$ & 2.9 & .19 & .32 & $<.16$ & .31 & 121 & 68 \\
\hline 3 & .76 & .39 & $<.18$ & $<.18$ & 1.0 & $<.18$ & .71 & $<.18$ & $<.18$ & 13 & 71 \\
\hline 3 & 10 & .28 & .92 & $<.20$ & 2.6 & $<.20$ & .72 & $<.20$ & .41 & 148 & 73 \\
\hline $3^{R}$ & 9.2 & .26 & .85 & $<.19$ & 2.1 & $<.19$ & .66 & $<.19$ & .26 & 159 & 72 \\
\hline 4 & 5.2 & .24 & 1.1 & .22 & 4.0 & .25 & .14 & $<.14$ & .35 & 93 & 70 \\
\hline 4 & 1.2 & .15 & .33 & .28 & 1.7 & $<.18$ & 1.6 & $<.18$ & $<.18$ & 15 & 74 \\
\hline 5 & 1.7 & .36 & .28 & $<.18$ & .60 & $<.18$ & 1.8 & $<.18$ & $<.18$ & 15 & 75 \\
\hline 5 & 6.0 & 1.0 & .87 & $<.17$ & 7.5 & $<.17$ & .28 & $<.17$ & .40 & 90 & 76 \\
\hline 9 & 8.1 & .31 & .83 & $<.18$ & 2.3 & $<.18$ & .16 & $<.18$ & .35 & 115 & 72 \\
\hline 9 & .79 & .22 & $<.18$ & $<.18$ & .77 & $<.18$ & .58 & $<.18$ & .21 & 15 & 72 \\
\hline 11 & 4.8 & .48 & .72 & $<.19$ & 24 & $<.19$ & .17 & $<.19$ & $<.19$ & 91 & 77 \\
\hline 11 & .81 & .15 & $<.18$ & $<.18$ & 1.3 & $<.18$ & .65 & $<.18$ & $<.18$ & 15 & 74 \\
\hline $11^{\mathbf{R}}$ & 1.8 & .20 & $<.20$ & $<.20$ & 2.3 & $<.20$ & 2.1 & $<.20$ & $<.20$ & 17 & 73 \\
\hline 12 & 11 & .17 & .79 & .17 & 5.3 & .38 & .49 & $<.10$ & .31 & 149 & 78 \\
\hline 12 & 1.1 & .25 & $<.09$ & $<.09$ & 1.1 & $<.09$ & .86 & $<.09$ & .18 & 13 & 77 \\
\hline 15 & 16 & .25 & .66 & .25 & 3.2 & .26 & .62 & $<.21$ & .75 & 111 & 76 \\
\hline 23 & 6.3 & .59 & .79 & $<.24$ & 7.6 & $<.24$ & .26 & $<.24$ & $<.24$ & 96 & 76 \\
\hline 23 & 2.5 & .13 & .15 & $<.12$ & .83 & $<.12$ & 1.2 & $<.12$ & $<.12$ & 20 & 68 \\
\hline 25 & 4.4 & .22 & .97 & $<.17$ & 8.1 & .99 & .20 & $<.17$ & .38 & 86 & 75 \\
\hline 25 & 1.5 & .08 & $<.16$ & $<.16$ & 1.6 & $<.16$ & 2.6 & $<.16$ & $<.16$ & 18 & 76 \\
\hline 31 & 3.6 & .30 & 1.0 & $<.18$ & 4.8 & 1.3 & .33 & $<.18$ & .26 & 294 & 74 \\
\hline 31 & 2.0 & .07 & $<.17$ & $<.17$ & 1.1 & $<.17$ & 1.5 & $<.17$ & $<.17$ & 36 & 73 \\
\hline 31 & 3.1 & .09 & $<.20$ & $<.20$ & 1.4 & $<.20$ & 2.6 & $<.20$ & $<.20$ & 45 & 76 \\
\hline 31 & 4.8 & .26 & 1.1 & $<.25$ & 5.6 & 2.4 & .33 & $<.25$ & $<.25$ & 595 & 76 \\
\hline 33 & .42 & $<.20$ & .11 & $<.02$ & .44 & $<.02$ & $<.10$ & $<.02$ & $<.10$ & 9.0 & 76 \\
\hline 33 & .84 & .14 & $<.21$ & $<.21$ & .89 & $<.21$ & 1.5 & $<.21$ & $<.21$ & 18 & 76 \\
\hline 33 & 5.4 & .28 & .97 & $<.16$ & 5.6 & .26 & $<.10$ & $<.16$ & .22 & 87 & 74 \\
\hline 34 & 7.6 & .38 & 1.3 & $<.22$ & 3.9 & .31 & .16 & $<.22$ & .41 & 157 & 78 \\
\hline 34 & 1.4 & .16 & $<.18$ & $<.18$ & .98 & $<.18$ & 1.2 & $<.18$ & $<.18$ & 16 & 71 \\
\hline 35 & 5.8 & .09 & .85 & $<.21$ & 4.4 & .30 & .20 & $<.21$ & $<.21$ & 150 & 75 \\
\hline 36 & 2.0 & .07 & .56 & $<.17$ & 3.3 & .22 & $<.10$ & $<.17$ & $<.17$ & 43 & 70 \\
\hline 38 & .56 & .04 & .66 & $<.22$ & 4.1 & $<.22$ & .24 & $<.22$ & $<.22$ & 127 & 74 \\
\hline 40 & 13 & .10 & 1.2 & $<.21$ & 4.4 & $<.21$ & .53 & $<.21$ & .63 & 131 & 77 \\
\hline 40 & 3.4 & .11 & $<.20$ & $<.20$ & .87 & $<.20$ & 2.8 & $<.20$ & $<.20$ & 25 & 79 \\
\hline 41 & 5.9 & .09 & 1.2 & .30 & 4.8 & .61 & .31 & $<.24$ & .42 & 204 & 77 \\
\hline 41 & 2.2 & .18 & $<.22$ & $<.22$ & 1.2 & $<.22$ & 3.7 & $<.22$ & $<.22$ & 23 & 77 \\
\hline
\end{tabular}

198 Water-Quality, Streambed-Sediment, and Biological Data from the Clark Fork-Pend Oreille and Spokane River Basins, Montana, Idaho, and Washington, 1998-2001 
Table 31. Organochlorine-compound concentration data for fish-tissue samples, Northern Rockies Intermontane Basins study unit, 1998-99

[Bold text denotes detected concentrations. Sample weight was 10 grams. Abbreviations: $\mu \mathrm{g} / \mathrm{kg}$, micrograms per kilogram, wet weight; R, replicate. Symbol: <, less than reporting level]

\begin{tabular}{|c|c|c|c|c|c|c|}
\hline $\begin{array}{l}\text { Site } \\
\text { num- } \\
\text { ber } \\
\text { (fig. 1) }\end{array}$ & Site name & Date & Time & $\begin{array}{c}\text { Body } \\
\text { part analyzed }\end{array}$ & Fish species & $\begin{array}{c}\text { Number } \\
\text { of fish } \\
\text { com- } \\
\text { posited }\end{array}$ \\
\hline 2 & Rock Creek near Clinton, Mont. & $08 / 11 / 98$ & 1320 & Whole fish & Mountain whitefish & 6 \\
\hline 3 & Clark Fork at Turah Bridge, near Bonner, Mont. & $08 / 12 / 98$ & 1300 & Whole fish & Largescale sucker & 8 \\
\hline $3^{\mathbf{R}}$ & Clark Fork at Turah Bridge, near Bonner, Mont. & $08 / 12 / 98$ & 1400 & Whole fish & Largescale sucker & 8 \\
\hline $3^{\mathbf{R}}$ & Clark Fork at Turah Bridge, near Bonner, Mont. & $08 / 12 / 98$ & 1500 & Whole fish & Largescale sucker & 6 \\
\hline 4 & Blackfoot River above Nevada Creek, near Helmville, Mont. & $08 / 18 / 98$ & 1200 & Whole fish & Largescale sucker & 8 \\
\hline 5 & Bitterroot River near Missoula, Mont. & $08 / 10 / 98$ & 1700 & Whole fish & Largescale sucker & 7 \\
\hline 9 & Clark Fork at St. Regis, Mont. & $09 / 08 / 98$ & 1500 & Whole fish & Largescale sucker & 6 \\
\hline 11 & Middle Fork Flathead River near West Glacier, Mont. & $07 / 21 / 98$ & 1300 & Whole fish & Mountain whitefish & 8 \\
\hline 12 & Flathead River at Perma, Mont. & $10 / 16 / 98$ & 0100 & Whole fish & Largescale sucker & 6 \\
\hline 15 & Priest River near Priest River, Idaho & $09 / 10 / 98$ & 1400 & Whole fish & Largescale sucker & 8 \\
\hline 23 & North Fork Coeur d'Alene River at Enaville, Idaho & $06 / 16 / 98$ & 1605 & Whole fish & Mountain whitefish & 5 \\
\hline 31 & South Fork Coeur d'Alene River near Pinehurst, Idaho & $06 / 17 / 98$ & 1625 & Whole fish & Rainbow trout & 5 \\
\hline 31 & South Fork Coeur d'Alene River near Pinehurst, Idaho & $08 / 17 / 99$ & 1200 & Whole fish & Brook trout & 5 \\
\hline 33 & St. Joe River at Red Ives Ranger Station, Idaho & $07 / 07 / 98$ & 1340 & Whole fish & Cutthroat trout & 5 \\
\hline 33 & St. Joe River at Red Ives Ranger Station, Idaho & $09 / 01 / 99$ & 1500 & Whole fish & Cutthroat trout & 5 \\
\hline 34 & St. Joe River at Calder, Idaho & $07 / 08 / 98$ & 1400 & Whole fish & Largescale sucker & 8 \\
\hline 35 & Spokane River near Post Falls, Idaho & $07 / 12 / 99$ & 1200 & Whole fish & Largescale sucker & 5 \\
\hline 36 & Spokane River above Liberty Bridge, near Otis Orchard, Wash. & $07 / 27 / 99$ & 1200 & Whole fish & Largescale sucker & 5 \\
\hline 38 & Spokane River at Sullivan Bridge, near Trentwood, Wash & $07 / 28 / 99$ & 1200 & Whole fish & Largescale sucker & 5 \\
\hline 40 & Hangman Creek at Spokane, Wash. & $06 / 19 / 98$ & 1500 & Whole fish & Largescale sucker & 8 \\
\hline 41 & Spokane River at Seven Mile Bridge, near Spokane, Wash. & $08 / 05 / 98$ & 1100 & Whole fish & Largescale sucker & 8 \\
\hline 41 & Spokane River at Seven Mile Bridge, near Spokane, Wash. & $08 / 05 / 98$ & 1130 & Fillet & Rainbow trout & 5 \\
\hline
\end{tabular}


Table 31. Organochlorine-compound concentration data for fish-tissue samples, Northern Rockies Intermontane Basins study unit, 1998-99 (Continued)

\begin{tabular}{|c|c|c|c|c|c|c|c|c|c|c|}
\hline $\begin{array}{c}\text { Site } \\
\text { num- } \\
\text { ber } \\
\text { (fig. 1) }\end{array}$ & $\begin{array}{l}\text { Aldrin } \\
(\mu \mathrm{g} / \mathbf{k g})\end{array}$ & $\begin{array}{c}\text { alpha-HCH } \\
(\mu \mathrm{g} / \mathrm{kg})\end{array}$ & $\begin{array}{c}\text { alpha- } \\
\text { HCH-d }_{6} \text {, } \\
\text { surrogate } \\
\text { (in percent) }\end{array}$ & $\begin{array}{c}\text { beta- } \\
\text { HCH } \\
(\mu \mathrm{g} / \mathbf{k g})\end{array}$ & $\begin{array}{c}\text { cis- } \\
\text { Chlor- } \\
\text { dane } \\
(\mu \mathrm{g} / \mathrm{kg})\end{array}$ & $\begin{array}{c}\text { cis- } \\
\text { Nona- } \\
\text { chlor } \\
(\mu \mathrm{g} / \mathbf{k g})\end{array}$ & $\begin{array}{c}\text { Dacthal } \\
(\mu \mathrm{g} / \mathrm{kg})\end{array}$ & $\begin{array}{c}\text { delta-HCH } \\
(\mu \mathbf{g} / \mathbf{k g})\end{array}$ & $\begin{array}{c}\text { Dieldrin } \\
(\mu \mathrm{g} / \mathbf{k g})\end{array}$ & $\begin{array}{l}\text { Endrin } \\
(\mu \mathrm{g} / \mathrm{kg})\end{array}$ \\
\hline 2 & $<5.0$ & $<5.0$ & 89 & $<5.0$ & $<<5.0$ & $<5.0$ & $<5.0$ & $<5.0$ & $<5.0$ & $<5.0$ \\
\hline 3 & $<5.0$ & $<5.0$ & 100 & $<5.0$ & $<5.0$ & $<5.0$ & $<5.0$ & $<5.0$ & $<5.0$ & $<5.0$ \\
\hline $3^{R}$ & $<5.0$ & $<5.0$ & 85 & $<5.0$ & $<5.0$ & $<5.0$ & $<5.0$ & $<5.0$ & $<5.0$ & $<5.0$ \\
\hline $3^{R}$ & $<5.0$ & $<5.0$ & 94 & $<5.0$ & $<5.0$ & $<5.0$ & $<5.0$ & $<5.0$ & $<5.0$ & $<6.0$ \\
\hline 4 & $<5.0$ & $<5.0$ & 82 & $<5.0$ & $<5.0$ & $<5.0$ & $<5.0$ & $<5.0$ & $<5.0$ & $<5.0$ \\
\hline 5 & $<5.0$ & $<5.0$ & 90 & $<5.0$ & $<5.0$ & $<5.0$ & $<5.0$ & $<5.0$ & $<5.0$ & $<17$ \\
\hline 9 & $<5.0$ & $<5.0$ & 80 & $<5.0$ & $<5.0$ & $<5.0$ & $<5.0$ & $<5.0$ & $<5.0$ & $<5.0$ \\
\hline 11 & $<5.0$ & $<5.0$ & 70 & $<5.0$ & $<5.0$ & $<5.0$ & $<5.0$ & $<5.0$ & $<5.0$ & $<5.0$ \\
\hline 12 & $<5.0$ & $<5.0$ & 83 & $<5.0$ & $<5.0$ & $<5.0$ & $<5.0$ & $<5.0$ & $<5.0$ & $<5.0$ \\
\hline 15 & $<5.0$ & $<5.0$ & 74 & $<5.0$ & $<5.0$ & $<5.0$ & $<5.0$ & $<5.0$ & $<5.0$ & $<5.0$ \\
\hline 23 & $<5.0$ & $<5.0$ & 77 & $<5.0$ & $<5.0$ & $<5.0$ & $<5.0$ & $<5.0$ & $<5.0$ & $<5.0$ \\
\hline 31 & $<5.0$ & $<5.0$ & 101 & $<5.0$ & $<5.0$ & $<5.0$ & $<5.0$ & $<5.0$ & $<5.0$ & $<5.0$ \\
\hline 31 & $<5.0$ & $<5.0$ & 90 & $<5.0$ & $<5.0$ & $<5.0$ & $<5.0$ & $<5.0$ & $<5.0$ & $<5.0$ \\
\hline 33 & $<5.0$ & $<5.0$ & 82 & $<5.0$ & $<5.0$ & $<5.0$ & $<5.0$ & $<5.0$ & $<5.0$ & $<5.0$ \\
\hline 33 & $<5.0$ & $<5.0$ & 121 & $<5.0$ & $<5.0$ & $<5.0$ & $<5.0$ & $<5.0$ & $<5.0$ & $<5.0$ \\
\hline 34 & $<5.0$ & $<5.0$ & 82 & $<5.0$ & $<5.0$ & $<5.0$ & $<5.0$ & $<5.0$ & $<5.0$ & $<5.0$ \\
\hline 35 & $<5.0$ & $<5.0$ & 100 & $<5.0$ & $<5.0$ & $<5.0$ & $<5.0$ & $<5.0$ & $<5.0$ & $<5.0$ \\
\hline 36 & $<5.0$ & $<5.0$ & 109 & $<5.0$ & $<5.0$ & $<5.0$ & $<5.0$ & $<5.0$ & $<5.0$ & $<5.0$ \\
\hline 38 & $<5.0$ & $<5.0$ & 103 & $<5.0$ & $<5.0$ & $<5.0$ & $<5.0$ & $<5.0$ & $<5.0$ & $<5.0$ \\
\hline 40 & $<5.0$ & $<5.0$ & 90 & $<5.0$ & $<5.0$ & $<5.0$ & $<5.0$ & $<5.0$ & $<5.0$ & $<5.0$ \\
\hline 41 & $<5.0$ & $<5.0$ & 89 & $<5.0$ & $<5.0$ & $<5.0$ & $<5.0$ & $<5.0$ & $<5.0$ & $<5.0$ \\
\hline 41 & $<5.0$ & $<5.0$ & 88 & $<5.0$ & $<5.0$ & $<5.0$ & $<5.0$ & $<5.0$ & $<5.0$ & $<5.0$ \\
\hline
\end{tabular}


Table 31. Organochlorine-compound concentration data for fish-tissue samples, Northern Rockies Intermontane Basins study unit, 1998-99 (Continued)

\begin{tabular}{|c|c|c|c|c|c|c|c|c|c|c|}
\hline $\begin{array}{l}\text { Site } \\
\text { num- } \\
\text { ber } \\
\text { (fig. 1) }\end{array}$ & $\begin{array}{c}\text { Heptachlor } \\
(\mu \mathrm{g} / \mathrm{kg})\end{array}$ & $\begin{array}{c}\text { Heptachlor } \\
\text { epoxide } \\
(\mu \mathrm{g} / \mathrm{kg})\end{array}$ & $\begin{array}{c}\text { Hexa- } \\
\text { chloro- } \\
\text { benzene } \\
(\mu \mathrm{g} / \mathrm{kg})\end{array}$ & $\begin{array}{c}\text { Lindane } \\
(\mu \mathrm{g} / \mathbf{k g})\end{array}$ & $\begin{array}{c}\text { Lipids } \\
\text { (percent) }\end{array}$ & $\begin{array}{c}\text { Mirex } \\
(\mu \mathrm{g} / \mathrm{kg})\end{array}$ & $\begin{array}{c}\text { o,p'-DDD } \\
(\mu \mathbf{g} / \mathbf{k g})\end{array}$ & $\begin{array}{c}\text { o,p'DDE } \\
(\mu \mathbf{g} / \mathbf{k g})\end{array}$ & $\begin{array}{c}\text { o.p'-DDT } \\
(\mu \mathrm{g} / \mathbf{k g})\end{array}$ & $\begin{array}{c}\text { o,p'- } \\
\text { Methoxy- } \\
\text { chlor } \\
(\mu \mathrm{g} / \mathbf{k g})\end{array}$ \\
\hline 2 & $<5.0$ & $<5.0$ & $<5.0$ & $<5.0$ & 9.1 & $<5.0$ & $<5.0$ & $<5.0$ & $<5.0$ & $<5.0$ \\
\hline 3 & $<5.0$ & $<5.0$ & $<5.0$ & $<5.0$ & 6.2 & $<5.0$ & $<5.0$ & $<5.0$ & $<5.0$ & $<5.0$ \\
\hline $3^{R}$ & $<5.0$ & $<5.0$ & $<5.0$ & $<5.0$ & 6.0 & $<5.0$ & $<5.0$ & $<5.0$ & $<5.0$ & $<5.0$ \\
\hline $3^{\mathbf{R}}$ & $<5.0$ & $<5.0$ & $<5.0$ & $<5.0$ & 5.7 & $<5.0$ & $<5.0$ & $<5.0$ & $<5.0$ & $<5.0$ \\
\hline 4 & $<5.0$ & $<5.0$ & $<5.0$ & $<5.0$ & 5.4 & $<5.0$ & $<5.0$ & $<5.0$ & $<5.0$ & $<5.0$ \\
\hline 5 & $<5.0$ & $<5.0$ & $<5.0$ & $<5.0$ & 7.5 & $<5.0$ & $<5.0$ & $<5.0$ & $<5.0$ & $<5.0$ \\
\hline 9 & $<5.0$ & $<5.0$ & $<5.0$ & $<5.0$ & 4.9 & $<5.0$ & $<5.0$ & $<5.0$ & $<5.0$ & $<5.0$ \\
\hline 11 & $<5.0$ & $<5.0$ & $<5.0$ & $<5.0$ & 7.2 & $<5.0$ & $<5.0$ & $<5.0$ & $<5.0$ & $<5.0$ \\
\hline 12 & $<5.0$ & $<5.0$ & $<5.0$ & $<5.0$ & 6.8 & $<5.0$ & $<5.0$ & $<5.0$ & $<5.0$ & $<5.0$ \\
\hline 15 & $<5.0$ & $<5.0$ & $<5.0$ & $<5.0$ & 3.4 & $<5.0$ & $<5.0$ & $<5.0$ & $<5.4$ & $<5.0$ \\
\hline 23 & $<5.0$ & $<5.0$ & $<5.0$ & $<5.0$ & 9.4 & $<5.0$ & $<5.0$ & $<5.0$ & $<5.0$ & $<5.0$ \\
\hline 31 & $<5.0$ & $<5.0$ & $<5.0$ & $<5.0$ & 7.1 & $<5.0$ & $<5.0$ & $<5.0$ & $<5.0$ & $<5.0$ \\
\hline 31 & $<5.0$ & $<5.0$ & $<5.0$ & $<5.0$ & 11 & $<5.0$ & $<5.0$ & $<5.0$ & $<5.0$ & $<5.0$ \\
\hline 33 & $<5.0$ & $<5.0$ & $<5.0$ & $<5.0$ & 8.9 & $<5.0$ & $<5.0$ & $<5.0$ & $<5.0$ & $<5.0$ \\
\hline 33 & $<5.0$ & $<5.0$ & $<5.0$ & $<5.0$ & 5.8 & $<5.0$ & $<5.0$ & $<5.0$ & $<5.0$ & $<5.0$ \\
\hline 34 & $<5.0$ & $<5.0$ & $<5.0$ & $<5.0$ & 3.9 & $<5.0$ & $<5.0$ & $<5.0$ & $<5.0$ & $<5.0$ \\
\hline 35 & $<5.0$ & $<5.0$ & $<5.0$ & $<5.0$ & 3.0 & $<5.0$ & $<5.0$ & $<5.0$ & $<5.0$ & $<5.0$ \\
\hline 36 & $<5.0$ & $<5.0$ & $<5.0$ & $<5.0$ & 5.7 & $<5.0$ & $<5.0$ & $<5.0$ & $<5.0$ & $<5.0$ \\
\hline 38 & $<5.0$ & $<5.0$ & $<5.0$ & $<5.0$ & 2.2 & $<5.0$ & $<5.0$ & $<5.0$ & $<5.0$ & $<5.0$ \\
\hline 40 & $<5.0$ & $<5.0$ & $<5.0$ & $<5.0$ & 2.6 & $<5.0$ & $<5.0$ & $<5.0$ & $<5.0$ & $<5.0$ \\
\hline 41 & $<5.0$ & $<5.0$ & $<5.0$ & $<5.0$ & 2.6 & $<5.0$ & $<5.0$ & $<5.0$ & $<5.0$ & $<5.0$ \\
\hline 41 & $<5.0$ & $<5.0$ & $<5.0$ & $<5.0$ & 14 & $<5.0$ & $<5.0$ & $<5.0$ & $<5.0$ & $<5.0$ \\
\hline
\end{tabular}


Table 31. Organochlorine-compound concentration data for fish-tissue samples, Northern Rockies Intermontane Basins study unit, 1998-99 Continued)

\begin{tabular}{|c|c|c|c|c|c|c|c|c|c|c|}
\hline $\begin{array}{c}\text { Site } \\
\text { num- } \\
\text { ber } \\
\text { (fig. 1) }\end{array}$ & $\begin{array}{c}\text { Oxy- } \\
\text { chlor- } \\
\text { dane } \\
(\mu \mathrm{g} / \mathrm{kg})\end{array}$ & $\begin{array}{c}\text { p,p'-DDD } \\
(\mu \mathbf{g} / \mathbf{k g})\end{array}$ & $\begin{array}{c}\mathbf{p}, \mathbf{p}^{\prime}-\mathbf{D D E} \\
(\mu \mathrm{g} / \mathbf{k g})\end{array}$ & $\begin{array}{c}\text { p,p'-DDT } \\
(\mu \mathbf{g} / \mathbf{k g})\end{array}$ & $\begin{array}{c}\text { p,p'- } \\
\text { Meth- } \\
\text { oxychlor } \\
(\mu \mathrm{g} / \mathrm{kg})\end{array}$ & $\begin{array}{l}\text { Penta- } \\
\text { chloro- } \\
\text { anisole } \\
(\mu \mathrm{g} / \mathrm{kg})\end{array}$ & $\begin{array}{c}\text { Poly- } \\
\text { chlorinated } \\
\text { biphenyls } \\
(\mu \mathrm{g} / \mathbf{k g})\end{array}$ & $\begin{array}{c}\text { Toxa- } \\
\text { phene } \\
(\mu \mathrm{g} / \mathrm{kg})\end{array}$ & $\begin{array}{c}\text { trans- } \\
\text { Chlor- } \\
\text { dane } \\
(\mu \mathrm{g} / \mathrm{kg})\end{array}$ & $\begin{array}{c}\text { trans- } \\
\text { Nona- } \\
\text { chlor } \\
(\mu \mathrm{g} / \mathrm{kg})\end{array}$ \\
\hline 2 & $<5.0$ & $<5.0$ & $<5.0$ & $<5.0$ & $<5.0$ & $<5.0$ & $<50$ & $<200$ & $<5.0$ & $<5.0$ \\
\hline 3 & $<5.0$ & $<5.0$ & $<5.0$ & $<5.0$ & $<5.0$ & $<5.0$ & $<50$ & $<200$ & $<5.0$ & $<5.0$ \\
\hline $3^{R}$ & $<5.0$ & $<5.0$ & $<5.0$ & $<5.0$ & $<5.0$ & $<5.0$ & 120 & $<200$ & $<5.0$ & $<5.0$ \\
\hline $3^{R}$ & $<5.0$ & $<5.0$ & $<5.0$ & $<5.0$ & $<5.0$ & $<5.0$ & $<50$ & $<200$ & $<5.0$ & $<5.0$ \\
\hline 4 & $<5.0$ & $<5.0$ & $<5.0$ & $<5.0$ & $<5.0$ & $<5.0$ & 160 & $<200$ & $<5.0$ & $<5.0$ \\
\hline 5 & $<5.0$ & $<5.0$ & 9.0 & $<5.0$ & $<5.0$ & $<5.0$ & 140 & $<200$ & $<5.0$ & $<5.0$ \\
\hline 9 & $<5.0$ & $<5.0$ & $<5.0$ & $<5.0$ & $<5.0$ & $<5.0$ & 220 & $<200$ & $<5.0$ & $<5.0$ \\
\hline 11 & $<5.0$ & $<5.0$ & $<5.0$ & $<5.0$ & $<5.0$ & $<5.0$ & $<5.0$ & $<5.0$ & $<5.0$ & $<5.0$ \\
\hline 12 & $<5.0$ & $<5.0$ & 6.6 & $<5.0$ & $<5.0$ & $<5.0$ & $<50$ & $<200$ & $<5.0$ & $<5.0$ \\
\hline 15 & $<5.0$ & $<5.0$ & 11 & $<5.0$ & $<5.0$ & $<5.0$ & 62 & $<200$ & $<5.0$ & $<5.0$ \\
\hline 23 & $<5.0$ & $<5.0$ & $<5.0$ & $<5.0$ & $<5.0$ & $<5.0$ & 150 & $<200$ & $<5.0$ & $<5.0$ \\
\hline 31 & $<5.0$ & $<5.0$ & 14 & $<5.0$ & $<5.0$ & $<5.0$ & 310 & $<200$ & $<5.0$ & $<5.0$ \\
\hline 31 & $<5.0$ & $<5.0$ & 8.7 & $<5.0$ & $<5.0$ & $<5.0$ & 200 & $<200$ & $<5.0$ & $<5.0$ \\
\hline 33 & $<5.0$ & $<5.0$ & $<5.0$ & $<5.0$ & $<5.0$ & $<5.0$ & $<50$ & $<200$ & $<5.0$ & $<5.0$ \\
\hline 33 & $<5.0$ & $<5.0$ & 5.0 & $<5.0$ & $<5.0$ & $<5.0$ & $<50$ & $<200$ & $<5.0$ & $<5.0$ \\
\hline 34 & $<5.0$ & $<5.0$ & 10 & $<5.0$ & $<5.0$ & $<5.0$ & $<50$ & $<200$ & $<5.0$ & $<5.0$ \\
\hline 35 & $<5.0$ & $<5.0$ & 11 & $<5.0$ & $<5.0$ & $<5.0$ & 270 & $<200$ & $<5.0$ & $<5.0$ \\
\hline 36 & $<5.0$ & $<5.0$ & 16 & $<5.0$ & $<5.0$ & $<5.0$ & 500 & $<200$ & $<5.0$ & $<5.0$ \\
\hline 38 & $<5.0$ & $<5.0$ & 12 & $<5.0$ & $<5.0$ & $<5.0$ & 310 & $<200$ & $<5.0$ & $<5.0$ \\
\hline 40 & $<5.0$ & $<5.0$ & 38 & 6.6 & $<5.0$ & $<5.0$ & 570 & $<200$ & $<5.0$ & $<5.0$ \\
\hline 41 & $<5.0$ & $<5.0$ & 20 & $<5.0$ & $<5.0$ & $<5.0$ & 140 & $<200$ & $<5.0$ & $<5.0$ \\
\hline 41 & $<5.0$ & $<5.0$ & 11 & $<5.0$ & $<5.0$ & $<5.0$ & 210 & $<200$ & $<5.0$ & $<5.0$ \\
\hline
\end{tabular}


Table 32. Benthic algae (periphyton) data for samples, Northern Rockies Intermontane Basins study unit, 1999-2001

[Abbreviations: $\mathrm{mg} / \mathrm{m}^{2}$, milligrams per square meter; $\mathrm{mg} / \mathrm{L}$, milligrams per liter; $\mathrm{g} / \mathrm{m}^{2}$, grams per square meter; $\mathrm{R}$, replicate. Symbol: <, less than reporting level]

\begin{tabular}{|c|c|c|c|c|c|}
\hline $\begin{array}{c}\text { Site } \\
\text { num- } \\
\text { ber } \\
\text { (fig.1) }\end{array}$ & Site name & Date & $\begin{array}{l}\text { Chloro- } \\
\text { phyll-a } \\
\left(\mathrm{mg} / \mathrm{m}^{2}\right)\end{array}$ & $\begin{array}{c}\text { Pheo- } \\
\text { phytin-a, } \\
\text { area- } \\
\text { weighted } \\
\text { average } \\
(\mathrm{mg} / \mathrm{L})\end{array}$ & $\begin{array}{c}\text { Biomass, } \\
\text { Peri- } \\
\text { phyton } \\
\text { ash } \\
\text { weight } \\
\left(\mathrm{g} / \mathrm{m}^{2}\right)\end{array}$ \\
\hline 3 & Clark Fork at Turah Bridge, near Bonner, Montana & $08 / 10 / 99$ & 4 & 2 & 5.3 \\
\hline 5 & Bitterroot River near Missoula, Montana & 08/11/99 & 25 & 15 & 14 \\
\hline 6 & St. Regis River above Rainy Creek, near Saltese, Montana & $08 / 23 / 00$ & 3 & 2 & 3 \\
\hline 7 & St. Regis River near Haugan, Montana & $08 / 23 / 00$ & 3 & 3 & 5 \\
\hline 8 & St. Regis River near St. Regis, Montana & $08 / 24 / 00$ & 4 & 2 & 5 \\
\hline 9 & Clark Fork at St. Regis, Montana & $08 / 24 / 99$ & 26 & 12 & 10 \\
\hline 12 & Flathead River at Perma, Montana & $08 / 25 / 99$ & 1 & 9 & 7 \\
\hline 14 & Lightning Creek at Clark Fork, Idaho & $08 / 02 / 99$ & $<1$ & 6 & 2 \\
\hline 15 & Priest River near Priest River, Idaho & $09 / 06 / 00$ & 13 & 4 & 9 \\
\hline 17 & North Fork Coeur d'Alene River above Shoshone Creek, near Prichard, Idaho & $09 / 11 / 00$ & 5 & 2 & 9 \\
\hline 18 & Prichard Creek near Murray, Idaho & $08 / 07 / 00$ & $<1$ & $<1$ & 2 \\
\hline 19 & East Fork Eagle Creek near mouth, near Prichard, Idaho & $08 / 09 / 00$ & 3 & 2 & 5 \\
\hline 20 & West Fork Eagle Creek below Settlers Grove, Idaho & $08 / 09 / 00$ & 17 & 4 & 10 \\
\hline 21 & Beaver Creek near mouth, near Murray, Idaho & $08 / 10 / 00$ & 1 & 1 & 4 \\
\hline 22 & Prichard Creek at mouth, at Prichard, Idaho & $08 / 08 / 00$ & 2 & $<1$ & 2 \\
\hline \multirow[t]{2}{*}{23} & North Fork Coeur d'Alene River at Enaville, Idaho & $07 / 14 / 99$ & 4 & 5 & 2 \\
\hline & & $09 / 12 / 00$ & 17 & 11 & 10 \\
\hline 24 & South Fork Coeur d'Alene River at Shoshone Park, near Mullan, Idaho & $08 / 22 / 00$ & 2 & 1 & 2 \\
\hline 26 & Canyon Creek near Burke, Idaho & $08 / 21 / 00$ & 4 & 2 & 4 \\
\hline 27 & Canyon Creek at Woodland Park, Idaho & $08 / 21 / 00$ & 9 & 4 & 8 \\
\hline 28 & South Fork Coeur d'Alene River at Silverton, Idaho & $08 / 22 / 00$ & 6 & 4 & 6 \\
\hline 29 & East Fork Pine Creek above Nabob Creek, near Pinehurst, Idaho & $08 / 10 / 00$ & $<1$ & $<1$ & 2 \\
\hline 30 & Pine Creek below Amy Gulch, near Pinehurst, Idaho & $08 / 10 / 00$ & 1 & 1 & 2 \\
\hline 31 & South Fork Coeur d'Alene River near Pinehurst, Idaho & $08 / 16 / 99$ & $<1$ & 6 & 2 \\
\hline $31^{\mathbf{R}}$ & & $08 / 16 / 99$ & $<1$ & 5 & 2 \\
\hline $31^{\mathbf{R}}$ & & $08 / 16 / 99$ & $<1$ & 6 & 4 \\
\hline 31 & & $09 / 12 / 00$ & 2 & 1 & 3 \\
\hline 31 & & $09 / 11 / 01$ & 24 & 8 & 11 \\
\hline 33 & St. Joe River at Red Ives Ranger Station, Idaho & $08 / 31 / 99$ & 4 & 8 & 5 \\
\hline $33^{\mathbf{R}}$ & & $08 / 31 / 99$ & 1 & 9 & 5 \\
\hline $33^{\mathbf{R}}$ & & $08 / 31 / 99$ & 2 & 4 & 5 \\
\hline 33 & & $09 / 13 / 00$ & 12 & 7 & 13 \\
\hline 33 & & $09 / 13 / 01$ & 46 & 17 & 17 \\
\hline 35 & Spokane River near Post Falls, Idaho & $07 / 26 / 99$ & 6 & $<1$ & 2 \\
\hline 35 & & $08 / 29 / 00$ & 15 & 4 & 12 \\
\hline 36 & Spokane River above Liberty Bridge, near Otis Orchard, Wash. & $07 / 27 / 99$ & 2 & .9 & 1 \\
\hline 37 & Spokane River at Greenacres, Wash. & $07 / 28 / 99$ & 2 & 3 & .4 \\
\hline 38 & Spokane River at Sullivan Bridge, near Trentwood, Wash. & $07 / 28 / 99$ & 4 & 6 & 4 \\
\hline 39 & Spokane River below Green Street, at Spokane, Wash. & $07 / 29 / 99$ & 10 & 5 & 6 \\
\hline 41 & Spokane River at Seven Mile Bridge, near Spokane Wash. & 07/30/99 & 94 & 5 & 25 \\
\hline
\end{tabular}

